HYDROLOGIC AND METEOROLOGIC DATA FOR THE BALD MOUNTAIN AREA

AROOSTOOK COUNTY, MAINE

JUNE 1979 THROUGH JUNE 1984

by Richard A. Fontaine

U.S. GEOLOGICAL SURVEY

Open-File Report 85-174

Prepared in cooperation with the

Maine Department of Environmental Protection

Augusta, Maine 


\author{
UNITED STATES DEPARTMENT OF THE INTERIOR \\ Donald Paul Hode1, Secretary \\ GEOLOGICAL SURVEY \\ Dallas L. Peck, Director
}

For additional information write to:

Chief, Maine Office

U.S. Geological Survey, WRD

26 Ganneston Drive

Augusta, Maine 04330
Copies of this report can be purchased from:

Open-File Services Section U.S. Geological Survey Box 25425, Federal Center Denver, Colorado 80225 


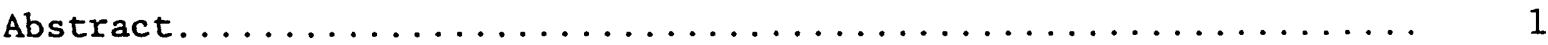

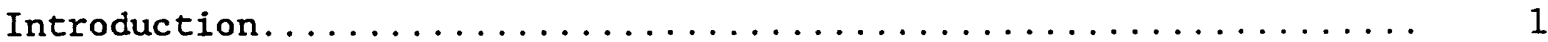

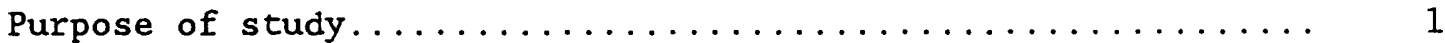

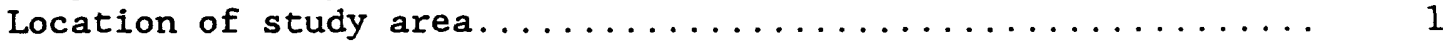

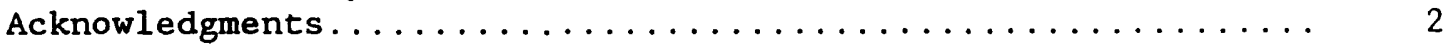

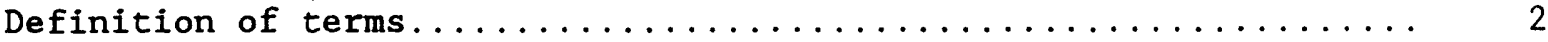

Downstream order, station number, and miscellaneous sites........ 6

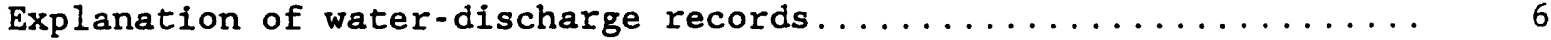

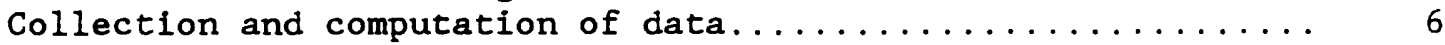

Accuracy of field data and computed results.............. 12

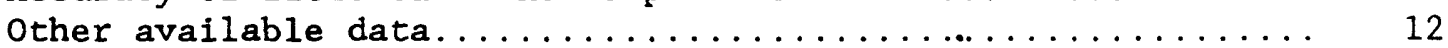

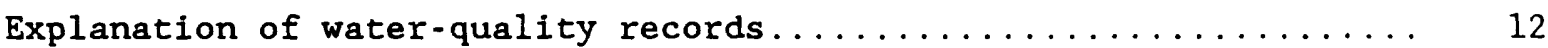

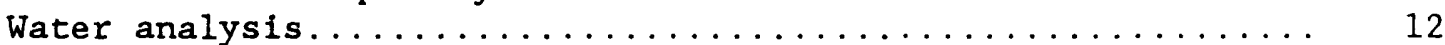

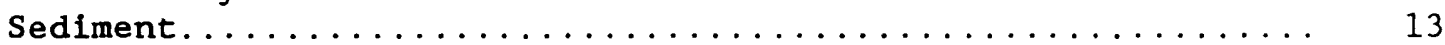

Explanation of meteorological records................. 14

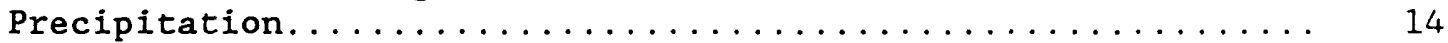

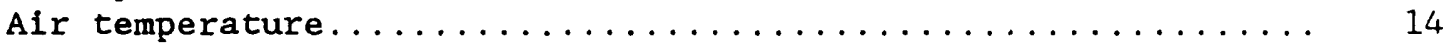

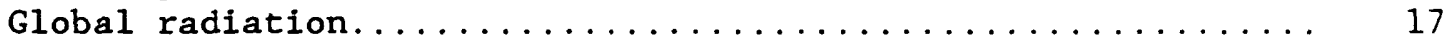

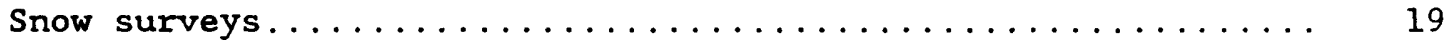

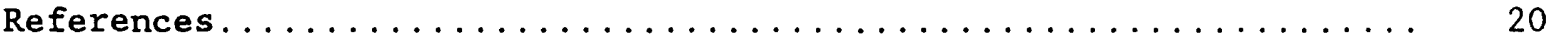

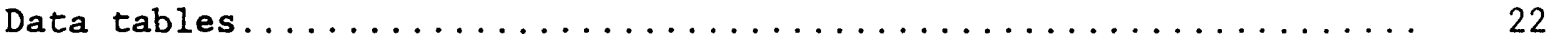

Figure 1. Map showing location of study area and data-collection sites in the Bald Mountain Area, Aroostook County,

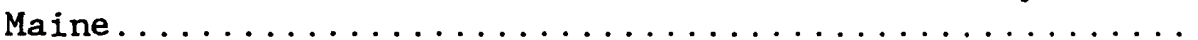

2. Graph showing discharge at gaging station 01012520 ,

Bald Mountain Brook near Bald Mountain, Maine,

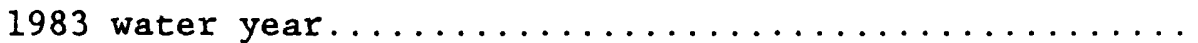

3. Graph showing monthly-total precipitation at rain gage 1 near Bald Mountain, Maine, 1983 water year.........

4. Graph showing monthly-mean air temperature at Bald Mountain, 1983 water year................ 
Table 1. Data-collection-site identifiers............... 7

2. Snow-survey site identifiers and characteristics....... 8

3. Daily-mean discharge at stream-gaging stations......... 23

4. Physical characteristics and concentrations of common chemical constituents in lakes and streams of the Bald Mountain watersheds................... 36

5. Summary of selected chemical and physical characteristics of water........................ 114

6. Suspended-sediment discharge for selected days in the Bald Mountain watersheds..................... 127

7. Daily-precipitation totals at continuous-recording gages.............................. 143

8. Storm periods from which unit precipitation data was

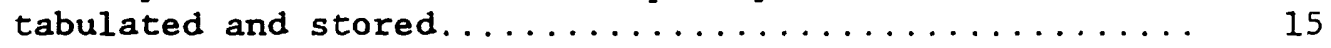

9. Daily air-temperature data in the Bald Mountain watersheds............................ 154 Daily global-radiation data in the Bald Mountain watersheds.............................. 162

11. Snow-survey data in the Bald Mountain watersheds....... 165 


\title{
HYDROLOGIC AND METEOROLOGIC DATA FOR THE BALD MOUNTAIN AREA, AROOSTOOK COUNTY, MAINE JUNE 1979 THROUGH JUNE 1984
}

\author{
by Richard A. Fontaine
}

\begin{abstract}
Hydrologic and meteorologic data were collected to assess the effects of a proposed open-pit copper mine in the Bald Mountain area of Maine. Data collected included streamflow and sediment discharge data at four locations, precipitation data at three locations, water-quality data at 14 locations, daily air-temperature data, global solar-radiation data, and snow survey data. The study was designed to provide background hydrologic information upstream from the basin to be mined, premining hydrologic conditions in the basin where excavation is planned, and premining hydrologic conditions downstream from the planned mining area. This study was a cooperative water-resources investigation between the Maine Department of Environmental Protection and U.S. Geological Survey. Data were collected from June 1979 through June 1984.
\end{abstract}

\section{INTRODUCTION}

Streamflow, water-quality, and meteorological data were collected as part of the Bald Mountain watershed-modeling study. These data were collected from 1979 through 1984 as part of a cooperative effort by the U.S. Geological Survey and Maine Department of Environmental Protection. The study was initiated in response to the discovery and planned mining of a large copper deposit in the western slopes of Bald Mountain. The deposit has been estimated to be 850 feet deep with a projected surface area of 22 acres. Plans call for the deposit to be developed as an open-pit mine.

\section{Purpose and Scope}

The purpose of the study was to describe watershed hydrology prior to any mining activity. A distributed parameter watershed-runoff model will be used to simulate surface-water flows. The model will be used to estimate impacts mining operations could have on the hydrology of surrounding basins. Water-quality data were collected and analyzed to evaluate background conditions in receiving streams.

Information in this report includes daily-mean stream and suspendedsediment discharges recorded at four locations in the study area. It includes daily precipitation totals recorded at three locations and waterquality determinations from samples taken at fourteen sampling sites. Also included are daily observations of air temperature and solar radiation as well as miscellaneous readings of snow depths and densities from thirteen locations.

\section{Location of Study Area}

Bald Mountain is located in Township 12 Range 8, Maine. The township is in central Aroostook County about 20 miles west of the towns of Portage and Ashland. Bald Mountain is located on the watershed divide in the headwaters of the Fish River and Machias River basins. The Fish River flows 
in a northerly direction from the Bald Mountain watersheds and joins the St. John River in Ft. Kent, Maine. The Machias River flows in an easterly direction from the Bald Mountain watersheds and joins the Aroostook River in Ashland, Maine. Figure 1 shows the locations of the Bald Mountain study area and data-collection sites.

\section{Acknowledgments}

Acknowledgment is made to Jeff Gammon and the entire staff of the Maine Department of Environmental Protection field office in Presque Isle for their invaluable assistance on the project. Special recognition is given to John Moulton and Carl Allen, who did most of the field work for the study often under adverse field conditions.

\section{DEFINITION OF TERMS}

Definition of terms related to streamflow, water quality, and other hydrologic data, as used in this report, are defined as follows:

Cfs-day is the volume of water represented by a flow of 1 cubic foot per second for 24 hours. It is equivalent to 86,400 cubic feet, about 646,000 gallons, or 2,447 cubic meters.

Control designates a feature downstream from the gage that determines the stage-discharge relation at the gage. This feature may be a natural constriction of the channel, an artificial structure, or a uniform cross section over a long reach of the channel.

Cubic foot per second ( $\mathrm{ft}^{3} / \mathrm{s}$; cfs in the water-quality tables) is the rate of discharge representing a volume of 1 cubic foot passing a given point during 1 second and is equivalent to 7.48 gallons per second or 448.8 gallons per minute or 0.2832 cubic meters per second.

Cubic foot per second per square $\mathrm{mile}\left[\left(\mathrm{ft}^{3} / \mathrm{s}\right) / \mathrm{mi}^{2}\right]$ is the average number of cubic feet of water flowing per second from each square mile of area drained, assuming that the runoff is distributed uniformly in time and area.

Discharge is the volume of water (or, more broadly, volume of fluid plus suspended sediment) that passes a given point within a given period of time.

Mean discharge (MEAN) is the arithmetic mean of individual daily mean discharges during a specific period.

Instantaneous discharge or streamflow is the discharge at a particular instant of time.

Dissolved refers to that material in a representative water sample which passed through a 0.45 -micrometer membrane filter. This is a convenient operational definition used by Federal agencies that collect water data. Determinations of "dissolved" constituents are made on subsamples of filtrate. 


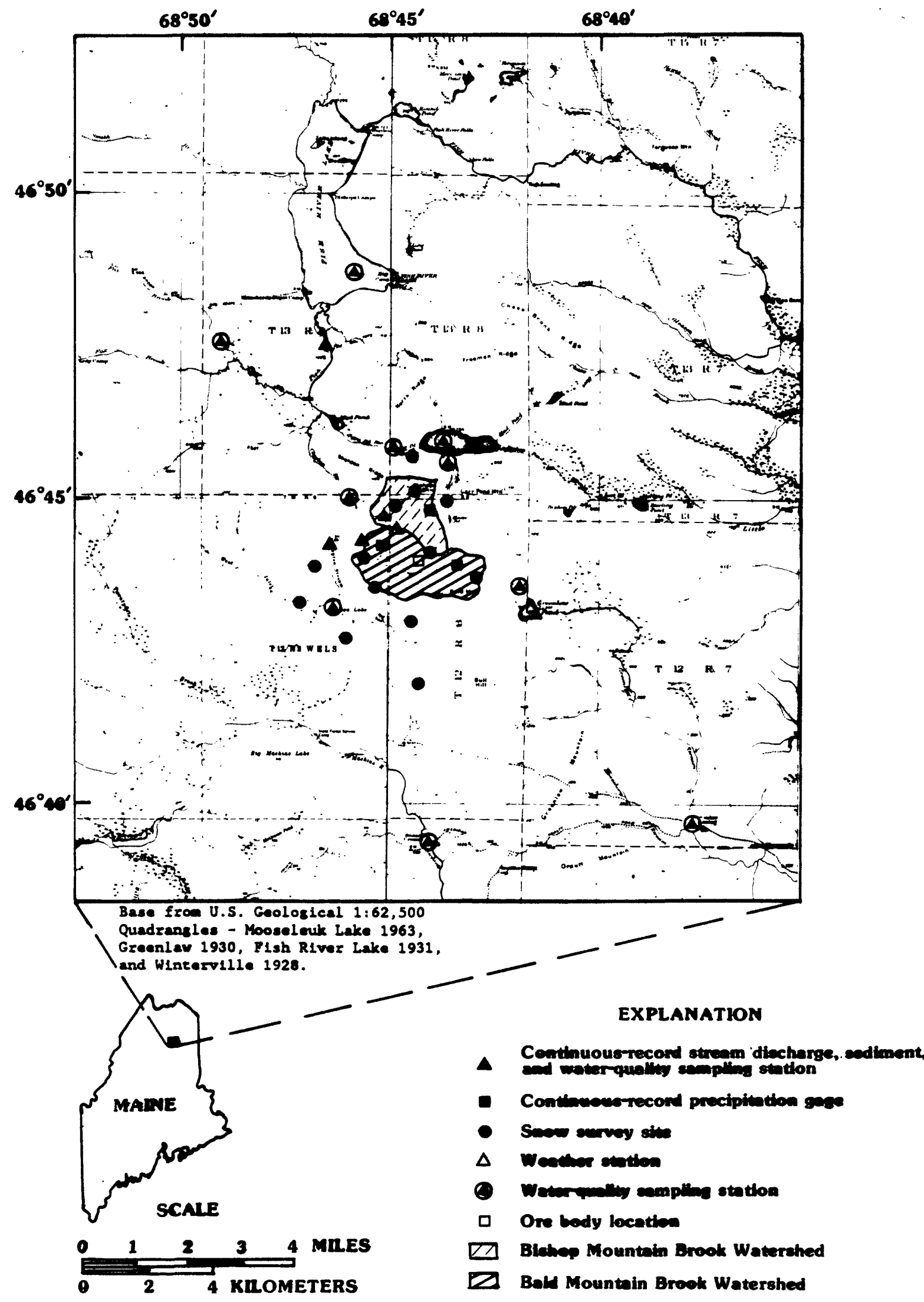

Figure 1.--Location of study area and data-collection sites in the Bald Mountain watersheds, Aroostook County, Maine. 
Drainage area of a stream at a specific location is that area, measured in a horizontal plane, enclosed by a topographic divide from which direct surface runoff from precipitation normally drains by gravity into the stream above the specified point. Figures of drainage area given herein include all closed basins, or noncontributing areas, within the area unless otherwise noted.

Drainage basin is a part of the surface of the earth that is occupied by a drainage system, which consists of a surface stream or a body of impounded surface water together with all tributary surface streams and bodies of impounded surface water.

Gage height is the water-surface elevation referred to some arbitrary gage datum. Gage height is often used interchangeably with the general term "stage", although gage height is more appropriate when used with a reading on a gage.

Gaging station is a particular site on a stream, lake, or reservoir where systematic observations of hydrologic data are obtained.

Langley is a unit of solar radiation equivalent to one gram calorie per square centimeter of irradiated surface.

Micrograms per liter $(\mu \mathrm{g} / \mathrm{L})$ is a unit expressing the concentration of chemical constituents in a sample as the mass (micrograms) of constituent per unit volume (liter) of sample. One thousand micrograms per liter is equivalent to one milligram per liter.

Milligrams per liter $(\mathrm{mg} / \mathrm{L})$ is a unit for expressing the concentration of chemical constituents in a sample. Milligrams per liter represents the mass of constituent per unit volume of sample.

National Geodetic Vertical Datum of 1929 (NGVD) is a geodetic datum derived from a general adjustment of the first order level nets of both the United States and Canada. It was formerly called "Sea Level Datum of 1929" or "mean sea level" in this series of reports. Although the datum was derived from the average sea level over a period of many years at 26 tide stations along the Atlantic, Gulf of Mexico, and Pacific Coasts, it does not necessarily represent local mean sea level at any particular place.

pH is a symbol denoting the negative logarithm (base 10) of the hydrogen ion concentration of a solution; $\mathrm{pH}$ values range from 0 to 14 --the lower the value, the more acid is the solution; i.e., the more hydrogen ions it contains.

Runoff in inches (in) shows the depth to which the drainage area would be covered if all the runoff for a given time period were uniformly distributed on it. 
Sediment is solid material that originates mostly from disintegrated rocks and is transported by, suspended in, or deposited from water; it includes chemical and biochemical precipitates and decomposed organic material such as humus. The quantity, characteristics, and cause of the occurrence of sediment in streams are influenced by environmental factors. Some major factors are degree of slope, length of slope, soil characteristics, land usage, and quantity and intensity of precipitation.

Suspended sediment is the sediment that at any given time is maintained in suspension by the upward components of turbulent currents or that exists in suspension as a colloid.

Specific conductance is a measure of the ability of a water to conduct an electrical current and is expressed in microsiemens per centimeter at $25^{\circ} \mathrm{C}\left(\mu \mathrm{S} / \mathrm{cm}\right.$ at $\left.25^{\circ} \mathrm{C}\right)$. Specific conductance is related to the type and concentration of ions in solution and can be used for estimating the dissolved-solids content of the water. Commonly, concentration of dissolved solids (in milligrams per liter) is about 65 percent of specific conductance (in $\mu \mathrm{S} / \mathrm{cm}$ at $25^{\circ} \mathrm{C}$ ). This relation is not constant from stream to stream or from well to well, and it may even vary in the same source with changes in composition of the water.

Stage-discharge relation is the relation between gage height (stage) and the volume of water per unit of time flowing in a channel.

Streamflow is the discharge that occurs in a natural channel. Although the term "discharge" can be applied to flow of a canal, the word "streamflow" uniquely describes discharge in a surface stream course. The term "streamflow" is more general than "runoff" as streamflow may be applied to discharge whether or not it is affected by diversion or regulation.

Tons per day is the quantity of a substance in solution or suspension that passes a stream section during a 24-hour day.

Total (as used in tables of chemical analysis):

Total, recoverable is the amount of a given constituent in solution after a representative water-suspended sediment sample has been digested by a method (usually using a dilute acid solution) that results in dissolution of only readily soluble substances. Complete dissolution of all particulate matter is not achieved by digestion treatment, and thus the determination represents something less than the "total" amount (that is, less than 95 percent) of the constituent present in the dissolved and suspended phases of the sample. To achieve comparability of analytical data, equivalent digestion procedures would be required of all laboratories performing such analyses because different digestion procedures are likely to produce different analytical results. 
Total is the total amount of a given constituent in a representative water-suspended sediment sample, regardless of the constituent's physical or chemical form. The term is used only when analytical procedure assures measurement of at least 95 percent of the constituent present in both the dissolved and suspended phases of the sample. Knowledge of the expected form of the constituent in the sample, as well as analytical methodology used, is required to judge when results should be reported as "total" (Note that the word "total" does double duty here, indicating the sample consists of a water-suspended sediment mixture and the analytical method determines all the constituent in the sample).

Turbidity is the opaqueness or reduced clarity of a fluid due to the presence of suspended matter.

Water year is the 12 -month period that begins with October 1 and ends with September 30 .

\section{DOWNSTREAY ORDER, STATION NUMBER,' AND MISCELIANEOUS SITES}

Since October 1, 1950, the order of listing hydrologic-station records in Survey reports is in a downstream direction along the main stream. All stations on a tributary entering upstream from a main-stream station are listed before that station. A station on a tributary that enters between two main-stream stations is listed between them.

As an added means of identification, each hydrologic station has been assigned a station number. These are in the same downstream order used in this report. Gaps are left in the series of numbers to allow for new stations that may be established; hence, the numbers are not consecutive. The complete eight-digit number for each station, such as 01012520, which appears just to the left of the station name, includes the two-digit part number "01" plus the six-digit downstream-order number "012520".

In this report, sampling sites on lakes, rain gage locations and snow sampling sites are identified by unique 13 digit numbers. The first six digits denote degrees, minutes, and seconds of latitude, the next seven digits denote degrees, minutes, and seconds of longitude.

A summary of data collection site identifiers for the streamgages, rain gages, water quality sampling sites, and the weather station operated as part of this study can be found in table 1 . Snow survey site identifiers and characteristics are summarized in table 2 .

\section{EXPLANATION OF WATER-DISCHARGE RECORDS}

\section{Collection and Computation of Data}

The base data collected at gaging stations consists of records of stage and measurements of discharge of streams and stage. In addtion, observations of factors affecting the stage-discharge relation, weather records, and other information are used to supplement base data in determining the daily flow. Records of stage are obtained from a waterstage recorder that gives a tape punched at selected time intervals. Streamgages on the Fish River and Clayton Stream recorded stages at 15minute intervals. Streamgages on Bald Mountain Brook and Bishop Mountain Brooks recorded stages at 5-minute intervals. The Clayton Stream gage was 
Table 1.--Data-collection-site identifiers.

\begin{tabular}{|c|c|c|}
\hline $\begin{array}{l}\text { Site } \\
\text { number }\end{array}$ & \multicolumn{2}{|l|}{$\begin{array}{l}\text { USGS station } \\
\text { identification } \\
\text { number }\end{array}$} \\
\hline & \multicolumn{2}{|r|}{ STREAM GAGES } \\
\hline $\begin{array}{l}1 \\
2 \\
3 \\
4\end{array}$ & $\begin{array}{l}01012515 \\
01012520 \\
01012525 \\
01012570\end{array}$ & $\begin{array}{l}\text { Clayton Stream at Outlet Clayton Lake } \\
\text { Bald Mountain Brook near Bald Mountain } \\
\text { Bishop Mountain Brook near Bishop Mountain } \\
\text { Fish River Lake at Inlet Fish River Lake } \\
\text { RAIN GAGES }\end{array}$ \\
\hline $\begin{array}{l}2 \\
3\end{array}$ & $\begin{array}{l}01012520 \\
4643500684414 \\
4644350684416\end{array}$ & $\begin{array}{l}\text { Rain gage } 1 \text { at Bald Mountain Brook near } \\
\text { Bald Mountain } \\
\text { Rain gage } 2 \text { at Bald Mountain } \\
\text { Rain gage } 3 \text { at Bishop Mountain }\end{array}$ \\
\hline & \multicolumn{2}{|c|}{ WATER QUALITY SAMPLING SITES } \\
\hline $\begin{array}{r}1 \\
2 \\
3 \\
4 \\
5 \\
6 \\
7 \\
8 \\
9 \\
10 \\
11 \\
12 \\
13 \\
14\end{array}$ & $\begin{array}{l}4648430684552 \\
01012520 \\
01012560 \\
01012570 \\
01012545 \\
01012515 \\
01016480 \\
01016410 \\
4645530684301 \\
01012530 \\
01012550 \\
01016460 \\
4643080684611 \\
01012525\end{array}$ & $\begin{array}{l}\text { Fish River Lake in Township } 13 \text { Range } 8 \\
\text { Bald Mountain Brook near Bald Mountain } \\
\text { North Branch Fox Brook near Fish River Lake } \\
\text { Fish River at Inlet Fish River Lake } \\
\text { Moose Pond Stream at Inlet Carr Pond } \\
\text { Clayton Stream at Outlet Clayton Lake } \\
\text { Greenlaw Stream at Greenlaw Crossing } \\
\text { Machias River at Russell Crossing } \\
\text { Carr Pond in Township } 13 \text { Range } 8 \\
\text { Clayton Stream at Sheldon Ridge } \\
\text { Carr Pond Stream at Outlet Carr Pond } \\
\text { Greenlaw Stream at Inlet Greenlaw Pond } \\
\text { Clayton Lake in Township } 12 \text { Range } 8 \\
\text { Bishop Mountain Brook near Bishop Mountain }\end{array}$ \\
\hline \multicolumn{3}{|c|}{ WEATHER STATION } \\
\hline 1 & 01012525 & Bishop Mountain Brook near Bishop Mountain \\
\hline
\end{tabular}


Table 2.--Snow-survey-site identifiers and characteristics

\begin{tabular}{|c|c|c|c|c|c|}
\hline $\begin{array}{l}\text { Site } \\
\text { number }\end{array}$ & $\begin{array}{l}\text { USGS } \\
\text { station } \\
\text { number }\end{array}$ & $\begin{array}{l}\text { Elevation } \\
\text { (feet NGVD) }\end{array}$ & Aspect & Slope & $\begin{array}{l}\text { Degree of } \\
\text { ground cover }\end{array}$ \\
\hline 1 & 4645440684414 & 800 & norther1y & $\operatorname{mild}$ & heavy \\
\hline 2 & 4644480684407 & 1150 & southwesterly & steep & moderate \\
\hline 3 & 4644450684510 & 870 & level ground & level ground & clear \\
\hline 4 & 4644400684354 & 1130 & level ground & leve1 ground & moderate \\
\hline 5 & 4644280684524 & 830 & level ground & level ground & clear \\
\hline 6 & 4644040684426 & 1040 & southwesterly & $\operatorname{mild}$ & moderate \\
\hline 7 & 4644500684523 & 950 & northeasterly & $\operatorname{mild}$ & moderate \\
\hline 8 & 4643480684325 & 1150 & northwesterly & steep & heavy \\
\hline 9 & 4641470684409 & 1020 & westerly & $\operatorname{mild}$ & moderate \\
\hline 10 & 4642400684600 & 1020 & northerly & $\operatorname{mild}$ & moderate \\
\hline 11 & 4643450684633 & 940 & easterly & $\operatorname{mild}$ & moderate \\
\hline 12 & 4643200684650 & 940 & southerly & $\operatorname{mild}$ & moderate \\
\hline 13 & 4642430684411 & 890 & level ground & level ground & heavy \\
\hline
\end{tabular}


located just upstream from the area to be impacted by the potential mine site. The Bald and Bishop gages are located on tributary streams that drain the mine site. The Fish River gage is located downstream from the study area. Measurements of discharge are made with a current meter, using the general methods adopted by the Geological Survey. These methods are described in standard textbooks, in Rantz and others (1982), and in Buchanan and Somers (1969).

For stream-gaging stations, rating tables giving the discharge for any stage are prepared from stage-discharge relation curves. If extensions to the rating curves are necessary to express discharge greater than measured, they are made on the basis of indirect measurements of peak discharge (such as slope-area or contracted-opening measurements, computation of flow over dams or weirs) and logarithmic plotting. The daily mean discharge is computed from gage heights and rating tables; then the monthly and yearly mean discharges are computed from the daily figures.

If the stage-discharge relation is subject to variability because of frequent or continual change in the physical features that form the control, the daily mean discharge is computed by the shifting-control method. In the shifting-control method correction factors, based on indiviual discharge measurements and notes by hydrologists and observers, are used in applying the gage heights to the rating tables. If the stage-discharge relation for a station is temporarily changed by the presence of aquatic growth or debris on the control, the daily mean discharge is computed by what is basically the shifting-control method.

At some stream-gaging stations the stage-discharge relation is affected by ice during the winter, and it becomes impossible to compute the discharge in the usual manner. Discharge for periods of ice effect is computed on the basis of the gage-height record and occasional winter discharge

measurements. Consideration is given to the available information on temperature and precipitation, notes by hydrologist, and comparable records of discharge for other stations in the same or nearby basins.

For some gaging stations, there are periods when no gage-height record is obtained or the recorded gage height is so faulty that it cannot be used to compute daily discharges. This happens when the recorder stops or otherwise fails to operate properly, intakes are plugged, the float is frozen in the well, or for various other reasons. For such periods, the daily discharges are estimated on the basis of recorded range in stage, prior and subsequent records, discharge measurements, weather records, and comparison with records for other stations in the same or nearby basins.

The data in this report generally comprise a description of the gaging station and tabulations of daily, monthly and yearly discharge. These data are presented in table 3 at the end of the report. Records are published by the water year, which begins on October 1 and ends on September 30. Figure 2 is an example of a typical discharge hydrograph for a gage in the Bald Mountain watershed for a water year. Discharge data in this report are also published in the annual series of reports, Water Resources Data--Maine (U.S. Geological Survey 1981-84). 


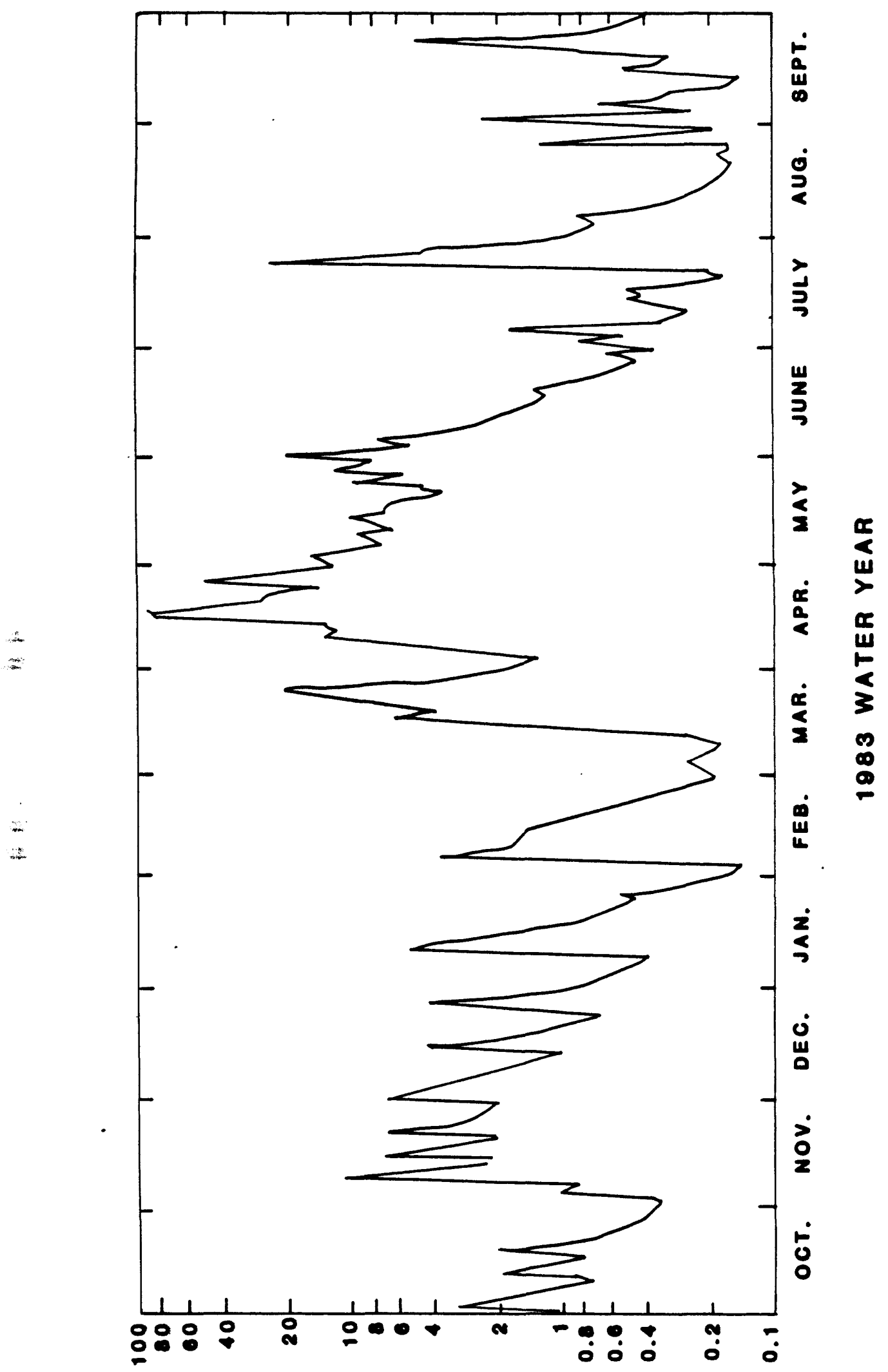

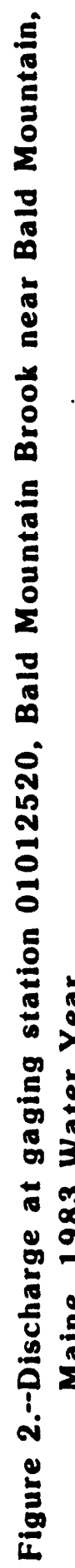

aNOOJS Y 
The description of the gaging station gives the location, draingage area, period of record, notations of revisions of previously published records, type and history of gages, general remarks, average discharge, and extremes of discharge. The location of the gaging station and the drainage area are obtained from the most accurate maps available. Periods for which there are published records for the present station or for stations generally equivalent to the present one are given under "PERIOD OF RECORD". The type of gage currently in use and datum of the present gage determinated from topographic maps are given under "GAGE". Information pertaining to the accuracy of the discharge records and to conditions that affect the natural flow at the gaging station is given under "REMARKS".

The following information is given under "EXTREMES": First, the extremes for the period of record, second, information available outside the period of record, and last, those for the current year. Unless otherwise qualified, the maximum discharge is the instantaneous maximum corresponding to the crest stage obtained by use of a water-stage recorder. If the maximum gage height did not occur on the same day as the maximum discharge it is given separately. Similarly, the minimum is the instantaneous minimum unless otherwise qualified.

For some stations, independent peak discharges above a selected base, including the maximum for the year, are listed with EXTREMES FOR THE CURRENT YEAR; with the time of occurence and corresponding gage heights are published in tabular format. The base discharge, which is given in the table heading, is selected so that an average of about three peaks a year will be presented. Time of day is expressed in 24-hour local standard time; for example, 12:30 a.m. is expressed 0030, 1:30 p.m. is 1330. The minimums for these stations are published in a separate paragraph following the table of peaks.

The daily table for stream-gaging stations gives the mean discharge for each day and is followed by monthly and yearly summaries. In the monthly summary below the daily table, the line headed "TOTAL" gives the sum of the daily figures. The line headed "MEAN" gives the average flow in cubic feet per second during the month. The lines headed "MAX" and "MIN" give the maximum and minimum daily discharges, respectively, for the month. Discharge for the month is also expressed in cubic feet per second per square mile (line headed "CFSM") and in inches (line headed "IN"). In the yearly summary below the monthly summary, the figures shown are the appropriate discharges for which the calendar and water years.

Attention is called to periods for which the discharge is computed or estimated by special methods because of no gage-height records, backwater from various sources, or other unusual conditions. Periods of no gageheight record are indicated if the period is continuous for a month or more or includes the maximum discharge for the year. Periods of backwater from an unusual source, or indefinite stage-discharge relation, or of any unusual condition at the gage site are indicated only if they are a month or more in length and the accuracy of the records is affected. 
Days on which the stage-discharge relation is affected by ice are not indicated. Methods used in computing discharge for various unusual conditions have been explained in preceding paragraphs.

\section{Accuracy of Field Data and Computed Results}

The accuracy of streamflow data depends primarily on (1) the stability of the stage-discharge relation or, if the control is unstable, the frequency of discharge measurements, and (2) the accuracy of observations of stage, measurements of discharge, and interpretations of records.

The station description under "REMARKS" states the degree of accuracy of the records. "EXCELLENT" means that about 95 percent of the daily discharges are within 5 percent of true value; "GOOD", within 10 percent; and "FAIR", with 15 percent. "POOR" means that daily discharges have less than "FAIR" accuracy.

Figures of daily mean discharge in this report are shown to the nearest hundredth of a cubic foot per second for discharges of less than $1 \mathrm{ft}^{3} / \mathrm{s}$; to tenths between 1.0 and $10 \mathrm{ft} / \mathrm{s}$; to whole numbers between 10 and 1,000 $\mathrm{ft}^{3} / \mathrm{s}$; and to 3 significant figures above $1,000 \mathrm{ft}^{3} / \mathrm{s}$. The number of significant figures used is based solely on the magnitude of the figure.

\section{Other Available Data}

Information of a more detailed nature than that published for most of the gaging stations such as discharge measurements, gage-height records, and rating tables is on file in the Maine state office. Also, most gagingstation records are available in computer-usable form and many statistical analyses have been made.

\section{EXPLANATION OF WATER-QUALITY RECORDS}

\section{Water Analysis}

Most methods for collecting and analyzing water samples are described in Rainwater and Thatcher (1960), Barnett and Mallory (1971), Goerlitz and Brown (1972), Stevens and others (1975), and Skougstad and others (1979). Study and interpretation of water quality data is summarized by Hem (1970).

One sample can define adequately the water quality at a given time if the mixture of solutes throughout the stream cross section is homogenous. However, the concentration of solutes at different locations in the cross section may vary widely with different rates of water discharge, depending on the source of material and the turbulence and mixing of the stream. Some streams must be sampled through several vertical sections to obtain a representative sample needed for an accurate mean concentration and for use in calculating load. 
Chemical-quality data published in this report are considered to be the most representative values available for the stations listed in table 1. The values reported represent water-quality conditions at the time sampling as much as possible, consistent with available sampling techniques and methods of analysis. Results of 502 samples from which a variety of physical characteristics and chemical constituents were observed are listed in table 4. A summary of these data for the 14 water-quality sampling sites is given in table 5. Included in the summary for each sampling site are the number of analyses, mean value and range for the most significant physical characteristics and chemical constituents. The mean value given for the characteristic $\mathrm{pH}$ is simply the mean value of the actual $\mathrm{pH}$ readings. Tables 4 and 5 are at the end of the report.

Water quality sampling sites were established to provide background data in watersheds that could potentially be impacted by a mining operation and also in basins that could serve as control or nonimpacted sites for purposes of trend identification.

\section{Sediment}

Daily mean suspended sediment concentrations and sediment discharges were computed for selected days at the four 6 tream gages. The sediment data are presented in table 6 at the end of the report.

Sediment samples were obtained at fixed points in stream cross-sections using automatic, pumping-type samplers. Coefficients were applied to the point samples to determine the mean concentration in the cross section. Coefficients were based on the correlation between point samples and samples collected using depth-integrating samplers at several verticals in the cross-section.

During periods of rapidly changing flow or rapidly changing concentration, samples may have been collected more frequently. The published sediment discharges for days of rapidly changing flow or concentration were computed by the subdivided day method (time-discharge weighted average). Therefore, for those days when the published sediment discharge value differs from the value computed as the product of discharge times mean concentration times 0.0027 , the reader can assume that the sediment discharge for that day was computed by the subdivided day method. These methods are described in greater detail by Guy (1970), Guy and Norman (1970) and Porterfield (1972).

For some periods when no samples were collected at the streamgages, daily loads of suspended sediment were estimated on the basis of water discharge, sediment concentrations observed immediately before and after the periods and suspended-sediment loads for other periods of similar discharge. These techniques are described in the previously listed references as well as by Colby (1956) and Guy (1964). 
Suspended-sediment samples were collected periodically at the 14 waterquality sampling stations. Although data collected periodically may represent conditions only at the time of observations, such data are useful in establishing seasonal relations between quality and streamflow in predicting long-term sediment-discharge characteristics of the stream. These data can be found in table 4 at the end of the report.

\section{EXPLANATION OF METEOROLOGIC RECORDS}

\section{Precipitation}

Three continuous-recording weighing-bucket type rain gages capable of recording to the nearest hundredth of an inch, were operated as part of this study. The gages were all equipped with shelter-type windshields and were operated throughout the year. The sites were selected to provide accurate temperal and spacial sampling of ralnfall in the Bald and Bishop basins. The gages were sited and operated according to the guidelines published by the U.S. Geological Survey (1977), Fisher and Hardy (1976), and Brakensiek and others (1979). These same publications served as the guides by which all the meteorological records noted in this report were obtained.

The gages are equipped with chart type recorders that provide a continuous pen trace of accumulated precipitation. During winter months, when precipitation is predominately in a solid form, the charts were geared such that data could be broken down into 3 hour time intervals. During the spring, summer and fall months the charts were geared such that data could be broken down into 15 minute time intervals. In this report, daily and monthly precipitation totals are given in table 7 , at the end of the report. For the selected storm periods listed in table 8 precipitation totals in 15 minute intervals were tabulated and stored in the U.S. Geological Survey's National Water data Storage and Retrieval System (WATSTORE) computer files. A graphical representation of the variation in monthly total precipitation for the 1983 water year is given in figure 3 .

To ensure accurate recording of precipitation totals the raingages were routinely calibrated following the manufacturer's recommended procedures. As an additional quality control step data recorded at the three gages was compared to ensure that no major recording errors had taken place.

\section{Air Temperature}

A continuous recording thermograph was also operated in the Bald Mountain watersheds. The thermograph was of the bimetallic type and was operated in a shelter similar to that used by the National Weather Service. Specifically the shelter had a solid top and louvered sides designed to protect the thermograph from precipitation and radiation. Louvered construction permits air to circulate around the instruments thus exposing them to the temperature of the surrounding air while reducing adverse temperature effects caused by radiation. 
Table 8.--Storm periods from which unit precipitation data was tabulated and stored

\begin{tabular}{|c|c|c|c|c|}
\hline Rain & n gage 1 & Rain & gage 2 & Rain gage 3 \\
\hline $\begin{array}{l}10-18 \text { tc } \\
10-22 \text { t } \\
11-17 t \\
06-02 \text { tc } \\
08-24 t\end{array}$ & $\begin{array}{l}\text { to } 10-21-81 \\
\text { to } 10-25-81 \\
\text { to } 11-23-81 \\
\text { to } 06-04-82 \\
\text { to } 08-26-82\end{array}$ & $\begin{array}{l}10-18 \text { tc } \\
10-22 \text { tc } \\
11-17 \text { tc } \\
06-02 \text { tc } \\
08-24 \text { tc }\end{array}$ & $\begin{array}{ll}=0 & 10-21-81 \\
=0 & 10-25-81 \\
=0 & 11-23-81 \\
=0 & 06-04-82 \\
=0 & 08-26-82\end{array}$ & $\begin{array}{lll}08-25 & \text { to } & 08-27-82 \\
09-02 & \text { to } & 09-05-82 \\
09-06 & \text { to } & 09-08-82 \\
09-14 & \text { to } & 09-18-82 \\
09-27 & \text { to } & 09-30-82\end{array}$ \\
\hline $\begin{array}{l}09-06 t \\
09-14 t \\
09-27 t \\
10-01 t \\
10-08 t\end{array}$ & $\begin{array}{l}\text { to } 09-08-82 \\
\text { to } 09-18-82 \\
\text { to } 09-29-82 \\
\text { to } 10-03-82 \\
\text { to } 10-10-82\end{array}$ & $\begin{array}{l}09-06 \text { tc } \\
09-14 \text { tc } \\
09-27 \text { tc } \\
10-01 \text { tc } \\
10-08 \text { tc }\end{array}$ & $\begin{array}{ll}=0 & 09-08-82 \\
=0 & 09-18-82 \\
=0 & 09-29-82 \\
=0 & 10-03-82 \\
=0 & 10-10-82\end{array}$ & $\begin{array}{l}10-01 \text { to } 10-04-82 \\
10-08 \text { to } 10-11-82 \\
10-14 \text { to } 10-16-82 \\
11-02 \text { to } 11-04-82 \\
11-05 \text { to } 11-07-82\end{array}$ \\
\hline $\begin{array}{l}10-13 t \\
11-02 t \\
11-05 t \\
11-13 t \\
11-21 t\end{array}$ & $\begin{array}{l}\text { to } 10-16-82 \\
\text { to } 11-04-82 \\
\text { to } 11-07-82 \\
\text { to } 11-15-82 \\
\text { to } 11-25-82\end{array}$ & $\begin{array}{l}10-13 \text { tc } \\
11-02 \text { tc } \\
11-05 \text { tc } \\
11-13 \text { tc } \\
11-21 \text { tc }\end{array}$ & $\begin{array}{ll}\text { co } & 10-16-82 \\
\text { co } & 11-04-82 \\
\text { co } & 11-07-82 \\
\text { =o } & 11-15-82 \\
\text { =o } & 11-25-82\end{array}$ & $\begin{array}{lll}11-13 & \text { to } 11-15-82 \\
11-21 & \text { to } & 11-25-82 \\
05-23 & \text { to } & 05-26-83 \\
05-27 & \text { to } & 05-29-83 \\
05-30 & \text { to } & 06-01-83\end{array}$ \\
\hline $\begin{array}{l}04-24 t \\
05-23 t \\
05-27 t \\
05-30 t \\
07-21 t\end{array}$ & $\begin{array}{l}\text { to } 04-28-83 \\
\text { to } 05-26-83 \\
\text { to } 05-29-83 \\
\text { to } 06-03-83 \\
\text { to } 07-24-83\end{array}$ & $\begin{array}{l}04-24 t \\
05-23 t \\
05-27 t \\
05-30 t \\
07-21 t c\end{array}$ & $\begin{array}{ll}\text { =o } & 04-28-83 \\
\text { =o } & 05-26-83 \\
\text { co } & 05-29-83 \\
\text { =o } & 06-03-83 \\
\text { co } & 07-24-83\end{array}$ & $\begin{array}{lll}06-06 & \text { to } 06-08-83 \\
06-14 & \text { to } 06-16-83 \\
07-21 & \text { to } 07-23-83 \\
08-27 & \text { to } 08-28-83 \\
08-31 \text { to } 09-02-83\end{array}$ \\
\hline $\begin{array}{ll}08-27 & t \\
08-31 & t \\
09-22 & t \\
11-03 & t \\
11-10 & t \\
11-24 & t\end{array}$ & $\begin{array}{ll}\text { to } & 08-28-83 \\
\text { to } & 09-02-83 \\
\text { to } & 09-24-83 \\
\text { to } & 11-09-83 \\
\text { to } 11-15-83 \\
\text { to } 11-28-83\end{array}$ & $\begin{array}{l}08-27 \text { tc } \\
08-31 \text { tc } \\
09-22 \text { tc } \\
11-03 \text { tc } \\
11-10 \text { tc } \\
11-24 \text { tc }\end{array}$ & $\begin{array}{ll}\text { =0 } & 08-28-83 \\
\text { =0 } & 09-02-83 \\
\text { =0 } & 09-24-83 \\
\text { =0 } & 11-09-83 \\
\text { =0 } & 11-15-83 \\
\text { =0 } & 11-28-83\end{array}$ & $\begin{array}{l}11-03 \text { to } 11-09-83 \\
11-10 \text { to } 11-15-83 \\
11-24 \text { to } 11-28-83\end{array}$ \\
\hline
\end{tabular}




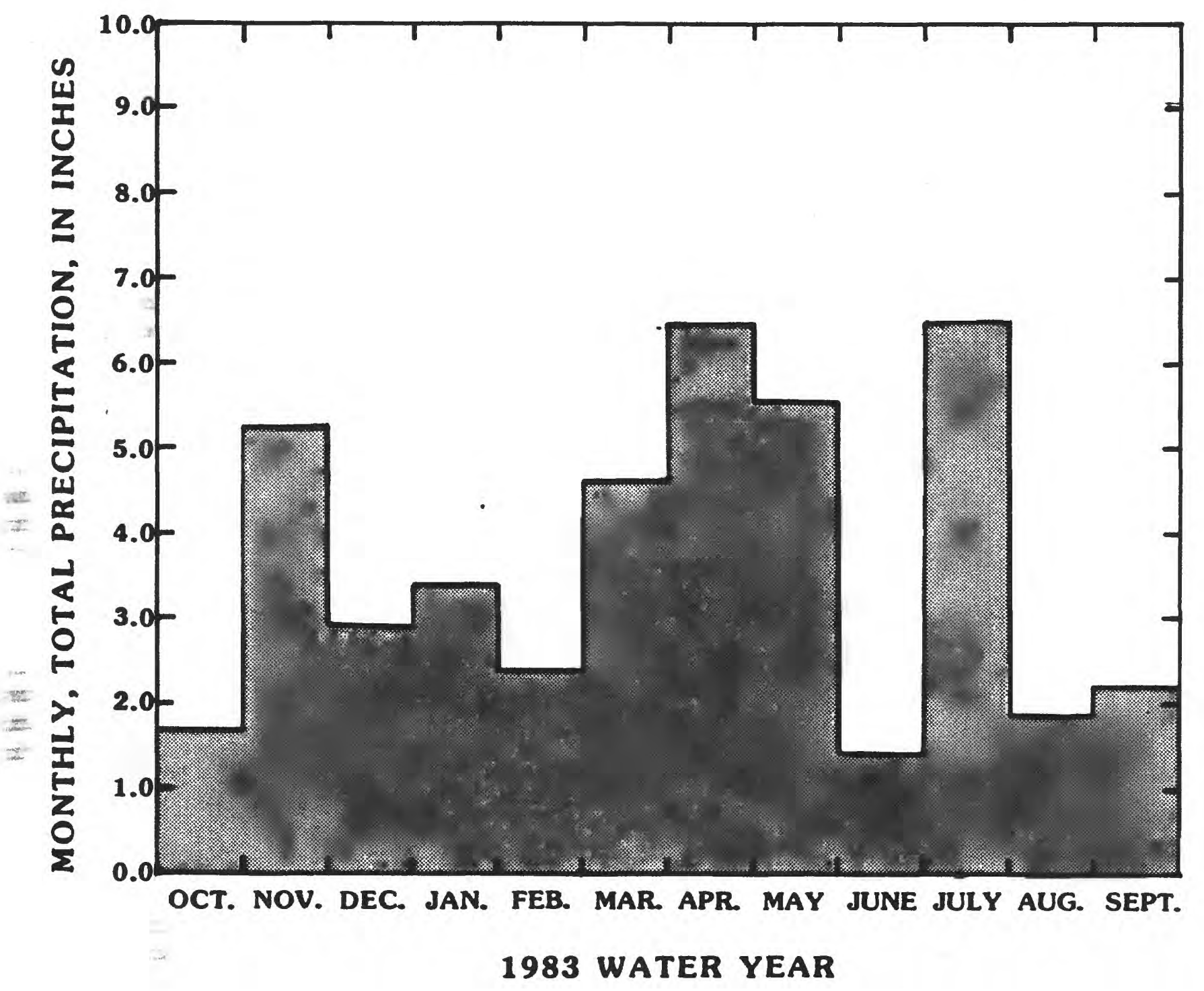

Figure 3.--Monthly total precipitation at rain gage 1 near Bald Mountain, Maine, 1983 water year. 
In a bimetallic thermometer the sensing element is a thin metallic bar that is fixed at one end and free on the other. The bar is made from two different metals that have dissimilar coefficients of expansion. When the temperature of the bar changes, the two metals expand or contract by differing amounts. The differences in the expansion or contraction of the metals creates movements in the free end of the sensing bar that is transferred to a recording pen.

Daily maximum and minimum air temperatures to the nearest degree, (Celsius) were picked off the continuous pen trace made by the recorder. The daily maximum and minimum were averaged to determine the daily mean temperature. These data are presented in table 9. Also included in the table are ranges and mean values, by months, for daily maximum, minimum, and mean air temperatures. A graphical representation of the variation in monthly mean air temperature for the 1983 water year is given in figure 4 .

To ensure accurate recording of air temperatures the recorder was routinely checked for accuracy against calibrated thermometers and recalibrated as required. As an additional quality control measure, the recorded temperatures were compared to surrounding National Weather Service Station data to detect gross recording errors.

\section{Global Radiation}

Global or the total downward direct and diffuse solar radiation as received on a horizontal surface was continuously recorded by a pyranograph in the Bald Mountain watersheds. The recorder was installed to ensure unobstructed exposure to the sun.

The measuring element of the pyranograph consists of two identical bimetallic strips. One of the metallic strips is blackened, while the other is highly polished and covered with a shield. The blackened strip is exposed to both the ambient temperature and the radiant energy of the sun. The shielded strip is exposed to the ambient temperature but shielded from the radiant energy. The metallic strips are mounted such that only the difference in temperature between them causes the recording pen to move. In this manner movement in the strips caused by ambient temperature changes results in no pen movement while temperature changes caused by radiant energy (global radiation) are recorded by the pen.

The scales on the recording chart are gram-calories per square centimeter per minute and time in minutes. To compute daily global radiation, the area under the curve for each day was computed using a digitizer and converted by applying a conversion factor.

The recording charts used in this study have a conversion factor of 851.61 gram calories per square centimeter per square inch or 851.61 Langleys per square inch. Daily global radiation data for the Bald Mountain watersheds were computed to the nearest Langley and are summarized in table 10 at the end of the report. Also included in table 10 are the mean, maximum and minimum daily values for each month. 


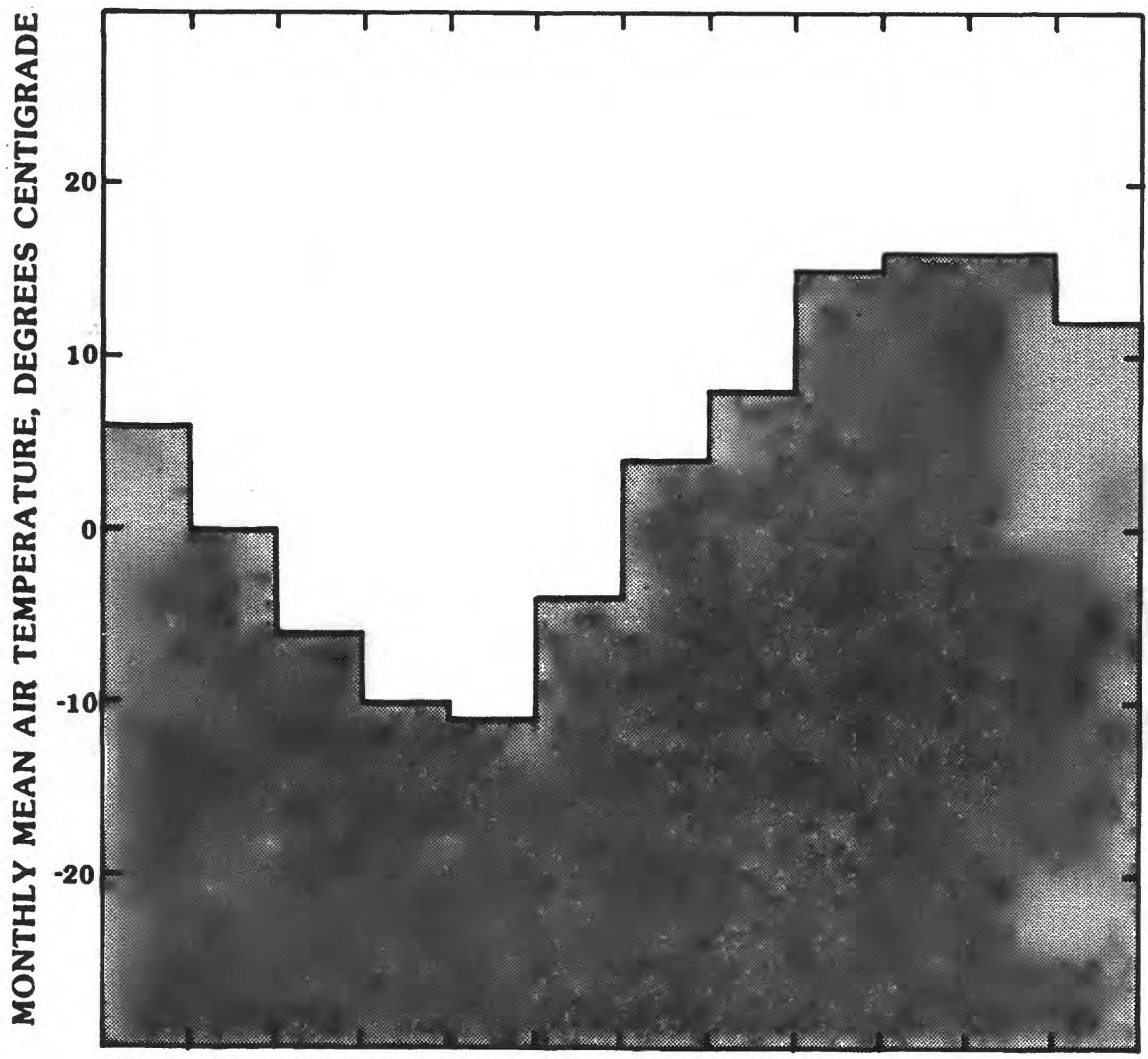

OCT. NOU. DEC. JAN. FEB. MAR. APR. MAY JUNE JULY AUG. SEPT. 1983 WATER YEAR

Figure 4.-Monthly mean air temperature in the Bald Mountain watersheds, 1983 water year. 
To ensure accurate recording of global radiation the recorder was routinely calibrated against National Weather Service instruments in their Caribou, Maine office. As an additional quality control measure the recorded data for the Bald Mountain watersheds was compared to National Weather Services data from the Caribou station to detect gross recording errors.

\section{Snow Surveys}

Periodic measurements of snow depths, densities, and water equivalents were collected for use in the calibration of the watershed model and to determine the variability of the snow mass. The volume and state of a snow mass can vary as a function of elevation, aspect, slope and degree of ground cover in watersheds. Thirteen snow survey sites were established in the Bald Mountain watersheds. The sites were selected to provide data over the range of the variables encountered in the basins. The site identifiers and characteristics are given in table 2 .

A Mt. Rose type snow sampler was used in this study. The sampler is a metallic tube that is graduated on the outside and constructed with a set inside diameter. The graduations on the outside of the tube are used to measure the total depth of the snow pack. The inside diameter of the tube is such that one ounce of core in the tube equals one inch of water equivalent. The empty tube is weighed, a snow core is taken, and the tube is weighed again. The difference between the readings represents the snow water equivalent. At snow courses, each time readings are taken the resultant value of snow depth and water equivalent reported is the average of ten readings. The use of ten readings at a site is a type of quality control used to increase the representativeness of the data.

Snow survey results are summarized in table 11 . Included in the table are the dates, depths and water equivalents for the observations. Snow densities are not included in the table, but can easily be computed as the water equivalent divided by the snow depth. Densities of 10 percent are common for newly fallen snow. Snow that is "ripe" or saturated with water and has reached it's holding capacity has a density in the vicinity of 30 percent. 


\section{REFERENCES}

Barnett, P. R., and Mallory, E. C., Jr., 1971, Determination of minor elements in water by emission spectroscopy: U.S. Geological Survey Techniques of Water-Resources Investigations, Book 5, Chap. A2, 31 p.

Benson, M. A., and Dalrymple, Tate, 1967, General field and office precedures for indirect discharge measurements: U.S. Geological Survey Techniques of Water-Resources Investigations, Book 3, Chap. A1, $30 \mathrm{p}$.

Brakensiek, D. D., Osborn, H. B., Rawls, W. J., coordinators, 1979, Field manual for research in agricultural hydrology: U.S. Department of Agricultural Handbook No. 224, 547 p.

Buchanan, T. J., and Somers, W. P., 1968, Stage measurement at gaging stations: U.S. Geological Survey Techniques of Water-Resources Investigations, Book 3, Chap. A7, 28 p.

Buchanan, T. J., and Somers, W. P., 1969, Discharge measurement at gaging . stations: U.S. Geological Survey Techniques of Water-Resources Investigations, Book 3, Chap. A8, 65 p.

Carter, R. W., and Davidian, Jacob, 1968, General procedure for gaging streams: U.S. Geological Survey Techniques of Water-Resources Investigations, Book 3, Chap. A6, 13 p.

Colby, B. R., 1956, Relationship of sediment to streamflow: U.S. Geological Survey, Quality of Water Branch, Open-File Report, 170 p.

Dalrymple, Tate, and Benson, M. A., 1967, Measurement of peak discharge by the slope-area method: U.S. Geological Survey Techniques of WaterResources Investigations, Book 3, Chap. A2, 12 p.

Fisher, W. C., and Hardy, C. E., 1976, Fire-weather observers' handbook: U.S. Department of Agriculture, Agriculture Handbook No. 494, 152 p.

Goerlitz, D. F., and Brown, Eugene, 1972, Methods for analysis of organic substances in water: U.S. Geological Survey Techniques of WaterResources Investigations, Book 5, Chap. A3, 40 p.

Guy, H. P., 1964, An analysis of some storm-period variables affecting stream sediment transport: U.S. Geological Survey Professional Paper $462-\mathrm{E}, 46 \mathrm{p}$.

Guy, H. P., 1969, Laboratory theory and methods for sediment analysis: U.S. Geological Survey Techniques of Water-Resources Investigations, Book 5, Chap. C1, $58 \mathrm{p}$.

Guy, H. P., 1970, Fluvial sediment concepts: U.S. Geological Survey Techniques of Water-Resources Investigations, Book 3, Chap. C1, 55 p. 
Guy, H. P., and Norman, V. W., 1970, Field methods for measurement of fluvial sediment: U.S. Geological Survey Techniques of Water-Resources Investigations, Book 3, Chap. C2, 59 p.

Hem, J. D., 1970, Study and interpretation of the Chemical characteristics of natural water: U.S. Geological Survey Water-Supply Paper 1473, $363 \mathrm{p}$.

Porterfield, G., 1972, Computation of fluvial-sediment discharge: U.S. Geological Sruvey Techniques of Water-Resources Investigations, Book 3 , Chap. C3, 66 p.

Rainwater, F. H., and Thatcher, L. L., 1960, Methods for collection and analysis of water samples: U.S. Geological Survey Water Supply Paper $1454,301 \mathrm{p}$.

Rantz, S. E., and others, 1982, Measurement and Computation of Streamflow: Volume 1 and Volume 2; U.S. Geological Survey Water-Supply Paper 2175, $631 \mathrm{p}$.

Skougstad, M. W., Fishman, M. J., Friedman, L. C., Erdmann, D. E., and Duncan, S. S., editors, 1979, Methods for determination of inorganic substances in water and fluvial sediments: U.S. Geological survey Techniques of Water-Resources Investigations, Book 5, Chap. A1, 626 p.

Stevens, H. H., Jr., Ficke, J. F., and Smoot, G. F., 1975, Water temperature--influential factors, field measurement, and data presentation: U.S. Geological Survey Techniques of Water-Resources Investigations, Book 1, Chap. D1, 65 p.

U.S. Geological Survey Office of Water Data Coordination, 1977, National handbook of recommended methods for water-data aquisition: Chapter 10 Hydrometeorological, $72 \mathrm{p}$.

U.S. Geological Survey, 1981-84, Water-Resources Data--Maine, water years 1981-84: U.S. Geological Survey water data reports (published annually). 
DATA TABLES 


\section{CLAYTON STREAM AT OUTLET CLAYTON LAKE, ME}

LOCATION.--Lat $46^{\circ} 43^{\circ} 50^{\prime \prime}$, Long $68^{\circ} 46^{\circ} 18^{\prime \prime}$, Aroostook County, Bydrologic Unit 01010003, on right bank $0.3 \mathrm{mi}$ (0.5 km) west from logging route $42,1.0 \mathrm{mi}(1.6 \mathrm{~km}$ ) west of $1 \mathrm{ts}$ intersection with the Carr Pond Road, and $18 \mathrm{mi}$ ( $29 \mathrm{~km}$ ) west of Portage.

DRAINAGE AREA. $--13.9 \mathrm{~mL}^{2}\left(36.0 \mathrm{~km}^{2}\right)$.

PERIOD OR RECORD.--July 16 to Sept. $30,1982$.

GAGE.-Water-stage recorder. Altitude of gage is $840 \mathrm{ft}(256 \mathrm{~m})$, from topographic map.

REMARKS. --Records fair.

EXTRMMES FOR CURRENT YEAR.--Peak discharges above base of $35 \mathrm{ft}^{3} / \mathrm{s}\left(0.99 \mathrm{~m}^{3} / \mathrm{s}\right)$ and maximum (

\begin{tabular}{|c|c|c|c|c|c|c|c|c|c|}
\hline Date & Time & $\begin{array}{l}\text { Discharge } \\
\left(\mathrm{ft}^{3 / \mathrm{s}}\right)\left(\mathrm{m}^{3} / \mathrm{s}\right)\end{array}$ & $\begin{array}{l}\text { Gage Beight } \\
\begin{array}{ll}\text { (ft) } & \text { (m) }\end{array}\end{array}$ & Date & Time & $\begin{array}{r}\text { Dis } \\
\left(f t^{3} / s\right.\end{array}$ & $\left(m^{3} / s\right)$ & $\begin{array}{l}\text { Gage } \\
(f t)\end{array}$ & $\begin{array}{l}\text { HeIght } \\
\text { (m) }\end{array}$ \\
\hline us. 27 & 1515 & 1.05 & 11.14 & Sept. & 0300 & $\star 58$ & 1.64 & 11.35 & 3.459 \\
\hline
\end{tabular}

Minimum discharge, $0.10 \mathrm{ft}^{3} / \mathrm{s}\left(0.003 \mathrm{~m}^{3} / \mathrm{s}\right)$ Aug. 9 , gage height, $9.87 \mathrm{ft}$ (3.008 $\left.\mathrm{m}\right)$.

DISCHARGE, IN CUBIC FEET PER SECOND, WATER YEAR OCTOBER 1981 TO SEPTEMBER 1982 MEAN VALUES

\begin{tabular}{|c|c|c|c|c|c|c|c|c|c|c|c|c|}
\hline DAY & OCT & Nov & DEC & JAN & FEB & MAR & APR & MAY & JUN & JUL & AUG & SEP \\
\hline 1 & --- & --- & --- & --- & --- & -- & --- & --- & --- & --- & .35 & 14 \\
\hline 2 & --- & --- & --- & --- & --- & --- & --- & --- & --- & --- & .28 & 25 \\
\hline 3 & --- & -- & --- & --- & --- & --- & --- & --- & --- & -- & .20 & 49 \\
\hline 4 & --- & --- & --- & --- & --- & --- & --- & --- & --- & -- & .14 & 56 \\
\hline 5 & --- & --- & --- & --- & -- & --- & --- & --- & -- & --- & .14 & 49 \\
\hline 6 & $=-$ & --- & --- & --- & --- & --- & --- & --- & -- & --- & .12 & 41 \\
\hline 7 & --- & --- & --- & --- & -- & --- & --- & --- & --- & --- & .11 & 41 \\
\hline 8 & -- & --- & --- & --- & --- & --- & -- & --- & --- & -- & .11 & 36 \\
\hline 9 & --- & --- & --- & --- & $-\cdots$ & --- & $\cdots$ & -- & $=--$ & -- & .11 & 31 \\
\hline 10 & --- & --- & --- & -- & -- & --- & -- & $=--$ & $=-$ & $\cdots$ & .72 & 26 \\
\hline 11 & --- & --- & --- & -- & --- & --- & -- & --- & --- & --- & .72 & 22 \\
\hline$\cdot 12$ & --- & -- & -- & -- & -- & --- & -- & --- & --- & --- & .65 & 19 \\
\hline 13 & -- & --- & --- & --- & --- & --- & --- & --- & --- & -- & .75 & 16 \\
\hline 14 & --- & --- & --- & --- & --- & --- & -- & -- & --- & -- & 1.1 & 14 \\
\hline 15 & --- & --- & --- & --- & --- & --- & -- & --- & --- & 28 & 1.3 & 13 \\
\hline 16 & --- & -- & -- & -- & --- & --- & -- & --- & --- & .42 & 1.2 & 16 \\
\hline 17 & --- & --- & --- & --- & --- & --- & --- & $=-$ & --- & .42 & 1.1 & 24 \\
\hline 18 & --- & --- & -- & --- & -- & --- & --- & -- & --- & .37 & .94 & 25 \\
\hline 19 & --- & --- & --- & --- & --- & --- & --- & --- & --- & .33 & .79 & 23 \\
\hline 20 & --- & --- & --- & --- & --- & --- & --- & --- & -- & .27 & .75 & 21 \\
\hline 21 & -- & -- & --- & --- & --- & --- & --- & --- & --- & .17 & .66 & 18 \\
\hline 22 & -- & --- & -- & -- & --- & -- & --- & -- & -- & .17 & .54 & \\
\hline 23 & --- & --- & --- & -- & $=-$ & -- & --- & -- & --- & .22 & .48 & 14 \\
\hline 24 & --- & --- & --- & -- & -- & --- & -- & -- & --- & .17 & .86 & 13 \\
\hline 25 & --- & --- & --- & --- & $=--$ & $=--$ & --- & --- & -- & .15 & 1.5 & 11 \\
\hline 26 & --- & -- & --- & --- & --- & -- & --- & -- & --- & .19 & 30 & 11 \\
\hline 27 & --- & --- & --- & --- & --- & -- & --- & -- & --- & .15 & 35 & 11 \\
\hline 28 & -- & --- & --- & -- & --- & -- & --- & -- & -- & .14 & 32 & 18 \\
\hline 29 & --- & -- & -- & -- & -- & -- & -- & -- & --- & .50 & 26 & 19 \\
\hline 30 & --- & -- & --- & --- & --- & --- & --- & -- & --- & .54 & 21 & 19 \\
\hline 31 & --- & --- & --- & --- & -- & -- & -- & -- & --- & .44 & 17 & \\
\hline TOTAL & --- & -- & -- & --- & --- & -- & --- & --- & --- & --- & 176.62 & 711 \\
\hline MEAN & --- & --- & -- & --- & --- & --- & -- & -- & --- & --- & 5.70 & 23.7 \\
\hline MAX & --- & --- & --- & $=-$ & -- & --- & -- & -- & --- & --- & 35 & 56 \\
\hline MIN & --- & --- & --- & -- & --- & --- & --- & --- & --- & --- & .11 & 11 \\
\hline CFSM & -- & --- & -- & -- & --- & --- & --- & -- & --- & -- & .41 & 1.71 \\
\hline IN. & -- & --- & --- & --- & --- & --- & -- & -- & 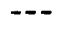 & --- & .47 & \\
\hline
\end{tabular}


LOCATION.--Iat $46^{\circ} 43^{\prime} 50^{\prime \prime}$, Iong $68^{\circ} 46^{\prime} 18^{\prime \prime}$, Aroostook County, Eydrolosic Unit 01010003, on right bank $0.3 \mathrm{mi}$ west

from $10881 \mathrm{ng}$ route $42,1.0 \mathrm{ml}$ west of $1 \mathrm{ts}$ intersection with the Carr Pond Road, and $18 \mathrm{ml}$ west of Portage.

DRAINAGE AREA. $--13.9 \mathrm{~mL}^{2}$.

PERIOD OF RECORD.--July 1982 to current year.

GAGE.--Water-stage recorder. Altitude of gage is $840 \mathrm{ft}$, from topographlc map.

RMMARRS.--Records good except for winter perlod, which are falr.

EXIREMES FOR PERIOD OF RECORD.--Maximum discharge, $409 \mathrm{ft}^{3} / \mathrm{s} \mathrm{Apr.} \mathrm{18,} 1983, \mathrm{gage} \mathrm{he} 1 \mathrm{ght}, 14.47 \mathrm{ft}$; minimum discharge, $0.10 \mathrm{ft}^{3} / \mathrm{s} \mathrm{Aug.} \mathrm{9,1982,} \mathrm{gage} \mathrm{helght,} 9.87 \mathrm{ft}$.

EXIREARS FOR CURRENT YEAR.--Peak discharges above base of $100 \mathrm{ft}^{3} / \mathrm{s}$ (revised) and maximum (*):

\begin{tabular}{|c|c|c|c|c|c|c|c|}
\hline Date & Time & $\begin{array}{c}\text { Discharge } \\
\left(\mathrm{ft}^{3} / \mathrm{s}\right)\end{array}$ & $\begin{array}{c}\text { Gage He Ight } \\
\text { (ft) }\end{array}$ & Date & Time & $\begin{array}{c}\text { Discharge } \\
\left(f t^{3} / s\right)\end{array}$ & $\begin{array}{c}\text { Gage HeIght } \\
\text { (ft) }\end{array}$ \\
\hline Apr. 18 & 1200 & $\star 409$ & 14.47 & Jun. 01 & 0300 & 124 & 12.16 \\
\hline Apr 26 & -- & 215 & -- & Jul. 22 & 0330 & 123 & 12.15 \\
\hline
\end{tabular}

Minimum discharge, $0.53 \mathrm{ft}^{3} / \mathrm{s}$ Aug. 26, 27, gage helght, $10.04 \mathrm{ft}$.

DISCEARGE, IN CUBIC FEET PER SECOND, WATER YEAR OCTOBER 1982 TO SEPTEMBER 1983 MEAN VALUES

\begin{tabular}{|c|c|c|c|c|c|c|c|c|c|c|c|c|}
\hline DAY & OCT & nov & DEC & JAN & $F E B$ & MAR & APR & MAY & JUN & JUL & AUG & SEP \\
\hline 1 & 20 & 7.4 & 32 & 9.5 & 3.9 & 3.0 & 23 & 115 & 123 & 4.3 & 31 & 22 \\
\hline 2 & 25 & 7.5 & 30 & 8.7 & 3.6 & 3.5 & 20 & 100 & 112 & 4.9 & 32 & 19 \\
\hline 3 & 25 & 9.5 & 30 & 8.0 & 3.4 & 3.9 & 18 & 110 & 95 & 6.2 & 31 & 18 \\
\hline 4 & 24 & 10 & 43 & 7.3 & 4.0 & 4.7 & 17 & 95 & 78 & 5.7 & 27 & 17 \\
\hline 5 & 22 & 30 & 52 & 6.7 & 5.2 & 4.3 & 17 & 84 & 63 & 10 & 23 & 15 \\
\hline 6 & 21 & 61 & 56 & 6.5 & 7.0 & 4.0 & 16 & 79 & 53 & 25 & 20 & 13 \\
\hline 7 & 18 & 69 & 57 & 6.0 & 8. 4 & 3.6 & 18 & 70 & 58 & 25 & 18 & 13 \\
\hline 8 & 16 & 67 & 52 & 5.8 & 7.9 & 3.4 & 28 & 60 & 54 & 22 & 17 & 13 \\
\hline 9 & 19 & 60 & 45 & 5.5 & 7.3 & 3.2 & 36 & 57 & 46 & 20 & 15 & 12 \\
\hline 10 & 19 & 51 & 37 & 5.2 & 6.7 & 3.0 & 46 & 59 & 39 & 17 & 13 & 11 \\
\hline 11 & 18 & 44 & 31 & 15 & 6.2 & 3.3 & 53 & 57 & 34 & 14 & 11 & 11 \\
\hline 12 & 17 & 38 & 27 & 43 & 5.8 & 4.0 & 70 & 52 & 30 & 12 & 9.4 & 9.7 \\
\hline 13 & 16 & 52 & 25 & 32 & 5.2 & 6.0 & 73 & 53 & 27 & 10 & 8.0 & 7.5 \\
\hline 14 & 17 & 66 & 20 & 24 & 4.9 & 9.5 & 80 & 58 & 24 & 9.3 & 7.0 & 3.2 \\
\hline 15 & 19 & 66 & 15 & 19 & 4.6 & 11 & 84 & 56 & 21 & 8.6 & 6.1 & 2.8 \\
\hline 16 & 19 & 61 & 18 & 16 & 4.4 & 9.8 & 87 & 52 & 19 & 8.3 & 5.2 & 2.4 \\
\hline 17 & 18 & 53 & 23 & 14 & 4.0 & 9.3 & 155 & 46 & 18 & 7.6 & 4.5 & 2.5 \\
\hline 18 & 17 & 46 & 33 & 12 & 3.8 & 9.0 & 369 & 41 & 18 & 6.5 & 4.1 & 2.9 \\
\hline 19 & 15 & 39 & 19 & 10 & 3.6 & 8.8 & 269 & 37 & 17 & 5.4 & 3.8 & 3.0 \\
\hline 20 & 14 & 34 & 16 & 9.0 & 3.5 & 12 & 208 & 35 & 15 & 4.7 & 3.4 & 3.0 \\
\hline 21 & 13 & 31 & 13 & 7.8 & 3.4 & 17 & 175 & 36 & 13 & 6.3 & 2.9 & 3.0 \\
\hline 22 & 12 & 35 & 12 & 7.0 & 3.2 & 25 & 140 & 36 & 12 & 92 & 2.6 & 16 \\
\hline 23 & 11 & 44 & 12 & 6.5 & 3.1 & 35 & 112 & 35 & 11 & 78 & 2.3 & 28 \\
\hline 24 & 11 & 58 & 11 & 7.1 & 3.0 & 53 & 99 & 45 & 9.8 & 75 & 1.9 & 28 \\
\hline 25 & 9.9 & 62 & 11 & 8.0 & 2.9 & 53 & 156 & 57 & 8.4 & 77 & 1.2 & 27 \\
\hline 26 & 9.4 & 59 & 14 & 6.8 & 2.9 & 49 & 205 & 57 & 7.4 & 65 & .59 & 26 \\
\hline 27 & 8.9 & 52 & 20 & 5.8 & 2.8 & 44 & 200 & 53 & 6.7 & 53 & 3.6 & 24 \\
\hline 28 & 8.6 & 43 & 15 & 5.2 & 2.7 & 39 & 170 & 73 & 6.4 & 48 & 4.2 & 22 \\
\hline 29 & 8.2 & 38 & 13 & 4.9 & $-\infty$ & 37 & 150 & 82 & 5.7 & 42 & 4.2 & 19 \\
\hline 30 & 8.0 & 35 & 12 & 4.3 & $-\cdots$ & 32 & 130 & 81 & 4.9 & 37 & 3.6 & 17 \\
\hline 31 & 7.6 & --- & 11 & 4.1 & -- & 27 & --- & 114 & --- & 32 & 11 & -- \\
\hline TOTAL & 486.6 & 1328.4 & 805 & 330.9 & 127.4 & 530.3 & 3224 & 1985 & 1029.3 & 831.8 & 327.59 & 411.0 \\
\hline MEAN & 15.7 & 44.3 & 26.0 & 10.7 & 4.55 & 17.1 & 107 & 64.0 & 34.3 & 26.8 & 10.6 & 13.7 \\
\hline MAX & 25 & 69 & 57 & 43 & 8.4 & 53 & 369 & 115 & 123 & 92 & 32 & 28 \\
\hline MIN & 7.6 & 7.4 & 11 & 4.1 & 2.7 & 3.0 & 16 & 35 & 4.9 & 4.3 & .59 & 2.4 \\
\hline CFSM & 1.13 & 3.19 & 1.87 & .77 & .33 & 1.23 & 7.73 & 4.61 & 2.47 & 1.93 & .76 & .99 \\
\hline IN. & 1.30 & 3.56 & 2.15 & .89 & .34 & 1.42 & 8.63 & 5.31 & 2.75 & 2.23 & .88 & 1.10 \\
\hline
\end{tabular}

CAL YR 1982 TOTAL 3540.27 MEAN 20.8 MAX 69 MIN .11 CFSM 1.50 IN. 9.47

WTR YR 1983 TOTAL 11417.24 MEAN 31.3 MAX 369 MIN .59 CFSM 2.25 IN. 30.6 
LOCATION.--Lat 46 $43^{\prime} 50^{\prime \prime}$, long $68^{\circ} 46^{\prime} 18^{\prime \prime}$, Aroostook County, Hydrologic Unit 01010003, on right bank 0.3 mi west

from logging route $42,1.0 \mathrm{ml}$ west of lts intersection with the Carr Pond Road, and $18 \mathrm{ml}$ west of Portage.

DRAINAGE AREA. $--13.9 \mathrm{mi}^{2}$.

PERIOD OF RECORD.--July 1982 to June 1984 (discontinued).

GAGE.-Water-stage recorder. Altitude of gage is $840 \mathrm{ft}$, from topographic map.

REMARKS.--Records good except those for winter period, which are fair.

EXIREMES FOR PERIOD OF RECORD.-Maximum dlscharge, $409 \mathrm{ft}^{3} / \mathrm{s}$ Apr. 18, 1983, 8age helght, $14.47 \mathrm{ft}$; minimum discharge, $0.10 \mathrm{ft}^{3} / \mathrm{s}$ Aug. $9,1982,828 \mathrm{e} \mathrm{helght,} 9.87 \mathrm{ft}$.

EXTREMES FOR CURRENT YEAR.--Peak discharges above base of $100 \mathrm{ft}^{3} / \mathrm{s}$ and maximum ( June 1984:

\begin{tabular}{|c|c|c|c|c|c|c|c|}
\hline Dete & Time & $\begin{array}{c}\text { DLscharge } \\
\left(f t^{3} / \mathrm{s}\right)\end{array}$ & $\begin{array}{c}\text { Gage Height } \\
\text { (ft) }\end{array}$ & Date & I Ime & $\begin{array}{c}\text { Discharge } \\
\left(\mathrm{ft}^{3} / \mathrm{s}\right)\end{array}$ & $\begin{array}{c}\text { Gage Height } \\
\text { (ft) }\end{array}$ \\
\hline Nov. 27 & 0330 & 136 & 12.27 & Apr. 25 & 1015 & $\star 228$ & 12.43 \\
\hline Apr. 17 & 1700 & 219 & 12.39 & Jun. 01 & unknown & 129 & 11.97 \\
\hline
\end{tabular}

Minlmum discherge not determined, occurred during perlod of backwater from ice: minlmum dally, 3.7 ft $3 / 5$ Mar. 20.

DISCHARGE, IN CUBIC FEET PER SECOND, WATER YEAR OCTOBER 1983 TO SEPTEMBER 1984 MEAN VALUES

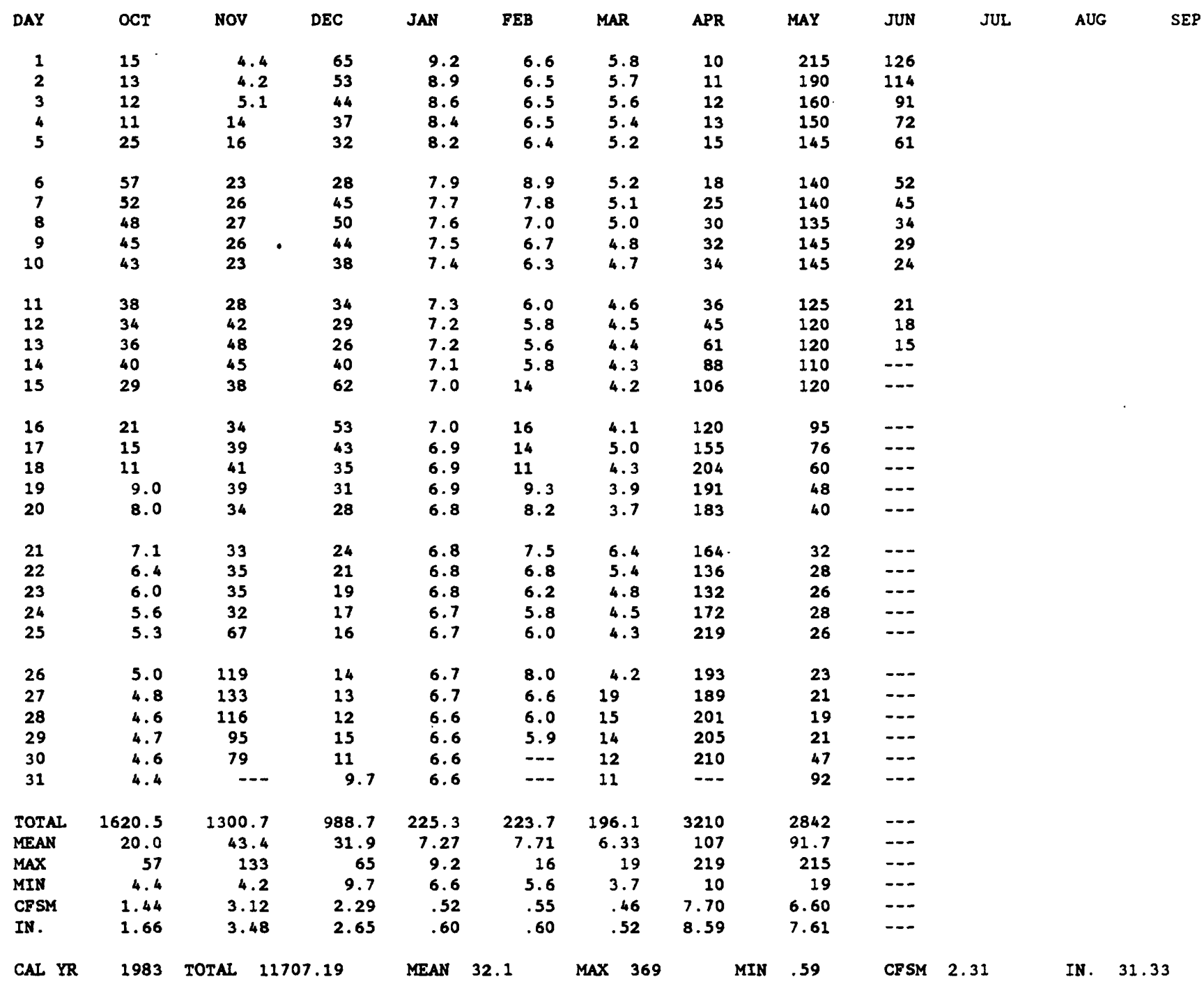


LOCATION.--Lat $46^{\circ} 44^{\prime} 23^{\prime \prime}$, Long 68 $45^{\prime} 21^{\prime \prime}$, Aroostook County, Bydrologic Unit 01010003, on left bank 30 ft ( 9 m) downstream from 1088 ing route $42,0.3 \mathrm{mi}(0.5 \mathrm{~km})$ west of its intersection with the Carr Pond Road and $18 \mathrm{mi}$ (29 $\mathrm{km}$ ) west of Portage.

DRAINAGE AREA. $--1.69 \mathrm{mi}^{2}\left(4.38 \mathrm{~km}^{2}\right)$.

PERIOD OF RECORD.--October 1980 to September 1981.

GAGE.--Water-stage recorder. Altitude of gage is $820 \mathrm{ft}$ (250 m), from topographic map.

RMMARKS.--Records good except those for winter period and period of no gage-helght record Aug. 19 to Sept. 21. EXTREMES FOR CURRENT YEAR.--Peak discharges above base of $25 \mathrm{ft}^{3} / \mathrm{s}\left(0.71 \mathrm{~m}^{3} / \mathrm{s}\right)$ and $\mathrm{max} i \mathrm{mum}(*)$ :

\begin{tabular}{|c|c|c|c|c|c|c|c|c|c|c|}
\hline Date & Time & $\begin{array}{c}\text { Discharge } \\
\left(\mathrm{ft}^{3} / \mathrm{s}\right) \quad\left(\mathrm{m}^{3} / \mathrm{s}\right)\end{array}$ & $\begin{array}{c}\text { Gage } \\
\text { (ft) }\end{array}$ & $\begin{array}{r}\text { He1ght } \\
(m)\end{array}$ & Date & Time & $\begin{array}{l}\text { DIsch } \\
\left(f t^{3 / s}\right)\end{array}$ & $\left(m^{3} / s\right)$ & $\begin{array}{l}\text { Gage } \\
(f t)\end{array}$ & $\begin{array}{r}\text { He Ight } \\
(m)\end{array}$ \\
\hline Feb. 20 & 2300 & lce $\mathrm{Jam}$ & $\star 6.30$ & 1.920 & Apr. 7 & 1330 & 28 & 0.79 & 5.41 & 1.649 \\
\hline Apr. 6 & 1400 & $\begin{array}{ll}70 & 1.98\end{array}$ & 6.10 & 1.859 & Aus. 17 & 2120 & 84 & 2.38 & 6.27 & 1.911 \\
\hline
\end{tabular}

Minimum discharge, $0.14 \mathrm{ft}^{3} / \mathrm{s}\left(0.004 \mathrm{~m}^{3} / \mathrm{s}\right) \mathrm{July} 24,25$, Aug. 4, 5, gage he1ght, $4.43 \mathrm{ft}$ (1.350 $\mathrm{m}$ ).

DISCBARGE, IN CUBIC FEET PER SECOND, WATER YEAR OCTOBER 1980 TO SEPTEMBER 1981 MEAN VALUES

\begin{tabular}{|c|c|c|c|c|c|c|c|c|c|c|c|c|}
\hline DAY & OCT & NOV & DEC & JAN & FEB & MAR & $\mathbf{A P R}$ & MAY & JUN & JUL & AUG & SEP \\
\hline 1 & 3.0 & 2.0 & 3.6 & .24 & .21 & 5.0 & 6.0 & 6.0 & 3.7 & .67 & .37 & .19 \\
\hline 2 & 2.8 & 1.9 & 1.7 & .24 & .25 & 4.1 & 15 & 5.3 & 2.3 & .51 & .21 & .18 \\
\hline 3 & 2.6 & 1.6 & 3.8 & .24 & 1.5 & 3.5 & 17 & 5.3 & 1.7 & .42 & .17 & .17 \\
\hline 4 & 2.5 & 1.4 & 18 & .24 & 5.3 & 3.1 & 12 & 4.6 & 1.7 & .38 & .16 & .17 \\
\hline 5 & 5.8 & 1.7 & 4.4 & .23 & 7.1 & 2.8 & 17 & 4.2 & 1.6 & .38 & 1.2 & .16 \\
\hline 6 & 2.5 & 1.6 & 2.6 & .23 & 5.5 & 2.6 & 25 & 4.1 & 1.5 & .46 & 38 & \\
\hline 7 & 2.0 & 1.4 & 1.7 & .23 & 4.7 & 2.4 & 21 & 3.4 & 1.6 & 1.6 & 32 & .16 \\
\hline 8 & 1.7 & 2.5 & 1.2 & .23 & 4.2 & 2.2 & 14 & 3.0 & 1.3 & 1.0 & 15 & .16 \\
\hline 9 & 1.6 & 2.3 & 1.6 & .23 & 3.8 & 2.1 & 13 & 2.7 & 1.2 & .70 & 9.2 & 7.1 \\
\hline 10 & 1.5 & 1.8 & .92 & .23 & 3.4 & 2.0 & 21 & 2.5 & 1.3 & .44 & 6.5 & 2.5 \\
\hline 11 & 1.4 & 1.9 & .60 & .22 & 3.2 & 2.1 & 16 & 2.4 & 1.1 & .36 & 5.4 & \\
\hline 12 & 2.3 & 2.1 & .44 & .22 & 6.8 & 2.1 & 12 & 2.4 & .93 & .30 & 5.8 & .26 \\
\hline 13 & 7.0 & 1.9 & .38 & .22 & 9.1 & 1.8 & 8. 7 & 6.3 & .79 & .27 & 5.1 & \\
\hline 14 & 4.5 & 1.7 & .36 & .22 & 6.8 & 1.5 & 6.8 & 7.1 & .69 & .49 & 3.5 & .24 \\
\hline 15 & 3.2 & 1.5 & .34 & .22 & 5.4 & 1.3 & 6.0 & 4.6 & .89 & .57 & 2.8 & .74 \\
\hline 16 & 2.5 & 1.3 & .33 & .22 & 4.8 & 1.2 & 8.5 & 4.4 & 1.4 & .29 & 9.9 & .54 \\
\hline 17 & 2.1 & 1.2 & .32 & .22 & 4.3 & 1.1 & 6.1 & 8.0 & 1.5 & .22 & 34 & \\
\hline 18 & 1.8 & 1.1 & .31 & .22 & 4.0 & .93 & 5.2 & 6.7 & 1.1 & .20 & 35 & .3 \\
\hline 19 & 1.7 & 1.1 & .30 & .22 & 4.1 & .83 & 5.9 & 4.9 & .70 & .18 & 14 & .35 \\
\hline 20 & 1.6 & 1.2 & .29 & .22 & 5.0 & .76 & 5.7 & 4.0 & .73 & .18 & 5.8 & 6.3 \\
\hline 21 & 1.5 & 1.0 & .29 & .22 & 6.6 & .71 & 4.6 & 3.5 & 1.6 & .19 & 3.0 & 2.4 \\
\hline 22 & 1.4 & .96 & .28 & .22 & 9.2 & .68 & 3.9 & 3.3 & 1.4 & .22 & 2.0 & 1.4 \\
\hline 23 & 1.3 & 1.0 & .27 & .22 & 10 & .68 & 3.6 & 3.1 & 2.3 & .16 & 1.4 & 2.9 \\
\hline 24 & 1.3 & .95 & .27 & .22 & 8.4 & .78 & 4.2 & 2.7 & 1.3 & .16 & 6.9 & 13 \\
\hline 25 & 1.2 & 3.3 & .27 & .22 & 7.1 & .90 & 5.4 & 2.4 & 1.0 & .15 & 4.2 & 12 \\
\hline 26 & 3.9 & 7.0 & .26 & .22 & 6.5 & .88 & 5.1 & 2.4 & 4.9 & .16 & 2.5 & 6.2 \\
\hline 27 & 4.7 & 15 & .26 & .22 & 7.5 & .98 & 5.0 & 2.6 & 3.4 & .37 & 1.4 & 4.5 \\
\hline 28 & 2.8 & 9.5 & .26 & .22 & 6.4 & 1.1 & 4.5 & 3.0 & 1.6 & .32 & .80 & 4.3 \\
\hline 29 & 2.1 & 4.0 & .25 & .21 & - & 1.2 & 5.2 & 3.7 & 1.1 & .22 & .42 & 3.6 \\
\hline 30 & 2.0 & 8.0 & .25 & .21 & --- & 1.5 & 7.9 & 2.8 & .81 & 1.9 & .29 & 3.0 \\
\hline 31 & 1.8 & --- & .25 & .21 & --- & 2.2 & --- & 3.3 & -- & 1.1 & .21 & --- \\
\hline TOTAL & 78.1 & 83.91 & 46.10 & 6.93 & 151.16 & 55.03 & 291.3 & 124.7 & 47.14 & 14.57 & 247.23 & 74.28 \\
\hline MEAN & 2.52 & 2.80 & 1.49 & .22 & 5.40 & 1.78 & 9.71 & 4.02 & 1.57 & .47 & 7.98 & 2.48 \\
\hline MAX & 7.0 & 15 & 18 & .24 & 10 & 5.0 & 25 & 8.0 & 4.9 & 1.9 & 38 & \\
\hline MIN & 1.2 & .95 & .25 & .21 & .21 & .68 & 3.6 & 2.4 & .69 & .15 & .16 & .16 \\
\hline CFSM & 1.49 & 1.66 & .88 & .13 & 3.19 & 1.05 & 5.75 & 2.38 & .93 & .28 & 4.72 & 1.47 \\
\hline IN. & 1.72 & 1.85 & 1.01 & .15 & 3.33 & 1.21 & 6.41 & 2.74 & 1.04 & .32 & 5.44 & \\
\hline
\end{tabular}

WTR YR 1981

TOTAL 1220.44

MEAN 3.34

MIN .15 CFSM $1.98 \quad$ IN. 26.9 


\section{BALD MOUNTAIN BROOK NEAR BALD MOUNTAIN, ME}

LOCATION.--Lat $46^{\circ} 44^{\prime} 23^{\prime \prime}$, Long $68^{\circ} 45^{\prime} 21^{\prime \prime}$, Aroostook County, Bydrologic Unit 0l010003, on left bank 30 ft (9 $\mathrm{m}$ ) downstream from 1088 ing route $42,0.3 \mathrm{mi}(0.5 \mathrm{~km}$ ) west of $1 \mathrm{ts}$ intersection with the Carr Pond Road and $18 \mathrm{ml}$ ( 29 kin) west of Portage.

DRAINAGE AREA. $--1.69 \mathrm{mi}^{2}(4.38 \mathrm{~km} 2)$.

PERIOD OR RECORD.--October 1980 to current year.

GAGE.--Water-stage recorder. Altitude of gage $13820 \mathrm{ft}$ ( $250 \mathrm{~m})$, from topographic map.

RMMARRS.--Records good except those for winter period, whlch are falr. No gage-height record Dec. 15 to Mar. 16.

EXTREMES FOR PERIOD OF RECORD.--Maximum discharge, $84 \mathrm{ft}^{3} / \mathrm{s}\left(2.38 \mathrm{~m}^{3} / \mathrm{s}\right)$ Aug. 17 . 1981 , gage helght, $6.27 \mathrm{ft}$

$(1.911 \mathrm{~m})$; maximum gage height, $6.30 \mathrm{ft}$ (1.920 m) Feb. 20, 1981 (backwater from lce); minlmum discharge,

$0.05 \mathrm{ft}^{3} / \mathrm{s}\left(0.001 \mathrm{~m}^{3} / \mathrm{s}\right) \mathrm{July} 17,1982$, gage height, $4.35 \mathrm{ft}(1.326 \mathrm{~m})$.

EXTREMES FOR CURRENT YEAR.--Peak discharges above base of $25 \mathrm{ft}^{3} / \mathrm{s}\left(0.71 \mathrm{~m}^{3} / \mathrm{s}\right)$ and maximum ( $)$ :

\begin{tabular}{|c|c|c|c|c|c|c|c|c|c|c|c|}
\hline Date & Time & $\begin{array}{c}\text { Discharge } \\
\left(\mathrm{ft}^{3} / \mathrm{s}\right)\left(\mathrm{m}^{3} / \mathrm{s}\right)\end{array}$ & $\begin{array}{l}\text { Gage } \\
(f t)\end{array}$ & $\begin{array}{r}\text { Height } \\
\text { (m) }\end{array}$ & Date & Ilme & & (s) & $\left(\mathrm{m}^{3} / \mathrm{s}\right)$ & $\begin{array}{l}\text { Gage } \\
\text { (ft) }\end{array}$ & $\begin{array}{r}\text { Helght } \\
\text { (m) }\end{array}$ \\
\hline Apr. 2 & 1610 & 1ce jam & $\star 6.16$ & 1.878 & Apr. 27 & 1240 & $\star$ & 51 & 1.44 & 5.82 & 1.774 \\
\hline
\end{tabular}

Minimun discharge, $0.05 \mathrm{ft}^{3} / \mathrm{s}\left(0.001 \mathrm{~m}^{3} / \mathrm{s}\right) \mathrm{July} 17$, gage height, $4.35 \mathrm{ft}$ (1.326 m).

DISCRARGE, IN CUBIC FEET PER SECOND, WATER YEAR OCTOBER 1981 TO SEPTEMBER 1982 MEAN VALUES

\begin{tabular}{|c|c|c|c|c|c|c|c|c|c|c|c|c|}
\hline DAY & OCT & NOV & DEC & JAN & FEB & MAR & APR & MAY & JUN & JUL & AUG & SEP \\
\hline 1 & 2.6 & 6.2 & 3.4 & 1.4 & .48 & .41 & 5.0 & 12 & .59 & .16 & .08 & .40 \\
\hline 2 & 2.2 & 5.8 & 1.4 & 1.4 & .48 & .40 & 7.4 & 11 & 1.3 & .12 & .07 & 1.9 \\
\hline 3 & 3.5 & 5.4 & 1.5 & 1.8 & .50 & .40 & 12 & 12 & 2.8 & .14 & .06 & 7.1 \\
\hline 4 & 5.1 & 4.8 & 1.8 & 2.5 & 1.1 & .40 & 7.8 & 13 & 1.6 & .14 & .06 & 4.4 \\
\hline 5 & 3.5 & 4.2 & 1.8 & 3.2 & 2.7 & .40 & 5.5 & 12 & .97 & .11 & .07 & 1.6 \\
\hline 6 & 2.5 & 3.9 & 2.4 & 4.2 & 2.3 & .40 & 4.5 & 13 & .71 & .08 & .08 & 1.1 \\
\hline 7 & 4.3 & 4.3 & 7.4 & 3.6 & 1.9 & .40 & 3.8 & 14 & .51 & .08 & .07 & 2.5 \\
\hline 8 & 15 & 4.3 & 7.7 & 3.0 & 1.6 & .40 & 3.5 & 14 & .46 & .07 & .08 & 1.4 \\
\hline 9 & 11 & 3.8 & 5.8 & 2.6 & 1.4 & .40 & 3.3 & 12 & .24 & .06 & .08 & .93 \\
\hline 10 & 7.2 & 3.1 & 5.1 & 2.2 & $1.2^{\circ}$ & .41 & 3.2 & 9.4 & .22 & .06 & .51 & .74 \\
\hline 11 & 5.8 & 2.7 & 4.5 & 1.9 & 1.1 & .41 & 3.3 & 7.9 & .20 & .06 & .24 & .58 \\
\hline 12 & 5.2 & 2.5 & 4.0 & 1.7 & .96 & .42 & 3.8 & 6.1 & .16 & .06 & .12 & .48 \\
\hline 13 & 4.5 & 2.3 & 3.6 & 1.5 & .87 & .58 & 4.2 & 5.6 & .16 & .06 & .14 & .39 \\
\hline 14 & 4.0 & 2.2 & 3.2 & 1.3 & .80 & .92 & 4.7 & 4.6 & .22 & .06 & .24 & .33 \\
\hline 15 & 3.5 & 2.1 & 2.9 & 1.1 & .74 & .80 & 4.5 & 4.0 & .22 & .06 & .22 & .42 \\
\hline 16 & 3.3 & 2.1 & 2.6 & 1.0 & .69 & .73 & 4.4 & 3.3 & .77 & .06 & .11 & 1.6 \\
\hline 17 & 3.1 & 2.1 & 2.5 & .96 & .65 & .67 & 7.0 & 2.8 & .69 & .07 & .08 & 4.0 \\
\hline 18 & 2.8 & 3.0 & 2.3 & .88 & .62 & .63 & 27 & 2.3 & .27 & .07 & .07 & 1.8 \\
\hline 19 & 8.4 & 3.7 & 2.2 & .84 & .58 & .61 & 15 & 2.0 & .18 & .06 & .07 & 1.2 \\
\hline 20 & 7.3 & 2.8 & 2.1 & .77 & .55 & .60 & 10 & 2.1 & .20 & .06 & .07 & .86 \\
\hline 21 & 4.9 & 4.8 & 2.0 & .74 & .53 & .79 & 15 & 2.1 & .18 & .06 & .06 & .69 \\
\hline 22 & 3.9 & 7.5 & 1.9 & .70 & .50 & .64 & 19 & 1.7 & .24 & $: 11$ & .06 & .59 \\
\hline 23 & 5.7 & 5.0 & 1.8 & .66 & .48 & .52 & 13 & 1.4 & .27 & .14 & .06 & .51 \\
\hline 24 & 20 & 3.3 & 1.8 & .63 & .46 & .45 & 18 & 1.2 & 1.2 & .08 & .44 & .55 \\
\hline 25 & 14 & 2.6 & 1.7 & .60 & .45 & .70 & 30 & 1.2 & .51 & .08 & .48 & .46 \\
\hline 26 & 9.7 & 2.4 & 1.6 & .57 & .44 & 1.3 & 37 & 1.0 & .27 & .16 & 12 & .41 \\
\hline 27 & 9.9 & 2.3 & 1.6 & .54 & .43 & 2.2 & 43 & .94 & .16 & .10 & 7.2 & .69 \\
\hline 28 & 16 & 2.3 & 1.5 & .52 & .42 & 4.0 & 35 & .86 & .12 & .08 & 1.2 & 3.3 \\
\hline 29 & 13 & 2.2 & 1.5 & .51 & --- & 6.8 & 25 & .85 & .14 & .34 & .78 & 1.7 \\
\hline 30 & 9.2 & 2.3 & 1.5 & .50 & $\ldots$ & 5.4 & 17 & .77 & .22 & .20 & .56 & .92 \\
\hline 31 & 7.2 & --- & 1.5 & .49 & --- & 4.4 & -. & .69 & -- & .10 & .47 & -- \\
\hline TOTAL & 218.3 & 106.0 & 86.6 & 44.31 & 24.93 & 37.59 & 391.9 & 175.81 & 15.78 & 3.09 & 25.83 & 43.55 \\
\hline MEAN & 7.04 & 3.53 & 2.79 & 1.43 & .89 & 1.21 & 13.1 & 5.67 & .53 & .10 & .83 & 1.45 \\
\hline MAX & 20 & 7.5 & 7.7 & 4.2 & 2.7 & 6.8 & 43 & 14 & 2.8 & .34 & 12 & 7.1 \\
\hline MIN & 2.2 & 2.1 & 1.4 & .49 & .42 & .40 & 3.2 & .69 & .12 & .06 & .06 & .33 \\
\hline CFSM & 4.17 & 2.09 & 1.65 & .85 & .53 & .72 & 7.73 & 3.36 & .31 & .06 & .49 & .86 \\
\hline IN. & 4.81 & 2.33 & 1.91 & .98 & .55 & .83 & 8.63 & 3.87 & .35 & .07 & .57 & .96 \\
\hline
\end{tabular}

CAL YR 1981 TOTAL 1423.22 MEAN 3.90 MAX 38 MIN .15 CFSM 2.31 IN. 31.3

WTR YR 1982 TOTAL 1173.67 MEAN 3.22 MAX 43 MIN .06 CFSM 1.90 IN. 25.8 
LOCATION,--Lat $46^{\circ} 44^{\prime} 23^{\prime \prime}$, Long $68^{\circ} 45^{\prime} 21^{\prime \prime}$, Aroostook County, Bydrologic Unit 01010003 , on left bank 30 ft downstream from logsing route $42,0.3 \mathrm{ml}$ west of 1 ts intersection with the Carr Pond Road and $18 \mathrm{ml}$ west of Portage.

DRAINAGE AREA, $--1.69 \mathrm{mi}^{2}$.

PERIOD OF RECORD.--October 1980 to current year.

GAGE.--Water-stage recorder. Altitude of gage is $820 \mathrm{ft}$, from topographlc map.

RDMARRS.--Records good except those for winter perlod, which are fair.

EXTREMES FOR PERIOD OF RECORD.--Maximum discharge, $198 \mathrm{ft}^{3} / \mathrm{s}$ Apr. 17, 1983 , gage helght, $7.57 \mathrm{ft}$; mintmum, $0.05 \mathrm{ft}^{3} / \mathrm{s}$ July 17,1982 , gage he1ght, $4.35 \mathrm{ft}$.

EXTREMES FOR CURRENT YEAR.--Peak discharges above base of $25 \mathrm{ft}^{3} / \mathrm{s}$ and maximum (*):

\begin{tabular}{|c|c|c|c|c|c|c|c|}
\hline Date & TIme & $\begin{array}{c}\text { D1scharge } \\
\left(\mathrm{ft}^{3} / \mathrm{s}\right)\end{array}$ & $\begin{array}{c}\text { Gage Height } \\
\text { (ft) }\end{array}$ & Date & TIme & $\begin{array}{c}\text { Discharge } \\
\left(\mathrm{ft}^{3} / \mathrm{s}\right)\end{array}$ & $\begin{array}{c}\text { Gage Helght } \\
\text { (ft) }\end{array}$ \\
\hline Apr. 17 & 2215 & • 198 & 7.57 & May & 0810 & 25 & 5.35 \\
\hline Apr. 25 & 2315 & 49 & 5.80 & Jul. 22 & 0925 & 40 & 5.65 \\
\hline
\end{tabular}

Minimum discharge, $0.11 \mathrm{ft}^{3} / \mathrm{s} \mathrm{Feb.} \mathrm{3,} \mathrm{gage} \mathrm{helght,} 4.41 \mathrm{ft}$.

DISCHARGE, IN CUBIC FEET PER SECOND, WATER YEAR OCTOBER 1982 TO SEPTEMBER 1983 MEAN VALUES

\begin{tabular}{|c|c|c|c|c|c|c|c|c|c|c|c|c|}
\hline DAY & OCT & NOV & DEC & JAN & FEB & MAR & APR & MAY & JUN & JUL & AUG & SEP \\
\hline 1 & 1.1 & .34 & 2.1 & .84 & .14 & .19 & 1.4 & 8.4 & 15 & .36 & 1.3 & 2.5 \\
\hline 2 & 3.6 & .37 & 1.9 & .74 & .13 & .19 & 1.3 & 8.9 & 10 & .59 & 1.2 & .94 \\
\hline 3 & 1.9 & 1.1 & 2.2 & .64 & .13 & .22 & 1.2 & 12 & 7.3 & .93 & 1.1 & .67 \\
\hline 4 & 1.3 & .80 & 5.4 & .57 & 1.0 & .27 & 1.1 & 10 & 5.9 & .48 & .85 & .44 \\
\hline 5 & 1.0 & 4.9 & 6.5 & .53 & 3.3 & .25 & 1.5 & 9.8 & 5.0 & .63 & .75 & .30 \\
\hline 6 & .86 & 11 & 5.0 & .48 & 2.1 & .23 & 2.3 & 8.4 & 4.3 & 1.9 & .69 & .24 \\
\hline 7 & .77 & 5.2 & 5.0 & .45 & 1.7 & .21 & 4.0 & 7.1 & 6.7 & .97 & .88 & .42 \\
\hline 8 & .91 & 3.5 & 3.5 & .43 & 1.5 & .20 & 6.1 & 6.4 & 5.7 & .54 & .66 & .66 \\
\hline 9 & 2.0 & 3.1 & 2.5 & .41 & 1.4 & .19 & 7.8 & 6.6 & 3.9 & .48 & .57 & .35 \\
\hline 10 & 1.5 & 2.5 & 1.8 & .39 & 1.3 & .18 & 13 & 7.8 & 3.1 & .34 & .44 & .33 \\
\hline 11 & 1.1 & 2.0 & 1.4 & .64 & 1.1 & .22 & 12 & 6.9 & 2.7 & .31 & .37 & .30 \\
\hline 12 & .93 & 2.0 & 1.3 & 5.8 & .92 & .28 & 14 & 6.0 & 2.4 & .28 & .33 & .28 \\
\hline 13 & .87 & 4.7 & 1.1 & 4.6 & .74 & 1.3 & 13 & 6.5 & 2.2 & .46 & .29 & .20 \\
\hline 14 & 1.3 & 6.9 & 1.0 & 3.0 & .61 & 7.4 & 13 & 8.1 & 2.0 & .55 & .24 & .18 \\
\hline 15 & 2.0 & 4.2 & .92 & 2.1 & .52 & 6.0 & 12 & 6.5 & 2.1 & .50 & .22 & .17 \\
\hline 16 & 1.5 & 3.1 & 1.3 & 1.5 & .45 & 5.2 & 14 & 6.1 & 1.8 & .56 & .21 & .16 \\
\hline 17 & 1.2 & 2.6 & 4.6 & 1.1 & .39 & 4.6 & 81 & 5.5 & 1.6 & .36 & .20 & .21 \\
\hline 18 & .99 & 2.3 & 3.0 & .88 & .34 & 4.2 & 88 & 4.2 & 1.7 & .25 & .20 & .49 \\
\hline 19 & .85 & 2.0 & 2.0 & .72 & .30 & 4.0 & 25 & 2.9 & 1.5 & .20 & .19 & .42 \\
\hline 20 & .74 & 1.8 & 1.3 & .60 & .28 & 6.0 & 24 & 2.9 & 1.2 & .23 & .16 & .33 \\
\hline 21 & .69 & 1.8 & .90 & .53 & .26 & 8.5 & 18 & 3.6 & 1.0 & .56 & .15 & .25 \\
\hline 22 & .64 & 3.1 & .68 & .48 & .24 & 12 & 13 & 3.5 & .85 & 25 & .14 & 2.4 \\
\hline 23 & .54 & 5.1 & .54 & .45 & .23 & 18 & 12 & 3.1 & .75 & 10 & .16 & 4.6 \\
\hline 24 & .51 & 6.5 & .46 & .52 & .22 & 13 & 9.4 & 6.0 & .66 & 5.3 & .14 & 1.5 \\
\hline 25 & .49 & 5.5 & .50 & .58 & .21 & 8.4 & 37 & 8.5 & .58 & 5.0 & .14 & .86 \\
\hline 26 & .47 & 3.3 & 1.4 & .50 & .21 & 6.0 & 32 & 5.7 & .50 & 3.4 & .12 & .69 \\
\hline 27 & .43 & 3.2 & 4.5 & .35 & .20 & 4.0 & 16 & 4.5 & .64 & 2.4 & 1.1 & .57 \\
\hline 28 & .44 & 6.2 & 3.3 & .27 & .20 & 3.0 & 12 & 10 & .59 & 2.3 & .59 & .50 \\
\hline 29 & .42 & 5.2 & 2.0 & .22 & -- & 2.4 & 11 & 9.5 & .44 & 1.9 & .29 & .44 \\
\hline 30 & .40 & 2.9 & 1.4 & .19 & $\cdots$ & 2.0 & 9.4 & 7.4 & .37 & 1.7 & .22 & .41 \\
\hline 31 & .38 & -- & 1.0 & .16 & $-\infty$ & 1.6 & -- & 19 & -- & 1.4 & 1.3 & $-\infty$ \\
\hline TOTAL & 31.83 & 107.21 & 70.50 & 30.67 & 20.12 & 120.23 & 505.5 & 221.8 & 92.48 & 69.88 & 15.20 & 21.81 \\
\hline MEAN & 1.03 & 3.57 & 2.27 & .99 & .72 & 3.88 & 16.8 & 7.15 & 3.08 & 2.25 & .49 & .73 \\
\hline MAX & 3.6 & 11 & 6.5 & 5.8 & 3.3 & 18 & 88 & 19 & 15 & 25 & 1.3 & 4.6 \\
\hline MIN & .38 & .34 & .46 & .16 & .13 & .18 & 1.1 & 2.9 & .37 & .20 & .12 & .16 \\
\hline CFSM & .61 & 2.11 & 1.35 & .59 & .43 & 2.29 & 9.97 & 4.23 & 1.82 & 1.33 & .29 & .43 \\
\hline IN. & .70 & 2.36 & 1.55 & .68 & .44 & 2.65 & 11.1 & 4.88 & 2.04 & 1.54 & .33 & .48 \\
\hline
\end{tabular}

CAL YR 1982 TOTAL 972.32 MEAN 2.66 MAX 43 MIN .06 CFSM 1.58 IN. 21.4 WTR YR 1983 TOTAL 1307.21 MEAN 3.58 MAX 88 MIN .12 CFSM 2.12 IN. 28.8 


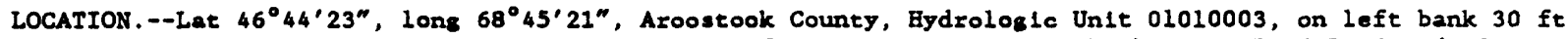
downstrean from logsing route $42,0.3 \mathrm{ml}$ west of $1 \mathrm{ts}$ Intersection with the Carr Pond Road and $18 \mathrm{ml}$ west of Portage.

DRAINAGE AREA. $--1.73 \mathrm{ml}^{2}$, revised.

PERIOD OF RECORD.--October 1980 to September 1984 (discont Inued).

GAGE.--Water-stage recorder. Alt1tude of gage is $820 \mathrm{ft}$, from topographlc map.

RMMARRs.--Records good except those for winter perlod, whlch are falr.

EXTREMS FOR PERIOD OF RECORD.--Maximum discharge, $198 \mathrm{ft} 3 / \mathrm{s}$ Apr. 17, 1983, gage helght, $7.57 \mathrm{ft}$; minlmum, $0.05 \mathrm{ft}^{3} / \mathrm{s}$ July 17,1982 , gage height, $4.35 \mathrm{ft}$.

EXTREAES FOR CURRENT YEAR.--Peak discharges above base of $25 \mathrm{ft}^{3} / \mathrm{s}$ and maximum (*):

\begin{tabular}{|c|c|c|c|c|c|c|c|}
\hline Date & Time & $\begin{array}{c}\text { Discharse } \\
\left(\mathrm{ft}^{3} / \mathrm{s}\right)\end{array}$ & $\begin{array}{c}\text { Gage Helght } \\
\text { (ft) }\end{array}$ & Date & Time & $\begin{array}{c}\text { Discharge } \\
\left(\mathrm{ft}^{3} / \mathrm{s}\right)\end{array}$ & $\begin{array}{c}\text { Gage Height } \\
(f t)\end{array}$ \\
\hline Nov. 26 & 0220 & 53 & 5.87 & Jul. 16 & 0900 & 30 & 5.45 \\
\hline Apr. 18 & 0355 & 47 & 5.77 & Aug. 15 & 1850 & * 195 & 7.55 \\
\hline Apr. 25 & 0210 & 47 & 5.77 & & & & \\
\hline
\end{tabular}

Minioum discharge, $0.27 \mathrm{ft}^{3} /$ sug $9-14$, gage he1ght, $4.49 \mathrm{ft}$.

DISCEARGE, IN CUBIC FEET PER SECOND, WATER YEAR OCTOBER 1983 TO SEPTEMBER 1984 MEAN VALUES

\begin{tabular}{|c|c|c|c|c|c|c|c|c|c|c|c|c|}
\hline DAY & OCT & NOV & $\mathrm{DEC}$ & JAN & FEB & MAR & APR & MAY & JUN & JUL & AUG & SEP \\
\hline 1 & .38 & .42 & 3.9 & 1.5 & .52 & .74 & 1.3 & 15 & 15 & 2.2 & .75 & 3.1 \\
\hline 2 & .34 & .40 & 3.4 & 1.4 & .52 & .68 & 1.4 & 9.1 & 8.0 & 1.9 & .65 & 2.3 \\
\hline 3 & .31 & .69 & 3.0 & 1.3 & .52 & .66 & 1.5 & 5.8 & 5.8 & 1.5 & .55 & 1.9 \\
\hline 4 & .30 & 3.6 & 2.6 & 1.3 & .52 & .64 & 1.6 & 5.3 & 4.7 & 1.3 & .48 & 1.8 \\
\hline 5 & 1.0 & 2.9 & 2.4 & 1.2 & 1.2 & .62 & 2.0 & 7.5 & 3.9 & 2.3 & .43 & 2.4 \\
\hline 6 & 6.9 & 3.8 & 2.2 & 1.2 & 1.1 & .60 & 2.6 & 9.5 & 3.3 & 2.8 & .38 & 2.0 \\
\hline 7 & 4.3 & 4.6 & 5.0 & 1.1 & .98 & .58 & 6.5 & 6.4 & 2.9 & 2.0 & .41 & 1.7 \\
\hline 8 & 2.0 & 3.3 & 12 & 1.0 & .87 & .56 & 5.9 & 5.2 & 2.7 & 2.8 & .42 & 1.5 \\
\hline 9 & 1.6 & 2.4 & 7.0 & 1.0 & .74 & .54 & 5.5 & 5.5 & 2.5 & 2.0 & .32 & 1.3 \\
\hline 10 & 1.3 & 1.9 & 4.3 & .95 & .64 & .53 & 5.2 & 5.3 & 2.3 & 1.4 & .29 & 1.1 \\
\hline 11 & 1.0 & 3.3 & 3.1 & .91 & .56 & .52 & 4.8 & 4.2 & 1.9 & 1.2 & .28 & 1.0 \\
\hline 12 & .92 & 6.5 & 2.5 & .87 & .50 & .51 & 6.1 & 3.9 & 1.7 & 2.3 & .29 & .88 \\
\hline 13 & 1.6 & 5.7 & 2.1 & .84 & .52 & .50 & 7.8 & 4.5 & 1.9 & 2.1 & .29 & .76 \\
\hline 14 & 2.1 & 3.6 & 11 & .80 & .60 & .48 & 13 & 4.1 & 2.5 & 1.5 & .51 & .66 \\
\hline 15 & 1.6 & 2.9 & 17 & .77 & 1.5 & .47 & 19 & 5.9 & 2.2 & 2.3 & 43 & .58 \\
\hline 16 & 1.1 & 2.5 & 9.0 & .75 & 4.6 & .46 & 25 & 4.8 & 1.6 & 19 & 35 & .51 \\
\hline 17 & .93 & 4.0 & 5.9 & .72 & 3.5 & .65 & 35 & 3.5 & 1.2 & 8.5 & 16 & .44 \\
\hline 18 & .86 & 4.4 & 4.7 & .69 & 2.6 & .60 & 30 & 2.8 & 1.1 & 4.9 & 9.7 & .39 \\
\hline 19 & .78 & 3.3 & 4.0 & .66 & 1.9 & .53 & 25 & 2.5 & 7.3 & 3.6 & 6.7 & .35 \\
\hline 20 & .71 & 2.8 & 3.6 & .64 & 1.4 & .43 & 23 & 2.5 & 5.1 & 3.0 & 5.6 & .80 \\
\hline 21 & .62 & 2.7 & 3.2 & .62 & 1.1 & .92 & 19 & 2.4 & 2.6 & 2.6 & 4.7 & .70 \\
\hline 22 & .54 & 3.4 & 2.9 & .60 & .86 & .82 & 16 & 2.1 & 2.1 & 2.2 & 3.9 & .62 \\
\hline 23 & .51 & 3.1 & 2.6 & .59 & .70 & .71 & 13 & 1.9 & 1.6 & 2.2 & 3.5 & .56 \\
\hline 24 & .57 & 2.9 & 2.4 & .58 & .58 & .62 & 31 & 2.6 & 1.3 & 2.5 & 3.9 & .51 \\
\hline 25 & .56 & 13 & 2.2 & .57 & .50 & .55 & 41 & 2.2 & 1.9 & 2.0 & 3.9 & .47 \\
\hline 26 & .48 & 35 & 2.1 & .56 & 1.1 & .50 & 26 & 1.8 & 5.2 & 1.6 & 3.0 & .44 \\
\hline 27 & .46 & 13 & 1.9 & .55 & .97 & 1.9 & 26 & 1.7 & 5.8 & 1.8 & 2.6 & .41 \\
\hline 28 & .46 & 7.8 & 1.8 & .54 & .87 & 1.7 & 26 & 1.4 & 4.0 & 1.6 & 2.3 & .38 \\
\hline 29 & .60 & 5.5 & 1.7 & .54 & .78 & 1.5 & 20 & 1.8 & 3.0 & 1.3 & 2.1 & .36 \\
\hline 30 & .54 & 4.5 & 1.6 & .53 & --- & 1.4 & 17 & 7.4 & 2.5 & 1.0 & 2.0 & .35 \\
\hline 31 & .46 & -- & 1.5 & .53 & -- & 1.2 & -- & 14 & --- & .84 & 3.1 & --- \\
\hline TOTAL & 35.83 & 153.91 & 132.6 & 25.81 & 32.75 & 23.12 & 457.2 & 152.6 & 107.6 & 88.24 & 157.05 & 30.27 \\
\hline MEAN & 1.16 & 5.13 & 4.28 & .83 & 1.13 & .75 & 15.2 & 4.92 & 3.59 & 2.85 & 5.07 & 1.01 \\
\hline MAX & 6.9 & 35 & 17 & 1.5 & 4.6 & 1.9 & 41 & 15 & 15 & 19 & 43 & 3.1 \\
\hline MIN & .30 & .40 & 1.5 & .53 & .50 & .43 & 1.3 & 1.4 & 1.1 & .84 & .28 & .35 \\
\hline CFSM & .68 & 3.04 & 2.53 & .49 & .67 & .44 & 9.02 & 2.91 & 2.12 & 1.68 & 3.00 & .60 \\
\hline IN. & .79 & 3.39 & 2.92 & .57 & .72 & .51 & 10.1 & 3.36 & 2.37 & 1.94 & 3.46 & .67 \\
\hline
\end{tabular}


01012525 BISBOP MOUNTAIN BROOR NEAR BISHOP MOUNTAIN, ME

LOCATION.--Lat $46^{\circ} 44^{\prime} 43^{\prime \prime}$, Long $68^{\circ} 45^{\prime} 11^{\prime \prime}$, Aroostook County, Hydrologic Unit 01010003, on left bank $0.3 \mathrm{mi}$ (0.5 km) north from $1088 \mathrm{ing}$ route $42,0.3 \mathrm{ml}(0.5 \mathrm{~km}$ ) west of lts intersection with the Carr Pond Road and $18 \mathrm{mi}$ ( $29 \mathrm{~km}$ ) west of Portage.

DRAINAGE AREA. $--1.04 \mathrm{mi}^{2}\left(2.69 \mathrm{~km}^{2}\right)$.

PERIOD OF RECORD.--Hov. 5, 1981 to Sept. 30, 1982.

GAGE.--Water-stage recorder. Altitude of gage $13820 \mathrm{ft}(250 \mathrm{~m})$, from topographic map.

REMARKS. --Records fair.

EXTREMES FOR CURRENT YEAR.--Peak discharges above base of $25 \mathrm{ft}^{3} / \mathrm{s}\left(0.71 \mathrm{~m}^{3} / \mathrm{s}\right)$ and maximums ( $(*)$ :

\begin{tabular}{|c|c|c|c|c|c|c|c|c|c|c|}
\hline Date & TIme & $\left(\mathrm{ft}^{3} / \mathrm{s}\right) \quad\left(\mathrm{m}^{3} / \mathrm{s}\right)$ & $\begin{array}{l}\text { Gage } \\
\text { (ft) }\end{array}$ & $\begin{array}{r}\text { Belght } \\
\text { (m) }\end{array}$ & Date & Time & $\left(f e^{\frac{D}{3} / s c h}\right.$ & $\left(m^{3} / s\right)$ & $\begin{array}{l}\text { Gage } \\
(f t)\end{array}$ & $\begin{array}{r}\text { Height } \\
\text { (m) }\end{array}$ \\
\hline $\begin{array}{l}\text { Mar. } 27 \\
\text { Apr. } 18\end{array}$ & 1205 & $\begin{array}{cc}\text { ice Jam } \\
26 & 0.74\end{array}$ & $\begin{array}{c}* 7.60 \\
--\end{array}$ & $\begin{array}{c}2.316 \\
--\end{array}$ & Apr. 27 & 0815 & $\star 61$ & 1.73 & 6.57 & 2.003 \\
\hline
\end{tabular}

Minimum discharge, $0.05 \mathrm{ft}^{3} / \mathrm{s}\left(0.001 \mathrm{~m}^{3} / \mathrm{s}\right) \mathrm{July} 10-12,15$, gage he1ght, 5.23 (1.594 $\mathrm{m}$ ).

DISCEARGE, IN CUBIC FEET PER SECOND, WATER YEAR OCTOBER 1981 TO SEPTEMBER 1982 MEAN VALUES

\begin{tabular}{|c|c|c|c|c|c|c|c|c|c|c|c|c|}
\hline DAY & OCT & NOV & DEC & JAN & FEB & MAR & APR & MAY & JUN & JUL & AUG & SEP \\
\hline 1 & --- & -- & .98 & .68 & .28 & .23 & 3.2 & 7.2 & .38 & .30 & .15 & .80 \\
\hline 2 & 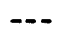 & --- & 1.1 & .67 & .27 & .22 & 5.0 & 5.0 & .50 & .24 & .14 & 7.3 \\
\hline 3 & --- & -- & 1.2 & 1.1 & .27 & .22 & 7.0 & 3.5 & 2.0 & .21 & .12 & 8.9 \\
\hline 4 &.- & $=-$ & 1.3 & 1.8 & 1.0 & .22 & 4.0 & 4.5 & 1.2 & .22 & .10 & 6.1 \\
\hline 5 & -- & 2.1 & 1.3 & 2.6 & 2.5 & .22 & 3.2 & 3.7 & .70 & .18 & .13 & 3.2 \\
\hline 6 & --- & 2.0 & 2.1 & 3.5 & 1.8 & .22 & 2.7 & 5.0 & .45 & .14 & .14 & 2.5 \\
\hline 7 & -- & 2.5 & 7.7 & 2.3 & 1.4 & .22 & 2.3 & 6.0 & .30 & .13 & .15 & 3.9 \\
\hline 8 & -- & 2.6 & 6.3 & 1.8 & 1.1 & .22 & 2.1 & 7.4 & .20 & .11 & .14 & 2.5 \\
\hline 9 & -- & 2.2 & 3.5 & 1.6 & .86 & .21 & 2.0 & 6.3 & .15 & .07 & .15 & 1.8 \\
\hline 10 & --- & 1.9 & 2.4 & 1.4 & .74 & .21 & 2.0 & 4.9 & .13 & .06 & 1.2 & 1.4 \\
\hline 11 & -- & 1.5 & 2.1 & 1.2 & .62 & .21 & 2.2 & 4.0 & .11 & .05 & .48 & 1.0 \\
\hline 12 & --- & 1.4 & 1.7 & 1.0 & .52 & .21 & 2.4 & 3.6 & .10 & .05 & .26 & .86 \\
\hline 13 & -- & 1.2 & 1.5 & .90 & .45 & .35 & 2.7 & 3.0 & .10 & .05 & .29 & .66 \\
\hline 14 & -- & 1.1 & 1.4 & .80 & .39 & .84 & 2.9 & 2.5 & .14 & .06 & .57 & .49 \\
\hline 15 & --- & 1.1 & 1.3 & .73 & .34 & .72 & 2.8 & 2.2 & .13 & .05 & .53 & .60 \\
\hline 16 & --- & 1.1 & 1.2 & .66 & .31 & .61 & 2.7 & 1.9 & .60 & .07 & .34 & 2.2 \\
\hline 17 &.- & 1.3 & 1.1 & .60 & .28 & .52 & 5.0 & 1.6 & .50 & .12 & .18 & 3.3 \\
\hline 18 & --- & 2.8 & 1.1 & .56 & .26 & .43 & 22 & 1.3 & .25 & .13 & .18 & 2.1 \\
\hline 19 & -- & 3.7 & 1.0 & .51 & .25 & .38 & 16 & 1.1 & .11 & .13 & .12 & 1.6 \\
\hline 20 & -- & 2.4 & .98 & .47 & .24 & .37 & 12 & 1.2 & .12 & .10 & .09 & 1.1 \\
\hline 21 & -- & 5.6 & .95 & .44 & .24 & .60 & 8.8 & 1.2 & .11 & .09 & .06 & .89 \\
\hline 22 & -- & 7.9 & .92 & .41 & .24 & .45 & 14 & 1.1 & .15 & .11 & .06 & .78 \\
\hline 23 & -- & 4.0 & .92 & .38 & .24 & .33 & 10 & .90 & .17 & .23 & .06 & .71 \\
\hline 24 & --- & 2.5 & .87 & .36 & .23 & .26 & 11 & .76 & .80 & .14 & .50 & .71 \\
\hline 25 & -- & 2.0 & .84 & .34 & .23 & .40 & 17 & .74 & .60 & .13 & 1.5 & .70 \\
\hline 26 & --- & 1.6 & .80 & .32 & .23 & .65 & 21 & .60 & .44 & .22 & 14 & .65 \\
\hline 27 & -- & 1.4 & .77 & .31 & .23 & 1.5 & 33 & .58 & .33 & .16 & 5.2 & .87 \\
\hline 28 & --- & 1.3 & .74 & .30 & .23 & 3.0 & 22 & .54 & .24 & .14 & 3.6 & 1.9 \\
\hline 29 & -- & 1.2 & .72 & .29 & -- & 5.0 & 16 & .52 & .26 & .70 & 2.2 & 1.9 \\
\hline 30 & --- & 1.1 & .70 & .29 & --- & 3.6 & 12 & .46 & .41 & .40 & 1.5 & 2.1 \\
\hline 31 & -- & -- & .69 & .28 & --- & 2.4 & -- & .42 & -- & .20 & 1.1 & -- \\
\hline TOTAL & --- & --- & 50.18 & 28.60 & 15.75 & 25.02 & 269.0 & 83.72 & 11.68 & 4.99 & 35.24 & 63.52 \\
\hline MEAN & -- & -- & 1.62 & .92 & .56 & .81 & 8.97 & 2.70 & .39 & .16 & 1.14 & 2.12 \\
\hline MAX & -- & --- & 7.7 & 3.5 & 2.5 & 5.0 & 33 & 7.4 & 2.0 & .70 & 14 & 8.9 \\
\hline MIN &.- & -- & .69 & .28 & .23 & .21 & 2.0 & .42 & .10 & .05 & .06 & .49 \\
\hline CFSM & -- & --- & 1.56 & .89 & .54 & .78 & 8.62 & 2.60 & .37 & .15 & 1.09 & 2.04 \\
\hline IN. & --- & -- & 1.79 & 1.02 & .56 & .89 & 9.62 & 2.99 & .42 & .18 & 1.26 & 2.27 \\
\hline
\end{tabular}


LOCATION.--Lat $46^{\circ} 44^{\circ} 43^{\prime \prime}$, Lons $68^{\circ} 45^{\prime} 11^{\prime \prime}$, Aroostook County, Bydrologic Unit 01010003, on left bank 0.3 mi north from logsing route $42,0.3 \mathrm{mi}$ west of its intersection with the Cart Pond Road and $18 \mathrm{mi}$ west of Portage.

DRAINAGE AREA. $--1.04 \mathrm{~mL}^{2}$.

PERIOD OR RECORD.--November 1981 to current year.

GAGE.--Water-stage recorder. Altitude of gage is $820 \mathrm{ft}$, from topographic map.

RMMARXS.--Records good except those for winter perlod, which are falr. No gage helght record Jan. 14 to Apr. 7 .

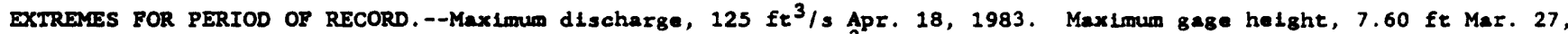
1982 (backwater from lce); minimum dally discharge, $0.04 \mathrm{ft} 3 / \mathrm{s} \mathrm{June} 27,1983$.

EXTREMES FOR CURREIT YEAR.--Peak discharges above base of $25 \mathrm{ft}^{3} / \mathrm{s}$ and maximum (*):

\begin{tabular}{|c|c|c|c|c|c|c|c|}
\hline Date & TIm & $\begin{array}{c}\text { Discharge } \\
\left(\mathrm{ft}^{3} / \mathrm{s}\right)\end{array}$ & $\begin{array}{c}\text { Gage Height } \\
\text { (ft) }\end{array}$ & Date & TImo & $\begin{array}{c}\text { Discharge } \\
\left(f \tau^{3} / s\right)\end{array}$ & $\begin{array}{c}\text { Gage Height } \\
\text { (ft) }\end{array}$ \\
\hline & -- & $\star 125$ & -- & Jul. 22 & 0445 & 41 & $\star 6.38$ \\
\hline
\end{tabular}

Minimum datly discharge, $0.04 \mathrm{ft}^{3} / \mathrm{s}$ June 27.

DISCBARGE, IN CUBIC FEET PER SECOND, WATER YEAR OCTOBER 1982 TO SEPTEMBER 1983 MEAN VALUES

\begin{tabular}{|c|c|c|c|c|c|c|c|c|c|c|c|c|}
\hline DAY & OCT & NOV & DEC & JAN & FEB & MAR & APR & MAY & JUN & JUL & AUG & SEP \\
\hline 1 & 3.4 & .60 & 2.2 & .41 & .17 & .16 & 1.5 & 5.2 & 4.5 & .06 & .96 & 3.1 \\
\hline 2 & 8.4 & .78 & 2.0 & .34 & .16 & .16 & 1.2 & 5.6 & 3.0 & .13 & .91 & 1.5 \\
\hline 3 & 5.3 & 1.9 & 4.0 & .30 & .15 & .18 & 1.1 & 8.2 & 2.2 & .21 & .85 & .83 \\
\hline 4 & 4.0 & 1.7 & 9.2 & .28 & .90 & .19 & 98 & 7.0 & $2.1^{\circ}$ & .17 & .70 & .60 \\
\hline 5 & 3.4 & 5.7 & 7.8 & .26 & 2.5 & .18 & .93 & 6.0 & 1.9 & .40 & .61 & .50 \\
\hline 6 & 1.9 & 5.3 & 6.4 & .24 & 1.9 & .17 & 2.0 & 4.9 & 1.9 & .81 & .54 & .37 \\
\hline 7 & 1.7 & 3.1 & 6.1 & .23 & 1.5 & .16 & 3.5 & 4.0 & 3.7 & .50 & .53 & .43 \\
\hline 8 & 2.1 & 2.7 & 4.6 & .22 & 1.2 & .15 & 6.0 & 3.6 & 2.4 & .29 & .45 & .50 \\
\hline 9 & 4.5 & 2.4 & 3.5 & .21 & 1.1 & .14 & 14 & 4.4 & 1.6 & .25 & .40 & .36 \\
\hline 10 & 3.9 & 2.1 & 2.1 & .20 & 1.0 & .35 & 13 & 5.2 & 1.1 & .18 & .34 & .41 \\
\hline 11 & 2.8 & 1.9 & 1.6 & 1.0 & .92 & .80 & 9.9 & 3.8 & 1.1 & .16 & .27 & .33 \\
\hline 12 & 2.3 & 1.9 & 1.3 & 6.0 & .78 & 1.5 & 12 & 3.3 & .93 & .15 & .25 & .30 \\
\hline 13 & 2.1 & 5.2 & 1.0 & 4.5 & .67 & 3.5 & 12 & 4.2 & .90 & .31 & .25 & .23 \\
\hline 14 & 3.1 & 4.1 & .83 & 3.0 & .57 & 7.0 & 12 & 4.2 & 1.3 & .43 & .23 & .18 \\
\hline 15 & 4.9 & 2.8 & .73 & 1.8 & .48 & 6.0 & 11 & 3.4 & 2.0 & .49 & .21 & .15 \\
\hline 16 & 4.1 & 2.4 & 1.5 & 1.2 & .40 & 5.0 & 12 & 3.3 & .98 & .54 & .22 & .13 \\
\hline 17 & 3.1 & 2.2 & 5.0 & .95 & .35 & 4.1 & 57 & 2.5 & .75 & .41 & .22 & .11 \\
\hline 18 & 2.4 & 2.1 & 2.1 & .78 & .30 & 3.3 & 70 & 2.3 & .65 & .25 & .21 & .34 \\
\hline 19 & 2.0 & 1.9 & 1.2 & .63 & .27 & 3.1 & 10 & 2.1 & .54 & .20 & .21 & .29 \\
\hline 20 & 1.5 & 1.8 & .82 & .54 & .25 & 5.0 & 9.8 & 2.2 & .38 & .23 & .18 & .24 \\
\hline 21 & .93 & 2.3 & .68 & .48 & .24 & 7.0 & 8.6 & 2.6 & .42 & 1.0 & .34 & .19 \\
\hline 22 & .89 & 4.3 & .57 & .42 & .22 & 10 & 7.9 & 2.5 & .29 & 23 & .17 & .75 \\
\hline 23 & .79 & 6.8 & .48 & .40 & .21 & 15 & 4.7 & 2.4 & .22 & 8.8 & .17 & 3.8 \\
\hline 24 & .74 & 8.8 & .40 & .43 & .20 & 12 & 2.9 & 5.5 & .12 & 5.8 & .17 & 2.1 \\
\hline 25 & .71 & 6.7 & .45 & .50 & .19 & 9.0 & 22 & 6.5 & .08 & 5.5 & .17 & 1.0 \\
\hline 26 & .71 & 4.5 & 1.7 & .43 & .18 & 7.0 & 18 & 3.9 & .05 & 3.8 & .18 & .48 \\
\hline 27 & .66 & 3.8 & 7.6 & .35 & .18 & 5.4 & 14 & 3.0 & .04 & 2.7 & 1.2 & .40 \\
\hline 28 & .68 & 3.7 & 2.8 & .29 & .17 & 4.0 & 12 & 8.3 & .08 & 2.7 & 1.1 & .35 \\
\hline 29 & .66 & 3.0 & 1.5 & .25 & -- & 2.8 & 8.3 & 6.1 & .05 & 2.2 & .75 & .31 \\
\hline 30 & .65 & 2.4 & .80 & .22 & --- & 2.2 & 6.0 & 5.1 & .05 & 1.8 & .55 & .28 \\
\hline 31 & .64 & -- & .51 & .19 & --- & 1.8 & -- & 7.6 & -- & 1.3 & 1.9 & -- \\
\hline TOTAX & 74.96 & 98.88 & 81.47 & 27.05 & 17.16 & 117.34 & 364.31 & 138.9 & 35.33 & 64.77 & 15.24 & 20.56 \\
\hline MEAN & 2.42 & 3.30 & 2.63 & .87 & .61 & 3.79 & 12.1 & 4.48 & 1.18 & 2.09 & .49 & .69 \\
\hline $\operatorname{MAX}$ & 8.4 & 8.8 & 9.2 & 6.0 & 2.5 & 15 & 70 & 8.3 & 4.5 & 23 & 1.9 & 3.8 \\
\hline MIN & .64 & .60 & .40 & .19 & .15 & .14 & .93 & 2.1 & .04 & .06 & .17 & .11 \\
\hline CFSM & 2.33 & 3.17 & 2.53 & .84 & .59 & 3.64 & 11.7 & 4.31 & 1.13 & 2.01 & .47 & .66 \\
\hline IN. & 2.68 & 3.54 & 2.91 & .97 & .61 & 4.20 & 13.0 & 4.97 & 1.26 & 2.32 & .55 & .74 \\
\hline
\end{tabular}

CAL YR 1982 TOTAL 792.82 MEAN 2.17 MAX 33 MIN .05 CFSM 2.09 IN. 28.4

WTR YR 1983 TOTAL 1055.96 MEAN 2.89 MAX 70 MIN .04 CFSM 2.78 IN. 37.8 
01012525 BISHOP MOUNTAIN BROOR NEAR BISHOP MOUNTAIN, ME

LOCATION.--Lat $46^{\circ} 44^{\prime} 43^{\prime \prime}$, long 68 $45^{\prime} 11^{\prime \prime}$, Aroostook County, Hydrologic Unit 01010003, on left bank 0.3 mi north from logglng route $42,0.3 \mathrm{ml}$ west of lts intersection with the Carr Pond Road and $18 \mathrm{ml}$ west of Portage.

DRAINAGE AREA. $--1.15 \mathrm{~mL}^{2}$, revised.

PERIOD OR RECORD.--November 1981 to September 1984 (discont1nued).

GAGE.--Water-stage recorder. Altitude of gage is $820 \mathrm{ft}$, from topographlc map.

RFMARKS.--Records good except those for winter perlod, which are fair.

EXIREMES FOR PERIOD OF RECORD.--Maximum discharge, $136 \mathrm{ft}^{3} / \mathrm{s}$ Aug. 15,1984 . Maximum 8 age height, $7.60 \mathrm{ft}$ Mar. 27 , 1982 (backwater from lce); minimum dally discharge, $0.04 \mathrm{ft} 3 / \mathrm{s}$ June $27,1983$.

EXTREMES FOR CURRENT YEAR.--Peak discharges above base of $25 \mathrm{ft}^{3} / \mathrm{s}$ and maximum (*):

\begin{tabular}{|c|c|c|c|c|c|c|c|}
\hline Dace & Time & $\begin{array}{c}\text { Discharge } \\
\left(f t^{3} / s\right)\end{array}$ & $\begin{array}{c}\text { Gage Helght } \\
\text { (ft) }\end{array}$ & Date & TIme & $\begin{array}{c}\text { Discharge } \\
\left(\mathrm{ft}^{3} / \mathrm{s}\right)\end{array}$ & $\begin{array}{c}\text { Gage Helght } \\
(f t)\end{array}$ \\
\hline $\begin{array}{l}\text { Nov. } 25 \\
\text { ApI. } 24\end{array}$ & $\begin{array}{l}1925 \\
1930\end{array}$ & $\begin{array}{l}26 \\
26\end{array}$ & $\begin{array}{l}6.21 \\
6.22\end{array}$ & Aug. 15 & 1840 & $\star 136$ & 7.09 \\
\hline
\end{tabular}

Minimum discharge, $0.13 \mathrm{ft}^{3} / \mathrm{s}$ Aug. 6, 7, 9-14, gage height, $3.35 \mathrm{ft}$.

DISCEARGE, IN CUBIC FEET PER SECOND, WATER YEAR OCTOBER 1983 TO SEPTEMBER 1984 MEAN VALUES

\begin{tabular}{|c|c|c|c|c|c|c|c|c|c|c|c|c|}
\hline DAY & OCT & NOV & DEC & JAN & FEB & MAR & APR & MAY & JUN & JUL & AUG & SEP \\
\hline 1 & .25 & .19 & 2.3 & .90 & .38 & .44 & .77 & 16 & 9.2 & 1.2 & .31 & .89 \\
\hline 2 & .22 & .18 & 1.9 & .86 & .38 & .42 & .84 & 12 & 5.7 & .88 & .27 & .63 \\
\hline 3 & .20 & .38 & 1.7 & .82 & .38 & .40 & .90 & 8.6 & 3.9 & .67 & .23 & .48 \\
\hline 4 & .19 & 2.4 & 1.6 & .78 & .38 & .38 & 1.0 & 8.0 & 3.0 & .55 & .20 & .46 \\
\hline 5 & .90 & 1.9 & 1.4 & .75 & .73 & .37 & 1.2 & 10 & 2.5 & .89 & .17 & .71 \\
\hline 6 & 4.2 & 2.7 & 1.3 & .72 & .70 & .36 & 1.6 & 11 & 2.1 & 1.0 & .15 & .61 \\
\hline 7 & 2.4 & 3.0 & 3.0 & .69 & .62 & .35 & 3.5 & 8.5 & 2.0 & .86 & .16 & .46 \\
\hline 8 & 1.4 & 2.0 & 7.0 & .66 & .52 & .34 & 3.2 & 7.0 & 1.9 & 1.4 & .17 & .39 \\
\hline 9 & 1.0 & 1.5 & 3.5 & .63 & .45 & .33 & 3.0 & 8.3 & 1.7 & .95 & .15 & .34 \\
\hline 10 & .78 & 1.1 & 1.7 & .61 & .39 & .32 & 2.8 & 7.0 & 1.3 & .67 & .14 & .30 \\
\hline 11 & .61 & 2.0 & 1.4 & .59 & .34 & .32 & 2.7 & 5.8 & 1.0 & .51 & .14 & .27 \\
\hline 12 & .58 & 4.9 & 1.3 & .57 & .30 & .31 & 3.3 & 5.6 & .86 & 1.2 & .14 & .25 \\
\hline 13 & .90 & 3.4 & 1.2 & .55 & .32 & .30 & 4.2 & 5.7 & .95 & 1.1 & .15 & .23 \\
\hline 14 & 1.3 & 1.9 & 5.0 & .53 & .34 & .29 & 6.0 & 3.5 & 1.2 & .77 & .22 & .22 \\
\hline 15 & 1.0 & 1.4 & 7.2 & .51 & .60 & .28 & 8.4 & 7.4 & 1.3 & 1.1 & 24 & .21 \\
\hline 16 & .70 & 1.2 & 5.9 & .50 & 3.0 & .28 & 12 & 5.2 & .94 & 8.9 & 13 & .20 \\
\hline 17 & .48 & 2.5 & 3.2 & .49 & 2.2 & .41 & 17 & 3.9 & .69 & 4.1 & 4.7 & .19 \\
\hline 18 & .35 & 2.7 & 2.5 & .48 & 1.6 & .35 & 15 & 3.3 & .62 & 2.0 & 2.5 & .18 \\
\hline 19 & .27 & 2.0 & 2.2 & .47 & 1.2 & .30 & 13 & 2.9 & 5.3 & 1.6 & 1.9 & .17 \\
\hline 20 & .24 & 1.6 & 2.0 & .46 & .90 & .26 & 13 & 2.8 & 2.8 & 1.2 & 1.6 & .50 \\
\hline 21 & .20 & 1.7 & 1.9 & .45 & .71 & .56 & 12 & 2.6 & 1.6 & .89 & 1.3 & .43 \\
\hline 22 & .18 & 2.2 & 1.7 & .44 & .58 & .49 & 11 & 2.1 & 1.3 & .76 & 1.0 & .38 \\
\hline 23 & .17 & 1.9 & 1.6 & .43 & .45 & .43 & 13 & 2.0 & .95 & .75 & .91 & .34 \\
\hline 24 & .19 & 1.8 & 1.4 & .42 & .35 & .38 & 18 & 2.8 & .73 & .97 & 1.1 & .31 \\
\hline 25 & .18 & 9.8 & 1.4 & .42 & .39 & .33 & 18 & 2.3 & 1.1 & .74 & 1.2 & .29 \\
\hline 26 & .16 & 11 & 1.3 & .41 & .70 & .30 & 15 & 1.9 & 3.5 & .62 & .85 & .27 \\
\hline 27 & .16 & 6.2 & 1.2 & .41 & .56 & 1.1 & 16 & 1.7 & 3.5 & .76 & .68 & .25 \\
\hline 28 & .16 & 4.0 & 1.1 & .40 & .50 & .98 & 15 & 1.4 & 2.2 & .75 & .56 & .23 \\
\hline 29 & .22 & 3.0 & 1.1 & .39 & .46 & .90 & 15 & 1.8 & 1.8 & .59 & .47 & .22 \\
\hline 30 & .22 & 2.6 & 1.0 & .39 & --- & .82 & 15 & 7.2 & 1.5 & .45 & .45 & .20 \\
\hline 31 & .20 & -- & .95 & .38 & $-\infty$ & .75 & -- & 11 & --- & .36 & .90 & --- \\
\hline TOTAL & 20.01 & 83.15 & 71.95 & 17.11 & 20.45 & 13.85 & 261.41 & 181.3 & 67.14 & 39.19 & 59.72 & 10.61 \\
\hline MEAN & .65 & 2.77 & 2.32 & .55 & .71 & .45 & 8.71 & 5.85 & 2.24 & 1.26 & 1.93 & .35 \\
\hline MAX & 4.2 & 11 & 7.2 & .90 & 3.0 & 1.1 & 28 & 16 & 9.2 & 8.9 & 24 & .89 \\
\hline MIN & .16 & .18 & .95 & .38 & .30 & .26 & .77 & 1.4 & .62 & .36 & .14 & .17 \\
\hline CESM & .62 & 2.67 & 2.23 & .53 & .68 & .43 & 8.38 & 5.62 & 2.15 & 1.22 & 1.85 & .34 \\
\hline IN. & .72 & 2.97 & 2.57 & .61 & .73 & .50 & 9.35 & 6.48 & 2.40 & 1.40 & 2.14 & .38 \\
\hline
\end{tabular}

CAL YR 1983 TOTAL 975.76 MEAN 2.67 MAX 70 MIN .04 CFSM 2.57 IN. 34.9

WTR YR 1984 TOTAL 845.88 MEAN 2.31 MAX 24 MIN .14 CFSM 2.22 IN. 30.3 
01012570 FISH RIVER AT INLET FISH RIVER LARE, ME

LOCATION,--Lat $46^{\circ} 47^{\prime} 22^{\prime \prime}$, Long $68^{\circ} 46^{\prime} 25^{\prime \prime}$, Aroostook County, Hydrologic Unit ol010003, on right bank 30 ft (9 m) upatream from Fox Brook tote Road and $20 \mathrm{ml}(32 \mathrm{~km}$ ) west of Portage.

DRAINAGE AREA. $--70.3 \mathrm{mi}^{2}\left(182.1 \mathrm{~km}^{2}\right)$.

PERIOD OF RECORD.--July 16 to Sept. 30, 1982.

GAGE.--Water-stage recorder. Altitude of gage is $720 \mathrm{ft}(219 \mathrm{~m})$, from topographic map.

RMMARKS. - Records fair.

EXTREMES FOR CURRENT YEAR.--Peak discharges above base of $300 \mathrm{ft}^{3} / \mathrm{s}(8.50 \mathrm{~m} / \mathrm{s})$ and maximum (*):

\begin{tabular}{|c|c|c|c|c|c|c|c|c|}
\hline Date & TImo & $\begin{array}{l}\text { Discharge } \\
(\mathrm{ft} / \mathrm{s}) \quad\left(\mathrm{m}^{3} / \mathrm{s}\right)\end{array}$ & $\begin{array}{l}\text { Gage HeIght } \\
\begin{array}{ll}\text { (ft) } & \text { (m) }\end{array}\end{array}$ & Date & IIme & $\begin{array}{l}\text { Discharge } \\
\left(\mathrm{et}^{3} / \mathrm{s}\right) \quad\left(\mathrm{m}^{3} / \mathrm{s}\right)\end{array}$ & $\begin{array}{l}\text { Gage } \\
(f t)\end{array}$ & $\begin{array}{r}\text { Height } \\
\text { (m) }\end{array}$ \\
\hline Aus. 26 & 1100 & $\star 437$ & 1.530 & Sept. 3 & 1830 & 9.80 & 4.32 & 1.378 \\
\hline
\end{tabular}

Minlmum dlacharge, $2.5 \mathrm{ft}^{3} / \mathrm{s}\left(0.071 \mathrm{~m}^{3} / \mathrm{s}\right) \mathrm{July} 25$, gage height, $1.26 \mathrm{ft}$ (0.384 $\left.\mathrm{m}\right)$.

DISCRARGE, IN CUBIC FEET PER SECOND, WATER YEAR OCTOBER 1981 TO SEPTEMBER 1982 MEAN VALUES

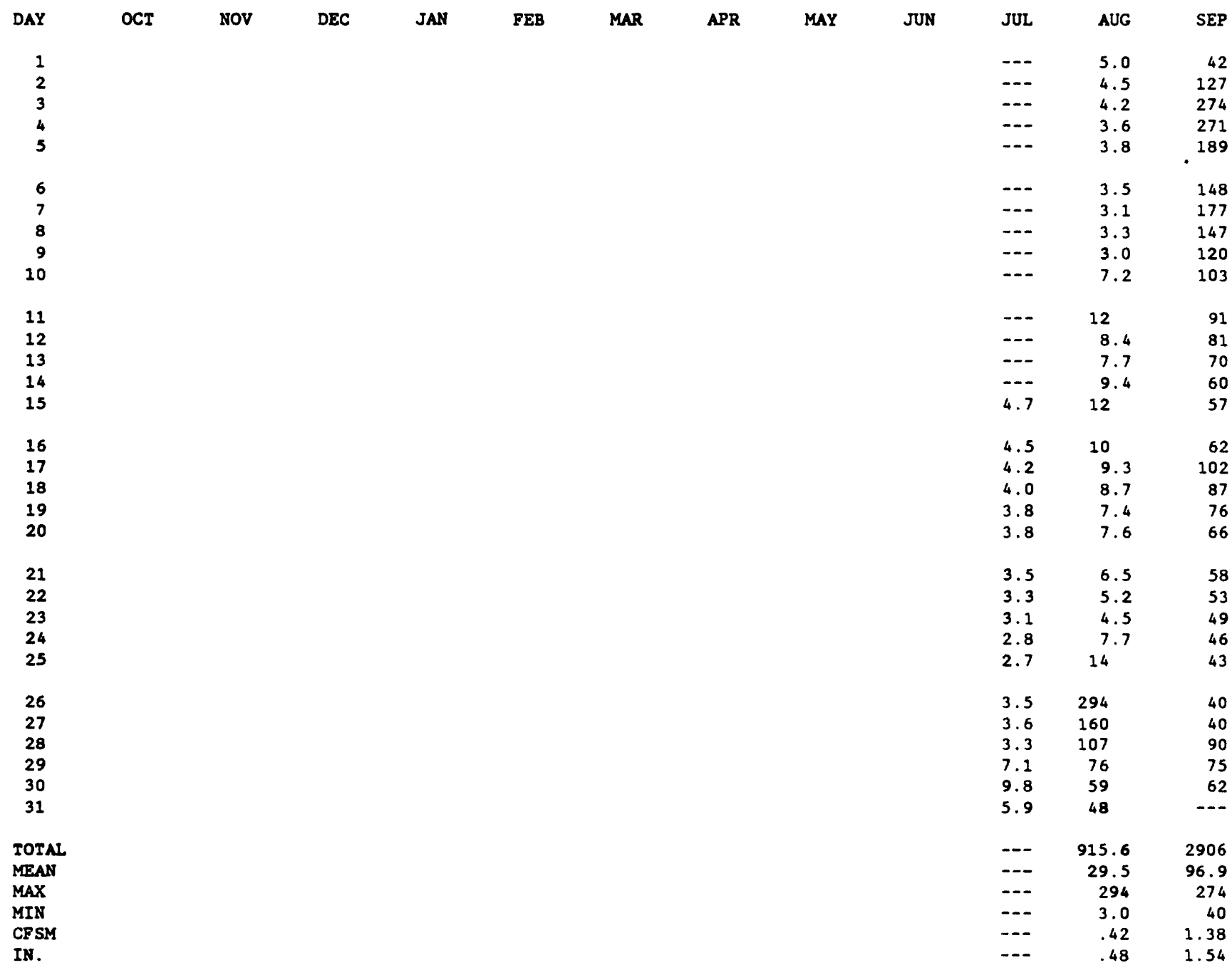


LOCATION.--Lat $46^{\circ} 47^{\prime} 22^{\prime \prime}$, Long $68^{\circ} 46^{\prime} 25^{\prime \prime}$, Aroostook County, Hydrologic Unit 01010003, on right bank 30 ft upstream from Fox Brook Tote Road and $20 \mathrm{ml}$ west of Portage.

DRAINAGE AREA. $--70.3 \mathrm{mi}^{2}$

PERIOD OE RECORD.--July 16 to current year.

GAGE.--Water-stage recorder. Altitude of gage $13720 \mathrm{ft}$, from topographlc map.

REMARKS.--Records good except those for winter perlod, which are falr.

EXTREMES FOR PERIOD OF RECORD.--Maximum discharge, $1650 \mathrm{ft}^{3} / \mathrm{s}$ Apr. 18, 1983, gage he1ght, $9.93 \mathrm{ft}$; minimum discharge $2.5 \mathrm{ft}^{3} / \mathrm{s} \mathrm{July} 25,1982$, gage helght, $1.26 \mathrm{ft}$.

EXTREMES FOR CURRENT YEAR.--Peak discharges above base of $550 \mathrm{ft}^{3} / \mathrm{s}$ (revised) and maximum (*):

\begin{tabular}{|c|c|c|c|c|c|c|c|}
\hline Date & TIme & $\begin{array}{c}\text { Discharge } \\
\left(\mathrm{ft}^{3} / \mathrm{s}\right)\end{array}$ & $\begin{array}{c}\text { Gage Helght } \\
\text { (ft) }\end{array}$ & Date & TIme & $\begin{array}{c}\text { Discharge } \\
\left(\mathrm{ft}^{3} / \mathrm{s}\right)\end{array}$ & $\begin{array}{c}\text { Gage Helght } \\
(f t)\end{array}$ \\
\hline Apr. 18 & 1015 & $\star 1650$ & 9.93 & May & 1500 & 823 & 6.81 \\
\hline Apr. 26 & 0615 & 1240 & 8.47 & Jul. 22 & 1430 & 554 & 5.70 \\
\hline May 28 & 1945 & 573 & 5.70 & & & $\cdot$ & \\
\hline
\end{tabular}

Minimum discharge, $2.8 \mathrm{ft}^{3} / \mathrm{s}$ August 26, gage he1ght, $1.29 \mathrm{ft}$.

DISCEARGE, IN CUBIC FEET PER SECOND, WATER YEAR OCTOBER 1982 TO SEPTEMBER 1983 MEAN VALUES

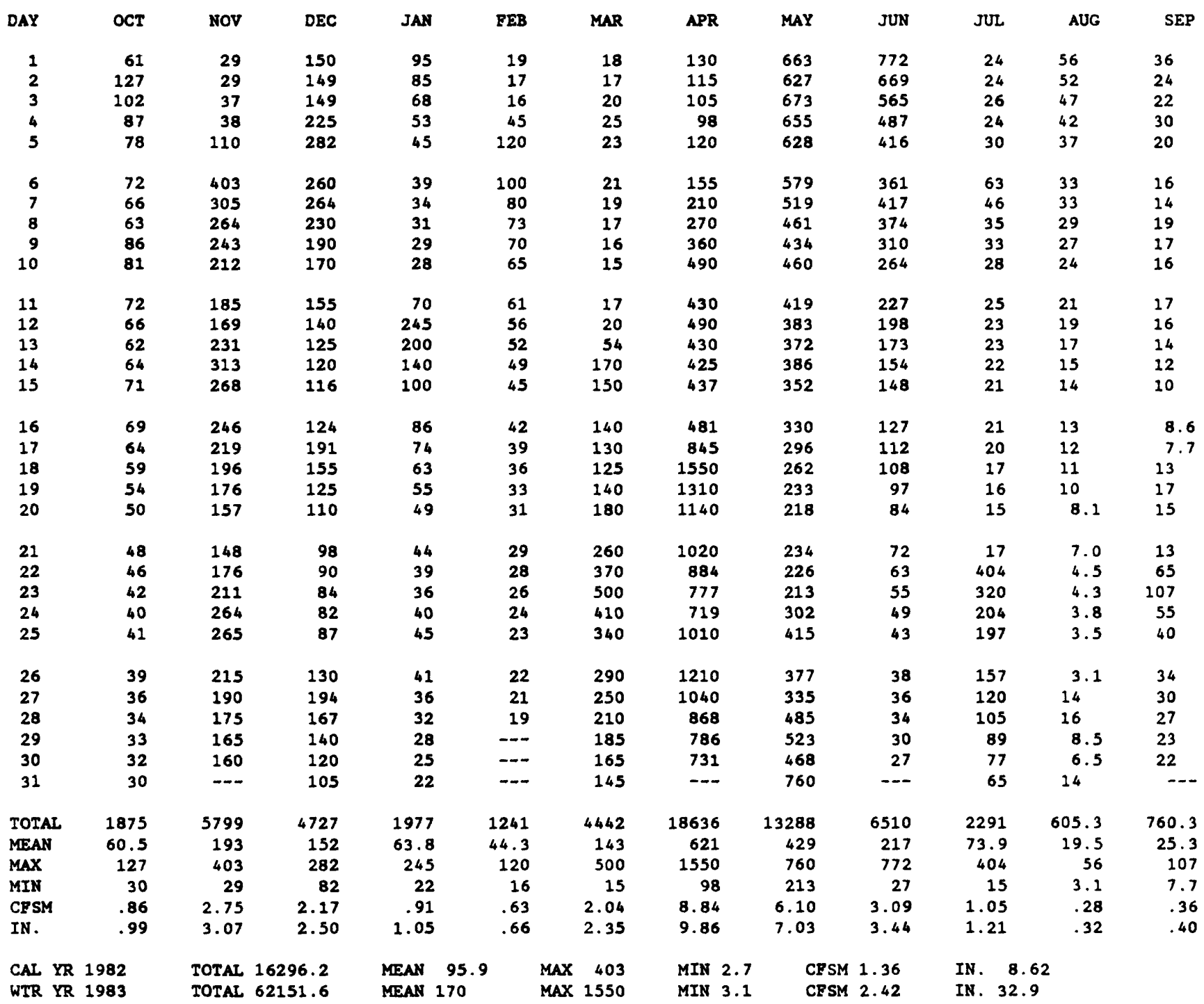


01012570 FISH RIVER AT INLET FISH RIVER LARE, ME

LOCATION.--Lat $46^{\circ} 47^{\prime} 22^{\prime \prime}$, long $68^{\circ} 46^{\prime} 23^{\prime \prime}$, Aroostook County, Hydrologle Unit 01010003, on right bank 30 ft upstrean from Fox Brook Tote Road and $20 \mathrm{ml}$ west of Portage.

DRAINAGE AREA. $-70.3 \mathrm{mi}^{2}$

PERIOD OF RECORD.--July 1982 to June 1984 (d1scontinued).

GAGE.--Water-stage recorder. Altitude of gage is $720 \mathrm{ft}$, from topographic map.

RMMARRS. - Records good except those for winter period, which are falr.

EXTREMES FOR PERIOD OF RECORD. - Maximum discharge, $1650 \mathrm{ft}^{3} / \mathrm{s}$ Apr. 18, 1983, gage height, $9.93 \mathrm{ft}$; minimum discharge $2.5 \mathrm{ft}^{3} / \mathrm{s}$ July 25,1982 , gage helght, $1.26 \mathrm{ft}$.

EXIREMES FOR CURRENT YEAR.--Peak discharges above base of $550 \mathrm{ft}^{3} / \mathrm{s}$ and maximum (*) during period October 1983 to June 1984:

\begin{tabular}{|c|c|c|c|c|c|c|c|}
\hline Date & TIme & $\begin{array}{c}\text { Discharge } \\
\left(\mathrm{ft}^{3} / \mathrm{s}\right)\end{array}$ & $\begin{array}{c}\text { Gage Helght } \\
\text { (ft) }\end{array}$ & Date & Time & $\begin{array}{c}\text { Discharge } \\
\left(\mathrm{ft}^{3} / \mathrm{s}\right)\end{array}$ & $\begin{array}{c}\text { Gage Helght } \\
(f t)\end{array}$ \\
\hline $\begin{array}{ll}\text { Nov. } 26 \\
\text { Apr. } 18\end{array}$ & $\begin{array}{l}1315 \\
0430\end{array}$ & $\begin{array}{r}954 \\
+\quad 1610\end{array}$ & $\begin{array}{l}7.34 \\
8.18\end{array}$ & $\begin{array}{cc}\text { May } & 01 \\
\text { Jun. } & 01\end{array}$ & $\begin{array}{l}0600 \\
0730\end{array}$ & $\begin{array}{r}1480 \\
755\end{array}$ & $\begin{array}{r}7.93 \\
26.70\end{array}$ \\
\hline
\end{tabular}

a Backwater from highway bridge construction.

Minimum discharge, $17 \mathrm{ft}^{3} / \mathrm{s}$ October 3,4 , gage helght, $1.66 \mathrm{ft}$.

DISCAARGE, IN CUBIC FEET PER SECOND, WATER YEAR OCTOBER 1983 TO SEPTEMBER 1984 MEAN VALUES

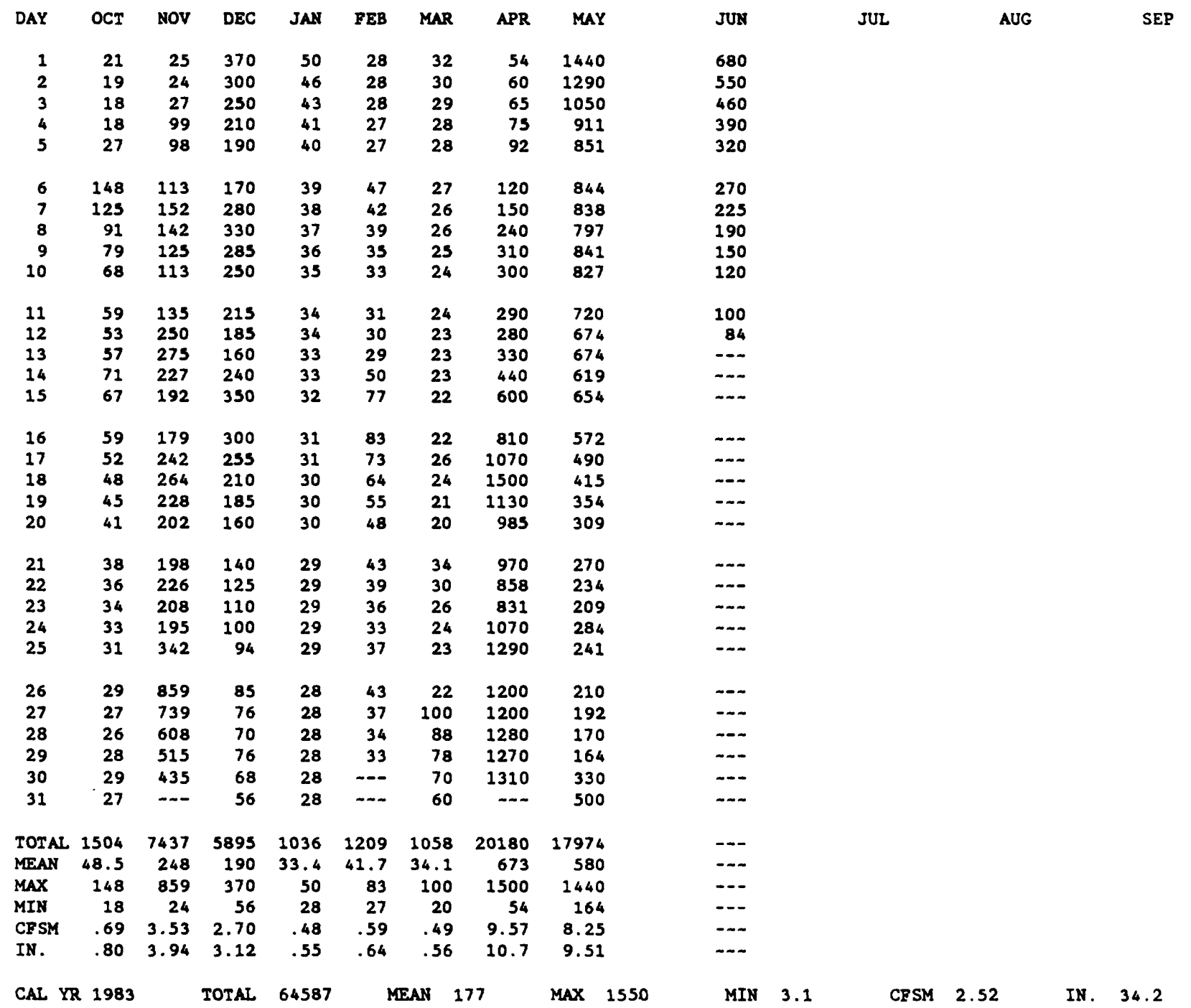


TAmB 4.--Physical characteristics and concentrations of common chemical constituents in lakes and streams of the Bald Mountain watersheds

01012515 CLAYTOA STREAM AT OUTLET CLAYTON LAKE ME SITE 6

\begin{tabular}{|c|c|c|c|c|c|c|c|c|c|c|}
\hline DATE & TIME & $\begin{array}{l}\text { STREAM- } \\
\text { FLOW, } \\
\text { INSTAN- } \\
\text { TANEOUS } \\
\text { (CFS) }\end{array}$ & $\begin{array}{l}\text { SPE- } \\
\text { CIFIC } \\
\text { CON- } \\
\text { DUCT- } \\
\text { ANCE } \\
\text { (US/CM) }\end{array}$ & $\begin{array}{c}\text { P日 } \\
\text { (STAND- } \\
\text { ARD } \\
\text { UNITS) }\end{array}$ & $\begin{array}{l}\text { TEMPER- } \\
\text { ATURE } \\
\text { WATER } \\
\text { (DEG C) }\end{array}$ & $\begin{array}{l}\text { COLOR } \\
\text { (PLAT- } \\
\text { INUM- } \\
\text { COBALT } \\
\text { UNITS) }\end{array}$ & $\begin{array}{l}\text { TUR- } \\
\text { BID- } \\
\text { ITY } \\
\text { (FTU) }\end{array}$ & $\begin{array}{l}\text { OXYGEN, } \\
\text { DIS- } \\
\text { SOLVED } \\
\text { (MG/L) }\end{array}$ & $\begin{array}{l}\text { OXYGEN } \\
\text { DEMAND, } \\
\text { BIO- } \\
\text { CHEM- } \\
\text { ICAL, } \\
5 \text { DAY } \\
\text { (MG/L) }\end{array}$ & $\begin{array}{l}\text { COLI- } \\
\text { FORM, } \\
\text { FECAL, } \\
0.45 \\
\text { UM-MF } \\
\text { (COLS. } 1 \\
100 \mathrm{ML})\end{array}$ \\
\hline \multicolumn{11}{|l|}{ AUG 1979} \\
\hline $07 \ldots$ & 1500 & -- & 41 & 6.8 & 21.0 & 55 & 1.5 & 8.3 & 0.3 & K11 \\
\hline$\underset{\text { SEP }}{21 \ldots}$ & 0900 & -- & 34 & 6.7 & 20.0 & 60 & 1.5 & 11.2 & 2.1 & -- \\
\hline $04 \ldots$ & 0900 & -- & 42 & 6.8 & 20.0 & 50 & 1.5 & 9.1 & .5 & $\mathrm{k} 6$ \\
\hline $\mathrm{OCT}^{18 \ldots}$ & 0900 & -- & 38 & 6.8 & 18.0 & 70 & 1.8 & 8.7 & -- & K9 \\
\hline $\begin{array}{l}22 \ldots \\
\text { JAN } 1980\end{array}$ & 1000 & -- & 42 & 6.3 & 10.0 & 70 & 1.6 & 9.6 & 1.2 & -- \\
\hline FEB & 1100 & -- & 54 & 6.8 & 0.0 & -- & 1.3 & 14.8 & - & -- \\
\hline $\begin{array}{l}19 \ldots \\
\text { MAR }\end{array}$ & 1100 & -- & 62 & 6.7 & 0.0 & 70 & 1.1 & 13.1 & 1.0 & K3 \\
\hline${ }_{A P R}^{20} \cdots$ & 1100 & -- & 52 & 6.4 & 2.0 & 60 & 1.7 & 12.0 & 2.8 & K3 \\
\hline$\stackrel{23}{23} \cdots$ & 1100 & $-\infty$ & 38 & 6.3 & 2.0 & 40 & 5.8 & 12.2 & 1.3 & $<1$ \\
\hline JUN & 1020 & -- & 30 & 7.0 & 14.0 & 35 & 1.5 & 9.8 & 2.1 & K1 \\
\hline JUL & 1120 & -- & 35 & 6.3 & 15.0 & 50 & 3.7 & 8.5 & .8 & -- \\
\hline AUG & 1120 & -- & 39 & 6.1 & 20.0 & 53 & 3.3 & 7.4 & -- & -- \\
\hline${ }_{\text {SEP }}^{29}$ & 1015 & -- & 40 & 6.3 & 20.0 & 45 & 1.4 & 7.6 & -- & -- \\
\hline${ }_{\mathrm{OCT}}^{29 \ldots}$ & 1020 & -- & 34 & 6.9 & 8.0 & 50 & 2.3 & 11.0 & -- & $-\infty$ \\
\hline $14 \ldots$ & 1030 & -- & 52 & 6.3 & 6.0 & 55 & 1.8 & 11.2 & -- & -- \\
\hline DATE & $\begin{array}{l}\text { ALRA- } \\
\text { LINITY } \\
\text { WAT WB } \\
\text { TOT FET } \\
\text { LAB } \\
\text { MG/L AS }\end{array}$ & $\begin{array}{l}\text { SOLIDS, } \\
\text { RESIDUE } \\
\text { AT } 105 \\
\text { DEG. C, } \\
\text { TOTAL }\end{array}$ & $\begin{array}{l}\text { SOLIDS, } \\
\text { VOLA- } \\
\text { TILE ON } \\
\text { IGNI- } \\
\text { TION, } \\
\text { TOTAL }\end{array}$ & $\begin{array}{l}\text { NITRO- } \\
\text { GEN, } \\
\text { NO2+NO3 } \\
\text { TOTAL } \\
\text { (MG/L }\end{array}$ & $\begin{array}{l}\text { NITRO- } \\
\text { GEN, } \\
\text { AMONIA } \\
\text { TOTAL } \\
\text { (MG /L }\end{array}$ & $\begin{array}{l}\text { NITRO- } \\
\text { GEN, } \\
\text { ORGANIC } \\
\text { TOTAL } \\
\text { (MG/L }\end{array}$ & $\begin{array}{l}\text { NITRO- } \\
\text { GEN, AM- } \\
\text { MONIA + } \\
\text { ORGANIC } \\
\text { TOTAL } \\
\text { (MG/L }\end{array}$ & $\begin{array}{l}\text { NITRO- } \\
\text { GEN, } \\
\text { TOTAL } \\
\text { (MG/L }\end{array}$ & $\begin{array}{l}\text { NITRO- } \\
\text { GEN, } \\
\text { TOTAL } \\
\text { (MG/L }\end{array}$ & $\begin{array}{l}\text { PHOS- } \\
\text { PHOROUS } \\
\text { TOTAL } \\
\text { (MG/L }\end{array}$ \\
\hline & $\mathrm{CACO} 3$ & (MG/L) & $(M G / L)$ & AS $N$ ) & AS N) & AS N) & AS N) & AS N) & AS NO3) & AS P) \\
\hline
\end{tabular}

AUG 1979

$07 \ldots$

$21 \ldots$

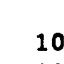

(a) 0.01

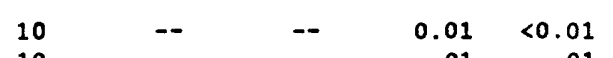

SEP

$04 \ldots$

$18 . .$.

OCT

$22 .$.

JAN 1980

$29 .$.

FEB
$19 . .$.
MAR

$20 .$. .

APR

$23 . .$.

MAY

$20 \ldots$

JUN

$17 \ldots$

10

8

11

JUL

13...

AUG

29 ...

SEP

$29 . .$.

OCT

$14 \ldots$

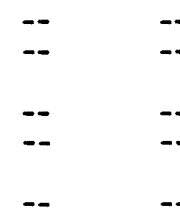

28

$28 \quad 73$

1250

--
--
--
-
-

$.01 \quad .01$

$<.01<.01$

.03

4

5

$11 \quad 67$

1060

$11 \quad 48$

1153

1456 
INI 4.--Physical characteristics and concentrations of comnon chemical constituents in lakes and streams of the Bald Mountain watersheds--Continued

01012515 CLAYTON STREAM AT OUTLET CLAYTON LARE ME SITE 6

\begin{tabular}{|c|c|c|c|c|c|c|c|c|}
\hline DATE & $\begin{array}{l}\text { ALUM- } \\
\text { INUM, } \\
\text { TOTAL } \\
\text { RECOV- } \\
\text { ERABLE } \\
\text { (UG/L }\end{array}$ & $\begin{array}{l}\text { ALUM- } \\
\text { INUM, } \\
\text { DIS- } \\
\text { SOLVED } \\
\text { (UG/L }\end{array}$ & $\begin{array}{l}\text { ARSENIC } \\
\text { TOTAL } \\
\text { (UG/L }\end{array}$ & $\begin{array}{l}\text { CADMIUM } \\
\text { TOTAL } \\
\text { RECOV- } \\
\text { ERABLE } \\
\text { (UG/L }\end{array}$ & $\begin{array}{l}\text { CERO- } \\
\text { MIUM, } \\
\text { TOTAL } \\
\text { RECOV- } \\
\text { ERABLE } \\
\text { (UG/L }\end{array}$ & $\begin{array}{l}\text { COPPER, } \\
\text { TOTAL } \\
\text { RECOV- } \\
\text { ERABLE } \\
\text { (UG/L }\end{array}$ & $\begin{array}{l}\text { COPPER, } \\
\text { DIS- } \\
\text { SOLVED } \\
\text { (UG/L }\end{array}$ & $\begin{array}{l}\text { IRON, } \\
\text { TOTAL } \\
\text { RECOV- } \\
\text { ERABLE } \\
\text { (UG/L }\end{array}$ \\
\hline & AS AL) & AS AL) & AS AS) & AS CD) & AS CR) & AS CU) & AS CU) & AS FE) \\
\hline
\end{tabular}

AUG 1979

07 ...

21 ..

SEP

$04 \ldots$

$18 \ldots$

OCT

22 ...

JAN 1980

29 ...

FEB

19...

MAR

20 ...

APR

23 ..

MAY

20 ...

JUस

$17 \ldots$

JUL

13 ..

AUG

29...

SEP

29...

OCT

14 ...

--
--
--

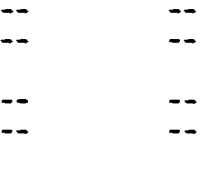

--
--
--

--
--
--
$<2$

$<6$

$<4$

$-$

190

$-\infty \quad--\quad<2$

$<2$

$<2$

$-$

$<2$

$<2$

$<2$

$-$

$<6$

$--$

$<4$

250

$<6$

$<4$

$<6$

$<4$

250

$-$

$<4$

250

$<5$

$<10$

$<20$

$<4$

$<5$

2

220

$<5$

2

290

4

320

$<4$

$-$

200

$<4$

$<6$

190

\begin{tabular}{|c|c|c|c|c|c|c|c|c|}
\hline DATE & $\begin{array}{l}\text { IRON, } \\
\text { DIS- } \\
\text { SOLVED } \\
\text { (UG/L }\end{array}$ & $\begin{array}{l}\text { LEAD, } \\
\text { TOTAL } \\
\text { RECOV- } \\
\text { ERABLE } \\
\text { (UG/L }\end{array}$ & $\begin{array}{l}\text { LEAD, } \\
\text { DIS- } \\
\text { SOLVED } \\
\text { (UG/L }\end{array}$ & $\begin{array}{l}\text { MERCURY } \\
\text { TOTAL } \\
\text { RECOV- } \\
\text { ERABLE } \\
\text { (UG/L }\end{array}$ & $\begin{array}{l}\text { ZINC, } \\
\text { TOTAL } \\
\text { RECOV- } \\
\text { ERABLE } \\
\text { (UG/L }\end{array}$ & $\begin{array}{l}\text { 2INC, } \\
\text { DIS- } \\
\text { SOLVED } \\
\text { (UG/L }\end{array}$ & $\begin{array}{l}\text { SEDI - } \\
\text { MENT, } \\
\text { SUS- } \\
\text { PENDED }\end{array}$ & $\begin{array}{c}\text { SEDI- } \\
\text { MENT, } \\
\text { DIS- } \\
\text { CHARGE, } \\
\text { SUS- } \\
\text { PENDED }\end{array}$ \\
\hline & AS FE) & AS PB) & AS PB) & AS HG) & AS $2 N$ ) & AS $2 N$ ) & (MG/L) & (T/DAY) \\
\hline
\end{tabular}

AUG 1979

$07 \ldots$

$21 \ldots$

SEP

$04 \ldots$

OCT

22 ...

JAN 1980

29...

FEB

$19 .$.

MAR

20...

APR

23 ...

MAY

20...

JUN

$17 .$.

$\begin{array}{ll}-- & <20 \\ - & \\ -- & <2 \\ -- & <20 \\ -- & <20 \\ -- & <20 \\ -- & <30 \\ -- & 30 \\ -- & <30 \\ -- & -- \\ -- & - \\ -- & -\end{array}$

JUL

13...

AUG

$29 .$.

SEP

29...

OCT

$14 .$.

$\begin{array}{lr}-- & 1.3 \\ -- & -- \\ -- & -- \\ -- & <0.2 \\ -- & <.2 \\ -- & <.2 \\ -- & -- \\ -- & -- \\ -- & -- \\ -- & .3 \\ -- & -- \\ -- & -- \\ -- & -\end{array}$

$$
\begin{gathered}
<2 \\
-- \\
-- \\
-- \\
-- \\
<2 \\
8 \\
2 \\
4 \\
30 \\
20 \\
5 \\
6 \\
6
\end{gathered}
$$

$<2 \quad-$

$-$

$<5$ 
TABIE 4.--Physical characteristics and concentrations of common chemical constituents in lakes and streams of the Bald Mountain watersheds-Continued

01012515 CLAYTON STREAM AT OUTLET CLAYTON LAKE ME SITE 6

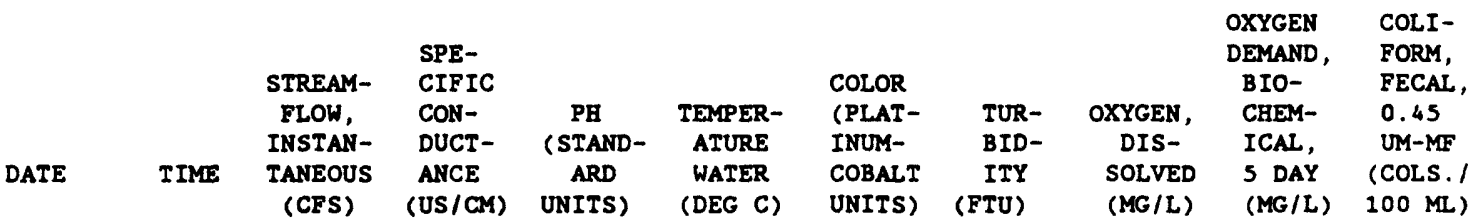

\begin{tabular}{|c|c|c|c|c|c|c|c|c|c|c|}
\hline${ }_{D E C}^{21 \ldots}$ & 1020 & - & 32 & 6.1 & 0.0 & 55 & 2.1 & 13.0 & -- & -- \\
\hline $\begin{array}{lll}12 & \ldots \\
\text { JAN } 1981\end{array}$ & 1045 & - & 50 & 6.1 & 0.0 & 65 & 1.5 & 13.1 & -- & - \\
\hline$\underset{\text { FEB }}{20} \ldots$ & 1100 & - & 53 & 6.1 & 0.0 & 60 & 1.8 & 11.6 & -- & -- \\
\hline $\operatorname{MAR}^{23 \ldots}$ & 1740 & - & 30 & -- & 2.0 & 70 & 3.4 & 12.2 & -- & -- \\
\hline${ }_{A P R}^{25} \cdots$ & 1015 & -- & 31 & 6.1 & 2.0 & 40 & 2.3 & 11.8 & -- & -- \\
\hline $\operatorname{MAY}^{14} \cdots$ & 1020 & -- & 23 & 6.1 & 2.0 & 45 & 3.1 & 11.4 & -- & -- \\
\hline JUN & 1107 & -- & 21 & 6.6 & 12.0 & 55 & 1.7 & 10.4 & -- & -- \\
\hline $\mathrm{JuL}^{10 \ldots}$ & 1000 & - & 31 & 6.6 & 13.0 & 50 & 1.7 & 9.3 & -- & -- \\
\hline${ }_{A \cup G}^{21} \ldots$ & 1000 & -- & 45 & 6.6 & -- & 50 & 1.8 & -- & -- & -- \\
\hline $\operatorname{SEP}^{24 \ldots}$ & 1210 & - & 30 & 6.1 & 19.0 & 100 & 1.4 & 7.9 & -- & -- \\
\hline${ }_{\mathrm{OCT}}^{22 \ldots}$ & 1345 & - & 30 & 7.0 & 13.0 & 90 & 1.9 & 9.5 & -- & -- \\
\hline Nov 20. & 1345 & - & $-\infty$ & 6.3 & 6.0 & 90 & 2.1 & 13.2 & -- & - \\
\hline${ }_{\text {DEC }}^{17 \ldots}$ & 1405 & -- & 35 & 6.1 & 4.0 & 60 & 1.8 & 10.5 & -- & -- \\
\hline $15 \ldots$ & 1240 & - & 58 & 6.2 & 1.0 & 60 & 1.5 & 13.4 & -- & -- \\
\hline DATE & $\begin{array}{l}\text { ALKA- } \\
\text { LINITY } \\
\text { WAT WB } \\
\text { TOT FET } \\
\text { LAB } \\
\text { MG/L AS } \\
\text { CACO3 }\end{array}$ & $\begin{array}{l}\text { SOLIDS, } \\
\text { RESIDUE } \\
\text { AT } 105 \\
\text { DEG. C, } \\
\text { TOTAL } \\
\text { (MG/L) }\end{array}$ & $\begin{array}{l}\text { SOLIDS, } \\
\text { VOLA- } \\
\text { TILE ON } \\
\text { IGNI- } \\
\text { TION, } \\
\text { TOTAL } \\
\text { (MG/L) }\end{array}$ & $\begin{array}{l}\text { NITRO- } \\
\text { GEN, } \\
\text { NO2+NO3 } \\
\text { TOTAL } \\
\text { (MG/L } \\
\text { AS N) }\end{array}$ & $\begin{array}{l}\text { NITRO- } \\
\text { GEN, } \\
\text { AMMONIA } \\
\text { TOTAL } \\
\text { (MG/L } \\
\text { AS N) }\end{array}$ & $\begin{array}{l}\text { NITRO- } \\
\text { GEN, } \\
\text { ORGANIC } \\
\text { TOTAL } \\
\text { (MG/L } \\
\text { AS N) }\end{array}$ & $\begin{array}{l}\text { NITRO- } \\
\text { GEN, AM- } \\
\text { MONIA + } \\
\text { ORGANIC } \\
\text { TOTAL } \\
\text { (MG/L } \\
\text { AS N) }\end{array}$ & $\begin{array}{l}\text { NITRO- } \\
\text { GEN, } \\
\text { TOTAL } \\
\text { (MG/L } \\
\text { AS N) }\end{array}$ & $\begin{array}{l}\text { NITRO- } \\
\text { GEN, } \\
\text { TOTAL } \\
\text { (MG/L } \\
\text { AS NO3) }\end{array}$ & $\begin{array}{l}\text { PHOS- } \\
\text { PHOROUS } \\
\text { TOTAL } \\
\text { (MG/L } \\
\text { AS P) }\end{array}$ \\
\hline
\end{tabular}

\begin{tabular}{|c|c|c|c|c|c|c|c|c|c|c|}
\hline DEC $21 \cdots$ & 11 & 66 & -- & -- & -- & -- & -- & -- & -- & 0.01 \\
\hline $\begin{array}{l}12 \ldots \\
\text { JAN } 1981\end{array}$ & 10 & 61 & - & - & - & - & -- & - & -- & .02 \\
\hline$\underset{\text { FEB }}{20 \ldots}$ & 14 & -- & -- & -- & -- & - & -- & -- & -- & .01 \\
\hline $\operatorname{MAR}^{23 \ldots}$ & 6 & - & -- & -- & -- & -- & - & -- & -- & .02 \\
\hline${ }_{A P R}^{25 \ldots}$ & -- & -- & -- & -- & -- & -- & -- & -- & -- & .01 \\
\hline $\operatorname{MAY}^{14 \ldots}$ & 10 & 45 & -- & -- & $-\infty$ & -- & -- & -- & -- & .02 \\
\hline JUN & 11 & 80 & - & -- & -- & -- & -- & -- & -- & .01 \\
\hline${ }_{\text {JUL }}^{10 \ldots}$ & 13 & 66 & $-\infty$ & -- & $-\infty$ & -- & -- & -- & -- & .01 \\
\hline AUG $^{21 \cdots}$ & 11 & 48 & -- & $-\infty$ & $-\infty$ & $-\infty$ & $-\infty$ & -- & -- & .02 \\
\hline SEP & 5 & 45 & -- & -- & -- & - & $-\infty$ & -- & -- & -- \\
\hline $\mathrm{OCT}^{22 \ldots}$ & 8 & 65 & -- & - & -- & -- & - & -- & -- & -- \\
\hline $\begin{array}{c}20 \ldots \\
\text { Nov }\end{array}$ & 6 & 42 & $-\infty$ & -- & - & -- & -- & -- & -- & .01 \\
\hline $\mathrm{DEC}^{17 \ldots}$ & 7 & 86 & -- & -- & -- & -- & -- & -- & $\cdots$ & .01 \\
\hline $15 \ldots$ & 9 & 36 & -- & -- & $-\infty$ & -- & -- & -- & -- & .01 \\
\hline
\end{tabular}


TAIR 4.--Physical characteristics and concentrations of common chemical constituents in lakes and streams of the Bald Mountain watersheds--Continued

01012515 CLAYTON STREAM AT OUTLET CLAYTON LAKE ME SITE 6

\begin{tabular}{|c|c|c|c|c|c|c|c|c|}
\hline$\cdot$ & $\begin{array}{l}\text { ALUM- } \\
\text { INUM, } \\
\text { TOTAL } \\
\text { RECOV- } \\
\text { ERABLE } \\
\text { (UG/L } \\
\text { AS AL) }\end{array}$ & $\begin{array}{l}\text { ALUM- } \\
\text { INUM, } \\
\text { DIS- } \\
\text { SOLVED } \\
\text { (UG/L } \\
\text { AS AL) }\end{array}$ & $\begin{array}{c}\text { ARSENIC } \\
\text { TOTAL } \\
\text { (UG/L } \\
\text { AS AS) }\end{array}$ & $\begin{array}{l}\text { CADMIUM } \\
\text { TOTAL } \\
\text { RECOV- } \\
\text { ERABLE } \\
\text { (UG/L } \\
\text { AS CD) }\end{array}$ & $\begin{array}{l}\text { CARO- } \\
\text { MIUM, } \\
\text { TOTAL } \\
\text { RECOV- } \\
\text { ERABLE } \\
\text { (UG/L } \\
\text { AS CR) }\end{array}$ & $\begin{array}{l}\text { COPPER, } \\
\text { TOTAL } \\
\text { RECOV- } \\
\text { ERABLE } \\
\text { (UG/L } \\
\text { AS CU) }\end{array}$ & $\begin{array}{l}\text { COPPER, } \\
\text { DIS- } \\
\text { SOLVED } \\
\text { (UG/L } \\
\text { AS CU) }\end{array}$ & $\begin{array}{l}\text { IRON, } \\
\text { TOTAL } \\
\text { RECOV- } \\
\text { ERABLE } \\
\text { (UG/L } \\
\text { AS FE) }\end{array}$ \\
\hline
\end{tabular}

NOV 1980

21 ...

DEC

$12 .$.

JAN 1981

$20 .$.

FEB

23...

MAR

25...

APR

$14 \ldots$.

MAY

$15 .$.

JUN

$10 .$.

JUL

$21 .$.

AUG

$24 \ldots$

SEP

22 ...

OCT

20...

Nov

$17 \ldots$

DEC

$15 .$.

AS AL)

$\begin{array}{llll}-- & -- & - & - \\ -- & -- & -- & - \\ -- & -- & -- & -- \\ -- & -- & -- & - \\ -- & -- & -- & - \\ -- & -- & -- & - \\ -- & -- & -- & - \\ -- & -- & -- \\ 180 & -- & -- & - \\ -- & -- & -\end{array}$

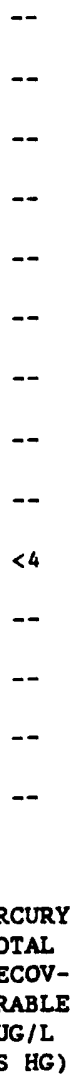

--
--
--
--
--
--
--
--
--
--
--
--
--
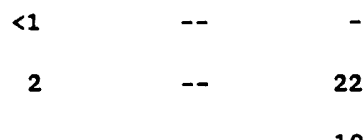

$1 \quad--\quad 330$

$1 \quad--\quad 210$

$1 \quad--\quad 260$

$<1 \quad--\quad 200$

$1 \quad--\quad 160$

$<1 \quad--\quad 240$

$1 \quad 250$

$<2 \quad--\quad 280$

$2 \quad--260$

$1 \quad--\quad 240$

$<1 \quad--\quad 180$

ATE

\begin{tabular}{|c|c|c|c|c|c|c|c|}
\hline $\begin{array}{l}\text { IRON, } \\
\text { DIS- } \\
\text { SOLVED } \\
\text { (UG/L }\end{array}$ & $\begin{array}{l}\text { LEAD, } \\
\text { TOTAL } \\
\text { RECOV- } \\
\text { ERABLE } \\
\text { (UG/L }\end{array}$ & $\begin{array}{l}\text { LEAD, } \\
\text { DIS- } \\
\text { SOLVED } \\
\text { (UG/L }\end{array}$ & $\begin{array}{l}\text { MERCURY } \\
\text { TOTAL } \\
\text { RECOV- } \\
\text { ERABLE } \\
\text { (UG/L }\end{array}$ & $\begin{array}{l}\text { ZINC, } \\
\text { TOTAL } \\
\text { RECOV- } \\
\text { ERABLE } \\
\text { (UG/L }\end{array}$ & $\begin{array}{l}\text { ZINC, } \\
\text { DIS- } \\
\text { SOLVED } \\
\text { (UG/L }\end{array}$ & $\begin{array}{l}\text { SEDI- } \\
\text { MENT, } \\
\text { SUS- } \\
\text { PENDED }\end{array}$ & $\begin{array}{l}\text { MENT, } \\
\text { DIS- } \\
\text { CHARGE, } \\
\text { SUS- } \\
\text { PENDED }\end{array}$ \\
\hline AS $F E$ ) & AS PB) & AS PB) & AS HG) & AS $2 \mathrm{~N}$ ) & AS $Z N)$ & (MG/L) & (T/DAY) \\
\hline
\end{tabular}
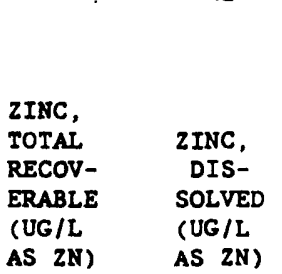

SEDIMENT,
DIS-

Nov 1980

21...

DEC

$12 \ldots$

JAN 1981

20 ...

FEB

23 ...

MAR

25...

APR

$14 \ldots$

MAY

15...

JUN

$10 .$.

JUL

21 ...

AUG

24 ...

SEP 22 .

OCT

20 ...

Nov

$17 \ldots$

DEC

15...

$<2$
--
--
--
--
--
--
--
--
$<4$
$<1$
--
--
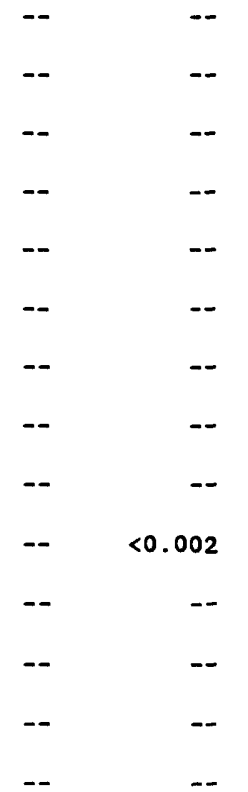

$<4$

$<0.002$

$$
5
$$

$$
5
$$

4

s3

--

$--$

--

$<5$

$<4$

9

5

6
5 
TAmL 4.--Physical characteristics and concentrations of common chemical constituents in lakes and streams of the Bald Mountain watersheds--Continued

01012515 CLAYTON STREAM AT OUTLET CLAYTON LARE ME SITE 6

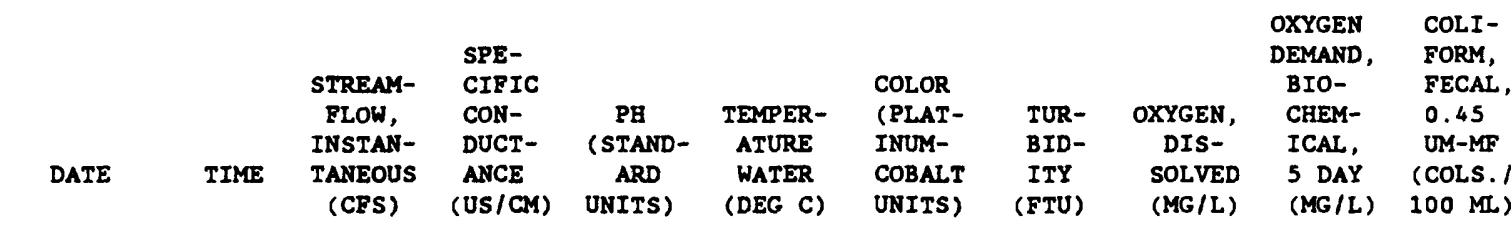

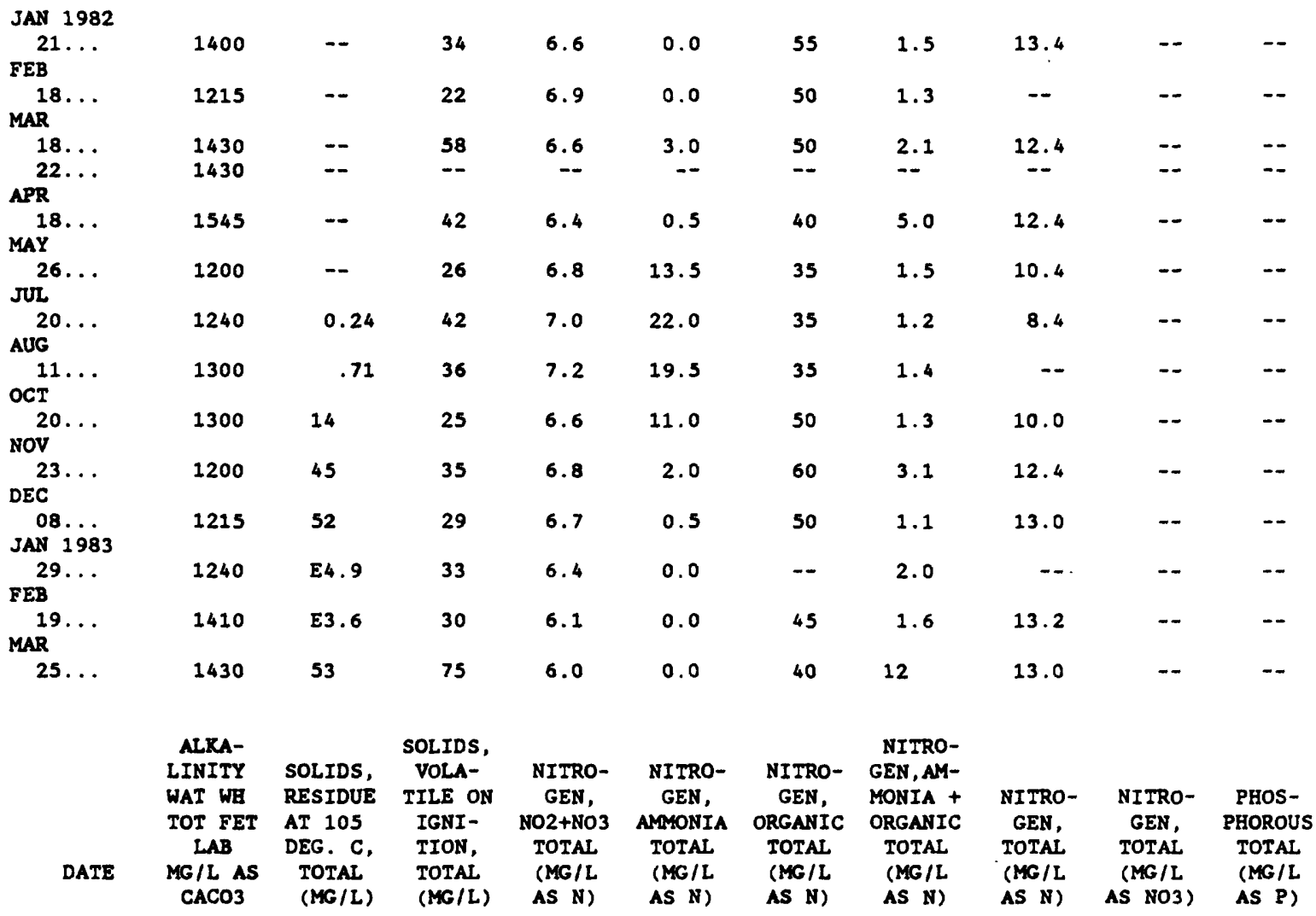

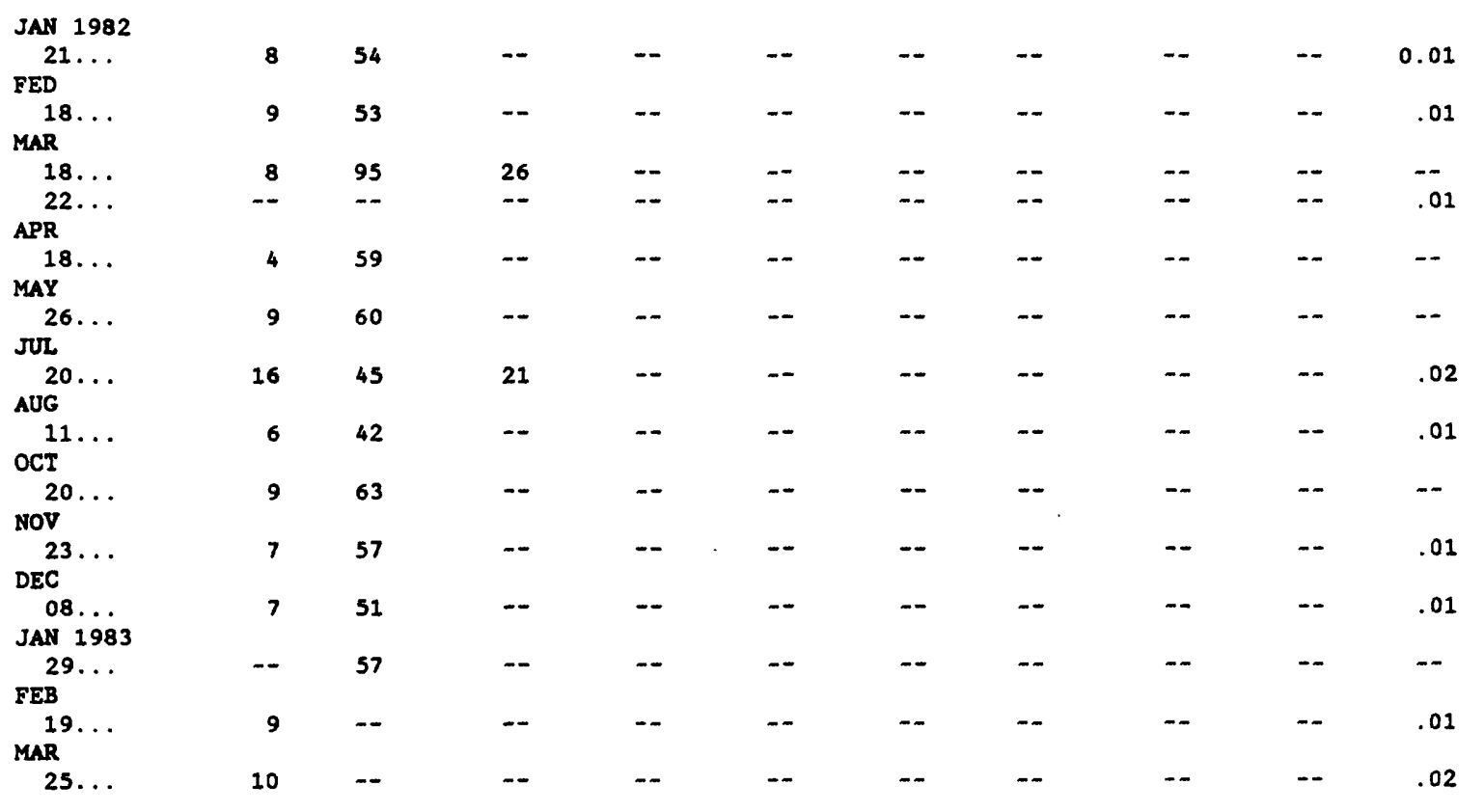


TAm: 4.--Physical characteristics and concentrations of common chemical constituents in lakes and streans of the Bald Mountain watersheds--Continued

01012515 CLAYTON STREAM AT OUTLET CLAYTON LARE ME SITE 6

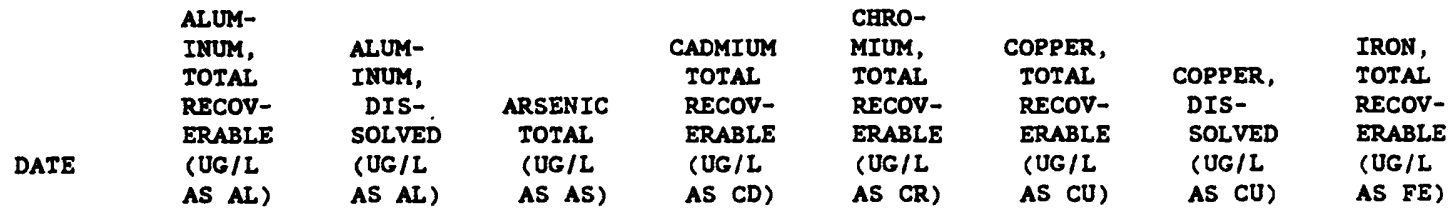

JAN 1982

$21 \ldots$

FEB

$18 \ldots$

MAR

18 ...

22 ...

APR

18 . .

MAY

26 ...

JUL.

20 ...

AUG

$11 .$.

OCT

20 ...

Nov

$23 .$.

DEC

$08 \ldots$

JAN 1983

29 ...

FEB

$19 \ldots$

MAR

25...

AS AI)

AS AL)

AS AS)

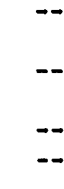

420

150
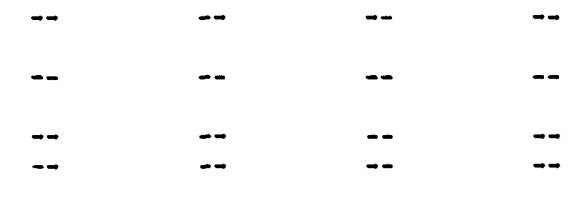

1
--2
2

$\begin{array}{rr}- & 180 \\ -- & 160 \\ -- & 170\end{array}$

$<100$.

$-$

$\rightarrow$

1

440

$-$

$-$

$<1$

$-$

140
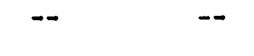

$<1$

120

3

160

$<1$

$--$

130

$<1$

$--$

230

$<1$

$--$

170

$<1$

$-150$

$-$

-

$-$

-.

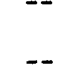

1

$-160$

1

730

$\begin{array}{ccll} & & & \\ & & & \\ & \text { IRON, } & \text { TOTAL, } & \text { LEAD, } \\ & \text { DIS- } & \text { RECOV- } & \text { DIS- } \\ & \text { SOLVED } & \text { ERABLE } & \text { SOLVED } \\ \text { DATE } & \text { (UG/L } & (U G / L & (U G / L \\ & \text { AS FE) } & \text { AS PB) } & \text { AS PB) }\end{array}$

MERCURY
TOTAL
RECOV-
ERABLE
(UG/L
AS HG)

ZINC,
TOTAL
RECOV-
ERABLE
(UG/L
AS $2 N$ )

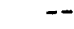

(5)

21...

FEB 18.

MAR

18 ...

$22 \ldots$

APR

18...

MAY

26 ...

JUL.

20...

AUG

OCT

20...

Nov

23...

DEC

08 ...

JAN 1983

29 ...

FEB

$19 \ldots$

MAR

25...

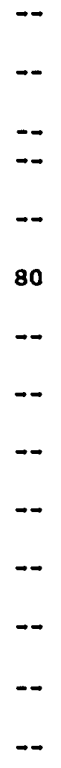

--
--
--
$<1$
$<1$
--
--
--
--
--
--

$\begin{array}{ll}-- & - \\ -- & -- \\ -- & -- \\ -- & -- \\ <1 & -- \\ -- & -- \\ -- & -- \\ -- & -- \\ -- & -- \\ -- & -\end{array}$

5

14

$--$

$<8$

$<5$

$<5$

$<8$

$-$

$<5$

$<5$

$<5$

9

$<5$
SEDIMENT,

$\begin{array}{rrr}\text { ZINC, } & \text { SEDI- } & \text { DIS- } \\ \text { DIS- } & \text { MENT, } & \text { CHARGE, }\end{array}$ SOLVED SUS(UG/L PENDED PENDED AS ZN) (MG/L) (T/DAY) 
rAme 4.--Physical characteristics and concentrations of common chemical constituents in lakes and streams of the Bald Mountain watersheds--Continued

01012515 CLAYTON STREAM AT OUTLET CLAYTON LARE ME SITE 6

\begin{tabular}{|c|c|c|c|c|c|c|c|c|c|c|}
\hline DATE & TIME & $\begin{array}{l}\text { STREAM- } \\
\text { FLOW, } \\
\text { INSTAN- } \\
\text { TANEOUS } \\
\text { (CES) }\end{array}$ & $\begin{array}{l}\text { SPE- } \\
\text { CIFIC } \\
\text { CON- } \\
\text { DUCT- } \\
\text { ANCE } \\
\text { (US/CM) }\end{array}$ & $\begin{array}{c}\text { PE } \\
\text { (STAND- } \\
\text { ARD } \\
\text { UNITS) }\end{array}$ & $\begin{array}{l}\text { TEMPER- } \\
\text { ATURE } \\
\text { WATER } \\
\text { (DEG C) }\end{array}$ & $\begin{array}{l}\text { COLOR } \\
\text { (PLAT- } \\
\text { INUM- } \\
\text { COBALT } \\
\text { UNITS) }\end{array}$ & $\begin{array}{l}\text { TUR- } \\
\text { BID- } \\
\text { ITY } \\
\text { (FTU) }\end{array}$ & $\begin{array}{c}\text { OXYGEN, } \\
\text { DIS- } \\
\text { SOLVED } \\
\text { (MG/L) }\end{array}$ & $\begin{array}{l}\text { OXYGEN } \\
\text { DEMAND, } \\
\text { BIO- } \\
\text { CHEM- } \\
\text { ICAL, } \\
\text { S DAY } \\
\text { (MG/L) }\end{array}$ & $\begin{array}{l}\text { COLI- } \\
\text { FORM, } \\
\text { FECAL, } \\
0.45 \\
\text { UM-MF } \\
\text { (COLS.I } \\
100 \mathrm{ML} \text { ) }\end{array}$ \\
\hline
\end{tabular}

\begin{tabular}{|c|c|c|c|c|c|c|c|}
\hline $\begin{array}{c}\text { APR } 1983 \\
19 \ldots\end{array}$ & 1400 & 253 & 16 & 6.6 & 3.5 & 40 & 11 \\
\hline $26 \ldots$ & 1100 & 206 & -- & -- & $-\infty$ & $=-$ & -- \\
\hline \multicolumn{8}{|l|}{ MAY } \\
\hline $19 \ldots$ & 1200 & 37 & -- & -- & -- & -- & -- \\
\hline \multicolumn{8}{|l|}{ JUN } \\
\hline $14 \ldots$ & 1530 & 23 & -- & 6.8 & 24.0 & 30 & 1.7 \\
\hline \multicolumn{8}{|l|}{ JUL } \\
\hline${ }_{A U G}^{19 \ldots}$ & 1600 & 3.4 & 40 & 6.9 & 22.0 & 35 & -- \\
\hline $30 \ldots$ & 1150 & 3.6 & 29 & $-\infty$ & 20.0 & 40 & 2.1 \\
\hline \multicolumn{8}{|l|}{ OCT } \\
\hline $06 \ldots$ & 1200 & 62 & -- & 6.3 & 11.0 & 70 & 2.5 \\
\hline $25 \ldots$ & 1415 & 3.4 & 28 & 7.1 & 7.5 & so & 0.9 \\
\hline \multicolumn{8}{|l|}{ MAR 1984} \\
\hline $29 \ldots$ & 1630 & E14 & 33 & 6.7 & 1.5 & 40 & 3.7 \\
\hline $\mathbf{A P R}$ & & & & & & & \\
\hline $\mathrm{MAY}^{30 \ldots}$ & 1300 & E210 & 13 & 6.8 & 5.3 & 50 & .9 \\
\hline $17 \ldots$ & 1554 & E76 & 29 & -- & 10.0 & 50 & 1.6 \\
\hline $30 \ldots$ & 1200 & 48 & 24 & 6.8 & - & 65 & 2.2 \\
\hline
\end{tabular}

\begin{tabular}{|c|c|c|c|c|c|c|c|c|c|c|}
\hline DATE & $\begin{array}{l}\text { ALRA- } \\
\text { LINITY } \\
\text { WAT WB } \\
\text { TOT FET } \\
\text { LAB } \\
\text { MG/L AS } \\
\text { CACO3 }\end{array}$ & $\begin{array}{l}\text { SOLIDS, } \\
\text { RESIDUE } \\
\text { AT } 105 \\
\text { DEG. C, } \\
\text { TOTAL } \\
\text { (MG/L) }\end{array}$ & $\begin{array}{l}\text { SOLIDS, } \\
\text { VOLA- } \\
\text { TILE ON } \\
\text { IGNI- } \\
\text { TION, } \\
\text { TOTAL } \\
\text { (MG/L) }\end{array}$ & $\begin{array}{l}\text { NITRO- } \\
\text { GEN, } \\
\text { NO2+NO3 } \\
\text { TOTAL } \\
\text { (MG/L } \\
\text { AS N) }\end{array}$ & $\begin{array}{l}\text { NITRO- } \\
\text { GEN, } \\
\text { AMONIA } \\
\text { TOTAL } \\
\text { (MG/L } \\
\text { AS N) }\end{array}$ & $\begin{array}{l}\text { NITRO- } \\
\text { GEN, } \\
\text { ORGANIC } \\
\text { TOTAL } \\
\text { (MG/L } \\
\text { AS N) }\end{array}$ & $\begin{array}{l}\text { NITRO- } \\
\text { GEN,AM- } \\
\text { MONIA + } \\
\text { ORGANIC } \\
\text { TOTAL } \\
\text { (MG/L } \\
\text { AS N) }\end{array}$ & $\begin{array}{l}\text { NITRO- } \\
\text { GEN, } \\
\text { TOTAL } \\
\text { (MG/L } \\
\text { AS N) }\end{array}$ & $\begin{array}{l}\text { NITRO- } \\
\text { GEN, } \\
\text { TOTAL } \\
\text { (MG/L } \\
\text { AS NO3) }\end{array}$ & $\begin{array}{l}\text { PHOS- } \\
\text { PHOROUS } \\
\text { TOTAL } \\
\text { (MG/L } \\
\text { AS P) }\end{array}$ \\
\hline APR 1983 & & & & & & & & & & \\
\hline $19 \ldots$ & 3 & -- & -- & -- & -- & -- & 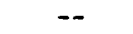 & -- & -- & -- \\
\hline $26 \ldots$ & 15 & -- & -- & -- & -- & -- & - & -- & -- & 0.03 \\
\hline MAY & & & & & & & & & & \\
\hline${ }_{\text {JUN }}^{19 \ldots}$ & -- & -- & -- & -- & $\cdots$ & -- & -- & $\cdots$ & -- & .01 \\
\hline${ }_{\pi}^{14} \ldots$ & 8 & -- & - & -- & -- & -- & -- & -- & -- & -- \\
\hline${ }_{\text {AUG }}^{19 \ldots}$ & 12 & -- & - & -- & -- & -- & -- & -- & -- & .02 \\
\hline $\mathrm{OCT}^{30 \ldots}$ & 13 & -- & -- & -- & -- & -- & -- & -- & -- & .02 \\
\hline $06 \ldots$ & 10 & 87 & -- & - & -- & -- & -- & -- & -- & -- \\
\hline $\begin{array}{c}25 \ldots \\
\operatorname{MAR} 1984\end{array}$ & 10 & 40 & -- & -- & -- & - & - & -- & -- & .01 \\
\hline $\begin{array}{l}29 \ldots \\
A P R\end{array}$ & 9 & 61 & -- & -- & -- & -- & -- & -- & -- & -- \\
\hline $\operatorname{MAY}_{\operatorname{MA}}^{30 \ldots}$ & 3 & 45 & -- & -- & -- & -- & -- & -- & -- & -- \\
\hline $17 \ldots$ & 7 & 55 & -- & -- & -- & -- & -- & -- & -- & -- \\
\hline $30 \ldots$ & 4 & 41 & -- & -- & -- & -- & -- & -- & -- & .01 \\
\hline
\end{tabular}


TAm. 4.--Physical characteristics and concentrations of common chemical constituents in lakes and streams of the Bald Mountain watersheds--Continued

01012515 CLAYTON STREAM AT OUTLET CLAYTON LAKE ME SITE 6

DATE

APR 1983

$19 \ldots$

$26 \ldots$

MAY

19...

JUN

$14 \ldots$

JUL

19.

AUG

$30 .$.

OCT

$06 .$.

25...

MAR 1984

$29 \ldots$

APR

$30 \ldots$

MAY

$17 . .$.

$30 .$.

\section{ALUM-}

INUM, ALUM-

TOTAL INUM,

RECOV- DIS-

ERABLE SOLVED

(UG/L

AS AL)

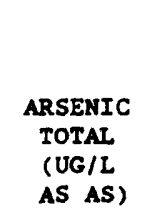

CERO-

MIUM, COPPER,

TOTAL TOTAL COPPER, TOTAL

RECOV- RECOV- DIS- RECOV-

ERABLE ERABLE SOLVED ERABLE

(UG/L (UG/L (UG/L (UG/L

AS CR)

AS CU)

AS FE)

\begin{tabular}{|c|c|c|c|c|c|c|c|}
\hline-- & -- & -- & -- & -- & $<1$ & -- & 690 \\
\hline-- & -- & -- & -- & -- & $<1$ & -- & 590 \\
\hline-- & -- & -- & -- & -- & 1 & -- & 180 \\
\hline-- & -- & -- & -- & -- & -- & $\rightarrow$ & -- \\
\hline-- & -- & -- & -- & -- & 1 & -- & 200 \\
\hline-- & -- & - & -- & -- & 1 & -- & 180 \\
\hline-- & -- & -- & -- & -- & -- & -- & - \\
\hline- & -- & -- & -- & -- & $<1$ & -- & 140 \\
\hline-- & -- & -- & -- & -- & 1 & -- & 220 \\
\hline- & -- & -- & -- & -- & $<1$ & -- & 100 \\
\hline-- & -- & -- & -- & -- & -- & -- & - \\
\hline-- & -- & -- & -- & -- & $<1$ & -- & 160 \\
\hline
\end{tabular}

\begin{tabular}{|c|c|c|c|c|c|c|c|c|}
\hline DATE & $\begin{array}{l}\text { IRON, } \\
\text { DIS- } \\
\text { SOLVED } \\
\text { (UG/L }\end{array}$ & $\begin{array}{l}\text { LEAD, } \\
\text { TOTAL } \\
\text { RECOV- } \\
\text { ERABLE } \\
\text { (UG/L }\end{array}$ & $\begin{array}{l}\text { LEAD, } \\
\text { DIS- } \\
\text { SOLVED } \\
\text { (UG/L }\end{array}$ & $\begin{array}{l}\text { MERCURY } \\
\text { TOTAL } \\
\text { RECOV- } \\
\text { ERABLE } \\
\text { (UG/L }\end{array}$ & $\begin{array}{l}\text { ZINC, } \\
\text { TOTAL } \\
\text { RECOV- } \\
\text { ERABLE } \\
\text { (UG/L }\end{array}$ & $\begin{array}{l}\text { ZINC, } \\
\text { DIS- } \\
\text { SOLVED } \\
\text { (UG/L }\end{array}$ & $\begin{array}{l}\text { SEDI- } \\
\text { MENT, } \\
\text { SUS- } \\
\text { PENDED }\end{array}$ & $\begin{array}{l}\text { SEDI- } \\
\text { MENT, } \\
\text { DIS- } \\
\text { CHARGE, } \\
\text { SUS- } \\
\text { PENDED }\end{array}$ \\
\hline & AS FE) & AS PB) & AS $\mathrm{PB}$ ) & AS HG) & AS $Z N)$ & AS $Z N$ ) & (MG/L) & (T/DAY) \\
\hline
\end{tabular}

APR 1983

$19 \ldots$

MAY

$19 . .$.

JUN

$14 \ldots$

JUL

$19 .$.

AUG

$30 .$.

ocr

$06 .$.

$25 . .$.

MAR 1984

29 ..

APR

$30 .$.

MAY

$17 \ldots$

\begin{tabular}{|c|c|c|}
\hline-- & -- & - \\
\hline-- & -- & -- \\
\hline-- & $<1$ & -- \\
\hline-- & -- & -- \\
\hline-- & -- & -- \\
\hline-- & -- & -- \\
\hline-- & -- & -- \\
\hline-- & -- & -- \\
\hline-- & $<1$ & -- \\
\hline-- & $<1$ & -- \\
\hline- & -- & - \\
\hline-- & $<1$ & -- \\
\hline
\end{tabular}

--

6
$<5$

$<5$

$<1$

$--\quad<5$

15

9

6

0.05

$<5$

.54

$<5$

$<5$

$--$

3
1

.01

.15

2.3

.41 
TABIR 4.--Physical characteristics and concentrations of commond chemical constituents in lakes and streams of the Bald Mountain watershed--Continued

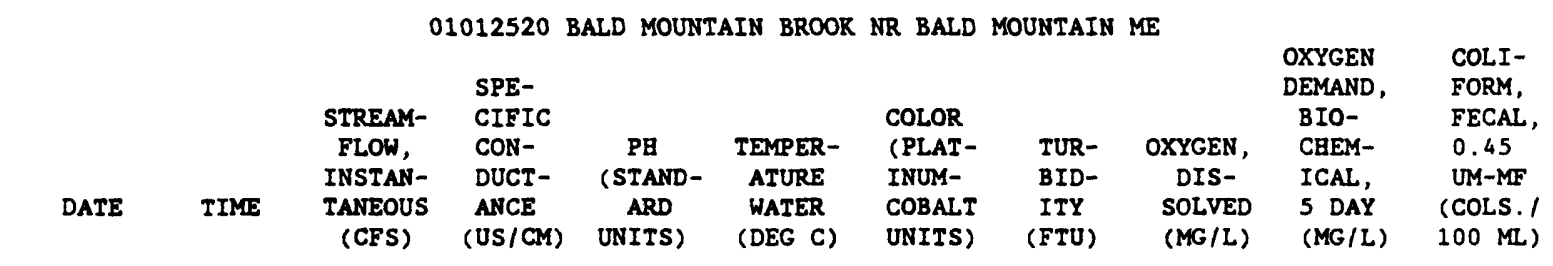

\begin{tabular}{|c|c|c|c|c|c|c|c|c|c|c|}
\hline \multicolumn{11}{|l|}{ JUN 1979} \\
\hline $11 \ldots$ & 0900 & -- & 35 & 7.0 & 13.0 & -- & 0.5 & 8.9 & 0.8 & $\mathrm{~K} 2$ \\
\hline $26 \ldots$ & 0900 & -- & 48 & 6.8 & 13.0 & -- & .8 & 10.0 & .6 & $\mathrm{~K} 14$ \\
\hline \multicolumn{11}{|l|}{ JUI } \\
\hline $17 \ldots$ & 0915 & -- & -- & 6.9 & 17.0 & -- & 1.1 & 9.2 & .6 & K8 \\
\hline $24 \ldots$ & 0915 & -- & 78 & -- & 20.0 & -- & 1.5 & 8.8 & .5 & KS \\
\hline \multicolumn{11}{|l|}{ AUG } \\
\hline $07 \ldots$ & 0900 & -- & 66 & 7.1 & 18.0 & $>70$ & 1.3 & 8.5 & .4 & $\mathrm{~K} 13$ \\
\hline$\cdot 21 \ldots$ & 0930 & -- & 53 & 6.9 & 18.0 & $>70$ & 2.6 & 11.5 & 1.5 & -- \\
\hline \multicolumn{11}{|l|}{ SEP } \\
\hline $04 \ldots$ & 0930 & -- & 68 & 7.0 & 18.0 & 50 & 1.5 & 9.0 & .5 & $\mathrm{~K} 7$ \\
\hline $18 \ldots$ & 0930 & -- & 46 & 6.5 & 13.0 & 70 & 1.8 & 9.4 & -- & -- \\
\hline \multicolumn{11}{|l|}{ OCT } \\
\hline FEB $22 \ldots$ & 1100 & -- & 58 & 6.9 & 10.0 & 55 & 1.0 & 10.1 & .5 & -- \\
\hline $19 \ldots$ & 1330 & -- & 115 & 6.7 & 0.0 & 20 & 3.7 & 10.9 & .7 & $\mathrm{~K} 1$ \\
\hline \multicolumn{11}{|l|}{ APR } \\
\hline $23 \ldots$ & 1130 & -- & 32 & 6.5 & 1.0 & 45 & 2.7 & 12.1 & .4 & - \\
\hline MAY & & & & & & & & & & \\
\hline JUN & 1315 & -- & \multicolumn{8}{|c|}{ JUN } \\
\hline $17 \ldots$ & 1400 & -- & 49 & 6.8 & 10.0 & 35 & 1.4 & 11.2 & $<.1$ & -- \\
\hline JUL & & & & & & & & & & \\
\hline $13 \ldots$ & 1330 & -- & 50 & 6.3 & 14.0 & 60 & 2.9 & 9.5 & -- & -- \\
\hline \multicolumn{11}{|l|}{ AUG } \\
\hline $\operatorname{SEP}^{14} \cdots$ & 1145 & -- & 78 & 7.0 & 14.0 & 35 & 1.3 & 9.6 & -- & - \\
\hline $29 .$. & 1145 & -- & 48 & 6.7 & 5.0 & 70 & 2.6 & 11.8 & -- & -- \\
\hline
\end{tabular}

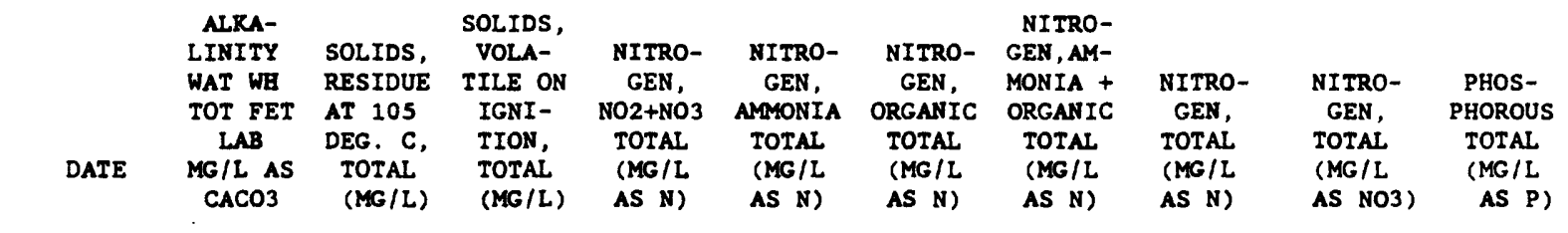

JUN 1979

\begin{tabular}{|c|c|c|c|c|c|c|c|c|c|c|}
\hline $11 \ldots$ & 20 & -- & -- & $<0.01$ & $<0.01$ & -- & -- & -- & -- & $<0.01$ \\
\hline $26 \ldots$ & 9 & -- & -- & $<.01$ & $<.01$ & -- & -- & -- & -- & -- \\
\hline \multicolumn{11}{|l|}{ JUL } \\
\hline $17 \ldots$ & 17 & -- & -- & .01 & $<.01$ & -- & 0.3 & 0.31 & 1.4 & .01 \\
\hline $24 \ldots$ & 22 & -- & -- & .03 & $<.01$ & -- & -- & - & -- & -- \\
\hline \multicolumn{11}{|l|}{ AUG } \\
\hline $07 \ldots$ & 18 & -- & -- & .04 & $<.01$ & -- & -- & -- & -- & -- \\
\hline $21 \ldots$ & 18 & -- & -- & .06 & $<.01$ & -- & -- & -- & -- & -- \\
\hline \multicolumn{11}{|l|}{ SEP } \\
\hline $04 \ldots$ & 20 & -- & -- & .04 & $<.01$ & -- & -- & -- & -- & -- \\
\hline $18 \ldots$ & 21 & -- & -- & $<.01$ & $<.01$ & $=$ & -- & -- & -- & -- \\
\hline \multicolumn{11}{|l|}{ OCT } \\
\hline $22 \ldots$ & 14 & -- & -- & .04 & $<.01$ & -- & -- & -- & -- & -- \\
\hline FEB 1980 & & & & & & & & & & \\
\hline $19 \ldots$ & 30 & 78 & -- & .22 & -- & -- & .1 & .32 & 1.4 & .02 \\
\hline \multicolumn{11}{|l|}{ APR } \\
\hline $23 \ldots$ & 6 & 67 & -- & .32 & $<.01$ & -- & .4 & .72 & 3.2 & .02 \\
\hline \multicolumn{11}{|l|}{ MAY } \\
\hline $20 \ldots$ & 5 & 42 & -- & .42 & .03 & 0.07 & .1 & .52 & 2.3 & .01 \\
\hline \multicolumn{11}{|l|}{ JUN } \\
\hline $17 \ldots$ & 11 & 86 & -- & .37 & .01 & .19 & .2 & .57 & 2.5 & .01 \\
\hline $13 \ldots$ & 11 & 68 & -- & -- & -- & -- & -- & -- & -- & .01 \\
\hline \multicolumn{11}{|l|}{ AUG } \\
\hline $14 \ldots$ & 26 & 88 & -- & -- & -- & -- & -- & - & -- & .01 \\
\hline \multicolumn{11}{|l|}{ SEP } \\
\hline $29 .$. & 18 & 72 & -- & -- & -- & -- & $=$ & -- & -- & .03 \\
\hline
\end{tabular}


FWT 4.--Physical characteristics and concentrations of commond chemical constituents in lakes and streams of the Bald Mountain watershed--Continued

01012520 BALD MOUNTAIX BROOK MR BALD MOUNTAIM ME

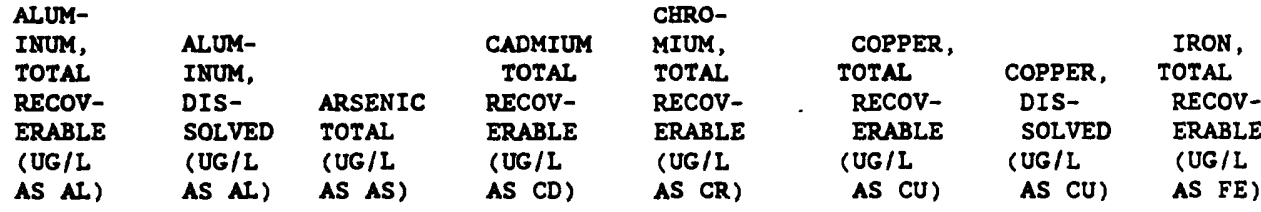

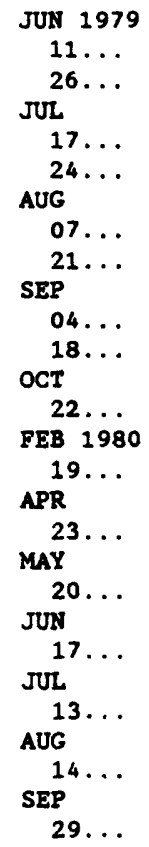

$\begin{array}{lll}-- & -- & -- \\ -- & -- & -- \\ -- & -- & -- \\ -- & -- & -- \\ -- & -- & -- \\ -- & -- & -- \\ -- & -- & -- \\ -- & -- & -- \\ -- & -- & -- \\ -- & -- & -- \\ -- & -- & -- \\ -- & -- & -- \\ -- & -- & -- \\ -- & -- & -\end{array}$

$\begin{array}{ll}2 & <6 \\ -- & -- \\ 2 & <6 \\ 2 & <6 \\ -- & <6 \\ -- & - \\ -- & - \\ -- & - \\ 2 & <6 \\ 2 & 6 \\ 2 & <5 \\ 2 & <20 \\ 2 & <5 \\ -- & - \\ -- & - \\ -- & \end{array}$

$<6$
--
$<6$
$<6$
$<6$
--
--
--
66
$<5$
20
$<5$

$\begin{array}{lll}<4 & -- & -- \\ -- & -- & - \\ <4 & -- & 240 \\ <4 & -- & 120 \\ <4 & -- & 200\end{array}$

$--\quad--\quad 200$

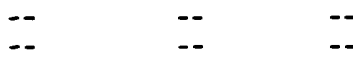

$<4 \quad--\quad 120$

$4 \quad--\quad 260$

$<10$

$<4$

8

100

$<5$

$<2$

160

$<4$

120

$<4$

320

DATE

\begin{tabular}{lllllllr} 
& LEAD, & \multicolumn{2}{c}{ MERCURY } & ZINC, & MENT, \\
IRON, & TOTAL & LEAD, & TOTAL & TOTAL & 2INC, & SEDI- & DIS- \\
DIS- & RECOV- & DIS- & RECOV- & RECOV- & DIS- & MENT, & CHARGE, \\
SOLVED & ERABLE & SOLVED & ERABLE & ERABLE & SOLVED & SUS- & SUS- \\
(UG/L & (UG/L & (UG/L & (UG/L & (UG/L & (UG/L & PENDED & PENDED \\
AS FE) & AS PB) & AS PB) & AS HG) & AS ZN) & AS 2N) & (MG/L) & (T/DAY)
\end{tabular}

JUN 1979

$11 .$.

26 ...

JUL

$17 .$.

$24 \ldots$

AUG

$07 .$.

21...

SEP

$04 \ldots$.

$18 \ldots$

OCT

22 ...

FEB 1980

$19 .$.

APR

$23 .$.

MAY

20...

JUN

$17 \ldots$

JUL.

$13 .$.

AUG

$14 \ldots$

SEP

29 ...

\begin{tabular}{|c|c|c|c|c|c|c|c|}
\hline-- & $<20$ & -- & -- & 20 & - & -- & -- \\
\hline-- & -- & -- & -- & -- & -- & 3 & 0.0 \\
\hline-- & $<20$ & -- & 0.4 & $<2$ & -- & 2 & 0.0 \\
\hline-- & $<20$ & -- & .3 & 2 & -- & 2 & 0.0 \\
\hline-- & $<20$ & -- & .3 & $<2$ & -- & 1 & 0.0 \\
\hline-- & - & $=$ & $=-$ & -- & $=$ & 2 & 0.0 \\
\hline-- & -- & -- & -- & -- & -- & 1 & 0.0 \\
\hline-- & -- & -- & -- & -- & -- & -- & -- \\
\hline-- & $<20$ & -- & $<.2$ & -- & -- & 1 & 0.0 \\
\hline-- & 20 & -- & .2 & 8 & -- & -- & -- \\
\hline-- & $<30$ & -- & -- & 4 & -- & 5 & 0.0 \\
\hline-- & 30 & -- & -- & 10 & -- & 2 & 0.0 \\
\hline-- & $<30$ & -- & $<.2$ & 8 & -- & 1 & 0.0 \\
\hline-- & -- & -- & $=-$ & 3 & -- & -- & -- \\
\hline-- & -- & -- & -- & 2 & -- & -- & -- \\
\hline-- & -- & -- & -- & $<2$ & -- & -- & -- \\
\hline
\end{tabular}


InI 4.--Physical characteristics and concentrations of commond chemical constituents In lakes and streams of the Bald Mountain watershed--Continued

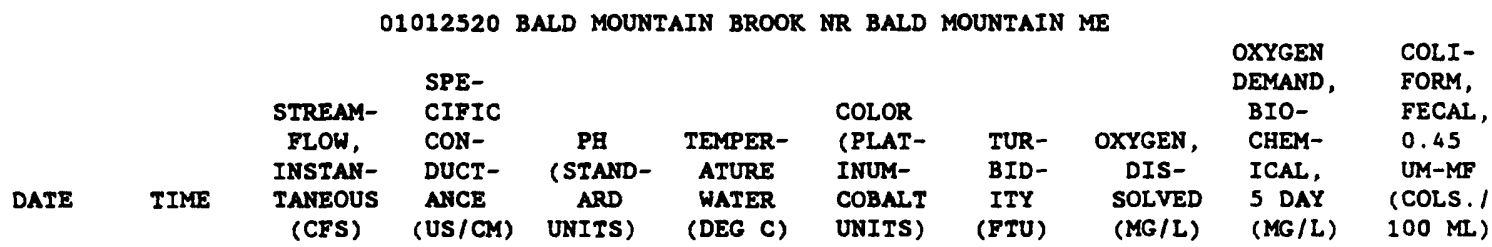

\begin{tabular}{|c|c|c|c|c|c|c|c|c|c|c|}
\hline $\operatorname{NOV}^{14 \ldots}$ & 1145 & 54.5 & 55 & 6.2 & 5.0 & 50 & 6.4 & 11.3 & -- & -- \\
\hline $\mathrm{DEC}^{21 \cdots}$ & 1145 & 1.1 & 185 & 6.6 & 0.0 & 35 & 1.8 & 13.4 & -- & -- \\
\hline $\begin{array}{l}\text { DEC } \\
12 \ldots\end{array}$ & & & & & & & & & & \\
\hline $\begin{array}{c}12 \ldots \\
\text { JAN } 1981\end{array}$ & 1330 & 0.46 & 55 & 6.2 & 0.0 & 45 & 1.6 & 13.2 & -- & -- \\
\hline$\underset{\text { FEB }}{20} \cdots$ & 1230 & $\begin{array}{c}E .22 \\
.\end{array}$ & 68 & 6.6 & 0.0 & 30 & 5.4 & 13.7 & -- & -- \\
\hline $\operatorname{MAR}^{23} \cdots$ & 1230 & E10 & 25 & 6.0 & 2.0 & 90 & 7.5 & 12.1 & -- & -- \\
\hline${ }_{A P R}^{18 \ldots}$ & 1015 & E. 93 & 55 & 6.6 & 2.0 & 20 & 0.7 & 13.2 & -- & -- \\
\hline $\operatorname{MAY}^{14 \ldots}$ & 1315 & E6.8 & 41 & 6.1 & 0.0 & 45 & 2.1 & 12.0 & -- & -- \\
\hline Jus & 1405 & 4.6 & 31 & 6.1 & 6.0 & 90 & 1.5 & 11.5 & -- & -- \\
\hline$\pi^{10} \cdot$ & 1415 & 1.3 & 42 & 6.9 & 9.0 & 20 & .9 & 11.0 & -- & -- \\
\hline AUG $21 \ldots$ & 1415 & .19 & 97 & 7.1 & -- & 45 & 3.5 & -- & -- & -- \\
\hline $\operatorname{SEP}^{24} \cdots$ & 1430 & E6.9 & 40 & 6.1 & 13.0 & 80 & 1.2 & 8.2 & -- & -- \\
\hline $\mathrm{OCr}^{22 \cdots}$ & 1305 & 1.3 & 30 & 7.0 & 9.0 & 90 & 1.6 & 10.1 & -- & -- \\
\hline Nov 20. & 1305 & 6.9 & 41 & 6.3 & 6.0 & 80 & 1.5 & 12.4 & -- & -- \\
\hline $17 \ldots$ & 1230 & 2.1 & 66 & 6.6 & 3.0 & 30 & 1.9 & 11.0 & -- & -- \\
\hline DATE & $\begin{array}{l}\text { ALKA- } \\
\text { LINITY } \\
\text { WAT WB } \\
\text { TOT FET } \\
\text { LAB } \\
\text { MG/L AS } \\
\text { CACO3 }\end{array}$ & $\begin{array}{l}\text { SOLIDS, } \\
\text { RESIDUE } \\
\text { AT } 105 \\
\text { DEG. C, } \\
\text { TOTAL } \\
\text { (MG/L) }\end{array}$ & $\begin{array}{l}\text { SOLIDS, } \\
\text { VOLA- } \\
\text { IILE ON } \\
\text { IGNI- } \\
\text { TION, } \\
\text { TOTAL } \\
\text { (MG/L) }\end{array}$ & $\begin{array}{l}\text { NITRO- } \\
\text { GEN, } \\
\text { NO2+NO3 } \\
\text { TOTAL } \\
\text { (MG/L } \\
\text { AS N) }\end{array}$ & $\begin{array}{l}\text { NITRO- } \\
\text { GEN, } \\
\text { AMONIA } \\
\text { TOTAL } \\
\text { (MG/L } \\
\text { AS N) }\end{array}$ & $\begin{array}{l}\text { NITRO- } \\
\text { GEN, } \\
\text { ORGANIC } \\
\text { TOTAL } \\
\text { (MG/L } \\
\text { AS N) }\end{array}$ & $\begin{array}{l}\text { NITRO- } \\
\text { GEN, AM- } \\
\text { MONIA + } \\
\text { ORGANIC } \\
\text { TOTAL } \\
\text { (MG /L } \\
\text { AS N) }\end{array}$ & $\begin{array}{l}\text { NITRO- } \\
\text { GEN, } \\
\text { TOTAL } \\
\text { (MG / L } \\
\text { AS N) }\end{array}$ & $\begin{array}{l}\text { NITRO- } \\
\text { GEN, } \\
\text { TOTAL } \\
\text { (MG/L } \\
\text { AS NO3) }\end{array}$ & $\begin{array}{l}\text { PHOS- } \\
\text { PHOROUS } \\
\text { TOTAL } \\
\text { (MG/L } \\
\text { AS P) }\end{array}$ \\
\hline
\end{tabular}

OCT 1980

\begin{tabular}{|c|c|c|c|c|c|c|c|c|c|c|}
\hline $14 \ldots$ & 24 & 71 & -- & -- & -- & -- & -- & -- & -- & 0.02 \\
\hline NOV & & & & & & & & & & \\
\hline $21 \ldots$ & 16 & 64 & -- & -- & $-\infty$ & -- & -- & - & -- & $<.01$ \\
\hline DEC & & & & & & & & & & \\
\hline JAN 12.981 & 12 & 58 & -- & -- & -- & -- & - & -- & -- & .01 \\
\hline $20 \ldots$ & 29 & -- & -- & -- & -- & -- & -- & -- & -- & .01 \\
\hline $\operatorname{MAR}^{23} \cdots$ & 5 & -- & -- & -- & -- & -- & -- & $-\infty$ & -- & .03 \\
\hline${ }_{A P R}^{18 \ldots}$ & 18 & 83 & -- & -- & -- & -- & -- & -- & -- & $<.01$ \\
\hline $\operatorname{MAY}^{14 \ldots}$ & 12 & 49 & -- & -- & -- & - & -- & -- & -- & .01 \\
\hline JUN & 9 & 57 & -- & -- & -- & -- & -- & -- & -- & .01 \\
\hline $\mathrm{JUL}^{10 \ldots}$ & 19 & 66 & -- & -- & -- & -- & -- & -- & -- & $<.01$ \\
\hline AUG & 35 & 99 & -- & -- & -- & -- & -- & -- & -- & -- \\
\hline $\operatorname{SEP}^{24 \cdots}$ & 9 & 51 & -- & -- & -- & -- & -- & -- & -- & -- \\
\hline${ }_{\text {OCT }}^{22}$ & 11 & 70 & -- & -- & $-\infty$ & -- & -- & -- & -- & -- \\
\hline $\operatorname{sov}^{20} \cdots$ & 6 & 65 & -- & -- & -- & -- & -- & -- & -- & .01 \\
\hline $17 \ldots$ & 12 & 88 & -- & -- & -- & -- & -- & -- & -- & $<.01$ \\
\hline
\end{tabular}


TAx. 4.--Physical characteristics and concentrations of commond chemical constituents in lakes and streams of the Bald Mountain watershed--Continued

01012520 BALD MOUNTAIN BROOK NR BALD MOUNTAIN ME

DATE

$$
\begin{gathered}
\text { OCT } 1980 \\
14 \ldots \\
\text { NOV } \\
21 \ldots \\
\text { DEC } \\
12 \ldots \\
\text { JAN } 1981 \\
20 \ldots \\
\text { FEB } \\
23 \ldots \\
\text { MAR } \\
18 \ldots \\
\text { APR } \\
14 \ldots \\
\text { MAY } \\
15 \ldots \\
\text { JUN } \\
10 \ldots \\
\text { JUL } \\
21 \ldots \\
\text { AUG } \\
24 \ldots \\
\text { SEP } \\
22 \ldots \\
\text { OCT } \\
20 \ldots \\
\text { NOV } \\
17 \ldots \\
\end{gathered}
$$

\begin{tabular}{|c|c|c|}
\hline 2 & -- & 460 \\
\hline$<1$ & -- & - \\
\hline 1 & - & 160 \\
\hline-- & - & 280 \\
\hline 3 & -- & 740 \\
\hline 3 & - & 90 \\
\hline 3 & - & 230 \\
\hline$<1$ & -- & 160 \\
\hline 1 & -- & 120 \\
\hline 2 & -- & 290 \\
\hline 16 & -- & 150 \\
\hline$<2$ & -- & 280 \\
\hline 1 & -- & 170 \\
\hline$<8$ & -- & 220 \\
\hline
\end{tabular}

$\begin{array}{lll}\text { ALUM- } & & \\ \text { INUM, } & \text { ALUM- } & \\ \text { TOTAL } & \text { INUM, } & \\ \text { RECOV- } & \text { DIS- } & \text { ARSENIC } \\ \text { ERABLE } & \text { SOLVED } & \text { TOTAL } \\ \text { (UG/L } & \text { (UG/L } & \text { (UG/L } \\ \text { AS AL) } & \text { AS AL) } & \text { AS AS) }\end{array}$

$\begin{array}{ll} & \text { CERO- } \\ \text { CADMIUM } & \text { MIUM, } \\ \text { TOTAL } & \text { TOTAL } \\ \text { RECOV- } & \text { RECOV- } \\ \text { ERABLE } & \text { ERABLE } \\ \text { (UG/L } & \text { (UG/L } \\ \text { AS CD) } & \text { AS CR) }\end{array}$
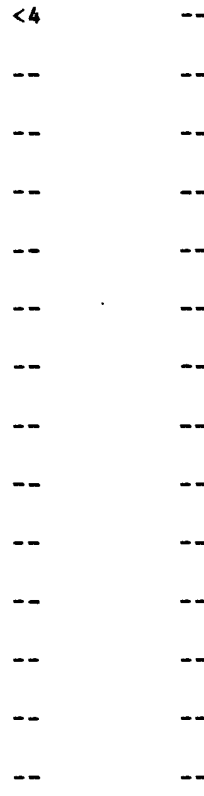

--
--
--
--
--
--
--
--
--
--

--

$\begin{array}{lll} & \text { LEAD, } & \\ \text { IRON, } & \text { TOTAL } & \text { LEAD, } \\ \text { DIS- } & \text { RECOV- } & \text { DIS- } \\ \text { SOLVED } & \text { ERABLE } & \text { SOLVED } \\ \text { (UG/L } & \text { (UG/L } & \text { (UG/L } \\ \text { AS FE) } & \text { AS PB) } & \text { AS PB) }\end{array}$

MERCURY
TOTAL.
RECOV-
ERABLE
(UG/L
AS HG)

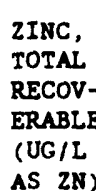

ZINC,
DIS-
SOLVED
(UG/L
AS ZN)
SEDI-
MENT, DIS-

DATE AS FE)

OCT 1980

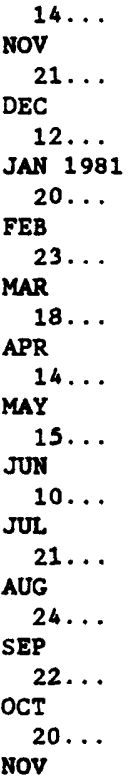

$17 \ldots$

$\begin{array}{ll}-- & <2 \\ - & - \\ - & - \\ -- & - \\ -- & - \\ -- & - \\ -- & - \\ -- & - \\ - & - \\ - & - \\ - & -\end{array}$

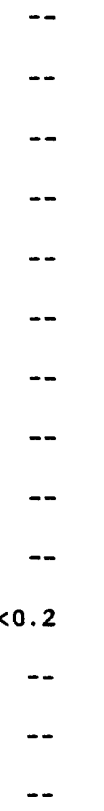

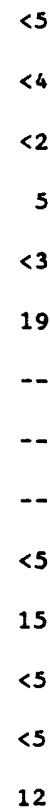

12 
TAra 4.--Physical characteristics and concentrations of commond chemical constituents in lakes and streams of the Bald Mountain watershed--Continued

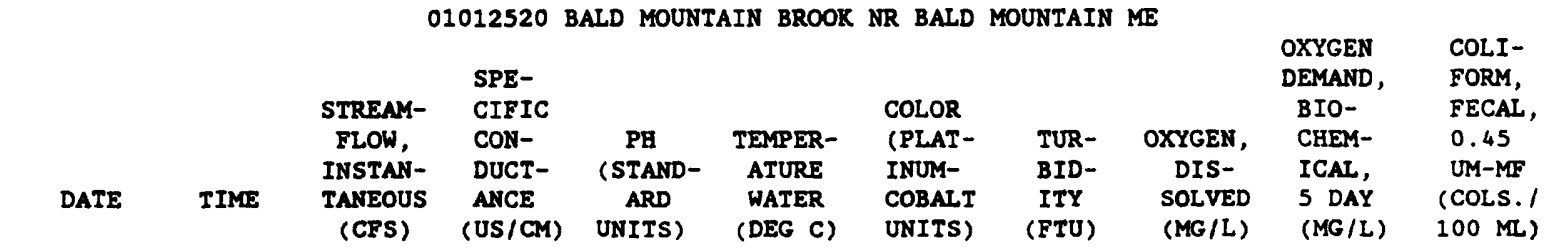

DEC 1981

$15 .$.

JAN 1982

$21 .$.

FEB

18...

MAR
$18 \ldots$

$18 \ldots 1400$

APR

$18 \ldots \quad 1105$

$18 \ldots 1305$

$18 \ldots .1520$

$19 .$.

MAY

03... 1340

26... 1305

JUL.

20... 1258

AUG

$11 \ldots \quad 1330$

SEP

$13 \ldots 1530$

OCT

$20 . .$.

NOV $22 \ldots$

DEC

$08 .$.

1430

1430

1240

E2. 9

EO. $74 \quad 48$

E. $62 \quad 49$

E.63 150

\section{E27}

\section{E27}

E27

E27

$12.96 \quad 42$

$55 \quad 6.2$

$--$

$--\quad \cdots$

6.7

.06105

7.1

.16

65

7.0

$\begin{array}{lll}.38 & 89 & 7.1\end{array}$

$.69 \quad 49$

3.3

3.3

ALXA-

LINITY

WAT WA

TOT FET

SOLIDS,

SOLIDS, VOLA-
RISIDUE TILE ON

AT 105 IGNI-

MG/L AS

TION,

TOTAL

CACO3

(MG/L)
6.7

6.5

6.5
DATE (MG/L)
0.0

0.0

0.0

2.0

.5

--

1.5

11.0

19.5

14.5

18.0

9.0

2.0

35

25

1.1

1.0

25

40

60

$-$

2.8

$-$

25

0.8

35

60

50

55

85

15

35

3.6
2.5

12.8

10.6

$-$

11.8

10.2

10.8

8. 8

9.4

11.8

12.6

13.2

DEC 1981

$15 \ldots$

JAN 1982

21 ...

FEB

18...

MAR

18 ...

10

71

15

APR

18 ...

18 ...

$18 \ldots$

$19 \ldots$

MAY

$03 . .$.

26...

19

16

81

62

119

7

82

--

$-$

-- $\quad-$

NITRO-
GEN,
NO2+NO3

NITRO-

GEN, AM-

O2+NO3 AMONIA ORGANIC ORGANIC

(MG/L TOTAL TOTAL TOTAL

AS N) AS N)

AS N)

AS $N$ )

$\begin{array}{ccc}\text { NITRO- } & \text { NITRO- } & \text { PHOS- } \\ \text { GEN, } & \text { GEN, } & \text { PHOROUS } \\ \text { TOTAL } & \text { TOTAL } & \text { TOTAL } \\ \text { (MG /L } & \text { (MG/L } & \text { (MG /L } \\ \text { AS N) } & \text { AS NO3) } & \text { AS P) }\end{array}$

JUL

20 ...

14

$--$

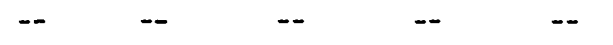

$-$

$-\quad<0.01$

AUG

11...

40

112

29

80

$-$

$-$

$--$

$--$

$--$

$--\quad<.01$

SEP

OCT

$20 \ldots$

13

91

32

$-$

$--$

$-$

.01

Nov

22 ..

DEC

08 ...

16

72

76

69

$\because=$

$\because=$

$-$

$--\quad--$

$--$

-- $\quad .01$

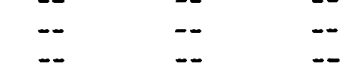

--

$\overline{--}$

$--\quad .02$

$\begin{array}{ll}-- & -- \\ -- & --\end{array}$

--

29

$--$

-- $\quad--$

.01

.03

.01

$-$

--

.01 
TNI. 4. - Physical characteristics and concentrations of conmond chemical constituents in lakes and streams of the Bald Mountain watershed--Continued

01012520 BALD MOUNTAIN BROOK MR BALD MOUNTAIN ME

ALUM- $\quad$ CHRO-

INUM,

TOTAL

RECOV-

ERABLE

ALUM-

INUM,

(UG/L

DIS-

ARSENIC

TOTAL

AS AL)

AS AL) AS AS )

$\begin{array}{ll}\text { CADMIUM } & \text { MIUM, } \\ \text { TOTAL } & \text { TOTAL } \\ \text { RECOV- } & \text { RECOV- } \\ \text { ERABLE } & \text { ERABLE } \\ \text { (UG/L } & \text { (UG/L } \\ \text { AS CD) } & \text { AS CR) }\end{array}$

COPPER,
TOTAL
RECOV-
ERABLE
(UG/L

COPPER,

IRON,

DIS-

SOLVED ERABLE

(UG/L UUG/L

DEC 1981

$15 \ldots$

JAN 1982

$21 \ldots$

FEB

$18 .$.

MAR

$18 . .$.

APR

$18 .$.

$18 .$.

$18 \ldots$

$19 .$.

MAY

$03 . .$.

26...

JUL

20 ...

AUG

$11 \ldots$

SEP

$13 \ldots$

OCT

$20 \ldots$

NOV

22 ...

DEC

$08, \ldots$

$\begin{array}{cccc}-- & -- & -- & - \\ -- & -- & - & - \\ -- & -- & -- & - \\ -- & -- & -- & - \\ 320 & - & -- & - \\ 330 & -- & -- & - \\ 280 & -- & -- & - \\ 360 & -- & -- & - \\ 290 & <100 & -- & - \\ 110 & <100 & -- & - \\ -- & -- & -- \\ -- & -- & - & - \\ -- & -- & - & - \\ -- & -- & - & - \\ -- & -- & - & - \\ -- & -- & - & -\end{array}$

-
-
--
--
--
--
--
--
-
-
-

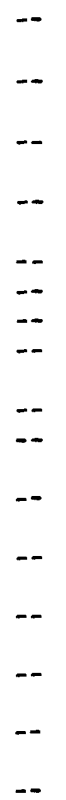

$<1$

AS $\mathrm{CU}$ )

AS FE)

DATE

DIS- RECOV-

SOLVED

ERABLE

(UG/L

(UG/L

AS PB)

DIS-

(UG/L

AS PB)

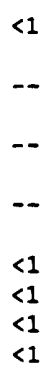

--

DEC 1981

JAN 1982

$21 \ldots$

FEB

$18 \ldots$

MAR

$18 \ldots$

APR

$18 .$.

$18 .$.

$18 .$.

$19 .$.

MAY

$03 \ldots$

26...

गय

20 ...

AUG

$11 \ldots$

SEP

$13 .$.

ocT

20...

NOV

22 ...

DEC

$08 .$.

\begin{tabular}{|c|c|c|c|c|c|c|c|}
\hline -- & $<1$ & -- & -- & $<5$ & -- & -- & -- \\
\hline -- & - & -- & -- & $<5$ & -- & -- & -- \\
\hline -- & -- & -- & -- & 6 & -- & -- & - \\
\hline- & $\cdots$ & -- & $\cdots$ & 5 & -- & 1 & 0.00 \\
\hline -- & $<1$ & -- & -- & $<8$ & -- & 5 & -- \\
\hline - & $<1$ & -- & -- & $<8$ & -- & - & -- \\
\hline-- & $<1$ & -- & -- & $<8$ & -- & - & -- \\
\hline -- & $<1$ & -- & -- & $<8$ & -- & - & - \\
\hline 60 & $<1$ & $<1$ & -- & $<5$ & $<5$ & 1 & -- \\
\hline 60 & $<1$ & $<1$ & - & $<5$ & $<5$ & - & -- \\
\hline- & $<1$ & - & -- & $<1$ & -- & 2 & 0.00 \\
\hline- & - & - & - & $<8$ & -- & 2 & 0.00 \\
\hline-- & - & -- & -- & $<5$ & -- & 2 & 0.00 \\
\hline & -- & -- & -- & -- & -- & 1 & 0.00 \\
\hline - & -- & -- & -- & 10 & -- & 7 & .06 \\
\hline- & -- & -- & -- & 6 & -- & 2 & .02 \\
\hline
\end{tabular}


TAx. 4.--Physical characteristics and concentrations of commond chemical constituents in lakes and streams of the Bald Mountain watershed--Continued

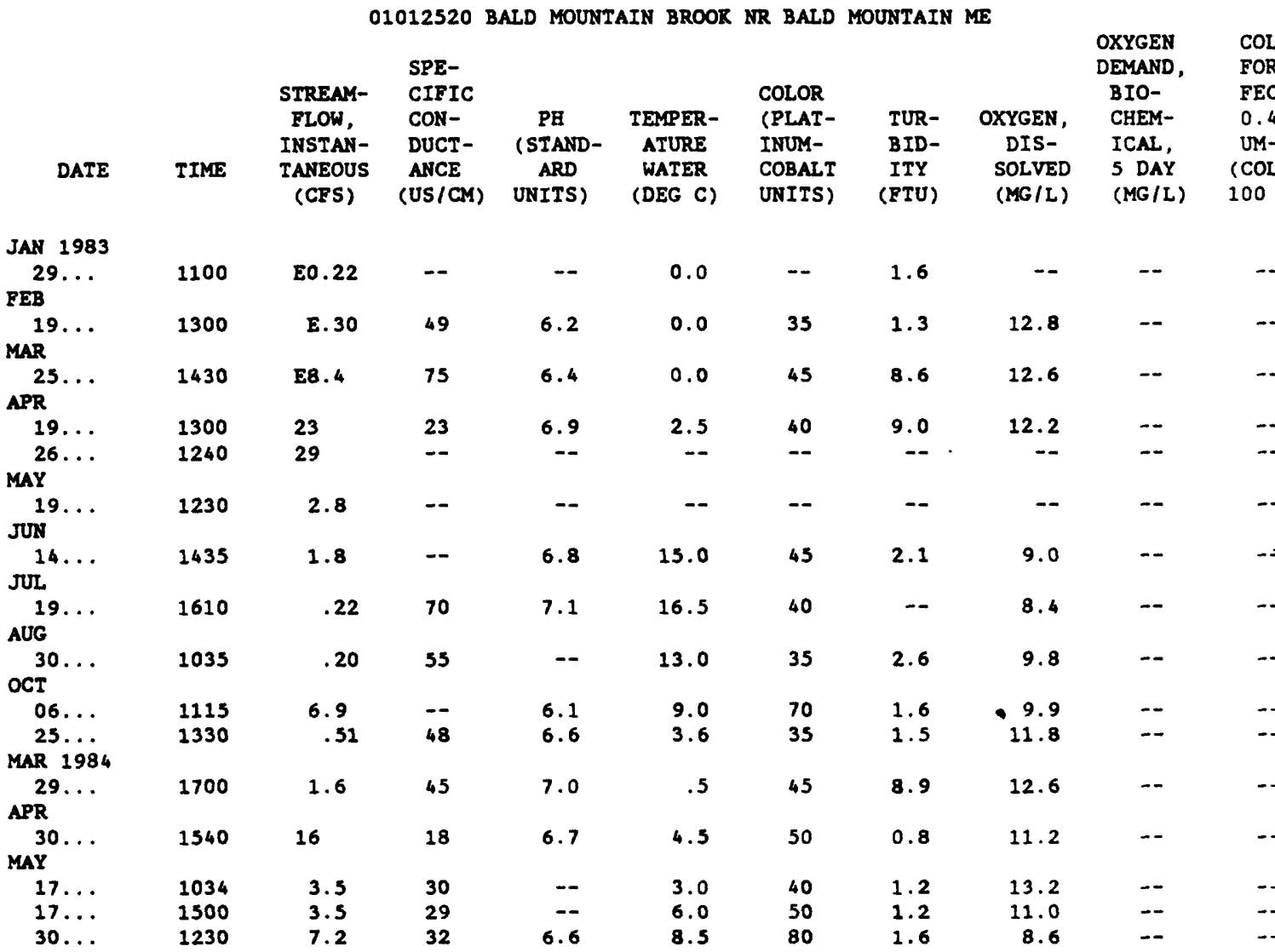

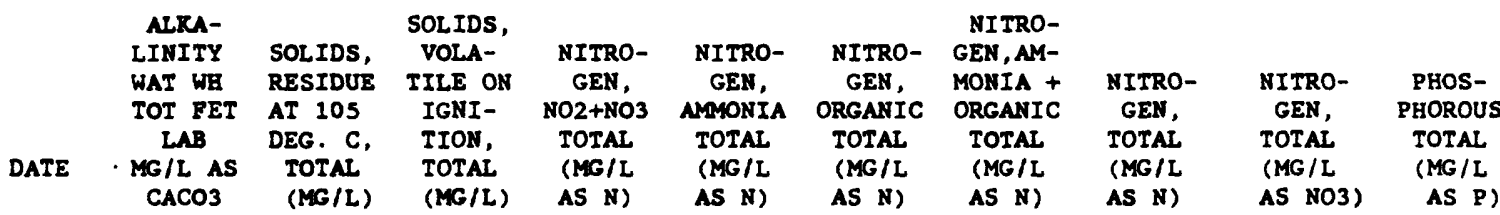

\begin{tabular}{|c|c|c|c|c|c|c|c|c|c|c|}
\hline $\begin{array}{c}\text { JAN } 1983 \\
29 \ldots \\
\text { FEB }\end{array}$ & -- & -- & -- & -- & -- & -- & -- & -- & -- & $<0.01$ \\
\hline $\operatorname{MAR}_{\operatorname{MAR}}^{19 \ldots}$ & 23 & 57 & -- & -- & -- & -- & -- & -- & -- & $<.01$ \\
\hline${ }_{A P R}^{25 \cdots}$ & 12 & -- & -- & -- & -- & -- & -- & -- & -- & .02 \\
\hline $\begin{array}{l}19 \ldots \\
26 \ldots\end{array}$ & $\begin{array}{r}4 \\
16\end{array}$ & -- & $=$ & $=$ & $=$ & $=$ & $\begin{array}{l}-- \\
--\end{array}$ & $=$ & -- & $\begin{array}{l}-. \\
.04\end{array}$ \\
\hline MAY & & & & & & & & & & \\
\hline JUN $^{19 \ldots}$ & -- & -- & -- & -- & -- & - & -- & -- & -- & .02 \\
\hline $\mathrm{JUL}^{14 \ldots}$ & 10 & -- & -- & -- & -- & -- & -- & -- & -- & .01 \\
\hline${ }_{A \cup G}^{19 \ldots}$ & 32 & -- & -- & - & -- & -- & -- & -- & -- & .01 \\
\hline $\mathrm{ocT}^{30 \ldots}$ & 28 & -- & -- & -- & -- & -- & -- & -- & -- & .01 \\
\hline $06 \ldots$ & 8 & 118 & -- & -- & -- & -- & -- & -- & -- & .01 \\
\hline $\begin{array}{c}25 \ldots \\
\operatorname{MaR} 1984\end{array}$ & 21 & 54 & -- & -- & -- & -- & -- & -- & - & .01 \\
\hline$\underset{A P R}{29 \ldots}$ & 13 & 74 & -- & -- & $-\infty$ & -- & -- & -- & -- & -- \\
\hline$\underset{\operatorname{MAY}}{30}$ & 2 & 49 & -- & -- & -- & -- & -- & -- & -- & -- \\
\hline $17 \ldots$ & 8 & 63 & -- & - & - & -- & -- & -- & -- & -- \\
\hline $17 \ldots$ & 6 & 68 & -- & - & - & -- & -- & -- & -- & $\overline{-1}$ \\
\hline $30 \ldots$ & 4 & 30 & 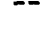 & - & $\rightarrow$ & $\cdots$ & -- & -- & -- & .02 \\
\hline
\end{tabular}


T.IT 4.--Physical characteristics and concentrations of commond chemical constituents in lakes and streams of the Bald Mountain watershed--Continued

01012520 BALD MOUNTAIN BROOK NR BALD MOUNTAIN ME

ATE ALUMINUM, ALUMTOTAL RECOVERABLE (UG/L AS AL)

\begin{tabular}{|c|c|c|c|}
\hline $\begin{array}{l}\text { ALUM- } \\
\text { INUM, }\end{array}$ & & $\begin{array}{l}\text { CADMIUM } \\
\text { TOTAL }\end{array}$ & $\begin{array}{l}\text { CHRO- } \\
\text { MIUY, } \\
\text { TOTAL }\end{array}$ \\
\hline $\begin{array}{l}\text { DIS- } \\
\text { SOLVED } \\
\text { (UG/L }\end{array}$ & $\begin{array}{l}\text { ARSENIC } \\
\text { TOTAL } \\
\text { (UG/L }\end{array}$ & $\begin{array}{l}\text { RECOV- } \\
\text { ERABLE } \\
\text { (UG/L }\end{array}$ & $\begin{array}{l}\text { RECOV- } \\
\text { ERABLE } \\
\text { (UG/L }\end{array}$ \\
\hline AS AL) & AS AS) & AS CD) & AS (R) \\
\hline
\end{tabular}

COPPER,
TOTAL
RECOV-
ERABLE
(UG/L
AS CU)

$\begin{array}{cc} & \text { IRON, } \\ \text { COPPER, } & \text { TOTAL } \\ \text { DIS- } & \text { RECOV- } \\ \text { SOLVED } & \text { ERABLE } \\ \text { (UG/L } & \text { (UG/L } \\ \text { AS CU) } & \text { AS FE) }\end{array}$

JAN 1983

$29 .$.

FEB

$19 . \ldots$

MAR

25...

APR

$19 . .$.

$26 . .$.

MAY

$19 .$.

JUN

$14 \ldots$

JUL

$19 .$.

AUG

$30 . .$.

OCT

$06 . .$.

25 ...

MAR 1984

29 ...

APR

$30 \ldots$

MAY

$17 \ldots$

$17 .$.

$30 \ldots$

\begin{tabular}{|c|c|c|c|}
\hline-- & -- & -- & -- \\
\hline-- & - & -- & -- \\
\hline-- & -- & -- & -- \\
\hline-- & -- & -- & -- \\
\hline-- & -- & -- & -- \\
\hline-- & -- & -- & -- \\
\hline-- & -- & -- & -- \\
\hline-- & -- & -- & -- \\
\hline-- & -- & -- & -- \\
\hline-- & -- & -- & - \\
\hline-- & -- & -- & -- \\
\hline-- & -- & -- & -- \\
\hline-- & -- & -- & -- \\
\hline-- & -- & -- & -- \\
\hline-- & -- & -- & -- \\
\hline-- & -- & -- & -- \\
\hline
\end{tabular}

$\begin{array}{lccc}-- & <1 & -- & 80 \\ -- & <1 & -- & 100 \\ - & 2 & -- & 920 \\ -- & <1 & -- & 580 \\ -- & 1 & -- & 330 \\ -- & 1 & -- & 170 \\ -- & 1 & -- & 170 \\ -- & 3 & -- & 100 \\ -- & 1 & -- & 160 \\ -- & 1 & -- & 280 \\ -- & <1 & -- & 140 \\ -- & 2 & -- & 580 \\ -- & -- & -- & -- \\ - & -- & - & -150\end{array}$

DATE

\begin{tabular}{|c|c|c|c|}
\hline $\begin{array}{l}\text { IRON, } \\
\text { DIS- } \\
\text { SOLVED } \\
\text { (UG/L }\end{array}$ & $\begin{array}{l}\text { LEAD, } \\
\text { TOTAL } \\
\text { RECOV- } \\
\text { ERABLE } \\
\text { (UG/L }\end{array}$ & $\begin{array}{l}\text { LEAD, } \\
\text { DIS- } \\
\text { SOLVED } \\
\text { (UG/L }\end{array}$ & $\begin{array}{l}\text { TOTAL } \\
\text { RECOV- } \\
\text { ERABLE } \\
\text { (UG/L }\end{array}$ \\
\hline AS FE) & AS $\mathbf{P B}$ ) & AS PB) & AS EG) \\
\hline
\end{tabular}
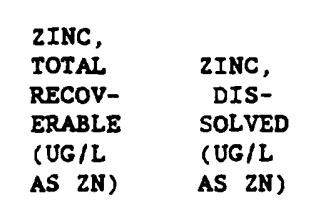

SEDI-

MENT,

MENI,

CHARGE

MENT,
SUS -

SUS -
PENDED

(MG/L) - (T/DAY)

JAN 1983

$29 .$.

FEB

19...

MAR

25...

APR

19...

26 ...

MAY

$19 .$.

JUN

$14 .$.

JuL

19 . . .

AUG

30 ...

OCT

$06 . .$.

25...

MAR 1984

29 ...

APR

$30 .$.

MAY

17 ...

$17 \ldots$

$30 .$.

$\begin{array}{lll}-- & - & - \\ -- & - & - \\ -- & - & - \\ -- & -- & - \\ -- & 2 & - \\ -- & -- & - \\ -- & -- & -- \\ -- & -- & -- \\ -- & -- & -- \\ -- & -- & -1 \\ -- & - & - \\ -- & -1 & - \\ -- & -1 & -\end{array}$

$\begin{array}{ll}-- & < \\ -- & <5 \\ -- & 1 \\ -- & <5 \\ -- & <1 \\ -- & \\ -- & 1 \\ -- & 38 \\ -- & - \\ -- & - \\ -- & \end{array}$

$\begin{array}{llcc}<5 & -- & 1 & 0.00 \\ <5 & -- & -- & -- \\ 8 & -- & -- & -- \\ 5 & -- & -- & .39 \\ 10 & -- & 5 & -- \\ <5 & -- & -- & -- \\ <10 & -- & -- & -- \\ 6 & -- & -- & 0.00 \\ <5 & -- & 4 & .06 \\ 12 & -- & 3 & .01 \\ 38 & -- & 6 & .02 \\ 9 & -- & 6 & .08 \\ -- & -- & 2 & .01 \\ -- & -- & 1 & \end{array}$


TAIIE 4. --Physical characterlstics and concentrations of commond chemical constltuents in lakes and straams of the Bald Mountain watershed--Continued

01012525 BISHOP MOUNTAIN BROOK NR BISHOP MTN ME

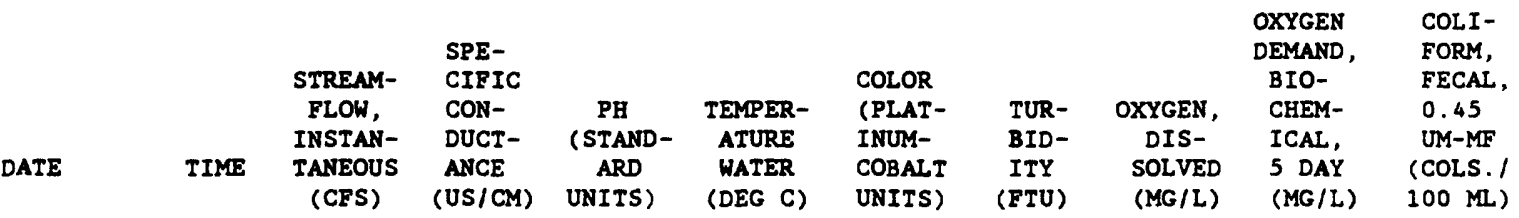

\begin{tabular}{|c|c|c|c|c|c|c|c|c|}
\hline$\underset{S E P}{29 \ldots}$ & 1245 & -- & 60 & 6.7 & 16.0 & 25 & 1.0 & 8.8 \\
\hline $\operatorname{OCT}^{29 \cdots}$ & 1245 & -- & 38 & 6.2 & 5.0 & 70 & 2.1 & 10.8 \\
\hline $\operatorname{NOV}^{14 \ldots}$ & 1245 & -- & 61 & 6.2 & 5.0 & 65 & 2.6 & 11.0 \\
\hline DEC $21 \ldots$ & 1300 & -- & 155 & 6.8 & 0.0 & 40 & 1.5 & 12.9 \\
\hline $\begin{array}{l}12 \ldots \\
\text { JAN } 1981\end{array}$ & 1400 & -- & 115 & 6.0 & 0.0 & 60 & 1.4 & 13.1 \\
\hline${ }_{\mathrm{FEB}}^{20} \ldots$ & 1315 & -- & 58 & 6.6 & 0.0 & 30 & 2.3 & 23.8 \\
\hline $\operatorname{MAR}^{23} \cdots$ & 1355 & -- & 22 & 6.0 & 2.0 & 80 & 4.2 & 11.4 \\
\hline${ }_{A P R}^{18 \ldots}$ & 1115 & -- & 46 & 6.5 & 1.0 & 30 & 1.9 & 13.1 \\
\hline $\operatorname{MAY}^{14} \cdots$ & 1345 & -- & 55 & 6.1 & 0.0 & 45 & 2.6 & 11.8 \\
\hline JUN & 1220 & -- & 28 & 6.1 & 6.0 & 70 & 1.0 & 10.7 \\
\hline $\mathrm{JUL}^{10 \ldots}$ & 1440 & -- & 40 & 6.2 & 10.0 & 45 & 2.1 & 8. 9 \\
\hline${ }_{A U G}^{21} \cdots$ & 1440 & -- & 88 & 6.3 & 16.0 & 180 & 3.2 & -- \\
\hline $\operatorname{SEP}^{24} \cdots$ & 1345 & $=-$ & 33 & 6.1 & 14.0 & 80 & 1.2 & 7.6 \\
\hline $22 \ldots$ & 1250 & -- & 25 & 6.8 & 10.0 & 90 & 2.0 & 9.4 \\
\hline
\end{tabular}

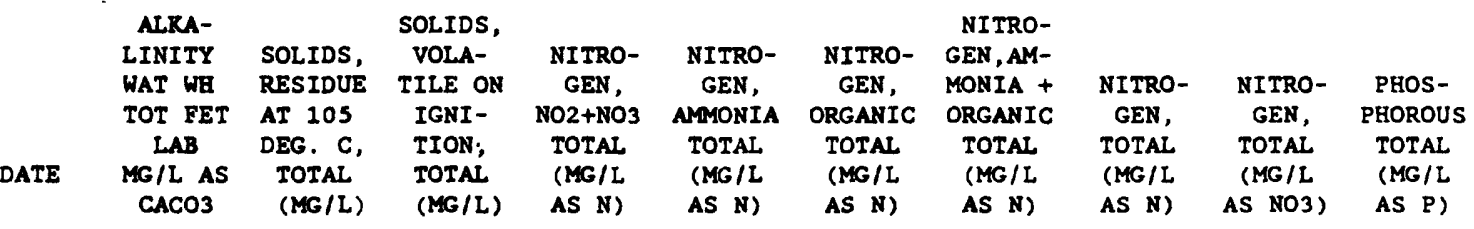

AUG 1980

\begin{tabular}{|c|c|c|}
\hline $29 \ldots$ & 21 & 57 \\
\hline \multicolumn{3}{|l|}{ SEP } \\
\hline $29 \ldots$ & 10 & 64 \\
\hline \multicolumn{3}{|l|}{ OCT } \\
\hline $14 \ldots$ & 12 & 81 \\
\hline \multicolumn{3}{|l|}{ Nov } \\
\hline $21 \ldots$ & 13 & 77 \\
\hline \multicolumn{3}{|l|}{ DEC } \\
\hline $12 \ldots$ & 16 & 59 \\
\hline \multicolumn{3}{|l|}{ JAN 1981} \\
\hline $20 \ldots$ & 23 & - \\
\hline \multicolumn{3}{|l|}{ FEB } \\
\hline $23 \ldots$ & 6 & - \\
\hline \multicolumn{3}{|l|}{ MAR } \\
\hline $18 \ldots$ & 17 & 54 \\
\hline \multicolumn{3}{|l|}{ APR } \\
\hline $14 \ldots$ & 12 & 51 \\
\hline \multicolumn{3}{|l|}{ MAY } \\
\hline $15 \ldots$ & 8 & 108 \\
\hline \multicolumn{3}{|l|}{ JUN } \\
\hline $10 \ldots$ & 18 & 70 \\
\hline \multicolumn{3}{|l|}{ JuL } \\
\hline $21 \ldots$ & 44 & 92 \\
\hline \multicolumn{3}{|l|}{ AUG } \\
\hline $24 \ldots$ & 8 & 52 \\
\hline \multicolumn{3}{|l|}{ SEP } \\
\hline $22 \ldots$ & 11 & 58 \\
\hline
\end{tabular}


ram: 4.--Physical characteristics and concentrations of commond chemical constituents in lakes and streans of the Bald Mountain watershed--Continued

01012525 BISHOP MOUNTAIN BROOK NR BISHOP MTN ME

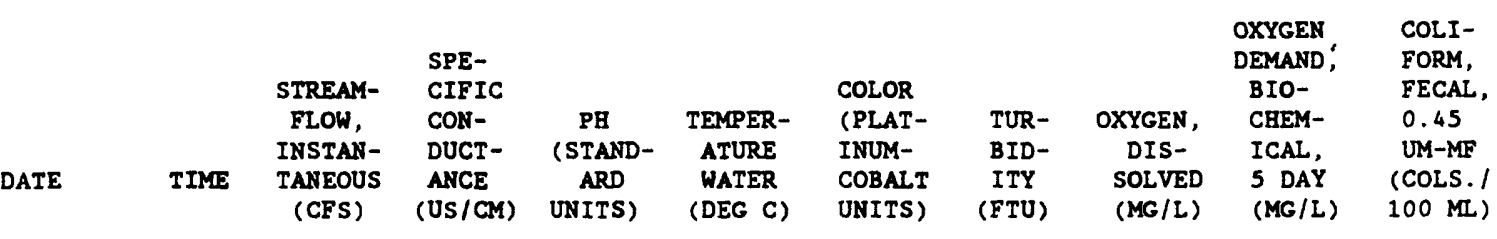

OCT 1981

20 Nov

$17 \ldots$

DEC

$15 \ldots$

JAN 1982

21 ...

FEB

$18 \ldots$

MAR

$18 \ldots$

APR

$18 \ldots$

$18 \ldots$

$18 \ldots$

$19 .$.

MAY

$03 .$.

26...

JUL

20...

AUG

$11 \ldots$

SEP

$13 \ldots$

OCT

$20 .$.

$\begin{array}{ccc}1250 & -- & 39 \\ 1210 & 1.3 & 58 \\ 1130 & E 1.3 & 34 \\ 1110 & E 0.44 & 44 \\ 1100 & E .26 & 37 \\ 1300 & E .43 & 59 \\ 1135 & E 22 & -- \\ 1335 & E 22 & -- \\ 1605 & E 22 & -- \\ 1535 & E 16 & -- \\ 1450 & E 3.5 & -- \\ 1400 & E .60 & 33 \\ 1035 & .13 & 93 \\ 1400 & .42 & 70 \\ 1400 & .55 & 58 \\ 1400 & 1.8 & 38\end{array}$

\begin{abstract}
6.1
\end{abstract}
5.0

6.4

3.0

6.2

0.0

6.7

0.0

7.0

0.0

6.5

3.0

6.1

--
--
--

$\begin{array}{rr}100 & 18 \\ -- & -- \\ -- & -- \\ -- & --\end{array}$

$\begin{array}{rr}-5 & 1.0 \\ 6.5 & 10.0\end{array}$

35

$6.7 \quad 21.0$

6.6

17.0

80

1.8

10.8

5.9

19.0

70

1.9

6.7

1.5

7.2

$6.9 \quad 10.0$

100

2.2

60

1.3

8.4

10.4

ALRA-

LINITY

WAT WB

TOT FET

LAB

DATE MG/L AS CACO3

$\begin{array}{lccccc}\text { SOLIDS, } & & & \\ \text { SOLIDS, } & \text { VOLA- } & \text { NITRO- } & \text { NITRO- } & \text { NITRO- } & \text { GEN, AM- } \\ \text { RESIDUE } & \text { TILE ON } & \text { GEN, } & \text { GEN, } & \text { GEN, } & \text { MONIA + } \\ \text { AT 105 } & \text { IGNI- } & \text { NO2+NO3 } & \text { NOMONIA } & \text { ORGANIC } & \text { ORGANIC } \\ \text { DEG. C, } & \text { TION, } & \text { TOTAL } & \text { TOTAL } & \text { TOTAL } & \text { TOTAL } \\ \text { TOTAL } & \text { TOTAL } & \text { (MG/L } & \text { (MG/L } & \text { (MG/L } & \text { (MG/L } \\ \text { (MG/L) } & \text { (MG/L) } & \text { AS N) } & \text { AS N) } & \text { AS N) } & \text { AS N) }\end{array}$

NITRO- NITRO- PHOS-

GEN, GEN, PHOROUS

TOTAL TOTAL TOTAL

(MG/L (MG/L (MG/L

AS N) AS NO3) AS P)

OCT 1981

20 . .
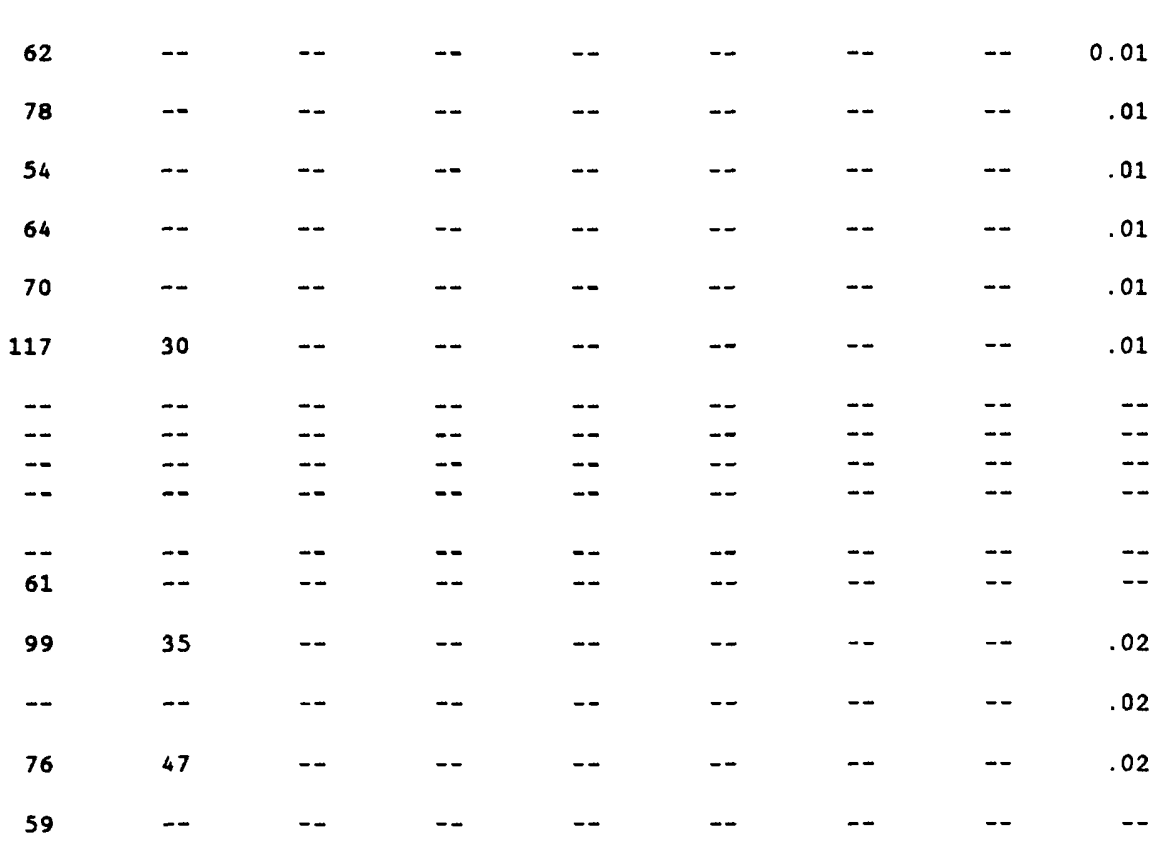
IATE 4.--Physlcal characteristics and concentrations of comond chemical constituents in lakes and streams of the Bald Mountain watershed--Continued

01012525 BISHOP MOUNTAIN BROOK NR BISHOP MTN ME

\begin{tabular}{|c|c|c|c|c|c|c|c|c|c|}
\hline TIME & $\begin{array}{l}\text { STREAM- } \\
\text { FLOW, } \\
\text { INSTAN- } \\
\text { TANEOUS } \\
\text { (CES) }\end{array}$ & $\begin{array}{l}\text { SPE- } \\
\text { CIFIC } \\
\text { CON- } \\
\text { DUCT- } \\
\text { ANCE } \\
\text { (US/CM) }\end{array}$ & $\begin{array}{c}\text { PH } \\
\text { (STAND- } \\
\text { ARD } \\
\text { UNITS) }\end{array}$ & $\begin{array}{c}\text { TEMPER- } \\
\text { ATURE } \\
\text { WATER } \\
\text { (DEG C) }\end{array}$ & $\begin{array}{l}\text { COLOR } \\
\text { (PLAT- } \\
\text { INUM- } \\
\text { COBALI } \\
\text { UNITS) }\end{array}$ & $\begin{array}{l}\text { TUR- } \\
\text { BID- } \\
\text { ITY } \\
\text { (FTU) }\end{array}$ & $\begin{array}{c}\text { OXYGEN, } \\
\text { DIS- } \\
\text { SOLVED } \\
\text { (MG/L) }\end{array}$ & $\begin{array}{l}\text { OXYGEN } \\
\text { DEMAND, } \\
\text { BIO- } \\
\text { CHEM- } \\
\text { ICAL, } \\
\text { 5 DAY } \\
\text { (MG/L) }\end{array}$ & $\begin{array}{l}\text { COLI- } \\
\text { FORM, } \\
\text { FECAL, } \\
0.45 \\
\text { UM-MF } \\
\text { (COLS. ' } \\
100 \mathrm{ML} \text { ) }\end{array}$ \\
\hline
\end{tabular}

NOV 1982

\begin{tabular}{|c|c|}
\hline $22 \ldots$ & 1600 \\
\hline DEC & \\
\hline $08 \ldots$ & 1130 \\
\hline JAN 1983 & \\
\hline $29 \ldots$ & 1030 \\
\hline FEB & \\
\hline $19 \ldots$ & 1345 \\
\hline MAR & \\
\hline $25 \ldots$ & 1430 \\
\hline APR & \\
\hline $19 \ldots$ & 1130 \\
\hline $26 \ldots$ & 1330 \\
\hline MAY & \\
\hline 19. & 1200 \\
\hline JUN & \\
\hline $14 \ldots$ & 1250 \\
\hline JUL & \\
\hline $19 \ldots$ & 1555 \\
\hline AUG & \\
\hline $30 \ldots$ & 1020 \\
\hline OCT & \\
\hline $06 \ldots$ & 1400 \\
\hline $25 \ldots$ & 1153 \\
\hline MAR 1984 & \\
\hline $29 \ldots$ & 1645 \\
\hline APR & \\
\hline $30 \ldots$ & 1355 \\
\hline
\end{tabular}

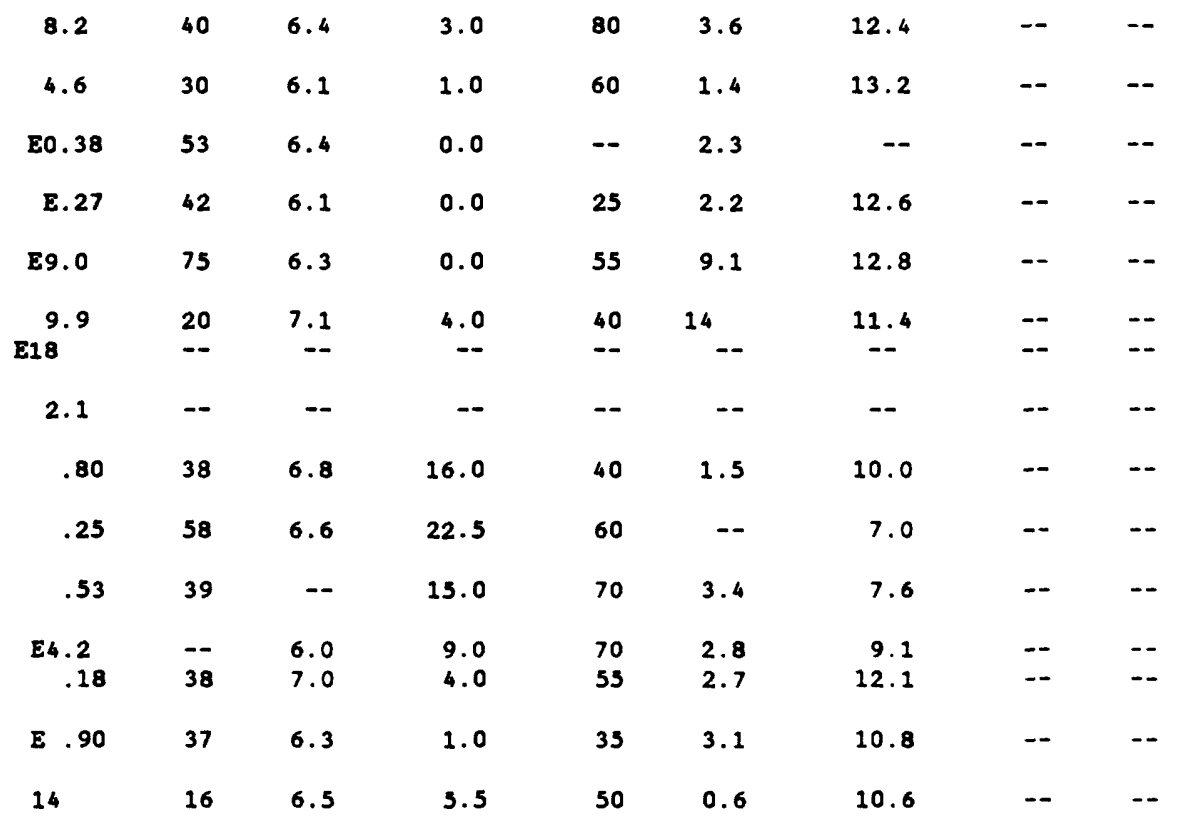

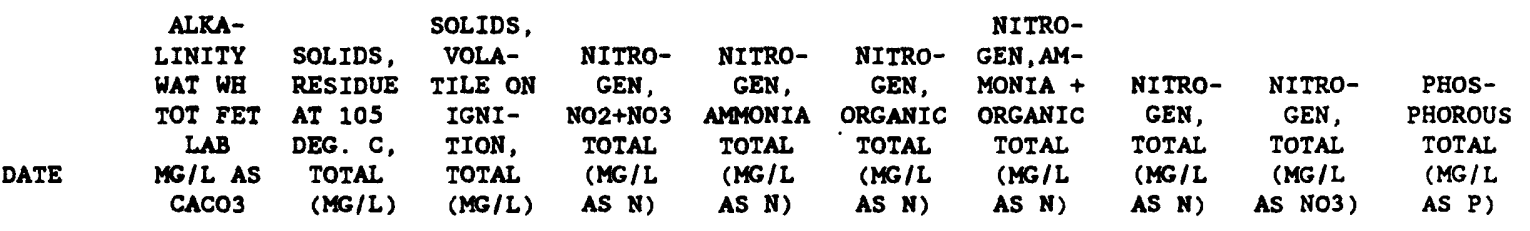

Nov 1982

$22 \ldots$
DEC
OB $\ldots$
JAN 198

JAN 1983

29 ..

FEB

$19 .$.

MAR

25...

APR

$19 .$.

26 ...

MAY

$19 .$.

JUN

$14 \ldots$

JUL. $19 .$.

AUG

$30 .$.

OCT

$06 .$.

$25 .$.

MAR 1984

29 . .

APR

$30 .$.

\begin{tabular}{|c|c|c|}
\hline 3 & 61 & -- \\
\hline 4 & 63 & - \\
\hline-- & 80 & -- \\
\hline 26 & 55 & -- \\
\hline 14 & -- & -- \\
\hline 3 & -- & -- \\
\hline 13 & 45 & -- \\
\hline-- & -- & - \\
\hline 16 & -- & $=-$ \\
\hline 20 & -- & - \\
\hline 22 & -- & -- \\
\hline 8 & 112 & -- \\
\hline 16 & 55 & -- \\
\hline 10 & 62 & -- \\
\hline 3 & 46 & -- \\
\hline
\end{tabular}

\begin{tabular}{|c|c|}
\hline-- & -- \\
\hline-- & -- \\
\hline -- & -- \\
\hline-- & -- \\
\hline-- & -- \\
\hline-- & -- \\
\hline-- & -- \\
\hline-- & -- \\
\hline-- & - \\
\hline-- & -- \\
\hline-- & -- \\
\hline-- & -- \\
\hline-- & -- \\
\hline-- & -- \\
\hline & - \\
\hline
\end{tabular}

\begin{tabular}{|c|c|c|c|}
\hline-- & -- & -- & 0.01 \\
\hline-- & -- & -- & .01 \\
\hline - & -- & -- & .01 \\
\hline- & -- & -- & $<.01$ \\
\hline- & -- & - & .02 \\
\hline- & -- & -- & -- \\
\hline - & -- & -- & .04 \\
\hline - & -- & -- & .01 \\
\hline - & -- & -- & .01 \\
\hline- & -- & - & .02 \\
\hline - & -- & -- & .02 \\
\hline - & -- & -- & .02 \\
\hline & -- & -- & .01 \\
\hline- & -- & -- & -- \\
\hline & -- & -- & -- \\
\hline
\end{tabular}


TABIB 4.--Physical characteristics and concentrations of commond chemical constituents in lakes and streams of the Bald Mountain watershed--Continued

01012525 BISEOP MOUNTAIN BROOK NR BISHOP MTN ME

AUG 1980

29. .

SEP

$29 .$.

OCT

$14 \ldots$

NoV

$21 \ldots$

DEC

12 ...

JAN 1981

$20 .$.

FEB

$23 \ldots$

MAR

$18 \ldots$

APR

14 . .

MAY

$15 \ldots$

JUN

$10 \ldots$

JUL

21...

AUG

24...

SEP

22 ...

ALUM-

INUM, ALUM-

TOTAL

RECOV-

ERABLE

(UG/L

INUM,
DIS-

SOLVED

(UG/L

AS AL)

AS AL)

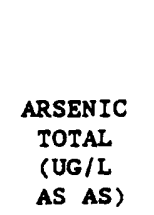

$\begin{array}{cl} & \text { CERO- } \\ \text { CADMIUM } & \text { MIUM, } \\ \text { TOTAL } & \text { TOTAL } \\ \text { RECOV- } & \text { RECOV- } \\ \text { ERABLE } & \text { ERABLE } \\ \text { (UG/L } & \text { (UG/L } \\ \text { AS CD) } & \text { AS CR) }\end{array}$

COPPER,

TOTAI

RECOV-

IRON,

TOTAL

ERABLE SOLVED

AS AS )

AS CD)

AS CR)

(UG/L

(UG/L

AS CU)

AS $\mathrm{CU})$

AS FE)

$\begin{array}{rrrrr}-- & -- & <4 & -- & 560 \\ -- & -- & <4 & -- & 410 \\ -- & -- & <6 & -- & 270 \\ -- & -- & <1 & -- & -- \\ -- & -- & 2 & -- & 160 \\ -- & -- & -- & -- & 130 \\ -- & -- & <1 & -- & 380 \\ -- & -- & 3 & -- & 140 \\ -- & -- & 1 & -- & 230 \\ -- & -- & <1 & -- & 160 \\ -- & -- & <1 & -- & 190 \\ -- & -- & <2 & -- & 850 \\ <4 & -- & 16 & -- & 60 \\ -- & -- & <2 & -- & 430\end{array}$

\begin{tabular}{|c|c|c|c|c|c|c|c|c|}
\hline ITE & $\begin{array}{l}\text { IRON, } \\
\text { DIS- } \\
\text { SOLVED } \\
\text { (UG/L }\end{array}$ & $\begin{array}{l}\text { LEAD, } \\
\text { TOTAL } \\
\text { RECOV- } \\
\text { ERABLE } \\
\text { (UG/L }\end{array}$ & $\begin{array}{l}\text { LEAD, } \\
\text { DIS- } \\
\text { SOLVED } \\
\text { (UG/I }\end{array}$ & $\begin{array}{l}\text { MERCURY } \\
\text { IOTAL } \\
\text { RECOV- } \\
\text { ERABLE } \\
\text { (UG/L }\end{array}$ & $\begin{array}{l}\text { ZINC, } \\
\text { TOTAL } \\
\text { RECOV- } \\
\text { ERABLE } \\
\text { (UG/L }\end{array}$ & $\begin{array}{l}\text { ZINC, } \\
\text { DIS- } \\
\text { SOLVED } \\
\text { (UG/L }\end{array}$ & $\begin{array}{l}\text { SEDI- } \\
\text { MENT, } \\
\text { SUS- } \\
\text { PENDED }\end{array}$ & $\begin{array}{c}\text { SEDI- } \\
\text { MENT, } \\
\text { DIS- } \\
\text { CHARGE , } \\
\text { SUS- } \\
\text { PENDED }\end{array}$ \\
\hline & & & & & & & & \\
\hline
\end{tabular}

AUG 1980

29..

SEP

29...

OCT

14 ...

NOV

21...

DEC

12 ...

JAN 1981

20 ...

FEB

$23 \ldots$

MAR

$18 \ldots$

APR

14 ..

MAY

$15 \ldots$

JUN

$10 \ldots$

JUL

21...

AUG

24...

SEP

22 ...

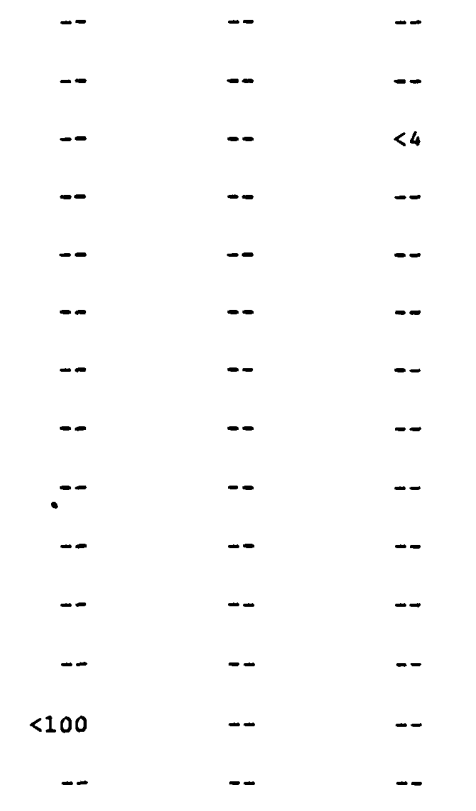


TABI. 4.--Physical characteristics and concentrations of commond chemical constituents in lakes and streams of the Bald Mountain watershed--Continued

01012525 BISHOP MOUNTAIN BROOK NR BISHOP MTN ME

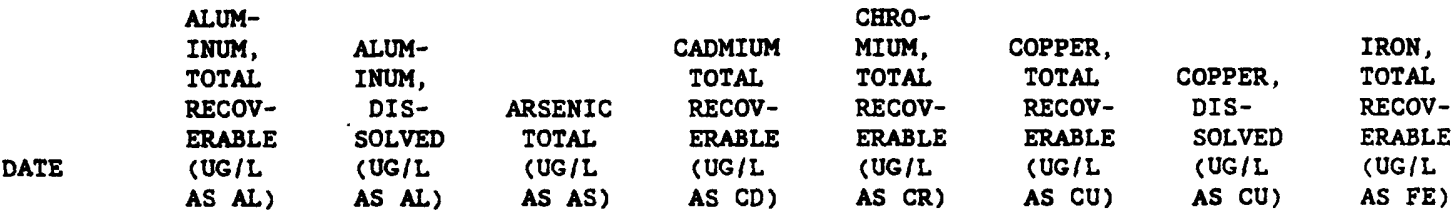

OCT 1981

20...

Nov

$17 .$.

DEC

$15 .$.

JAN 1982

$21 \ldots$

FEB

$18 \ldots$

MAR

$18 .$.

APR

18...

$18 .$.

18...

$19 . .$.

MAY

$03 . .$.

26...

JUL

$20 \ldots$

AUG

11...

SEP

$13 . .$.

OCT

20...

\begin{tabular}{|c|c|}
\hline-- & - \\
\hline-- & - \\
\hline- & -- \\
\hline- & -- \\
\hline -- & -- \\
\hline -- & -- \\
\hline 1900 & -- \\
\hline 1600 & -- \\
\hline 1300 & $=$ \\
\hline 740 & -- \\
\hline 210 & $<100$ \\
\hline 240 & $<100$ \\
\hline-- & -- \\
\hline-- & -. \\
\hline-- & - \\
\hline- & - \\
\hline
\end{tabular}

$\begin{array}{lll}-- & -- & - \\ -- & -- & - \\ -- & -- & - \\ -- & - & - \\ -- & - & - \\ -- & -- & - \\ -- & -- & - \\ -- & -- & - \\ -- & -- & - \\ -- & - & - \\ -- & -- & - \\ -- & -- & - \\ -- & - & - \\ -- & -\end{array}$

--
--
--
--
--
--
--
--
--
--
--

$5 \quad-140$

$1 \quad--\quad 260$

$<1 \quad--\quad 160$

$<1 \quad--\quad 180$

$1 \quad--\quad 150$

$1 \quad--\quad 340$

$4 \quad--\quad 1700$

$10 \quad 1700$

$\begin{array}{rrr}1 & -- & 1400\end{array}$

$<1 \quad 2 \quad 240$

$\begin{array}{llll}-- & 6 & -- & 660\end{array}$

$\begin{array}{llll}- & 2 & -- & 560\end{array}$

$\begin{array}{llll}- & <1 & - & 260\end{array}$

DATE

OCT 1981

20...

NOV

$17 .$.

DEC

$15 .$.

JAN 1982

$21 .$.

FEB

$18 \ldots$

MAR

18 ...

APR

$18 .$.

18...

18...

19.

MAY

$03 .$.

26...

गU.

20...

AUG

$11 \ldots$

SEP

$13 .$.

OCT

$20 .$.

\begin{tabular}{|c|c|c|c|c|c|c|c|}
\hline $\begin{array}{l}\text { IRON, } \\
\text { DIS- } \\
\text { SOLVED } \\
\text { (UG/L } \\
\text { AS FE) }\end{array}$ & $\begin{array}{l}\text { LEAD, } \\
\text { TOTAL } \\
\text { RECOV- } \\
\text { ERABLE } \\
\text { (UG/L } \\
\text { AS PB) }\end{array}$ & $\begin{array}{l}\text { LEAD, } \\
\text { DIS- } \\
\text { SOLVED } \\
\text { (UG/L } \\
\text { AS PB) }\end{array}$ & $\begin{array}{l}\text { MERCURY } \\
\text { TOTAL } \\
\text { RECOV- } \\
\text { ERABLE } \\
\text { (UG/L } \\
\text { AS BG) }\end{array}$ & $\begin{array}{l}\text { ZINC, } \\
\text { TOTAL } \\
\text { RECOV- } \\
\text { ERABLE } \\
\text { (UG/L } \\
\text { AS ZN) }\end{array}$ & $\begin{array}{l}\text { ZINC, } \\
\text { DIS- } \\
\text { SOLVED } \\
\text { (UG/L } \\
\text { AS ZN) }\end{array}$ & $\begin{array}{l}\text { SEDI- } \\
\text { MENT, } \\
\text { SUS- } \\
\text { PENDED } \\
\text { (MG/L) }\end{array}$ & $\begin{array}{r}\text { SEDI- } \\
\text { MENT, } \\
\text { DIS- } \\
\text { CHARGE } \\
\text { SUS- } \\
\text { PENDE } \\
\text { (T/DAY }\end{array}$ \\
\hline
\end{tabular}

\begin{tabular}{|c|c|c|c|c|c|c|c|}
\hline-- & -- & -- & -- & $<5$ & -- & -- & - \\
\hline-- & $<1$ & -- & -- & $<5$ & -- & -- & -- \\
\hline-- & $<1$ & -- & -- & $<5$ & -- & -- & -- \\
\hline-- & -- & -- & -- & $<5$ & -- & -- & -- \\
\hline-- & -- & -- & -- & 5 & -- & -- & -- \\
\hline -- & -- & -- & -- & $<5$ & -- & 1 & 0.00 \\
\hline -- & $<1$ & -- & -- & 9 & - & 10 & .59 \\
\hline -- & $<1$ & - & -- & $<8$ & -- & -- & -- \\
\hline -- & $<1$ & -- & -- & 10 & -- & -- & - \\
\hline-- & $<1$ & - & - & $<8$ & - & -- & -- \\
\hline 80 & $<1$ & 1 & -- & $<5$ & $<5$ & 2 & .02 \\
\hline 40 & $<1$ & 3 & -- & $<5$ & $<5$ & -- & -- \\
\hline -- & -- & -- & -- & 6 & -- & 2 & .00 \\
\hline -- & -- & -- & -- & $<8$ & - & 6 & .01 \\
\hline -- & -- & -- & -- & $<5$ & -- & 2 & .0 \\
\hline- & -- & -- & -- & 13 & -- & 2 & \\
\hline
\end{tabular}


TABIB 4.--Physical characteristics and concentrations of commond chemical constituents in lakes and streams of the Bald Mountain watershed--Continued

01012525 BISHOP MOUNTAIN BROOK NR BISHOP MTN ME

\begin{tabular}{|c|c|c|c|c|c|c|c|c|}
\hline DATE & $\begin{array}{l}\text { ALUM- } \\
\text { INUM, } \\
\text { TOTAL } \\
\text { RECOV- } \\
\text { ERABLE } \\
\text { (UG/L }\end{array}$ & $\begin{array}{l}\text { ALUM- } \\
\text { INUM, } \\
\text { DIS- } \\
\text { SOLVED } \\
\text { (UG/L }\end{array}$ & $\begin{array}{c}\text { ARSENIC } \\
\text { TOTAL } \\
\text { (UG/L }\end{array}$ & $\begin{array}{l}\text { CADMIUM } \\
\text { TOTAL } \\
\text { RECOV- } \\
\text { ERABLE } \\
\text { (UG/L }\end{array}$ & $\begin{array}{l}\text { CERO- } \\
\text { MIUM, } \\
\text { TOTAL } \\
\text { RECOV- } \\
\text { ERABLE } \\
\text { (UG/L }\end{array}$ & $\begin{array}{c}\text { COPPER, } \\
\text { TOTAL } \\
\text { RECOV- } \\
\text { ERABLE } \\
\text { (UG/L }\end{array}$ & $\begin{array}{l}\text { COPPER, } \\
\text { DIS- } \\
\text { SOLVED } \\
\text { (UG/L }\end{array}$ & $\begin{array}{l}\text { IRON, } \\
\text { TOTAL } \\
\text { RECOV- } \\
\text { ERABLE } \\
\text { (UG/L }\end{array}$ \\
\hline & AS AI) & AS AL) & AS AS) & AS CD) & AS CR) & AS CU) & AS CU) & AS FE) \\
\hline
\end{tabular}

NOV 1982

$22 \ldots$
DEC
$08 \ldots$
JAN 1983
$29 \ldots$
FEB
$19 \ldots$
MAR
$25 \ldots$
APR
$19 \ldots$
$26 \ldots$
MAY
$19 \ldots$
JUN
$14 \ldots$
JUL.
$19 \ldots$
AUG
$30 \ldots$
OCT
$06 \ldots$
$25 \ldots$
MAR 1984
$29 \ldots$
APR
$30 \ldots$

$\begin{array}{llll}-- & -- & -- & - \\ -- & -- & -- & -- \\ -- & -- & -- & -- \\ -- & -- & -- & -- \\ -- & -- & -- & -- \\ -- & -- & -- & -- \\ -- & -- & -- & -- \\ -- & -- & -- & -- \\ -- & -- & -- & -- \\ -- & -- & -- & -- \\ -- & -- & -- & -- \\ -- & -- & -- & -- \\ -- & -- & - & -- \\ -- & -- & -- & -- \\ -- & -- & -- & --\end{array}$

$\begin{array}{rrrr}-- & <1 & -- & 310 \\ -- & 1 & -- & 180 \\ -- & <1 & -- & 150 \\ -- & 1 & -- & 180 \\ -- & 1 & -- & 620 \\ -- & <1 & -- & 880 \\ -- & 1 & -- & 710 \\ -- & 1 & -- & 150 \\ -- & 1 & -- & 150 \\ -- & 2 & -- & 270 \\ -- & 1 & -- & 540 \\ -- & 1 & -- & 400 \\ -- & <1 & -- & 250 \\ -- & <1 & -- & 200 \\ -- & -- & -- & --\end{array}$

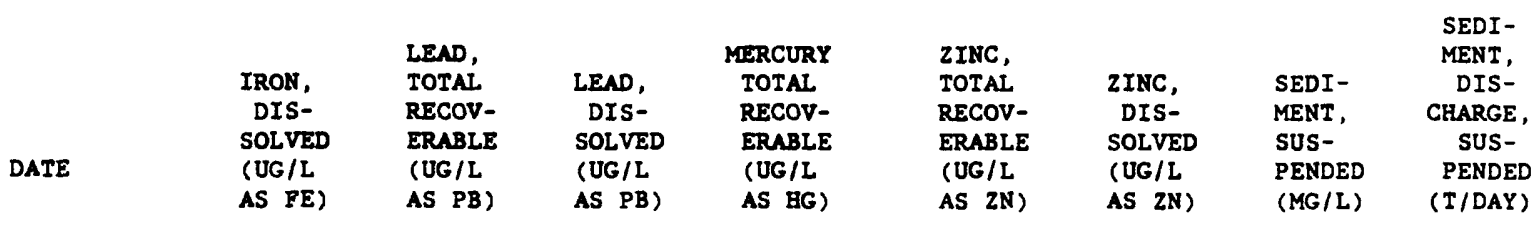

Nov 1982

$22 \ldots$
DEC
$08 \ldots$

JAN 1983

29 ...

FEB

$19 .$.

MAR
25.

APR

19...

26 ...

MAY

19...

JoN $14 \ldots$

JuL

19 ...

AUG

$30 .$.

OCT

$06 . .$.

25 ...

MAR 1984

29 . .

APR

$30 .$.

$\begin{array}{cccc}-- & -- & -- & - \\ -- & -- & -- & - \\ -- & -- & -- & - \\ -- & -- & -- & - \\ -- & -- & -- & - \\ -- & -- & -- & - \\ -- & -- & -- & - \\ -- & <1 & -- & - \\ -- & -- & -- & - \\ -- & -- & -- & - \\ -- & -- & -- & - \\ -- & -- & -- & -- \\ -- & -- & -- & -- \\ -- & <1 & -- & - \\ -- & -- & -- & --\end{array}$


ranal 4.--Physlcal characteristics and concentrations of commond chemical constituents in lakes and streams of the Bald Mountain watershed--Continued

01012525 BISHOP MOUNTAIN BROOR NR BISHOP MTN ME

\begin{tabular}{|c|c|c|c|c|c|c|c|c|c|c|}
\hline DATE & TIME & $\begin{array}{l}\text { STREAY- } \\
\text { FLOW, } \\
\text { INSTAN- } \\
\text { TANEOUS } \\
\text { (CFS) }\end{array}$ & $\begin{array}{l}\text { SPE- } \\
\text { CIFIC } \\
\text { CON- } \\
\text { DUCT- } \\
\text { ANCE } \\
\text { (US/CM) }\end{array}$ & $\begin{array}{c}\text { PE } \\
\text { (STAND- } \\
\text { ARD } \\
\text { UNITS) }\end{array}$ & $\begin{array}{c}\text { TEMPER- } \\
\text { ATURE } \\
\text { WATER } \\
\text { (DEG C) }\end{array}$ & $\begin{array}{l}\text { COLOR } \\
\text { (PLAT- } \\
\text { INUM- } \\
\text { COBALT } \\
\text { UNITS) }\end{array}$ & $\begin{array}{c}\text { TUR- } \\
\text { BID- } \\
\text { ITY } \\
\text { (FTU) }\end{array}$ & $\begin{array}{c}\text { OXYGEN, } \\
\text { DIS- } \\
\text { SOLVED } \\
\text { (MG/L) }\end{array}$ & $\begin{array}{l}\text { OXYGEN } \\
\text { DEMAND, } \\
\text { BIO- } \\
\text { CHEM- } \\
\text { ICAL, } \\
\text { 5 DAY } \\
\text { (MG /L) }\end{array}$ & $\begin{array}{l}\text { COLI- } \\
\text { FORM, } \\
\text { FECAL, } \\
0.45 \\
\text { UM-MF } \\
\text { (COLS. } \\
100 \mathrm{ML})\end{array}$ \\
\hline
\end{tabular}

MAY 1984

$30 .$.

1310

$\begin{array}{ll}7.5 & 27\end{array}$

6.50

9.0

80

2.4

8.9

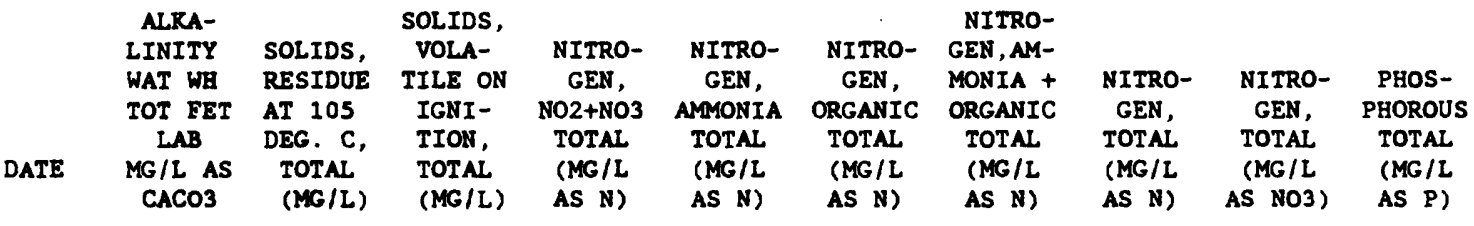

MAY 1984

$30 \ldots$

30

(2)

AS $N$ )

AS N)

AS N)

AS $N$ )

AS $\mathrm{NO3}$

AS P)

30. 
IAnTs 4.--Physlcal character1st1cs and concentraticns cf commond chemical constiuents In lakes and streams of the Bald Mountain watershed--Continued

01012525 BISHOP MOUNTAIN BROOR NR BISHOP MTN ME

\begin{tabular}{|c|c|c|c|c|c|c|c|c|}
\hline DATE & $\begin{array}{l}\text { ALUM- } \\
\text { INUM, } \\
\text { TOTAL } \\
\text { RECOV- } \\
\text { ERABLE } \\
\text { (UG/L }\end{array}$ & $\begin{array}{l}\text { ALUM- } \\
\text { INUM, } \\
\text { DIS- } \\
\text { SOLVED } \\
\text { (UG/L }\end{array}$ & $\begin{array}{l}\text { ARSENIC } \\
\text { TOTAL } \\
\text { (UG/L }\end{array}$ & $\begin{array}{l}\text { CADMIUM } \\
\text { TOTAL } \\
\text { RECOV- } \\
\text { ERABLE } \\
\text { (UG/L }\end{array}$ & $\begin{array}{l}\text { CERO- } \\
\text { MIUM, } \\
\text { TOTAL } \\
\text { RECOV- } \\
\text { ERABLE } \\
\text { (UG /L }\end{array}$ & $\begin{array}{l}\text { COPPER, } \\
\text { TOTAL } \\
\text { RECOV- } \\
\text { ERABLE } \\
\text { (UG/L }\end{array}$ & $\begin{array}{l}\text { COPPER, } \\
\text { DIS- } \\
\text { SOLVED } \\
\text { (UG /L }\end{array}$ & $\begin{array}{l}\text { IRON, } \\
\text { TOTAL } \\
\text { RECOV- } \\
\text { ERABLE } \\
\text { (UG/L }\end{array}$ \\
\hline & AS AL) & AS AL) & AS AS) & AS $(D)$ & AS CR) & AS (U) & AS (U) & AS FE) \\
\hline
\end{tabular}

MAY 1984

$30 .$.

AS AL)

AS AL)

AS AS)

AS CD)

AS CR )

AS CU)

AS CU)

AS FE)

$--\quad--$

$-$

1

--

200

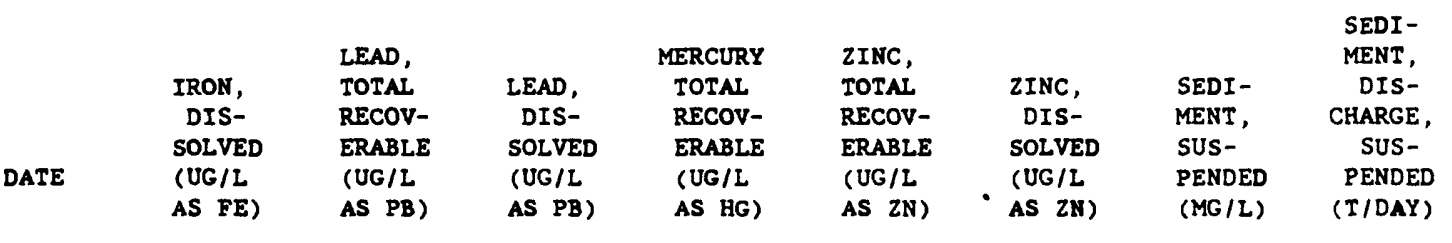

MAY 1984

$30 \ldots$

$<1$

$<5$

2

0.04 
TA.T. 4,--Physleal characterlstics and concentrations of commond chemlcal constituents in lakes and streams of the Bald Mountaln watershed--Continued

01012530 CLAYTON STREAM AT SHELDON RIDGE ME

\begin{tabular}{|c|c|c|c|c|c|c|c|c|c|c|}
\hline DATE & IIME & $\begin{array}{l}\text { STREAM- } \\
\text { FLOW, } \\
\text { INSTAN- } \\
\text { TANEOUS } \\
\text { (CFS) }\end{array}$ & $\begin{array}{l}\text { SPE- } \\
\text { CIFIC } \\
\text { CON- } \\
\text { DUCI- } \\
\text { ANCE } \\
\text { (US/CM) }\end{array}$ & $\begin{array}{c}\text { PE } \\
\text { (STAND- } \\
\text { ARD } \\
\text { UNITS) }\end{array}$ & $\begin{array}{l}\text { TEMPER- } \\
\text { ATURE } \\
\text { WATER } \\
\text { (DEG C) }\end{array}$ & $\begin{array}{l}\text { COLOR } \\
\text { (PLAT- } \\
\text { INUM- } \\
\text { COBALT } \\
\text { UNITS) }\end{array}$ & $\begin{array}{l}\text { IUR- } \\
\text { BID- } \\
\text { ITY } \\
\text { (FTU) }\end{array}$ & $\begin{array}{c}\text { OXYGEN, } \\
\text { DIS- } \\
\text { SOLVED } \\
\text { (MG/L) }\end{array}$ & $\begin{array}{l}\text { OXYGEN } \\
\text { DEMAND, } \\
\text { BIO- } \\
\text { CHEM- } \\
\text { ICAL, } \\
5 \text { DAY } \\
\text { (MG/L) }\end{array}$ & $\begin{array}{l}\text { COLI- } \\
\text { FORM, } \\
\text { FECAL, } \\
0.45 \\
\text { UM-MF } \\
\text { (COLS. I } \\
100 \mathrm{ML} \text { ) }\end{array}$ \\
\hline $\begin{array}{c}\text { JAN } 1980 \\
29 \ldots \\
\text { FEB }\end{array}$ & 1130 & -- & 58 & 6.8 & 0.0 & -- & 1.6 & 14.2 & -- & R3 \\
\hline${ }_{\text {MAR }}^{19 \ldots}$ & 1130 & -- & 80 & 6.7 & 0.0 & 60 & 5.0 & 12.8 & 1.0 & K1 \\
\hline$\underset{A P R}{20 \ldots}$ & 1130 & -- & 50 & 6.4 & 0.0 & 60 & 5.8 & 12.6 & 3.7 & K8 \\
\hline${ }_{\operatorname{MAY}}^{23} \ldots$ & 1130 & -- & 40 & 6.3 & 2.0 & 35 & 5.1 & 12.2 & 1.1 & $<1$ \\
\hline${ }_{\text {JUN }}^{20} \cdots$ & 1100 & -- & 35 & 7.0 & 14.0 & 40 & 1.8 & 10.0 & 2.1 & K2 \\
\hline JuL $17 \ldots$ & 1200 & -- & 45 & 6.8 & 14.0 & 40 & 1.8 & 10.2 & 0.6 & -- \\
\hline AUG & 1200 & -- & 39 & 6.8 & 20.0 & 55 & 2.4 & 8.7 & -- & -- \\
\hline SEP & 1100 & -- & 45 & 7.0 & 17.0 & 45 & 1.2 & 9.5 & -- & -- \\
\hline $\mathrm{OCT}^{29 \ldots}$ & 1300 & -- & 40 & 6.7 & 7.0 & 45 & 1.6 & 11.2 & -- & -- \\
\hline nov $14 \ldots$ & 1100 & -- & 62 & 6.6 & 6.0 & 60 & 3.0 & 11.6 & -- & -- \\
\hline${ }_{D E C}^{21 \cdots}$ & 1100 & -- & 42 & 6.2 & 0.0 & 50 & 2.6 & 12.9 & -- & -- \\
\hline $\begin{array}{c}12 \ldots \\
\text { FEB } 1981\end{array}$ & 1130 & $=-$ & 75 & 6.1 & 0.0 & 65 & 1.6 & 12.7 & -- & - \\
\hline$\underset{\operatorname{MaR}}{23} \ldots$ & 1755 & -- & 32 & -- & 2.0 & 70 & 3.7 & 12.3 & -- & - \\
\hline $25 \ldots$ & 1040 & -- & 33 & 6.2 & 1.0 & 50 & 2.6 & 12.9 & -- & -- \\
\hline DATE & $\begin{array}{l}\text { ALKA- } \\
\text { LINITY } \\
\text { WAT WH } \\
\text { TOT FET } \\
\text { LAB } \\
\text { MG/L AS } \\
\text { CACO3 }\end{array}$ & $\begin{array}{l}\text { SOLIDS, } \\
\text { RESIDUE } \\
\text { AT 105 } \\
\text { DEG. C, } \\
\text { TOTAL } \\
\text { (MG/L) }\end{array}$ & $\begin{array}{l}\text { SOLIDS, } \\
\text { VOLA- } \\
\text { IILE ON } \\
\text { IGNI- } \\
\text { TION, } \\
\text { TOTAL } \\
\text { (MG/L) }\end{array}$ & $\begin{array}{l}\text { NITRO- } \\
\text { GEN, } \\
\text { NO2+NO3 } \\
\text { TOTAL } \\
\text { (MG/L } \\
\text { AS N) }\end{array}$ & $\begin{array}{l}\text { NITRO- } \\
\text { GEN, } \\
\text { AMMONIA } \\
\text { TOTAL } \\
\text { (MG/L } \\
\text { AS N) }\end{array}$ & $\begin{array}{l}\text { NITRO- } \\
\text { GEN, } \\
\text { ORGANIC } \\
\text { TOTAL } \\
\text { (MG/L } \\
\text { AS N) }\end{array}$ & $\begin{array}{l}\text { NITRO- } \\
\text { GEN, AM- } \\
\text { MONIA + } \\
\text { ORGANIC } \\
\text { TOTAL } \\
\text { (MG/L } \\
\text { AS N) }\end{array}$ & $\begin{array}{l}\text { NITRO- } \\
\text { GEN, } \\
\text { TOTAL } \\
\text { (MG/L } \\
\text { AS N) }\end{array}$ & $\begin{array}{l}\text { NITRO- } \\
\text { GEN, } \\
\text { TOTAL } \\
\text { (MG/L } \\
\text { AS NO3) }\end{array}$ & $\begin{array}{l}\text { PHOS- } \\
\text { PHOROUS } \\
\text { TOTAL } \\
\text { (MG/L } \\
\text { AS P) }\end{array}$ \\
\hline JAN 1980 & & & & & & & & & & \\
\hline$\underset{\text { FEB }}{29} \ldots$ & -- & 20 & -- & -- & -- & -- & -- & -- & $\cdots$ & 0.01 \\
\hline $\operatorname{MAR}^{19} \ldots$ & 22 & 74 & -- & 0.15 & -- & -- & 0.2 & 0.35 & 1.5 & .02 \\
\hline$\underset{A P R}{20} \ldots$ & 9 & 65 & -- & -- & -- & -- & .4 & -- & -- & .03 \\
\hline $\operatorname{MAY}^{23} \ldots$ & 4 & 28 & -- & .28 & 0.03 & 0.27 & .3 & .58 & 2.6 & .02 \\
\hline JUN & 7 & 55 & -- & .22 & $<.01$ & -- & .2 & .42 & 1.9 & .02 \\
\hline JUL $^{17 \ldots}$ & 12 & 68 & -- & .14 & $<.01$ & -- & .2 & .34 & 1.5 & .04 \\
\hline${ }_{A U G}^{13} \ldots$ & 10 & 54 & -- & -- & -- & -- & -- & -- & -- & .01 \\
\hline $\operatorname{SEP}^{29 \ldots}$ & 15 & 48 & -- & -- & -- & -- & -- & -- & -- & .01 \\
\hline${ }_{\text {OCT }}^{29 \ldots}$ & 17 & 53 & -- & -- & -- & -- & -- & -- & -- & .02 \\
\hline $\operatorname{Nov}^{14} \ldots$ & 11 & 65 & -- & -- & -- & -- & -- & -- & -- & .01 \\
\hline $\begin{array}{l}21 \ldots \\
\text { DEC }\end{array}$ & 12 & 66 & -- & -- & -- & - & -- & -- & -- & .01 \\
\hline $\begin{array}{c}12 \ldots \\
\text { FEB } 1981\end{array}$ & 10 & 62 & -- & -- & -- & -- & -- & -- & -- & .02 \\
\hline$\underset{\text { MAR }}{23 \ldots}$ & 10 & -- & -- & -- & -- & -- & -- & -- & -- & -- \\
\hline $25 \ldots$ & -- & -- & -- & -- & -- & -- & -- & -- & -- & .01 \\
\hline
\end{tabular}


IABI. 4.--Physical characteristics and concentrations of commond chemical constituants in lakes and streams of the Bald Mountain watershed--Continued

01012530 CLAYTON STREAM AT SHELDON RIDGE ME

DATE

JAN 1980

29 ...

FEB

$19 \ldots$

MAR

$20 \ldots$

APR

23...

MAY

20 ...

JUN

$17 \ldots$

JUL

$13 \ldots$

AUG

29...

SEP

29...

OCT

$14 \ldots$

NOV

21 ...

DEC

12 ...

FEB 1981

$23 \ldots$

MAR

25 ...

JAN 1980

29. .

FEB

$19 .$.

$20 \ldots$

APR

$23 \ldots$

MAY

20...

JUN

17 ...

JUL

$13 .$.

AUG

29...

SEP

$29 .$.

OCT

$14 \ldots$

NOV

$21 \ldots$

DEC

$12 \ldots$

FEB 1981

$23 \ldots$

MAR

25 ...
ALUM-

INUM, ALUM-

TOTAL INUM,

RECOV- DIS-

TRABLE SOLVED

S AL)

(UG/L

AS $\mathbf{A}$ )
ARSENIC

TOTAL

AS AS)

CADMIUM
TOTAL
RECOV-
ERABLE
(UG/L
AS CD)

CERO-

MIUM,

RECOV-

ERABLE

(UG/L)

AS CR)

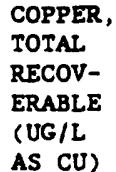

COPPER,

IRON

TOTAL

RECOV-

SOLVED ERABLE

(UG/L) (UG/L

AS CU)

AS FE)

\begin{tabular}{|c|c|c|c|c|}
\hline$<2$ & $<6$ & $<4$ & -- & - \\
\hline$<2$ & $<6$ & $<4$ & -- & 450 \\
\hline-- & -- & $<4$ & - & - \\
\hline$<2$ & $<5$ & $<10$ & - & - \\
\hline$<2$ & $<20$ & $<4$ & -- & - \\
\hline$<2$ & $<5$ & 2 & -- & 180 \\
\hline -- & $<5$ & $<2$ & -- & 200 \\
\hline-- & -- & $<4$ & -- & 180 \\
\hline-- & - & $<4$ & - & 170 \\
\hline-- & - & 1 & - & 230 \\
\hline- & - & 1 & - & -- \\
\hline- & - & 2 & $\cdots$ & 220 \\
\hline - & -- & -- & -- & $\cdots$ \\
\hline- & -- & 1 & -- & 220 \\
\hline
\end{tabular}

\begin{tabular}{|c|c|c|c|c|c|c|c|c|}
\hline ATE & $\begin{array}{l}\text { IRON, } \\
\text { DIS- } \\
\text { SOLVED } \\
\text { (UG/L }\end{array}$ & $\begin{array}{l}\text { LEAD, } \\
\text { TOTAL } \\
\text { RECOV- } \\
\text { ERABLE } \\
\text { (UG/L }\end{array}$ & $\begin{array}{l}\text { LEAD, } \\
\text { DIS- } \\
\text { SOLVED } \\
\text { (UG/L }\end{array}$ & $\begin{array}{l}\text { MERCURY } \\
\text { TOTAL } \\
\text { RECOV- } \\
\text { ERABLE } \\
\text { (UG/L }\end{array}$ & $\begin{array}{l}\text { ZINC, } \\
\text { TOTAL } \\
\text { RECOV- } \\
\text { ERABLE } \\
\text { (UG/L }\end{array}$ & $\begin{array}{l}\text { ZINC, } \\
\text { DIS- } \\
\text { SOLVED } \\
\text { (UG/L }\end{array}$ & $\begin{array}{l}\text { SEDI - } \\
\text { MENT, } \\
\text { SUS- } \\
\text { PENDED }\end{array}$ & $\begin{array}{c}\text { SEDI - } \\
\text { MENT, } \\
\text { DIS - } \\
\text { CHARGE, } \\
\text { SUS - } \\
\text { PENDED }\end{array}$ \\
\hline & AS $F E$ ) & AS PB) & AS PB) & AS BG) & AS $Z N$ ) & AS $\mathrm{ZN}$ ) & (MG/L) & (T/DAY) \\
\hline
\end{tabular}

\begin{tabular}{|c|c|c|c|c|c|c|c|}
\hline-- & $<20$ & -- & $<0.2$ & 2 & -- & -- & -- \\
\hline-- & $<20$ & -- & $<.2$ & 6 & -- & -- & -- \\
\hline-- & $<20$ & -- & -- & 3 & -- & 7 & 0.0 \\
\hline-- & $<30$ & -- & - & 4 & - & 5 & 0.0 \\
\hline-- & $<30$ & -- & -- & 11 & -- & 3 & 0.0 \\
\hline-- & $<30$ & -- & $<.2$ & 120 & -- & 2 & 0.0 \\
\hline-- & -- & -- & -- & 2 & -- & -- & -- \\
\hline-- & -- & -- & -- & 4 & -- & -- & -- \\
\hline-- & -- & - & -- & $<2$ & -- & -- & -- \\
\hline-- & -- & -- & -- & $<5$ & -- & -- & -- \\
\hline-- & $<2$ & - & -- & -- & -- & -- & -- \\
\hline-- & -- & - & -- & 4 & - & -- & - \\
\hline-- & -- & - & -- & -- & -- & 2 & 0.0 \\
\hline-- & - & -- & -- & $<3$ & -- & 4 & 0.0 \\
\hline
\end{tabular}


TAmR 4.--Physical characteristics and concentrations of commond chemical constituents in lakes and streams of the Bald Mountain watershed--Continued

01012530 CLAYTON STREAM AT SHELDON RIDGE ME

\begin{tabular}{|c|c|c|c|c|c|c|c|c|c|c|}
\hline DATE & TIME & $\begin{array}{l}\text { STREAM- } \\
\text { FLOW, } \\
\text { INSTAN- } \\
\text { TANEOUS } \\
\text { (CRS) }\end{array}$ & $\begin{array}{l}\text { SPE- } \\
\text { CIFIC } \\
\text { CON- } \\
\text { DUCT- } \\
\text { ANCE } \\
\text { (US/CM) }\end{array}$ & $\begin{array}{c}\text { PB } \\
\text { (STAND- } \\
\text { ARD } \\
\text { UNITS) }\end{array}$ & $\begin{array}{l}\text { TEMPER- } \\
\text { ATURE } \\
\text { WATER } \\
\text { (DEG C) }\end{array}$ & $\begin{array}{l}\text { COLOR } \\
\text { (PLAT- } \\
\text { INUM- } \\
\text { COBALT } \\
\text { UNITS) }\end{array}$ & $\begin{array}{l}\text { TUR- } \\
\text { BID- } \\
\text { ITY } \\
\text { (FTU) }\end{array}$ & $\begin{array}{c}\text { OXYGEN, } \\
\text { DIS- } \\
\text { SOLVED } \\
\text { (MG/L) }\end{array}$ & $\begin{array}{l}\text { OXYGEN } \\
\text { DEMAND, } \\
\text { BIO- } \\
\text { CHEM- } \\
\text { ICAL, } \\
5 \text { DAY } \\
\text { (MG/L) }\end{array}$ & $\begin{array}{l}\text { COLI- } \\
\text { FORM, } \\
\text { FECAL, } \\
0.45 \\
\text { UM-MF } \\
\text { (COLS. I } \\
100 \mathrm{ML} \text { ) }\end{array}$ \\
\hline
\end{tabular}

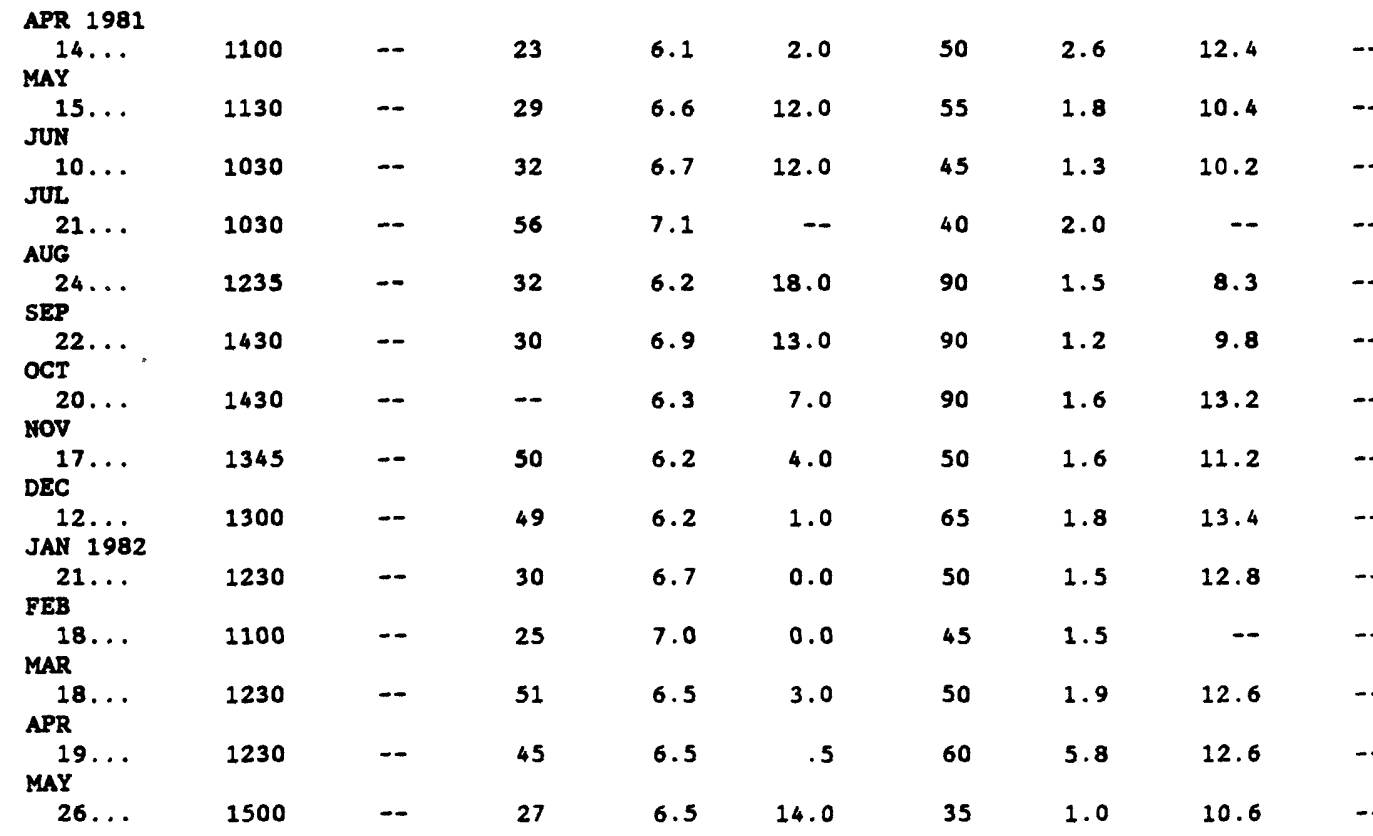

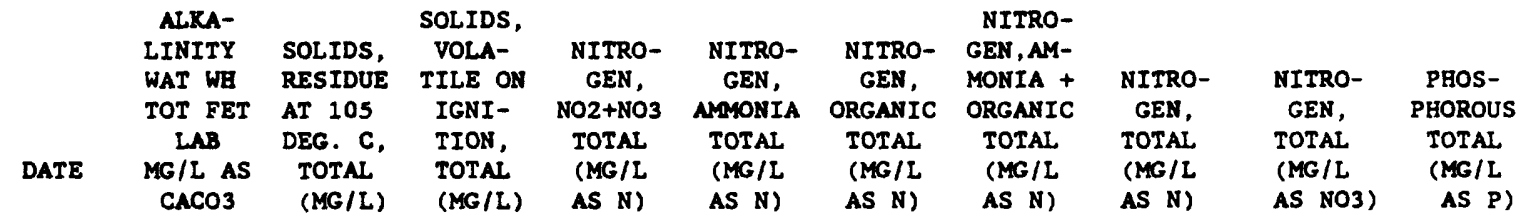

APR 1981

$14 \ldots$

MAY

JuN

10 ...

1500

$6.5 \quad 14.0$

(n)

(100 
TAnal 4.--Physlcal characteristics and concentrations of commond chemical constituents in lakes and streams of the Bald Mountain watershed--Continued

01012530 CLAYTON STREAM AT SHELDON RIDGE ME

ALUM-

INUM, ALUM-

TOTAL INUM,

RECOV-

ERABLE

(UG/L

AS AL)

DIS -

(UG/L

AS AL) AS AS) AS CD) AS CR)

$\begin{array}{cl} & \text { CHRO- } \\ \text { CADMIUM } & \text { MIUM, } \\ \text { TOTAL } & \text { TOTAL } \\ \text { RECOV- } & \text { RECOV- } \\ \text { ERABLE } & \text { ERABLE } \\ \text { (UG/L } & \text { (UG/L } \\ \text { AS CD) } & \text { AS CR) }\end{array}$

COPPER,
TOTAL
RECOV-
ERABLE
(UG/L
AS CU)

COPPER, TOTAL,

DIS- RECOV-

SOLVED ERABLE

AS CU)

AS CUY AS FE

APR 1981

$14 \ldots$

$15 \ldots$

JuN

$10 \ldots$

JUL

21 ...

AUG

24...

SEP

22 ...

OCT

20...

NOV

17 ...

DEC

12 ...

JAN 1982

21 ...

FEB

$18 \ldots$

MAR

$18 \ldots$

APR

19...

MAY

26 ...

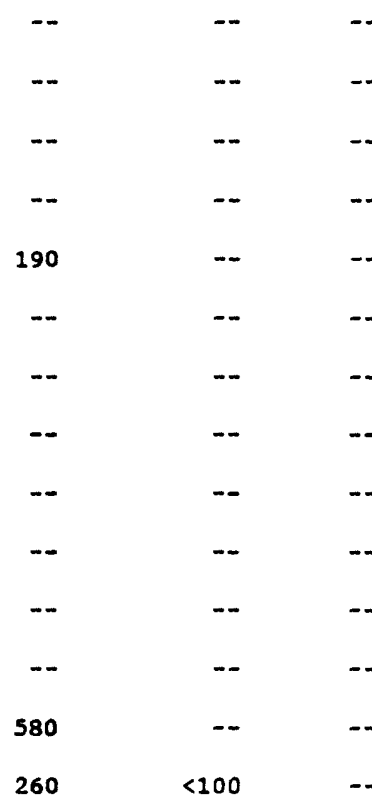

--
--
--
--
--
--
--
--
--

--
--
--
--
--
--
--
--
-

\begin{tabular}{|c|c|}
\hline 1 & $=-$ \\
\hline 1 & -- \\
\hline 5 & $-\infty$ \\
\hline 2 & -- \\
\hline 12 & -- \\
\hline$<2$ & $-\infty$ \\
\hline 8 & -- \\
\hline 1 & -- \\
\hline$<1$ & - \\
\hline 2 & -- \\
\hline 1 & -- \\
\hline 1 & -- \\
\hline 1 & -- \\
\hline 2 & $<1$ \\
\hline
\end{tabular}

DATE

$\begin{array}{clc} & \text { LEAD, } \\ \text { IRON, } & \text { TOTAL } & \text { LEAD, } \\ \text { DIS- } & \text { RECOV- } & \text { DIS- } \\ \text { SOLVED } & \text { ERABLE } & \text { SOLVED } \\ \text { (UG/L } & \text { (UG/L } & (U G / L \\ \text { AS FE) } & \text { AS PB) } & \text { AS PB) }\end{array}$

MERCURY
TOTAL
RECOV-
ERABLE
(UG/L
AS HG)

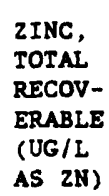

2INC,
DIS-
SOLVED
(UG/L
AS ZN)

SEDI-

MENT,

MENI,

SEDI-

MENT,

SUS-

PENDED

CHARGE,

SUS-

PENDED

(T/DAY)

APR 1981

$14 \ldots$

MAY

$15 .$.

JuN

$10 .$.

गU

21 ...

AUG

24...

SEP

22 ...

OCT

$20 .$.

Nov

$17 \ldots$

DEC

12 ...

JAN 1982

21 ...

FEB

18...

MAR

$18 \ldots$

APR

$19 .$.

MAY

$26 .$.

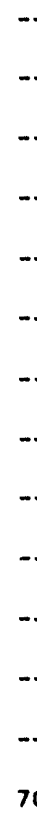

70

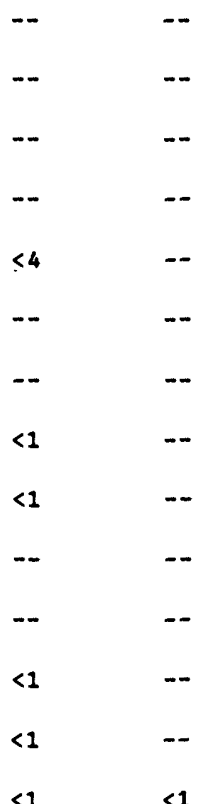

$<0.2$

5

$<4$

(MG/L)

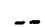

0.0

30.0

4

0.0

$--$

$<5$

$<5$

6

$<5$

$<5$

6

$<5$

25

$-12$

0.0

$-$

$<5$

$<5$

0.0 
TAME 4.--Physical characteristics and concentrations of commond chemical constituents in lakes and streams of the Bald Mountain watershed--Continued

01012530 CLAYTON STREAM AT SEELDON RIDGE ME

\begin{tabular}{|c|c|c|c|c|c|c|c|c|c|}
\hline TIME & $\begin{array}{l}\text { STREAM- } \\
\text { FLOW, } \\
\text { INSTAN- } \\
\text { TANEOUS }\end{array}$ & $\begin{array}{l}\text { SPE- } \\
\text { CIFIC } \\
\text { CON- } \\
\text { DUCT- } \\
\text { ANCE }\end{array}$ & $\begin{array}{c}\text { PH } \\
\text { (STAND- } \\
\text { ARD }\end{array}$ & $\begin{array}{l}\text { TEMPER- } \\
\text { ATURE } \\
\text { WATER }\end{array}$ & $\begin{array}{l}\text { COLOR } \\
\text { (PLAT- } \\
\text { INUM- } \\
\text { COBALT }\end{array}$ & $\begin{array}{l}\text { IUR- } \\
\text { BID- } \\
\text { ITY }\end{array}$ & $\begin{array}{l}\text { OXYGEN, } \\
\text { DIS- } \\
\text { SOLVED }\end{array}$ & $\begin{array}{l}\text { OXYGEN } \\
\text { DEMAND, } \\
\text { BIO- } \\
\text { CHEM- } \\
\text { ICAL, } \\
5 \text { DAY }\end{array}$ & $\begin{array}{l}\text { COLI- } \\
\text { FORM, } \\
\text { FECAL, } \\
0.45 \\
\text { UM-MF } \\
\text { COOLS. }\end{array}$ \\
\hline & (CFS) & (US/CM) & UNITS) & (DEG C) & UNITS) & (FTU) & $(M G / L)$ & (MG/L) & $100 \mathrm{ML})$ \\
\hline
\end{tabular}

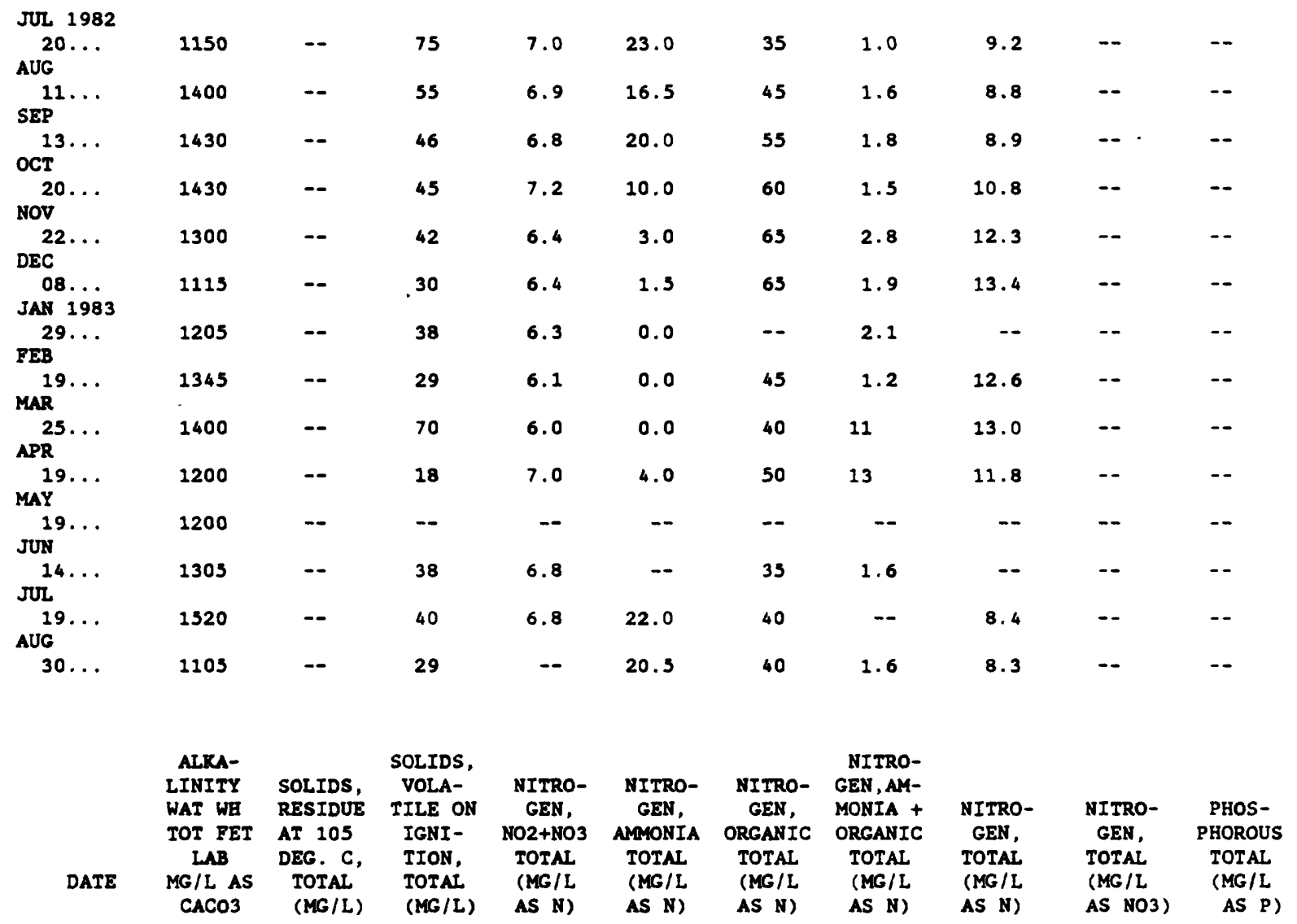

JUL 1982

\begin{tabular}{|c|c|c|c|c|c|c|c|c|c|c|}
\hline AUG $^{20 \ldots}$ & 23 & 75 & 26 & -- & -- & -- & -- & -- & -- & 0.02 \\
\hline $\operatorname{SEP}_{11}^{11}$ & 12 & 45 & -- & -- & -- & -- & -- & -- & -- & .01 \\
\hline $\mathrm{OCT}^{13 \ldots}$ & 8 & 71 & 50 & -- & -- & -- & -- & -- & -- & .02 \\
\hline${ }_{\text {Nov }}^{20} .$. & 10 & 39 & -- & -- & -- & -- & -- & -- & -- & -- \\
\hline${ }_{\mathrm{DEC}}^{22} \cdots$ & 7 & 62 & -- & -- & -- & -- & -- & -- & -- & .02 \\
\hline $\begin{array}{c}08 \ldots \\
\text { JAN } 1983\end{array}$ & 3 & 64 & -- & -- & -- & -- & -- & -- & -- & .01 \\
\hline$\underset{\text { FEB }}{29} \ldots$ & -- & 58 & -- & -- & -- & -- & -- & -- & -- & .01 \\
\hline $\operatorname{MAR}^{19} \ldots$ & 14 & 35 & -- & -- & -- & -- & -- & -- & -- & $<.01$ \\
\hline$\underset{A P R}{25 \ldots}$ & 10 & -- & -- & -- & -- & -- & -- & -- & -- & .02 \\
\hline $\operatorname{MAY}^{19} \ldots$ & 3 & -- & -- & -- & -- & -- & -- & -- & -- & -- \\
\hline $\mathrm{JUN}^{19 \ldots}$ & -- & -- & -- & -- & -- & -- & -- & -- & -- & .01 \\
\hline JuL $^{14 \ldots}$ & 12 & -- & -- & -- & -- & -- & -- & -- & -- & .01 \\
\hline${ }_{A \cup G}^{19} \ldots$ & 23 & -- & -- & -- & -- & -- & -- & -- & -- & .01 \\
\hline $30 \ldots$ & 14 & -- & -- & -- & -- & -- & -- & -- & -- & $<.01$ \\
\hline
\end{tabular}


TNIT 4.--Physleal characterlstics and concentrations of commond chemical constituents In lakes and streams of the Bald Mountain watershed--Cont Inued

01012530 CLAYTON STREAM AT SHELDON RIDGE ME

\begin{tabular}{|c|c|c|c|c|c|c|c|}
\hline $\begin{array}{l}\text { ALUM- } \\
\text { INUM, } \\
\text { TOTAL }\end{array}$ & $\begin{array}{l}\text { ALUM- } \\
\text { INUM, }\end{array}$ & & $\begin{array}{l}\text { CADMIUM } \\
\text { TOTAL }\end{array}$ & $\begin{array}{l}\text { CERO- } \\
\text { MIUM, } \\
\text { TOTAL }\end{array}$ & $\begin{array}{l}\text { COPPER, } \\
\text { TOTAL }\end{array}$ & COPPER, & $\begin{array}{l}\text { IRON, } \\
\text { TOTAL }\end{array}$ \\
\hline $\begin{array}{l}\text { RECOV- } \\
\text { ERABLE } \\
\text { (UG/L }\end{array}$ & $\begin{array}{l}\text { DIS- } \\
\text { SOLVED } \\
\text { (UG/L }\end{array}$ & $\begin{array}{l}\text { ARSENIC } \\
\text { TOTAL } \\
\text { (UG/L }\end{array}$ & $\begin{array}{l}\text { RECOV- } \\
\text { ERABLE } \\
\text { (UG/L }\end{array}$ & $\begin{array}{l}\text { RECOV- } \\
\text { ERABLE } \\
\text { (UG/L }\end{array}$ & $\begin{array}{l}\text { RECOV- } \\
\text { ERABLE } \\
\text { (UG/L }\end{array}$ & $\begin{array}{l}\text { DIS- } \\
\text { SOLVED } \\
\text { (UG/L }\end{array}$ & $\begin{array}{l}\text { RECOV- } \\
\text { ERABLE } \\
\text { (UG/L }\end{array}$ \\
\hline AS AL) & AS AL) & AS AS) & AS CD) & AS (R) & AS CU) & AS CU) & AS FE) \\
\hline
\end{tabular}

AS CD)
AS FE)

$\begin{array}{llll}-- & <1 & -- & 80 \\ -- & 3 & -- & 150 \\ -- & 2 & -- & 240 \\ -- & <1 & -- & 180 \\ -- & <1 & -- & 270 \\ -- & <1 & -- & 200 \\ -- & <1 & -- & 170 \\ -- & <1 & -- & 150 \\ -- & 1 & -- & 740 \\ -- & <1 & -- & 700 \\ -- & 2 & -- & 260 \\ -- & <1 & -- & 210 \\ -- & 1 & -- & 200 \\ -- & 1 & -- & 160\end{array}$

DATE

IRON, TOTAR

DIS-

SOLVED

(UG/L

AS FE)

RECOV-

ERABLE

(UG/L

AS PB)

$\begin{array}{ll}-- \\ -- \\ -- \\ - & - \\ -- & - \\ - & - \\ -- & - \\ -- & - \\ -- & - \\ -- & - \\ -- & -\end{array}$

--
--
--
--
--
--
--
--
--
--
--
--
--
--

DATE

JUL 1982

20..

AUG

11...

SEP

13 ..

OCT

20 ..

NOV

22 ...

DEC

08 ...

JAN 1983

29...

FEB

19..

MAR

25 ..

APR

$19 .$.

MAY

19...

JUN

$14 \ldots$

JUL.

19. .

AUG

$30 .$.
JUL 1982

20 ...

AUG

11.

SEP

$13 .$.

OCI

20 ...

Nov

22 ...

DEC

$08 .$.

JAN 1983

29 ...

FEB

19...

MAR

25...

APR

19...

MAY

19...

JUN

$14 \ldots$

JUL

$19 .$.

AUG

30 ..

\begin{tabular}{|c|c|c|c|c|c|c|c|}
\hline-- & -- & -- & -- & $<5$ & - & 1 & 0.0 \\
\hline-- & -- & -- & -- & $<8$ & -- & 1 & 0.0 \\
\hline-- & -- & -- & -- & $<5$ & -- & 3 & 0.0 \\
\hline-- & -- & -- & -- & 11 & -- & 3 & 0.0 \\
\hline -- & -- & -- & -- & $<5$ & -- & 4 & 0.0 \\
\hline- & -- & -- & -- & $<5$ & -- & 4 & 0.0 \\
\hline -- & -- & -- & -- & $<5$ & - & 2 & 0.0 \\
\hline-- & -- & -- & -- & 8 & -- & -- & -- \\
\hline-- & -- & -- & -- & $<5$ & -- & -- & -. \\
\hline-- & -- & -- & -- & 8 & - & -- & -- \\
\hline-- & 2 & -- & -- & $<5$ & -- & -- & -- \\
\hline-- & -- & -- & -- & $<11$ & -- & -- & -- \\
\hline -- & -- & -- & -- & 7 & -- & -- & -- \\
\hline-- & -- & -- & -- & 12 & -- & 2 & 0.0 \\
\hline
\end{tabular}


TAns 4.-Physical characteristics and concentrations of commond chemical constituents in lakes and streams of the Bald Mountain watershed--Continued

01012530 CLAYTON STREAM AT SHELDON RIDGE ME

\begin{tabular}{|c|c|c|c|c|c|c|c|c|c|c|}
\hline DATE & TIME & $\begin{array}{l}\text { STREAM- } \\
\text { FLOW, } \\
\text { INSTAN- } \\
\text { IANEOUS } \\
\text { (CFS) }\end{array}$ & $\begin{array}{l}\text { SPE- } \\
\text { CIFIC } \\
\text { CON- } \\
\text { DUCI- } \\
\text { ANCE } \\
\text { (US/CM) }\end{array}$ & $\begin{array}{c}\text { PE } \\
\text { (STAND- } \\
\text { ARD } \\
\text { UNITS) }\end{array}$ & $\begin{array}{l}\text { TEMPER- } \\
\text { ATURE } \\
\text { WATER } \\
\text { (DEG C) }\end{array}$ & $\begin{array}{l}\text { COLOR } \\
\text { (PLAT- } \\
\text { INUM- } \\
\text { COBALT } \\
\text { UNITS) }\end{array}$ & $\begin{array}{l}\text { TUR- } \\
\text { BID- } \\
\text { ITY } \\
\text { (FTU) }\end{array}$ & $\begin{array}{c}\text { OXYGEN, } \\
\text { DIS- } \\
\text { SOLVED } \\
\text { (MG/L) }\end{array}$ & $\begin{array}{l}\text { OXYGEN } \\
\text { DEMAND, } \\
\text { BIO- } \\
\text { CHEM- } \\
\text { ICAL, } \\
5 \text { DAY } \\
\text { (MG/L) }\end{array}$ & $\begin{array}{l}\text { COLI- } \\
\text { FORM, } \\
\text { FECAL, } \\
0.45 \\
\text { UM-MF } \\
\text { (COLS.l } \\
100 \mathrm{ML} \text { ) }\end{array}$ \\
\hline OCT 1983 & & & & & & & & & & \\
\hline $06 \ldots$ & 0930 & -- & -- & 6.4 & 9.0 & 60 & 3.5 & 10.2 & -- & -- \\
\hline $\begin{array}{c}25 \ldots \\
M A R \\
1984\end{array}$ & 1300 & -- & 30 & 7.1 & 4.0 & 45 & 0.9 & -- & -- & -- \\
\hline$\underset{A P R}{29 \ldots}$ & 1515 & -- & 34 & 6.9 & 2.5 & 45 & 2.9 & 12.2 & -- & -- \\
\hline $\operatorname{MAY}^{30 \ldots}$ & 1425 & $\cdots$ & 17 & 6.7 & 7.5 & 50 & 1.0 & 11.0 & -- & -- \\
\hline $\begin{array}{l}18 \ldots \\
30 \ldots\end{array}$ & $\begin{array}{l}1312 \\
1245\end{array}$ & -- & $\begin{array}{l}28 \\
27\end{array}$ & $6 . \overline{-.}$ & $\begin{array}{l}11.0 \\
10.0\end{array}$ & $\begin{array}{r}50 \\
100\end{array}$ & $\begin{array}{l}1.6 \\
2.2\end{array}$ & 12.0 & -- & -- \\
\hline DATE & $\begin{array}{l}\text { ALIXA- } \\
\text { LINITY } \\
\text { WAT WE } \\
\text { TOT FET } \\
\text { LAB } \\
\text { MG/L AS } \\
\text { CACO3 }\end{array}$ & $\begin{array}{l}\text { SOLIDS, } \\
\text { RESIDUE } \\
\text { AT } 105 \\
\text { DEG. C, } \\
\text { TOTAL } \\
\text { (MG/L) }\end{array}$ & $\begin{array}{l}\text { SOLIDS, } \\
\text { VOLA- } \\
\text { TILE ON } \\
\text { IGNI- } \\
\text { TION, } \\
\text { TOTAL } \\
\text { (MG/L) }\end{array}$ & $\begin{array}{l}\text { NITRO- } \\
\text { GEN, } \\
\text { NO2+NO3 } \\
\text { TOTAL } \\
\text { (MG/L } \\
\text { AS N) }\end{array}$ & $\begin{array}{l}\text { NITRO- } \\
\text { GEN, } \\
\text { AMOMONIA } \\
\text { TOTAL } \\
\text { (MG/L } \\
\text { AS N) }\end{array}$ & $\begin{array}{l}\text { NITRO- } \\
\text { GEN, } \\
\text { ORGANIC } \\
\text { TOTAL } \\
\text { (MG/L } \\
\text { AS N) }\end{array}$ & $\begin{array}{l}\text { NITRO- } \\
\text { GEN,AM- } \\
\text { MONIA + } \\
\text { ORGANIC } \\
\text { TOTAL } \\
\text { (MG /L } \\
\text { AS N) }\end{array}$ & $\begin{array}{l}\text { NITRO- } \\
\text { GEN, } \\
\text { TOTAL } \\
\text { (MG/L } \\
\text { AS N) }\end{array}$ & $\begin{array}{l}\text { NITRO- } \\
\text { GEN, } \\
\text { TOTAL } \\
\text { (MG/L } \\
\text { AS NO3) }\end{array}$ & $\begin{array}{l}\text { PHOS- } \\
\text { PHOROUS } \\
\text { TOTAL } \\
\text { (MG/L } \\
\text { AS P) }\end{array}$ \\
\hline OCT 1983 & & & & & & & & & & \\
\hline $06 \ldots$ & 16 & 80 & -- & -- & -- & -- & -- & -- & -- & 0.02 \\
\hline $\begin{array}{r}23 \ldots \\
\operatorname{MAR} 1984\end{array}$ & 13 & 38 & -- & -- & -- & -- & -- & -- & -- & .01 \\
\hline$\underset{A P R}{29 \ldots}$ & 8 & 56 & -- & -- & -- & -- & -- & -- & -- & -- \\
\hline${ }_{\mathrm{MAY}}^{30 \ldots}$ & 2 & 47 & -- & -- & -- & -- & -- & -- & -- & -- \\
\hline $18 \ldots$ & 8 & 51 & -- & -- & -- & -- & -- & -- & -- & -- \\
\hline $30 \ldots$ & 3 & 43 & -- & -- & -- & -- & -- & -- & - & .01 \\
\hline
\end{tabular}


TNBIE 4.--Physical characteristics and concentrations of comnond chemical constituents in lakes and streams of the Bald Mountain watershed--Continued

01012530 CLAYTON STREAM AT SHELDON RIDGE ME

\begin{tabular}{|c|c|c|c|c|c|c|c|c|}
\hline DATE & $\begin{array}{l}\text { ALUM- } \\
\text { INUM, } \\
\text { TOTAL } \\
\text { RECOV- } \\
\text { ERABLE } \\
\text { (UG/L }\end{array}$ & $\begin{array}{l}\text { ALUM- } \\
\text { INUM, } \\
\text { DIS- } \\
\text { SOLVED } \\
\text { (UG/L }\end{array}$ & $\begin{array}{l}\text { ARSENIC } \\
\text { TOTAL } \\
\text { (UG/L }\end{array}$ & $\begin{array}{l}\text { CADMIUM } \\
\text { TOTAL } \\
\text { RECOV- } \\
\text { ERABLE } \\
\text { (UG/L }\end{array}$ & $\begin{array}{l}\text { CHRO- } \\
\text { MIUM, } \\
\text { TOTAL } \\
\text { RECOV- } \\
\text { ERABLE } \\
\text { (UG/L }\end{array}$ & $\begin{array}{l}\text { COPPER, } \\
\text { TOTAL } \\
\text { RECOV- } \\
\text { ERABLE } \\
\text { (UG/L }\end{array}$ & $\begin{array}{l}\text { COPPER, } \\
\text { DIS- } \\
\text { SOLVED } \\
\text { (UG/L }\end{array}$ & $\begin{array}{l}\text { IRON, } \\
\text { TOTAL } \\
\text { RECOV- } \\
\text { ERABLE } \\
\text { (UG/L }\end{array}$ \\
\hline OCT 1983 & AS AL) & AS AL) & AS AS) & AS $(D)$ & AS CR) & AS CU) & AS $(U)$ & AS FE) \\
\hline $06 \ldots$ & -- & -- & -- & -- & -- & 1 & -- & 370 \\
\hline $\begin{array}{c}25 \ldots \\
\operatorname{MAR} 1984\end{array}$ & - & -- & -- & -- & - & $<1$ & -- & 160 \\
\hline$\underset{A P R}{29 \ldots}$ & -- & -- & -- & -- & $-\infty$ & $<1$ & -- & 200 \\
\hline $\operatorname{MAX}^{30} \cdot$ & -- & -- & -- & -- & -- & $<1$ & -- & 60 \\
\hline $\begin{array}{l}18 \ldots \\
30 \ldots\end{array}$ & -- & -- & -- & - & -- & -- & -- & 210 \\
\hline DATE & $\begin{array}{l}\text { IRON, } \\
\text { DIS- } \\
\text { SOLVED } \\
\text { (UG/L } \\
\text { AS FE) }\end{array}$ & $\begin{array}{l}\text { LEAD, } \\
\text { TOTAL } \\
\text { RECOV- } \\
\text { ERABLE } \\
\text { (UG/I } \\
\text { AS PB) }\end{array}$ & $\begin{array}{l}\text { LEAD, } \\
\text { DIS- } \\
\text { SOLVED } \\
\text { (UG/L } \\
\text { AS PB) }\end{array}$ & $\begin{array}{l}\text { MERCURY } \\
\text { TOTAL } \\
\text { RECOV- } \\
\text { ERABLE } \\
\text { (UG/L } \\
\text { AS HG) }\end{array}$ & $\begin{array}{l}\text { ZINC, } \\
\text { TOTAL } \\
\text { RECOV- } \\
\text { ERABLE } \\
\text { (UG/L } \\
\text { AS 2N) }\end{array}$ & $\begin{array}{l}\text { ZINC, } \\
\text { DIS- } \\
\text { SOLVED } \\
\text { (UG/L } \\
\text { AS ZN) }\end{array}$ & $\begin{array}{l}\text { SEDI- } \\
\text { MENT, } \\
\text { SUS- } \\
\text { PENDED } \\
\text { (MG/L) }\end{array}$ & $\begin{array}{c}\text { SEDI- } \\
\text { MENT, } \\
\text { DIS- } \\
\text { CHARGE, } \\
\text { SUS- } \\
\text { PENDED } \\
\text { (T/DAY) }\end{array}$ \\
\hline OCI 1983 & & & & & & & & \\
\hline $06 \ldots$ & -- & -- & -- & -- & 5 & -- & 9 & 0.0 \\
\hline $\begin{array}{c}25 \ldots \\
\operatorname{MAR} 1984\end{array}$ & -- & -- & -- & -- & $<5$ & -- & 1 & 0.0 \\
\hline${ }_{A P R}^{29} \cdots$ & -- & $<1$ & -- & -- & $<6$ & -- & 4 & 0.0 \\
\hline${ }_{\text {MAY }}^{30}$ & -- & $<1$ & -- & -- & $<5$ & -- & 5 & 0.0 \\
\hline $18 \ldots$ & -- & - & $=-$ & -- & - & -- & 2 & 0.0 \\
\hline $30 \ldots$ & -- & $<1$ & -- & -- & $<5$ & -- & 3 & 0.0 \\
\hline
\end{tabular}


TA.I. 4.--Physical characteristics and concentrations of commond chemical constituents in lakes and streams of the Bald Mountain watershed (Continued)

01012545 MOOSE POND STREAM AT INLET CARR POND ME

\begin{tabular}{|c|c|c|c|c|c|c|c|c|c|}
\hline TIME & $\begin{array}{l}\text { STREAM- } \\
\text { FLOW, } \\
\text { INSTAN- } \\
\text { TANEOUS }\end{array}$ & $\begin{array}{l}\text { SPE- } \\
\text { CIFIC } \\
\text { CON- } \\
\text { DUCT- } \\
\text { ANCE }\end{array}$ & $\begin{array}{c}\text { PB } \\
\text { (STAND- } \\
\text { ARD }\end{array}$ & $\begin{array}{l}\text { TEMPER- } \\
\text { ATURE } \\
\text { WATER }\end{array}$ & $\begin{array}{l}\text { COLOR } \\
\text { (PLAT- } \\
\text { INUM- } \\
\text { COBALT }\end{array}$ & $\begin{array}{l}\text { TUR- } \\
\text { BID- } \\
\text { ITY }\end{array}$ & $\begin{array}{c}\text { OXYGEN, } \\
\text { DIS- } \\
\text { SOLVED }\end{array}$ & $\begin{array}{l}\text { OXYGEN } \\
\text { DEMAND, } \\
\text { BIO- } \\
\text { CHEM- } \\
\text { ICAL, } \\
5 \text { DAY }\end{array}$ & $\begin{array}{l}\text { COLI- } \\
\text { FORM, } \\
\text { FECAL, } \\
0.45 \\
\text { UM-MF } \\
\text { (COLS. }\end{array}$ \\
\hline & (CFS) & (US/CM) & UNITS) & (DEG C) & UNITS) & (FTU) & $(M G / L)$ & $(M G / L)$ & $100 \mathrm{ML})$ \\
\hline
\end{tabular}

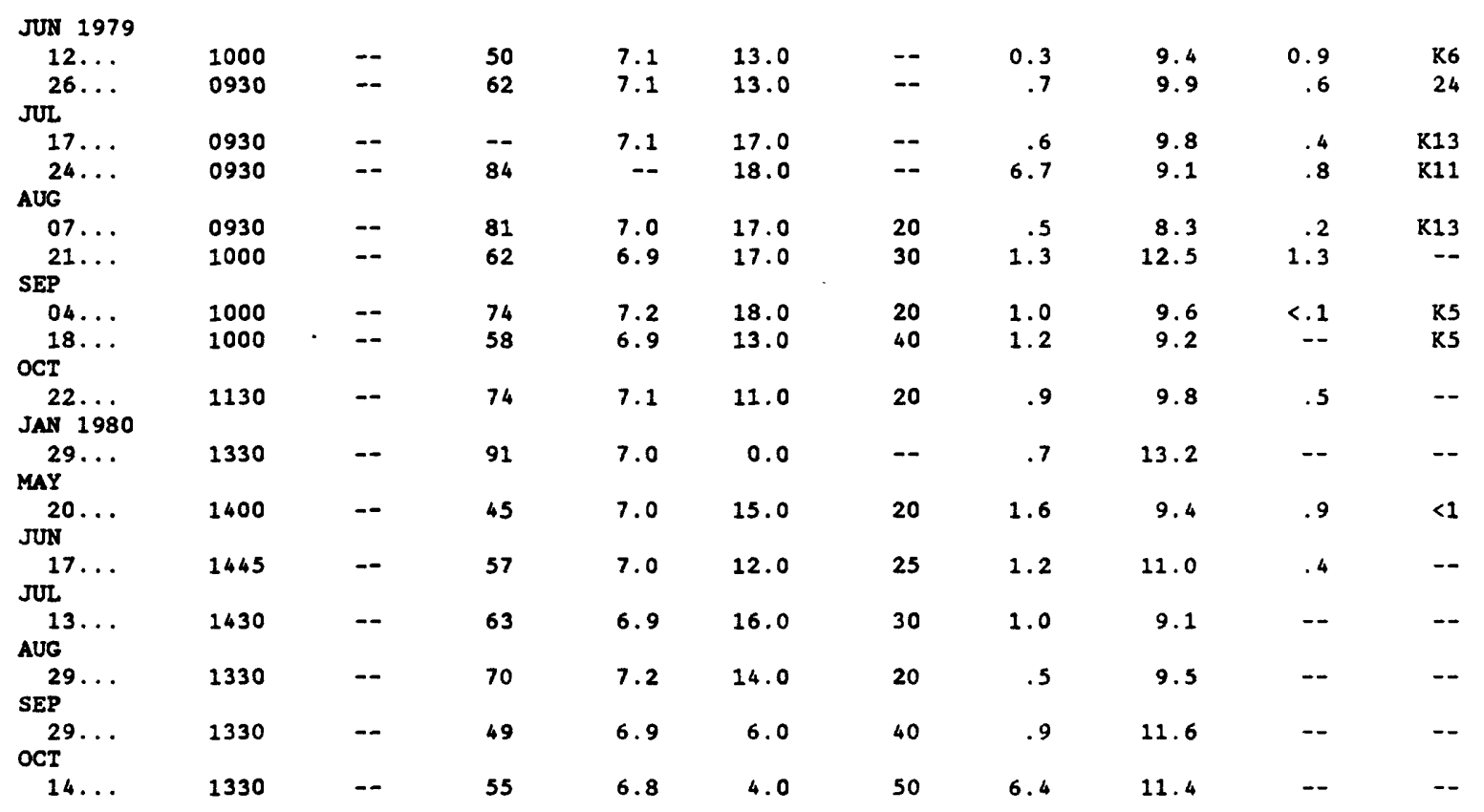

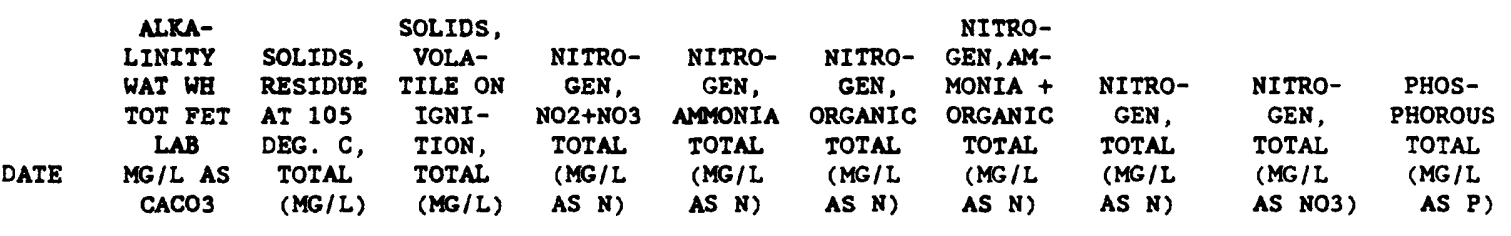

JUN 1979

\begin{tabular}{|c|c|c|c|c|c|c|c|c|c|c|}
\hline $12 \ldots$ & 13 & -- & -- & 0.01 & $<0.01$ & -- & -- & -- & -- & $<0.01$ \\
\hline $26 \ldots$ & 15 & -- & -- & .02 & $<.01$ & -- & -- & -- & -- & -- \\
\hline \multicolumn{11}{|l|}{ J几L } \\
\hline $17 \ldots$ & 28 & -- & -- & .05 & .02 & 0.08 & 0.1 & 0.15 & 0.66 & $<.01$ \\
\hline $24 \ldots$ & 28 & -- & -- & .07 & $<.01$ & -- & -- & -- & -- & -- \\
\hline \multicolumn{11}{|l|}{ AUG } \\
\hline $07 \ldots$ & 26 & -- & -- & .06 & $<.01$ & -- & -- & -- & -- & -- \\
\hline $21 \ldots$ & 1 & -- & -- & .05 & .04 & -- & -- & -- & -- & -- \\
\hline \multicolumn{11}{|l|}{ SEP } \\
\hline $04 \ldots$ & 26 & -- & -- & .04 & $<.01$ & -- & -- & -- & -- & -- \\
\hline $18 \ldots$ & 26 & -- & -- & $<.01$ & $<.01$ & -- & -- & -- & -- & -- \\
\hline \multicolumn{11}{|l|}{ oCT } \\
\hline JAN 1980 & 24 & - & -- & .03 & .01 & -- & -- & -- & -- & -- \\
\hline $\operatorname{MAY}^{29 \ldots}$ & -- & -- & -- & -- & -- & -- & -- & -- & -- & $<.01$ \\
\hline${ }_{\text {JNN }}^{20 \ldots}$ & 10 & -- & -- & .14 & $<.01$ & -- & .2 & .34 & 1.5 & .01 \\
\hline${ }_{\text {JUL }}^{17 \ldots}$ & 22 & -- & -- & .12 & $<.01$ & -- & .1 & .22 & .97 & .01 \\
\hline AUG & 22 & -- & -- & -- & -- & -- & -- & -- & -- & $<.01$ \\
\hline $\operatorname{SEP}^{29 \ldots}$ & 28 & 66 & -- & -- & -- & -- & -- & -- & -- & $<.01$ \\
\hline OCT & 30 & 68 & -- & -- & -- & -- & -- & -- & -- & .01 \\
\hline $14 \ldots$ & 24 & 71 & -- & -- & -- & -- & -- & -- & -- & .02 \\
\hline
\end{tabular}


The. 4.--Physical characteristics and concontrations of commond chemical constituents In Iakes and streams of the Bald Mountain watershed (Continued)

01012545 MOOSE POND STREAM AT INLET CARR POND ME

\begin{tabular}{|c|c|c|c|c|c|c|c|c|}
\hline DATE & $\begin{array}{l}\text { ALUM- } \\
\text { INUM, } \\
\text { TOTAL } \\
\text { RECOV- } \\
\text { ERABLE } \\
\text { (UG/L } \\
\text { AS AL) }\end{array}$ & $\begin{array}{l}\text { ALUM- } \\
\text { INUM, } \\
\text { DIS- } \\
\text { SOLVED } \\
\text { (UG/L } \\
\text { AS AL) }\end{array}$ & $\begin{array}{l}\text { ARSENIC } \\
\text { TOTAL } \\
\text { (UG/L } \\
\text { AS AS) }\end{array}$ & $\begin{array}{l}\text { CADMIUM } \\
\text { TOTAL } \\
\text { RECOV- } \\
\text { ERABLE } \\
\text { (UG/L } \\
\text { AS CD) }\end{array}$ & $\begin{array}{l}\text { CHRO- } \\
\text { MIUM, } \\
\text { TOTAL } \\
\text { RECOV- } \\
\text { ERABLE } \\
\text { (UG/L } \\
\text { AS CR) }\end{array}$ & $\begin{array}{l}\text { COPPER, } \\
\text { TOTAL } \\
\text { RECOV- } \\
\text { ERABLE } \\
\text { (UG/L } \\
\text { AS CU) }\end{array}$ & $\begin{array}{l}\text { COPPER, } \\
\text { DIS- } \\
\text { SOLVED } \\
\text { (UG/L } \\
\text { AS CU) }\end{array}$ & $\begin{array}{l}\text { IRON, } \\
\text { TOTAL } \\
\text { RECOV- } \\
\text { ERABLE } \\
\text { (UG/L } \\
\text { AS FE) }\end{array}$ \\
\hline \multicolumn{9}{|l|}{ JUN 1979} \\
\hline $12 \ldots$ & -- & -- & -- & $<2$ & $<6$ & $<4$ & -- & -- \\
\hline JuL $26 \ldots$ & -- & -- & -- & -- & -- & -- & -- & -- \\
\hline $17 \ldots$ & - & -- & -- & $<2$ & $<6$ & $<4$ & -- & 70 \\
\hline${ }_{A U G}^{24} \ldots$ & -- & -- & -- & $<2$ & $<6$ & $<4$ & -- & 50 \\
\hline $07 \ldots$ & -- & -- & -- & -- & $<6$ & $<4$ & -- & 80 \\
\hline$\underset{\text { SEP }}{21} \cdots$ & -- & -- & -- & -- & -- & -- & -- & -- \\
\hline $04 \ldots$ & -- & -- & -- & -- & -- & -- & -- & -- \\
\hline${ }_{\mathrm{OCT}}^{18 \ldots}$ & -- & -- & -- & -- & -- & -- & -- & -- \\
\hline $\begin{array}{c}22 \ldots \\
\text { JAN } 1980\end{array}$ & -- & -- & -- & -- & $<6$ & $<4$ & -- & 50 \\
\hline$\underset{M A Y}{29 \ldots}$ & -- & -- & -- & $<2$ & $<6$ & $<4$ & -- & -- \\
\hline Juns & -- & -- & -- & $<2$ & $<20$ & $<4$ & -- & -- \\
\hline JUL $17 \ldots$ & -- & -- & -- & $<2$ & $<5$ & 4 & -- & 220 \\
\hline${ }_{A U G}^{13 \ldots}$ & -- & -- & -- & -- & $<5$ & $<2$ & -- & 40 \\
\hline $\begin{array}{l}29 \ldots \\
\text { SEP }\end{array}$ & -- & -- & -- & -- & -- & 6 & -- & 120 \\
\hline${ }_{\text {OCT }}^{29 \ldots}$ & -- & -- & -- & -- & -- & $<4$ & -- & 70 \\
\hline $14 \ldots$ & -- & -- & $<4$ & -- & -- & 2 & -- & 460 \\
\hline DATE & $\begin{array}{l}\text { IRON, } \\
\text { DIS- } \\
\text { SOLVED } \\
\text { (UG/L } \\
\text { AS FE) }\end{array}$ & $\begin{array}{l}\text { LEAD, } \\
\text { TOTAL } \\
\text { RECOV- } \\
\text { ERABLE } \\
\text { (UG/L } \\
\text { AS PB) }\end{array}$ & $\begin{array}{l}\text { LEAD, } \\
\text { DIS- } \\
\text { SOLVED } \\
\text { (UG/L } \\
\text { AS PB) }\end{array}$ & $\begin{array}{l}\text { MERCURY } \\
\text { TOTAL } \\
\text { RECOV- } \\
\text { ERABLE } \\
\text { (UG/L } \\
\text { AS HG) }\end{array}$ & $\begin{array}{l}\text { ZINC, } \\
\text { TOTAL } \\
\text { RECOV- } \\
\text { ERABLE } \\
\text { (UG/L } \\
\text { AS ZN) }\end{array}$ & $\begin{array}{l}\text { ZINC, } \\
\text { DIS- } \\
\text { SOLVED } \\
\text { (UG/L } \\
\text { AS ZN) }\end{array}$ & $\begin{array}{l}\text { SEDI- } \\
\text { MENT, } \\
\text { SUS- } \\
\text { PENDED } \\
\text { (MG/L) }\end{array}$ & $\begin{array}{c}\text { SEDI- } \\
\text { MENT, } \\
\text { DIS- } \\
\text { CHARGE, } \\
\text { SUS- } \\
\text { PENDED } \\
\text { (T/DAY) }\end{array}$ \\
\hline
\end{tabular}

\footnotetext{
JUN 1979

$12 \ldots$

26...

JUL.

$17 . .$.

$24 \ldots$

AUG

$07 .$.

21..

SEP

$04 \ldots$

$18 \ldots$

OCT

22 ...

JAN 1980

29 ...

MAY

$20 \ldots$

JUN

$17 \ldots$

JUL

13...

AUG

29...

SEP

29...

OCT
}

$<20$

$<20$

$<20$

$\begin{array}{ll}-- & <20 \\ -- & --\end{array}$

--

$--\quad-$

$--\quad<20$

$<20$

$<30$

$<30$

$-$
$-$

0.6

.3

.2

$--$

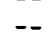

$<.2$

$<.2$

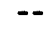

$<.2$

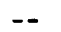

$-$
$-$

$-$

--

--

--

$--$

$-$

$-$

$-$

$-$

9

$<2$

$<5$ 
TA.r. 4.--Physical characteristics and concentrations of comnond chemical constituents in lakes and streams of the Bald Mountain watershed (Continued)

01012545 MOOSE POND STREAM AT INLET CARR POND ME

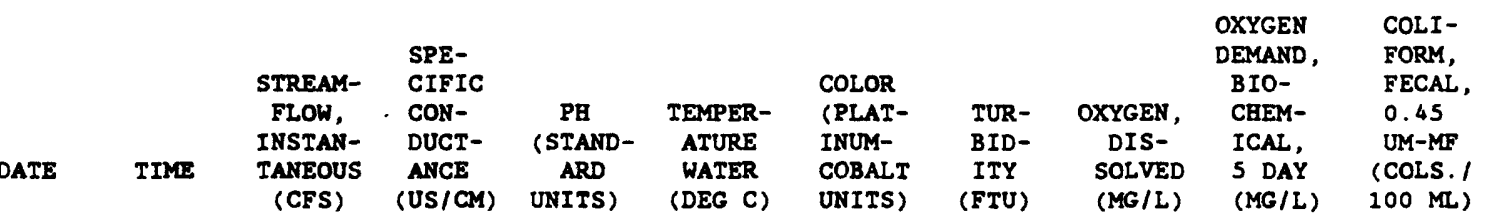

\begin{tabular}{|c|c|c|c|c|c|c|c|c|c|c|}
\hline FEB $12 \ldots$ & 1445 & -- & 39 & 7.0 & 0.0 & 10 & 0.7 & 12.9 & -- & -- \\
\hline${ }_{\text {MAR }}^{23 \ldots}$ & 1545 & -- & 30 & 6.2 & 3.0 & 45 & 3.8 & 12.6 & -- & -- \\
\hline${ }_{\text {APR }}^{18 \ldots}$ & 1240 & -- & 53 & 6.7 & 2.0 & 10 & .8 & 13.2 & -- & -- \\
\hline $\operatorname{MAX}^{14 \ldots}$ & 1410 & -- & 60 & 6.7 & 2.0 & 25 & 1.4 & 12.0 & -- & $=-$ \\
\hline $\mathrm{JUN}^{15 \ldots}$ & 1530 & - & 30 & 6.9 & 9.0 & 45 & 1.1 & $10: 6$ & -- & -- \\
\hline JUL $10 \ldots$ & 1215 & $=$ & 50 & 7.0 & 9.0 & 15 & 1.2 & 10.8 & -- & -- \\
\hline$\stackrel{21 \ldots}{A U G}$ & 1215 & -- & 82 & 7.1 & 14.0 & 25 & 1.0 & -- & -- & -- \\
\hline $\operatorname{SEP}^{24 \ldots}$ & 1730 & -- & 40 & 6.7 & 13.0 & 40 & 2.3 & 9.7 & -- & -- \\
\hline $\mathrm{OCT}^{22 \ldots}$ & 1210 & -- & 60 & 7.4 & 8. 0 & 30 & .7 & 11.4 & -- & -- \\
\hline${ }_{\text {NOV }}^{20 \ldots}$ & 1210 & -- & 40 & 6.7 & 5.0 & 40 & 1.0 & 12.2 & -- & -- \\
\hline $\begin{array}{l}17 \ldots \\
\text { MAR } 1982\end{array}$ & 1015 & -- & 40 & 6.8 & 4.0 & 10 & 1.2 & 10.5 & -- & -- \\
\hline$\underset{A P R}{26 \ldots}$ & 0830 & -- & 60 & 7.0 & -- & 20 & 1.0 & -- & -- & -- \\
\hline${ }_{\text {JN }}^{19}$ & 1430 & -- & 48 & 6.7 & 2.0 & 40 & 3.5 & -- & -- & -- \\
\hline $01 \ldots$ & 1135 & - & 67 & 6.9 & 12.0 & 35 & .8 & 8. 6 & -- & -- \\
\hline DATE & $\begin{array}{l}\text { ALRA- } \\
\text { LINITY } \\
\text { WAT WH } \\
\text { TOI FET } \\
\text { LAB } \\
\text { MG/L AS } \\
\text { CACO3 }\end{array}$ & $\begin{array}{l}\text { SOLIDS, } \\
\text { RESIDUE } \\
\text { AT } 105 \\
\text { DEG. C, } \\
\text { TOTAL } \\
\text { (MG/L) }\end{array}$ & $\begin{array}{l}\text { SOLIDS, } \\
\text { VOLA- } \\
\text { IILE ON } \\
\text { IGNI- } \\
\text { IION, } \\
\text { TOTAL } \\
\text { (MG/L) }\end{array}$ & $\begin{array}{l}\text { NITRO- } \\
\text { GEN, } \\
\text { NO2+NO3 } \\
\text { TOTAL } \\
\text { (MG/L } \\
\text { AS N) }\end{array}$ & $\begin{array}{l}\text { NITRO- } \\
\text { GEN, } \\
\text { AMONIA } \\
\text { IOTAL } \\
\text { (MG/L } \\
\text { AS N) }\end{array}$ & $\begin{array}{l}\text { NITRO- } \\
\text { GEN, } \\
\text { ORGANIC } \\
\text { TOTAL } \\
\text { (MG/L } \\
\text { AS N) }\end{array}$ & $\begin{array}{l}\text { NITRO- } \\
\text { GEN, AM- } \\
\text { MONIA + } \\
\text { ORGANIC } \\
\text { TOTAL } \\
\text { (MG/L } \\
\text { AS N) }\end{array}$ & $\begin{array}{l}\text { NITRO- } \\
\text { GEN, } \\
\text { TOTAL } \\
\text { (MG / I } \\
\text { AS N) }\end{array}$ & $\begin{array}{l}\text { NITRO- } \\
\text { GEN, } \\
\text { TOTAL } \\
\text { (MG/L } \\
\text { AS NO3) }\end{array}$ & $\begin{array}{l}\text { PHOS- } \\
\text { PHOROUS } \\
\text { TOTAL } \\
\text { (MG/L } \\
\text { AS P) }\end{array}$ \\
\hline
\end{tabular}

DEC 1980

$12 \ldots$

FEB 1981

23 .

MAR

18 ...

APR

$14 \ldots$

MAY

15 ...

JUN

10 ...

JUL.

21...

AUG

24...

SEP

OCT 22.

20...

\begin{tabular}{|c|c|c|c|c|c|c|c|c|c|}
\hline 23 & -- & -- & -- & -- & -- & -- & -- & -- & 0.01 \\
\hline 12 & -- & -- & -- & -- & -- & -- & -- & -- & .02 \\
\hline 24 & 40 & $=-$ & -- & -- & -- & -- & -- & -- & $<.01$ \\
\hline 14 & 48 & -- & -- & -- & -- & -- & -- & -- & .02 \\
\hline 17 & 52 & -- & -- & -- & -- & -- & -- & -- & $<.01$ \\
\hline 20 & 67 & -- & -- & -- & -- & -- & -- & -- & .01 \\
\hline 36 & 65 & -- & -- & -- & -- & -- & -- & -- & .01 \\
\hline 10 & -- & $=-$ & $=$ & -- & -- & $=$ & -- & -- & -- \\
\hline 21 & 71 & -- & -- & -- & -- & -- & -- & -- & -- \\
\hline 12 & 64 & $=-$ & $=-$ & -- & -- & -- & -- & -- & .01 \\
\hline 17 & 68 & -- & -- & -- & -- & -- & -- & -- & $<.01$ \\
\hline 26 & 75 & $-\infty$ & -- & -- & -- & -- & -- & -- & -- \\
\hline-- & 61 & - & -- & -- & -- & -- & -- & $=-$ & -- \\
\hline 22 & 84 & - & -- & -- & -- & $=-$ & -- & -- & -- \\
\hline
\end{tabular}


Tanra 4.--Physical characteristics and concentrations of commond chemical constituents in lakes and streams of the Bald Mountain watershed (Continued)

01012545 MOOSE POND STREAM AT INLET CARR POND ME

\begin{tabular}{|c|c|c|c|c|c|c|c|c|}
\hline $\operatorname{ATE}$ & $\begin{array}{l}\text { ALUM- } \\
\text { INUM, } \\
\text { TOTAL } \\
\text { RECOV- } \\
\text { ERABLE } \\
\text { (UG/L }\end{array}$ & $\begin{array}{l}\text { ALUM- } \\
\text { INUM, } \\
\text { DIS- } \\
\text { SOLVED } \\
\text { (UG/L }\end{array}$ & $\begin{array}{l}\text { ARSENIC } \\
\text { TOTAL } \\
\text { (UG/L }\end{array}$ & $\begin{array}{l}\text { CADMIUM } \\
\text { TOTAL } \\
\text { RECOV- } \\
\text { ERABLE } \\
\text { (UG/L }\end{array}$ & $\begin{array}{l}\text { CHRO- } \\
\text { MIUM, } \\
\text { TOTAL } \\
\text { RECOV- } \\
\text { ERABLE } \\
\text { (UG/L }\end{array}$ & $\begin{array}{l}\text { COPPER, } \\
\text { TOTAL } \\
\text { RECOV- } \\
\text { ERABLE } \\
\text { (UG / L }\end{array}$ & $\begin{array}{l}\text { COPPER, } \\
\text { DIS- } \\
\text { SOLVED } \\
\text { (UG/L }\end{array}$ & $\begin{array}{l}\text { IRON, } \\
\text { TOTAL } \\
\text { RECOV- } \\
\text { ERABLE } \\
\text { (UG/L }\end{array}$ \\
\hline & AS AL) & AS AL) & AS AS) & AS $C D)$ & AS CR) & AS CU) & AS CU) & AS FE) \\
\hline
\end{tabular}

DEC 1980

$12 \ldots$

FEB 1981

23...

MAR

$18 \ldots$

APR

$14 \ldots$

MAY

15...

JUN

10 ...

गU.

21...

AUG

$24 \ldots$

SEP

22...

OCT

$20 \ldots$

Nov

$17 \ldots$

MAR 1982

26 ...

APR

19 ...

JUN

$01 .$.

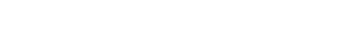$$
\text { 1... }
$$

$\begin{array}{ccccc}-- & -- & - & -- & - \\ -- & -- & -- & -- & - \\ -- & -- & -- & -- & - \\ -- & -- & -- & -- & - \\ -- & -- & -- & -- & - \\ -- & -- & -- & -- & - \\ -- & -- & -- & -- & - \\ <100 & -- & -- & <4 & - \\ -- & -- & -- & -- & - \\ -- & -- & -- & -- & - \\ -- & -- & -- & -- & - \\ -- & -- & -- & -- & - \\ 560 & -- & -- & -- & - \\ <100 & <100 & -- & -- & -\end{array}$

$\begin{array}{rrrr}-- & 2 & -- & 100 \\ -- & 2 & -- & 380 \\ -- & 1 & -- & 60 \\ -- & 2 & -- & 400 \\ -- & 1 & -- & 100 \\ -- & 2 & -- & 100 \\ -- & 2 & -- & 100 \\ -- & 12 & -- & 150 \\ -- & <2 & -- & 40 \\ -- & 3 & -- & 70 \\ - & -- & -- & -- \\ -- & -- & -- & -- \\ - & 2 & -- & 510 \\ - & <1 & <1 & 70\end{array}$

DATE

$\begin{array}{clc} & & \\ \text { IRON, } & \text { TOTAL } & \text { LEAD, } \\ \text { DIS- } & \text { RECOV- } & \text { DIS- } \\ \text { SOLVED } & \text { ERABLE } & \text { SOLVED } \\ \text { (UG/L } & \text { (UG/L } & \text { (UG/L } \\ \text { AS FE) } & \text { AS PB) } & \text { AS PB) }\end{array}$
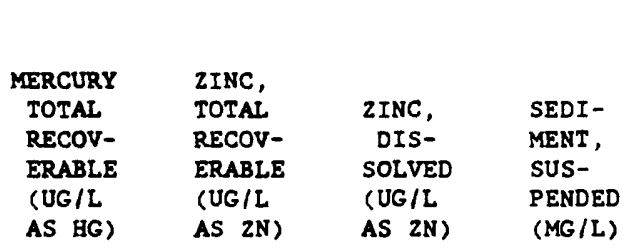

SEDI-

MENT,

DIS-

CHARGE,

SUS-

PENDED

(T/DAY)

DEC 1980

12 ..

FEB 1981

23 ...

MAR

$18 \ldots$

APR

$14 \ldots$

MAY

$15 \ldots$

JUN

$10 \ldots$

गU

21...

AUG

$24 \ldots$

SEP

22 ...

OCT

20 ...

Nov

$17 \ldots$

MAR 1982

26 ...

APR

$19 .$.

\begin{tabular}{|c|c|c|c|c|c|c|}
\hline$-\infty$ & -- & - & 10 & -- & -- & -- \\
\hline-- & -- & -- & 7 & -- & 10 & 0.0 \\
\hline-- & -- & -- & S5 & -- & 2 & 0.0 \\
\hline$-\infty$ & -- & $\cdots$ & -- & -- & -- & -- \\
\hline-- & -- & - & -- & -- & -- & -- \\
\hline- & -- & -- & -- & -- & -- & -- \\
\hline-- & - & - & $<5$ & -- & 3 & 0.0 \\
\hline$<4$ & $=$ & $<0.2$ & $<4$ & -- & -- & -- \\
\hline- & - & - & $<5$ & -- & -- & -- \\
\hline-- & - & $-\infty$ & $<5$ & $\cdots$ & -- & -- \\
\hline-- & -- & -- & -- & -- & -- & -- \\
\hline-- & -- & - & -- & -- & 2 & 0.0 \\
\hline$<1$ & -- & -- & $<8$ & -- & 17 & 0.0 \\
\hline$<1$ & $<1$ & -- & 5 & $<5$ & -- & -- \\
\hline
\end{tabular}


INI. 4.--Physical characteristics and concentrations of commond chemical constituents in lakes and streams of the Bald Mountain watershed--Continued

01012550 CARR POND STREAM AT OUTLET CARR POND ME

\begin{tabular}{|c|c|c|c|c|c|c|c|c|c|c|}
\hline DATE & IIME & $\begin{array}{l}\text { STREAM- } \\
\text { FLOW, } \\
\text { INSTAN- } \\
\text { TANEOUS } \\
\text { (CFS) }\end{array}$ & $\begin{array}{l}\text { SPE- } \\
\text { CIFIC } \\
\text { CON- } \\
\text { DUCT- } \\
\text { ANCE } \\
\text { (US/CM) }\end{array}$ & $\begin{array}{c}\text { PB } \\
\text { (STAND- } \\
\text { ARD } \\
\text { UNITS) }\end{array}$ & $\begin{array}{l}\text { TEMPER- } \\
\text { ATURE } \\
\text { WATER } \\
\text { (DEG C) }\end{array}$ & $\begin{array}{l}\text { COLOR } \\
\text { (PLAT- } \\
\text { INUM- } \\
\text { COBALT } \\
\text { UNITS) }\end{array}$ & $\begin{array}{l}\text { TUR- } \\
\text { BID- } \\
\text { ITY } \\
\text { (FTU) }\end{array}$ & $\begin{array}{c}\text { OXYGEN, } \\
\text { DIS- } \\
\text { SOLVED } \\
\text { (MG/L) }\end{array}$ & $\begin{array}{l}\text { OXYGEN } \\
\text { DEMAND, } \\
\text { BIO- } \\
\text { CHEM- } \\
\text { ICAL, } \\
5 \text { DAY } \\
(M G / L)\end{array}$ & $\begin{array}{l}\text { COLI- } \\
\text { FORM, } \\
\text { FECAL, } \\
0.45 \\
\text { UM-MF } \\
\text { (COLS.' } \\
100 \mathrm{ML} \text { ) }\end{array}$ \\
\hline JAN 1980 & & & & & & & & & & \\
\hline$\stackrel{29}{29} \ldots$ & 1415 & -- & 66 & 7.0 & 0.0 & -- & 0.6 & 14.6 & -- & -- \\
\hline $\operatorname{MAR}^{19 \ldots}$ & 1415 & -- & 71 & 6.7 & 0.0 & 40 & .9 & 13.5 & 0.7 & $<1$ \\
\hline${ }_{A P R}^{20} \cdots$ & 1315 & -- & 58 & 7.1 & 0.0 & 40 & 3.3 & 12.8 & .7 & $<1$ \\
\hline $\operatorname{MAY}^{23} \cdots$ & 1315 & -- & 26 & 6.7 & 3.0 & 30 & 2.8 & 13.2 & .6 & $<1$ \\
\hline JUN $20 \ldots$ & 1500 & -- & 45 & 7.0 & 15.0 & 25 & 1.5 & 10.6 & 1.6 & -- \\
\hline $\mathrm{JUL}^{17 \ldots}$ & 1545 & -- & 42 & 7.1 & 17.0 & 35 & 1.3 & 10.5 & $<.1$ & -- \\
\hline${ }_{A U G}^{13 \ldots}$ & 1500 & -- & 50 & 7.0 & 22.0 & 30 & 2.6 & 9.0 & -- & -- \\
\hline $\begin{array}{c}29 \ldots \\
\text { SEP }\end{array}$ & 1430 & -- & 53 & 7.4 & 24.0 & 35 & 1.6 & 8.9 & -- & -- \\
\hline OCT $^{29 \ldots}$ & 1430 & -- & 49 & 7.0 & 12.0 & 30 & 1.3 & 9.8 & -- & -- \\
\hline $\operatorname{mov}^{14} \ldots$ & 1430 & -- & 63 & 7.0 & 9.0 & 25 & 1.4 & 10.2 & -- & -- \\
\hline${ }_{D E C}^{21} \cdots$ & 1430 & -- & 76 & 7.1 & 1.0 & 30 & 1.0 & 13.2 & -- & -- \\
\hline $\begin{array}{c}12 \ldots \\
\text { JAN } 1981\end{array}$ & 1600 & -- & 120 & 6.7 & 0.0 & 40 & 1.2 & 13.0 & -- & -- \\
\hline$\underset{\text { FEB }}{20 \ldots}$ & 1500 & -- & 54 & 6.6 & 1.0 & 35 & 1.7 & 13.9 & -- & -- \\
\hline $23 \ldots$ & 1445 & -- & 40 & 6.7 & 3.0 & 40 & 2.2 & 12.6 & -- & -- \\
\hline
\end{tabular}

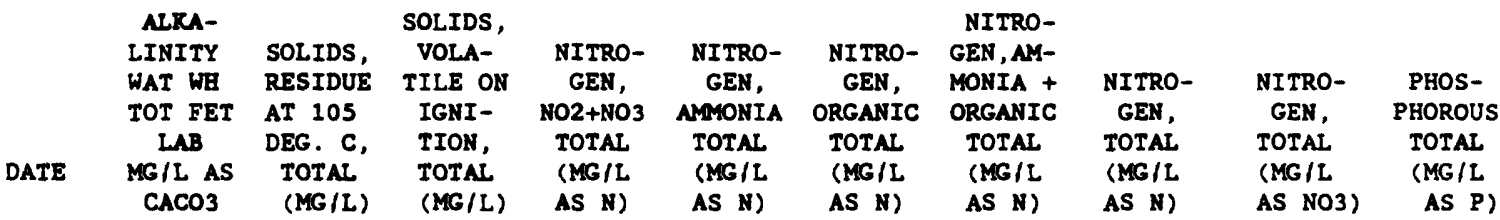

\begin{tabular}{|c|c|c|c|c|c|c|c|c|c|c|}
\hline $\begin{array}{c}\text { JAN } 1980 \\
29 \ldots \\
\text { FEB }\end{array}$ & -- & 4 & -- & -- & -- & -- & -- & -- & -- & 0.01 \\
\hline $\operatorname{MAR}^{19} \ldots$ & 21 & 65 & -- & 0.05 & -- & -- & 0.2 & 0.25 & 1.1 & $<.01$ \\
\hline$\underset{A P R}{20 \ldots}$ & 17 & 63 & -- & .07 & 0.02 & 0.18 & .2 & .27 & 1.2 & .01 \\
\hline${ }_{\text {MAY }}^{23} \ldots$ & 9 & 38 & -- & .09 & $<.01$ & -- & .2 & .29 & 1.3 & .01 \\
\hline JUN & 13 & 98 & -- & .08 & $<.01$ & -- & .1 & .18 & 0.80 & .01 \\
\hline Jur $17 \ldots$ & 15 & 79 & -- & .04 & $<.01$ & -- & .1 & .14 & .62 & .01 \\
\hline${ }_{A U G}^{13} \cdots$ & 15 & 58 & -- & -- & -- & -- & -- & -- & -- & .01 \\
\hline $\operatorname{sEP}^{29} \cdots$ & 17 & 50 & -- & -- & -- & -- & -- & -- & -- & $<.01$ \\
\hline $\mathrm{OCT}^{29}$ & 20 & 53 & -- & -- & -- & -- & -- & -- & -- & .02 \\
\hline $\operatorname{Nov}^{14 \ldots}$ & 23 & 62 & -- & -- & -- & -- & -- & -- & -- & .01 \\
\hline${ }_{D E C}^{21} \cdots$ & 21 & 65 & -- & -- & -- & -- & -- & -- & -- & .01 \\
\hline $\begin{array}{l}12 \ldots \\
\text { JAN } 1981\end{array}$ & 25 & 54 & -- & -- & -- & -- & -- & -- & -- & .01 \\
\hline$\underset{\text { FEB }}{20}$ & 26 & -- & -- & -- & -- & -- & -- & -- & -- & .01 \\
\hline $23 \ldots$ & 21 & -- & -- & -- & -- & -- & -- & -- & -- & .01 \\
\hline
\end{tabular}


TNmE 4.--Physical characteristics and concentrations of commond chemical constituents in lakes and streams of the Bald Mountain watershed--Continued

01012550 CARR POND STREAM AT OUTLET CARR POND ME

DATE

JAN 1980

29.

FEB

19 .

MAR

20 ...

APR

23...

MAY

20 . . .

JUN

17 ...

JUL

13.

AUG

29 ..

SEP

29...

OCT

$14 \ldots$

Nov

$21 \ldots$

DEC

$12 \ldots$

JAN 1981

$20 \ldots$

FEB

$23 \ldots$
ALUM-

INUM, ALUM-

TOTAL

RECOV-

ERABLE

(UG/L

AS AL)
ALUM-

DIS-

SOLVED

(UG/L

AS AL)

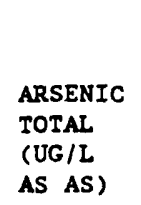

$\begin{array}{cl} & \text { CHRO- } \\ \text { CADMIUM } & \text { MIUM, } \\ \text { TOTAL } & \text { TOTAL } \\ \text { RECOV- } & \text { RECOV- } \\ \text { ERABLE } & \text { ERABLE } \\ \text { (UG/L } & \text { (UG/L } \\ \text { AS CD) } & \text { AS CR) }\end{array}$

COPPER,
TOTAL
RECOV-
ERABLE
(UG/L
AS CU)

COPPER

IRON,

TOTAL

DIS- RECOV-

SOLVED ERABLE

(UG/L

(UG/L

AS CU)

$\begin{array}{rrrrrrrr}-- & -- & - & <2 & <6 & <4 & -- & -- \\ -- & -- & - & <2 & <6 & <4 & - & 60 \\ -- & -- & -- & -- & -- & <4 & -- & - \\ -- & -- & -- & <2 & <5 & <10 & -- & -- \\ -- & -- & -- & <2 & <20 & <4 & -- & - \\ -- & -- & -- & <2 & <5 & 3 & -- & 60 \\ -- & -- & -- & -- & <5 & <2 & -- & 60 \\ -- & -- & -- & -- & -- & <4 & -- & 30 \\ -- & -- & -- & -- & -- & <4 & -- & 20 \\ -- & -- & <4 & -- & -- & 1 & -- & 20 \\ -- & -- & -- & -- & -- & <1 & -- & -- \\ -- & -- & -- & -- & -- & <1 & -- & 80 \\ -- & -- & -- & -- & -- & -- & - & 30\end{array}$

DATE

\begin{tabular}{|c|c|c|c|c|c|c|c|c|}
\hline & $\begin{array}{l}\text { IRON, } \\
\text { DIS- } \\
\text { SOLVED } \\
\text { (UG/L }\end{array}$ & $\begin{array}{l}\text { LEAD, } \\
\text { TOTAL } \\
\text { RECOV- } \\
\text { ERABLE } \\
\text { (UG /L }\end{array}$ & $\begin{array}{l}\text { LEAD, } \\
\text { DIS- } \\
\text { SOLVED } \\
\text { (UG/L }\end{array}$ & $\begin{array}{l}\text { MERCURY } \\
\text { TOTAL } \\
\text { RECOV- } \\
\text { ERABLE } \\
\text { (UG/L }\end{array}$ & $\begin{array}{l}\text { ZINC, } \\
\text { TOTAL } \\
\text { RECOV- } \\
\text { ERABLE } \\
\text { (UG/L }\end{array}$ & $\begin{array}{l}\text { 2INC, } \\
\text { DIS- } \\
\text { SOLVED } \\
\text { (UG/L }\end{array}$ & $\begin{array}{l}\text { SEDI- } \\
\text { MENT, } \\
\text { SUS - } \\
\text { PENDED }\end{array}$ & $\begin{array}{r}\text { MENT, } \\
\text { DIS- } \\
\text { CHARGE, } \\
\text { SUS- } \\
\text { PENDED }\end{array}$ \\
\hline & AS $F E$ ) & AS PB) & AS PB) & AS HG) & As $2 \mathrm{~N}$ ) & AS ZN) & (MG/L) & ( $T / D A Y)$ \\
\hline
\end{tabular}

JAN 1980
$29 \ldots$

29.

FEB 19.

MAR

$20 .$.

APR

$23 . .$.

MAY

$20 \ldots$

JUN

$17 \ldots$

JUL

13 ...

AUG

29...

SEP

29...

OCT

$14 \ldots$

NOV

$21 \ldots$

DEC

12 ...

JAN 1981

20 ...

FEB

$23 \ldots$

\begin{tabular}{|c|c|c|c|c|}
\hline-- & $<20$ & -- & 0.4 & $<2$ \\
\hline-- & $<20$ & -- & $<.2$ & 14 \\
\hline-- & $<20$ & - & -- & $<2$ \\
\hline-- & $<30$ & -- & -- & 2 \\
\hline-- & $<30$ & -- & -- & 22 \\
\hline-- & $<30$ & -- & $<.2$ & 2 \\
\hline-- & -- & -- & -- & 2 \\
\hline-- & -- & -- & -- & $<2$ \\
\hline- & -- & -- & -- & $<2$ \\
\hline-- & -- & -- & -- & $<5$ \\
\hline-- & $<2$ & - & -- & $<4$ \\
\hline-- & -- & -- & - & $<2$ \\
\hline- & -- & -- & -- & $<4$ \\
\hline & -- & - & -- & $<3$ \\
\hline
\end{tabular}

$\begin{array}{ccc}- & -- & - \\ -- & -- & - \\ - & 1 & 0.0 \\ -- & 2 & 0.0 \\ - & 1 & 0.0 \\ - & 3 & 0.0 \\ - & -- & -- \\ - & -- & -- \\ - & -- & -- \\ - & -- & -- \\ - & -- & - \\ - & -- & -- \\ - & -- & -- \\ - & 1 & 0.0\end{array}$


TA.T 4.--Physical characteristics and concentrations of commond chemical constituents in lakes and streams of the Bald Mountain watershed--Continued

01012550 CARR POND STREAM AT OUTLET CARR POND ME

\begin{tabular}{|c|c|c|c|c|c|c|c|c|c|c|}
\hline ATE & TIME & $\begin{array}{l}\text { STREAM- } \\
\text { FLOW, } \\
\text { INSTAN- } \\
\text { TANEOUS }\end{array}$ & $\begin{array}{l}\text { SPE- } \\
\text { CIFIC } \\
\text { CON- } \\
\text { DUCT- } \\
\text { ANCE }\end{array}$ & $\begin{array}{c}\text { PB } \\
\text { (STAND- } \\
\text { ARD }\end{array}$ & $\begin{array}{l}\text { TEMPER- } \\
\text { ATURE } \\
\text { WATER }\end{array}$ & $\begin{array}{l}\text { COLOR } \\
\text { (PLAT- } \\
\text { INUM- } \\
\text { COBALT }\end{array}$ & $\begin{array}{l}\text { TUR- } \\
\text { BID- } \\
\text { ITY }\end{array}$ & $\begin{array}{c}\text { OXYGEN, } \\
\text { DIS- } \\
\text { SOLVED }\end{array}$ & $\begin{array}{l}\text { OXYGEN } \\
\text { DEMAND, } \\
\text { BIO- } \\
\text { CHEM- } \\
\text { ICAL, } \\
5 \text { DAY }\end{array}$ & $\begin{array}{l}\text { COLI- } \\
\text { FORM, } \\
\text { FECAL, } \\
\text { O. } 45 \\
\text { UM-MF } \\
\text { (COLS. ) }\end{array}$ \\
\hline & & (CFS) & (US/CM) & (NITS) & (DEG C) & UNITS) & (FTU) & $(M G / L)$ & $(M G / L)$ & $100 \mathrm{ML})$ \\
\hline
\end{tabular}

\begin{tabular}{|c|c|c|c|c|c|c|c|c|c|c|}
\hline${ }_{A P R}^{18 \ldots}$ & 1155 & -- & 50 & 6.5 & 2.0 & 20 & 1.0 & 13.8 & -- & - \\
\hline $\mathrm{MAY}^{14 \ldots}$ & 1500 & - & 61 & 6.7 & 1.0 & 35 & 1.4 & 12.0 & -- & -- \\
\hline $\operatorname{JUN}_{N}^{15}$ & 1510 & -- & 30 & 6.9 & 13.0 & 35 & 0.9 & 9.2 & -- & -- \\
\hline $\mathrm{JUL}^{10 \ldots}$ & 1315 & -- & 43 & 7.4 & 13.0 & 20 & 1.3 & 10.8 & -- & -- \\
\hline AUG $21 \cdots$ & 1315 & -- & 58 & 7.1 & 21.0 & 25 & 1.1 & -- & -- & -- \\
\hline $\operatorname{SEP}^{24 \ldots}$ & 1550 & - & 50 & 7.0 & 19.0 & 60 & .9 & 8.6 & -- & -- \\
\hline $\operatorname{ocr}^{22} \cdots$ & 1145 & -- & 32 & 7.0 & 15.0 & 50 & 1.1 & 8.8 & -- & -- \\
\hline Nov $20 \ldots$ & 1145 & - & 42 & 6.8 & 6.0 & 50 & 1.1 & 12.2 & -- & -- \\
\hline $\begin{array}{l}17 \ldots \\
\text { MAR } 1982\end{array}$ & 1110 & -- & 85 & 6.8 & 4.0 & 30 & 1.3 & 10.5 & -- & -- \\
\hline${ }_{A P R}^{26} \cdots$ & 0830 & -- & 34 & 7.0 & $-\infty$ & 40 & .6 & $=$ & $=-$ & $\cdots$ \\
\hline${ }_{\mathrm{MAY}}^{18} \cdots$ & 1400 & -- & -- & 6.7 & 4.5 & 30 & 3.0 & 10.8 & -- & -- \\
\hline JU & 1300 & -- & -- & -- & 2.0 & -- & 15 & 12.1 & -- & -- \\
\hline $01 \ldots$ & 1200 & -- & 48 & 7.2 & 21.0 & 35 & 1.3 & 10.3 & -- & -- \\
\hline
\end{tabular}

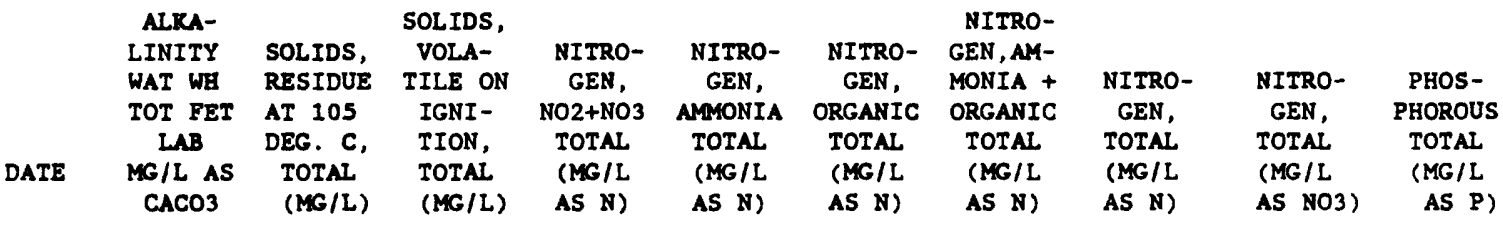

\begin{tabular}{|c|c|c|c|c|c|c|c|c|c|c|}
\hline $18 \ldots$ & 20 & 69 & -- & -- & -- & -- & -- & -- & -- & 0.01 \\
\hline APR & & & & & & & & & & \\
\hline $\operatorname{MAY}^{14} \cdots$ & 18 & 47 & -- & -- & -- & -- & -- & -- & -- & .01 \\
\hline JuN & 22 & 57 & -- & -- & -- & -- & -- & -- & -- & $<.01$ \\
\hline JUL & 23 & 56 & -- & -- & -- & -- & -- & -- & - & .01 \\
\hline AUG & 16 & 70 & -- & -- & -- & -- & -- & -- & -- & .01 \\
\hline $\operatorname{SEP}^{24} \cdots$ & 14 & 37 & -- & -- & -- & -- & -- & -- & -- & -- \\
\hline OCT & -- & 62 & -- & -- & -- & -- & -- & -- & -- & -- \\
\hline Nov $20 .$. & 15 & 45 & -- & -- & -- & -- & -- & -- & -- & .01 \\
\hline MAR $17 \dot{982}$ & 13 & 82 & -- & -- & -- & -- & -- & -- & -- & .01 \\
\hline$\underset{A P R}{26 \ldots}$ & 15 & 71 & 49 & -- & -- & -- & -- & -- & -- & -- \\
\hline${ }_{M A Y}^{18} \ldots$ & 14 & 74 & $=-$ & -- & -- & -- & -- & -- & -- & -- \\
\hline Jun & -- & -- & - & -- & -- & -- & - & -- & -- & -- \\
\hline $01 \ldots$ & 15 & 52 & -- & -- & -- & -- & -- & -- & -- & -- \\
\hline
\end{tabular}


IN.T 4.--Physical characteristics and concentrations of commond chemical constituents in lakes and streams of the Bald Mountain watershed--Continued

01012550 CARR POND STREAM AT OUTLET CARR POND ME

\begin{tabular}{|c|c|c|c|c|c|c|c|c|}
\hline ATE & $\begin{array}{l}\text { ALUM- } \\
\text { INUM, } \\
\text { TOTAL } \\
\text { RECOV- } \\
\text { ERABLE } \\
\text { (UG/L }\end{array}$ & $\begin{array}{l}\text { ALUM- } \\
\text { INUM, } \\
\text { DIS- } \\
\text { SOLVED } \\
\text { (UG/L }\end{array}$ & $\begin{array}{l}\text { ARSENIC } \\
\text { TOTAL } \\
\text { (UG/L }\end{array}$ & $\begin{array}{l}\text { CADMIUM } \\
\text { TOTAL } \\
\text { RECOV- } \\
\text { ERABLE } \\
\text { (UG/L }\end{array}$ & $\begin{array}{l}\text { CERO- } \\
\text { MIUM, } \\
\text { TOTAI } \\
\text { RECOV- } \\
\text { ERABLE } \\
\text { (UG/L }\end{array}$ & $\begin{array}{l}\text { COPPER, } \\
\text { TOTAL } \\
\text { RECOV- } \\
\text { ERABLE } \\
\text { (UG /L }\end{array}$ & $\begin{array}{l}\text { COPPER, } \\
\text { DIS- } \\
\text { SOLVED } \\
\text { (UG/L }\end{array}$ & $\begin{array}{l}\text { IRON, } \\
\text { TOTAL } \\
\text { RECOV- } \\
\text { ERABLE } \\
\text { (UG/L }\end{array}$ \\
\hline & AS AL) & AS AL) & AS AS) & AS CD) & AS CR) & AS CU) & AS CU) & AS FE) \\
\hline
\end{tabular}

MAR 1981

$18 \ldots$

APR

$14 \ldots$

MAY

15...

JUN

$10 \ldots$

JUL

$21 .$.

AUG

$24 \ldots$

SEP

22 ...

OCT

$20 .$.

Nov

17 ..

MAR 1982

26...

APR

$18 \ldots$

MAY

$03 .$.

JUN

$01 .$.

\begin{tabular}{|c|c|}
\hline-- & - \\
\hline-- & - \\
\hline-- & -- \\
\hline-- & -- \\
\hline-- & -- \\
\hline$<100$ & - \\
\hline-- & - \\
\hline-- & - \\
\hline-- & -- \\
\hline-- & -- \\
\hline 150 & -- \\
\hline 120 & $<100$ \\
\hline 130 & $<100$ \\
\hline
\end{tabular}

$\begin{array}{ll}- & - \\ - & - \\ - & - \\ -- & - \\ <4 & - \\ -- & - \\ -- & - \\ -- & - \\ -- & - \\ -- & - \\ - & -\end{array}$

$\begin{array}{lll}1 & -- & 60 \\ 1 & -- & 180 \\ <1 & -- & 80 \\ <1 & -- & 70 \\ <2 & -- & 50 \\ 6 & -- & 60 \\ <2 & -- & 70 \\ 1 & -- & 80 \\ -- & -- & -- \\ -- & -- & -- \\ 1 & -1 & 210 \\ <1 & -100 \\ <1 & -1 & 90\end{array}$

IRON, LEAD,

RECOV-

LEAD, TOTAL
DIS-

ZINC,

TOTAL.

RECOV-

ERABLE

ERABLE

SOLVED ERABLE SOLVED

(UG/L

(UG/L)

(UG/L)

(UG/L

AS $\mathbf{2 N}$ )

ZINC,
DIS-
SOLVED
(UG/L
AS ZN)

SEDI -

MENT,

DIS-

AS EE)

AS $P B$ )

AS PB)

AS BG)

MAR 1981

$18 \ldots$

APR

$14 \ldots$

MAY

15...

JUN

10 ...

JUL

21...

AUG

$24 \ldots$

SEP

22 ...

OCI

20 ...

NOV

17 ...

MAR 1982

26 ...

APR

$18 \ldots$

MAY

03...

JUN

$01 .$.

\begin{tabular}{|c|c|c|}
\hline-- & -- & -- \\
\hline-- & -- & -- \\
\hline-- & -- & -- \\
\hline-- & -- & - \\
\hline- & - & - \\
\hline$<4$ & -- & $<0.2$ \\
\hline- & - & - \\
\hline-- & - & - \\
\hline-- & -- & - \\
\hline- & -- & - \\
\hline$<1$ & - & -- \\
\hline$<1$ & $<1$ & - \\
\hline$<1$ & $<1$ & -- \\
\hline
\end{tabular}

$\begin{array}{ccc}-- & 1 & 0.0 \\ -- & -- & - \\ - & 2 & 0.0 \\ - & 6 & 0.0 \\ - & 3 & 0.0 \\ - & -- & -- \\ - & -- & -- \\ -- & -- & -- \\ -- & -- & 0.0 \\ -5 & 1 & 0.0 \\ -5 & 5 & 0.0\end{array}$


Table 4.--Physical characteristics and concentrations of common chemical constituents in lakes and streams of the Bald Mountain watersheds--Continued

01012560 NORTH BRANCH FOX BROOK NR FISH RIVER LK, ME

\begin{tabular}{|c|c|c|c|c|c|c|c|c|c|}
\hline TIME & $\begin{array}{l}\text { STREAM- } \\
\text { FLOW, } \\
\text { INSTAN- } \\
\text { TANEOUS } \\
\text { (CFS) }\end{array}$ & $\begin{array}{l}\text { SPE- } \\
\text { CIFIC } \\
\text { CON- } \\
\text { DUCT- } \\
\text { ANCE } \\
\text { (US/CM) }\end{array}$ & $\begin{array}{c}\text { PH } \\
\text { (STAND- } \\
\text { ARD } \\
\text { UNITS) }\end{array}$ & $\begin{array}{l}\text { TEMPER- } \\
\text { ATURE } \\
\text { WATER } \\
\text { (DEG C) }\end{array}$ & $\begin{array}{l}\text { COLOR } \\
\text { (PLAT- } \\
\text { INUM- } \\
\text { COBALT } \\
\text { UNITS) }\end{array}$ & $\begin{array}{l}\text { TUR- } \\
\text { BID- } \\
\text { ITY } \\
\text { (FTU) }\end{array}$ & $\begin{array}{c}\text { OXYGEN, } \\
\text { DIS- } \\
\text { SOLVED } \\
\text { (MG/L) }\end{array}$ & $\begin{array}{l}\text { OXYGEN } \\
\text { DEMAND, } \\
\text { BIO- } \\
\text { CHEM- } \\
\text { ICAL, } \\
5 \text { DAY } \\
\text { (MG } / L)\end{array}$ & $\begin{array}{l}\text { COLI- } \\
\text { FORM, } \\
\text { FECAL, } \\
0.45 \\
\text { UM-MF } \\
\text { (COLS. ' } \\
\text { 100 ML) }\end{array}$ \\
\hline
\end{tabular}

\begin{tabular}{|c|c|c|c|c|c|c|c|c|c|c|}
\hline \multicolumn{11}{|l|}{ JUN 1979} \\
\hline $11 \ldots$ & 1100 & -- & 43 & 7.2 & 12.0 & -- & 0.4 & 9.6 & 0.6 & K6 \\
\hline $26 \ldots$ & 1100 & -- & 58 & 7.1 & 14.0 & -- & 1.0 & 10.0 & .4 & $K_{4}$ \\
\hline \multicolumn{11}{|l|}{ JUL } \\
\hline $17 \ldots$ & 1130 & -- & -- & 7.2 & 18.0 & -- & 1.0 & 9.8 & .8 & 68 \\
\hline $24 \ldots$ & 1130 & -- & 72 & 7.2 & 20.0 & -- & .5 & 8.9 & .2 & K7 \\
\hline \multicolumn{11}{|l|}{ AUG } \\
\hline $07 \ldots$ & 1130 & -- & 75 & 7.2 & 17.0 & 10 & 1.4 & 8.6 & $<.1$ & 21 \\
\hline $21 \ldots$ & 1100 & -- & 62 & 7.1 & 17.0 & 10 & .6 & 12.6 & .2 & $=$ \\
\hline \multicolumn{11}{|l|}{ SEP } \\
\hline $04 \ldots$ & 1130 & -- & 74 & 7.2 & 18.0 & 5 & .8 & 11.2 & $<.1$ & K2 \\
\hline $18 .$. & 1130 & -- & 54 & 7.2 & 14.0 & 25 & 1.5 & 9.8 & -- & K9 \\
\hline \multicolumn{11}{|l|}{ OCT } \\
\hline $\begin{array}{l}22 \ldots \\
\text { JAN } 1980\end{array}$ & 1030 & -- & 60 & 7.1 & 10.0 & 20 & .7 & 10.5 & .4 & - \\
\hline FEB & 1300 & -- & 62 & 7.0 & 0.0 & -- & 1.6 & -- & -- & -- \\
\hline${ }_{A P R}^{19} \ldots$ & 1300 & -- & 71 & 6.9 & 0.0 & 10 & 1.1 & 13.2 & .4 & -- \\
\hline$\underset{\text { MAY }}{23} \ldots$ & 1100 & -- & 35 & 6.8 & 2.0 & 25 & 2.1 & 13.3 & .6 & -- \\
\hline JUN $20 .$. & 1230 & -- & 35 & 7.1 & 10.0 & 20 & 1.2 & 10.5 & .9 & K1 \\
\hline $\begin{array}{l}17 \ldots \\
\text { APR } 1982\end{array}$ & 1300 & -- & 45 & 6.9 & 14.0 & 20 & 1.1 & 11.0 & $<.1$ & -- \\
\hline $22 \ldots$ & 1200 & -- & -- & -- & $\cdots$ & -- & -- & -- & -- & -- \\
\hline $03 \ldots$ & 1135 & -- & -- & -- & -- & -- & -- & -- & -- & -- \\
\hline
\end{tabular}

\begin{tabular}{|c|c|c|c|c|c|c|c|c|c|}
\hline $\begin{array}{l}\text { ALRA- } \\
\text { LINITY }\end{array}$ & SOLIDS, & $\begin{array}{l}\text { SOLIDS, } \\
\text { VOLA- }\end{array}$ & NITRO- & NITRO- & NITRO- & $\begin{array}{l}\text { NITRO- } \\
\text { GEN , AM- }\end{array}$ & & & \\
\hline $\begin{array}{l}\text { WAT WB } \\
\text { TOT FET } \\
\text { LAB } \\
\text { MG/L AS } \\
\text { CACO3 }\end{array}$ & $\begin{array}{l}\text { RESIDUE } \\
\text { AT } 105 \\
\text { DEG. C, } \\
\text { TOTAL } \\
\text { (MG/L) }\end{array}$ & $\begin{array}{l}\text { TILE ON } \\
\text { IGNI- } \\
\text { TION, } \\
\text { TOTAL } \\
\text { (MG/L) }\end{array}$ & $\begin{array}{c}\text { GEN, } \\
\text { NO2+NO3 } \\
\text { TOTAL } \\
\text { (MG/L } \\
\text { AS N) }\end{array}$ & $\begin{array}{l}\text { GEN, } \\
\text { AMPONIA } \\
\text { TOTAL } \\
\text { (MG/L } \\
\text { AS N) }\end{array}$ & $\begin{array}{l}\text { GEN, } \\
\text { ORGANIC } \\
\text { TOTAL } \\
\text { (MG/L } \\
\text { AS N) }\end{array}$ & $\begin{array}{l}\text { MONIA + } \\
\text { ORGANIC } \\
\text { TOTAL } \\
\text { (MG/L } \\
\text { AS N) }\end{array}$ & $\begin{array}{l}\text { NITRO- } \\
\text { GEN, } \\
\text { TOTAL } \\
\text { (MG/L } \\
\text { AS N) }\end{array}$ & $\begin{array}{l}\text { NITRO- } \\
\text { GEN, } \\
\text { TOTAL } \\
\text { (MG/L } \\
\text { AS NO3) }\end{array}$ & $\begin{array}{l}\text { PHOS- } \\
\text { PHOROUS } \\
\text { TOTAL } \\
\text { (MG / L } \\
\text { AS P) }\end{array}$ \\
\hline
\end{tabular}

JUN 1979

\begin{tabular}{|c|c|c|c|c|c|c|c|c|c|c|}
\hline $11 \ldots$ & 13 & -- & -- & 0.01 & $<0.01$ & -- & -- & -- & -- & 0.03 \\
\hline $26 \ldots$ & 17 & -- & -- & .01 & $<.01$ & -- & -- & -- & -- & -- \\
\hline \multicolumn{11}{|l|}{ JUL } \\
\hline $17 \ldots$ & 24 & -- & - & .10 & $<.01$ & -- & $<0.10$ & -- & -- & $<.01$ \\
\hline $24 \ldots$ & 26 & -- & -- & .13 & $<.01$ & -- & -- & -- & -- & -- \\
\hline \multicolumn{11}{|l|}{ AUG } \\
\hline $07 \ldots$ & 26 & -- & -- & .16 & .01 & -- & -- & -- & -- & -- \\
\hline $21 \ldots$ & 28 & -- & - & .13 & $<.01$ & -- & -- & -- & - & -- \\
\hline \multicolumn{11}{|l|}{ SEP } \\
\hline $04 \ldots$ & 20 & -- & -- & .12 & $<.01$ & -- & -- & $\cdots$ & -- & -- \\
\hline $18 \ldots$ & 27 & -- & -- & .04 & $<.01$ & $-\cdot$ & -- & $\cdots$ & -- & -- \\
\hline \multicolumn{11}{|l|}{ OCT } \\
\hline $\begin{array}{l}22 \ldots \\
\text { JAN } 1980\end{array}$ & 20 & -- & -- & .05 & .01 & -- & -- & -- & $\cdots$ & -- \\
\hline$\underset{\text { FEB }}{29 \ldots}$ & -- & 54 & \multicolumn{6}{|c|}{ FEB } & & .10 \\
\hline $19 \ldots$ & 24 & 55 & -- & .24 & -- & -- & .10 & 0.34 & 1.5 & .01 \\
\hline \multicolumn{11}{|l|}{ APR } \\
\hline $23 \ldots$ & 9 & 33 & $-\infty$ & .13 & .02 & 0.08 & .10 & .23 & 1.0 & .01 \\
\hline \multicolumn{11}{|l|}{ MAY } \\
\hline \multicolumn{9}{|l|}{ JUN } & 0.93 & .01 \\
\hline $\begin{array}{c}17 \ldots \\
A P R \\
1982\end{array}$ & 19 & 84 & -- & .13 & .01 & -- & $<.10$ & -- & -- & $<.01$ \\
\hline $22 \ldots$ & -- & 32 & -- & -- & -- & -- & -- & -- & -- & -- \\
\hline MAY & & & & & & & & & & \\
\hline $03 \ldots$ & -- & -- & -- & -- & -- & -- & -- & -- & -- & -- \\
\hline
\end{tabular}


Iable 4.--Physical characteristics and concentrations of common chemical constituents in lakes and streams of the Bald Mountain watersheds--Continued

01012560 NORTE BRANCE FOX BROOK NR FISE RIVER LK, ME

DATE

JUN 1979

$$
11 \ldots
$$

26

$17 \ldots$

$24 \ldots$

AUG

$07 .$.

21 .

SEP

$04 \ldots$.

$18 \ldots$

OCT

22 ...

JAN 1980

29 ...

FEB

$19 \ldots$

APR

$23 . .$.

MAY

20...

JUN $17 \ldots$

APR 1982

22 . .

MAY

$03 .$.

DATE

JUN 1979

$11 \ldots$

JUL.

$17 .$.

$24 \ldots$

AUG

$07 .$.

$21 \ldots$

SEP

$04 \ldots$

$18 \ldots$

OCT

22 ...

JAN 1980

29 ...

FEB

$19 .$.

APR

23...

MAY

20 . . .

JUN

$17 .$.

APR 1982

22. . .

MAY

$03 .$.

$\begin{array}{lllll}\text { ALUM- } & & & & \text { CHRO- } \\ \text { INUM, } & \text { ALUM- } & & \text { CADMIUM } & \text { MIUM, } \\ \text { TOTAL } & \text { INUM, } & & \text { TOTAL } & \text { TOTAL } \\ \text { RECOV- } & \text { DIS- } & \text { ARSENIC } & \text { RECOV- } & \text { RECOV- } \\ \text { ERABLE } & \text { SOLVED } & \text { TOTAL } & \text { ERABLE } & \text { ERABLE } \\ \text { (UG/L } & \text { (UG/L } & \text { (UG/L } & \text { (UG/L } & \text { (UG/L } \\ \text { AS AL) } & \text { AS AL) } & \text { AS AS) } & \text { AS CD) } & \text { AS CR) }\end{array}$

$\begin{array}{lll}\text { COPPER, } & & \text { IRON, } \\ \text { TOTAL } & \text { COPPER, } & \text { TOTAL } \\ \text { RECOV- } & \text { DIS- } & \text { RECOV- } \\ \text { ERABLE } & \text { SOLVED } & \text { ERABLE } \\ \text { (UG/L } & \text { (UG/L } & \text { (UG/L } \\ \text { AS CU) } & \text { AS CU) } & \text { AS FE) }\end{array}$

\begin{tabular}{|c|c|c|c|c|}
\hline$<2$ & $<6$ & $<4$ & -- & -- \\
\hline-- & -- & -- & -- & -- \\
\hline$<2$ & $<6$ & $<4$ & -- & 150 \\
\hline$<2$ & $<6$ & $<4$ & -- & 40 \\
\hline-- & $<6$ & $<4$ & -- & 30 \\
\hline-- & -- & -- & -- & -- \\
\hline-- & -- & - & -- & -- \\
\hline-- & -- & -- & - & -- \\
\hline$<2$ & $<6$ & 66 & -- & 320 \\
\hline$<2$ & $<6$ & $<4$ & - & - \\
\hline$<2$ & $<6$ & 10 & -- & 80 \\
\hline$<2$ & $<5$ & $<10$ & -- & $\cdots$ \\
\hline$<2$ & $<20$ & $<4$ & -- & -- \\
\hline$<2$ & $<5$ & $<2$ & -- & 130 \\
\hline- & -- & 2 & -- & 1100 \\
\hline & -- & $<1$ & $<1$ & 100 \\
\hline
\end{tabular}

SEDI -

MENT

DIS-

CHARGE,

SUS-

PENDED

( $T / D A Y$ )

$\begin{array}{clll} & \text { LEAD, } & & \text { MERCURY } \\ \text { IRON, } & \text { TOTAL } & \text { LEAD, } & \text { TOTAL } \\ \text { DIS- } & \text { RECOV- } & \text { DIS- } & \text { RECOV- } \\ \text { SOLVED } & \text { ERABLE } & \text { SOLVED } & \text { ERABLE } \\ \text { (UG/L } & \text { (UG/L } & \text { (UG/L } & \text { (UG/L } \\ \text { AS FE) } & \text { AS PB) } & \text { AS PB) } & \text { AS HG) }\end{array}$

RECOV- DIS-

ERABLE

(UG/L

As $2 N$ )
(UG/L

AS $2 N$ )
MENT

SUS-

(MG/L)
0.0

0.0

0.0

0.0

0.0

0.0

0.0

$\begin{array}{rrr}-- & 3 & 0.0\end{array}$

$<.2$

$<.2$

.2

$--$

$-$

$<.2$

$<8$

$<5$

$<5$
0.0

0.0

0.0

0.0 
Imble 4.--Physical characteristics and concentrations of common chamical constituents in lakes and streams of the Bald Mountain watersheds--Continued

01012560 NORTA BRANCB FOX BROOK NR FISA RIVER LK, ME

\begin{tabular}{|c|c|c|c|c|c|c|c|c|c|c|}
\hline DATE & TIME & $\begin{array}{l}\text { STREAM- } \\
\text { FLOW, } \\
\text { INSTAN- } \\
\text { TANEOUS } \\
\text { (CFS) }\end{array}$ & $\begin{array}{l}\text { SPE- } \\
\text { CIFIC } \\
\text { CON- } \\
\text { DUCT- } \\
\text { ANCE } \\
\text { (US/CM) }\end{array}$ & $\begin{array}{c}\text { PE } \\
\text { (STAND- } \\
\text { ARD } \\
\text { UNITS) }\end{array}$ & $\begin{array}{c}\text { TEMPER- } \\
\text { ATURE } \\
\text { WATER } \\
\text { (DEG C) }\end{array}$ & $\begin{array}{l}\text { COLOR } \\
\text { (PLAT- } \\
\text { INUY- } \\
\text { COBALT } \\
\text { UNITS) }\end{array}$ & $\begin{array}{l}\text { TUR- } \\
\text { BID- } \\
\text { ITY } \\
\text { (FTU) }\end{array}$ & $\begin{array}{c}\text { OXYGEN, } \\
\text { DIS- } \\
\text { SOLVED } \\
\text { (MG/L) }\end{array}$ & $\begin{array}{c}\text { OXYGEN } \\
\text { DEMAND, } \\
\text { BIO- } \\
\text { CHEM- } \\
\text { ICAL, } \\
5 \text { DAY } \\
\text { (MG/L) }\end{array}$ & $\begin{array}{l}\text { COLI- } \\
\text { FORM, } \\
\text { FECAL, } \\
0.45 \\
\text { UM-MF } \\
\text { (COLS. I } \\
100 \mathrm{ML} \text { ) }\end{array}$ \\
\hline $\begin{array}{c}\text { IUN } 1982 \\
01 \ldots\end{array}$ & 1645 & -- & 52 & 7.1 & 16.5 & 25 & 0.5 & 9.1 & - & -- \\
\hline DATE & $\begin{array}{l}\text { ALRA- } \\
\text { LINITY } \\
\text { WAT WH } \\
\text { TOT FET } \\
\text { LAB } \\
\text { MG/L AS } \\
\text { CACO3 }\end{array}$ & $\begin{array}{l}\text { SOLIDS, } \\
\text { RESIDUE } \\
\text { AT } 105 \\
\text { DEG. C, } \\
\text { TOTAL } \\
\text { (MG/L) }\end{array}$ & $\begin{array}{l}\text { SOLIDS, } \\
\text { VOLA- } \\
\text { TILE ON } \\
\text { IGNI- } \\
\text { TION, } \\
\text { TOTAL } \\
\text { (MG/L) }\end{array}$ & $\begin{array}{c}\text { NITRO- } \\
\text { GEN, } \\
\text { NO2+NO3 } \\
\text { TOTAL } \\
\text { (MG/L } \\
\text { AS N) }\end{array}$ & $\begin{array}{l}\text { NITRO- } \\
\text { GEN, } \\
\text { AMOONIA } \\
\text { TOTAL } \\
\text { (MG/L } \\
\text { AS N) }\end{array}$ & $\begin{array}{l}\text { NITRO- } \\
\text { GEN, } \\
\text { ORGANIC } \\
\text { TOTAL } \\
\text { (MG/L } \\
\text { AS N) }\end{array}$ & $\begin{array}{l}\text { NITRO- } \\
\text { GEN, AM- } \\
\text { MONIA + } \\
\text { ORGANIC } \\
\text { TOTAL } \\
\text { (MG /L } \\
\text { AS N) }\end{array}$ & $\begin{array}{l}\text { NITRO- } \\
\text { GEN, } \\
\text { TOTAL } \\
\text { (MG/L } \\
\text { AS N) }\end{array}$ & $\begin{array}{l}\text { NITRO- } \\
\text { GEN, } \\
\text { TOTAL } \\
\text { (MG/L } \\
\text { AS NO3) }\end{array}$ & $\begin{array}{l}\text { PHOS- } \\
\text { PHOROUS } \\
\text { TOTAL } \\
\text { (MG/L } \\
\text { AS P) }\end{array}$ \\
\hline
\end{tabular}

JUN 1982

$01 .$. .

17

49 
Table 4.--Physical characteristics and concentrations of common chemical constituents in lakes and streams of the Bald Mountain watersheds--Continued

01012560 NORTE BRANCE FOX BROOK NR FISE RIVER LR, ME

DATE

JUN 1982

$01 \ldots$

ATE

JUN 1982

$01 \ldots$
ALUMINUM, ALUMTOTAL RECOV ERABLE (UG/L AS AL)

120

$<100$ INUM, DISVED TOTAL (UG/L (UG/L AS. AL)

AS AS )

$\begin{array}{cl}\text { CADMIUM } & \text { CHRO- } \\ \text { MOTUL, } & \text { TOTAL } \\ \text { RECOV- } & \text { RECOV- } \\ \text { ERABLE } & \text { ERABLE } \\ \text { (UG/L } & \text { (UG/L } \\ \text { AS CD) } & \text { AS CR) }\end{array}$

$--$

$-$

COPPER, TOTAL COPPER RECOV- DIS- RECOVERABLE SOLVED ERABLE (UG/L (UG/L (UG/L AS CU) AS CU) AS FE)
$<1$

60

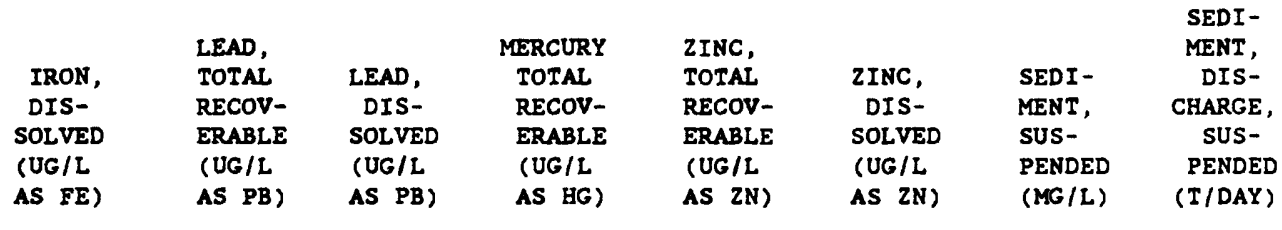

$<5$

$<5$ 
The 4.--Physical characteristics and concentrations of commond chemical constituents in lakes and streams of the Bald Mountain watershed--Continued

01012570 FISH RIVER AT INLET FISE RIVER LARE ME

\begin{tabular}{|c|c|c|c|c|c|c|c|c|c|c|}
\hline DATE & TIME & $\begin{array}{l}\text { STREAY- } \\
\text { FLOW, } \\
\text { INSTAN- } \\
\text { TANEOUS } \\
\text { (CFS) }\end{array}$ & $\begin{array}{l}\text { SPE- } \\
\text { CIFIC } \\
\text { CON- } \\
\text { DUCT- } \\
\text { ANCE } \\
\text { (US/CM) }\end{array}$ & $\begin{array}{c}\text { PE } \\
\text { (STAND- } \\
\text { ARD } \\
\text { UNITS) }\end{array}$ & $\begin{array}{l}\text { TEMPER- } \\
\text { ATURE } \\
\text { WATER } \\
\text { (DEG C) }\end{array}$ & $\begin{array}{l}\text { COLOR } \\
\text { (PLAT- } \\
\text { INUM- } \\
\text { COBALT } \\
\text { UNITS) }\end{array}$ & $\begin{array}{l}\text { TUR- } \\
\text { BID- } \\
\text { ITY } \\
\text { (FTU) }\end{array}$ & $\begin{array}{c}\text { OXYGEN, } \\
\text { DIS- } \\
\text { SOLVED } \\
\text { (MG/L) }\end{array}$ & $\begin{array}{l}\text { OXYGEN } \\
\text { DEMAND, } \\
\text { BIO- } \\
\text { CEEM- } \\
\text { ICAL, } \\
\text { S DAY } \\
\text { (MG/L) }\end{array}$ & $\begin{array}{l}\text { COLI- } \\
\text { FORM, } \\
\text { FECAL, } \\
0.45 \\
\text { UM-MF } \\
\text { (COLS. / } \\
100 \mathrm{ML} \text { ) }\end{array}$ \\
\hline
\end{tabular}

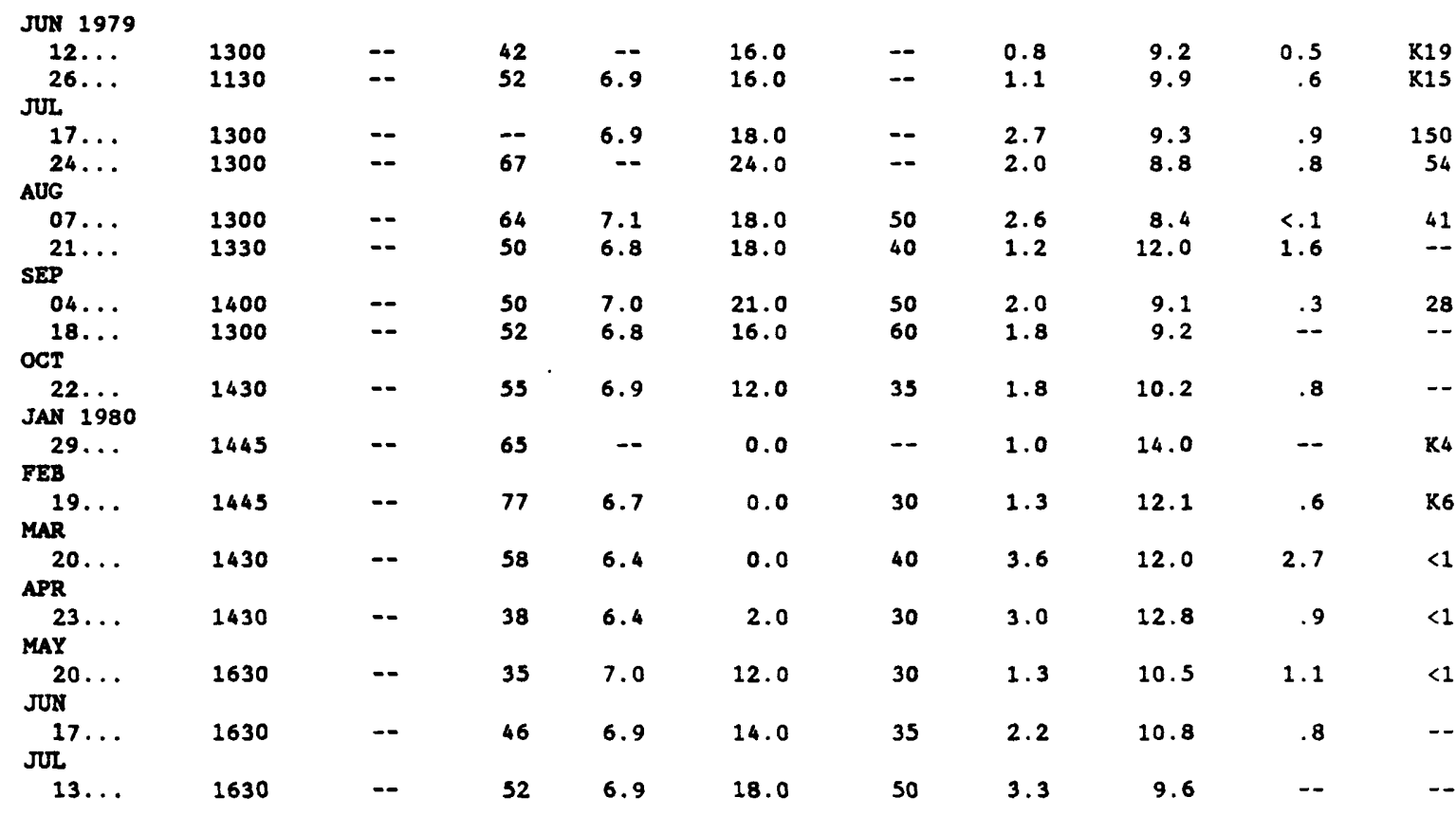

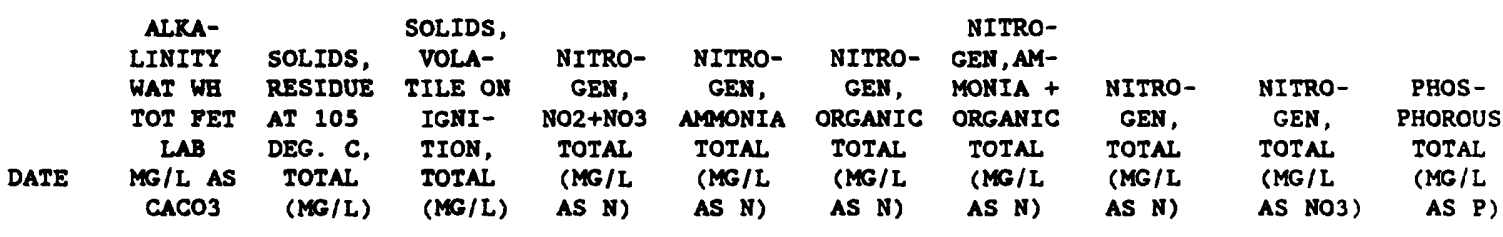

JUR 1979

$12 \ldots$

JuL.

17 ...

24...

AUG

$07 .$.

21...

SEP

$04 \ldots$

$18 \ldots$

OCT

22...

JAN 1980

29...

FEB
$19 .$.

MAR

20...

APR

$23 . .$.

MAY

20...

JUN

17 ...

$13 \ldots$

\begin{tabular}{|c|c|c|c|c|c|c|c|c|c|}
\hline 10 & -- & -- & 0.02 & $<0.01$ & -- & -- & -- & -- & 0.01 \\
\hline 15 & -- & -- & $<.01$ & $<.01$ & -- & -- & -- & -- & -- \\
\hline 19 & -- & -- & .14 & .01 & 0.09 & 0.1 & 0.24 & 1.1 & .01 \\
\hline 22 & -- & -- & .03 & .01 & -- & -- & -- & -- & -- \\
\hline 21 & -- & - & .08 & .02 & -- & - & -- & -- & -- \\
\hline 20 & -- & - & .05 & .01 & -- & -- & -- & -- & -- \\
\hline 17 & -- & -- & .02 & $<.01$ & -- & -- & -- & -- & -- \\
\hline 23 & -- & -- & .03 & $<.01$ & -- & - & -- & -- & -- \\
\hline 9 & -- & -- & .04 & .01 & -- & -- & -- & -- & -- \\
\hline-- & 16 & -- & -- & -- & -- & -- & -- & -- & .01 \\
\hline 22 & 73 & -- & .18 & -- & -- & .1 & .28 & 1.2 & $<.01$ \\
\hline 2 & 50 & -- & .45 & .03 & .17 & .2 & .63 & 2.9 & .01 \\
\hline 9 & -- & -- & .18 & .01 & .19 & .2 & .38 & 1.7 & .01 \\
\hline 9 & 47 & -- & .21 & .02 & .28 & .3 & .51 & 2.3 & .01 \\
\hline 21 & 71 & -- & .12 & $<.01$ & -- & .1 & .22 & 0.97 & .01 \\
\hline 17 & -- & - & -- & -- & -- & -- & -- & -- & .01 \\
\hline
\end{tabular}


TABIE 4.--Physical characteristics and concentrations of commond chemical constituents in lakes and streams of the Bald Mountain watershed--Continued

01012570 FISH RIVER AT INLET FISH RIVER LAKE ME

\begin{tabular}{|c|c|c|c|c|c|c|c|}
\hline $\begin{array}{l}\text { ALUM- } \\
\text { INUM, } \\
\text { TOTAL }\end{array}$ & $\begin{array}{l}\text { ALUM- } \\
\text { INUM, }\end{array}$ & & $\begin{array}{l}\text { CADMIUM } \\
\text { TOTAL }\end{array}$ & $\begin{array}{l}\text { CHRO- } \\
\text { MIUM, } \\
\text { TOTAL }\end{array}$ & $\begin{array}{l}\text { COPPER, } \\
\text { TOTAL }\end{array}$ & COPPER, & $\begin{array}{l}\text { IRON, } \\
\text { TOTAL }\end{array}$ \\
\hline $\begin{array}{l}\text { RECOV- } \\
\text { ERABLE } \\
\text { (UG/L }\end{array}$ & $\begin{array}{l}\text { DIS- } \\
\text { SOLVED } \\
\text { (UG/L }\end{array}$ & $\begin{array}{l}\text { ARSENIC } \\
\text { TOTAL } \\
\text { (UG/L }\end{array}$ & $\begin{array}{l}\text { RECOV- } \\
\text { ERABLE } \\
\text { (UG/L }\end{array}$ & $\begin{array}{l}\text { RECOV- } \\
\text { ERABLE } \\
\text { (UG/L }\end{array}$ & $\begin{array}{l}\text { RECOV- } \\
\text { ERABLE } \\
\text { (UG/L }\end{array}$ & $\begin{array}{l}\text { DIS- } \\
\text { SOLVED } \\
\text { (UG/L }\end{array}$ & $\begin{array}{l}\text { RECOV- } \\
\text { ERABLE } \\
\text { (UG / L }\end{array}$ \\
\hline AS AL) & As $\mathbf{A L})$ & AS AS) & AS CD) & AS CR) & AS CU) & AS CU) & AS FE) \\
\hline
\end{tabular}

JUN 1979

$12 \ldots$

26 ...

JUL

17 ...

$24 \ldots$

AUG

$07 \ldots$

21 ...

SEP

$04 \ldots$

$18 \ldots$

OCT

22 ...

JAN 1980

29...

FEB

19...

MAR

20 ...

APR

23 ...

MAY

20 ...

JUN

$17 \ldots$

JuL

$13 .$.

$\begin{array}{llll}-- & -- & -- & <2 \\ -- & -- & -- & <2 \\ -- & -- & -- & <2 \\ -- & -- & -- & -2 \\ -- & -- & -- & -- \\ -- & -- & -- & -- \\ -- & -- & -- & <2 \\ -- & -- & -- & <2 \\ -- & -- & -- & <2 \\ -- & -- & -- & -- \\ -- & -- & -- & <2 \\ -- & -- & -- & <2 \\ -- & -- & -- & -- \\ -- & -- & -- & \end{array}$

$\begin{array}{lccc}<6 & <4 & -- & -- \\ -- & -- & -- & -- \\ <6 & <4 & -- & 250 \\ <6 & <4 & -- & 210 \\ <6 & <4 & -- & 220 \\ -- & -- & -- & -- \\ -- & -- & -- & -- \\ -- & -- & -- & -- \\ <6 & <4 & -- & 130 \\ <6 & <4 & -- & -- \\ <6 & 6 & -- & 160 \\ -- & <4 & -- & -- \\ <5 & <10 & -- & -- \\ <20 & 4 & -- & -- \\ <5 & 2 & -- & 230 \\ <5 & <2 & -- & 250\end{array}$

$\begin{array}{lll} & \text { LEAD, } & \\ \text { IRON, } & \text { TOTAL } & \text { LEAD, } \\ \text { DIS- } & \text { RECOV- } & \text { DIS- } \\ \text { SOLVED } & \text { ERABLE } & \text { SOLVED } \\ \text { (UG/L } & \text { (UG/L } & \text { (UG/L } \\ \text { AS FE) } & \text { AS PB) } & \text { AS PB) }\end{array}$

MERCURY

TOTAL

2INC,

TOTAL

ERABLE ERABLE

(UG/L

(UG/L

AS HG)

AS ZN)

ZINC,
DIS-
SOLVED
(UG/L
AS ZN)

SEDI -

MENT,

DIS-

CHARGE,

SUS -

PENDED

JUN 1979

$12 \ldots$
$26 \ldots$

JUL

$17 \ldots$

$24 \ldots$

AUG

$07 . .$.

21 ...

SEP

$04 \ldots$

$18 \ldots$

OCT

22...

JAN 1980

$29 .$. .

FEB

$19 .$.

MAR

20 ...

APR

23...

$<20$

$--\quad<2$

$+2$

19

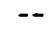

PENDED

( $T / D A Y$ )

MAY

20 ...

$->\quad<20$

$<20 \quad \ldots \quad 0.3$

$+-\quad<20$

$\begin{array}{ll}-- & 0.3 \\ -- & <.2\end{array}$

$--\quad<20$

$<20$

$--$

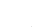

JUN

$17 \ldots$

JUL.

13...

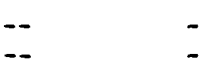

$-$

$<20 \quad--\quad<.2$

30

$--\quad<.2$

$<20$

$--\quad<.2$

$<20$

$-$

$<30$

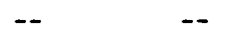

$<30$

$-$

$<2$

$<.2$

20

2

0.0

0.0

0.0

0.0

0.0

0.0

$<30$

$--$

- 
TABIS 4.--Physical characteristics and concentrations of commond chemical constituents in lakes and streams of the Bald Mountain watershed--Continued

01012570 FISE RIVER AT INLET FISH RIVER LARE ME

\begin{tabular}{|c|c|c|c|c|c|c|c|c|c|c|}
\hline DATE & TIME & $\begin{array}{l}\text { STREAM- } \\
\text { FLOW, } \\
\text { INSTAN- } \\
\text { TANEOUS } \\
\text { (CFS) }\end{array}$ & $\begin{array}{l}\text { SPE- } \\
\text { CIFIC } \\
\text { CON- } \\
\text { DUCT- } \\
\text { ANCE } \\
\text { (US/CM) }\end{array}$ & $\begin{array}{c}\text { PB } \\
\text { (STAND- } \\
\text { ARD } \\
\text { UNITS) }\end{array}$ & $\begin{array}{c}\text { TEMPER- } \\
\text { ATURE } \\
\text { WATER } \\
\text { (DEG C) }\end{array}$ & $\begin{array}{l}\text { COLOR } \\
\text { (PLAT- } \\
\text { INUM- } \\
\text { COBALT } \\
\text { UNITS) }\end{array}$ & $\begin{array}{l}\text { TUR- } \\
\text { BID- } \\
\text { ITY } \\
\text { (FTU) }\end{array}$ & $\begin{array}{c}\text { OXYGEN, } \\
\text { DIS- } \\
\text { SOLVED } \\
\text { (MG/L) }\end{array}$ & $\begin{array}{l}\text { OXYGEN } \\
\text { DEMAND, } \\
\text { BIO- } \\
\text { CHEM- } \\
\text { ICAL, } \\
5 \text { DAY } \\
\text { (MG/L) }\end{array}$ & $\begin{array}{l}\text { COLI- } \\
\text { FORM, } \\
\text { FECAL, } \\
0.45 \\
\text { UM-MF } \\
\text { (COLS. } \\
100 \mathrm{ML} \text { ) }\end{array}$ \\
\hline
\end{tabular}

\begin{tabular}{|c|c|c|c|c|c|c|c|c|c|c|}
\hline $\begin{array}{l}\text { AUG } 1980 \\
29 \ldots \\
\text { SEP }\end{array}$ & 1600 & -- & 60 & 7.0 & 22.0 & 45 & 3.2 & 8.5 & -- & -- \\
\hline $\mathrm{OCT}^{29 \ldots}$ & 1600 & -- & 50 & 6.7 & 6.0 & 30 & 1.7 & 11.3 & -- & -- \\
\hline $\operatorname{Nov}^{14} \ldots$ & 1600 & -- & 70 & 6.7 & 5.0 & 40 & 1.1 & 11.4 & -- & -- \\
\hline DEC $21 \ldots$ & 1600 & -- & 47 & 6.4 & 0.0 & 35 & 1.5 & 12.8 & -- & -- \\
\hline $\begin{array}{c}12 \ldots \\
\text { JAN } 1981\end{array}$ & 1630 & -- & 78 & 6.6 & 0.0 & 42 & 1.2 & 12.9 & -- & -- \\
\hline FEB $20 \ldots$ & 1545 & -- & 80 & 6.7 & 0.0 & 30 & 2.6 & 13.5 & -- & -- \\
\hline $\operatorname{MAR}^{23} \ldots$ & 1645 & -- & 30 & 6.3 & 2.0 & 65 & 3.5 & 12.5 & -- & -- \\
\hline${ }_{A P R}^{18 \ldots}$ & 1300 & -- & 73 & 6.5 & 1.0 & 20 & 1.1 & 12.4 & -- & -- \\
\hline $\operatorname{MAY}^{14 \ldots}$ & 1630 & -- & 30 & 6.5 & -- & 40 & 2.0 & -- & -- & -- \\
\hline JUN & 1605 & -. & 30 & 6.7 & 11.0 & 45 & 1.8 & 10.3 & -- & -- \\
\hline $\mathrm{JUL}^{10 \ldots}$ & 1100 & -- & 40 & 6.7 & 10.0 & 30 & 2.4 & 10.3 & -- & -- \\
\hline AUG & 1100 & -- & 69 & 7.0 & 16.0 & 35 & 2.6 & -- & -- & -- \\
\hline$\underset{\operatorname{SEP}}{24} \ldots$ & 1730 & -- & 46 & 6.3 & 18.0 & 70 & 1.5 & 8.2 & -- & -- \\
\hline $22 \ldots$ & 1110 & -- & 30 & 7.2 & 11.0 & 60 & 1.7 & 10.0 & -- & -- \\
\hline DATE & $\begin{array}{l}\text { ALKA- } \\
\text { LINITY } \\
\text { WAT WB } \\
\text { TOT FET } \\
\text { LAB } \\
\text { MG/L AS } \\
\text { CACO3 }\end{array}$ & $\begin{array}{l}\text { SOLIDS, } \\
\text { RESIDUE } \\
\text { AT } 105 \\
\text { DEG. C, } \\
\text { TOTAL } \\
\text { (MG/L) }\end{array}$ & $\begin{array}{l}\text { SOLIDS, } \\
\text { VOLA- } \\
\text { TILE ON } \\
\text { IGNI- } \\
\text { TION, } \\
\text { TOTAL } \\
\text { (MG/L) }\end{array}$ & $\begin{array}{l}\text { NITRO- } \\
\text { GEN, } \\
\text { NO2+NO3 } \\
\text { TOTAL } \\
\text { (MG/L } \\
\text { AS N) }\end{array}$ & $\begin{array}{l}\text { NITRO- } \\
\text { GEN, } \\
\text { AMONIA } \\
\text { TOTAL } \\
\text { (MG/L } \\
\text { AS N) }\end{array}$ & $\begin{array}{l}\text { NITRO- } \\
\text { GEN, } \\
\text { ORGANIC } \\
\text { TOTAL } \\
\text { (MG/L } \\
\text { AS N) }\end{array}$ & $\begin{array}{l}\text { NITRO- } \\
\text { GEN, AM- } \\
\text { MONIA + } \\
\text { ORGANIC } \\
\text { TOTAL } \\
\text { (MG/L } \\
\text { AS N) }\end{array}$ & $\begin{array}{l}\text { NITRO- } \\
\text { GEN, } \\
\text { TOTAL } \\
\text { (MG/L } \\
\text { AS N) }\end{array}$ & $\begin{array}{l}\text { NITRO- } \\
\text { GEN, } \\
\text { TOTAL } \\
\text { (MG/L } \\
\text { AS NO3) }\end{array}$ & $\begin{array}{l}\text { PHOS- } \\
\text { PHOROUS } \\
\text { TOTAL } \\
\text { (MG } / L \\
\text { AS P) }\end{array}$ \\
\hline
\end{tabular}

\begin{tabular}{|c|c|c|c|c|c|c|c|c|c|c|}
\hline $\begin{array}{c}\text { AUG } 1980 \\
29 \ldots \\
\text { SEP }\end{array}$ & 21 & 85 & -- & -- & -- & -- & -- & -- & -- & 0.01 \\
\hline${ }_{\text {OCT }}^{29 . \ldots}$ & 18 & 60 & -- & -- & -- & -- & -- & -- & -- & .02 \\
\hline Nov $14 \ldots$ & 20 & 61 & -- & -- & -- & -- & -- & -- & -- & .01 \\
\hline${ }_{D E C}^{21} \ldots$ & 17 & 63 & -- & -- & -- & -- & -- & -- & -- & .01 \\
\hline JAN 12 igi & 19 & 51 & -- & -- & -- & -- & -- & -- & -- & .01 \\
\hline$\underset{\text { FEB }}{20 \ldots}$ & 28 & -- & -- & -- & -- & -- & -- & -- & -- & .02 \\
\hline $\operatorname{MAR}^{23} \cdots$ & 9 & -- & -- & -- & -- & -- & -- & -- & -- & .01 \\
\hline${ }_{A P R}^{18 \ldots}$ & 16 & 74 & -- & -- & -- & -- & -- & -- & -- & .01 \\
\hline $\operatorname{MAY}_{\operatorname{MA}}^{14 \ldots}$ & 14 & 35 & -- & -- & -- & -- & -- & -- & -- & .01 \\
\hline $\operatorname{JuN}^{15 \ldots}$ & 15 & 56 & -- & -- & -- & -- & -- & -- & -- & .02 \\
\hline JUL $10 \ldots$ & 18 & 59 & -- & -- & -- & -- & -- & -- & -- & -- \\
\hline${ }_{A U C}^{21} \ldots$ & 31 & 58 & -- & -- & -- & -- & -- & -- & -- & -- \\
\hline $\operatorname{SEP}_{\operatorname{SEP}}^{24 \ldots}$ & 12 & 51 & -- & -- & -- & -- & -- & -- & -- & .01 \\
\hline $22 \ldots$ & 15 & 56 & -- & -- & -- & -- & -- & -- & -- & -- \\
\hline
\end{tabular}


TABLE 4.--Physical characteristics and concentrations of comond chemical constituents in lakes and streams of the Bald Mountain watershed--Continued

01012570 FISE RIVER AT INLET FISE RIVER LAKE ME

\begin{tabular}{|c|c|c|c|c|c|c|c|c|}
\hline TE & $\begin{array}{l}\text { ALUM- } \\
\text { INUM, } \\
\text { TOTAL } \\
\text { RECOV- } \\
\text { ERABLE } \\
\text { (UG/L }\end{array}$ & $\begin{array}{l}\text { ALUM- } \\
\text { INUM, } \\
\text { DIS- } \\
\text { SOLVED } \\
\text { (UG/L }\end{array}$ & $\begin{array}{l}\text { ARSENIC } \\
\text { TOTAL } \\
\text { (UG/L }\end{array}$ & $\begin{array}{l}\text { CADMIUM } \\
\text { TOTAL } \\
\text { RECOV- } \\
\text { ERABLE } \\
\text { (UG/L }\end{array}$ & $\begin{array}{l}\text { CERO- } \\
\text { MIUM, } \\
\text { TOTAL } \\
\text { RECOV- } \\
\text { ERABLE } \\
\text { (UG/L }\end{array}$ & $\begin{array}{l}\text { COPPER, } \\
\text { TOTAL } \\
\text { RECOV- } \\
\text { ERABLE } \\
\text { (UG/L }\end{array}$ & $\begin{array}{l}\text { COPPER, } \\
\text { DIS- } \\
\text { SOLVED } \\
\text { (UG/L }\end{array}$ & $\begin{array}{l}\text { IRON, } \\
\text { TOTAL } \\
\text { RECOV- } \\
\text { ERABLE } \\
\text { (UG/L }\end{array}$ \\
\hline & AS $A L)$ & AS AL) & AS AS) & AS CD) & AS CR) & AS CU) & AS CU) & AS FE) \\
\hline
\end{tabular}

AUG 1980

29. .

SEP

29...

OCT

$14 \ldots$

Nov

DEC

12 ...

JAN 1981

$20 \ldots$

FEB
$23 .$.

MAR

18 ...

APR

$14 \ldots$

MAY

15...

JUN

10 ...

JUL

$21 \ldots$

AUG

$24 \ldots$

SEP

22 ..

\begin{tabular}{|c|c|c|c|}
\hline-- & -- & -- & -- \\
\hline -- & -- & -- & -- \\
\hline -- & -- & $<4$ & - \\
\hline -- & -- & -- & -- \\
\hline -- & -- & -- & -- \\
\hline -- & -- & -- & -- \\
\hline -- & -- & -- & -- \\
\hline -- & -- & -- & -- \\
\hline -- & -- & -. & -- \\
\hline -- & -- & -- & -- \\
\hline -- & -- & -- & -- \\
\hline -- & -- & -- & -- \\
\hline$<100$ & -- & -- & $<4$ \\
\hline -- & -- & -- & -- \\
\hline
\end{tabular}

$\begin{array}{ll}-- & < \\ -- & < \\ -- & < \\ -- & < \\ -- & - \\ -- & \\ -- & \\ -- & \\ -- & \\ -- & \\ -- & < \\ -- & < \\ -- & \\ -- & -\end{array}$

$<4$

$--\quad 350$

$<4 \quad--\quad 170$

$<6 \quad--\quad 110$

$<1 \quad--\quad-$

$-\infty \quad--\quad 250$

$5 \quad 310$

$1 \quad 130$

$1 \quad \cdots \quad 180$

$1 \quad 280$

$2 \quad-\quad 270$

$<2 \quad--\quad 830$

$<2 \quad--\quad 220$

$1 \quad 210$

\begin{tabular}{|c|c|c|c|c|c|c|c|c|}
\hline DATE & $\begin{array}{l}\text { IRON, } \\
\text { DIS- } \\
\text { SOLVED } \\
\text { (UG/L }\end{array}$ & $\begin{array}{l}\text { LEAD, } \\
\text { TOTAL } \\
\text { RECOV- } \\
\text { ERABLE } \\
\text { (UG/L }\end{array}$ & $\begin{array}{l}\text { LEAD, } \\
\text { DIS- } \\
\text { SOLVED } \\
\text { (UG/L }\end{array}$ & $\begin{array}{l}\text { MERCURY } \\
\text { TOTAL } \\
\text { RECOV- } \\
\text { ERABLE } \\
\text { (UG/L }\end{array}$ & $\begin{array}{l}\text { ZINC, } \\
\text { TOTAL } \\
\text { RECOV- } \\
\text { ERABLE } \\
\text { (UG/L }\end{array}$ & $\begin{array}{l}\text { ZINC, } \\
\text { DIS- } \\
\text { SOLVED } \\
\text { (UG/L }\end{array}$ & $\begin{array}{l}\text { SEDI- } \\
\text { MENT, } \\
\text { SUS- } \\
\text { PENDED }\end{array}$ & $\begin{array}{l}\text { SEDI - } \\
\text { MENT, } \\
\text { DIS- } \\
\text { CHARGE, } \\
\text { SUS- } \\
\text { PENDED }\end{array}$ \\
\hline & AS FE) & $A S$ PB) & AS PB) & AS EG) & AS ZN) & AS $Z N$ ) & (MG/L) & (T/DAY) \\
\hline
\end{tabular}

AUG 1980

$29 . \ldots$

SEP

29...

OCT

$14 \ldots$

Nov

21...

DEC

$12 \ldots$

JAN 1981

$20 \ldots$

FEB

$23 \ldots$

MAR

18...

APR

$14 \ldots$

MAY

15 ...

JWN

10 ...

JUL

21...

AUG

$24 \ldots$

22...

\begin{tabular}{|c|c|c|c|c|c|c|c|}
\hline-- & -- & -- & -- & 4 & -- & -- & -- \\
\hline-- & -- & -- & -- & 3 & -- & -- & -- \\
\hline-- & -- & -- & -- & $<5$ & -- & -- & -- \\
\hline-- & $<2$ & -- & $=-$ & 10 & -- & -- & -- \\
\hline-- & - & -- & -- & 20 & -- & -- & -- \\
\hline-- & - & - & -- & $<3$ & -- & -- & -- \\
\hline- & -- & -- & -- & 7 & -- & 1 & 0.0 \\
\hline-- & -- & -- & -- & -- & -- & 2 & 0.0 \\
\hline-- & -- & -- & -- & -- & -- & -- & -- \\
\hline-- & -- & - & -- & 51 & -- & 3 & 0.0 \\
\hline-- & -- & -- & -- & 6 & -- & 7 & 0.0 \\
\hline-- & -- & -- & -- & $<5$ & $=$ & 18 & 0.0 \\
\hline-- & $<4$ & -- & $<2.0$ & $<4$ & -- & - & -- \\
\hline-- & -- & -- & -- & -- & -- & -- & -- \\
\hline
\end{tabular}


TABI. 4.--Physical characteristics and concentrations of commond chemical constituents in lakes and streams of the Bald Mountain watershed--Continued

01012570 FISB RIVER AT INLET FISH RIVER LAKE ME

\begin{tabular}{|c|c|c|c|c|c|c|c|c|c|c|}
\hline ATE & IIME & $\begin{array}{l}\text { STREAM- } \\
\text { FLOW, } \\
\text { INSTAN- } \\
\text { TANEOUS }\end{array}$ & $\begin{array}{l}\text { SPE- } \\
\text { CIFIC } \\
\text { CON- } \\
\text { DUCT- } \\
\text { ANCE }\end{array}$ & $\begin{array}{c}\text { PH } \\
\text { (STAND- } \\
\text { ARD }\end{array}$ & $\begin{array}{c}\text { TEMPER- } \\
\text { ATURE } \\
\text { WATER }\end{array}$ & $\begin{array}{l}\text { COLOR } \\
\text { (PLAT- } \\
\text { INUM- } \\
\text { COBALT }\end{array}$ & $\begin{array}{l}\text { TUR- } \\
\text { BID- } \\
\text { ITY }\end{array}$ & $\begin{array}{c}\text { OXYGEN, } \\
\text { DIS- } \\
\text { SOLVED }\end{array}$ & $\begin{array}{l}\text { OXYGEN } \\
\text { DEMAND, } \\
\text { BIO- } \\
\text { CBEM- } \\
\text { ICAL, } \\
5 \text { DAY }\end{array}$ & $\begin{array}{l}\text { COLI- } \\
\text { FORM, } \\
\text { FECAL, } \\
0.45 \\
\text { UM-MF } \\
\text { (COLS. }\end{array}$ \\
\hline & & (CFS) & (US/CM) & UNITS) & (DEG C) & UNITS) & (FTU) & (MG/L) & (MG/L) & $100 \mathrm{ML})$ \\
\hline
\end{tabular}

\begin{tabular}{|c|c|c|c|c|c|c|c|c|c|c|}
\hline $\operatorname{Nov}^{20 \ldots}$ & 1110 & -- & 45 & 6.7 & 5.0 & 65 & 1.9 & 13.0 & -- & -- \\
\hline $\begin{array}{c}17 \ldots \\
\text { MAR } 1982\end{array}$ & 0930 & -- & 41 & 6.6 & 3.0 & 30 & 1.6 & 11.2 & -- & -- \\
\hline APR & 1000 & -- & 32 & 6.8 & $-\infty$ & 35 & 1.3 & -- & -- & -- \\
\hline MAY & 1140 & -- & 45 & 6.6 & 0.0 & -- & 6.5 & 12.6 & - & $\rightarrow$ \\
\hline JUN & 1245 & -- & -- & -- & 2.0 & -- & -- & 12.5 & -- & -- \\
\hline SEP & 1630 & -- & 52 & 6.7 & 19.0 & 35 & 1.8 & 9.0 & - & - \\
\hline OCT $^{13 \ldots}$ & 1230 & 71 & 53 & 6.8 & 18.5 & 45 & 1.5 & 9.1 & - & -- \\
\hline${ }_{\text {Nov }}^{20} \ldots$ & 1230 & 50 & 34 & 6.8 & 8.0 & 45 & 1.3 & 10.6 & -- & -- \\
\hline DEC & 1300 & 180 & 32 & 6.6 & 4.0 & 35 & 2.3 & 12.6 & -- & -- \\
\hline $\begin{array}{c}08 \ldots \\
\text { JAN } 1983\end{array}$ & 1335 & $\mathbf{E 2 3 0}$ & 29 & 6.7 & .5 & 50 & 1.1 & 13.0 & -- & -- \\
\hline FEB & 0930 & E28 & 43 & 6.3 & 0.0 & -- & 2.2 & - & -- & $-\infty$ \\
\hline $\operatorname{MAR}^{19 \ldots}$ & - 1150 & E33 & 36 & 6.0 & 0.0 & 30 & 1.2 & 11.8 & -- & -- \\
\hline $25 \ldots$ & 1315 & E340 & 50 & 6.2 & 0.0 & 40 & 5.0 & 13.0 & -- & -- \\
\hline DATE & $\begin{array}{l}\text { ALKA- } \\
\text { LINITY } \\
\text { WAT WR } \\
\text { TOT FET } \\
\text { LAB } \\
\text { MG/L AS } \\
\text { CACO3 }\end{array}$ & $\begin{array}{l}\text { SOLIDS, } \\
\text { RESIDUE } \\
\text { AT 10S } \\
\text { DEG. C, } \\
\text { TOTAL } \\
\text { (MG/L) }\end{array}$ & $\begin{array}{l}\text { SOLIDS, } \\
\text { VOLA- } \\
\text { TILE ON } \\
\text { IGNI- } \\
\text { IION, } \\
\text { TOTAL } \\
\text { (MG/L) }\end{array}$ & $\begin{array}{l}\text { NITRO- } \\
\text { GEN, } \\
\text { NO2+NO3 } \\
\text { TOTAL } \\
\text { (MG/L } \\
\text { AS N) }\end{array}$ & $\begin{array}{l}\text { NITRO- } \\
\text { GEN, } \\
\text { AMANIA } \\
\text { TOTAL } \\
\text { (MG/L } \\
\text { AS N) }\end{array}$ & $\begin{array}{l}\text { NITRO- } \\
\text { GEN, } \\
\text { ORGANIC } \\
\text { IOTAL } \\
\text { (MG/L } \\
\text { AS N) }\end{array}$ & $\begin{array}{l}\text { NITRO- } \\
\text { GEN, AM- } \\
\text { MONIA + } \\
\text { ORGANIC } \\
\text { TOTAL } \\
\text { (MG/L } \\
\text { AS N) }\end{array}$ & $\begin{array}{l}\text { NITRO- } \\
\text { GEN, } \\
\text { TOIAL } \\
\text { (MG/L } \\
\text { AS } N \text { ) }\end{array}$ & $\begin{array}{l}\text { NITRO- } \\
\text { GEN, } \\
\text { TOTAL } \\
\text { (MG/L } \\
\text { AS NO3) }\end{array}$ & $\begin{array}{l}\text { PHOS- } \\
\text { PHOROUS } \\
\text { TOTAL } \\
\text { (MG/L } \\
\text { AS P) }\end{array}$ \\
\hline
\end{tabular}
OCT 1981

\begin{tabular}{|c|c|}
\hline $20 \ldots$ & 9 \\
\hline Nov & \\
\hline $17 \ldots$ & 13 \\
\hline MAR 1982 & \\
\hline $26 \ldots$ & 15 \\
\hline APR & \\
\hline $18 \ldots$ & 7 \\
\hline MAY & \\
\hline $03 \ldots$ & - \\
\hline JUN & \\
\hline $01 \ldots$ & 16 \\
\hline SEP & \\
\hline $13 \ldots$ & 12 \\
\hline OCI & \\
\hline $20 \ldots$ & 18 \\
\hline Nov & \\
\hline $22 \ldots$ & 11 \\
\hline DEC & \\
\hline $08 \ldots$ & 7 \\
\hline JAN 1983 & \\
\hline $29 \ldots$ & -- \\
\hline FEB & \\
\hline $19 \ldots$ & 18 \\
\hline MAR & \\
\hline $25 \ldots$ & 13 \\
\hline
\end{tabular}


TABIE 4.--Physical characteristics and concentrations of commond chemical constituents In lakes and streams of the Bald Mountain watershed--Continued

01012570 FISH RIVER AT INLET FISH RIVER. LAKE ME

DATE

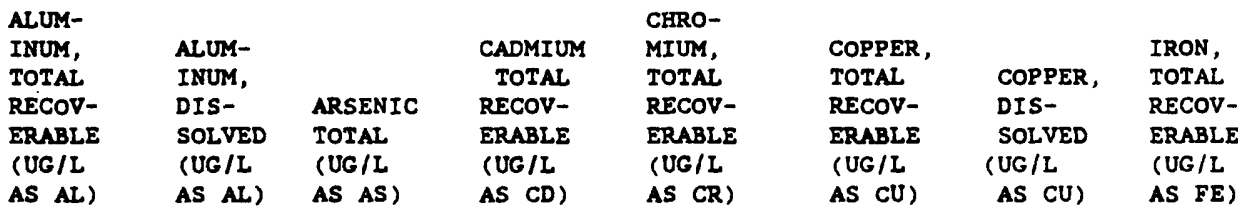

OCT 1981

$20 \ldots$

NOV

$17 \ldots$

MAR 1982

26 ...

APR

18 ...

MAY

$03 .$.

JUN

$01 \ldots$

SEP

$13 .$.

OCT

20 ...

Nov

22 ...

DEC

08...

JAN 1983

29 ...

FEB

$19 \ldots$

MAR

25 ...

AS AL)

AS AL) AS AS )

AS CD)

$\begin{array}{cccc}-- & -- & -- \\ -- & -- & -- & -- \\ -- & -- & -- & -- \\ 550 & -- & -- & -- \\ 200 & <100 & -- & -- \\ <100 & <100 & -- & -- \\ -- & -- & -- & -- \\ -- & -- & -- & - \\ -- & -- & -- & -- \\ -- & -- & -- & - \\ -- & -- & -- & -- \\ -- & -- & -- & -\end{array}$

--
--
--
--
-
--
--
--
--
--
--
--
--

$\begin{array}{ccc}-- & -- & -- \\ -- & -- & - \\ - & -- & - \\ 1 & -- & 530 \\ 1 & 2 & 190 \\ 1 & 2 & 120 \\ 3 & -- & 160 \\ <1 & -- & 130 \\ 1 & -- & 130 \\ <1 & -- & 120 \\ <1 & -- & 130 \\ 1 & -- & 120 \\ 1 & -- & 330\end{array}$

DATE

$\begin{array}{llll} & \text { LEAD, } & & \text { MERCURY } \\ \text { IRON, } & \text { TOTAL } & \text { LEAD, } & \text { TOTAL } \\ \text { DIS- } & \text { RECOV- } & \text { DIS- } & \text { RECOV- } \\ \text { SOLVED } & \text { ERABLE } & \text { SOLVED } & \text { ERABLE } \\ \text { (UG/L } & \text { (UG/L } & \text { (UG/L } & \text { (UG/L } \\ \text { AS FE) } & \text { AS PB) } & \text { AS PB) } & \text { AS HG) }\end{array}$

ZINC,

TOTAL

RECOV -

ERABLE

(UG/L

AS $2 N$ )

ZINC,

DIS-

SOLVED

(UG/L

AS $\mathrm{ZN}$ )

330

OCT 1981

$20 \ldots$

Nov

$17 .$.

MAR 1982

26...

APR

18...

MAY

$03 \ldots$

JUN

01...

SEP

$13 .$.

OCT

$20 .$.

NOV

22...

DEC

$08 . .$.

JAN 1983

29 ...

FEB

$19 . \ldots$

MAR

25 ...

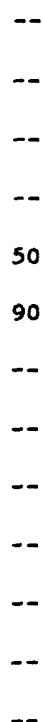

\begin{tabular}{|c|c|c|c|}
\hline- & -- & -- & -- \\
\hline- & -- & -- & -- \\
\hline- & - & - & $m$ \\
\hline 1 & - & -- & $<8$ \\
\hline$<1$ & $<1$ & -- & $<5$ \\
\hline$<1$ & $<1$ & -- & $<5$ \\
\hline -- & -- & -- & $<5$ \\
\hline -- & -- & $\cdots$ & $<6$ \\
\hline-- & - & -- & $<5$ \\
\hline-- & -- & -- & $<5$ \\
\hline- & -- & -- & $<5$ \\
\hline-- & - & $\cdots$ & 5 \\
\hline & -- & -- & $<5$ \\
\hline
\end{tabular}

0.0

0.0

0.0

5

- 2

.38

.27

1.5

1.2

.15

2

$-$ 
TAm. 4.--Physical characteristics and concentrations of commond chemical constituents in lakes and streams of the Bald Mountain watershed--Continued

01012570 FISH RIVER AT INLET FISH RIVER LAKE ME

\begin{tabular}{|c|c|c|c|c|c|c|c|c|c|c|}
\hline DATE & TIME & $\begin{array}{l}\text { STREAM- } \\
\text { FLOW, } \\
\text { INSTAN- } \\
\text { TANEOUS } \\
\text { (CFS) }\end{array}$ & $\begin{array}{l}\text { SPE- } \\
\text { CIFIC } \\
\text { CON- } \\
\text { DUCT- } \\
\text { ANCE } \\
\text { (US/CM) }\end{array}$ & $\begin{array}{c}\text { PH } \\
\text { (STAND- } \\
\text { ARD } \\
\text { UNITS) }\end{array}$ & $\begin{array}{c}\text { TEMPER- } \\
\text { ATURE } \\
\text { WATER } \\
\text { (DEG C) }\end{array}$ & $\begin{array}{l}\text { COLOR } \\
\text { (PLAT- } \\
\text { INUM- } \\
\text { COBALT } \\
\text { UNITS) }\end{array}$ & $\begin{array}{l}\text { TUR- } \\
\text { BID- } \\
\text { ITY } \\
\text { (FTU) }\end{array}$ & $\begin{array}{c}\text { OXYGEN, } \\
\text { DIS- } \\
\text { SOLVED } \\
\text { (MG/L) }\end{array}$ & $\begin{array}{l}\text { OXYGEN } \\
\text { DEMAND, } \\
\text { BIO- } \\
\text { CHEM- } \\
\text { ICAL, } \\
\text { S DAY } \\
\text { (MG/L) }\end{array}$ & $\begin{array}{l}\text { COLI- } \\
\text { FORM, } \\
\text { FECAL, } \\
0.45 \\
\text { UM-MF } \\
\text { (COLS. } \\
100 \mathrm{ML} \text { ) }\end{array}$ \\
\hline
\end{tabular}

\begin{tabular}{|c|c|c|c|c|c|c|c|c|c|c|}
\hline \multicolumn{11}{|l|}{ APR 1983} \\
\hline $19 \ldots$ & 1000 & 1330 & 24 & 6.2 & 2.0 & 30 & 12 & 12.8 & -- & -- \\
\hline $26 \ldots$ & 1515 & 1210 & -- & -- & - & -- & -- & - & -- & - \\
\hline \multicolumn{11}{|l|}{ MAY } \\
\hline $19 \ldots$ & 1500 & 231 & -- & -- & -- & $-\infty$ & $-\infty$ & -- & -- & - \\
\hline \multicolumn{11}{|l|}{ JUN } \\
\hline $14 \ldots$ & 1055 & 152 & 35 & 7.0 & 18.0 & 35 & 2.1 & 9.2 & -- & - \\
\hline \multicolumn{11}{|l|}{ JUL } \\
\hline${ }_{A U G}^{19}$ & 1630 & 15 & 62 & 7.1 & 23.5 & 40 & -- & 8.6 & -- & -- \\
\hline $30 \ldots$ & 0910 & 6 & 56 & -- & 18.0 & 35 & 1.6 & 8.6 & -- & -- \\
\hline \multicolumn{11}{|l|}{ oct } \\
\hline $06 \ldots$ & 1005 & 158 & -- & 6.3 & 13.5 & 30 & 2.8 & 9.1 & -- & - \\
\hline $\begin{array}{l}25 \ldots \\
\operatorname{MAR} 1984\end{array}$ & 1430 & 32 & 38 & 7.0 & 5.0 & 30 & 1.4 & 11.1 & -- & - \\
\hline$\underset{A P R}{29 \ldots}$ & 1200 & E78 & 38 & 7.0 & 0.5 & 25 & 1.2 & 11.4 & -- & - \\
\hline $\operatorname{MAY}^{25} \cdots$ & 0955 & 1310 & 19 & 7.2 & 1.5 & so & 1.3 & -- & -- & - \\
\hline $17 \ldots$ & 1100 & 495 & 40 & -- & 7.0 & 45 & 1.6 & 12.0 & -- & -- \\
\hline $30 \ldots$ & 1140 & 324 & 34 & 7.3 & 10.0 & 50 & 5.7 & 10.2 & -- & - \\
\hline
\end{tabular}

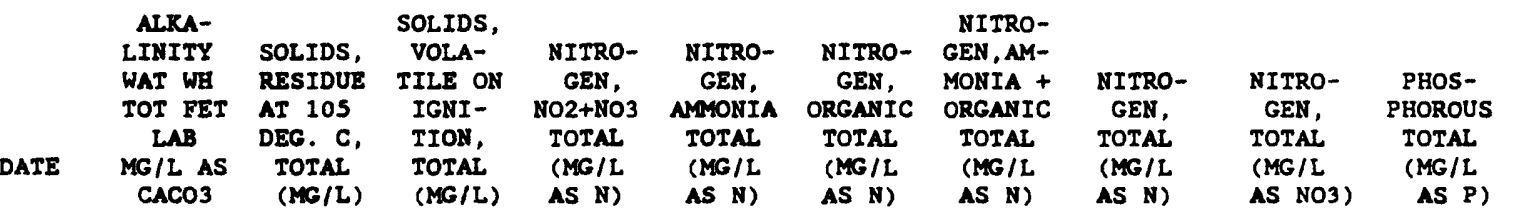

\begin{tabular}{|c|c|c|c|c|c|c|c|c|c|c|}
\hline APR 1983 & & & & & & & & & & \\
\hline $19 .$. & 6 & -- & -- & -- & -- & -- & - & -- & - & -- \\
\hline $\operatorname{mAY}^{26 .}$ & 15 & 10 & -- & -- & -- & -- & -- & -- & -- & 0.01 \\
\hline $19 \ldots$ & -- & -- & -- & -- & -- & -- & -- & -- & -- & .01 \\
\hline $14 \ldots$ & 16 & -- & -- & -- & -- & -- & -- & -- & -- & .01 \\
\hline JUL & 3 & & & & & & & & & \\
\hline AUG $19 .$. & 23 & $\cdots$ & $\cdots$ & -- & -- & -- & -- & $\cdots$ & -- & .02 \\
\hline $30 .$. & 28 & -- & -- & -- & -- & -- & -- & -- & - & $<.01$ \\
\hline $06 \ldots$ & 17 & 94 & -- & -- & -- & -. & -- & -- & -- & .01 \\
\hline $25 \ldots$ & 18 & 40 & -- & -- & -- & -- & -- & -- & -- & .01 \\
\hline $\begin{array}{c}\text { MAR 1984 } \\
29 \ldots\end{array}$ & & & & & & & & & & \\
\hline $\mathrm{APR}_{\mathrm{APR}}^{29 . \cdots}$ & 8 & 50 & - & $\ldots$ & 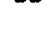 & 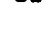 & - & $-\infty$ & - & -- \\
\hline $25 \ldots$ & 4 & 44 & -- & -- & -- & -- & -- & -- & -- & -- \\
\hline MAY & 11 & & & s & & -8 & - & - & 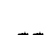 & \\
\hline $17 \ldots$ & 6 & 46 & -. & $\ldots$ & - & .- & - & -- & -. & .01 \\
\hline
\end{tabular}


TAx. 4.--Physical characteristics and concentrations of commond chemical constituents in lakes and streams of the Bald Mountain watershed--Continued

01012570 FISE RIVER AT INLET FISE RIVER LARE ME

DATE

APR 1983

$19 .$.

MAY

19..

JUN

$14 \ldots$

JUL

19...

AUG

$30 . .$.

oct

06.

$25 \ldots$

MAR 1984

29 ...

APR

25 ...

MAY

$17 .$.

30 ...

DATE

APR 1983

19 ...

26 ...

MAY

$19 .$.

JUN

$14 \ldots$

JUL.

19...

AUG

$30 .$.

OCT

$06 .$.

$25 \ldots$

MAR 1984

29. . .

APR

25 ...

MAY

$17 .$.

$30 \ldots$
ALUM-

INUM,

TOTAL

RECOV-

ERABLE

(UG/L

AS AL)

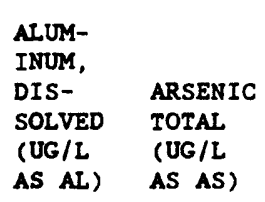

$\begin{array}{cl} & \text { CHRO- } \\ \text { CADMIUM } & \text { MIUM, } \\ \text { TOTAL } & \text { TOTAL } \\ \text { RECOV- } & \text { RECOV- } \\ \text { ERABLE } & \text { ERABLE } \\ \text { (UG/L } & \text { (UG/L } \\ \text { AS CD) } & \text { AS CR) }\end{array}$

\begin{tabular}{|c|c|c|}
\hline-- & -- & -- \\
\hline- & -- & -- \\
\hline$=-$ & -- & -- \\
\hline-- & -- & -- \\
\hline-- & -- & -- \\
\hline-- & -- & -- \\
\hline-- & -- & - \\
\hline-- & - & \\
\hline-- & -- & - \\
\hline-- & -- & -- \\
\hline-- & -- & \\
\hline & -- & \\
\hline
\end{tabular}

$\begin{array}{ll}-- & \\ -- & - \\ -- & - \\ -- & - \\ -- & - \\ -- & - \\ -- & - \\ -- & -\end{array}$

--
--
--
--
--
--
--
--

$\begin{array}{cl} & \text { LEAD, } \\ \text { IRON, } & \text { TOTAL } \\ \text { DIS- } & \text { RECOV- } \\ \text { SOLVED } & \text { ERABLE } \\ \text { (UG/L } & \text { (UG/L } \\ \text { AS FE) } & \text { AS PB) }\end{array}$

LEAD,
DIS-
SOLVED
(UG/L
AS PB)

列

MERCURY
TOTAL
RECOV-
ERABLE
(UG/L
AS HG)

ZINC,

TOTAL

RECOV-

ERABLE

(UG/L

AS 2N)

ZINC

DIS-

SOLVED

(UG/L

AS 2N)

MENT,

SUS-

PENDED

(MG/L)

$\because$

$<5$
$<5$

$<5$

--

6

$<1$

$<5$

$<11$

8

$<5$

24

$-$

$--$

$-$

$--$

-.

$<1$

$-$

$<5$

$-$

$--$

$->$

--
--
--

$-$ 
TAMS 4.--Physical characteristics and concentrations of comnond chemical constituents in lakes and streams of the Bald Mountain watershed--Continued

01016410 MACEIAS RIVER AT RUSSELL CROSSING ME

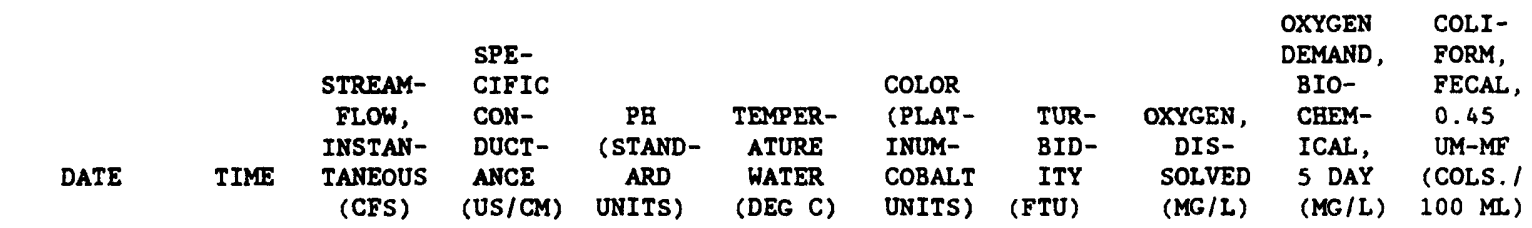

\begin{tabular}{|c|c|c|c|c|c|c|c|c|c|c|}
\hline OCT 1979 & & & & & & & & & & \\
\hline $\begin{array}{r}12 \ldots \\
\text { JAN } 1980\end{array}$ & 0930 & -- & 50 & 7.1 & 10.0 & 60 & 2.0 & 11.1 & 0.4 & -- \\
\hline FEB $01 \ldots$ & 0930 & -- & 64 & 6.8 & 0.0 & -- & 1.9 & 14.0 & -- & - \\
\hline $\operatorname{MAR}^{19 \ldots}$ & 0900 & -- & 75 & 6.8 & 0.0 & 40 & 2.3 & 12.0 & .7 & K2 \\
\hline$\stackrel{20 \ldots}{A P R}$ & 0945 & -- & 260 & 6.7 & 0.0 & 40 & 2.4 & 12.0 & 2.0 & $<1$ \\
\hline$\underset{\text { MAY }}{04 . .}$ & 0930 & -- & 35 & 6.7 & 2.0 & 45 & 5.7 & 13.1 & 3.4 & $<1$ \\
\hline JUN & 1000 & -- & 34 & 6.8 & 12.0 & 35 & 2.3 & 10.0 & 1.4 & $\mathrm{KI}$ \\
\hline JUL $^{17 \ldots}$ & 1030 & -- & 35 & 7.0 & 14.0 & 35 & 2.3 & 10.5 & .8 & -- \\
\hline${ }_{A U G}^{13} \ldots$ & 1030 & -- & 42 & 6.9 & 18.0 & 35 & 1.5 & 9.0 & $\cdots$ & -- \\
\hline $\operatorname{SEP}^{29 . \cdots}$ & 0930 & -- & 50 & 7.2 & 18.0 & 35 & 1.7 & 9.7 & -- & -- \\
\hline OCT & 0930 & -- & 38 & 6.9 & 6.0 & 45 & 3.3 & 11.6 & -- & -- \\
\hline $\operatorname{Nov}^{14 \ldots}$ & 0915 & -- & 42 & 6.2 & 5.0 & 65 & 2.5 & 11.8 & -- & -- \\
\hline${ }_{\text {DEC }}^{21 \ldots}$ & 0930 & -- & 37 & 6.7 & 0.0 & 40 & 1.6 & 13.4 & -- & - \\
\hline $\begin{array}{l}12 \ldots \\
\text { JAN } 1981\end{array}$ & 0945 & -- & 130 & 6.8 & 0.0 & 35 & 1.2 & 13.2 & -- & -- \\
\hline $20 \ldots$ & 0945 & -- & 58 & 6.5 & 0.0 & 45 & 2.3 & 12.0 & -- & -- \\
\hline DATE & $\begin{array}{l}\text { ALRA- } \\
\text { LINITY } \\
\text { WAT WB } \\
\text { TOT FET } \\
\text { LAB } \\
\text { MG/L AS } \\
\text { CACO3 }\end{array}$ & $\begin{array}{l}\text { SOLIDS, } \\
\text { RESIDUE } \\
\text { AT } 105 \\
\text { DEG. C, } \\
\text { TOTAL } \\
\text { (MG/L) }\end{array}$ & $\begin{array}{l}\text { SOLIDS, } \\
\text { VOLA- } \\
\text { TILE ON } \\
\text { IGNI- } \\
\text { TION, } \\
\text { TOTAL } \\
\text { (MG/L) }\end{array}$ & $\begin{array}{l}\text { NITRO- } \\
\text { GEN, } \\
\text { NO2+NO3 } \\
\text { TOTAL } \\
\text { (MG/L } \\
\text { AS N) }\end{array}$ & $\begin{array}{l}\text { NITRO- } \\
\text { GEN, } \\
\text { AMPONIA } \\
\text { TOTAL } \\
\text { (MG/L } \\
\text { AS N) }\end{array}$ & $\begin{array}{l}\text { NITRO- } \\
\text { GEN, } \\
\text { ORGANIC } \\
\text { TOTAL } \\
\text { (MG/L } \\
\text { AS N) }\end{array}$ & $\begin{array}{l}\text { NITRO- } \\
\text { GEN,AM- } \\
\text { MONIA + } \\
\text { ORGANIC } \\
\text { TOTAL } \\
\text { (MG/L } \\
\text { AS N) }\end{array}$ & $\begin{array}{l}\text { NITRO- } \\
\text { GEN, } \\
\text { TOTAL } \\
\text { (MG/L } \\
\text { AS N) }\end{array}$ & $\begin{array}{l}\text { NITRO- } \\
\text { GEN, } \\
\text { TOTAL } \\
\text { (MG/L } \\
\text { AS NO3) }\end{array}$ & $\begin{array}{l}\text { PHOS- } \\
\text { PHOROUS } \\
\text { TOTAL } \\
\text { (MG/L } \\
\text { AS P) }\end{array}$ \\
\hline
\end{tabular}

OCT 1979

12 ...

JAN 1980

$01 .$. .

FEB

19...

.

20...

APR

$04 \ldots$

MAY

20 ...

JUN

$17 .$.

JUL

$13 .$.

AUG

29...

SEP

OCT

14 ...

Nov

$21 .$.

DEC

$12 .$.

JAN 1981

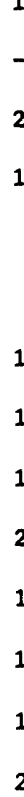

-
62
67
38
55
65
55
50
60
67
63
51
--
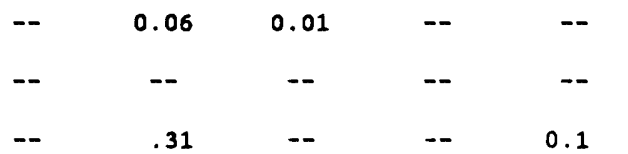

$\begin{array}{ccc}-- & -- & -- \\ -- & -- & 0.01 \\ 0.41 & 1.8 & .02 \\ .54 & 2.4 & .01 \\ .50 & 2.2 & .02 \\ .43 & 1.9 & -- \\ .30 & 1.3 & .01 \\ -- & -- & .01 \\ -- & -- & <.01 \\ -- & -- & -- \\ \ldots & .- & .01 \\ -- & -- & .01 \\ -- & -- & .01 \\ -- & -- & .01\end{array}$


TABIS 4.--Physical characteristics and concentrations of commond chemical constituents In lakes and streams of the Bald Mountain watershed--Continued

01016410 MACEIAS RIVER AT RUSSELL CROSSING ME

DATE

OCT 1979

JAN 1980

01 . .

FEB

19...

MAR

$20 \ldots$

APR

$04 .$.

MAY

20 ...

JUN

17 ...

JUL.

$13 \ldots$

AUG

29...

SEP

29...

OCT

$14 \ldots$

NoV

21 ...

DEC

12 ...

JAN 1981

20...

ocr 1979

12 ...

JAN 1980

$01 \ldots$

FEB

19...

MAR

20 ...

APR

04...

MAY

20...

JUN

$17 .$.

JUL

$13 .$.

AUG

29 ...

SEP

29 ...

OCT

$14 \ldots$

Nov

21 ..

DEC

12 ...

JAN 1981

20 ...
ALUM-

INUM,

TOTAL.

RECOV-

ERABLE

(UG/L

AS AL)

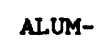

INUM,

DIS-

SOLVED

(UG/L

AS AL)

ARSENIC

TOTAL.

(UG/L

AS AS)

$\begin{array}{cl} & \text { CHRO- } \\ \text { CADMIUM } & \text { MIUM, } \\ \text { TOTAL } & \text { TOTAL } \\ \text { RECOV- } & \text { RECOV- } \\ \text { ERABLE } & \text { ERABLE } \\ \text { (UG/L } & \text { (UG/L } \\ \text { AS CD) } & \text { AS CR) }\end{array}$

COPPER,
TOTAL
RECOV-
ERABLE
(UG/L
AS CU)

COPPER,

IRON

TOTAL

RECOV-

ERABLE

$\begin{array}{ll}\text { (UG /L } & \text { (UG/L } \\ \text { AS CU) } & \text { AS FE) }\end{array}$

$\begin{array}{lrrrr}<2 & <6 & <4 & -- & 240 \\ <2 & <6 & <4 & -- & -- \\ <2 & <6 & <4 & -- & 950 \\ -- & -- & <4 & -- & -- \\ <2 & <5 & <10 & -- & -- \\ <2 & <20 & <4 & -- & -- \\ <2 & <5 & <2 & -- & 160 \\ -- & <5 & 2 & -- & 180 \\ -- & -- & <4 & -- & 120 \\ -- & -- & <4 & -- & 300 \\ -- & -- & <6 & -- & 230 \\ -- & -- & <1 & -- & -- \\ -- & -- & 2 & -- & 150 \\ -- & -- & -- & -- & 150\end{array}$

240

950

$-$

60

180

20

230

$-$

150

$\begin{array}{lllllllr} & \text { LEAD, } & & \text { MERCURY } & \text { ZINC, } & & \text { MENT, } \\ \text { IRON, } & \text { TOTAL } & \text { LEAD, } & \text { TOTAL } & \text { TOTAL } & \text { ZINC, } & \text { SEDI- } & \text { DIS- } \\ \text { DIS- } & \text { RECOV- } & \text { DIS- } & \text { RECOV- } & \text { RECOV- } & \text { DIS- } & \text { MENT, } & \text { CHARGE, } \\ \text { SOLVED } & \text { ERABLE } & \text { SOLVED } & \text { ERABLE } & \text { ERABLE } & \text { SOLVED } & \text { SUS- } & \text { SUS- } \\ \text { (UG/L } & \text { (UG/L } & \text { (UG/L } & \text { (UG/L } & \text { (UG/L } & \text { (UG/L } & \text { PENDED } & \text { PENDED } \\ \text { AS FE) } & \text { AS PB) } & \text { AS PB) } & \text { AS HG) } & \text { AS ZN) } & \text { AS ZN) } & \text { (MG/L) } & \text { (T/DAY) }\end{array}$

$\begin{array}{lrrrrrrr}-- & 20 & -- & <0.2 & -- & -- & -- & -- \\ -- & <20 & -- & .2 & <2 & -- & -- & -- \\ -- & <20 & -- & <.2 & 5 & -- & -- & -- \\ -- & <20 & -- & -- & <2 & -- & 1 & 0.0 \\ -- & <30 & -- & -- & 3 & -- & 2 & 0.0 \\ -- & <30 & -- & -- & 52 & -- & 3 & 0.0 \\ -- & <30 & -- & <.2 & 4 & -- & 2 & 0.0 \\ -- & -- & -- & -- & 4 & -- & -- & -- \\ -- & -- & -- & -- & 7 & -- & -- & - \\ -- & -- & -- & -- & 2 & -- & -- & -- \\ -- & -- & -- & -- & <5 & -- & -- & -- \\ -- & <2 & -- & -- & <4 & -- & -- & -- \\ -- & -- & -- & -- & 3 & -- & -- & -- \\ -- & -- & -- & -- & 6 & -- & -- & -\end{array}$


TNIT 4.--Physical characteristics and concentrations of commond chemical constituents in lakes and streams of the Bald Mountain watershed--Continued

01016410 MACHIAS RIVER AT RUSSELL CROSSING ME

\begin{tabular}{|c|c|c|c|c|c|c|c|c|c|}
\hline TIME & $\begin{array}{l}\text { STREAM- } \\
\text { FLOW, } \\
\text { INSTAN- } \\
\text { TANEOUS } \\
\text { (CFS) }\end{array}$ & $\begin{array}{l}\text { SPE- } \\
\text { CIFIC } \\
\text { CON- } \\
\text { DUCT- } \\
\text { ANCE } \\
\text { (US/CM) }\end{array}$ & $\begin{array}{c}\text { PE } \\
\text { (STAND- } \\
\text { ARD } \\
\text { UNITS) }\end{array}$ & $\begin{array}{c}\text { TEMPER- } \\
\text { ATURE } \\
\text { WATER } \\
\text { (DEG C) }\end{array}$ & $\begin{array}{l}\text { COLOR } \\
\text { (PLAT- } \\
\text { INUM- } \\
\text { COBALT } \\
\text { UNITS) }\end{array}$ & $\begin{array}{l}\text { TUR- } \\
\text { BID- } \\
\text { ITY } \\
\text { (FTU) }\end{array}$ & $\begin{array}{c}\text { OXYGEN, } \\
\text { DIS- } \\
\text { SOLVED } \\
\text { (MG/L) }\end{array}$ & $\begin{array}{c}\text { OXYGEN } \\
\text { DEMAND, } \\
\text { BIO- } \\
\text { CHEM- } \\
\text { ICAL, } \\
5 \text { DAY } \\
\text { (MG/L) }\end{array}$ & $\begin{array}{l}\text { COLI- } \\
\text { FORM, } \\
\text { FECAL } \\
0.45 \\
\text { UM-MF } \\
\text { (COLS. } \\
100 \mathrm{ML} \text { ) }\end{array}$ \\
\hline
\end{tabular}

\begin{tabular}{|c|c|c|c|c|c|c|c|c|c|c|}
\hline FEB 1981 & & & & & & & & & & \\
\hline $\operatorname{MAR}_{\operatorname{MaR}}^{23}$ & 1820 & -- & 38 & -- & 2.0 & 45 & 2.8 & 12.2 & -- & -- \\
\hline$\underset{A P R}{25} \ldots$ & 0930 & -- & 30 & 6.7 & 2.0 & 35 & 1.0 & 14.1 & -- & -- \\
\hline $\operatorname{MAY}^{14 \ldots}$ & 0930 & - & 25 & 6.2 & 1.0 & 35 & 3.1 & 12.2 & -- & -- \\
\hline Jur $15 \ldots$ & 0910 & -- & 29 & 6.7 & 9.0 & 50 & 1.5 & 11.8 & -- & -- \\
\hline$\pi^{10 \ldots}$ & 0845 & -- & 33 & 6.8 & 11.0 & 45 & 2.2 & 10.4 & -- & -- \\
\hline AUG & 0845 & -- & 56 & 7.3 & -- & 40 & 1.0 & -- & -- & -- \\
\hline$\underset{\operatorname{SEP}}{24 \ldots}$ & 1125 & -- & 32 & 6.6 & 17.0 & 90 & 1.4 & 8.9 & -- & -- \\
\hline $\mathrm{OCT}^{22 \ldots}$ & 1600 & -- & 52 & 7.3 & 9.0 & 60 & 5.3 & 11.3 & -- & -- \\
\hline Nov $20 .$. & 1600 & -- & -- & 6.6 & 7.0 & 60 & 2.4 & 11.2 & -- & -- \\
\hline $\begin{array}{l}17 \ldots \\
\operatorname{MAR} 1982\end{array}$ & 1450 & -- & 50 & 6.7 & 3.0 & 30 & 1.5 & 10.4 & -- & -- \\
\hline$\underset{A P R}{26 \ldots}$ & 1130 & -- & 31 & 7.0 & -- & 35 & 2.5 & -- & -- & -- \\
\hline JuN $18 \ldots$ & 1720 & -- & 30 & 6.6 & 2.5 & 40 & 2.0 & 10.8 & -- & -- \\
\hline$\underset{\text { OCT }}{01 \ldots}$ & 1800 & -- & 41 & 6.7 & 20.0 & 35 & 1.5 & 9.2 & -- & -- \\
\hline $06 \ldots$ & 1400 & -- & -- & 6.6 & 12.5 & 60 & 6.1 & 9.8 & -- & -- \\
\hline DATE & $\begin{array}{l}\text { ALRA- } \\
\text { LINITY } \\
\text { WAT WR } \\
\text { TOT FET } \\
\text { LAB } \\
\text { MG } / 2 \text { AS } \\
\text { CACO3 }\end{array}$ & $\begin{array}{l}\text { SOLIDS, } \\
\text { RESIDUE } \\
\text { AT } 105 \\
\text { DEG. C, } \\
\text { TOTAL } \\
\text { (MG/L) }\end{array}$ & $\begin{array}{l}\text { SOLIDS, } \\
\text { VOLA- } \\
\text { TILE ON } \\
\text { IGNI- } \\
\text { TION, } \\
\text { TOTAL } \\
\text { (MG/L) }\end{array}$ & $\begin{array}{l}\text { NITRO- } \\
\text { GEN, } \\
\text { NO2+NO3 } \\
\text { TOTAL } \\
\text { (MG/L } \\
\text { AS N) }\end{array}$ & $\begin{array}{l}\text { NITRO- } \\
\text { GEN, } \\
\text { AMONIA } \\
\text { TOTAL } \\
\text { (MG/L } \\
\text { AS N) }\end{array}$ & $\begin{array}{l}\text { NITRO- } \\
\text { GEN, } \\
\text { ORGANIC } \\
\text { TOTAL } \\
\text { (MG/L } \\
\text { AS N) }\end{array}$ & $\begin{array}{l}\text { NITRO- } \\
\text { GEN, AM- } \\
\text { MONIA + } \\
\text { ORGANIC } \\
\text { TOTAL } \\
\text { (MG/L } \\
\text { AS N) }\end{array}$ & $\begin{array}{l}\text { NITRO- } \\
\text { GEN, } \\
\text { TOTAL } \\
\text { (MG/L } \\
\text { AS N) }\end{array}$ & $\begin{array}{l}\text { NITRO- } \\
\text { GEN, } \\
\text { TOTAL } \\
\text { (MG/L } \\
\text { AS NO3) }\end{array}$ & $\begin{array}{l}\text { PHOS- } \\
\text { PHOROUS } \\
\text { TOTAL } \\
\text { (MG/L } \\
\text { AS P) }\end{array}$ \\
\hline
\end{tabular}

FEB 1981

23...

MAR

$25 .$.

$14 \ldots$

MAY

JUN $15 .$.

(MG/L)

(MG/L)

AS N)

AS N)

AS N)

AS N)

AS $N$ )

AS NO3)

AS P)

Jur. 10

21...

AUG
$24 \ldots$

SEP

22 ...

OCT

20...

Nov

$17 .$.

MAR 1982

12

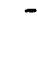

26 . .

APR

$18 \ldots$

JUN

$01 .$.

OCT

06 ...

$$
-
$$
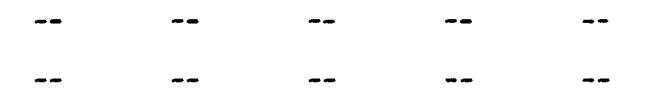

$--$

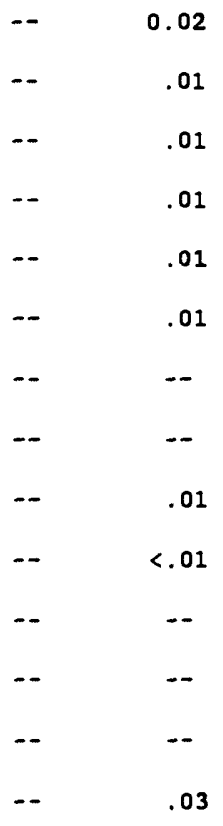


ThI 4.--Physical characteristics and concentrations of commond chemical constituents in lakes and streams of the Bald Mountain watershed--Continued

01016410 MACHIAS RIVER AT RUSSELI CROSSING ME

ALUM-

INUM, ALUM-

TOTAL INUM,

RECOV- DIS-

ERABLE

(UG/L

SOLVED

TOTAL

AS AL) AS AL) AS AS) AS CD) AS CR)

$\begin{array}{cl} & \text { CHRO- } \\ \text { CADMIUM } & \text { MIUM, } \\ \text { TOTAL } & \text { TOTAL } \\ \text { RECOV- } & \text { RECOV- } \\ \text { ERABLE } & \text { ERABLE } \\ \text { (UG/L } & \text { (UG/L } \\ \text { AS CD) } & \text { AS CR) }\end{array}$

COPPER, TOTAL

RECOV-

ERABLE

(UG/L

AS CU)

IRON,

DIS- TOTAL

SOLVED ERABLE

(UG/L (UG/L

FEB 1981

23...

MAR

25...

APR

$14 \ldots$

MAY

$15 .$.

JUN

10 ...

JUL

21 ...

AUG

$24 \ldots$

SEP

22 ...

OCT

20 ...

NOV

$17 . .$.

MAR 1982

26 ...

APR

$18 \ldots$

JUN

$01 .$.

OCT

06 ...

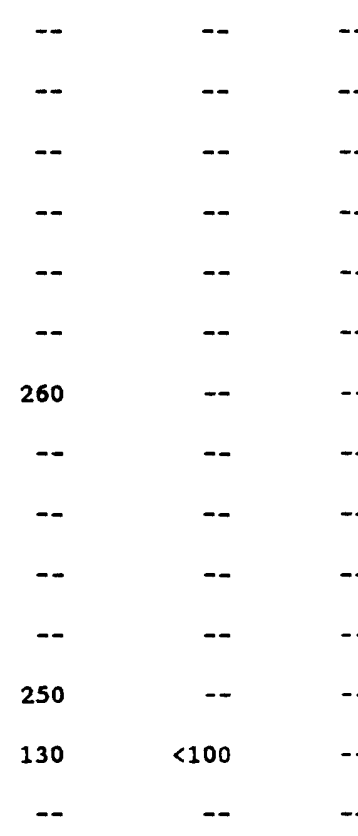

\begin{tabular}{|c|c|c|c|}
\hline-- & 1 & -- & 300 \\
\hline-- & 2 & -- & 110 \\
\hline-- & 1 & $-\infty$ & 260 \\
\hline-- & $<1$ & -- & 160 \\
\hline-- & $<1$ & -- & 220 \\
\hline-- & $<10$ & -- & 130 \\
\hline-- & 12 & -- & 270 \\
\hline-- & 2 & -- & 280 \\
\hline$=-$ & $=-$ & -- & - \\
\hline-- & -- & $=$ & -- \\
\hline- & $-\sim$ & -- & -- \\
\hline-- & $<1$ & -- & 220 \\
\hline-- & $<1$ & 13 & 100 \\
\hline & $<1$ & $-\sim$ & 580 \\
\hline
\end{tabular}

\section{IRON,}

SOLVED

(UG/L.

LEAD,

TOTAL LEAD, TOTAL

RECOV-

ERABLE

(UG/L

DIS-

SOLVED

(UG/L

AS PB)

RECOV-

ERABLE

(UG/L

AS BG)

FEB 1981

$23 \ldots$

MAR

25 . .

APR

$14 \ldots$

MAY

$15 \ldots$

Jus

10...

JUL

$21 .$.

AUG

24 ..

SEP

22 ...

OCT

20...

Nov

$17 \ldots$

MAR 1982

26 ...

APR

$18 \ldots$

JUN

$01 . .$.

OCT

06 ...

\begin{tabular}{|c|c|c|c|}
\hline-- & -- & -- & -- \\
\hline-- & -- & -- & -- \\
\hline-- & -- & - & - \\
\hline-- & -- & -- & -- \\
\hline-- & -- & -- & -- \\
\hline-- & -- & - & $=$ \\
\hline-- & $<4$ & -- & $<0.2$ \\
\hline-- & -- & -- & -- \\
\hline-- & -- & -- & -- \\
\hline-- & -- & $=$ & $=-$ \\
\hline-- & -- & - & -- \\
\hline-- & $<1$ & -- & -- \\
\hline 70 & $<1$ & 7 & -- \\
\hline & $\approx$ & $\sim$ & \\
\hline
\end{tabular}

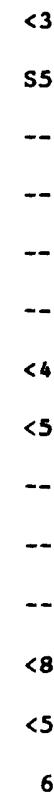

$<3$
S5
--
--
--
--
$<4$
$<5$
--
--
$<8$
65
ZINC,

RECOV-

ERABLE

(UG/L

AS 2N)
$<1$

580

\section{0 \\ 20 \\ 70 \\ 80 \\ 20 \\ 00}

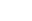

SEDI -

MENT,

DIS-

CHARGE,

SUS -

PENDED

(T/DAY) 
TABIR 4.--Physical characteristics and concentrations of commond chemical constituents in lakes and streams of the Bald Mountain watershed (Continued)

01016460 GREENLAW STREAM AT INLET GREENLAW POND ME

\begin{tabular}{|c|c|c|c|c|c|c|c|c|c|c|}
\hline DATE & TIME & $\begin{array}{l}\text { STREAM- } \\
\text { FLOW, } \\
\text { INSTAN- } \\
\text { TANEOUS } \\
\text { (CFS) }\end{array}$ & $\begin{array}{l}\text { SPE- } \\
\text { CIFIC } \\
\text { CON- } \\
\text { DUCT- } \\
\text { ANCE } \\
\text { (US/CM) }\end{array}$ & $\begin{array}{c}\text { PA } \\
\text { (STAND- } \\
\text { ARD } \\
\text { UNITS) }\end{array}$ & $\begin{array}{l}\text { TEMPER- } \\
\text { ATURE } \\
\text { WATER } \\
\text { (DEG C) }\end{array}$ & $\begin{array}{l}\text { COLOR } \\
\text { (PLAT- } \\
\text { INUN- } \\
\text { COBALT } \\
\text { UNITS) }\end{array}$ & $\begin{array}{l}\text { TUR- } \\
\text { BID- } \\
\text { ITY } \\
\text { (FTU) }\end{array}$ & $\begin{array}{c}\text { OXYGEN, } \\
\text { DIS- } \\
\text { SOLVED } \\
\text { (MG/L) }\end{array}$ & $\begin{array}{l}\text { OXYGEN } \\
\text { DEMAND, } \\
\text { BIO- } \\
\text { CHEM- } \\
\text { ICAL, } \\
5 \text { DAY } \\
\text { (MG/L) }\end{array}$ & $\begin{array}{l}\text { COLI- } \\
\text { FORM, } \\
\text { FECAL, } \\
0.45 \\
\text { UM-MF } \\
\text { (COLS. / } \\
100 \mathrm{ML} \text { ) }\end{array}$ \\
\hline JUN 1980 & & & & & & & & & & \\
\hline $\mathrm{JUL}^{17 \cdots}$ & 0930 & -- & 42 & 6.9 & 2.0 & 25 & 1.0 & 9.3 & $<0.1$ & -- \\
\hline $13 \ldots$ & 0930 & -- & 48 & 6.9 & 20.0 & 25 & 3.2 & 8.2 & -- & -- \\
\hline 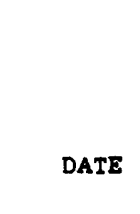 & $\begin{array}{l}\text { ALRA- } \\
\text { LINITY } \\
\text { WAT WA } \\
\text { TOT FET } \\
\text { LAB } \\
\text { MG/L AS } \\
\text { CACO3 }\end{array}$ & $\begin{array}{l}\text { SOLIDS, } \\
\text { RESIDUE } \\
\text { AT } 105 \\
\text { DEG. C, } \\
\text { TOTAL } \\
\text { (MG/L) }\end{array}$ & $\begin{array}{l}\text { SOLIDS, } \\
\text { VOLA- } \\
\text { TILE ON } \\
\text { IGNI- } \\
\text { TION, } \\
\text { TOTAL } \\
\text { (MG/L) }\end{array}$ & $\begin{array}{l}\text { NITRO- } \\
\text { GEN, } \\
\text { NO2+NO3 } \\
\text { TOTAL } \\
\text { (MG/L } \\
\text { AS N) }\end{array}$ & $\begin{array}{l}\text { NITRO- } \\
\text { GEN, } \\
\text { AMMONIA } \\
\text { TOTAL } \\
\text { (MG/L } \\
\text { AS N) }\end{array}$ & $\begin{array}{l}\text { NITRO- } \\
\text { GEN, } \\
\text { ORGANIC } \\
\text { TOTAL } \\
\text { (MG/L } \\
\text { AS N) }\end{array}$ & $\begin{array}{l}\text { NITRO- } \\
\text { GEN, AM- } \\
\text { MONIA + } \\
\text { ORGANIC } \\
\text { TOTAL } \\
\text { (MG /L } \\
\text { AS N) }\end{array}$ & $\begin{array}{l}\text { NITRO- } \\
\text { GEN, } \\
\text { TOTAL } \\
\text { (MG/L } \\
\text { AS N) }\end{array}$ & $\begin{array}{l}\text { NITRO- } \\
\text { GEN, } \\
\text { TOTAL } \\
\text { (MG/L } \\
\text { AS NO3) }\end{array}$ & $\begin{array}{l}\text { PHOS- } \\
\text { PHOROUS } \\
\text { TOTAL } \\
\text { (MG/L } \\
\text { AS P) }\end{array}$ \\
\hline JUN 1980 & & & & & & & & & & \\
\hline $\mathrm{JUL}^{17 \ldots}$ & 16 & 69 & -- & $<0.01$ & 0.01 & 0.19 & 0.2 & -- & -- & 0.01 \\
\hline $13 \ldots$ & 18 & 46 & -- & -- & -- & -- & -- & -- & -- & .01 \\
\hline
\end{tabular}


INA. 4.--Physical cheracteristics and concentrations of commond chemical constituents In lakes and streans of the Bald Mountain watershed (Continued)

01016460 GREENLAW STREAM AT INLET GREENLAW POND ME

DATE

JUN 1980

$17 \ldots$

JUL

$13 \ldots$

DATE

JUN 1980

$17 \ldots$

JUL

$13 .$.
ALUM-

INUM, ALUM-

TOTAL INUM,

RECOV- DIS-

ERABLE

(UG/L

AS AL)

SOLVE

(UG/L

AS AL)

ARSENIC

TOTAL

(UG/L

AS AS)

$\begin{array}{cc} & \text { CERO- } \\ \text { CADMIUM } & \text { MIUM, } \\ \text { TOTAL } & \text { TOTAL } \\ \text { RECOV- } & \text { RECOV } \\ \text { ERABLE } & \text { ERABL } \\ \text { (UG/L } & \text { (UG/L } \\ \text { AS CD) } & \text { AS CR }\end{array}$

COPPER,

RECOV

ERABLE SOLVED ERABLE

(UG/L (UG/L (UG/L

AS CU) AS CU)

A CR) AS CU) AS CU) AS FE)

$<5$

2

$-$

10

$-$

$<5$

$<2$

50

SEDI -

$\begin{array}{clll}\text { MERCURY } & \text { ZINC, } & \text { MENT, } \\ \text { TOTAL } & \text { TOTAL } & \text { ZINC, }\end{array}$

IRON, TOTAL LEAD, TOTAL TOTAL TINC,

SOLVED ERABLE SOLVED ERABLE ERABLE SOLVED SUS-

(UG/L (UG/L (UG/L (UG/L (UG/L (UG/L

SUS -

AS FE) AS PB) AS PB) AS HG) AS ZN

AS ZN)

(MG/L)

(T/DAY) 
TArr 4.--Physical characteristics and concentrations of commcnd chemical constituents in lakes and streams of the Bald Mountain watershed--Continued

01016480 GREENLAW STREAM AT GREENLAW CROSSING ME

\begin{tabular}{|c|c|c|c|c|c|c|c|c|c|c|}
\hline DATE & TIME & $\begin{array}{l}\text { STREAM- } \\
\text { FLOW, } \\
\text { INSTAN- } \\
\text { TANEOUS } \\
\text { (CFS) }\end{array}$ & $\begin{array}{l}\text { SPE- } \\
\text { CIFIC } \\
\text { CON- } \\
\text { DUCT- } \\
\text { ANCE } \\
\text { (US/CM) }\end{array}$ & $\begin{array}{c}\text { PE } \\
\text { (STAND- } \\
\text { ARD } \\
\text { UNITS) }\end{array}$ & $\begin{array}{l}\text { TEMPER- } \\
\text { ATURE } \\
\text { WATER } \\
\text { (DEG C) }\end{array}$ & $\begin{array}{l}\text { COLOR } \\
\text { (PLAT- } \\
\text { INUM- } \\
\text { COBALT } \\
\text { UNITS) }\end{array}$ & $\begin{array}{l}\text { TUR- } \\
\text { BID- } \\
\text { ITY } \\
\text { (FTU) }\end{array}$ & $\begin{array}{c}\text { OXYGEN, } \\
\text { DIS- } \\
\text { SOLVED } \\
\text { (MG/L) }\end{array}$ & $\begin{array}{c}\text { OXYGEN } \\
\text { DEMAND, } \\
\text { BIO- } \\
\text { CHEM- } \\
\text { ICAL, } \\
5 \text { DAY } \\
\text { (MG/L) }\end{array}$ & $\begin{array}{l}\text { COLI- } \\
\text { FORM, } \\
\text { FECAL, } \\
0.45 \\
\text { UM-MF } \\
\text { (COLS. I } \\
100 \mathrm{ML} \text { ) }\end{array}$ \\
\hline
\end{tabular}

\begin{tabular}{|c|c|c|c|c|c|c|c|c|c|c|}
\hline $\begin{array}{l}22 \ldots \\
\text { JAN } 1980\end{array}$ & 0900 & -- & 63 & 7.1 & 12.0 & 60 & 1.4 & 10.0 & 0.4 & -- \\
\hline$\stackrel{F E B}{29} \ldots^{29}$ & 0915 & $=-$ & 104 & 7.1 & 0.0 & -- & 1.6 & 14.6 & $=$ & -- \\
\hline $\operatorname{MAR}^{19 \ldots}$ & 0900 & -- & 120 & 7.1 & 1.0 & 20 & 1.0 & 12.4 & .6 & $\mathbf{K} 3$ \\
\hline$\underset{A P R}{20 \ldots}$ & 0930 & -- & 93 & 6.7 & 0.0 & 30 & 5.9 & 12.8 & 2.6 & K3 \\
\hline $\mathrm{MAY}^{04 \ldots}$ & 0900 & -- & 40 & 6.7 & 1.0 & 40 & 1.3 & 13.3 & .9 & K2 \\
\hline Jun & 0845 & -- & 40 & 6.9 & 8.0 & 40 & 0.9 & 11.0 & 1.4 & $<1$ \\
\hline $\operatorname{JuL}^{17 \ldots}$ & 0845 & -- & 55 & 7.1 & 12.0 & 45 & 1.6 & 10.8 & .5 & -- \\
\hline AUG $^{13 \ldots}$ & 0900 & -- & 49 & 6.8 & 18.0 & 70 & 2.2 & 11.1 & -- & - \\
\hline $\operatorname{SEP}^{29 \ldots}$ & 0845 & -- & 70 & 7.1 & 14.0 & 45 & 1.6 & 10.2 & -- & -- \\
\hline OCI $^{29 \ldots}$ & 0915 & -- & 40 & 6.7 & 4.0 & 110 & 1.4 & 12.0 & -- & -- \\
\hline Nov $14 \ldots$ & 0845 & -- & 45 & 6.7 & 5.0 & 120 & 2.6 & 12.2 & -- & -- \\
\hline${ }_{\text {DEC }}^{21} \cdots$ & 0845 & -- & 41 & 6.7 & 0.0 & 50 & 1.0 & 12.9 & -- & -- \\
\hline $\begin{array}{l}12 \ldots \\
\text { JAN } 1981\end{array}$ & 0900 & -- & 110 & 6.3 & 0.0 & 55 & 1.0 & 13.0 & -- & - \\
\hline $20 \ldots$ & 0915 & -- & 150 & 6.7 & 0.0 & 30 & 1.0 & 12.3 & -- & -- \\
\hline
\end{tabular}

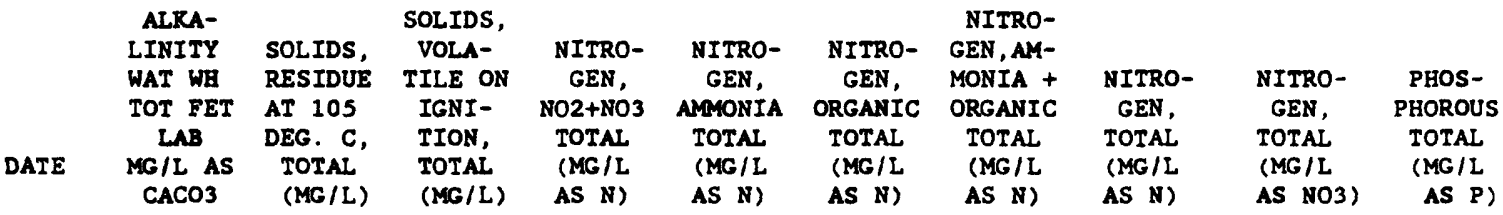

OCT 1979

29.

FEB

19...

20..

6.7

0.030

1.0

12.3

APR

$04 \ldots$

$20 \ldots 11$

JN

$17 \ldots 22$

0.03

0.01

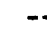

13 ...
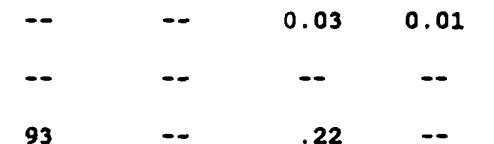

$$
-
$$$$
--
$$$$
-
$$$$
-
$$$$
0.01
$$$$
46
$$$$
52
$$$$
=
$$$$
.47 \quad .04
$$$$
0.16
$$$$
<0.1
$$$$
<.01
$$

$$
38
$$$$
\ldots .14
$$$$
.01
$$$$
.19
$$$$
.2
$$$$
0.67
$$$$
3.0
$$$$
.01
$$$$
64
$$$$
-
$$$$
.09
$$$$
<.01
$$$$
-
$$

.2

.34

1.5

1.3

$$
88
$$$$
.1
$$$$
<.01
$$$$
.2
$$$$
.29
$$$$
1.3
$$ 
TA.I. 4.--Physical characteristics and concentrations of comond chemlcal constituents in lakes and streams of the Bald Mountain watershed--Continued

01016480 GREENLAW STREAM AT GREENLAW CROSSING ME

\begin{tabular}{|c|c|c|c|c|c|c|c|}
\hline $\begin{array}{l}\text { ALUM- } \\
\text { INUM, } \\
\text { TOTAL }\end{array}$ & $\begin{array}{l}\text { ALUM- } \\
\text { INUM, }\end{array}$ & & $\begin{array}{l}\text { CADMIUM } \\
\text { TOTAL }\end{array}$ & $\begin{array}{l}\text { CERO- } \\
\text { MIUM, } \\
\text { TOTAL }\end{array}$ & $\begin{array}{l}\text { COPPER, } \\
\text { TOTAL. }\end{array}$ & COPPER, & $\begin{array}{l}\text { IRON, } \\
\text { IOTAL }\end{array}$ \\
\hline $\begin{array}{l}\text { RECOV- } \\
\text { ERABLE } \\
\text { (UG/L }\end{array}$ & $\begin{array}{l}\text { DIS- } \\
\text { SOLVED } \\
\text { (UG/L }\end{array}$ & $\begin{array}{l}\text { ARSENIC } \\
\text { TOTAL } \\
\text { (UG/L }\end{array}$ & $\begin{array}{l}\text { RECOV- } \\
\text { ERABLE } \\
\text { (UG/L }\end{array}$ & $\begin{array}{l}\text { RECOV- } \\
\text { ERABLE } \\
\text { (UG/L }\end{array}$ & $\begin{array}{l}\text { RECOV- } \\
\text { ERABLE } \\
\text { (UG/L }\end{array}$ & $\begin{array}{l}\text { DIS- } \\
\text { SOLVED } \\
\text { (UG/L }\end{array}$ & $\begin{array}{l}\text { RECOV- } \\
\text { ERABLE } \\
\text { (UG/L }\end{array}$ \\
\hline AS AL) & AS $A L$ ) & AS AS) & AS $(D)$ & AS (R) & AS CU) & AS CU) & AS FE) \\
\hline
\end{tabular}

OCT 1979

22 .

JAN 1980

$29 .$.

FEB

$19 .$.

MAR

20...

APR

$04 \ldots$

MAY

20...

Jun

$17 \ldots$

JuL

13...

AUG

$29 .$.

SEP

29...

ocT

$14 \ldots$

NOV

21 ...

DEC

$12 .$.

JAN 1981

20 ...

$\begin{array}{lll}-- & - & - \\ -- & - & - \\ -- & - & -- \\ - & -- & -- \\ -- & -- & -- \\ -- & -- & -- \\ -- & -- & -- \\ -- & -- & - \\ -- & - & - \\ -- & - & -\end{array}$

\begin{tabular}{|c|c|c|c|c|}
\hline$<2$ & $<6$ & $<4$ & -- & 150 \\
\hline$<2$ & $<6$ & $<4$ & -- & -- \\
\hline$<2$ & $<6$ & $<4$ & -- & 60 \\
\hline-- & -- & $<4$ & -- & -- \\
\hline$<2$ & $<5$ & $<10$ & -- & -- \\
\hline$<2$ & $<20$ & 5 & -- & -- \\
\hline$<2$ & $<5$ & $<2$ & -- & 140 \\
\hline-- & $<5$ & $<2$ & -- & 400 \\
\hline-- & -- & $<4$ & -- & 160 \\
\hline-- & -- & $<4$ & -- & 160 \\
\hline- & - & $<1$ & -- & 250 \\
\hline-- & $-\infty$ & $<1$ & -- & \\
\hline-- & -- & 2 & -- & 140 \\
\hline-- & -- & -- & -- & 60 \\
\hline
\end{tabular}

DATE

$\begin{array}{cll} & \text { LEAD, } & \\ \text { IRON, } & \text { TOTAL } & \text { LEAD, } \\ \text { DIS- } & \text { RECOV- } & \text { DIS- } \\ \text { SOLVED } & \text { ERABLE } & \text { SOLVED } \\ \text { (UG /L } & \text { (UG/L } & \text { (UG/L } \\ \text { AS FE) } & \text { AS PB) } & \text { AS PB) }\end{array}$

$\begin{array}{ll}\text { MERCURY } & \text { ZINC, } \\ \text { TOTAL } & \text { TOTAL } \\ \text { RECOV- } & \text { RECOV- } \\ \text { ERABLE } & \text { ERABLE } \\ \text { (UG/L } & \text { (UG/L } \\ \text { AS HG) } & \text { AS ZN) }\end{array}$

$<20$

$<0.2$

22 . . .

JAN 1980

29 ...

FEB

$19 \ldots$

MAR

20 ...

APR

$04 \ldots$

MAY

20 ...

JUN

17 ...

JUL $13 .$.

AUG

$29 .$.

SEP

$29 . .$.

OCT

$14 \ldots$

Nov

21 ...

DEC

$12 \ldots$

JAN 1981

20 ...

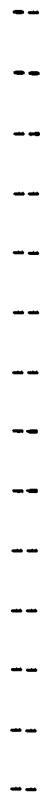

$<20$

$<.2$

$<.2$

$<20$

$--$

$<30$

$<30$

$-$

$<30+-$

3

ZINC,

DIS-

SOLVED

(UG/L

AS ZN)

SEDI -

MENT,

DIS-

60

$\begin{array}{lr}\text { MENT, } & \text { CHARGE } \\ \text { SUS- } & \text { SUS- }\end{array}$

PENDED PENDED

(MG/L) (T/DAY) 
TARIB 4,--Physical characteristics and concentrations of commond chamical constituants in lakes and streams of the Bald Mountain watershed--Continued

01016480 GREENLAW STREAM AT GREENLAW CROSSING ME

\begin{tabular}{|c|c|c|c|c|c|c|c|c|c|c|}
\hline DATE & TIME & $\begin{array}{l}\text { STREAM- } \\
\text { FLOW, } \\
\text { INSTAN- } \\
\text { TANEOUS } \\
\text { (CFS) }\end{array}$ & $\begin{array}{l}\text { SPE- } \\
\text { CIFIC } \\
\text { CON- } \\
\text { DUCT- } \\
\text { ANCE } \\
\text { (US/CM) }\end{array}$ & $\begin{array}{c}\text { PH } \\
\text { (STAND- } \\
\text { ARD } \\
\text { UNITS) }\end{array}$ & $\begin{array}{l}\text { TEMPER- } \\
\text { ATURE } \\
\text { WATER } \\
\text { (DEG C) }\end{array}$ & $\begin{array}{l}\text { COLOR } \\
\text { (PLAT- } \\
\text { INUM- } \\
\text { COBALT } \\
\text { UNITS) }\end{array}$ & $\begin{array}{l}\text { TUR- } \\
\text { BID- } \\
\text { ITY } \\
\text { (FTU) }\end{array}$ & $\begin{array}{c}\text { OXYGEN, } \\
\text { DIS- } \\
\text { SOLVED } \\
\text { (MG/L) }\end{array}$ & $\begin{array}{l}\text { OXYGEN } \\
\text { DEMAND, } \\
\text { BIO- } \\
\text { CBEM- } \\
\text { ICAL, } \\
5 \text { DAY } \\
\text { (MG/L) }\end{array}$ & $\begin{array}{l}\text { COLI- } \\
\text { FORM, } \\
\text { FECAL, } \\
0.45 \\
\text { UM-MF } \\
\text { (COLS. / } \\
\text { 100 ML) }\end{array}$ \\
\hline
\end{tabular}

FEB 1981

$23 \ldots$

MAR

$18 \ldots$

APR

$14 \ldots$

MAY

$15 \ldots$

10 ...

JUL.

$21 \ldots$

AUG

$24 \ldots$

SEP

$22 \ldots$

OCT 20

nov

$17 \ldots$

MAR 1982

26 ...

APR

$18 \ldots$

MAY

$03 .$.

JUN

$01 .$.

1840

- 30

$1840 \quad 62$

$0845 \quad-2 \quad 30$

0850

$0820 \quad--\quad 45$

0820

1050

1630

1630

1515

1210

1345

1200

1820
$-$

6.7

6.7

6.7

6.9

84

42

$--\quad 7.4$

$-\quad 6.6$

49

39

48

6.8

6.5

$-$

67

7.1

7.3

6.7
2.0

2.0

1.0

7.0

10.0

17.0

8. 0

6.0

4.0

$--$

1.5

4. 0

19.0

ALKA-

LINITY

SOLIDS,

WAT WH RESIDUE TILE ON

TOT FET AT 105

AT 105

DATE DEG. $C$,

IGNI-

IION

$\mathrm{CACO}$ (MG/L)

TOTAL

(MG/L)

4.0

1.2

1.4

0.9

1.6

1.2

85

1.0

90

1.9

110

1.2

30

40

50

2.9

2. 9

1.5

$-$

35

.8
8.5

8.5

11.5

12.9

12.8

12. 3

10.9

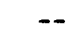

8.9

11.4

12.0

10.0

11.

12.

.5

FEB 1981

$23 \ldots$

$18 \ldots$

16

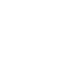

(1)

APR

$14 \ldots$

MAY

$15 \ldots$

JUN

$10 \ldots$

$21 \ldots$

$21 \ldots$
AUG
$24 \ldots$

24... 14

SEP

$22 \ldots 18$

OCT

$20 \ldots \quad 11$

Nov

$17 \ldots$

MAR 1982

26 ...

APR

MAY

$03 .$.

JUN

$01 \ldots$
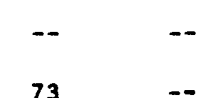

NITRO- NITRO- NITRO- NITRONO2+NO3 AMONIA ORGANIC ORGANIC TOTAL TOTAL TOTAL TOTAL

AS N) AS N) AS N)

26

13

14

28

37

14

15

6

73

54

92

61

62

54

66

67

98

76

9

70

26 
TAmE 4,--Physical characteristics and concentrations of commond chemical constituents in lakes and streams of the Bald Mountain watershed--Continued

01016480 GREENLAW STREAM AT GREENLAW CROSSING ME

\begin{tabular}{|c|c|c|c|c|c|c|c|c|}
\hline DATE & $\begin{array}{l}\text { ALUM- } \\
\text { INUM, } \\
\text { TOTAL } \\
\text { RECOV- } \\
\text { ERABLE } \\
\text { (UG/L }\end{array}$ & $\begin{array}{l}\text { ALUM- } \\
\text { INUM, } \\
\text { DIS- } \\
\text { SOLVED } \\
\text { (UG/L }\end{array}$ & $\begin{array}{l}\text { ARSENIC } \\
\text { TOTAL } \\
\text { (UG/L }\end{array}$ & $\begin{array}{l}\text { CADMIUM } \\
\text { TOTAL } \\
\text { RECOV- } \\
\text { ERABLE } \\
\text { (UG/L }\end{array}$ & $\begin{array}{l}\text { CHRO- } \\
\text { MIUM, } \\
\text { TOTAL } \\
\text { RECOV- } \\
\text { ERABLE } \\
\text { (UG/L }\end{array}$ & $\begin{array}{l}\text { COPPER, } \\
\text { TOTAL } \\
\text { RECOV- } \\
\text { ERABLE } \\
\text { (UG/L }\end{array}$ & $\begin{array}{l}\text { COPPER, } \\
\text { DIS- } \\
\text { SOLVED } \\
\text { (UG/L }\end{array}$ & $\begin{array}{l}\text { IRON, } \\
\text { TOTAL } \\
\text { RECOV- } \\
\text { ERABLE } \\
\text { (UG/L }\end{array}$ \\
\hline & AS AL) & AS $\mathbf{A L}$ ) & AS AS) & AS CD) & AS CR) & AS CU) & AS $(U)$ & AS FE) \\
\hline
\end{tabular}

FEB 1981

$23 \ldots$
MAR
$18 \ldots$
APR
$14 \ldots$
MAY
$15 \ldots$
JUN
$10 \ldots$
JUL
$21 \ldots$
AUG
$24 \ldots$
SEP
$22 \ldots$
OCT
$20 \ldots$
NOV
$17 \ldots$
MAR 1982
$26 \ldots$
APR
$18 \ldots$
MAY
$03 \ldots$
JUN
$01 \ldots$

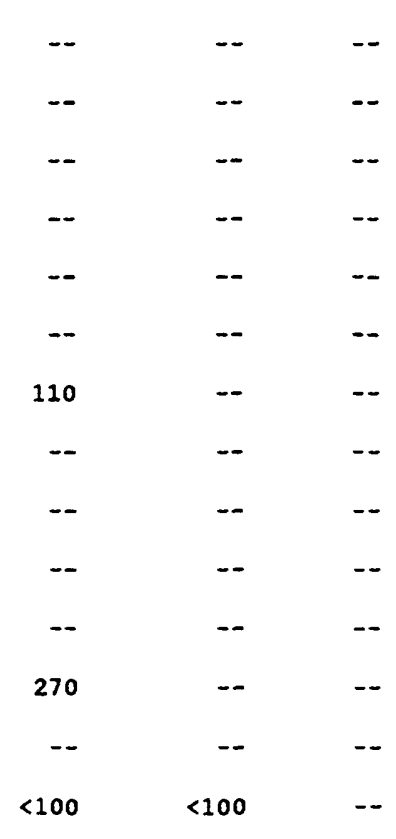

(

$\begin{array}{rrrr}- & 1 & -- & 400 \\ - & 3 & - & 90 \\ -- & 1 & -- & 120 \\ - & 1 & -- & 160 \\ - & 1 & -- & 130 \\ - & <10 & -- & 140 \\ -- & 6 & -- & 150 \\ -- & <8 & -- & 170 \\ -- & -- & -- & -- \\ -- & -- & -- & -- \\ -- & -- & -- & -- \\ -- & 1 & -- & 260 \\ -- & -- & -- & -- \\ -- & <1 & <1 & 80\end{array}$

DATE

\begin{tabular}{|c|c|c|c|c|c|c|c|c|}
\hline & $\begin{array}{l}\text { IRON, } \\
\text { DIS- } \\
\text { SOLVED } \\
\text { (UG/L } \\
\text { AS FE) }\end{array}$ & $\begin{array}{l}\text { LEAD, } \\
\text { TOTAL } \\
\text { RECOV- } \\
\text { ERABLE } \\
\text { (UG/L } \\
\text { AS PB) }\end{array}$ & $\begin{array}{l}\text { LEAD, } \\
\text { DIS- } \\
\text { SOLVED } \\
\text { (UG/L } \\
\text { AS PB) }\end{array}$ & $\begin{array}{l}\text { MERCURY } \\
\text { TOTAL } \\
\text { RECOV- } \\
\text { ERABLE } \\
\text { (UG/L } \\
\text { AS HG) }\end{array}$ & $\begin{array}{l}\text { ZINC, } \\
\text { TOTAL } \\
\text { RECOV- } \\
\text { ERABLE } \\
\text { (UG/L } \\
\text { AS ZN) }\end{array}$ & $\begin{array}{l}\text { ZINC, } \\
\text { DIS- } \\
\text { SOLVED } \\
\text { (UG/L } \\
\text { AS } 2 N)\end{array}$ & $\begin{array}{l}\text { SEDI- } \\
\text { MENT, } \\
\text { SUS- } \\
\text { PENDED } \\
\text { (MG/L) }\end{array}$ & $\begin{array}{l}\text { MENT, } \\
\text { DIS- } \\
\text { CHARGE, } \\
\text { SUS- } \\
\text { PENDED } \\
\text { (T/DAY) }\end{array}$ \\
\hline
\end{tabular}

FEB 1981

$23 .$.

MAR

$18 .$.

APR

$14 \ldots$

MAY

$15 . .$.

JUN

$10 \ldots$

JUL

AUG

24 ...

SEP

22...

OCT

20...

Nov

$17 .$.

MAR 1982

$26 \ldots$

APR

18 ...

MAY

03... JUN

$01 .$.
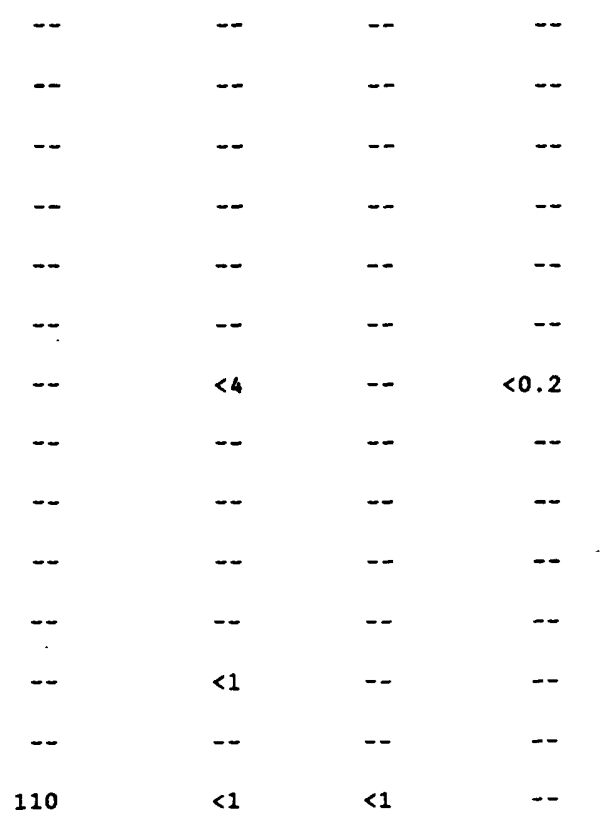

$\begin{array}{cccc}<3 & -- & 2 & 0.0 \\ 510 & -- & 4 & 0.0 \\ -- & -- & -- & -- \\ -- & -- & 4 & 0.0 \\ -- & -- & -- & -- \\ -- & -- & 1 & 0.0 \\ <4 & -- & -- & -- \\ <5 & -- & -- & -- \\ -- & -- & -- & -- \\ -- & -- & -- & -- \\ -- & -- & 2 & 0.0 \\ <8 & -- & 8 & 0.0 \\ -- & -- & 1 & 0.0 \\ <5 & <5 & -- & --\end{array}$


TABIB 4.--Physical characteristics and concentrations of commond chemical constituents in lakes and streams of the Bald Mountain watershed--Continued

01016480 GREENLAW STREAM AT GREENLAW CROSSING ME

\begin{tabular}{|c|c|c|c|c|c|c|c|c|c|c|}
\hline DATE & TIME & $\begin{array}{l}\text { STREAM- } \\
\text { FLOW, } \\
\text { INSTAN- } \\
\text { TANEOUS } \\
\text { (CFS) }\end{array}$ & $\begin{array}{l}\text { SPE- } \\
\text { CIFIC } \\
\text { CON- } \\
\text { DUCT- } \\
\text { ANCE } \\
\text { (US/CM) }\end{array}$ & $\begin{array}{c}\text { PH } \\
\text { (STAND- } \\
\text { ARD } \\
\text { UNITS) }\end{array}$ & $\begin{array}{l}\text { TEMPER- } \\
\text { ATURE } \\
\text { WATER } \\
\text { (DEG C) }\end{array}$ & $\begin{array}{l}\text { COLOR } \\
\text { (PLAT- } \\
\text { INUM- } \\
\text { COBALT } \\
\text { UNITS) }\end{array}$ & $\begin{array}{l}\text { TUR- } \\
\text { BID- } \\
\text { ITY } \\
\text { (FTU) }\end{array}$ & $\begin{array}{c}\text { OXYGEN, } \\
\text { DIS- } \\
\text { SOLVED } \\
\text { (MG/L) }\end{array}$ & $\begin{array}{l}\text { OXYGEN } \\
\text { DEMAND, } \\
\text { BIO- } \\
\text { CHEM- } \\
\text { ICAL, } \\
5 \text { DAY } \\
(M G / L)\end{array}$ & $\begin{array}{l}\text { COLI- } \\
\text { FORM, } \\
\text { FECAL, } \\
0.45 \\
\text { UM-MF } \\
\text { (COLS. } \\
100 \mathrm{ML})\end{array}$ \\
\hline $\begin{array}{c}\text { CT } 1982 \\
06 \ldots\end{array}$ & 1430 & -- & -- & 6.3 & 10.5 & 70 & 2.2 & 9.9 & -- & -- \\
\hline DATE & $\begin{array}{l}\text { ALRA- } \\
\text { LINITY } \\
\text { WAT WH } \\
\text { TOT FET } \\
\text { LAB } \\
\text { MG/L AS } \\
\text { CACO3 }\end{array}$ & $\begin{array}{l}\text { SOLIDS, } \\
\text { RESIDUE } \\
\text { AT } 105 \\
\text { DEG. C, } \\
\text { TOTAL } \\
\text { (MG/L) }\end{array}$ & $\begin{array}{l}\text { SOLIDS, } \\
\text { VOLA- } \\
\text { TILE ON } \\
\text { IGNI- } \\
\text { TION, } \\
\text { TOTAL } \\
\text { (MG/L) }\end{array}$ & $\begin{array}{l}\text { NITRO- } \\
\text { GEN, } \\
\text { NO2+NO3 } \\
\text { TOTAL } \\
\text { (MG/L } \\
\text { AS N) }\end{array}$ & $\begin{array}{l}\text { NITRO- } \\
\text { GEN, } \\
\text { AMMONIA } \\
\text { TOTAL } \\
\text { (MG/L } \\
\text { AS N) }\end{array}$ & $\begin{array}{l}\text { NITRO- } \\
\text { GEN, } \\
\text { ORGANIC } \\
\text { TOTAL } \\
\text { (MG/L } \\
\text { AS N) }\end{array}$ & $\begin{array}{l}\text { NITRO- } \\
\text { GEN,AM- } \\
\text { MONIA + } \\
\text { ORGANIC } \\
\text { TOTAL } \\
\text { (MG/L } \\
\text { AS N) }\end{array}$ & $\begin{array}{l}\text { NITRO- } \\
\text { GEN, } \\
\text { TOTAL } \\
\text { (MG/L } \\
\text { AS N) }\end{array}$ & $\begin{array}{l}\text { NITRO- } \\
\text { GEN, } \\
\text { TOTAL } \\
\text { (MG/L } \\
\text { AS NO3) }\end{array}$ & $\begin{array}{l}\text { PHOS- } \\
\text { PHOROUS } \\
\text { TOTAL } \\
\text { (MG/L } \\
\text { AS P) }\end{array}$ \\
\hline
\end{tabular}

OCT 1982

$06 .$.

1694 
TAmB 4.--Physlcal characteristics and concentrations of commond chemical constituents In lakes and streams of the Bald Mountain watershed--Continued

01016480 GREENLAW STREAM AT GREENLAW CROSSING ME

DATE

OCT 1982

$06 . .$.

DATE

OCT 1982

$06 .$.
ALUM-

INUM, ALUM-

TOTAL INUM,

RECOV - DIS-

AS AL)

SOLVED

(UG/I

AS AL)

AS AS)

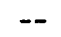

$--$

$--$

LEAD

TOTAI

DIS- RECOV-

SOLVED ERABLE

(UG/L (UG/L

AS FE)

AS PB)

LEAD,

DIS-

SOLVED

(UG/L

AS PB)

CADMIUM
TOTAL
RECOV-
ERABLE
(UG /L
AS CD)

CHRO-

MIUM, COPPER,

TOTAL TOTAL

RECOV-

ERABLE

(UG/L

AS CR)

RECOV-

ERABLE

(UG/L

AS CU)

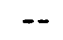

$<1$

250

$-$

$-$

$<1$

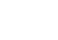

SOLVED ERABLE

(UG/L

(UG/L

AS FE)

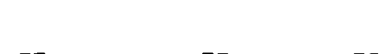

$\begin{array}{ll}\text { MERCURY } & \text { ZINC, } \\ \text { TOTAL } & \text { TOTAL } \\ \text { RECOV- } & \text { RECOV- } \\ \text { ERABLE } & \text { ERABLE } \\ \text { (UG/L } & \text { (UG/L } \\ \text { AS HG) } & \text { AS ZN) }\end{array}$

$\begin{array}{ll} & \\ \text { ZINC, } & \text { SEDI- } \\ \text { DIS- } & \text { MENT, } \\ \text { SOLVED } & \text { SUS- } \\ \text { (UG /L } & \text { PENDED } \\ \text { AS ZN) } & \text { (MG/L) }\end{array}$

SEDI-

MENT,

DIS-

CHARGE,

SUS-

PENDED

( $I$ /DAY)

$-$

$-$

$--$

6

3

0.0 


\begin{tabular}{|c|c|c|c|c|c|c|c|c|c|c|}
\hline DATE & TIME & $\begin{array}{l}\text { SAM- } \\
\text { PLING } \\
\text { DEPTH } \\
\text { (FEET) }\end{array}$ & $\begin{array}{l}\text { SPE- } \\
\text { CIFIC } \\
\text { CON- } \\
\text { DUCT- } \\
\text { ANCE } \\
\text { (US/CM) }\end{array}$ & $\begin{array}{c}\text { PH } \\
\text { (STAND- } \\
\text { ARD } \\
\text { UNITS) }\end{array}$ & $\begin{array}{l}\text { TEMPER- } \\
\text { ATURE } \\
\text { WATER } \\
\text { (DEG C) }\end{array}$ & $\begin{array}{l}\text { COLOR } \\
\text { (PLAT- } \\
\text { INUM- } \\
\text { COBALI } \\
\text { UNITS) }\end{array}$ & $\begin{array}{l}\text { TUR- } \\
\text { BID- } \\
\text { ITY } \\
\text { (FTU) }\end{array}$ & $\begin{array}{c}\text { OXYGEN, } \\
\text { DIS- } \\
\text { SOLVED } \\
\text { (MG/L) }\end{array}$ & $\begin{array}{l}\text { OXYGEN } \\
\text { DEMAND, } \\
\text { BIO- } \\
\text { CHEM- } \\
\text { ICAL, } \\
5 \text { DAY } \\
\text { (MG/L) }\end{array}$ & $\begin{array}{l}\text { COLI- } \\
\text { FORM, } \\
\text { FECAL, } \\
0.45 \\
\text { UM-MF } \\
\text { (COLS.I } \\
100 \mathrm{ML} \text { ) }\end{array}$ \\
\hline
\end{tabular}

\begin{tabular}{|c|c|c|c|c|c|c|c|c|c|c|}
\hline $\begin{array}{c}\text { JAN } 1980 \\
29 \ldots \\
\text { FEB }\end{array}$ & 1030 & -- & 56 & 7.0 & 0.0 & -- & 1.9 & -- & -- & -- \\
\hline FEB & & & & & & & & & & \\
\hline $19 \ldots$ & 1030 & -- & 53 & 6.7 & 0.0 & 70 & 1.0 & 13.2 & 0.8 & $<1$ \\
\hline $\begin{array}{l}\text { MAR } \\
20 \ldots\end{array}$ & 1030 & -- & 62 & 6.7 & 0.0 & 90 & -- & 127 & 5 & -- \\
\hline APR & (200 & & 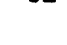 & s. & . & 0 & & 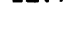 & $\cdot 0$ & \\
\hline$\underset{\operatorname{MAY}}{23} \ldots$ & 1030 & -- & 30 & 6.3 & 1.0 & 35 & 2.3 & 12.8 & 1.5 & $<1$ \\
\hline JUN & 1000 & -- & 30 & 6.6 & 13.0 & 40 & 1.4 & 10.3 & 1.3 & $<1$ \\
\hline${ }_{\text {JUL }}^{17 \ldots}$ & 1100 & -- & 30 & 6.7 & 16.0 & 45 & 2.0 & 10.2 & .7 & -- \\
\hline AUG $^{13} \ldots$ & 1100 & -- & 33 & 7.0 & 20.0 & 45 & 3.4 & 8.9 & -- & -- \\
\hline$\underset{S E P}{29 \ldots}$ & 1000 & -- & 40 & 6.4 & 22.0 & 35 & 0.9 & 8.6 & -- & - \\
\hline $\mathrm{OCT}^{29 \ldots}$ & 1000 & -- & 35 & 6.8 & 11.0 & 45 & 2.6 & 11.4 & - & - \\
\hline $\operatorname{Nov}^{14 \ldots}$ & 1000 & -- & 48 & 6.6 & 13.0 & 55 & 2.2 & 10.6 & -- & -- \\
\hline${ }_{\mathrm{DEC}}^{21} \cdots$ & 1000 & -- & 28 & 6.2 & 1.0 & 45 & 2.0 & 12.5 & -- & -- \\
\hline $\begin{array}{c}12 \ldots \\
\text { JAN } 1981\end{array}$ & 1015 & -- & 145 & 6.4 & 1.0 & 50 & 1.4 & 12.7 & - & -- \\
\hline${ }_{\text {FEB }}^{20} \ldots$ & 1030 & -- & 90 & 6.7 & 0.0 & 45 & 2.6 & 13.3 & $\cdots$ & -- \\
\hline $23 \ldots$ & 1715 & -- & 18 & -- & 2.0 & 15 & 2.6 & 12.2 & -- & - \\
\hline
\end{tabular}

\begin{tabular}{|c|c|c|c|c|c|c|c|c|c|}
\hline $\begin{array}{l}\text { ALKA- } \\
\text { LINITY } \\
\text { WAT WE } \\
\text { TOT FET } \\
\text { LAB } \\
\text { MG/L AS } \\
\text { CACO3 }\end{array}$ & $\begin{array}{l}\text { SOLIDS, } \\
\text { RESIDUE } \\
\text { AT } 105 \\
\text { DEG. C, } \\
\text { TOTAL } \\
\text { (MG/L) }\end{array}$ & $\begin{array}{l}\text { SOLIDS, } \\
\text { VOLA- } \\
\text { TILE ON } \\
\text { IGNI- } \\
\text { TION, } \\
\text { TOTAL } \\
\text { (MG/L) }\end{array}$ & $\begin{array}{l}\text { NITRO- } \\
\text { GEN, } \\
\text { NO2+NO3 } \\
\text { TOTAL } \\
\text { (MG/L } \\
\text { AS N) }\end{array}$ & $\begin{array}{l}\text { NITRO- } \\
\text { GEN, } \\
\text { AMYONIA } \\
\text { TOTAL } \\
\text { (MG/L } \\
\text { AS N) }\end{array}$ & $\begin{array}{l}\text { NITRO- } \\
\text { GEN, } \\
\text { ORGANIC } \\
\text { TOTAL } \\
\text { (MG/L } \\
\text { AS N) }\end{array}$ & $\begin{array}{l}\text { NIIRO- } \\
\text { GEN, AM- } \\
\text { MONIA + } \\
\text { ORGANIC } \\
\text { TOTAL } \\
\text { (MG/L } \\
\text { AS N) }\end{array}$ & $\begin{array}{l}\text { NITRO- } \\
\text { GEN, } \\
\text { TOTAL } \\
\text { (MG /L } \\
\text { AS N) }\end{array}$ & $\begin{array}{l}\text { NITRO- } \\
\text { GEN, } \\
\text { TOTAL } \\
\text { (MG/L } \\
\text { AS NO3) }\end{array}$ & $\begin{array}{l}\text { PHOS- } \\
\text { PHOROUS } \\
\text { TOTAL } \\
\text { (MG / } \\
\text { AS P) }\end{array}$ \\
\hline
\end{tabular}

JAN 1980

29.

FEB 19.

MAR

20 . .

23 ...

MAY

20 ...

Jun

17 ...

JUL

AUG

29... 10

SEP

29... 14

OCT

$14 \ldots 13$

Nov

21... 15

DEC

$12 \ldots$

JAN 1981

$20 \ldots 23$

EB

$23 \ldots$

$\begin{array}{ll}-- & - \\ 26 & - \\ -- & - \\ 40 & - \\ 74 & - \\ 68 & - \\ 37 & -- \\ 47 & -- \\ 58 & - \\ 68 & - \\ 42 & - \\ -- & - \\ -- & -\end{array}$

$\begin{array}{cccc}0.08 & -- & -- & \\ 0.0 & -- & 0.2 \\ .25 & 0.01 & 0.29 & .16 \\ .18 & .04 & .48 & .02 \\ .21 & .02 & .06 & .24\end{array}$

$0.2 \quad 0.28$

1.2

0.02

.3

.55

2.4

.01

.2

.38

1.7

.02

.5

.71

3.1

.02

.38

1.7

.02

.01

$<.01$

.04

.01

.01

.02

.01

.02 
TABI. 4. --Physical characteristics and concentrations of commond chemical constituents in lakes and streams of the Bald Mountain watershed--Continued

464308068461100 CLAYTON LARE IN TOWNSHIP 12 RANGE 8 ME

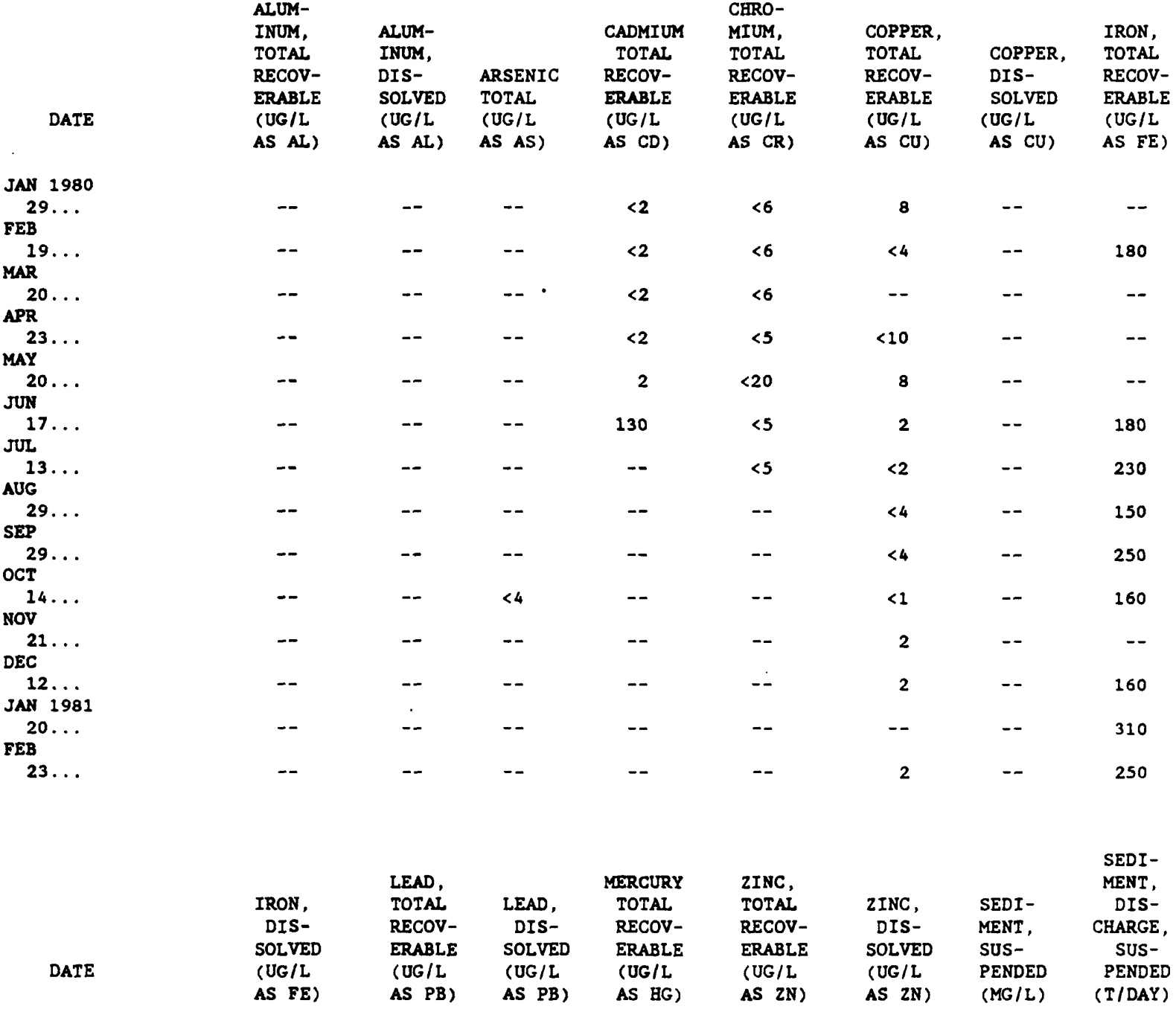

\footnotetext{
JAN 1980

$29 .$.

FEB

19...

MAR

$20 \ldots$

APR

23...

MAY

20...

JUN

17 . .

JUL.

$13 .$.

AUG

29 ...

SEP

29...

ocr

$14 \ldots$

Nov

$21 \ldots$

DEC

$12 \ldots$

JAN 1981

$20 .$.

FEB

23...
}

\begin{tabular}{|c|c|c|c|c|}
\hline 0.2 & 59 & -- & -- & -- \\
\hline .2 & 5 & -- & -- & -- \\
\hline$<.2$ & -- & -- & -- & -- \\
\hline-- & 8 & -- & $<1$ & -- \\
\hline-- & 51 & -- & 1 & 0.0 \\
\hline$<.2$ & 3 & -- & 3 & 0.0 \\
\hline-- & 3 & -- & -- & -- \\
\hline-- & 11 & -- & -- & -- \\
\hline-- & 2 & -- & -- & -- \\
\hline-- & $<5$ & -- & -- & -- \\
\hline-- & -- & -- & -- & -- \\
\hline-- & 6 & -- & -- & -- \\
\hline-- & 16 & $=-$ & -- & -- \\
\hline-- & 8 & -- & 1 & 0.0 \\
\hline
\end{tabular}


TABIB 4.--Physical characteristics and concentrations of commond chemical constituents in lakes and streams of the Bald Mountain watershed--Continued

464308068461100 CLAYTON LAKE IN TOWNSHIP 12 RANGE 8 ME

\begin{tabular}{|c|c|c|c|c|c|c|c|c|c|c|}
\hline DATE & TIME & $\begin{array}{l}\text { SAM- } \\
\text { PLING } \\
\text { DEPTH } \\
\text { (EEET) }\end{array}$ & $\begin{array}{l}\text { SPE- } \\
\text { CIFIC } \\
\text { CON- } \\
\text { DUCT- } \\
\text { ANCE } \\
\text { (US/CM) }\end{array}$ & $\begin{array}{c}\text { PH } \\
\text { (STAND- } \\
\text { ARD } \\
\text { UNITS) }\end{array}$ & $\begin{array}{l}\text { TEMPER- } \\
\text { ATURE } \\
\text { WATER } \\
\text { (DEG C) }\end{array}$ & $\begin{array}{l}\text { COLOR } \\
\text { (PLAT- } \\
\text { INUM- } \\
\text { COBALT } \\
\text { UNITS) }\end{array}$ & $\begin{array}{l}\text { TUR- } \\
\text { BID- } \\
\text { ITY } \\
\text { (ETU) }\end{array}$ & $\begin{array}{c}\text { OXYGEN, } \\
\text { DIS- } \\
\text { SOLVED } \\
\text { (MG/L) }\end{array}$ & $\begin{array}{l}\text { OXYGEN } \\
\text { DEMAND, } \\
\text { BIO- } \\
\text { CHEM- } \\
\text { ICAL, } \\
5 \text { DAY } \\
\text { (MG/L) }\end{array}$ & $\begin{array}{l}\text { COLI- } \\
\text { FORM, } \\
\text { FECAL, } \\
0.45 \\
\text { UM-MF } \\
\text { (COLS. I } \\
100 \mathrm{ML} \text { ) }\end{array}$ \\
\hline
\end{tabular}

\begin{tabular}{|c|c|c|c|c|c|c|c|c|c|}
\hline${ }_{A P R}^{19 \ldots}$ & 1515 & -- & 29. & 6.5 & 0.0 & 35 & 1.9 & 13.0 & -- \\
\hline $\operatorname{MAY}^{14 \ldots}$ & 1000 & - & 20 & 6.1 & 0.0 & 45 & 2.2 & 12.0 & -- \\
\hline${ }_{\text {JuN }}^{15}$ & 1000 & -- & 32 & 6.7 & 12.0 & 60 & 1.6 & 10.2 & -- \\
\hline $\mathrm{JUL}^{10 \ldots}$ & 0930 & -- & 31 & 6.7 & 14.0 & 45 & 1.8 & 9.2 & -- \\
\hline${ }_{A U G}^{21} \cdots$ & 0930 & -- & 42 & 6.7 & -- & 45 & 1.2 & -- & -- \\
\hline SEP & 1250 & $\ldots$ & 32 & 6.2 & 19.0 & 100 & 1.4 & 8.3 & -- \\
\hline $\mathrm{OCT}^{22} \cdots$ & 1500 & $\ldots$ & 30 & 7.0 & 14.0 & 90 & 1.6 & 9.1 & -- \\
\hline $\mathrm{NOV}^{30} \ldots$ & 1500 & -- & -- & 6.3 & 7.0 & 90 & 2.2 & 13.2 & -- \\
\hline $17 \ldots$ & 1435 & -- & 59 & 6.1 & 3.0 & 50 & 1.8 & 10.4 & -- \\
\hline
\end{tabular}

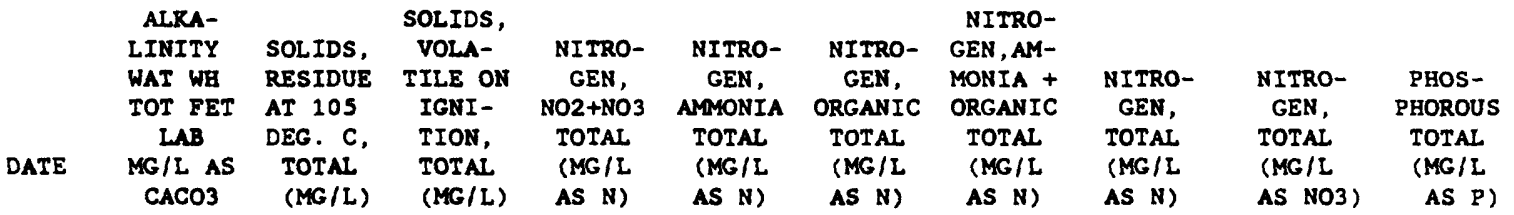

MAR 1981

$19 .$.

APR

14 ...

MAY

15...

JUN

$10 .$.

JUL

21...

AUG

24 ...

SEP

22 ..

OCT

$30 .$.

NOV

$17 .$.

$\begin{array}{rr}11 & 29 \\ 6 & 4 \\ 10 & 9 \\ 13 & 6 \\ 12 & 5 \\ 7 & 53 \\ 8 & 6 \\ 6 & 5 \\ 9 & 78\end{array}$

\begin{tabular}{|c|c|c|c|c|c|c|c|c|}
\hline 29 & -- & -- & -- & -- & -- & -- & -- & 0.01 \\
\hline 41 & -- & -- & -- & -- & -- & -- & -- & .01 \\
\hline 96 & -- & -- & -- & -- & -- & -- & -- & .01 \\
\hline 66 & - & -- & -- & - & -- & - & -- & .02 \\
\hline 53 & -- & -- & - & - & - & -- & -- & .01 \\
\hline 53 & -- & -- & -- & -- & -- & -- & -- & - \\
\hline 64 & -- & -- & -- & -- & -- & -- & -- & $=$ \\
\hline 54 & -- & -- & -- & -- & -- & - & -- & - \\
\hline 78 & -- & -- & -- & - & -- & -- & - & .01 \\
\hline
\end{tabular}


TABIE 4.--Physical characteristics and concentrations of commond chemical constituents in lakes and streams of the Bald Mountain watershed--Continued

464308068461100 CLAYTON LAKE IN TOWNSHIP 12 RANGE 8 ME

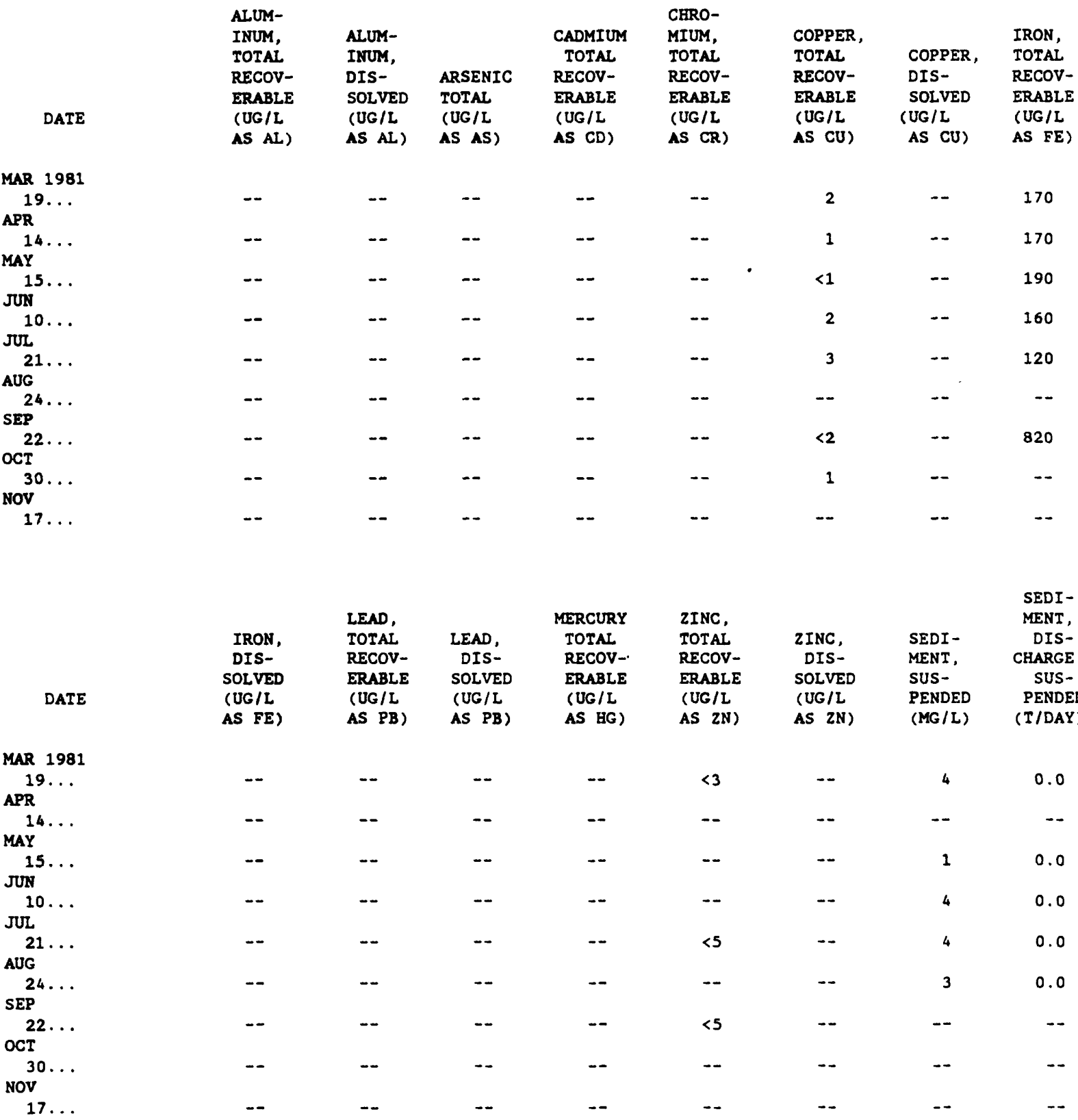


TAIT 4.--Physical characteristics and concentrations of commond chemical constituents In lakes and streans of the Bald Mountain watershed--Continued

464533068430100 CARR POND IN TOWNSEIP 13 RANGE 8 ME

WATER QUALITY DATA

\begin{tabular}{|c|c|c|c|c|c|c|c|c|c|c|}
\hline DATE & TIME & $\begin{array}{l}\text { SAM- } \\
\text { PLING } \\
\text { DEPTH } \\
\text { (EEET) }\end{array}$ & $\begin{array}{l}\text { SPE- } \\
\text { CIFIC } \\
\text { CON- } \\
\text { DUCT- } \\
\text { ANCE } \\
\text { (US/CM) }\end{array}$ & $\begin{array}{c}\text { PH } \\
\text { (STAND- } \\
\text { ARD } \\
\text { (NNITS) }\end{array}$ & $\begin{array}{l}\text { TEMPER- } \\
\text { ATURE } \\
\text { WATER } \\
\text { (DEG C) }\end{array}$ & $\begin{array}{l}\text { COLOR } \\
\text { (PLAT- } \\
\text { INUM- } \\
\text { COBALT } \\
\text { UNITS) }\end{array}$ & $\begin{array}{l}\text { TUR- } \\
\text { BID- } \\
\text { ITY } \\
\text { (FTU) }\end{array}$ & $\begin{array}{c}\text { OXYGEN, } \\
\text { DIS- } \\
\text { SOLVED } \\
\text { (MG/L) }\end{array}$ & $\begin{array}{l}\text { OXYGEN } \\
\text { DEMAND, } \\
\text { BIO- } \\
\text { CHEM- } \\
\text { ICAL, } \\
5 \text { DAY } \\
(M G / L)\end{array}$ & $\begin{array}{l}\text { COLI- } \\
\text { FORM, } \\
\text { FECAL, } \\
0.45 \\
\text { UM-MF } \\
\text { (COLS. I } \\
100 \mathrm{ML})\end{array}$ \\
\hline JUN 1979 & & & & & & & & & & \\
\hline $\begin{array}{l}12 \ldots \\
26 \ldots\end{array}$ & 1100 & -- & 45 & 7.4 & 16.0 & -- & 0.7 & 9.8 & 0.9 & -- \\
\hline $\mathrm{Ju}^{26 \ldots}$ & 1030 & -- & 32 & 7.1 & 20.0 & 35 & 1.0 & 9.8 & .7 & -- \\
\hline $17 \ldots$ & 1030 & -- & 22 & 7.1 & 23.0 & -- & .6 & 9.0 & .6 & -- \\
\hline AUG $24 \ldots$ & 1100 & -- & 36 & -- & 26.0 & 30 & 1.2 & 8.7 & .6 & -- \\
\hline$\underset{\text { SEP }}{07 . .}$ & 1045 & -- & 37 & 7.0 & 24.0 & 40 & -- & 8.0 & $<.1$ & -- \\
\hline $04 \ldots$ & 1030 & -- & 70 & 7.1 & 20.0 & 40 & 1.3 & 9.1 & .4 & -- \\
\hline $\mathrm{OCT}^{18 \ldots}$ & 1015 & -- & 36 & 6.8 & 16.0 & 40 & 1.2 & 9.7 & -- & -- \\
\hline $\begin{array}{l}22 \\
\text { JAN } 1980\end{array}$ & 1300 & -- & 57 & 6.7 & 12.0 & 35 & 1.0 & 9.8 & .8 & -- \\
\hline$\underset{F E B}{29 \ldots}$ & 1400 & -- & 71 & 7.1 & 0.0 & -- & 1.1 & -- & -- & -- \\
\hline $\operatorname{MAR}^{19 \ldots}$ & 1400 & -- & 74 & 6.7 & 0.0 & 40 & .6 & 12.8 & .6 & $<1$ \\
\hline$\underset{A P R}{20 \ldots}$ & 1300 & -- & 70 & 6.7 & 0.0 & 35 & -- & 14.0 & 1.0 & -- \\
\hline${ }_{\mathrm{MAY}}^{23} \ldots$ & 1300 & -- & 12 & 6.7 & 2.0 & 20 & 1.9 & 12.6 & .9 & -- \\
\hline${ }_{\text {JUN }}^{20} \cdots$ & 1445 & -- & 45 & 7.1 & 16.0 & 25 & 1.0 & 10.5 & 1.0 & $<1$ \\
\hline${ }_{\text {JUL }}^{17 \ldots}$ & 1530 & -- & 48 & 7.1 & 17.0 & 35 & 1.6 & 10.5 & 1.4 & -- \\
\hline $13 \ldots$ & 1443 & -- & 45 & 7.1 & 20.0 & 20 & 1.5 & 9.0 & -- & -- \\
\hline DATE & $\begin{array}{l}\text { ALRA- } \\
\text { LINITY } \\
\text { WAT WH } \\
\text { TOT FET } \\
\text { LAB } \\
\text { MG/L AS } \\
\text { CACO3 }\end{array}$ & $\begin{array}{l}\text { SOLIDS, } \\
\text { RESIDUE } \\
\text { AT } 103 \\
\text { DEG. C, } \\
\text { TOTAL } \\
\text { (MG/L) }\end{array}$ & $\begin{array}{l}\text { SOLIDS, } \\
\text { VOLA- } \\
\text { TILE ON } \\
\text { IGNI- } \\
\text { TION, } \\
\text { TOTAL } \\
\text { (MG/L) }\end{array}$ & $\begin{array}{l}\text { NITRO- } \\
\text { GEN, } \\
\text { NO2+NO3 } \\
\text { TOTAL } \\
\text { (MG/L } \\
\text { AS N) }\end{array}$ & $\begin{array}{l}\text { NITRO- } \\
\text { GEN, } \\
\text { AMMONIA } \\
\text { TOTAL } \\
\text { (MG /L } \\
\text { AS N) }\end{array}$ & $\begin{array}{l}\text { NITRO- } \\
\text { GEN, } \\
\text { ORGANIC } \\
\text { TOTAL } \\
\text { (MG/L } \\
\text { AS N) }\end{array}$ & $\begin{array}{l}\text { NITRO- } \\
\text { GEN, AM- } \\
\text { MONIA + } \\
\text { ORGANIC } \\
\text { TOTAL } \\
\text { (MG/L } \\
\text { AS N) }\end{array}$ & $\begin{array}{l}\text { NITRO- } \\
\text { GEN, } \\
\text { TOTAI } \\
\text { (MG/L } \\
\text { AS N) }\end{array}$ & $\begin{array}{l}\text { NITRO- } \\
\text { GEN, } \\
\text { TOTAL } \\
\text { (MG/L } \\
\text { AS NO3) }\end{array}$ & $\begin{array}{l}\text { PHOS- } \\
\text { PHOROUS } \\
\text { TOTAL } \\
\text { (MG / } \\
\text { AS P) }\end{array}$ \\
\hline JUN 1979 & & & & & & & & & & \\
\hline $12 \ldots$ & 10 & -- & -- & -- & -- & -- & -- & -- & -- & 0.01 \\
\hline $26 \ldots$ & 13 & -- & -- & 0.01 & 0.02 & -- & -- & -- & -- & .01 \\
\hline JoL & & & & & & & & & & \\
\hline $17 \ldots$ & 19 & -- & -- & .03 & .01 & 0.09 & 0.1 & 0.13 & 0.58 & .01 \\
\hline $24 \ldots$ & 17 & -- & -- & .01 & .01 & -- & -- & -- & -- & .01 \\
\hline AUG & & & & & & & & & & \\
\hline $\begin{array}{l}07 \ldots \\
\text { SEP }\end{array}$ & 16 & -- & -- & .01 & .01 & -- & -- & -- & -- & .02 \\
\hline $04 \ldots$ & 16 & -- & -- & $<.01$ & $<.01$ & -- & -- & -- & -- & -- \\
\hline $\mathrm{OCT}^{18 \ldots}$ & 28 & -- & -- & .01 & $<.01$ & -- & -- & -- & -- & .01 \\
\hline $\begin{array}{c}22 \ldots \\
\text { JAN } 1980\end{array}$ & 16 & -- & -- & .03 & .01 & -- & -- & -- & -- & .02 \\
\hline FEB & -- & -- & -- & -- & -- & -- & -- & -- & -- & .01 \\
\hline $\operatorname{MAR}^{19 \ldots}$ & 21 & -- & -- & .06 & -- & -- & $<.1$ & -- & -- & .01 \\
\hline$\underset{A P R}{20 \ldots}$ & 1 & -- & -- & .35 & .07 & .53 & .6 & .95 & 4.2 & $<.01$ \\
\hline$\underset{\operatorname{MAY}}{23} \cdots$ & 3 & -- & -- & .07 & .02 & .18 & .2 & .27 & 1.2 & .01 \\
\hline JUN $20 \ldots$ & 14 & -- & -- & .11 & $<.01$ & -- & .2 & .31 & 1.4 & .02 \\
\hline${ }_{J u}^{17 \ldots}$ & 13 & -- & -- & .08 & $<.01$ & -- & .1 & .18 & .8 & .02 \\
\hline $13 \ldots$ & 15 & -- & -- & -- & -- & -- & -- & -- & -- & .02 \\
\hline
\end{tabular}


rame 4.--Physical characteristics and concentrations of commond chemical constituents in lakes and streams of the Bald Mountain watershed--Continued

464553068430100 CARR POND IN TOWNSHIP 13 RANGE 8 ME

WATER QUALITY DATA

\begin{tabular}{|c|c|c|c|c|c|c|c|c|}
\hline DATE & $\begin{array}{l}\text { ALUM- } \\
\text { INUM, } \\
\text { TOTAL } \\
\text { RECOV- } \\
\text { ERABLE } \\
\text { (UG/L }\end{array}$ & $\begin{array}{l}\text { ALUM- } \\
\text { INUM, } \\
\text { DIS- } \\
\text { SOLVED } \\
\text { (UG/L }\end{array}$ & $\begin{array}{l}\text { ARSENIC } \\
\text { TOTAL } \\
\text { (UG/L }\end{array}$ & $\begin{array}{l}\text { CADMIUM } \\
\text { TOTAL } \\
\text { RECOV- } \\
\text { ERABLE } \\
\text { (UG/L }\end{array}$ & $\begin{array}{l}\text { CHRO- } \\
\text { MIUM, } \\
\text { TOTAL } \\
\text { RECOV- } \\
\text { ERABLE } \\
\text { (UG/L }\end{array}$ & $\begin{array}{l}\text { COPPER, } \\
\text { TOTAL } \\
\text { RECOV- } \\
\text { ERABLE } \\
\text { (UG/L }\end{array}$ & $\begin{array}{l}\text { COPPER, } \\
\text { DIS- } \\
\text { SOLVED } \\
\text { (UG/L }\end{array}$ & $\begin{array}{l}\text { IRON, } \\
\text { TOTAL } \\
\text { RECOV- } \\
\text { ERABLE } \\
\text { (UG/L }\end{array}$ \\
\hline & AS $\mathbf{A L}$ ) & AS $\mathbf{A L}$ ) & AS AS) & AS CD) & AS CR) & AS CU) & As CU) & AS $F E$ ) \\
\hline
\end{tabular}

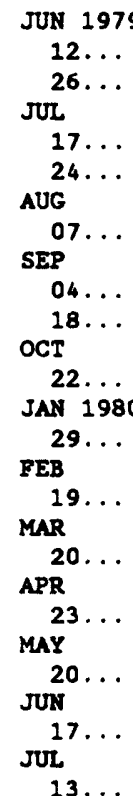

DATE

$\begin{array}{lll}-- & -- & - \\ -- & - & - \\ -- & - & - \\ -- & -- & - \\ -- & - & - \\ - & - & - \\ -- & - & - \\ -- & -- & - \\ -- & -- & - \\ -- & -- & - \\ -- & -- \\ -- & -- \\ -- & -\end{array}$

--
--
-
-
--
--
--
--
-
-
-
-
-

--
--
--

$\begin{array}{ll}<2 & <6 \\ -- & -\end{array}$

$<2$

$$
<6
$$$$
<2
$$

$<2$

$<6$

$<6$

$<2$

$<2$

$<6$

$<2$

$<5$

$<20$

$<5$

$<5$

$<5$

$<4$

$--\quad 50$

6

$-$

$<4$

$-$

80

$<2$

$<4$
$<4$
$<4$
--
$<10$
$<4$
$<2$
$<2$

2 INC,
DIS-
SOLVED
(UG/L
AS $\mathrm{ZN}$ )

$--$

--

$--\quad 60$

--

$--\quad 60$

$+-\quad \quad-$

--

--

$--\quad 60$

$--\quad<5$

40

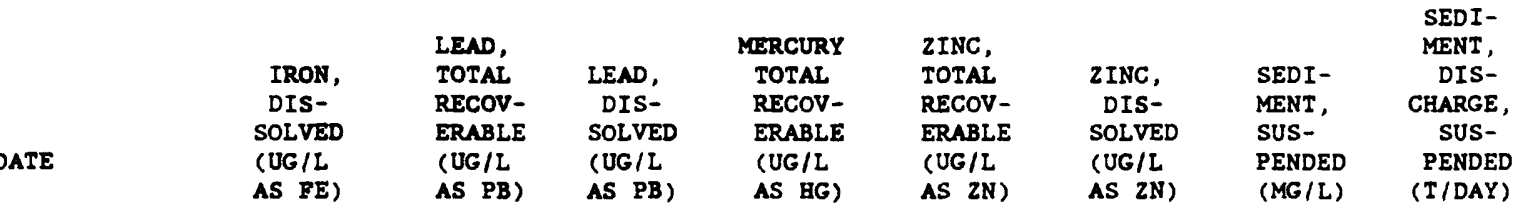

JUN 1979

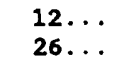

JUL.

$17 .$.

$24 \ldots$

AUG

$07 . .$.

SEP

$04 \ldots$

$18 .$.

OCT

22 ...

JAN 1980

$29 .$.

FEB

$19 .$.

MAR

20...

APR

23...

MAY

$20 .$.

JUN

$17 . .$.

$\begin{array}{lll}-- & -- & - \\ -- & -- & - \\ -- & <20 & - \\ -- & -- & - \\ -- & 310 & -- \\ -- & -- & -- \\ -- & -- & -- \\ -- & 70 & -- \\ -- & <20 & - \\ -- & <20 & -- \\ -- & <20 & - \\ -- & <30 & -- \\ -- & <30 & - \\ -- & <30 & -- \\ -- & -- & --\end{array}$

$--$

$-$

0.3

--

2

$\begin{array}{lr}-- & <1 \\ -- & 3\end{array}$

0.0

JUL

13... 
TA.T. 4.--Physical characteristics and concentrations of commond chemical constituents in lakes and streans of the Bald Mountain watershed--Continued

464553068430100 CARR POND IN TOWNSHIP 13 RANGE $8 \mathrm{ME}$

WATER QUALITY DATA

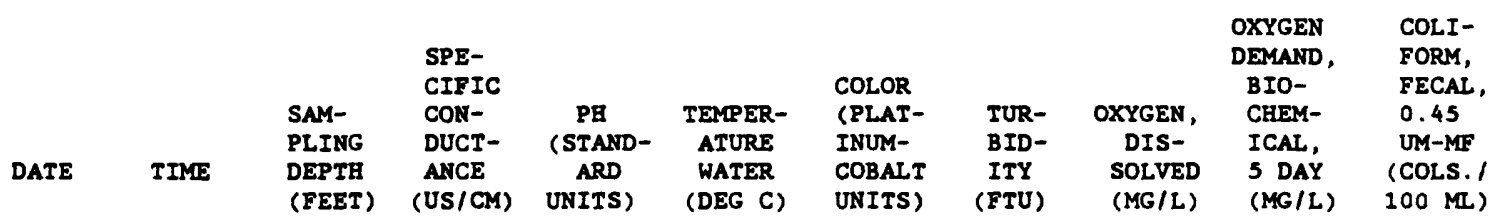

\begin{tabular}{|c|c|c|c|c|c|c|c|c|c|c|}
\hline $\operatorname{SEP}^{29 \ldots}$ & 1415 & -- & 48 & 7.4 & -- & 25 & 0.8 & 8.8 & -- & -- \\
\hline $\begin{array}{l}29 \ldots \\
\text { oct }\end{array}$ & 1415 & -- & 48 & 6.9 & 14.0 & 25 & 1.2 & 10.8 & -- & -- \\
\hline $\operatorname{NOV}^{14 \ldots}$ & 1400 & -- & 50 & 6.9 & 8.0 & 25 & .9 & 10.3 & -- & -- \\
\hline DEC $21 \cdots$ & 1415 & -- & 65 & 7.0 & 1.0 & 30 & 1.2 & 12.4 & -- & -- \\
\hline $\begin{array}{l}12 \ldots \\
\text { JAN } 1981\end{array}$ & 1530 & -- & 125 & 6.8 & 1.0 & 40 & 1.0 & 12.2 & -- & -- \\
\hline $\begin{array}{l}20 \ldots \\
\mathrm{FBB}\end{array}$ & 1430 & -- & 95 & 6.6 & 0.0 & 35 & 1.1 & 13.3 & -- & -- \\
\hline $\operatorname{MAR}^{23} \cdots$ & 1430 & -- & 15 & 6.7 & 2.0 & 10 & 1.5 & 12.6 & -- & -- \\
\hline${ }_{A P R}^{18 \ldots}$ & 1415 & -- & 75 & 6.5 & 1.0 & 70 & 1.0 & 13.4 & -- & -- \\
\hline $\operatorname{MAY}^{14 \ldots}$ & 1445 & -- & 65 & 6.7 & 1.0 & 35 & 1.4 & 12.0 & -- & -- \\
\hline $\begin{array}{l}15 \ldots \\
\text { JUN }\end{array}$ & 1500 & -- & 38 & 7.0 & 9.0 & 30 & .6 & 10.6 & -- & -- \\
\hline JUL $^{10}$ & 1245 & -- & 45 & 7.1 & 12.0 & 20 & 1.1 & 10.3 & -- & -- \\
\hline $\operatorname{SEP}^{21} \cdots$ & 1245 & -- & 58 & 7.3 & 21.0 & 25 & 1.0 & -- & -- & -- \\
\hline OCT $22 \ldots$ & 1145 & -- & 31 & 7.1 & 14.0 & 50 & 1.5 & 8.4 & -- & -- \\
\hline $20 \ldots$ & 1145 & -- & 48 & 6.7 & 7.0 & 55 & 1.3 & 10.8 & -- & -- \\
\hline DATE & $\begin{array}{l}\text { ALKA- } \\
\text { LINITY } \\
\text { WAT WH } \\
\text { TOT FET } \\
\text { LAB } \\
\text { MG/L AS } \\
\text { CACO3 }\end{array}$ & $\begin{array}{l}\text { SOLIDS, } \\
\text { RESIDUE } \\
\text { AT } 105 \\
\text { DEG. C, } \\
\text { TOTAL } \\
\text { (MG/L) }\end{array}$ & $\begin{array}{l}\text { SOLIDS, } \\
\text { VOLA- } \\
\text { TILE ON } \\
\text { IGNI- } \\
\text { TION, } \\
\text { TOTAL } \\
\text { (MG/L) }\end{array}$ & $\begin{array}{l}\text { NITRO- } \\
\text { GEN, } \\
\text { NO2+NO3 } \\
\text { TOTAL } \\
\text { (MG/L } \\
\text { AS N) }\end{array}$ & $\begin{array}{l}\text { NITRO- } \\
\text { GEN, } \\
\text { AMMONIA } \\
\text { TOTAL } \\
\text { (MG/L } \\
\text { AS N) }\end{array}$ & $\begin{array}{l}\text { NITRO- } \\
\text { GEN, } \\
\text { ORGANIC } \\
\text { TOTAL } \\
\text { (MG/L } \\
\text { AS N) }\end{array}$ & $\begin{array}{l}\text { NITRO- } \\
\text { GEN, AM- } \\
\text { MONIA + } \\
\text { ORGANIC } \\
\text { TOTAL } \\
\text { (MG/L } \\
\text { AS N) }\end{array}$ & $\begin{array}{l}\text { NITRO- } \\
\text { GEN, } \\
\text { TOTAL } \\
\text { (MG/L } \\
\text { AS N) }\end{array}$ & $\begin{array}{l}\text { NITRO- } \\
\text { GEN, } \\
\text { TOTAL } \\
\text { (MG/L } \\
\text { AS NO3) }\end{array}$ & $\begin{array}{l}\text { PHOS- } \\
\text { PHOROUS } \\
\text { TOTAL } \\
\text { (MG/L } \\
\text { AS P) }\end{array}$ \\
\hline
\end{tabular}

AUG 1980

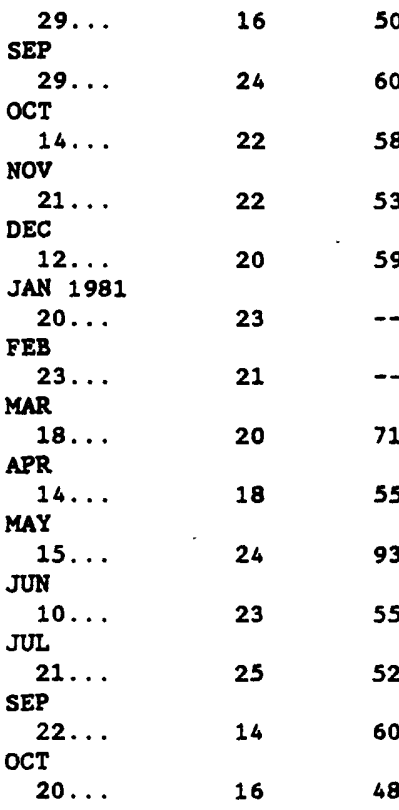


TABIB 4.--Physical characteristics and concentrations of commond chemical constituents In lakes and streams of the Bald Mountain watershed--Continued

464553068430100 CARR POND IN TOWNSHIP 13 RANGE 8 ME

WATER QUALITY DATA

\begin{tabular}{|c|c|c|c|c|c|c|c|c|}
\hline ATE & $\begin{array}{l}\text { ALUM- } \\
\text { INUM, } \\
\text { TOTAL } \\
\text { RECOV- } \\
\text { ERABLE } \\
\text { (UG/L }\end{array}$ & $\begin{array}{l}\text { ALUM- } \\
\text { INUM, } \\
\text { DIS- } \\
\text { SOLVED } \\
\text { (UG/L }\end{array}$ & $\begin{array}{l}\text { ARSENIC } \\
\text { TOTAL } \\
\text { (UG/L }\end{array}$ & $\begin{array}{l}\text { CADMIUM } \\
\text { TOTAL } \\
\text { RECOV- } \\
\text { ERABLE } \\
\text { (UG/L }\end{array}$ & $\begin{array}{l}\text { CERO- } \\
\text { MIUM, } \\
\text { TOTAL } \\
\text { RECOV- } \\
\text { ERABLE } \\
\text { (UG/L }\end{array}$ & $\begin{array}{l}\text { COPPER, } \\
\text { TOTAL } \\
\text { RECOV- } \\
\text { ERABLE } \\
\text { (UG/L }\end{array}$ & $\begin{array}{l}\text { COPPER, } \\
\text { DIS- } \\
\text { SOLVED } \\
\text { (UG/L }\end{array}$ & $\begin{array}{l}\text { IRON, } \\
\text { TOTAL } \\
\text { RECOV- } \\
\text { ERABLE } \\
\text { (UG /L }\end{array}$ \\
\hline & AS AL) & AS AL) & AS AS) & AS $(D)$ & AS CR) & AS CU) & AS (U) & AS FE) \\
\hline
\end{tabular}

$$
\begin{gathered}
\text { AUG } 1980 \\
29 \ldots \\
\text { SEP } \\
29 \ldots \\
\text { OCT } \\
14 \ldots \\
\text { NOV } \\
21 \ldots \\
\text { DEC } \\
12 \ldots \\
\text { JAN } 1981 \\
20 \ldots \\
\text { FEB } \\
23 \ldots \\
\text { MAR } \\
18 \ldots \\
\text { APR } \\
14 \ldots \\
\text { MAY } \\
15 \ldots \\
\text { JUN } \\
10 \ldots \\
\text { JUL } \\
21 \ldots \\
\text { SEP } \\
22 \ldots \\
\text { OCT } \\
20 \ldots \\
\end{gathered}
$$

\begin{tabular}{|c|c|c|}
\hline$<4$ & -- & 50 \\
\hline$<4$ & -- & 30 \\
\hline 8 & -- & 30 \\
\hline 6 & -- & $=$ \\
\hline 1 & -- & 90 \\
\hline-- & -- & 50 \\
\hline 2 & -- & 50 \\
\hline 1 & -- & 80 \\
\hline 1 & -- & 100 \\
\hline$<1$ & - & 80 \\
\hline 4 & - & 70 \\
\hline$<2$ & -- & 50 \\
\hline$<2$ & - & 80 \\
\hline 1 & -- & 80 \\
\hline
\end{tabular}

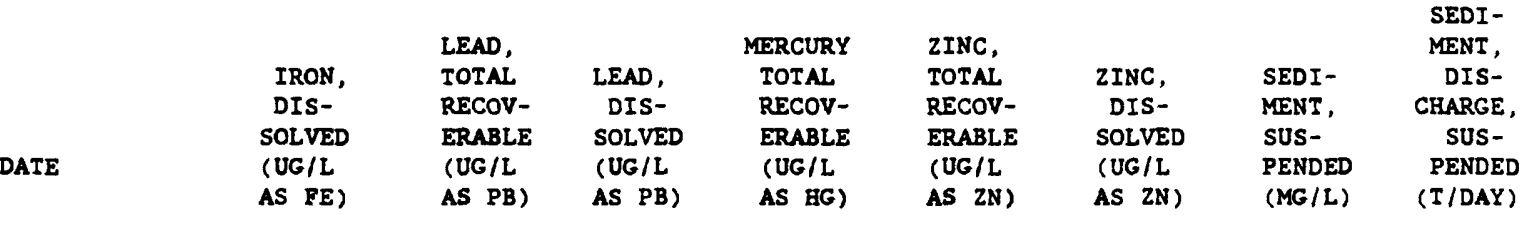

--

AUG 1980

$29 \ldots$
SEP
$29 \ldots$
OCT
$14 \ldots$
NOV
$21 \ldots$
DEC
$12 \ldots$
JAN 1981
$20 \ldots$
FEB
$23 \ldots$
MAR $\ldots$
$18 \ldots$
APR
$14 \ldots$
MAY
$15 \ldots$
JUN
$10 \ldots$
JUL
$21 \ldots$
SEP
$22 \ldots$
OCT
$20 \ldots$

$\begin{array}{lll}-- & - & - \\ -- & - & - \\ -- & - & - \\ -- & <2 & - \\ -- & -- & -- \\ -- & -- & -- \\ -- & -- & - \\ -- & -- \\ -- & -- \\ -- & -- & - \\ -- & - & -\end{array}$


IAIDE 4.--Physical characteristics and concentrations of commond chemical constituents in lakes and streams of the Bald Mountain watershed--Continued

464553068430100 CARR POND IN TOWNSHIP 13 RANGE 8 ME

WATER QUALITY DATA

\begin{tabular}{|c|c|c|c|c|c|c|c|c|c|c|}
\hline DATE & TIME & $\begin{array}{l}\text { SAM- } \\
\text { PLIKG } \\
\text { DEPTH } \\
\text { (EEET) }\end{array}$ & $\begin{array}{l}\text { SPE- } \\
\text { CIFIC } \\
\text { CON- } \\
\text { DUCT- } \\
\text { ANCE } \\
\text { (US/CM) }\end{array}$ & $\begin{array}{c}\text { PE } \\
\text { (STAND- } \\
\text { ARD } \\
\text { UNITS) }\end{array}$ & $\begin{array}{l}\text { TEMPER- } \\
\text { ATURE } \\
\text { WATER } \\
\text { (DEG C) }\end{array}$ & $\begin{array}{l}\text { COLOR } \\
\text { (PLAT- } \\
\text { INUM- } \\
\text { COBALT } \\
\text { UNITS) }\end{array}$ & $\begin{array}{l}\text { TUR- } \\
\text { BID- } \\
\text { ITY } \\
\text { (FTU) }\end{array}$ & $\begin{array}{c}\text { OXYGEN, } \\
\text { DIS- } \\
\text { SOLVED } \\
\text { (MG/L) }\end{array}$ & $\begin{array}{l}\text { OXYGEN } \\
\text { DEMAND, } \\
\text { BIO- } \\
\text { CBEM- } \\
\text { ICAL, } \\
5 \mathrm{DAY} \\
\text { (MG/L) }\end{array}$ & $\begin{array}{l}\text { COLI- } \\
\text { FORM, } \\
\text { FECAL, } \\
0.45 \\
\text { UM-MF } \\
\text { (COLS./ } \\
100 \mathrm{ML})\end{array}$ \\
\hline \multicolumn{11}{|l|}{ MAR 1982} \\
\hline $\begin{array}{l}26 \ldots \\
26 \ldots\end{array}$ & $\begin{array}{l}0800 \\
0810\end{array}$ & $\begin{array}{r}3.3 \\
49.2\end{array}$ & $\begin{array}{l}33 \\
33\end{array}$ & $\begin{array}{l}7.0 \\
6.9\end{array}$ & -- & $\begin{array}{l}35 \\
40\end{array}$ & $\begin{array}{r}0.5 \\
.6\end{array}$ & -- & -- & -- \\
\hline \multicolumn{11}{|l|}{ JUN } \\
\hline $\begin{array}{l}01 \ldots \\
01 \ldots\end{array}$ & $\begin{array}{l}1200 \\
1210\end{array}$ & $\begin{array}{r}1.0 \\
65.6\end{array}$ & $\begin{array}{l}48 \\
50\end{array}$ & $\begin{array}{l}6.8 \\
6.7\end{array}$ & $\begin{array}{l}2.0 \\
6.0\end{array}$ & $\begin{array}{l}35 \\
35\end{array}$ & $\begin{array}{r}.8 \\
1.3\end{array}$ & $\begin{array}{l}9.4 \\
9.2\end{array}$ & -- & -- \\
\hline DATE & $\begin{array}{l}\text { ALKA- } \\
\text { LINITY } \\
\text { WAT WR } \\
\text { TOT FET } \\
\text { LAB } \\
\text { MG/L AS } \\
\text { CACO3 }\end{array}$ & $\begin{array}{l}\text { SOLIDS, } \\
\text { RESIDUE } \\
\text { AT 105 } \\
\text { DEG. C, } \\
\text { TOTAL } \\
\text { (MG/L) }\end{array}$ & $\begin{array}{l}\text { SOLIDS, } \\
\text { VOLA- } \\
\text { TILE ON } \\
\text { IGNI- } \\
\text { TION, } \\
\text { TOTAL } \\
\text { (MG/L) }\end{array}$ & $\begin{array}{l}\text { NITRO- } \\
\text { GEN, } \\
\text { NO2+NO3 } \\
\text { TOTAL } \\
\text { (MG/L } \\
\text { AS N) }\end{array}$ & $\begin{array}{l}\text { NITRO- } \\
\text { GEN, } \\
\text { AMONIA } \\
\text { TOTAL } \\
\text { (MG/L } \\
\text { AS N) }\end{array}$ & $\begin{array}{l}\text { NITRO- } \\
\text { GEN, } \\
\text { ORGANIC } \\
\text { TOTAL } \\
\text { (MG/L } \\
\text { AS N) }\end{array}$ & $\begin{array}{l}\text { NITRO- } \\
\text { GEN, AM- } \\
\text { MONIA + } \\
\text { ORGANIC } \\
\text { TOTAL } \\
\text { (MG/L } \\
\text { AS N) }\end{array}$ & $\begin{array}{l}\text { NITRO- } \\
\text { GEN, } \\
\text { TOTAL } \\
\text { (MG/L } \\
\text { AS N) }\end{array}$ & $\begin{array}{l}\text { NITRO- } \\
\text { GEN, } \\
\text { TOTAL } \\
\text { (MG/L } \\
\text { AS NO3) }\end{array}$ & $\begin{array}{l}\text { PHOS- } \\
\text { PHOROUS } \\
\text { TOTAL } \\
\text { (MG/L } \\
\text { AS P) }\end{array}$ \\
\hline \multicolumn{11}{|l|}{ MAR 1982} \\
\hline $26 \ldots$ & 16 & 62 & -- & - & -- & -- & -- & -- & -- & -- \\
\hline Jus $26 \ldots$ & \multicolumn{9}{|c|}{ JUस } & -- \\
\hline $01 \ldots$ & 15 & 54 & -- & -- & -- & -- & -- & -- & -- & -- \\
\hline $01 \ldots$ & 12 & 86 & -- & -- & -- & -- & -- & -- & -- & -- \\
\hline
\end{tabular}


TABIB 4.--Physical characteristics and concentrations of commond chemical constituents in lakes and streams of the Bald Mountain watershed--Continued

464553068430100 CARR POND IN TOWNSHIP 13 RANGE 8 ME

WATER QUALITY DATA

\begin{tabular}{|c|c|c|c|c|c|c|c|c|}
\hline DATE & $\begin{array}{l}\text { ALUM- } \\
\text { INUM, } \\
\text { TOTAL } \\
\text { RECOV- } \\
\text { ERABLE } \\
\text { (UG/L }\end{array}$ & $\begin{array}{l}\text { ALUM- } \\
\text { INUM, } \\
\text { DIS- } \\
\text { SOLVED } \\
\text { (UG/I }\end{array}$ & $\begin{array}{l}\text { ARSENIC } \\
\text { TOTAL } \\
\text { (UG/L }\end{array}$ & $\begin{array}{l}\text { CADMIUM } \\
\text { TOTAL } \\
\text { RECOV- } \\
\text { ERABLE } \\
\text { (UG/L }\end{array}$ & $\begin{array}{l}\text { CHRO- } \\
\text { MIUM, } \\
\text { TOTAL } \\
\text { RECOV- } \\
\text { ERABLE } \\
\text { (UG/L }\end{array}$ & $\begin{array}{l}\text { COPPER, } \\
\text { TOTAL } \\
\text { RECOV- } \\
\text { ERABLE } \\
\text { (UG/L }\end{array}$ & $\begin{array}{l}\text { COPPER, } \\
\text { DIS- } \\
\text { SOLVED } \\
\text { (UG/L }\end{array}$ & $\begin{array}{l}\text { IRON, } \\
\text { TOTAL } \\
\text { RECOV- } \\
\text { ERABLE } \\
\text { (UG/L }\end{array}$ \\
\hline & AS AL). & AS $A L$ ) & AS AS) & AS $C D$ ) & AS CR) & AS CU) & AS CU) & AS EE) \\
\hline \multicolumn{9}{|l|}{ MAR 1982} \\
\hline $26 \ldots$ & -- & -- & -- & -- & -- & -- & -- & -- \\
\hline $26 \ldots$ & -- & -- & -- & -- & -- & -- & -- & -- \\
\hline \multicolumn{9}{|l|}{ JUN } \\
\hline $01 \ldots$ & $<100$ & $<100$ & -- & -- & -- & 2 & $<1$ & 60 \\
\hline $01 \ldots$ & $<100$ & $<100$ & -- & -- & -- & 6 & 4 & 70 \\
\hline DATE & $\begin{array}{l}\text { IRON, } \\
\text { DIS- } \\
\text { SOLVED } \\
\text { (UG/L } \\
\text { AS FE) }\end{array}$ & $\begin{array}{l}\text { LEAD, } \\
\text { TOTAL } \\
\text { RECOV- } \\
\text { ERABLE } \\
\text { (UG/L } \\
\text { AS PB) }\end{array}$ & $\begin{array}{l}\text { LEAD, } \\
\text { DIS- } \\
\text { SOLVED } \\
\text { (UG/I } \\
\text { AS PB) }\end{array}$ & $\begin{array}{l}\text { MERCURY } \\
\text { TOTAL } \\
\text { RECOV- } \\
\text { ERABLE } \\
\text { (UG/L } \\
\text { AS HC) }\end{array}$ & $\begin{array}{l}\text { ZINC, } \\
\text { TOTAL } \\
\text { RECOV- } \\
\text { ERABLE } \\
\text { (UG/L } \\
\text { AS ZN) }\end{array}$ & $\begin{array}{l}\text { ZINC, } \\
\text { DIS- } \\
\text { SOLVED } \\
\text { (UG/I }\end{array}$ & $\begin{array}{l}\text { SEDI- } \\
\text { MENT, } \\
\text { SUS- } \\
\text { PENDED }\end{array}$ & $\begin{array}{c}\text { SEDI- } \\
\text { MENT, } \\
\text { DIS- } \\
\text { CHARGE, } \\
\text { SUS- } \\
\text { PENDED }\end{array}$ \\
\hline \multicolumn{9}{|l|}{ MAR 1982} \\
\hline $26 \ldots$ & -- & -- & -- & -- & -- & -- & -- & -- \\
\hline $26 \ldots$ & -- & -- & -- & -- & -- & -- & 1 & 0.0 \\
\hline \multicolumn{9}{|l|}{ JUN } \\
\hline $01 \ldots$ & 50 & $<1$ & $<1$ & -- & $<5$ & $<5$ & -- & -- \\
\hline $01 \ldots$ & 60 & $<1$ & $<1$ & -- & $<5$ & 9 & -- & -- \\
\hline
\end{tabular}


TARE 4.--Physical characteristics and concentrations of commond chemical constituents in lakes and streams of the Bald Mountain watershed--Continued

464843068455200 FISH RIVER LARE IN TOWNSHIP 13 RANGE 8 ME

\begin{tabular}{|c|c|c|c|c|c|c|c|c|c|c|}
\hline DATE & TIME & $\begin{array}{l}\text { SAM- } \\
\text { PLING } \\
\text { DEPTH } \\
\text { (EEET) }\end{array}$ & $\begin{array}{l}\text { SPE- } \\
\text { CIFIC } \\
\text { CON- } \\
\text { DUCT- } \\
\text { ANCE } \\
\text { (US/CM) }\end{array}$ & $\begin{array}{c}\text { PB } \\
\text { (STAND- } \\
\text { ARD } \\
\text { UNITS) }\end{array}$ & $\begin{array}{l}\text { TEMPER- } \\
\text { ATURE } \\
\text { WATER } \\
\text { (DEG C) }\end{array}$ & $\begin{array}{l}\text { COLOR } \\
\text { (PLAT- } \\
\text { INUM- } \\
\text { COBALT } \\
\text { UNITS) }\end{array}$ & $\begin{array}{l}\text { TUR- } \\
\text { BID- } \\
\text { ITY } \\
\text { (ETU) }\end{array}$ & $\begin{array}{c}\text { OXYGEN, } \\
\text { DIS- } \\
\text { SOLVED } \\
\text { (MG/L) }\end{array}$ & $\begin{array}{l}\text { OXYGEN } \\
\text { DEMAND, } \\
\text { BIO- } \\
\text { CHEM- } \\
\text { ICAL, } \\
5 \text { DAY } \\
\text { (MG/L) }\end{array}$ & $\begin{array}{l}\text { COLI- } \\
\text { FORM, } \\
\text { FECAL, } \\
0.45 \\
\text { UM-MF } \\
\text { (COLS. / } \\
100 \mathrm{ML} \text { ) }\end{array}$ \\
\hline JUN 1979 & & & & & & & & & & \\
\hline JUL $^{26 \ldots}$ & 1300 & - & 42 & 7.0 & 20.0 & 35 & 1.8 & 9.5 & 0.5 & -- \\
\hline $17 \ldots$ & 1400 & -- & -- & 7.0 & 22.0 & -- & 3.3 & 9.4 & .5 & -- \\
\hline${ }_{A U G}^{24} \ldots$ & 1400 & -- & 46 & -- & 24.0 & 30 & 1.2 & 9.1 & .4 & -- \\
\hline $07 \ldots$ & 1400 & -- & 46 & 7.1 & 24.0 & 30 & -- & 7.9 & -- & -- \\
\hline$\underset{\operatorname{SEP}}{21} \cdots$ & 1300 & -- & 40 & 7.1 & 20.0 & 30 & 1.2 & 11.2 & 1.8 & -- \\
\hline $\mathrm{OCT}^{04 \ldots}$ & 1330 & -- & 47 & -- & 22.0 & 35 & 1.5 & 8.9 & .6 & -- \\
\hline $\operatorname{MAR} 1980$ & 1330 & -- & 50 & 6.5 & 10.0 & 40 & 1.5 & 10.4 & 1.0 & -- \\
\hline$\underset{A P R}{20 \ldots}$ & 1400 & -- & 40 & 4.5 & 0.0 & 30 & -- & 14.2 & 3.1 & -- \\
\hline $\operatorname{MAY}^{23} \ldots$ & 1400 & - & 29 & 6.7 & 4.0 & 25 & 2.9 & 12.6 & .5 & -- \\
\hline $\mathrm{JUL}^{20 \cdots}$ & 1540 & -- & 40 & 7.0 & 18.0 & 25 & 2.1 & 10.0 & 1.7 & -- \\
\hline${ }_{A U G}^{13 \ldots}$ & 1345 & -- & 42 & 6.7 & 20.0 & 30 & 1.3 & 9.7 & -- & -- \\
\hline$\underset{S E P}{29 \ldots}$ & 1515 & -- & 45 & 7.1 & 21.0 & 25 & 1.0 & 8.9 & -- & -- \\
\hline $\mathrm{OCT}^{29 \ldots}$ & 1515 & -- & 49 & 6.7 & 12.0 & 30 & 1.4 & 9.8 & -- & -- \\
\hline $\operatorname{Nov}^{14 \ldots}$ & 1515 & -- & 70 & 7.0 & 8.0 & 35 & 2.3 & 10.8 & -- & -- \\
\hline $21 \ldots$ & 1515 & -- & 40 & 6.8 & 1.0 & 25 & 1.1 & 13.2 & -- & -- \\
\hline DATE & $\begin{array}{l}\text { ALRA- } \\
\text { LINITY } \\
\text { WAT WH } \\
\text { TOT FET } \\
\text { LAB } \\
\text { MG/L AS } \\
\text { CACO3 }\end{array}$ & $\begin{array}{l}\text { SOLIDS, } \\
\text { RESIDUE } \\
\text { AT } 105 \\
\text { DEG. C, } \\
\text { TOTAL } \\
\text { (MG/L) }\end{array}$ & $\begin{array}{l}\text { SOLIDS, } \\
\text { VOLA- } \\
\text { TILE ON } \\
\text { IGNI- } \\
\text { TION, } \\
\text { TOTAL } \\
\text { (MG/L) }\end{array}$ & $\begin{array}{l}\text { NITRO- } \\
\text { GEN, } \\
\text { NO2+NO3 } \\
\text { TOTAL } \\
\text { (MG/L } \\
\text { AS N) }\end{array}$ & $\begin{array}{l}\text { NITRO- } \\
\text { GEN, } \\
\text { AMPONIA } \\
\text { TOTAL } \\
\text { (MG/L } \\
\text { AS N) }\end{array}$ & $\begin{array}{l}\text { NITRO- } \\
\text { GEN, } \\
\text { ORGANIC } \\
\text { TOTAL } \\
\text { (MG/L } \\
\text { AS N) }\end{array}$ & $\begin{array}{l}\text { NITRO- } \\
\text { GEN,AM- } \\
\text { MONIA + } \\
\text { ORGANIC } \\
\text { TOTAL } \\
\text { (MG/L } \\
\text { AS N) }\end{array}$ & $\begin{array}{l}\text { NITRO- } \\
\text { GEN, } \\
\text { TOTAL } \\
\text { (MG/L } \\
\text { AS N) }\end{array}$ & $\begin{array}{l}\text { NITRO- } \\
\text { GEN, } \\
\text { TOTAL } \\
\text { (MG/L } \\
\text { AS NO3) }\end{array}$ & $\begin{array}{l}\text { PHOS- } \\
\text { PHOROUS } \\
\text { TOTAL } \\
\text { (MG/L } \\
\text { AS P) }\end{array}$ \\
\hline JUN 1979 & & & & & & & & & & \\
\hline JUL $26 \ldots$ & 10 & - & - & $<0.01$ & 0.01 & -- & $\cdots$ & $\cdots$ & -- & 0.01 \\
\hline $17 \ldots$ & -- & -- & -- & $<.10$ & .34 & 0.06 & 0.4 & -- & -- & .05 \\
\hline AUG $^{24 \ldots}$ & 12 & - & -- & $<.10$ & .02 & -- & -- & -- & -- & .01 \\
\hline $07 \ldots$ & 11 & -- & -- & .01 & $<.01$ & -- & -- & -- & -- & .01 \\
\hline$\underset{\operatorname{SEP}}{21 \ldots}$ & 12 & -- & -- & .01 & .01 & -- & -- & -- & -- & .02 \\
\hline${ }_{O C T}{ }^{24 \ldots}$ & 12 & -- & - & .01 & .01 & -- & $\because-$ & -- & -- & .01 \\
\hline $\begin{array}{l}22 \ldots \\
\text { MAR } 1980\end{array}$ & 12 & -- & -- & .02 & .01 & -- & -- & -- & -- & .02 \\
\hline$\underset{A P R}{20 \ldots}$ & -- & -- & -- & .79 & .14 & .16 & .3 & 1.1 & 4.8 & .02 \\
\hline$\underset{\operatorname{MAY}}{23} \cdots$ & 17 & -- & -- & .12 & .02 & .18 & .2 & 0.32 & 1.4 & .01 \\
\hline $\mathrm{JUL}^{20 \ldots}$ & 10 & -- & -- & .01 & $<.01$ & -- & .2 & .21 & 0.93 & .02 \\
\hline${ }_{A U G}^{13} \cdots$ & 12 & -- & -- & -- & -- & -- & -- & -- & -- & .01 \\
\hline $\operatorname{SEP}^{29 \ldots}$ & 19 & 40 & -- & -- & -- & -- & -- & -- & -- & $<.01$ \\
\hline $\mathrm{OCT}^{29} \ldots$ & 21 & 43 & -- & -- & -- & -- & -- & -- & -- & .03 \\
\hline Nov $14 \ldots$ & 14 & 46 & -- & -- & -- & - & -- & -- & -- & .01 \\
\hline $21 \ldots$ & 43 & 58 & -- & -- & -- & -- & -- & -- & -- & .01 \\
\hline
\end{tabular}


IABx 4.-Physical characteristics and concentrations of commond chemical constituents in lakes and streams of the Bald Mountain watershed--Continued

464843068455200 FISH RIVER LAKE IN TOWNSHIP 13 RANGE 8 ME

JUN 1979

26 ..

JUL.

$17 \ldots$

$24 \ldots$

AUG

$07 \ldots$

$21 \ldots$

SEP

$04 \ldots$

OCT

22...

MAR 1980

20 ...

APR

$23 .$.

MAY

20...

JUL

$13 \ldots$

AUG

$29 \ldots$

SEP

29 ...

OCT

$14 \ldots$

NOV

21 ...

DATE

ALUM-

INUM, ALUM-

TOTAL INUM,

RECOV- DIS-

ERABLE SOLVED

(UG/L

AS AL)

(UG/L

AS AL)

ARSENIC

TOTAL

(UG/L

AS AS)

CADMIUM
TOTAC
RECOV-
ERABLE
(UG/L
AS CD)

CERO-

MIUM,

TOTAL

RECOV-

ERABLE

(UG/L

AS CR)

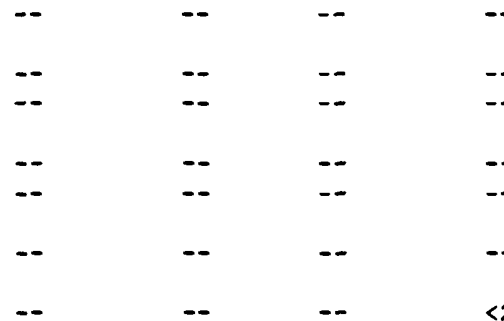

$\begin{array}{lll}- & - \\ -- & - \\ -- & - \\ -- & -\end{array}$

$<2$

$<6$

$<2$

$<6$

$<2$

$<20$

$\begin{array}{lll}\text { COPPER, } & & \text { IRON, } \\ \text { TOTAL } & \text { COPPER, } & \text { TOTAL } \\ \text { RECOV- } & \text { DIS- } & \text { RECOV- } \\ \text { ERABLE } & \text { SOLVED } & \text { ERABLE } \\ \text { (UG/L } & \text { (UG/L } & \text { (UG/L } \\ \text { AS CU) } & \text { AS CU) } & \text { AS FE) }\end{array}$

AS CU)

AS $F E$ )

$\begin{array}{lll}-- & -- & -- \\ -- & -- & -- \\ -- & -- & \\ <4 & -- & 90 \\ -- & -- & -- \\ -- & -- & -- \\ <4 & -- & 200\end{array}$

$-$

200

$<10$
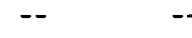

$<4$

$--$

4

40

$<4$

80

$<1$

90

3

\begin{tabular}{|c|c|c|c|c|c|c|c|}
\hline $\begin{array}{l}\text { IRON, } \\
\text { DIS- } \\
\text { SOLVED } \\
\text { (UG/L }\end{array}$ & $\begin{array}{l}\text { LEAD, } \\
\text { TOTAL } \\
\text { RECOV- } \\
\text { ERABLE } \\
\text { (UG / L }\end{array}$ & $\begin{array}{l}\text { LEAD, } \\
\text { DIS- } \\
\text { SOLVED } \\
\text { (UG/L }\end{array}$ & $\begin{array}{l}\text { MERCURY } \\
\text { TOTAL } \\
\text { RECOV- } \\
\text { ERABLE } \\
\text { SUG/L }\end{array}$ & $\begin{array}{l}\text { ZINC, } \\
\text { TOTAL } \\
\text { RECOV- } \\
\text { ERABLE } \\
\text { (UG /L }\end{array}$ & $\begin{array}{l}\text { ZINC, } \\
\text { DIS- } \\
\text { SOLVED } \\
\text { (UG/L }\end{array}$ & $\begin{array}{l}\text { SEDI- } \\
\text { MENT, } \\
\text { SUS- } \\
\text { PENDED }\end{array}$ & $\begin{array}{c}\text { SEDI- } \\
\text { MENT, } \\
\text { DIS- } \\
\text { CHARGE, } \\
\text { SUS- } \\
\text { PENDED }\end{array}$ \\
\hline AS FE) & AS PB) & AS PB) & AS HG) & AS $\mathrm{ZN}$ ) & AS $2 N$ ) & (MG/L) & (T/DAY) \\
\hline
\end{tabular}

JUN 1979

26 . .

गU.

$17 .$.

24 ...

AUG

$07 .$.

21 ...

SEP

$04 \ldots$

OCT

22 ...

MAR 1980

$20 \ldots$

APR

$23 .$.

MAX

20 ...

JiL

$13 .$.

AUG

29...

SEP

$29 . .$.

OCT

$14 \ldots$

21 ...

\begin{tabular}{|c|c|}
\hline-- & - \\
\hline-- & -- \\
\hline- & -- \\
\hline- & 60 \\
\hline$-=$ & -- \\
\hline$=-$ & - \\
\hline-- & 90 \\
\hline-- & $<20$ \\
\hline-- & $<30$ \\
\hline-- & $<30$ \\
\hline-- & -- \\
\hline-- & -- \\
\hline-- & -- \\
\hline-- & -- \\
\hline-- & $<2$ \\
\hline
\end{tabular}

$\begin{array}{cccc}-- & - & 6 & 0.0 \\ -- & -- & 8 & 0.0 \\ 3 & -- & 1 & 0.0 \\ -- & -- & -- & -0 \\ -- & -- & 3 & 0.0 \\ - & -- & 4 & 0.0 \\ -- & -- & -- & -- \\ 17 & -- & 4 & 0.0 \\ 21 & -- & 2 & 0.0 \\ -- & -- & -- & -- \\ 4 & -- & -- & -- \\ <2 & -- & -- & -1 \\ 15 & - & - & -1\end{array}$


TAm 4.--Physical characteristics and concentrations of commond chemlcal constituents in lakes and streams of the Bald Mountain watershed--Continued

464843068455200 FISH RIVER LAKE IN TOWNSEIP 13 RANGE 8 ME

\begin{tabular}{|c|c|c|c|c|c|c|c|c|c|c|}
\hline DATE & TIME & $\begin{array}{l}\text { SAM- } \\
\text { PLING } \\
\text { DEPTH } \\
\text { (FEET) }\end{array}$ & $\begin{array}{l}\text { SPE- } \\
\text { CIFIC } \\
\text { CON- } \\
\text { DUCT- } \\
\text { ANCE } \\
\text { (US/CM) }\end{array}$ & $\begin{array}{c}\text { PH } \\
\text { (STAND- } \\
\text { ARD } \\
\text { UNITS) }\end{array}$ & $\begin{array}{l}\text { TEMPER- } \\
\text { ATURE } \\
\text { WATER } \\
\text { (DEG C) }\end{array}$ & $\begin{array}{l}\text { COLOR } \\
\text { (PLAT- } \\
\text { INUM- } \\
\text { COBALT } \\
\text { UNITS) }\end{array}$ & $\begin{array}{l}\text { TUR- } \\
\text { BID- } \\
\text { ITY } \\
\text { (FTU) }\end{array}$ & $\begin{array}{c}\text { OXYGEN, } \\
\text { DIS- } \\
\text { SOLVED } \\
\text { (MG/L) }\end{array}$ & $\begin{array}{l}\text { OXYGEN } \\
\text { DEMAND, } \\
\text { BIO- } \\
\text { CHEM- } \\
\text { ICAL, } \\
5 \text { DAY } \\
\text { (MG/L) }\end{array}$ & $\begin{array}{l}\text { COLI- } \\
\text { FORM, } \\
\text { FECAL, } \\
0.45 \\
\text { UM-MF } \\
\text { (COLS.I } \\
100 \mathrm{ML})\end{array}$ \\
\hline
\end{tabular}

\begin{tabular}{|c|c|c|c|c|c|c|c|c|c|c|}
\hline $\begin{array}{l}23 \ldots \\
\operatorname{MAR}\end{array}$ & 1145 & - & 30 & 6.7 & 1.0 & 35 & 2.1 & 12.2 & -- & -- \\
\hline${ }_{A P R}^{19 \ldots}$ & 1215 & - & -- & 6.5 & 0.0 & 25 & 1.4 & 13.2 & -- & -- \\
\hline $\operatorname{MAY}^{14 \ldots}$ & 1540 & -- & 55 & 6.7 & 1.0 & 35 & 2.1 & 11.4 & -- & -- \\
\hline JUN $15 \ldots$ & 1700 & - & 30 & 6.6 & 13.0 & 30 & 1.2 & 10.6 & -- & - \\
\hline JuL $^{10 \ldots}$ & 1130 & -- & 36 & 6.9 & 13.0 & 20 & 1.4 & 10.2 & -- & -- \\
\hline$\underset{\operatorname{sEP}}{21} \cdots$ & 1130 & -- & 49 & 7.1 & 21.0 & 30 & 1.5 & -- & -- & - \\
\hline $\mathrm{OCT}^{22} \cdots$ & 0950 & - & 28 & -- & $\cdots$ & 55 & 1.6 & -- & -- & - \\
\hline$\underset{\text { Nov }}{20 . .}$ & 0950 & -- & 28 & -- & 7.0 & 60 & 1.4 & 8.0 & -- & -- \\
\hline $\operatorname{MAR}^{17} 1 \ddot{1982}$ & 0845 & -- & 28 & 6.8 & 1.0 & 30 & 1.3 & 10.6 & -- & -- \\
\hline $25 \ldots$ & 1245 & 3.28 & 30 & 7.0 & 0.0 & 45 & 0.61 & 12.2 & -- & -- \\
\hline${ }_{\text {JUN }}^{25 . \cdots}$ & 1300 & 39.4 & 32 & 6.7 & 2.5 & 40 & 1.5 & 5.7 & -- & -- \\
\hline $\begin{array}{l}01 \ldots \\
01 \ldots\end{array}$ & $\begin{array}{l}1200 \\
1205\end{array}$ & $\begin{array}{r}0.0 \\
39.4\end{array}$ & $\begin{array}{l}39 \\
39\end{array}$ & $\begin{array}{l}6.8 \\
6.7\end{array}$ & $\begin{array}{r}16.0 \\
9.0\end{array}$ & $\begin{array}{l}35 \\
35\end{array}$ & -- & $\begin{array}{r}11.0 \\
4.0\end{array}$ & -- & -- \\
\hline
\end{tabular}

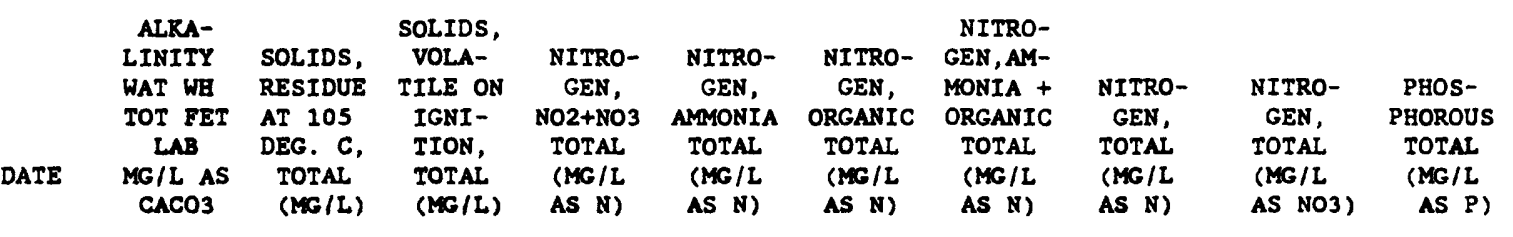

FEB 1981

MAR $23 .$.

19...

17

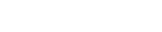

APR
$14 \ldots$
$\operatorname{MAY}$

17

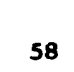

$--$

15 ...

17

74

--

$--$

$-$

$-$

$\begin{array}{llll}-- & -- & -- & 0.02 \\ -- & -- & -- & .01 \\ -- & -- & -- & .01 \\ -- & -- & -- & .01 \\ -- & -- & -- & .01 \\ -- & -- & -- & .01 \\ -- & -- & -- & -- \\ -- & -- & -- & .02 \\ -- & -- & -- & .01 \\ -- & -- & -- & -- \\ -- & -- & -- & -- \\ -- & -- & -- & -- \\ -- & -- & -- & --\end{array}$


TAm 4.--Physlcel characterlstics and concentrations of commond chemlcal constituents In lakes and streams of the Bald Mountain watershed--Continued

464843068455200 FISA RIVER LARE IN TOWNSAIP 13 RANGE 8 ME

\begin{tabular}{|c|c|c|c|c|c|c|c|c|}
\hline DATE & $\begin{array}{l}\text { ALUM- } \\
\text { INUM, } \\
\text { TOTAL } \\
\text { RECOV- } \\
\text { ERABLE } \\
\text { (UG/L }\end{array}$ & $\begin{array}{l}\text { ALUM- } \\
\text { INUM, } \\
\text { DIS- } \\
\text { SOLVED } \\
\text { (UG/I }\end{array}$ & $\begin{array}{l}\text { ARSENIC } \\
\text { TOTAL } \\
\text { SUG/L }\end{array}$ & $\begin{array}{l}\text { CADMIUM } \\
\text { TOTAL } \\
\text { RECOV- } \\
\text { ERABLE } \\
\text { (UG/L }\end{array}$ & $\begin{array}{l}\text { CHRO- } \\
\text { MIUM, } \\
\text { TOTAL } \\
\text { RECOV- } \\
\text { ERABLE } \\
\text { (UG/L }\end{array}$ & $\begin{array}{l}\text { COPPER, } \\
\text { TOTAL } \\
\text { RECOV- } \\
\text { ERABLE } \\
\text { (UG/L }\end{array}$ & $\begin{array}{l}\text { COPPER, } \\
\text { DIS- } \\
\text { SOLVED } \\
\text { (UG/L }\end{array}$ & $\begin{array}{l}\text { IRON, } \\
\text { TOTAL } \\
\text { RECOV- } \\
\text { ERABLE } \\
\text { (UG/L }\end{array}$ \\
\hline & AS AL) & AS AL) & AS AS ) & AS $(D)$ & AS CR) & AS CU) & AS CU) & AS $F E$ ) \\
\hline FEB 1981 & & & & & & & & \\
\hline$\stackrel{\operatorname{MAR}}{23} \cdots^{23}$ & -- & -- & -- & -- & -- & 3 & -- & 240 \\
\hline${ }_{A P R}^{19 \ldots}$ & -- & -- & -- & -- & -- & 1 & -- & 160 \\
\hline $\mathrm{MAY}^{14 \ldots}$ & - & $=$ & -- & $=$ & -- & 1 & -- & 260 \\
\hline JuN & -- & -- & -- & -- & -- & 2 & -- & 90 \\
\hline${ }_{\text {JUL }}^{10 \ldots}$ & -- & -- & -- & -- & -- & $<6$ & -- & 80 \\
\hline$\stackrel{21 \ldots}{S E P}$ & $-\infty$ & -- & -- & -- & -- & $<2$ & -- & 40 \\
\hline $\mathrm{OCI}^{22 \ldots}$ & -- & $-=$ & -- & -- & -- & $<2$ & -- & 190 \\
\hline$\stackrel{20 \ldots}{\text { Nov }}$ & -- & -- & -- & -- & -- & 5 & -- & 140 \\
\hline MAR $17 \ddot{982}$ & -- & -- & -- & -- & -- & -- & -- & -- \\
\hline $25 \ldots$ & -- & -- & -- & -- & -- & -- & -- & -- \\
\hline JUN $25 \ldots$ & -- & -- & -- & $=-$ & -- & -- & -- & -- \\
\hline $\begin{array}{l}01 \ldots \\
01 \ldots\end{array}$ & $\begin{array}{l}<100 \\
<100\end{array}$ & $\begin{array}{l}<100 \\
<100\end{array}$ & $\begin{array}{l}-- \\
--\end{array}$ & -- & $\begin{array}{l}-- \\
--\end{array}$ & $\begin{array}{r}<1 \\
3\end{array}$ & $\begin{array}{r}2 \\
<1\end{array}$ & $\begin{array}{r}80 \\
120\end{array}$ \\
\hline DATE & $\begin{array}{l}\text { IRON, } \\
\text { DIS- } \\
\text { SOLVED } \\
\text { (UG/L } \\
\text { AS FE) }\end{array}$ & $\begin{array}{l}\text { LEAD, } \\
\text { TOTAL } \\
\text { RECOV- } \\
\text { ERABLE } \\
\text { (UG/L } \\
\text { AS PB) }\end{array}$ & $\begin{array}{l}\text { LEAD, } \\
\text { DIS- } \\
\text { SOLVED } \\
\text { (UG/L } \\
\text { AS PB) }\end{array}$ & $\begin{array}{l}\text { MERCURY } \\
\text { TOTAL } \\
\text { RECOV- } \\
\text { ERABLE } \\
\text { (UG/L } \\
\text { AS HG) }\end{array}$ & $\begin{array}{l}\text { ZINC, } \\
\text { TOTAL } \\
\text { RECOV- } \\
\text { ERABLE } \\
\text { (UG/I } \\
\text { AS ZN) }\end{array}$ & $\begin{array}{l}\text { ZINC, } \\
\text { DIS- } \\
\text { SOLVED } \\
\text { (UG/L } \\
\text { AS ZN) }\end{array}$ & $\begin{array}{l}\text { SEDI- } \\
\text { MENT, } \\
\text { SUS- } \\
\text { PENDED } \\
\text { (MG/L) }\end{array}$ & $\begin{array}{l}\text { SEDI - } \\
\text { MENT, } \\
\text { DIS- } \\
\text { CHARGE, } \\
\text { SUS- } \\
\text { PENDED } \\
\text { (T/DAY) }\end{array}$ \\
\hline
\end{tabular}

FEB 1981

23...

MAR

19...

APR

$14 \ldots$

MAY

$15 .$.

JUN

$10 \ldots$

JUL

21...

SEP

22 ...

OCT

20 ...

Nov

$17 . .$.

MAR 1982

25 ...

$25 \ldots$

JUN

$01 .$.

(1)

COPPER

TOTAL

RABLE

S FE) 
Table 5.--Summary of selected chemical and physical characteristics of water

01012515 Clayton Stream at Outlet Clayton Lake

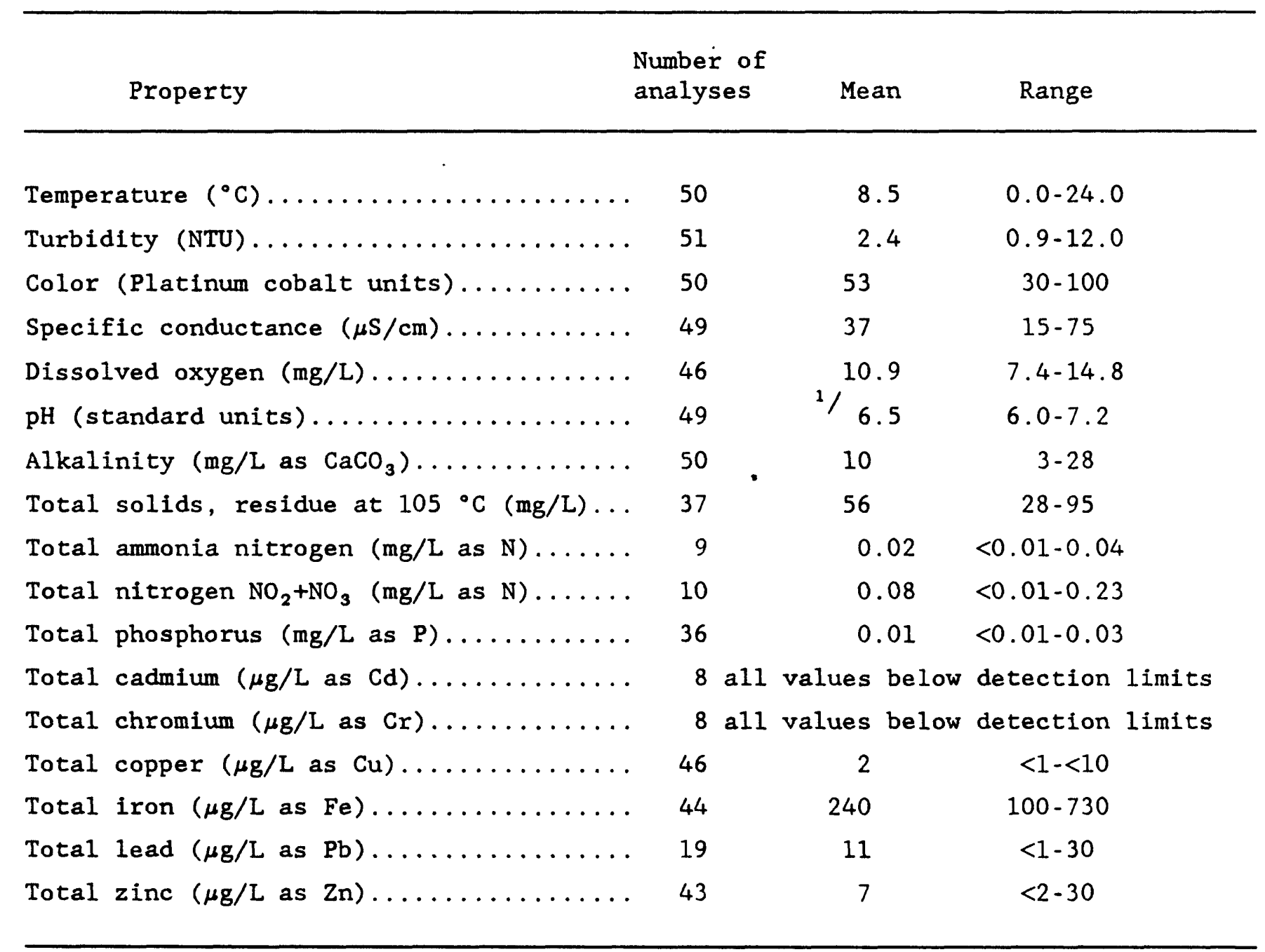

1/ Mean of $\mathrm{pH}$ readings 
Table 5.--Summary of selected chemical and physical characteristics of water--Continued

01012520 Bald Mountain Brook near Bald Mountain

\begin{tabular}{|c|c|c|c|}
\hline Property & $\begin{array}{l}\text { Number of } \\
\text { analyses }\end{array}$ & Mean & Range \\
\hline Temperature $\left({ }^{\circ} \mathrm{C}\right) \ldots \ldots \ldots \ldots \ldots \ldots$ & 53 & 7.7 & $0.0-20.0$ \\
\hline Turbidity (NTU) $\ldots \ldots \ldots \ldots \ldots \ldots \ldots$ & 53 & 2.7 & $0.5-15.0$ \\
\hline Color (Platinum cobalt units).......... & 49 & 50 & $20-90$ \\
\hline Specific conductance $(\mu \mathrm{S} / \mathrm{cm}) \ldots \ldots \ldots \ldots$ & 50 & 57 & $18-185$ \\
\hline Dissolved oxygen $(\mathrm{mg} / \mathrm{L}) \ldots \ldots \ldots \ldots \ldots$ & 51 & 11.0 & $7.2-13.7$ \\
\hline $\mathrm{pH}($ standard units $) \ldots \ldots \ldots \ldots \ldots$ & 49 & $1 / 6.7$ & $6.0-7.8$ \\
\hline Alkalinity $\left(\mathrm{mg} / \mathrm{L}\right.$ as $\left.\mathrm{CaCO}_{3}\right) \ldots \ldots \ldots$ & 54 & 16 & $2-40$ \\
\hline Total solids, residue at $105{ }^{\circ} \mathrm{C}(\mathrm{mg} / \mathrm{L}) \ldots$ & 38 & 72 & $33-119$ \\
\hline Total ammonia nitrogen (mg/L as $N) \ldots \ldots$ & 12 & $<0.01$ & $<0.01-0.03$ \\
\hline Total nitrogen $\mathrm{NO}_{2}+\mathrm{NO}_{3}(\mathrm{mg} / \mathrm{L}$ as $\mathrm{N}) \ldots \ldots$ & 13 & 0.12 & $<0.01-0.42$ \\
\hline Total phosphorus (mg/L as $P$ ) $\ldots \ldots \ldots \ldots$ & 39 & 0.01 & $<0.01-0.04$ \\
\hline Total cadmium $(\mu \mathrm{g} / \mathrm{L}$ as $\mathrm{Cd}) \ldots \ldots \ldots \ldots$ & 8 & 2 & $2-2$ \\
\hline Total chromium $(\mu \mathrm{g} / \mathrm{L}$ as $\mathrm{Cr}) \ldots \ldots \ldots$ & 10 & 7 & $<5-20$ \\
\hline Total copper $(\mu \mathrm{g} / \mathrm{L}$ as $\mathrm{Cu}) \ldots \ldots \ldots \ldots$ & 53 & 3 & $<1-16$ \\
\hline Total iron $(\mu \mathrm{g} / \mathrm{L}$ as $\mathrm{Fe}) \ldots \ldots$ & 50 & 256 & $50-920$ \\
\hline Total lead $(\mu \mathrm{g} / \mathrm{L}$ as $\mathrm{Pb}) \ldots \ldots$ & 23 & 10 & $<1-30$ \\
\hline Total zinc $(\mu \mathrm{g} / \mathrm{L}$ as $\mathrm{Zn}) \ldots \ldots \ldots$ & 50 & 8 & $<1-20$ \\
\hline Total aluminum $(\mu \mathrm{g} / \mathrm{L}$ as $\mathrm{Al}) \ldots \ldots \ldots \ldots$ & 7 & 256 & $100-360$ \\
\hline
\end{tabular}

$1 /$ Mean of $\mathrm{pH}$ readings 
Table 5.--Summary of selected chemical and physical characteristics of water--Continued

0102525 Bishop Mountain Brook near Bishop Mountain

\begin{tabular}{|c|c|c|c|}
\hline Property & $\begin{array}{l}\text { Number of } \\
\text { analyses }\end{array}$ & Mean & Range \\
\hline Temperature $\left({ }^{\circ} \mathrm{C}\right) \ldots \ldots \ldots \ldots \ldots$ & 40 & 6.6 & $0.0-22.5$ \\
\hline Turbidity (NTU) $\ldots \ldots \ldots \ldots \ldots \ldots \ldots$ & 39 & 3.1 & $0.6-18.0$ \\
\hline Color (Platinum cobalt units).......... & 39 & 61 & $25-180$ \\
\hline Specific conductance $(\mu \mathrm{S} / \mathrm{cm}) \ldots \ldots \ldots \ldots$ & 38 & 50 & $16-155$ \\
\hline Dissolved oxygen $(\mathrm{mg} / \mathrm{L}) \ldots \ldots \ldots \ldots$ & 36 & 10.7 & $6.7-13.8$ \\
\hline $\mathrm{pH}($ standard units $) \ldots \ldots \ldots \ldots \ldots$ & 39 & $1 / 6.4$ & $5.9-7.1$ \\
\hline Alkalinity $\left(\mathrm{mg} / \mathrm{L}\right.$ as $\left.\mathrm{CaCO}_{3}\right) \ldots \ldots \ldots \ldots$ & 40 & 14 & $3-44$ \\
\hline Total solids, residue at $105{ }^{\circ} \mathrm{C}(\mathrm{mg} / \mathrm{L}) \ldots$ & 32 & 68 & $45-117$ \\
\hline Total phosphorus (mg/L as P).......... & 33 & 0.01 & $<0.01-0.04$ \\
\hline Total copper $(\mu \mathrm{g} / \mathrm{L}$ as $\mathrm{Cu}) \ldots \ldots \ldots \ldots$ & 44 & 2 & $<1-16$ \\
\hline Total iron $(\mu \mathrm{g} / \mathrm{L}$ as $\mathrm{Fe}) \ldots \ldots \ldots \ldots \ldots$ & 44 & 425 & $60-1700$ \\
\hline Total lead $(\mu \mathrm{g} / \mathrm{L}$ as $\mathrm{Pb}) \ldots \ldots \ldots \ldots$ & 13 al1 & alues bel & detection limits \\
\hline Total zinc $(\mu \mathrm{g} / \mathrm{L}$ as $\mathrm{Zn}) \ldots \ldots$ & 42 & 6 & $<3-13$ \\
\hline Total aluminum $\mu \mathrm{g} / \mathrm{L}$ as $\mathrm{Al}) \ldots \ldots \ldots$ & 7 & 870 & $<100-1900$ \\
\hline
\end{tabular}

$1 /$ Mean of $\mathrm{pH}$ readings 
Table 5.--Summary of selected chemical and physical characteristics of water--Continued

01012530 Clayton Stream at Sheldon Ridge

\begin{tabular}{|c|c|c|c|}
\hline Property & $\begin{array}{l}\text { Number of } \\
\text { analyses }\end{array}$ & Mean & Range \\
\hline Temperature $\left({ }^{\circ} \mathrm{C}\right) \ldots \ldots \ldots \ldots \ldots$ & 45 & 7.4 & $0.0-23.0$ \\
\hline Turbidity (NTU) $\ldots \ldots \ldots \ldots \ldots \ldots \ldots$ & 46 & 2.6 & $0.9-13.0$ \\
\hline Color (Platinum cobalt units).......... & 45 & 54 & $35-100$ \\
\hline Specific conductance $(\mu \mathrm{S} / \mathrm{cm}) \ldots \ldots \ldots \ldots$ & 45 & 41 & $17-80$ \\
\hline Dissolved oxygen $(m g / L) \ldots \ldots \ldots \ldots$ & 41 & 11.3 & $8.3-14.2$ \\
\hline $\mathrm{pH}($ standard units $) \ldots \ldots \ldots \ldots \ldots \ldots$ & 44 & $1 / 6.6$ & $6.0-7.2$ \\
\hline Alkalinity $\left(\mathrm{mg} / \mathrm{L}\right.$ as $\left.\mathrm{CaCO}_{3}\right) \ldots \ldots \ldots \ldots$ & 44 & 10 & $2-23$ \\
\hline Total solids, residue at $105{ }^{\circ} \mathrm{C}(\mathrm{mg} / \mathrm{L}) \ldots$ & 40 & 58 & $20-95$ \\
\hline Total phosphorus (mg/L as $\mathrm{P}) \ldots \ldots \ldots \ldots$ & 38 & 0.01 & $<0.01-0.04$ \\
\hline Total cadmium $(\mu \mathrm{g} / \mathrm{L}$ as $\mathrm{Cd}) \ldots \ldots \ldots \ldots$ & 6 al1 & samples be & w detection limits \\
\hline Total chromium $(\mu \mathrm{g} / \mathrm{L}$ as $\mathrm{Cr}) \ldots \ldots \ldots \ldots$ & 6 all & samples be & w detection limits \\
\hline Total copper $(\mu \mathrm{g} / \mathrm{L}$ as $\mathrm{Cu}) \ldots \ldots \ldots$ & 46 & 2 & $<1-12$ \\
\hline Total iron $(\mu \mathrm{g} / \mathrm{L}$ as $\mathrm{Fe}) \ldots \ldots \ldots \ldots$ & 41 & 255 & $60-740$ \\
\hline Total lead $(\mu \mathrm{g} / \mathrm{L}$ as $\mathrm{Pb}) \ldots \ldots \ldots \ldots$ & 17 & 10 & $<1-<30$ \\
\hline Total zinc $(\mu \mathrm{g} / \mathrm{L}$ as $\mathrm{Zn}) \ldots \ldots \ldots$ & 42 & 9 & $<2-120$ \\
\hline
\end{tabular}

1/ Mean of $\mathrm{pH}$ readings 
Table 5.--Summary of selected chemical and physical characteristics of water--Continued

01012545 Moose Pond Stream at Inlet Carr Pond

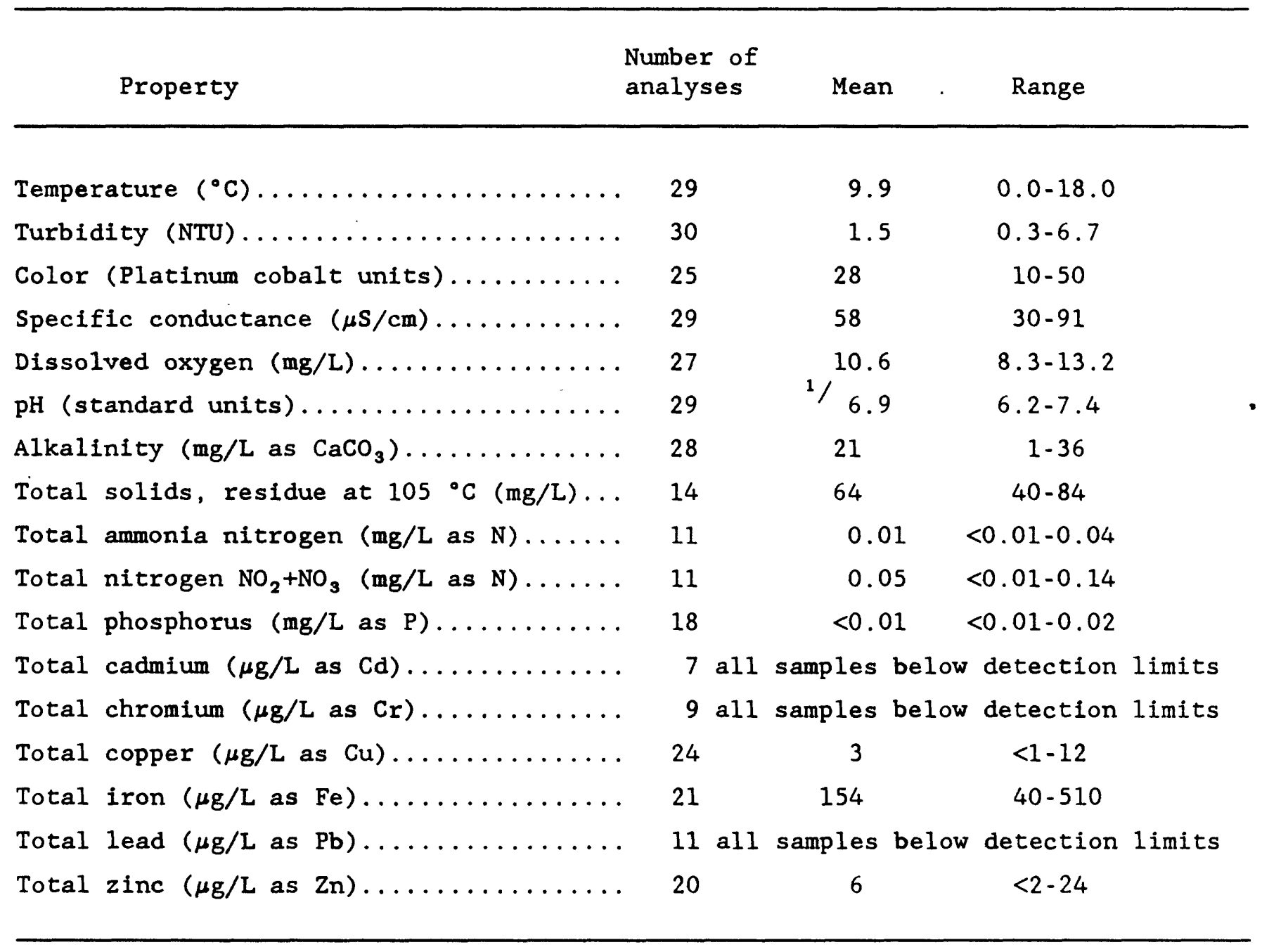

1/ Mean of $\mathrm{pH}$ readings 
Table 5.--Summary of selected chemical and physical characteristics of water--Continued

$$
01012550 \text { Carr Pond Stream at Outlet Carr Pond }
$$

\begin{tabular}{|c|c|c|c|}
\hline Property & $\begin{array}{l}\text { Number of } \\
\text { analyses }\end{array}$ & Mean & Range \\
\hline Temperature $\left({ }^{\circ} \mathrm{C}\right) \ldots \ldots \ldots \ldots \ldots \ldots$ & 26 & 8.8 & $0.0-24.0$ \\
\hline 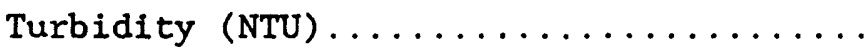 & 27 & 2.0 & $0.6-15.0$ \\
\hline Color (Platinum cobalt units)......... & 25 & 35 & $20-60$ \\
\hline Specific conductance $(\mu \mathrm{S} / \mathrm{cm}) \ldots \ldots \ldots \ldots$ & 25 & 54 & $26-120$ \\
\hline Dissolved oxygen $(\mathrm{mg} / \mathrm{L}) \ldots \ldots \ldots \ldots \ldots$ & 25 & 11.4 & $8.6-14.6$ \\
\hline pH $($ standard units) $\ldots \ldots \ldots \ldots \ldots \ldots$ & 26 & $1 / 6.9$ & $6.5-7.4$ \\
\hline Alkalinity $\left(\mathrm{mg} / \mathrm{L}\right.$ as $\left.\mathrm{CaCO}_{3}\right) \ldots \ldots \ldots \ldots$ & 24 & 18 & $9-26$ \\
\hline Total solids, residue at $105{ }^{\circ} \mathrm{C}(\mathrm{mg} / \mathrm{L}) \ldots$ & 24 & 59 & $4-98$ \\
\hline Total phosphorus (mg/L as $\mathrm{P}) \ldots \ldots \ldots \ldots$ & 21 & 0.01 & $<0.01-0.02$ \\
\hline Total cadmium $(\mu \mathrm{g} / \mathrm{L}$ as $\mathrm{Cd}) \ldots \ldots \ldots \ldots$ & 6 a11 & samples be & w detection limits \\
\hline Total chromium $(\mu \mathrm{g} / \mathrm{L}$ as $\mathrm{Cr}) \ldots \ldots \ldots \ldots$ & 6 al1 & samples be & w detection limits \\
\hline Total copper $(\mu \mathrm{g} / \mathrm{L}$ as $\mathrm{Cu}) \ldots \ldots \ldots \ldots$ & 24 & 2 & $<1-<10$ \\
\hline Total iron $(\mu \mathrm{g} / \mathrm{L}$ as $\mathrm{Fe}) \ldots \ldots \ldots \ldots$ & 20 & 78 & $20-210$ \\
\hline Total lead $(\mu \mathrm{g} / \mathrm{L}$ as $\mathrm{Pb}) \ldots \ldots \ldots \ldots$ & 11 a11 & samples be & w detection limits \\
\hline Total zinc $(\mu \mathrm{g} / \mathrm{L}$ as $\mathrm{Zn}) \ldots \ldots \ldots \ldots$ & 22 & 5 & $<2-22$ \\
\hline
\end{tabular}

$1 /$ Mean of $\mathrm{pH}$ readings 
Table 5.--Summary of selected chemical and physical characteristics of water--Continued

01012560 North Branch Fox Brook near Fish River Lake

\begin{tabular}{|c|c|c|c|}
\hline Property & $\begin{array}{l}\text { Number of } \\
\text { analyses }\end{array}$ & Mean & Range \\
\hline Temperature $\left({ }^{\circ} \mathrm{C}\right) \ldots \ldots \ldots \ldots \ldots \ldots$ & 15 & 12.2 & $0.0-20.0$ \\
\hline Turbidity $(\mathrm{NTU}) \ldots \ldots \ldots \ldots \ldots \ldots \ldots$ & 15 & 1.0 & $0.4-2.1$ \\
\hline Color (Platinum cobalt units).......... & 10 & 17 & $5-25$ \\
\hline Specific conductance $(\mu \mathrm{S} / \mathrm{cm}) \ldots \ldots \ldots \ldots$ & 14 & 57 & $35-75$ \\
\hline Dissolved oxygen $(\mathrm{mg} / \mathrm{L}) \ldots \ldots \ldots \ldots \ldots$ & 14 & 10.6 & $8.6-13.3$ \\
\hline $\mathrm{pH}($ standard units $) \ldots \ldots \ldots \ldots \ldots \ldots$ & 15 & $1 / 7.1$ & $6.8-7.2$ \\
\hline Alkalinity $\left(\mathrm{mg} / \mathrm{L}\right.$ as $\left.\mathrm{CaCO}_{3}\right) \ldots \ldots \ldots$ & 14 & 20 & $9-28$ \\
\hline Total solids, residue at $105^{\circ} \mathrm{C}(\mathrm{mg} / \mathrm{L}) \ldots$ & 7 & 47 & $25-84$ \\
\hline Total ammonia nitrogen $(\mathrm{mg} / \mathrm{L}$ as $\mathrm{N}) \ldots \ldots$ & 12 & $<0.01$ & $<0.01-0.02$ \\
\hline Total nitrogen $\mathrm{NO}_{2}+\mathrm{NO}_{3}(\mathrm{mg} / \mathrm{L}$ as $\mathrm{N}) \ldots \ldots$ & 13 & 0.10 & $0.01-0.24$ \\
\hline Total phosphorus (mg/L as $\mathrm{P}) \ldots \ldots \ldots \ldots$ & 7 & 0.03 & $<0.01-0.10$ \\
\hline Total cadmium $(\mu g / L$ as $\mathrm{Cd}) \ldots \ldots \ldots \ldots$ & 9 a11 & samples be & w detection limits \\
\hline Total chromium $(\mu \mathrm{g} / \mathrm{L}$ as $\mathrm{Cr}) \ldots \ldots \ldots \ldots$ & 10 all & samples be & w detection limits \\
\hline Total copper $(\mu \mathrm{g} / \mathrm{L}$ as $\mathrm{Cu}) \ldots \ldots \ldots \ldots$ & 13 & 9 & $<1-66$ \\
\hline Total iron $(\mu \mathrm{g} / \mathrm{L}$ as $\mathrm{Fe}) \ldots \ldots \ldots \ldots$ & 9 & 223 & $30-1100$ \\
\hline Total lead $(\mu \mathrm{g} / \mathrm{L}$ as $\mathrm{Pb}) \ldots \ldots \ldots \ldots$ & 13 & 18 & $<1-<30$ \\
\hline Total zinc $(\mu \mathrm{g} / \mathrm{L}$ as $\mathrm{Zn}) \ldots \ldots \ldots \ldots$ & 12 & 7 & $<2-20$ \\
\hline
\end{tabular}

1/ Mean of $\mathrm{pH}$ readings 
Table 5.--Summary of selected chemical and physical characteristics of water--Continued

01012570 Fish River at Inlet Fish River Lake

\begin{tabular}{|c|c|c|c|}
\hline Property & $\begin{array}{l}\text { Number of } \\
\text { analyses }\end{array}$ & Mean & Range \\
\hline Temperature $\left({ }^{\circ} \mathrm{C}\right) \ldots \ldots \ldots \ldots \ldots \ldots$ & 51 & 9.1 & $0.0-24.0$ \\
\hline Turbidity (NTU) $\ldots \ldots \ldots \ldots \ldots \ldots \ldots$ & 51 & 2.3 & $0.8-12.0$ \\
\hline Color (Platinum cobalt units) ......... & 45 & 40 & $20-70$ \\
\hline Specific conductance $(\mu \mathrm{S} / \mathrm{cm}) \ldots \ldots \ldots$ & 50 & 47 & $19-80$ \\
\hline Dissolved oxygen $(\mathrm{mg} / \mathrm{L}) \ldots \ldots \ldots \ldots \ldots$ & 48 & 10.9 & $8.2-14.0$ \\
\hline $\mathrm{pH}($ standard units $) \ldots \ldots \ldots \ldots \ldots$ & 47 & 1/ 6.7 & $6.0-7.3$ \\
\hline Alkalinity $\left(\mathrm{mg} / \mathrm{L}\right.$ as $\left.\mathrm{CaCO}_{3}\right) \ldots \ldots \ldots \ldots$ & 51 & 15 & $2-31$ \\
\hline Total solids, residue at $105^{\circ} \mathrm{C}(\mathrm{mg} / \mathrm{L}) \ldots$ & 35 & 58 & $10-94$ \\
\hline Total ammonia nitrogen $(\mathrm{mg} / \mathrm{L}$ as $\mathrm{N}) \ldots \ldots$ & 13 & 0.01 & $<0.01-0.03$ \\
\hline Total nitrogen $\mathrm{NO}_{2}+\mathrm{NO}_{3}(\mathrm{mg} / \mathrm{L}$ as $\mathrm{N}) \ldots \ldots$ & 14 & 0.11 & $<0.01-0.45$ \\
\hline Total phosphorus (mg/L as $\mathrm{P}) \ldots \ldots$ & 35 & 0.01 & $<0.01-0.02$ \\
\hline Total cadmium $(\mu \mathrm{g} / \mathrm{L}$ as $\mathrm{Cd}) \ldots \ldots \ldots \ldots$ & 11 al1 & samples be & w detection limits \\
\hline Total chromium $(\mu \mathrm{g} / \mathrm{L}$ as $\mathrm{Cr}) \ldots$ & 11 a11 & samples be & w detection limits \\
\hline Total copper $(\mu \mathrm{g} / \mathrm{L}$ as $\mathrm{Cu}) \ldots \ldots \ldots \ldots$ & 44 & 2 & $<1-<10$ \\
\hline Total iron $(\mu \mathrm{g} / \mathrm{L}$ as $\mathrm{Fe}) \ldots . .$. & 39 & 244 & $100-830$ \\
\hline Total lead $(\mu g / L$ as $\mathrm{Pb}) \ldots \ldots \ldots \ldots$ & 19 & 14 & $<1-30$ \\
\hline Total zinc $(\mu \mathrm{g} / \mathrm{L}$ as $\mathrm{Zn}) \ldots \ldots \ldots \ldots$ & 42 & 8 & $<2-51$ \\
\hline
\end{tabular}

$1 /$ Mean of $\mathrm{pH}$ readings 
Table 5.--Summary of selected chemical and physical characteristics of water--Continued

01016410 Machias River at Russel1 Crossing

\begin{tabular}{|c|c|c|c|}
\hline Property & $\begin{array}{l}\text { Number of } \\
\text { analyses }\end{array}$ & Mean & Range \\
\hline Temperature $\left({ }^{\circ} \mathrm{C}\right) \ldots \ldots \ldots \ldots \ldots \ldots$ & 26 & 7.0 & $0.0-20.0$ \\
\hline Turbidity (NTU) $\ldots \ldots \ldots \ldots \ldots \ldots \ldots$ & 28 & 2.4 & $1.0-6.1$ \\
\hline Color (Platinum cobalt units)......... & 27 & 45 & $30-90$ \\
\hline Specific conductance $(\mu \mathrm{S} / \mathrm{cm}) \ldots \ldots \ldots$ & 26 & 54 & $25-260$ \\
\hline Dissolved oxygen $(\mathrm{mg} / \mathrm{L}) \ldots \ldots \ldots \ldots \ldots$ & 26 & 11.4 & $8.9-14.1$ \\
\hline $\mathrm{pH}($ standard units $) \ldots \ldots \ldots \ldots \ldots$ & 27 & $1 / 6.8$ & $6.2-7.3$ \\
\hline Alkalinity $\left(\mathrm{mg} / \mathrm{L}\right.$ as $\left.\mathrm{CaCO}_{3}\right) \ldots \ldots \ldots \ldots$ & 26 & 14 & $4-23$ \\
\hline Total solids, residue at $105^{\circ} \mathrm{C}(\mathrm{mg} / \mathrm{L}) \ldots$ & 23 & 62 & $38-102$ \\
\hline Total phosphorus $(m g / L$ as $P) \ldots \ldots \ldots$ & 20 & 0.01 & $<0.01-0.03$ \\
\hline Total cadmium $(\mu \mathrm{g} / \mathrm{L}$ as $\mathrm{Cd}) \ldots \ldots \ldots \ldots$ & 7 all & samples be & w detection limits \\
\hline Total chromium $(\mu \mathrm{g} / \mathrm{L}$ as $\mathrm{Cr}) \ldots \ldots \ldots \ldots$ & 7 all & samples be & w detection limits \\
\hline Total copper $(\mu \mathrm{g} / \mathrm{L}$ as $\mathrm{Cu}) \ldots \ldots \ldots \ldots$ & 24 & 4 & $<1-12$ \\
\hline Total iron $(\mu g / L$ as $F e) \ldots \ldots \ldots \ldots$ & 21 & 256 & $100-950$ \\
\hline Total lead $(\mu \mathrm{g} / \mathrm{L}$ as $\mathrm{Pb}) \ldots \ldots \ldots \ldots$ & 11 & 16 & $<1-<30$ \\
\hline Total zinc $(\mu \mathrm{g} / \mathrm{L}$ as $\mathrm{zn}) \ldots \ldots \ldots \ldots$ & 20 & 7 & $<2-52$ \\
\hline
\end{tabular}

$1 /$ Mean of $\mathrm{pH}$ readings 
Table 5.--Summary of selected chemical and physical characteristics of water--Continued

01016480 Greenlaw Stream at Greenlaw Crossing

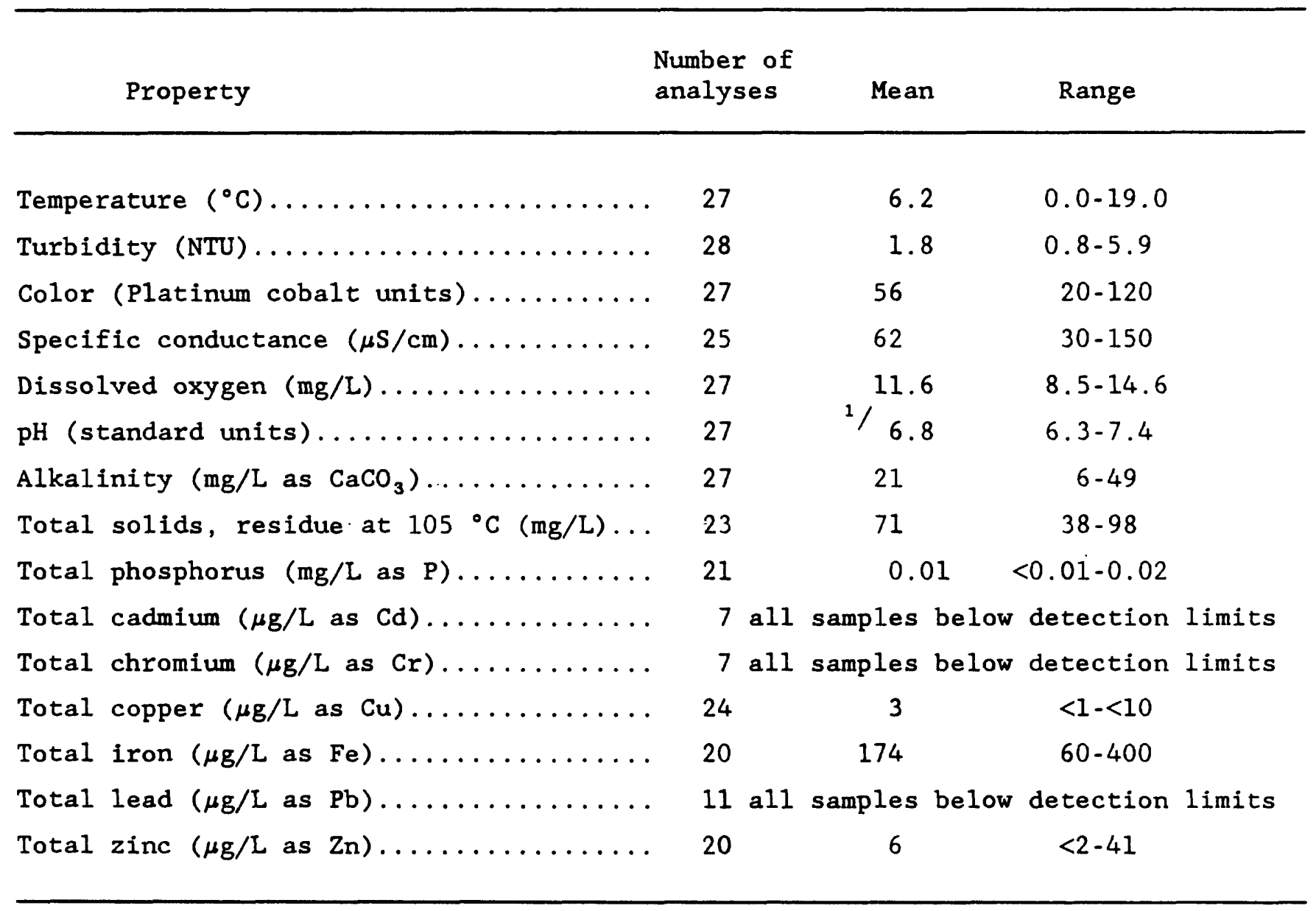

1/ Mean of $\mathrm{pH}$ readings 
Table 5.--Summary of selected chemical and physical characteristics of water--Continued

4643080684611 Clayton Lake in Township 12 Range 8

\begin{tabular}{|c|c|c|c|}
\hline Property & $\begin{array}{l}\text { Number of } \\
\text { analyses }\end{array}$ & Mean & Range \\
\hline Temperature $\left({ }^{\circ} \mathrm{C}\right) \ldots \ldots \ldots \ldots \ldots \ldots$ & 22 & 7.7 & $0.0-22.0$ \\
\hline 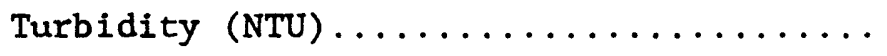 & 22 & 1.9 & $0.9-3.4$ \\
\hline Color (Platinum cobalt units)......... & 22 & 53 & $15-100$ \\
\hline Specific conductance $(\mu \mathrm{S} / \mathrm{cm}) \ldots \ldots \ldots$ & 22 & 44 & $18-145$ \\
\hline Dissolved oxygen $(\mathrm{mg} / \mathrm{L}) \ldots \ldots \ldots \ldots \ldots$ & 21 & 11.2 & $8.3-13.3$ \\
\hline $\mathrm{PH}($ standard units) $\ldots \ldots \ldots \ldots \ldots \ldots$ & 22 & $1 / 6.6$ & $6.1-7.0$ \\
\hline Alkalinity $\left(\mathrm{mg} / \mathrm{L}\right.$ as $\left.\mathrm{CaCO}_{3}\right) \ldots \ldots \ldots \ldots$ & 22 & 11 & $5-23$ \\
\hline Total solids, residue at $105^{\circ} \mathrm{C}(\mathrm{mg} / \mathrm{L}) \ldots$ & 18 & 55 & $26-96$ \\
\hline Total phosphorus (mg/L as $\mathrm{P}) \ldots \ldots \ldots \ldots$ & 20 & 0.02 & $<0.01-0.04$ \\
\hline Total chromium $(\mu \mathrm{g} / \mathrm{L}$ as $\mathrm{Cr}) \ldots \ldots \ldots \ldots$ & 7 al1 & samples be & w detection limits \\
\hline Total copper $(\mu \mathrm{g} / \mathrm{L}$ as $\mathrm{Cu}) \ldots \ldots \ldots \ldots$ & 19 & 3 & $<1-<10$ \\
\hline Total iron $(\mu \mathrm{g} / \mathrm{L}$ as $\mathrm{Fe}) \ldots \ldots \ldots \ldots \ldots$ & 15 & 233 & $120-820$ \\
\hline Total lead $(\mu \mathrm{g} / \mathrm{L}$ as $\mathrm{Pb}) \ldots \ldots \ldots \ldots \ldots$ & 6 & 22 & $<2-30$ \\
\hline Total zinc $(\mu \mathrm{g} / \mathrm{L}$ as $\mathrm{Zn}) \ldots \ldots \ldots$ & 15 & 13 & $<3-59$ \\
\hline
\end{tabular}

\section{1/ Mean of $\mathrm{pH}$ readings}


Table 5.--Summary of selected chemical and physical characteristics of water--Continued

4645530684301 Carr Pond in Townwhip 13 Range 8

\begin{tabular}{|c|c|c|c|}
\hline Property & $\begin{array}{l}\text { Number of } \\
\text { analyses }\end{array}$ & Mean & Range \\
\hline Temperature $\left({ }^{\circ} \mathrm{C}\right) \ldots \ldots \ldots \ldots \ldots \ldots$ & 30 & 10.4 & $0.0-26.0$ \\
\hline Turbidity (NTU) $\ldots \ldots \ldots \ldots \ldots \ldots \ldots$ & 31 & 1.1 & $0.5-1.9$ \\
\hline Color (Platinum cobalt units).......... & 30 & 34 & $10-70$ \\
\hline Specific conductance $(\mu \mathrm{S} / \mathrm{cm}) \ldots \ldots \ldots \ldots$ & 33 & 53 & $12-125$ \\
\hline Dissolved oxygen $(\mathrm{mg} / \mathrm{L}) \ldots \ldots \ldots \ldots \ldots$ & 29 & 10.6 & $8.0-14.0$ \\
\hline $\mathrm{pH},($ standard units $) \ldots \ldots \ldots \ldots \ldots \ldots$ & 32 & $1 / 6.9$ & $6.5-7.4$ \\
\hline Alkalinity $\left(\mathrm{mg} / \mathrm{L}\right.$ as $\left.\mathrm{CaCO}_{3}\right) \ldots \ldots \ldots \ldots$ & 32 & 17 & $1-28$ \\
\hline Total solids, residue at $105{ }^{\circ} \mathrm{C}(\mathrm{mg} / \mathrm{L}) \ldots$ & 16 & 61 & $48-93$ \\
\hline Total ammonia nitrogen (mg/L as $\mathrm{N}) \ldots \ldots$ & 11 & 0.02 & $<0.01-0.07$ \\
\hline Total nitrogen $\mathrm{NO}_{2}+\mathrm{NO}_{3}(\mathrm{mg} / \mathrm{L}$ as $\mathrm{N}) \ldots \ldots$ & 12 & 0.06 & $<0.01-0.35$ \\
\hline Total phosphorus $(\mathrm{mg} / \mathrm{L}$ as $\mathrm{P}) \ldots \ldots \ldots \ldots$ & 27 & 0.01 & $<0.01-0.02$ \\
\hline Total cadmium $(\mu \mathrm{g} / \mathrm{L}$ as $\mathrm{Cd}) \ldots \ldots \ldots \ldots$ & 9 all & samples be & w detection limits \\
\hline Total chromium $(\mu \mathrm{g} / \mathrm{L}$ as $\mathrm{Cr}) \ldots \ldots \ldots$ & 10 all & samples be & w detection limits \\
\hline Total copper $(\mu \mathrm{g} / \mathrm{L}$ as $\mathrm{Cu}) \ldots \ldots$ & 24 & 4 & $<1-<10$ \\
\hline Total iron $(\mu \mathrm{g} / \mathrm{L}$ as $\mathrm{Fe}) \ldots$ & 21 & 63 & $30-100$ \\
\hline Total zinc $(\mu \mathrm{g} / \mathrm{L}$ as $\mathrm{Zn}) \ldots \ldots \ldots \ldots$ & 21 & 7 & $<2-22$ \\
\hline
\end{tabular}

1/ Mean of $\mathrm{pH}$ readings 
Table 5.--Summary of selected chemical and physical characteristics of water--Continued

4648430684552 Fish River Lake in Township 13 Range 8

\begin{tabular}{|c|c|c|c|}
\hline Property & $\begin{array}{l}\text { Number of } \\
\text { analyses }\end{array}$ & Mean & Range \\
\hline Temperature $\left({ }^{\circ} \mathrm{C}\right) \ldots \ldots \ldots \ldots \ldots \ldots$ & 27 & 11.5 & $0.0-24.0$ \\
\hline Turbidity (NTU) $\ldots \ldots \ldots \ldots \ldots \ldots$ & 24 & 1.6 & $0.6-3.3$ \\
\hline Color (Platinum cobalt units) .......... & 27 & 33 & $20-60$ \\
\hline Specific conductance $(\mu \mathrm{S} / \mathrm{cm}) \ldots \ldots \ldots \ldots$ & 26 & 40 & $28-70$ \\
\hline Dissolved oxygen $(m g / L) \ldots \ldots \ldots \ldots \ldots$ & 26 & 10.2 & $4.0-14.2$ \\
\hline $\mathrm{pH}$ (standard units) $\ldots \ldots \ldots \ldots \ldots \ldots$ & 24 & $1 / 6.7$ & $4.5-7.1$ \\
\hline Alkalinity $\left(\mathrm{mg} / \mathrm{L}\right.$ as $\left.\mathrm{CaCO}_{3}\right) \ldots \ldots \ldots \ldots$ & 26 & 16 & $10-43$ \\
\hline Total solids, residue at $105{ }^{\circ} \mathrm{C}(\mathrm{mg} / \mathrm{L}) \ldots$ & 16 & 57 & $40-74$ \\
\hline Total ammonia nitrogen $(\mathrm{mg} / \mathrm{L}$ as $\mathrm{N}) \ldots \ldots$ & 10 & 0.06 & $<0.01-0.34$ \\
\hline Total nitrogen $\mathrm{NO}_{2}+\mathrm{NO}_{3}(\mathrm{mg} / \mathrm{L}$ as $\mathrm{N}) \ldots \ldots$ & 10 & 0.12 & $<0.01-0.79$ \\
\hline Total phosphorus $(\mathrm{mg} / \mathrm{L}$ as $\mathrm{P}) \ldots \ldots \ldots \ldots$ & 23 & 0.02 & $<0.01-0.05$ \\
\hline Total cadmium $(\mu \mathrm{g} / \mathrm{L}$ as $\mathrm{Cd}) \ldots \ldots \ldots \ldots$ & 4 al1 & samples be & w detection limits \\
\hline Total chromium $(\mu g / L$ as $\mathrm{Cr}) \ldots \ldots \ldots \ldots$ & 5 al1 & samples be & w detection limits \\
\hline Total copper $(\mu \mathrm{g} / \mathrm{L}$ as $\mathrm{Cu}) \ldots \ldots$ & 18 & 4 & $<1-<10$ \\
\hline Total iron $(\mu \mathrm{g} / \mathrm{L}$ as $\mathrm{Fe}) \ldots \ldots \ldots$ & 16 & 127 & $40-260$ \\
\hline Total zinc $(\mu \mathrm{g} / \mathrm{L}$ as $\mathrm{zn}) \ldots \ldots \ldots \ldots$ & 14 & 8 & $<2-21$ \\
\hline
\end{tabular}

$1 /$ Mean of $\mathrm{pH}$ readings 
Iable 6.--Suspended-sediment discharge for selected days in the Bald Mountain watersheds

$01012515 \mathrm{Clayton}$ Stream at outlet Clayton Lake, 1983 water year

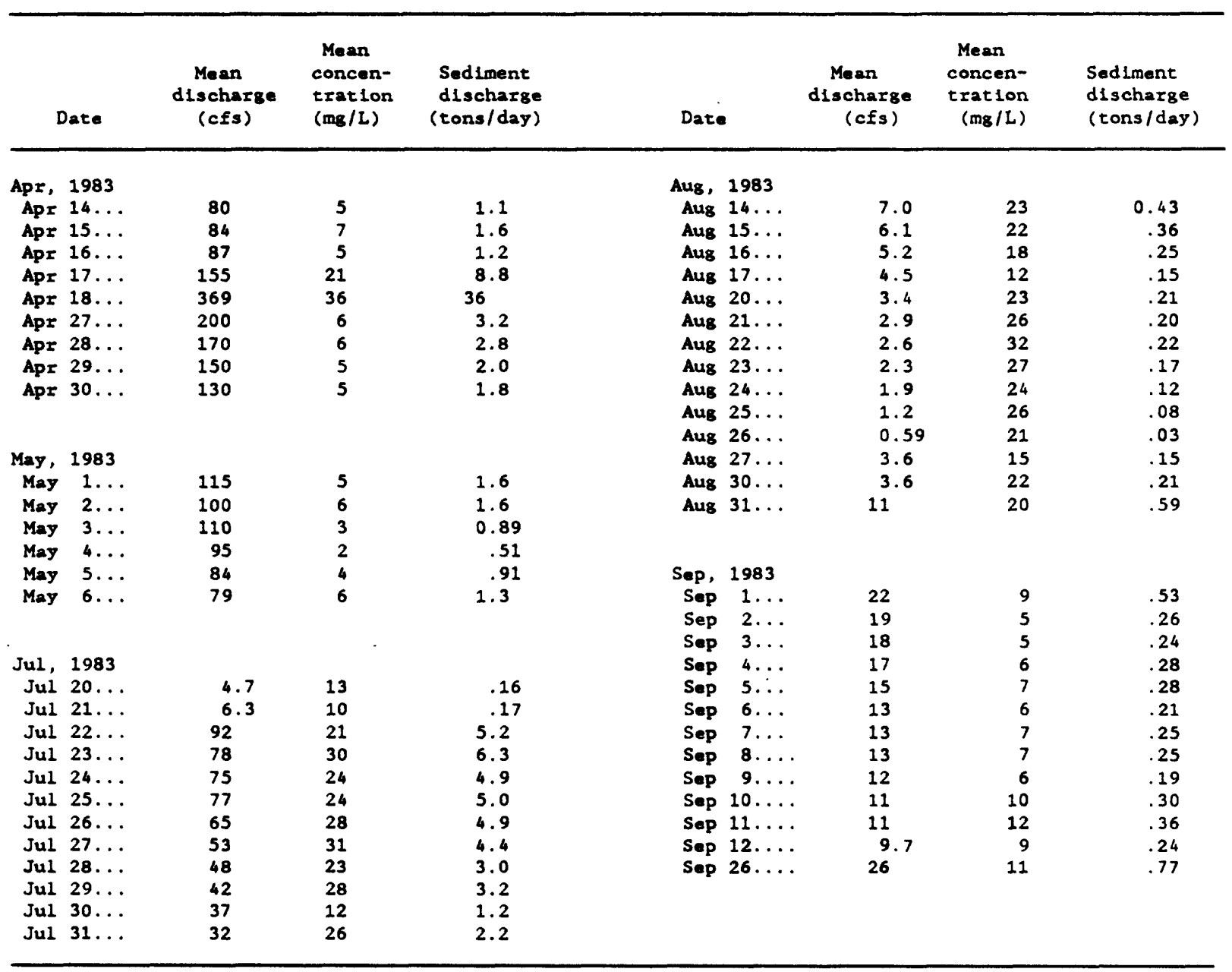


Table 6.--Suspended-sediment discharge for selected days in the Bald Mountain watersheds--Continued

01012515 Clayton Stream at outlet Clayton Lake, 1984 water year

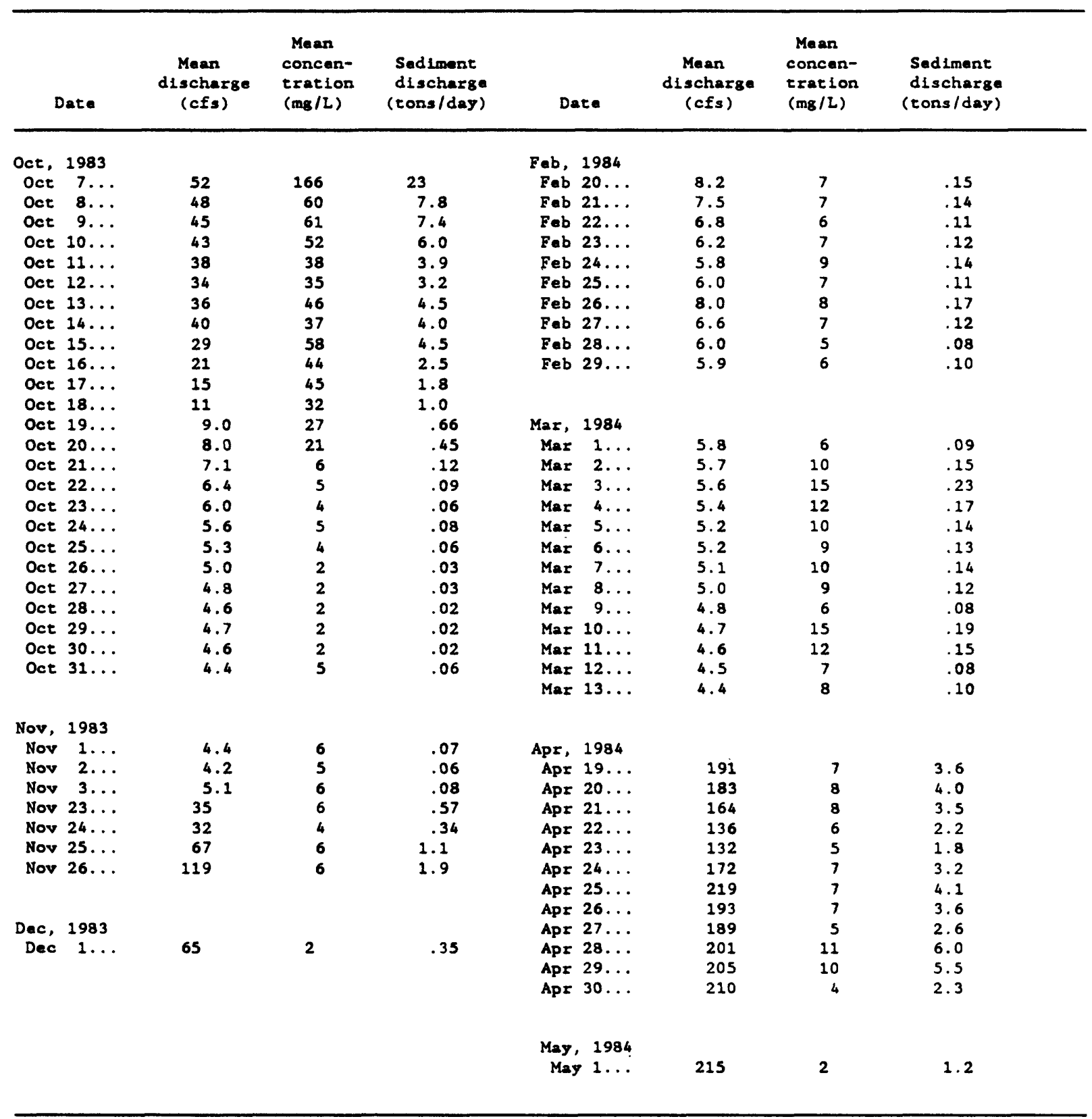


Table 6.--Suspended-sediment discharge for selected days in the Bald Mountain watersheds--Continued

01012520 Bald Mountain Brook neax Bald Mountain, 1981 water yeax

\begin{tabular}{|c|c|c|c|c|c|c|c|c|}
\hline & Date & $\begin{array}{c}\text { Mean } \\
\text { discharge } \\
\text { (cfs) }\end{array}$ & $\begin{array}{l}\text { Sediment } \\
\text { concen- } \\
\text { trat ion } \\
\text { (mg/L) }\end{array}$ & $\begin{array}{c}\text { Sediment } \\
\text { discharge } \\
\text { (tons/day) }\end{array}$ & Date & $\begin{array}{c}\text { Mean } \\
\text { discharge } \\
\text { (cfs) }\end{array}$ & $\begin{array}{l}\text { Mean } \\
\text { concen- } \\
\text { trat Lon } \\
\text { (mg/L) }\end{array}$ & $\begin{array}{c}\text { Sediment } \\
\text { discharge } \\
\text { (tons/day) }\end{array}$ \\
\hline \multirow{4}{*}{$\begin{array}{r}\text { Oct, } \\
\text { Oct }\end{array}$} & 1980 & & & & Mar, 1981-- & ntinued & & \\
\hline & $=31 \ldots$ & 01.8 & 3 & 0.01 & $\operatorname{Max} 20 \ldots$ & .76 & 4 & .01 \\
\hline & & & & & $\operatorname{Max} 21 \ldots$ & .71 & 5 & .01 \\
\hline & & & & & Mar $22 \ldots$ & .68 & 5 & .01 \\
\hline Nov, & 1980 & & & & $\operatorname{Max} 23 \ldots$ & .68 & 6 & .01 \\
\hline Nov & $1 \ldots$ & 2.0 & 2 & .01 & $\operatorname{Max} 24 \ldots$ & .78 & 5 & .01 \\
\hline Nov & $2 \ldots$ & 1.9 & 3 & .02 & $\operatorname{Max} 25 \ldots$ & .90 & 4 & .01 \\
\hline Nov & $3 \ldots$ & 1.6 & 5 & .02 & $\operatorname{Max} 26 \ldots$ & .88 & 2 & 0 \\
\hline Nov & $4 \ldots$ & 1.4 & 1 & 0 & $\operatorname{Mar} 27 \ldots$ & .98 & 5 & .01 \\
\hline \multirow[t]{3}{*}{ Nov } & $5 \ldots$ & 1.7 & 2 & .01 & $\operatorname{Max} 28 \ldots$ & 1.1 & 3 & .01 \\
\hline & & & & & Mar 29... & 1.2 & 9 & .03 \\
\hline & & & & & Mar $30 \ldots$ & 1.5 & 15 & .06 \\
\hline Feb, & 1981 & & & & Max $31 \ldots$ & 2.2 & 11 & .07 \\
\hline Feb & $10 \ldots$ & 3.4 & 2 & .02 & & & & \\
\hline Feb & $11 \ldots$ & 3.2 & 3 & .03 & & & & \\
\hline Feb & $12 \ldots$ & 6.8 & 4 & .07 & Apr, 1981 & & & \\
\hline Feb & $13 \ldots$ & 9.1 & 6 & .15 & Apr $1 \ldots$ & 6.0 & 8 & .13 \\
\hline Feb & $14 \ldots$ & 6.8 & 6 & .11 & $2 \ldots$ & 15 & 6 & .24 \\
\hline Feb & $15 \ldots$ & 5.4 & 6 & .09 & Apr $3 \ldots$ & 17 & 5 & .23 \\
\hline Feb & $16 \ldots$ & 4.8 & 5 & .06 & $8 \ldots$ & 14 & 6 & .23 \\
\hline Feb & $17 \ldots$ & 4.3 & 4 & .05 & Apr $\quad 9 \ldots$ & 13 & 11 & 0.39 \\
\hline Feb & $18 \ldots$ & 4.0 & 5 & .05 & $A p=10 \ldots$ & 21 & 10 & .59 \\
\hline Feb & $19 \ldots$ & 4.1 & 9 & .10 & $A p=11 \ldots$ & 16 & 6 & .26 \\
\hline Feb & $20 \ldots$ & 5.0 & 10 & .14 & $A p=12 \ldots$ & 12 & 6 & .19 \\
\hline Feb & $21 \ldots$ & 6.6 & 9 & .16 & Apr $13 \ldots$ & 8.7 & 5 & .12 \\
\hline Eeb & $22 \ldots$ & 9.2 & 6 & .15 & $A p=14 \ldots$ & 6.8 & 11 & .20 \\
\hline Feb & $23 \ldots$ & 10 & 6 & .16 & $A p=24 \ldots$ & 4.2 & 3 & .03 \\
\hline Feb & $24 \ldots$ & 8.4 & 4 & .09 & $A p=25 \ldots$ & 5.4 & 2 & .03 \\
\hline Feb & $25 \ldots$ & 7.1 & 3 & .06 & Apr $26 \ldots$ & 5.1 & 2 & .03 \\
\hline Feb & $26 \ldots$ & 6.5 & 22 & .17 & Ap $=27 \ldots$ & 5.0 & 2 & .03 \\
\hline Feb & $27 \ldots$ & 7.5 & 2 & .04 & $A p=28 \ldots$ & 4.5 & 2 & .02 \\
\hline \multirow[t]{2}{*}{ Feb } & $28 \ldots$ & 6.4 & 3 & .05 & Apr $29 \ldots$ & 5.2 & 7 & .09 \\
\hline & & & & & $A p=30 \ldots$ & 7.9 & 4 & .09 \\
\hline \multicolumn{9}{|c|}{ Mar, 1981} \\
\hline Max & $=1 \ldots$ & 5.0 & 8 & .11 & May, 1981 & & & \\
\hline Mar & $2 \ldots$ & 4.1 & 19 & .21 & May $1 \ldots$ & 6.0 & 2 & .03 \\
\hline Max & $=3 \ldots$ & 3.5 & 8 & .08 & $2 \ldots$ & 5.3 & 3 & .04 \\
\hline Max & $4 \ldots$ & 3.1 & 5 & .04 & $3 \ldots$ & 5.3 & 2 & .03 \\
\hline Mar & $5 \ldots$ & 2.8 & 5 & .04 & $4 \ldots$ & 4.6 & 2 & .02 \\
\hline Mar & $6 \ldots$ & 2.6 & 3 & 0.02 & $5 \ldots$ & 4.2 & 2 & .02 \\
\hline Mar & $7 \ldots$ & 2.4 & 3 & .02 & $6 \ldots$ & 4.1 & 1 & .01 \\
\hline Mar & $8 \ldots$ & 2.2 & 3 & .02 & May 7... & 3.4 & 2 & .02 \\
\hline Mar & $9 \ldots$ & 2.1 & 2 & .01 & May $13 \ldots$ & 6.3 & 15 & .24 \\
\hline Max & $10 \ldots$ & 2.0 & 3 & .02 & May $14 \ldots$ & 7.1 & 4 & .08 \\
\hline Max & $11 \ldots$ & 2.1 & 2 & .01 & May 15... & 4.6 & 2 & .02 \\
\hline Max & $12 \ldots$ & 2.1 & 2 & .02 & May $16 \ldots$ & 4.4 & 8 & .10 \\
\hline Mar & $13 \ldots$ & 1.8 & 3 & .01 & May $17 \ldots$ & 8.0 & 2 & .04 \\
\hline Max & $14 \ldots$ & 1.5 & 3 & .01 & May 18... & 6.7 & 1 & .02 \\
\hline Max & $15 \ldots$ & 1.3 & 3 & .01 & May 19... & 4.9 & 2 & .03 \\
\hline Mar & $16 \ldots$ & 1.2 & 4 & .01 & May $20 \ldots$ & 4.0 & 2 & .02 \\
\hline Mar & $17 \ldots$ & 1.1 & 4 & .01 & May 21... & 3.5 & 1 & .01 \\
\hline Max & $18 \ldots$ & .93 & 4 & .01 & & & & \\
\hline Max & $19 \ldots$ & .83 & 5 & .01 & & & & \\
\hline
\end{tabular}


Iable 6.--Suspended-sediment discharge for selected days in the Bald Mountain watersheds--Continued

01012520 Bald Mountain Brook near Bald Mountain, 1981 water year--continued

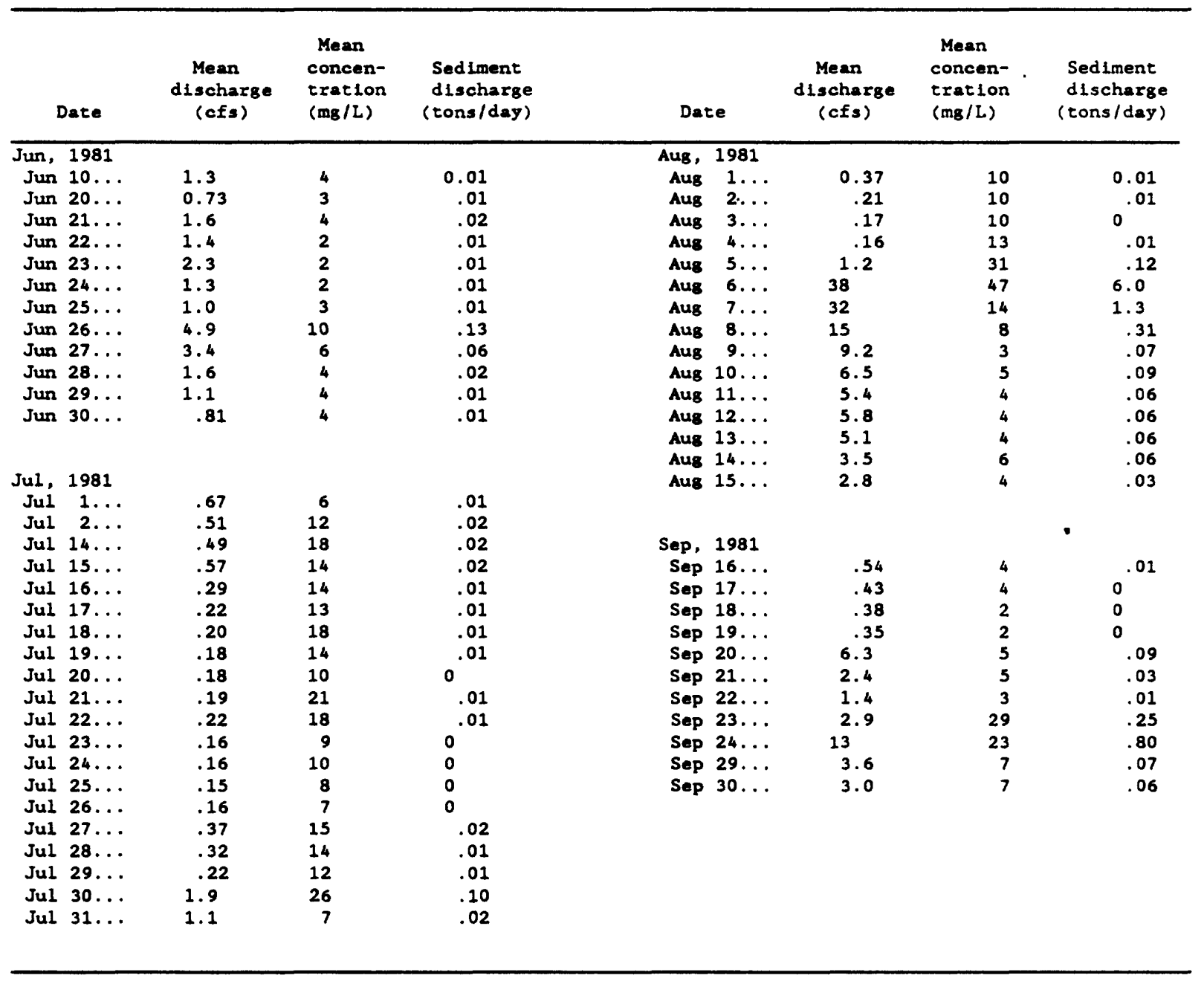


Table 6.--Suspended-sediment discharge for selected days in the Bald Mountain watersheds--Continued

01012520 Bald Mountain Brook near Bald Mountain, 1982 water year

\begin{tabular}{|c|c|c|c|c|c|c|c|c|}
\hline & Date & $\begin{array}{c}\text { Mean } \\
\text { discharge } \\
\text { (cfs) }\end{array}$ & $\begin{array}{l}\text { Mean } \\
\text { concen- } \\
\text { tration } \\
(\mathrm{mg} / \mathrm{L})\end{array}$ & $\begin{array}{l}\text { Sediment } \\
\text { discharge } \\
\text { (tons/day) }\end{array}$ & Date & $\begin{array}{c}\text { Mean } \\
\text { discharge } \\
\text { (cfs) }\end{array}$ & $\begin{array}{l}\text { Mean } \\
\text { concen- } \\
\text { tration } \\
\text { (mg/L) }\end{array}$ & $\begin{array}{l}\text { Sediment } \\
\text { discharge } \\
\text { (tons/day) }\end{array}$ \\
\hline Oct, & 1981 & & & & Nov, 1981 & & & \\
\hline Oct & $1 \ldots$ & 2.6 & 10 & 0.07 & Nov $21 \ldots$ & 4.8 & 15 & 0.19 \\
\hline Oct & $2 \ldots$ & 2.2 & 8 & .05 & Nov $22 \ldots$ & 7.5 & 17 & .34 \\
\hline Oct & $3 \ldots$ & 3.5 & 7 & .07 & Nov $23 \ldots$ & 5.0 & 19 & .26 \\
\hline Oct & $4 \ldots$ & 5.1 & 5 & .07 & Nov $24 \ldots$ & 3.3 & 12 & .11 \\
\hline Oct & s... & 3.5 & 6 & .06 & Nov $25 \ldots$ & 2.6 & 14 & .10 \\
\hline Oct & $6 \ldots$ & 2.5 & 5 & .03 & Nov $26 \ldots$ & 2.3 & 14 & .09 \\
\hline Oct & $7 \ldots$ & 4.3 & 7 & .10 & Nov $27 \ldots$ & 2.1 & 13 & .07 \\
\hline Oct & 8... & 15 & 8 & .31 & Nov 28... & 1.9 & 14 & .07 \\
\hline Oet & $9 .$. & 11 & 5 & .15 & Nov $29 \ldots$ & 1.7 & 8 & .04 \\
\hline Oct & $10 \ldots$ & 7.2 & 3 & .06 & Nov $30 \ldots$ & 1.5 & 10 & .04 \\
\hline Oct & $11 \ldots$ & 5.8 & 3 & .05 & & & & \\
\hline Oct & $12 \ldots$ & 5.2 & 4 & .06 & & & & \\
\hline Oct & $13 \ldots$ & 4.5 & 3 & .04 & Dec, 1981 & & & \\
\hline Oct & $14 \ldots$ & 4.0 & 1 & .01 & Dec $1 \ldots$ & 1.5 & 9 & .04 \\
\hline Oct & $15 \ldots$ & 3.5 & 3 & .03 & $2 \ldots$ & 1.4 & 3 & .01 \\
\hline Oct & $16 \ldots$ & 3.3 & 3 & .03 & $3 \ldots$ & 1.5 & 3 & .01 \\
\hline Oct & $17 \ldots$ & 3.1 & 3 & .03 & $4 \ldots$ & 1.8 & 2 & .01 \\
\hline Oct & $18 \ldots$ & 2.8 & 3 & .02 & $5 \ldots$ & 1.8 & 8 & .04 \\
\hline Oct & $19 \ldots$ & 8.5 & 12 & .26 & $6 \ldots$ & 2.4 & 3 & .02 \\
\hline Oct & $20 \ldots$ & 7.2 & 3 & .06 & $7 \ldots$ & 7.4 & 2 & .04 \\
\hline Oct & $21 \ldots$ & 4.9 & 1 & .01 & $8 \ldots$ & 7.7 & 8 & .17 \\
\hline Oct & $22 \ldots$ & 3.9 & 3 & .03 & Dec $9 . \ldots$ & 5.8 & 7 & .11 \\
\hline Oct & $23 \ldots$ & 5.2 & 5 & .07 & Dec $10 \ldots$ & 5.1 & 3 & .04 \\
\hline Oct & $24 \ldots$ & 20 & 11 & .59 & Dec $11 \ldots$ & 4.5 & 3 & .04 \\
\hline Oct & $25 \ldots$ & 14 & 4 & .15 & Dec $12 \ldots$ & 4.0 & 6 & .06 \\
\hline \multirow[t]{3}{*}{ Oct } & $26 \ldots$ & 9.8 & 4 & .11 & Dec $13 \ldots$ & 3.6 & 8 & .08 \\
\hline & & & & & Dec $14 \ldots$ & 3.2 & 5 & .04 \\
\hline & & & & & Dec $15 \ldots$ & 2.9 & 9 & .07 \\
\hline Nov, & 1981 & & & & Dec $16 \ldots$ & 2.6 & 26 & .18 \\
\hline Nov & $17 \ldots$ & 2.1 & 14 & .08 & Dec $17 \ldots$ & 2.5 & 12 & .08 \\
\hline Nov & $18 \ldots$ & 3.0 & 11 & .09 & Dec $18 \ldots$ & 2.3 & 9 & .06 \\
\hline Nov & $19 \ldots$ & 3.7 & 13 & .13 & Dec $19 \ldots$ & 2.2 & 10 & .06 \\
\hline \multirow[t]{2}{*}{ Nov } & $20 \ldots$ & 2.8 & 14 & .11 & Dec $20 \ldots$ & 2.1 & 3 & .02 \\
\hline & & & & & Dec $21 \ldots$ & 2.0 & 11 & .06 \\
\hline
\end{tabular}


Iable 6.--Suspended-sediment discharge for selected days in the Bald Mountain watersheds--Continued

01012520 Bald Mountain Brook near Bald Mountain, 1982 water year--continued

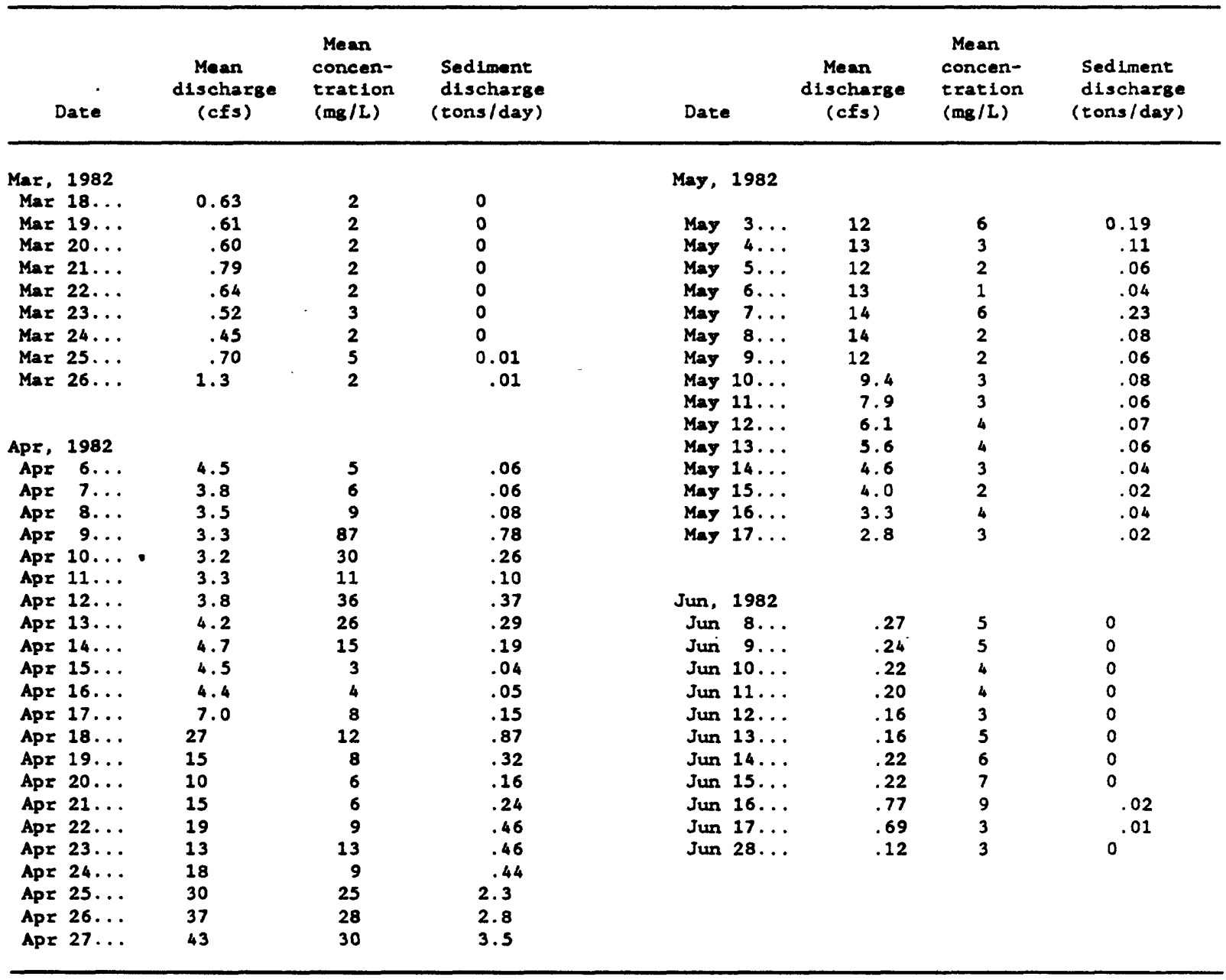


Table 6.--Suspended-sediment discharge for selected days in the Bald Mountain watersheds--Continued

01012520 Bald Mountain Brook near Bald Mountaln, 1982 water year--continued

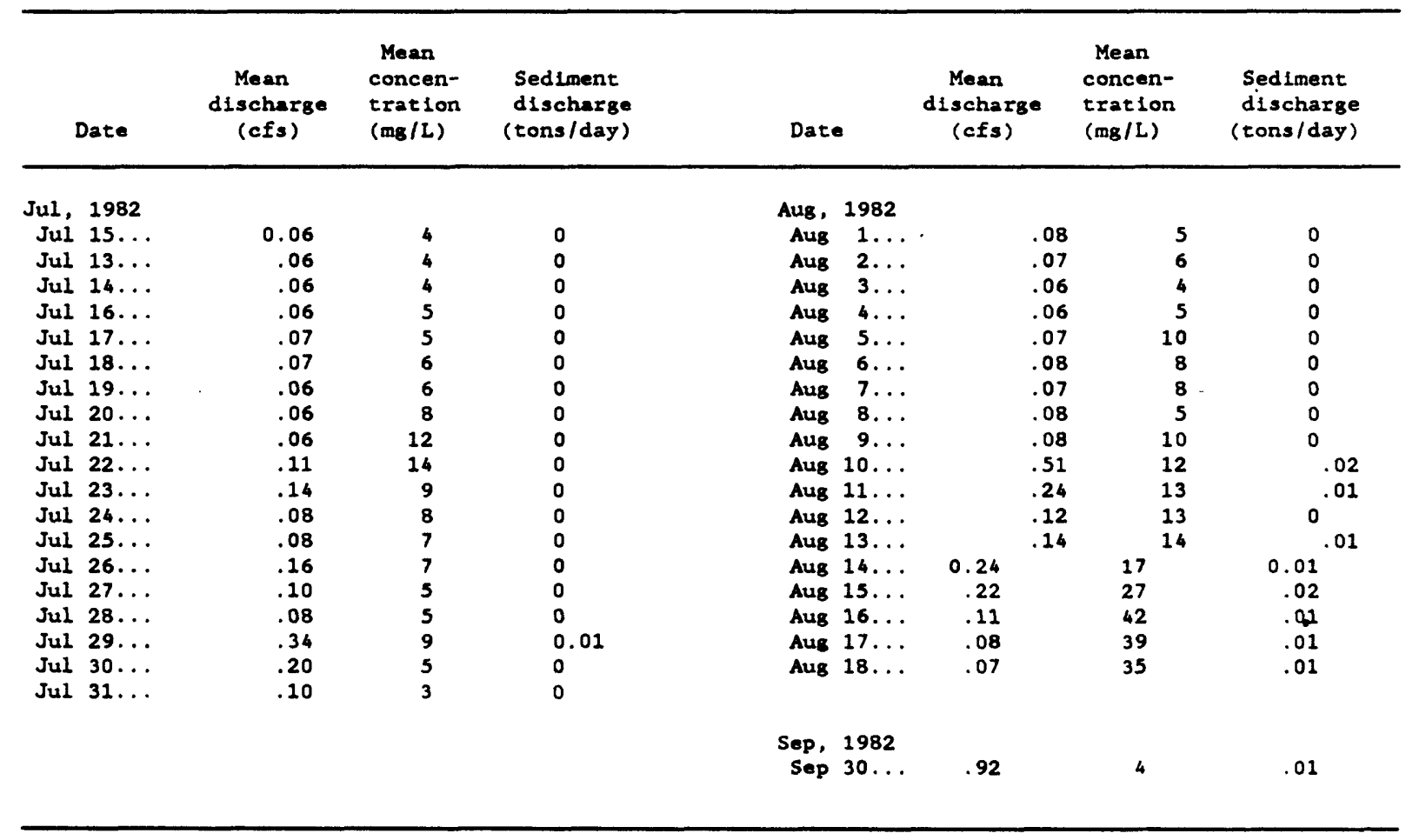


Table 6.--Suspended-sediment discharge for selected days in the Bald Mountain watersheds--Continued

01012520 Bald Mountain Brook near Bald Mountain, 1983 water year

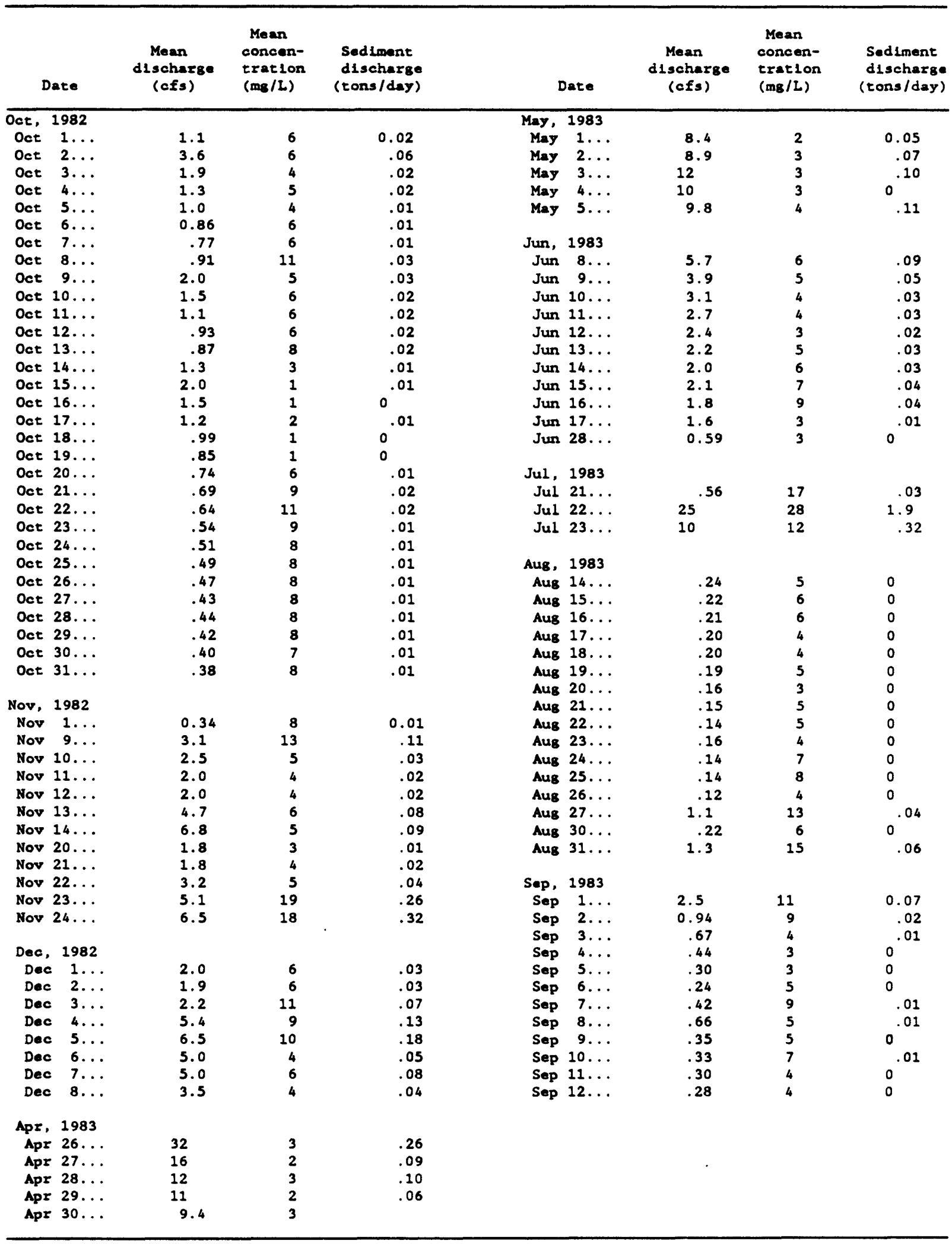


Table 6.--Suspended-sediment discharge for selected days in the Bald Mountain watersheds--Continued

01012520 Bald Mountain Brook near Bald Mountain, 1984 water year

\begin{tabular}{|c|c|c|c|c|c|c|c|c|}
\hline \multicolumn{2}{|r|}{ Date } & $\begin{array}{c}\text { Mean } \\
\text { discharge } \\
\text { (cfs) }\end{array}$ & $\begin{array}{l}\text { Mean } \\
\text { concen- } \\
\text { trat Ion } \\
\text { (mg/L) }\end{array}$ & $\begin{array}{c}\text { Sediment } \\
\text { discharge } \\
\text { (tons/day) }\end{array}$ & Date & $\begin{array}{c}\text { Mean } \\
\text { discharge } \\
\text { (cfs) }\end{array}$ & $\begin{array}{l}\text { Mean } \\
\text { concen- } \\
\text { tration } \\
(\mathrm{mg} / \mathrm{L})\end{array}$ & $\begin{array}{l}\text { Sediment } \\
\text { discharge } \\
\text { (tons/day) }\end{array}$ \\
\hline \multicolumn{5}{|c|}{ Oct, 1983} & \multicolumn{3}{|c|}{ Max, 1984--Continued } & \\
\hline Oct & $7 \ldots$ & 4.3 & 4 & 0.05 & $\operatorname{Mar} 4 \ldots$ & .64 & 10 & .02 \\
\hline Oct & $8 \ldots$ & 2.0 & 3 & .02 & $5 \ldots$ & .62 & 10 & .02 \\
\hline Oct & $9 \ldots$ & 1.6 & 3 & .01 & Mar $6 \ldots$ & .60 & 11 & .02 \\
\hline Oct & $10 \ldots$ & 1.3 & 2 & .01 & Mar $7 \ldots$ & .58 & 12 & .02 \\
\hline Oct & $11 \ldots$ & 1.0 & 2 & 0 & $8 \ldots$ & .56 & 10 & .02 \\
\hline Oct & $12 \ldots$ & 0.92 & 2 & 0 & Mar $9 \ldots$ &.$\dot{54}$ & 13 & .02 \\
\hline Oet & $13 \ldots$ & 1.6 & 2 & .01 & Mar $10 \ldots$ & .53 & 20 & .03 \\
\hline Oct & $14 \ldots$ & 2.1 & 2 & .01 & Mar $29 \ldots$ & 1.5 & 9 & .04 \\
\hline Oct & $15 \ldots$ & 1.6 & 2 & .01 & $\operatorname{Mar} 30 \ldots$ & 1.4 & 7 & .03 \\
\hline Oct & $16 \ldots$ & 1.1 & 2 & .01 & Mar $31 \ldots$ & 1.2 & 7 & .02 \\
\hline Oct & $17 \ldots$ & .93 & 2 & .01 & & & & \\
\hline Oct & $18 \ldots$ & .86 & 3 & .01 & Apr, 1984 & & & \\
\hline Oct & $19 \ldots$ & .78 & 3 & .01 & Apr $1 \ldots$ & 1.3 & 7 & .02 \\
\hline \multirow[t]{2}{*}{ Oct } & $20 \ldots$ & \multirow{2}{*}{\multicolumn{3}{|c|}{.71}} & Apr $2 \ldots$ & 1.4 & 5 & .02 \\
\hline & & & & & Apr $3 \ldots$ & 1.5 & 9 & .04 \\
\hline \multirow{2}{*}{$\begin{array}{l}\text { Nov, } \\
\text { Nov }\end{array}$} & 1983 & & & & $4 \ldots$ & 1.6 & 11 & .05 \\
\hline & $3 \ldots$ & .69 & 8 & .01 & $5 \ldots$ & 2.0 & 12 & .06 \\
\hline Nov & $4 \ldots$ & 3.6 & 7 & .07 & $6 \ldots$ & 2.6 & 25 & .18 \\
\hline Nov & $5 \ldots$ & 2.9 & 4 & .03 & $7 \ldots$ & 6.5 & 30 & .53 \\
\hline Nov & $6 \ldots$ & 3.8 & 5 & .05 & Apr $8 \ldots$ & 5.9 & 69 & 1.1 \\
\hline Nov & $7 \ldots$ & 4.6 & 7 & .09 & Apr $\quad 9 \ldots$ & 5.5 & 19 & .28 \\
\hline Nov & $8 \ldots$ & 3.3 & 7 & .06 & Apr $12 \ldots$ & 6.1 & 28 & .46 \\
\hline Nov & $9 \ldots$ & 2.4 & 5 & .03 & Apr $13 \ldots$ & 7.8 & 24 & .50 \\
\hline Nov & $10 \ldots$ & 1.9 & 2 & .01 & Apr $14 \ldots$ & 13 & 17 & .60 \\
\hline Nov & $11 \ldots$ & 3.3 & 7 & .06 & Apr $21 \ldots$ & 19 & 2 & .10 \\
\hline \multirow[t]{2}{*}{ Nov } & $12 \ldots$ & 6.5 & 12 & .21 & Apr $22 \ldots$ & 16 & 3 & .11 \\
\hline & & & & & Apr $23 \ldots$ & 13 & 13 & .53 \\
\hline Dec, & 1983 & & & & Apr $24 \ldots$ & 31 & 9 & .79 \\
\hline Dec & $1 \ldots$ & 3.9 & 3 & .03 & Apr $25 \ldots$ & 41 & 3 & .36 \\
\hline Dec & $2 \ldots$ & 3.4 & 7 & .06 & Apr $26 \ldots$ & 26 & 3 & .21 \\
\hline Dec & $3 \ldots$ & 3.0 & 7 & .06 & Apr $30 \ldots$ & 17 & 2 & .09 \\
\hline Dec & $4 \ldots$ & 2.6 & 5 & .04 & & & & \\
\hline Dec & 5... & 2.4 & 4 & .02 & May 1984 & & & \\
\hline Dec & $6 \ldots$ & 2.2 & 6 & .04 & May $1 \ldots$ & 15 & 3 & .12 \\
\hline Dec & $7 \ldots$ & 5.0 & 9 & .12 & $2 \ldots$ & 9.1 & 3 & .07 \\
\hline Dec & $10 \ldots$ & 4.3 & 9 & .10 & May 3... & 5.8 & 9 & .14 \\
\hline Dec & $13 \ldots$ & 2.1 & 9 & .05 & $4 \ldots$ & 5.3 & 4 & .06 \\
\hline \multirow[t]{2}{*}{ Dec } & $14 \ldots$ & 11 & 8 & .26 & 3... & 7.5 & 6 & .12 \\
\hline & & & & & $6 \ldots$ & 9.5 & 6 & .15 \\
\hline Feb, & 1984 & & & & $7 \ldots$ & 6.4 & 4 & .07 \\
\hline Feb & $15 \ldots$ & 1.3 & 3 & .01 & 8... & 5.2 & 8 & .11 \\
\hline Feb & $16 \ldots$ & 4.6 & 4 & .05 & May $9 .$. & 5.5 & 3 & .04 \\
\hline Feb & $17 \ldots$ & 3.5 & 6 & .06 & May $10 \ldots$ & 5.3 & 3 & .04 \\
\hline Feb & $18 \ldots$ & 2.6 & 4 & .03 & May $11 \ldots$ & 4.2 & 12 & .14 \\
\hline Feb & $19 \ldots$ & 1.9 & 3 & .02 & May $12 \ldots$ & 3.9 & 14 & .15 \\
\hline Fab & $20 \ldots$ & 1.4 & 2 & .01 & May 25... & 2.2 & 2 & .01 \\
\hline Fab & $21 \ldots$ & 1.1 & 22 & .06 & May 26... & 1.8 & 1 & .01 \\
\hline Fab & $22 \ldots$ & .86 & 8 & .02 & May $27 \ldots$ & 1.7 & 3 & .01 \\
\hline Feb & $23 \ldots$ & .70 & 7 & .01 & May 28... & 1.4 & 1 & 0 \\
\hline$F \cdot b$ & $24 \ldots$ & .58 & 21 & .03 & May 29... & 1.8 & 2 & .01 \\
\hline Fab & $25 \ldots$ & .30 & 7 & .01 & May $30 \ldots$ & 7.4 & 6 & .12 \\
\hline Feb & $26 \ldots$ & 1.1 & 3 & .01 & May $31 \ldots$ & 14 & 6 & .23 \\
\hline Feb & $27 \ldots$ & .97 & 10 & .02 & & & & \\
\hline Fab & $28 \ldots$ & .87 & 21 & .05 & Jun, 1984 & & & \\
\hline \multirow[t]{2}{*}{ Fab } & $29 \ldots$ & .78 & 17 & .04 & Jun $1 \ldots$ & 15 & 3 & .12 \\
\hline & & & & & Jun $2 \ldots$ & 8.0 & 2 & .04 \\
\hline Mar, & 1984 & & & & $3 \ldots$ & 5.8 & 2 & .03 \\
\hline Mar & $1 \ldots$ & .74 & 10 & .02 & $4 \ldots$ & 4.7 & 4 & .05 \\
\hline Mar & $2 \ldots$ & .68 & 10 & .02 & $5 \ldots$ & 3.9 & 3 & .03 \\
\hline Mar & $3 \ldots$ & .66 & 13 & .02 & & & & \\
\hline
\end{tabular}


Iable 6.--Suspended-sediment discharge for selected days in the Bald Mountain watersheds--Continued

01012525 B1shop Mountain Brook near B1shop Mountain, 1982 water year

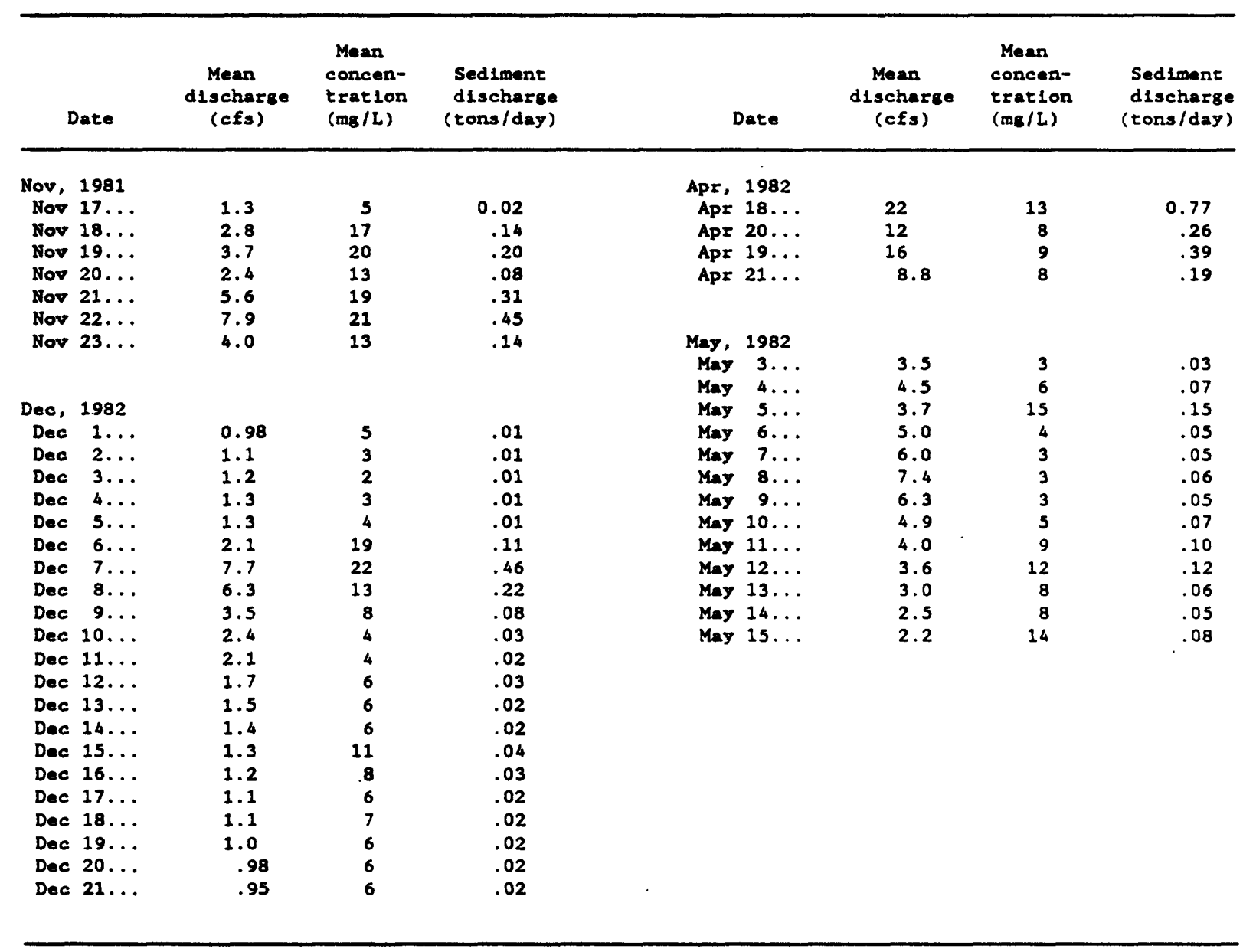


Iable 6.--Suspended-sediment discharge for selected days in the Bald Mountain watersheds--Continued

01012525 Bishop Mountain Brook near Bishop Mountain, 1983 water year

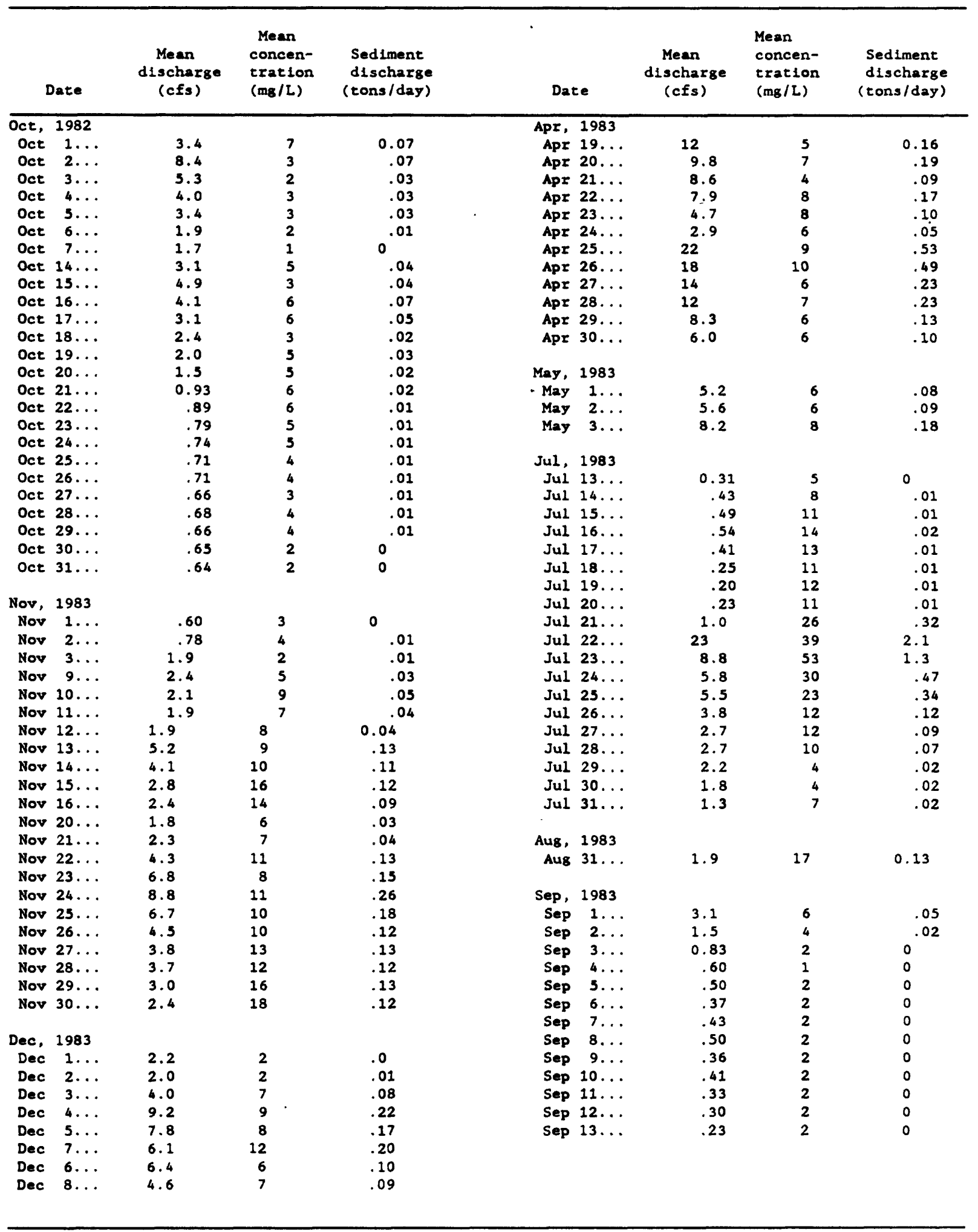


Table 6.--Suspended-sediment discharge for selected days in the Bald Mountaln watersheds--Continued

01012525 Bishop Mountain Brook near Bishop Mountain, 1984 water year

\begin{tabular}{|c|c|c|c|c|c|c|c|c|c|}
\hline & Date & $\begin{array}{c}\text { Mean } \\
\text { discharse } \\
\text { (cfs) }\end{array}$ & $\begin{array}{l}\text { Mean } \\
\text { concen- } \\
\text { tration } \\
(m g / L)\end{array}$ & $\begin{array}{c}\text { Sediment } \\
\text { discharge } \\
\text { (tons/day) }\end{array}$ & . & Date & $\begin{array}{c}\text { Mean } \\
\text { discharge } \\
\text { (cfs) }\end{array}$ & $\begin{array}{l}\text { Mean } \\
\text { concen- } \\
\text { tration } \\
(\mathrm{mg} / \mathrm{L})\end{array}$ & $\begin{array}{l}\text { Sediment } \\
\text { discharge } \\
\text { (tons/day) }\end{array}$ \\
\hline Oct, & 1983 & & & & & Dec, 1983 & & & \\
\hline Oct & $6 \ldots$ & 4.2 & 3 & 0.03 & & Dec $1 \ldots$ & 2.3 & 6 & .04 \\
\hline Oet & $7 \ldots$ & 2.4 & 11 & .07 & & Dec $2 \ldots$ & 1.9 & 10 & .05 \\
\hline Oct & $8 \ldots$ & 1.4 & 10 & .04 & & $3 \ldots$ & 1.7 & 10 & .05 \\
\hline Oct & $9 \ldots$ & 1.0 & 10 & .03 & & $4 \ldots$ & 1.6 & 12 & .05 \\
\hline Oct & $10 \ldots$ & 0.78 & 9 & .02 & & $5 \ldots$ & 1.4 & 14 & .05 \\
\hline Oct & $11 \ldots$ & .61 & 10 & .02 & & $6 \ldots$ & 1.3 & 12 & .04 \\
\hline Oct & $12 \ldots$ & .58 & 8 & .01 & & Dec $7 \ldots$ & 3.0 & 27 & .22 \\
\hline Oct & $13 \ldots$ & .90 & 9 & .02 & & Dec $10 \ldots$ & 1.7 & 36 & .16 \\
\hline Oet & $14 \ldots$ & 1.3 & 6 & .02 & & Dec $13 \ldots$ & 1.2 & 10 & .03 \\
\hline Oct & $21 \ldots$ & .20 & 6 & 0 & & Dec $14 \ldots$ & 5.0 & 10 & .14 \\
\hline Oct & $22 \ldots$ & .18 & 8 & 0 & & Dec $15 \ldots$ & 7.2 & 14 & .27 \\
\hline Oct & $23 \ldots$ & .17 & 7 & 0 & & Dec $27 \ldots$ & 1.2 & 20 & .06 \\
\hline Oct & $24 \ldots$ & .19 & 6 & 0 & & Dec $28 \ldots$ & 1.1 & 8 & .02 \\
\hline Oct & $25 \ldots$ & .18 & 7 & 0 & & Dec $29 \ldots$ & 1.1 & 6 & .02 \\
\hline Oct & $26 \ldots$ & .16 & 6 & 0 & & Dec $30 \ldots$ & 1.0 & 8 & .02 \\
\hline Oet & $27 \ldots$ & .16 & 5 & 0 & & Dec $31 \ldots$ & .95 & 9 & .02 \\
\hline Oct & $28 \ldots$ & .16 & 3 & 0 & & & & & \\
\hline Oct 2 & $29 \ldots$ & .22 & 5 & 0 & & & & & \\
\hline Oct 3 & $30 \ldots$ & .22 & 6 & 0 & & Jan, 1984 & & & \\
\hline \multirow[t]{3}{*}{ Oct } & $31 \ldots$ & .20 & 6 & 0 & & $\operatorname{Jan} 1 \ldots$ & .90 & 6 & .01 \\
\hline & & & & & & Jan $2 \ldots$ & .86 & 7 & .02 \\
\hline & & & & & & $\operatorname{Jan} 3 \ldots$ & .82 & 6 & .01 \\
\hline Nov, & 1983 & & & & & Jan $4 \ldots$ & .78 & 5 & .01 \\
\hline Nov & $1 \ldots$ & 0.19 & 3 & 0 & & $\operatorname{Jan} 5 \ldots$ & .75 & 5 & .01 \\
\hline Nov & $2 \ldots$ & .18 & 6 & 0 & & $\operatorname{Jan} 6 \ldots$ & .72 & 5 & .01 \\
\hline Nov & $3 \ldots$ & .38 & 15 & 0.02 & . & $7 \ldots$ & .69 & 5 & .01 \\
\hline Nov & $4 \ldots$ & 2.4 & 32 & .21 & & $\operatorname{Jan} 8 \ldots$ & .66 & 6 & .01 \\
\hline Nov & $6 \ldots$ & 2.7 & 11 & .08 & & $\operatorname{Jan} 9 \ldots$ & .63 & 5 & .01 \\
\hline Nov & $7 \ldots$ & 3.0 & 6 & .05 & & $\operatorname{Jan} 10 \ldots$ & .61 & 6 & .01 \\
\hline Nov & 8... & 2.0 & 4 & .02 & & & & & \\
\hline Nov & $9 \ldots$ & 1.5 & 4 & .02 & & & . & & \\
\hline Nov & $10 \ldots$ & 1.1 & 4 & .01 & & Fab, 1984 & & & \\
\hline Nov & $11 \ldots$ & 2.0 & 5 & .03 & & Feb $25 \ldots$ & .39 & 5 & .01 \\
\hline Nov & $12 \ldots$ & 4.9 & 30 & .40 & & Fab $26 \ldots$ & .70 & 5 & .01 \\
\hline Nov & $13 \ldots$ & 3.4 & 20 & .18 & & Fab $27 \ldots$ & .56 & 5 & .01 \\
\hline Nov & $23 \ldots$ & 1.9 & 15 & .08 & & Feb $28 \ldots$ & .50 & 5 & .01 \\
\hline Nov & $24 \ldots$ & 1.8 & 10 & .05 & & Feb $29 \ldots$ & .46 & 13 & .02 \\
\hline Nov & $25 \ldots$ & 9.8 & 19 & .50 & & & & & \\
\hline Nov & $26 \ldots$ & 11 & 12 & .36 & & & & & \\
\hline Nov & $27 \ldots$ & 6.2 & 7 & .12 & & & & & \\
\hline Nov & $28 \ldots$ & 4.0 & 5 & .05 & & & & & \\
\hline Nov & $29 \ldots$ & 3.0 & 9 & .07 & & & & & \\
\hline Nov & $30 \ldots$ & 2.6 & 6 & .04 & & & & & \\
\hline
\end{tabular}


Table 6.--Suspended-sediment discharge for selected days in the

Bald Mountain watersheds--Continued

01012525 Bishop Mountain Brook near B1shop Mountain, 1984 water year--continued

\begin{tabular}{|c|c|c|c|c|c|c|c|c|c|}
\hline \multicolumn{2}{|c|}{ Date } & $\begin{array}{c}\text { Mean } \\
\text { discharge } \\
\text { (cfs) }\end{array}$ & $\begin{array}{l}\text { Mean } \\
\text { concen- } \\
\text { tration } \\
\text { (mg/L) }\end{array}$ & $\begin{array}{r}\text { Sediment } \\
\text { discharge } \\
\text { (tons/day) }\end{array}$ & \multicolumn{2}{|c|}{ Date } & $\begin{array}{c}\text { Mean } \\
\text { discharge } \\
\text { (cfs) }\end{array}$ & $\begin{array}{l}\text { Mean } \\
\text { concen- } \\
\text { tration } \\
(\mathrm{mg} / \mathrm{L})\end{array}$ & $\begin{array}{l}\text { Sediment } \\
\text { discharge } \\
\text { (tons/day) }\end{array}$ \\
\hline \multicolumn{10}{|c|}{ Apr, 1984--Continued } \\
\hline Mar & $1 \ldots$ & .44 & 7 & .01 & Apr & $24 \ldots$ & 18 & 4 & .19 \\
\hline Mar & $2 \ldots$ & .42 & 7 & .01 & Apr & $25 \ldots$ & 18 & 3 & .15 \\
\hline Mar & 3... & .40 & 6 & .01 & Apr & $26 \ldots$ & 15 & 5 & .20 \\
\hline Mar & $4 \ldots$ & .38 & 6 & .01 & Apr & $27 \ldots$ & 16 & 6 & .26 \\
\hline Mar & $5 \ldots$ & .37 & 5 & 0 & Apr & $28 \ldots$ & 15 & 6 & .24 \\
\hline Mar & $6 \ldots$ & .36 & 11 & .01 & Apr & $29 \ldots$ & 15 & 4 & .16 \\
\hline Mar & $7 \ldots$ & .35 & 17 & .02 & Apr & $30 \ldots$ & 15 & 2 & .08 \\
\hline Mar & $8 \ldots$ & .34 & 14 & .01 & & & & & \\
\hline Mar & $9 \ldots$ & .33 & 9 & .01 & & & & & \\
\hline Mar & $10 \ldots$ & .32 & 11 & .01 & May, & 1984 & & & \\
\hline Mar & $11 \ldots$ & .32 & 15 & .01 & May & $1 \ldots$ & 16 & 2 & .09 \\
\hline Mar & $12 \ldots$ & .31 & 13 & .01 & May & $2 \ldots$ & 12 & 2 & .06 \\
\hline Mar & $29 \ldots$ & .90 & 7 & .02 & May & $3 \ldots$ & 8.6 & 2 & .05 \\
\hline Mar & $30 \ldots$ & .82 & 6 & .01 & May & $4 \ldots$ & 8.0 & 2 & .04 \\
\hline \multirow[t]{3}{*}{ Mar } & $31 \ldots$ & .75 & 4 & .01 & May & $5 \ldots$ & 10 & 2 & .05 \\
\hline & & & & & May & $6 \ldots$ & 11 & 1 & .03 \\
\hline & & & & & May & $7 \ldots$ & 8.5 & 1 & .02 \\
\hline Apr, & 1984 & & & & May & $24 \ldots$ & 2.8 & 15 & .11 \\
\hline Apr & $1 \ldots$ & .77 & 4 & .01 & May & $25 \ldots$ & 2.3 & 3 & .02 \\
\hline Apr & 2. & .84 & 4 & .01 & May & $26 \ldots$ & 1.9 & 4 & .02 \\
\hline Apr & 3... & .90 & 6 & .01 & May & $27 \ldots$ & 1.7 & 4 & .02 \\
\hline Apr & $13 \ldots$ & 4.2 & 8 & .09 & May & $28 \ldots$ & 1.4 & 4 & .02 \\
\hline Apr & $14 \ldots$ & 6.0 & 10 & .16 & May & $29 \ldots$ & 1.8 & 7 & .03 \\
\hline Apr & $15 . \therefore$ & 8.4 & 6 & .14 & May & $30 \ldots$ & 7.2 & 9 & .17 \\
\hline Apr & $16 \ldots$ & 12 & 3 & .10 & May & $31 \ldots$ & 11 & 3 & .09 \\
\hline Apr & $17 \ldots$ & 17 & 4 & .18 & & & & & \\
\hline Apr & $18 \ldots$ & 15 & 3 & .12 & & & & & \\
\hline Apr & $19 \ldots$ & 13 & 3 & .10 & Jun, & 1984 & $\cdot$ & & \\
\hline Apr & $20 \ldots$ & 13 & 3 & .10 & Jun & 1 & 9.2 & 3 & .07 \\
\hline Apr & $21 \ldots$ & 12 & 3 & .10 & Jun & 2 & 5.7 & 5 & .08 \\
\hline Apr & $22 \ldots$ & 11 & 3 & .09 & Jun & 3 & 3.9 & 5 & .05 \\
\hline \multirow[t]{2}{*}{ Apr } & $23 \ldots$ & 13 & 5 & .18 & Jun & 4 & 3.0 & 5 & .04 \\
\hline & & & & & Jun & 5 & 2.5 & 3 & .02 \\
\hline
\end{tabular}


Iable 6.--Suspended-sediment discharge for selected days in the

Bald Mountain watersheds--Continued

01012570 Fish River above inlet Fish River Lake, 1983 water year

\begin{tabular}{|c|c|c|c|c|c|c|c|c|}
\hline & Date & $\begin{array}{c}\text { Mean } \\
\text { d1scharge } \\
\text { (cfs) }\end{array}$ & $\begin{array}{l}\text { Mean } \\
\text { concen- } \\
\text { tration } \\
\text { (mg/L) }\end{array}$ & $\begin{array}{l}\text { Sediment } \\
\text { discharge } \\
\text { (tons/day) }\end{array}$ & Date & $\begin{array}{c}\text { Mean } \\
\text { discharge } \\
\text { (cfs) }\end{array}$ & $\begin{array}{c}\text { Mean } \\
\text { concen- } \\
\text { tration } \\
(\mathrm{mg} / \mathrm{L})\end{array}$ & $\begin{array}{l}\text { Sediment } \\
\text { discharge } \\
\text { (tons/day) }\end{array}$ \\
\hline Oct, & 1982 & & & & Мay, 1983 & & & \\
\hline Oct & $1 \ldots$ & 61 & 2 & 0.33 & May $1 \ldots$ & 663 & 5 & 9.0 \\
\hline Oct & $2 \ldots$ & 127 & 2 & .69 & May 2... & 627 & 5 & 8.5 \\
\hline Oct & $3 \ldots$ & 102 & 2 & .55 & May 3... & 673 & 9 & 16 \\
\hline Oct & $4 \ldots$ & 87 & 2 & .47 & May 4... & 655 & 6 & 11 \\
\hline Oct & $5 \ldots$ & 78 & 3 & .63 & $5 \ldots$ & 628 & 7 & 12 \\
\hline Oct & $6 \ldots$ & 72 & 2 & .39 & & & & \\
\hline Oct & $20 \ldots$ & 50 & 5 & .68 & Jul, 1983 & & & \\
\hline Oct & $21 \ldots$ & 48 & 3 & .39 & Ju1 $19 \ldots$ & 16 & 2 & .09 \\
\hline Oct & $22 \ldots$ & 46 & 4 & .50 & Jul $20 \ldots$ & 15 & 6 & .24 \\
\hline Oct & $23 \ldots$ & 42 & 3 & .34 & Jul $21 \ldots$ & 17 & 8 & .37 \\
\hline Oct & $24 \ldots$ & 40 & 3 & .32 & Ju1 22... & 404 & 13 & 14 \\
\hline Oct & $25 \ldots$ & 41 & 5 & .55 & Ju1 23... & 320 & 10 & 8.6 \\
\hline Oct & $26 \ldots$ & 39 & 2 & .21 & Ju1 $24 \ldots$ & 204 & 7 & 3.9 \\
\hline Oct & $27 \ldots$ & 36 & 5 & .49 & Jul $25 \ldots$ & 197 & 5 & 2.7 \\
\hline Oct & $28 \ldots$ & 34 & 5 & .46 & Ju1 $26 \ldots$ & 157 & 8 & 3.4 \\
\hline Oct & $29 \ldots$ & 33 & 5 & .45 & Ju1 $27 \ldots$ & 120 & 5 & 1.6 \\
\hline Oct & $30 \ldots$ & 32 & 8 & .69 & Jul 28... & 105 & 5 & 1.4 \\
\hline \multirow[t]{2}{*}{ Oct } & $31 \ldots$ & 30 & 5 & .40 & Ju1 $29 \ldots$ & 89 & 6 & 1.4 \\
\hline & & & & & Jul $30 \ldots$ & 77 & 6 & 1.2 \\
\hline Nov, & 1982 & & & & Ju1 $31 \ldots$ & 65 & 4 & .70 \\
\hline Nov & $1 \ldots$ & 29 & 5 & .39 & & & & \\
\hline Nov & $2 \ldots$ & 29 & 8 & .63 & Aug, 1983 & & & \\
\hline Nov & $9 \ldots$ & 243 & 2 & 1.3 & Aug $13 \ldots$ & 17 & 5 & 0.23 \\
\hline Nov & $10 \ldots$ & 212 & 2 & 1.1 & Aug $14 \ldots$ & 15 & 3 & .12 \\
\hline Nov & $11 \ldots$ & 185 & 2 & 1.0 & Aug $15 \ldots$ & 14 & 6 & .23 \\
\hline Nov & $12 \ldots$ & 169 & 2 & .91 & Aug $16 \ldots$ & 13 & 3 & .11 \\
\hline Nov & $13 \ldots$ & 231 & 2 & 1.2 & Aug $17 \ldots$ & 12 & 3 & .10 \\
\hline Nov & $14 \ldots$ & 313 & 3 & 2.5 & Aug $18 \ldots$ & 11 & 4 & .12 \\
\hline Nov & $15 \ldots$ & 268 & 3 & 2.2 & Aug $19 \ldots$ & 10 & 4 & .11 \\
\hline Nov & $16 \ldots$ & 246 & 3 & 2.0 & Aus $20 \ldots$ & 8.1 & 6 & .13 \\
\hline Nov & $17 \ldots$ & 219 & 2 & 1.2 & Aug $21 \ldots$ & 7.0 & 4 & .08 \\
\hline Nov & $18 \ldots$ & 196 & 3 & 1.6 & Aug $22 \ldots$ & 4.5 & 3 & .04 \\
\hline Nov & $19 \ldots$ & 176 & 3 & 1.4 & Aug $23 \ldots$ & 4.3 & 4 & .05 \\
\hline Nov & $22 \ldots$ & 176 & 7 & 3.3 & Aug $24 \ldots$ & 3.8 & 4 & .04 \\
\hline Nov & $23 \ldots$ & 211 & 8 & 4.6 & Aug $25 \ldots$ & 3.5 & 4 & .04 \\
\hline Nov & $24 \ldots$ & 264 & 9 & 6.4 & Aus $30 \ldots$ & 6.5 & 4 & .07 \\
\hline Nov & $25 \ldots$ & 265 & 8 & 5.7 & Aus $31 \ldots$ & 14 & 2 & .08 \\
\hline Nov & $26 \ldots$ & 215 & 7 & 4.1 & & & & \\
\hline \multirow[t]{2}{*}{ Nov } & $27 \ldots$ & 190 & 6 & 3.1 & & & & \\
\hline & & & & & Sep, 1983 & & & \\
\hline Dec, & 1982 & & & & Sep $1 \ldots$ & 36 & 2 & .19 \\
\hline Dec & $1 \ldots$ & 150 & 2 & 0.81 & $2 \ldots$ & 24 & 1 & .06 \\
\hline Dec & $2 \ldots$ & 149 & 3 & 1.2 & $3 \ldots$ & 22 & 1 & .06 \\
\hline Dec & $3 \ldots$ & 149 & 2 & .80 & Sep $4 \ldots$ & 30 & 1 & .08 \\
\hline Dec & $4 \ldots$ & 225 & 2 & 1.2 & Sep $5 \ldots$ & 20 & 1 & .05 \\
\hline Dec & $5 \ldots$ & 282 & 1 & .76 & Sep $13 \ldots$ & 14 & 4 & .15 \\
\hline Dec & $6 \ldots$ & 260 & 1 & .70 & Sep $14 \ldots$ & 12 & 5 & .16 \\
\hline Dec & $7 \ldots$ & 264 & 2 & 1.4 & Sep $15 \ldots$ & 10 & 7 & .19 \\
\hline \multirow[t]{2}{*}{ Dec } & 8... & 230 & 2 & 1.2 & Sep $16 \ldots$ & 8.6 & 7 & .16 \\
\hline & & & & & Sep $17 \ldots$ & 7.7 & 8 & .17 \\
\hline Apr, & 1983 & & & & Sep $18 \ldots$ & 13 & 6 & .21 \\
\hline Apr & $19 \ldots$ & 1310 & 9 & 32 & Sep $19 \ldots$ & 17 & 4 & .18 \\
\hline Apr & $26 \ldots$ & 1210 & 3 & 9.8 & $\operatorname{sep} 20 \ldots$ & 15 & 5 & .20 \\
\hline Apr & $27 \ldots$ & 1040 & 3 & 8.4 & Sep $21 \ldots$ & 13 & 5 & .18 \\
\hline Apt & $28 \ldots$ & 868 & 3 & 7.0 & Sep $22 \ldots$ & 65 & 9 & 1.6 \\
\hline Apr & $29 \ldots$ & 786 & 3 & 6.4 & Sep $23 \ldots$ & 107 & 12 & 3.5 \\
\hline \multirow[t]{2}{*}{ Apr } & $30 \ldots$ & 731 & 3 & 5.9 & Sep $24 \ldots$ & 55 & 8 & 1.2 \\
\hline & & & & & Sep $25 \ldots$ & 40 & 7 & .76 \\
\hline
\end{tabular}


Iable 6.--Suspended-sediment discharge for selected days in the

Bald Mountain watersheds--Continued

01012570 Fish River above Inlet Fish River Lake, 1984 water year

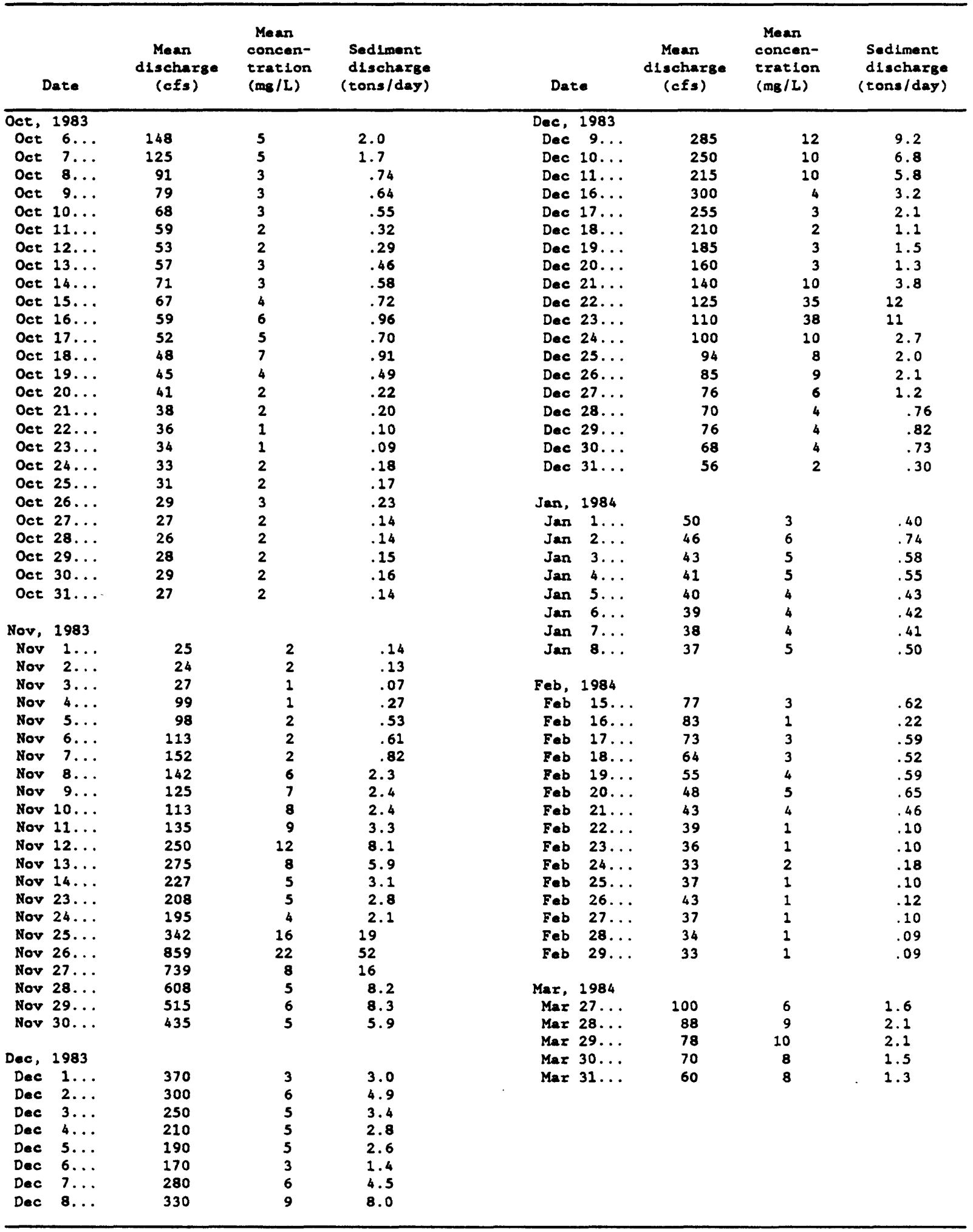


Table 6.--Suspended-sediment discharge for selected days in the Bald Mountain watersheds--Continued

01012570 Fish River above Inlet Fish River Lake, 1984 water year--continued

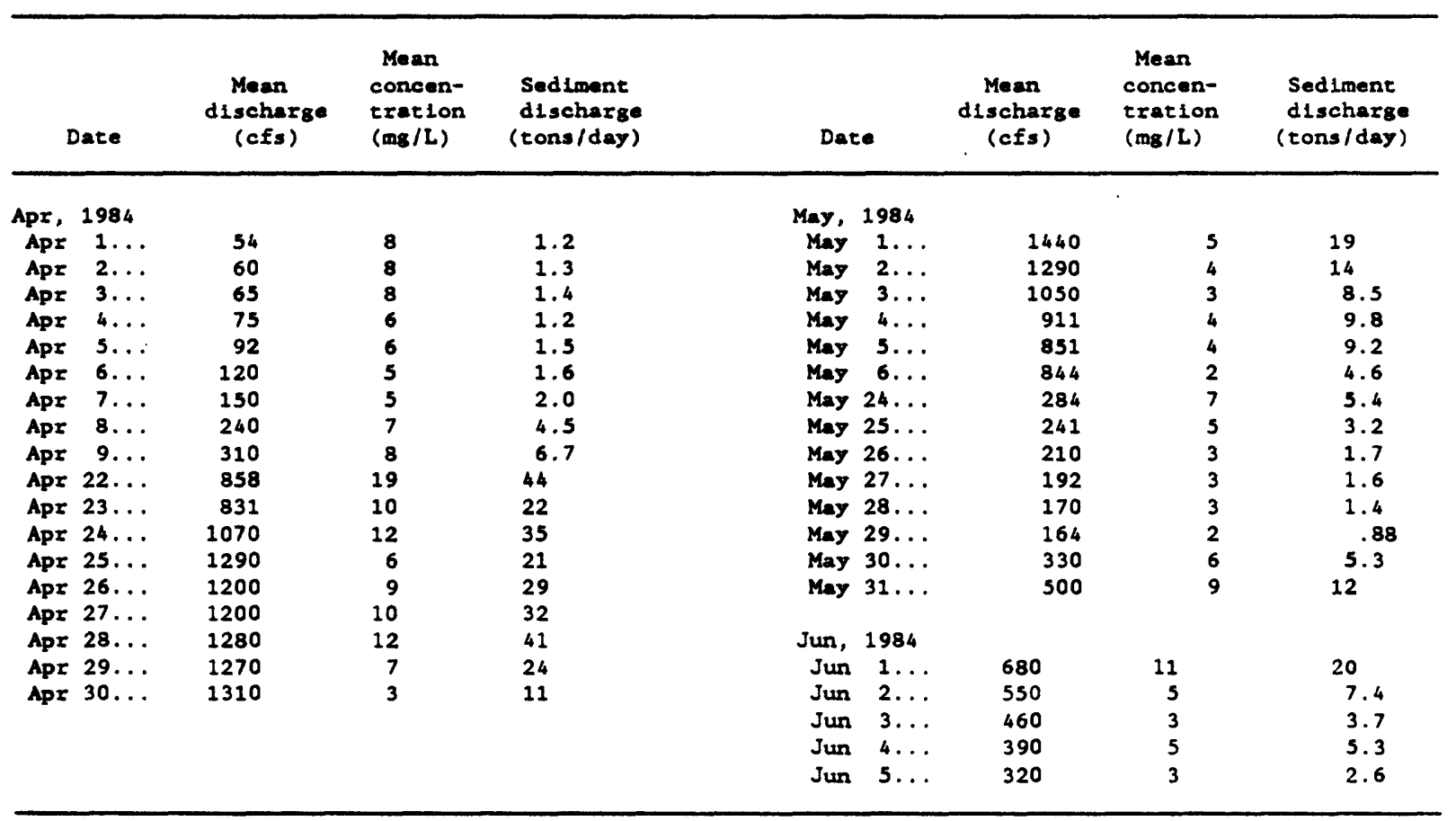


Table 7.--Daily precipitation totals at continuous-recording gages

01012520 Rain Gage 1 near Bald Mountain, ME

Precipitation totals, in inches

\begin{tabular}{|c|c|c|c|c|c|c|c|c|c|c|c|c|}
\hline \multirow[b]{2}{*}{ Day } & \multicolumn{3}{|c|}{1980} & \multicolumn{9}{|c|}{1981} \\
\hline & OCT & NOV & $\mathrm{DEC}$ & JAN & FEB & MAR & APR & MAY & JUN & JUL & AUG & SEP \\
\hline $\begin{array}{l}1 \\
2 \\
3 \\
4 \\
5\end{array}$ & & $\begin{array}{l}- \\
- \\
-- \\
--\end{array}$ & $\begin{array}{r}0.05 \\
.18 \\
1.17 \\
.18 \\
.00\end{array}$ & $\begin{array}{r}0.00 \\
.25 \\
.02 \\
.00 \\
.01\end{array}$ & $\begin{array}{r}0.03 \\
.83 \\
.02 \\
.00 \\
.00\end{array}$ & $\begin{array}{r}0.15 \\
.00 \\
.03 \\
.00 \\
.05\end{array}$ & $\begin{array}{r}0.00 \\
.31 \\
.00 \\
.04 \\
.23\end{array}$ & $\begin{array}{r}0.00 \\
.07 \\
.00 \\
.00 \\
.00\end{array}$ & $\begin{array}{r}0.00 \\
.00 \\
.00 \\
.01 \\
.00\end{array}$ & $\begin{array}{r}0.00 \\
.00 \\
.03 \\
.00 \\
.00\end{array}$ & $\begin{array}{r}0.00 \\
.00 \\
.00 \\
.00 \\
1.80\end{array}$ & $\begin{array}{r}0.00 \\
.00 \\
.00 \\
.00 \\
.00\end{array}$ \\
\hline $\begin{array}{r}6 \\
7 \\
8 \\
9 \\
10\end{array}$ & & $\begin{array}{l}- \\
- \\
-- \\
-- \\
--\end{array}$ & $\begin{array}{l}.00 \\
.00 \\
.27 \\
.00 \\
.05\end{array}$ & $\begin{array}{l}.00 \\
.47 \\
.00 \\
.00 \\
.30\end{array}$ & $\begin{array}{l}.00 \\
.00 \\
.22 \\
.18 \\
.00\end{array}$ & $\begin{array}{l}.06 \\
.07 \\
.00 \\
.00 \\
.00\end{array}$ & $\begin{array}{l}.02 \\
.00 \\
.00 \\
.23 \\
.00\end{array}$ & $\begin{array}{l}.00 \\
.00 \\
.00 \\
.00 \\
.00\end{array}$ & $\begin{array}{l}.12 \\
.03 \\
.00 \\
.18 \\
.00\end{array}$ & $\begin{array}{l}.57 \\
.00 \\
.09 \\
.00 \\
.01\end{array}$ & $\begin{array}{r}3.65 \\
.05 \\
.07 \\
.06 \\
.03\end{array}$ & $\begin{array}{l}.00 \\
.00 \\
.00 \\
.51 \\
.09\end{array}$ \\
\hline $\begin{array}{l}11 \\
12 \\
13 \\
14 \\
15\end{array}$ & & $\begin{array}{l}- \\
- \\
\cdots \\
- \\
-\end{array}$ & $\begin{array}{l}.30 \\
.07 \\
.08 \\
.02 \\
.02\end{array}$ & $\begin{array}{l}.05 \\
.00 \\
.20 \\
.00 \\
.00\end{array}$ & $\begin{array}{l}.25 \\
.20 \\
.00 \\
.00 \\
.00\end{array}$ & $\begin{array}{l}.00 \\
.00 \\
.03 \\
.05 \\
.00\end{array}$ & $\begin{array}{l}.00 \\
.05 \\
.00 \\
.28 \\
.00\end{array}$ & $\begin{array}{l}.04 \\
.16 \\
.94 \\
.03 \\
.00\end{array}$ & $\begin{array}{l}.00 \\
.00 \\
.00 \\
.00 \\
.10\end{array}$ & $\begin{array}{l}.00 \\
.00 \\
.20 \\
.15 \\
.00\end{array}$ & $\begin{array}{l}.32 \\
.00 \\
.00 \\
.00 \\
.14\end{array}$ & $\begin{array}{l}.01 \\
.00 \\
.00 \\
.24 \\
.01\end{array}$ \\
\hline $\begin{array}{l}16 \\
17 \\
18 \\
19 \\
20\end{array}$ & & $\begin{array}{r}\cdots \\
\cdots \\
0.00 \\
0.00\end{array}$ & $\begin{array}{l}.48 \\
.05 \\
.00 \\
.03 \\
.00\end{array}$ & $\begin{array}{l}.00 \\
.00 \\
.00 \\
.00 \\
.00\end{array}$ & $\begin{array}{l}.00 \\
.00 \\
.00 \\
.07 \\
.07\end{array}$ & $\begin{array}{r}.00 \\
1.37 \\
.22 \\
.19 \\
.05\end{array}$ & $\begin{array}{l}.00 \\
.02 \\
.21 \\
.00 \\
.00\end{array}$ & $\begin{array}{l}.52 \\
.50 \\
.00 \\
.00 \\
.00\end{array}$ & $\begin{array}{l}.24 \\
.06 \\
.00 \\
.00 \\
.15\end{array}$ & $\begin{array}{l}.00 \\
.01 \\
.00 \\
.00 \\
.00\end{array}$ & $\begin{array}{r}1.19 \\
2.45 \\
.06 \\
.00 \\
.00\end{array}$ & $\begin{array}{l}.00 \\
.01 \\
.03 \\
.53 \\
.16\end{array}$ \\
\hline $\begin{array}{l}21 \\
22 \\
23 \\
24 \\
25\end{array}$ & & $\begin{array}{l}.00 \\
.00 \\
.00 \\
.20 \\
.63\end{array}$ & $\begin{array}{l}.00 \\
.02 \\
.08 \\
.24 \\
.03\end{array}$ & $\begin{array}{l}.00 \\
.14 \\
.06 \\
.00 \\
.00\end{array}$ & $\begin{array}{l}.10 \\
.01 \\
.00 \\
.05 \\
.00\end{array}$ & $\begin{array}{l}.00 \\
.00 \\
.00 \\
.00 \\
.00\end{array}$ & $\begin{array}{l}.00 \\
.00 \\
.00 \\
.41 \\
.05\end{array}$ & $\begin{array}{l}.00 \\
.07 \\
.00 \\
.00 \\
.00\end{array}$ & $\begin{array}{l}.41 \\
.39 \\
.01 \\
.00 \\
.17\end{array}$ & $\begin{array}{l}.05 \\
.00 \\
.00 \\
.00 \\
.00\end{array}$ & $\begin{array}{l}.00 \\
.00 \\
.48 \\
.00 \\
.00\end{array}$ & $\begin{array}{r}.00 \\
.06 \\
1.25 \\
.80 \\
.02\end{array}$ \\
\hline $\begin{array}{l}26 \\
27 \\
28 \\
29 \\
30 \\
31\end{array}$ & & $\begin{array}{l}.32 \\
.03 \\
.67 \\
.17 \\
.03 \\
.-\end{array}$ & $\begin{array}{l}.00 \\
.02 \\
.02 \\
.16 \\
.04 \\
.01\end{array}$ & $\begin{array}{l}.02 \\
.02 \\
.00 \\
.00 \\
.00 \\
.00\end{array}$ & $\begin{array}{l}.48 \\
.14 \\
.08 \\
-- \\
-- \\
--\end{array}$ & $\begin{array}{l}.00 \\
.00 \\
.06 \\
.20 \\
.00 \\
.34\end{array}$ & $\begin{array}{l}.00 \\
.00 \\
.00 \\
.66 \\
.93 \\
.-\end{array}$ & $\begin{array}{l}.12 \\
.16 \\
.41 \\
.08 \\
.05 \\
.36\end{array}$ & $\begin{array}{l}.80 \\
.00 \\
.00 \\
.00 \\
.01 \\
.-\end{array}$ & $\begin{array}{l}.00 \\
.31 \\
.00 \\
.57 \\
.00 \\
.01\end{array}$ & $\begin{array}{l}.00 \\
.03 \\
.00 \\
.00 \\
.31 \\
.00\end{array}$ & $\begin{array}{l}.00 \\
.16 \\
.02 \\
.05 \\
.00 \\
-.\end{array}$ \\
\hline Total & & - & 3.30 & 1.54 & 2.73 & 3.80 & 2.56 & 3.47 & 2.85 & 2.31 & 10.73 & 3.95 \\
\hline
\end{tabular}


Table 7. - Daily precipitation totals at continuous-recording gages--Continued 01012520 Rain Gage 1 near Bald Mountain, ME.--Continued

Precipitation totals, in inches

1981

Day OCT NOV DEC JAN FEB MAR APR MAY JUN JUL AUG SEP

\begin{tabular}{|c|c|c|c|c|c|c|c|c|c|c|c|c|}
\hline $\begin{array}{l}1 \\
2 \\
3 \\
4 \\
5\end{array}$ & $\begin{array}{r}0.00 \\
.05 \\
.58 \\
.03 \\
.00\end{array}$ & $\begin{array}{r}0.00 \\
.00 \\
.00 \\
.00 \\
.00\end{array}$ & $\begin{array}{r}0.00 \\
.11 \\
.24 \\
.00 \\
.00\end{array}$ & $\begin{array}{r}0.71 \\
.22 \\
.00 \\
.97 \\
.02\end{array}$ & $\begin{array}{r}0.85 \\
.00 \\
.63 \\
.05 \\
.10\end{array}$ & $\begin{array}{r}0.03 \\
.08 \\
.02 \\
.00 \\
.39\end{array}$ & $\begin{array}{r}0.46 \\
.03 \\
.20 \\
.28 \\
.21\end{array}$ & $\begin{array}{r}0.07 \\
.03 \\
.00 \\
.00 \\
.00\end{array}$ & $\begin{array}{r}0.00 \\
.82 \\
.00 \\
.00 \\
.00\end{array}$ & $\begin{array}{r}0.00 \\
.14 \\
.12 \\
.00 \\
.00\end{array}$ & $\begin{array}{r}0.05 \\
.00 \\
.00 \\
.00 \\
.15\end{array}$ & $\begin{array}{r}0.00 \\
1.25 \\
.71 \\
.00 \\
.00\end{array}$ \\
\hline $\begin{array}{r}6 \\
7 \\
8 \\
9 \\
10\end{array}$ & $\begin{array}{l}.01 \\
.91 \\
.77 \\
.06 \\
.00\end{array}$ & $\begin{array}{l}.24 \\
.17 \\
.00 \\
.00 \\
.00\end{array}$ & $\begin{array}{l}.70 \\
.49 \\
.01 \\
.05 \\
.24\end{array}$ & $\begin{array}{l}.00 \\
.03 \\
.01 \\
.02 \\
.00\end{array}$ & $\begin{array}{l}.12 \\
.00 \\
.00 \\
.07 \\
.05\end{array}$ & $\begin{array}{l}.00 \\
.52 \\
.19 \\
.01 \\
.00\end{array}$ & $\begin{array}{l}.00 \\
.57 \\
.00 \\
.00 \\
.00\end{array}$ & $\begin{array}{l}.00 \\
.00 \\
.00 \\
.00 \\
.19\end{array}$ & $\begin{array}{l}.00 \\
.00 \\
.00 \\
.00 \\
.00\end{array}$ & $\begin{array}{l}.00 \\
.00 \\
.00 \\
.00 \\
.00\end{array}$ & $\begin{array}{l}.01 \\
.07 \\
.00 \\
.11 \\
.69\end{array}$ & $\begin{array}{l}.46 \\
.00 \\
.00 \\
.00 \\
.00\end{array}$ \\
\hline $\begin{array}{l}11 \\
12 \\
13 \\
14 \\
15\end{array}$ & $\begin{array}{l}.00 \\
.00 \\
.00 \\
.00 \\
.00\end{array}$ & $\begin{array}{l}.07 \\
.00 \\
.00 \\
.00 \\
.00\end{array}$ & $\begin{array}{l}.11 \\
.00 \\
.00 \\
.00 \\
.09\end{array}$ & $\begin{array}{l}.00 \\
.00 \\
.00 \\
.13 \\
.02\end{array}$ & $\begin{array}{l}.00 \\
.00 \\
.00 \\
.00 \\
.00\end{array}$ & $\begin{array}{l}.08 \\
.00 \\
.34 \\
.04 \\
.00\end{array}$ & $\begin{array}{l}.00 \\
.00 \\
.23 \\
.02 \\
.00\end{array}$ & $\begin{array}{l}.05 \\
.00 \\
.00 \\
.00 \\
.00\end{array}$ & $\begin{array}{l}.00 \\
.00 \\
.00 \\
.18 \\
.00\end{array}$ & $\begin{array}{l}.00 \\
.00 \\
.00 \\
.00 \\
.00\end{array}$ & $\begin{array}{l}.00 \\
.00 \\
.20 \\
.03 \\
.00\end{array}$ & $\begin{array}{l}.00 \\
.00 \\
.00 \\
.08 \\
.09\end{array}$ \\
\hline $\begin{array}{l}16 \\
17 \\
18 \\
19 \\
20\end{array}$ & $\begin{array}{l}.07 \\
.02 \\
.42 \\
.66 \\
.00\end{array}$ & $\begin{array}{l}.03 \\
.17 \\
.78 \\
.09 \\
.30\end{array}$ & $\begin{array}{l}.93 \\
.00 \\
.00 \\
.65 \\
.15\end{array}$ & $\begin{array}{l}.01 \\
.04 \\
.00 \\
.00 \\
.02\end{array}$ & $\begin{array}{l}.00 \\
.00 \\
.00 \\
.00 \\
.00\end{array}$ & $\begin{array}{l}.00 \\
.00 \\
.00 \\
.00 \\
.00\end{array}$ & $\begin{array}{l}.00 \\
.05 \\
.11 \\
.00 \\
.00\end{array}$ & $\begin{array}{l}.00 \\
.00 \\
.00 \\
.04 \\
.11\end{array}$ & $\begin{array}{l}.63 \\
.00 \\
.00 \\
.00 \\
.08\end{array}$ & $\begin{array}{l}.00 \\
.00 \\
.00 \\
.11 \\
.00\end{array}$ & $\begin{array}{l}.00 \\
.00 \\
.00 \\
.00 \\
.00\end{array}$ & $\begin{array}{l}.81 \\
.02 \\
.03 \\
.00 \\
.00\end{array}$ \\
\hline $\begin{array}{l}21 \\
22 \\
23 \\
24 \\
25\end{array}$ & $\begin{array}{l}.02 \\
.33 \\
.96 \\
.67 \\
.00\end{array}$ & $\begin{array}{l}.75 \\
.00 \\
.00 \\
.01 \\
.00\end{array}$ & $\begin{array}{l}.00 \\
.18 \\
.41 \\
.02 \\
.00\end{array}$ & $\begin{array}{l}.00 \\
.00 \\
.37 \\
.13 \\
.00\end{array}$ & $\begin{array}{l}.25 \\
.30 \\
.04 \\
.03 \\
.00\end{array}$ & $\begin{array}{l}.00 \\
.36 \\
.11 \\
.02 \\
.00\end{array}$ & $\begin{array}{l}.25 \\
.00 \\
.00 \\
.00 \\
.00\end{array}$ & $\begin{array}{l}.00 \\
.00 \\
.00 \\
.00 \\
.00\end{array}$ & $\begin{array}{l}.14 \\
.10 \\
.51 \\
.08 \\
.00\end{array}$ & $\begin{array}{l}.00 \\
.31 \\
.01 \\
.00 \\
.25\end{array}$ & $\begin{array}{r}.00 \\
.00 \\
.05 \\
.58 \\
1.43\end{array}$ & $\begin{array}{l}.00 \\
.05 \\
.00 \\
.00 \\
.00\end{array}$ \\
\hline $\begin{array}{l}26 \\
27 \\
28 \\
29 \\
30 \\
31\end{array}$ & $\begin{array}{l}.20 \\
.45 \\
.63 \\
.00 \\
.00 \\
.00\end{array}$ & $\begin{array}{l}.00 \\
.23 \\
.05 \\
.02 \\
.00 \\
.-\end{array}$ & $\begin{array}{l}.00 \\
.09 \\
.23 \\
.22 \\
.00 \\
.00\end{array}$ & $\begin{array}{l}.00 \\
.00 \\
.04 \\
.02 \\
.16 \\
.16\end{array}$ & $\begin{array}{l}.00 \\
.00 \\
.01 \\
-- \\
-- \\
-.\end{array}$ & $\begin{array}{l}.45 \\
.05 \\
.00 \\
.00 \\
.04 \\
.01\end{array}$ & $\begin{array}{l}.07 \\
.23 \\
.00 \\
.00 \\
.09 \\
.-\end{array}$ & $\begin{array}{l}.00 \\
.00 \\
.00 \\
.00 \\
.00 \\
.00\end{array}$ & $\begin{array}{l}.00 \\
.00 \\
.00 \\
.15 \\
.01 \\
.-\end{array}$ & $\begin{array}{l}.01 \\
.00 \\
.28 \\
.37 \\
.00 \\
.00\end{array}$ & $\begin{array}{l}.69 \\
.16 \\
.00 \\
.00 \\
.00 \\
.00\end{array}$ & $\begin{array}{l}.00 \\
.73 \\
.01 \\
.00 \\
.00 \\
.-\end{array}$ \\
\hline Total & 6.84 & 2.91 & 4.95 & 3.00 & 2.50 & 2.74 & 2.80 & 0.49 & 2.70 & 1.60 & 4.04 & 4.24 \\
\hline
\end{tabular}


Table 7.-- Daily precipitation totals at continuous-recording gages--Continued

01012520 Rain Gage 1 near Bald Mountain, ME.--Continued

Precipitation totals, in inches

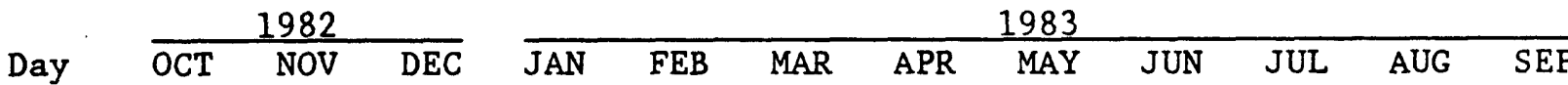

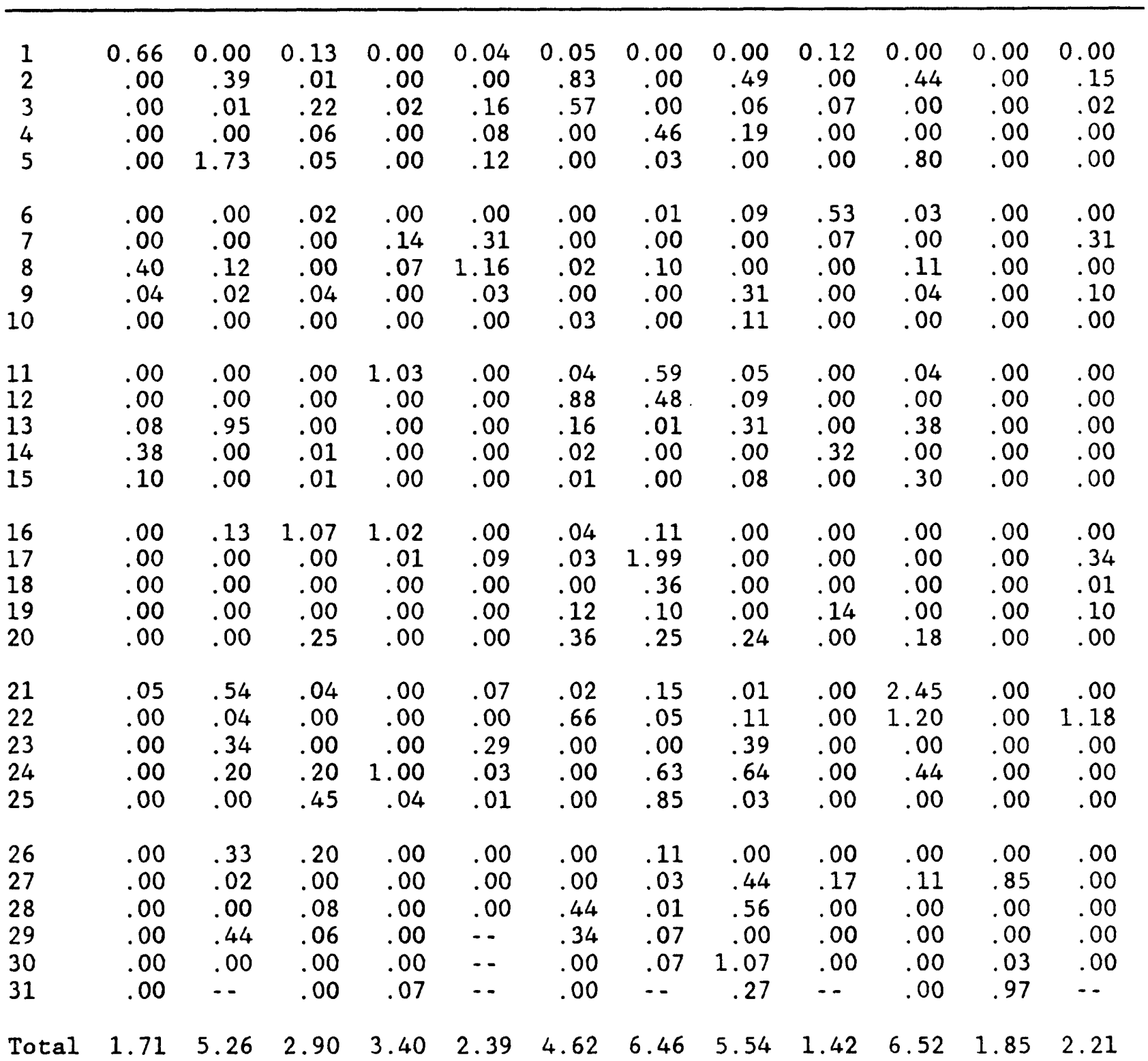


Table 7.-. Daily precipitation totals at continuous-recording gages--Continued 01012520 Rain Gage 1 near Bald Mountain, ME.--Continued

\begin{tabular}{|c|c|c|c|c|c|c|c|c|c|c|c|c|}
\hline \multirow[b]{2}{*}{ Day } & \multicolumn{12}{|c|}{$\begin{array}{c}\text { Precipitation totals, in inches } \\
1984 \\
\end{array}$} \\
\hline & OCT & NOV & DEC & JAN & FEB & MAR & APR & MAY & JUN & JUL & AUG & SEP \\
\hline $\begin{array}{l}1 \\
2 \\
3 \\
4 \\
5\end{array}$ & $\begin{array}{r}0.00 \\
.00 \\
.00 \\
.04 \\
1.18\end{array}$ & $\begin{array}{r}0.00 \\
.00 \\
.76 \\
.15 \\
.36\end{array}$ & $\begin{array}{r}0.00 \\
.00 \\
.00 \\
.00 \\
.12\end{array}$ & $\begin{array}{r}0.00 \\
.00 \\
.00 \\
.00 \\
.00\end{array}$ & $\begin{array}{r}0.01 \\
.00 \\
.16 \\
.02 \\
1.34\end{array}$ & $\begin{array}{r}0.01 \\
.00 \\
.00 \\
.00 \\
.08\end{array}$ & $\begin{array}{r}0.00 \\
.00 \\
.00 \\
.00 \\
.10\end{array}$ & $\begin{array}{r}0.00 \\
.00 \\
.00 \\
.82 \\
.35\end{array}$ & $\begin{array}{r}0.10 \\
.00 \\
.00 \\
.00 \\
.00\end{array}$ & & & \\
\hline $\begin{array}{r}6 \\
7 \\
8 \\
9 \\
10\end{array}$ & $\begin{array}{l}.32 \\
.01 \\
.08 \\
.00 \\
.00\end{array}$ & $\begin{array}{l}.43 \\
.03 \\
.00 \\
.00 \\
.00\end{array}$ & $\begin{array}{r}.91 \\
1.17 \\
.00 \\
.00 \\
.00\end{array}$ & $\begin{array}{l}.05 \\
.05 \\
.06 \\
.02 \\
.26\end{array}$ & $\begin{array}{l}.28 \\
.00 \\
.02 \\
.00 \\
.02\end{array}$ & $\begin{array}{l}.03 \\
.00 \\
.00 \\
.00 \\
.00\end{array}$ & $\begin{array}{l}.19 \\
.29 \\
.32 \\
.00 \\
.18\end{array}$ & $\begin{array}{l}.00 \\
.00 \\
.25 \\
.15 \\
.00\end{array}$ & $\begin{array}{l}.00 \\
.04 \\
.11 \\
.00 \\
.00\end{array}$ & & & \\
\hline $\begin{array}{l}11 \\
12 \\
13 \\
14 \\
15\end{array}$ & $\begin{array}{l}.00 \\
.01 \\
.40 \\
.08 \\
.00\end{array}$ & $\begin{array}{l}.94 \\
.22 \\
.02 \\
.00 \\
.08\end{array}$ & $\begin{array}{l}.00 \\
.39 \\
.86 \\
.78 \\
.26\end{array}$ & $\begin{array}{l}.05 \\
.00 \\
.00 \\
.06 \\
.00\end{array}$ & $\begin{array}{l}.01 \\
.02 \\
.00 \\
.00 \\
.34\end{array}$ & $\begin{array}{r}.04 \\
.00 \\
.35 \\
1.26 \\
.00\end{array}$ & $\begin{array}{l}.00 \\
.01 \\
.02 \\
.00 \\
.00\end{array}$ & $\begin{array}{l}.00 \\
.33 \\
.02 \\
.63 \\
.00\end{array}$ & $\begin{array}{l}.00 \\
.00 \\
\cdots \\
\cdots \\
\cdots\end{array}$ & & & \\
\hline $\begin{array}{l}16 \\
17 \\
18 \\
19 \\
20\end{array}$ & $\begin{array}{l}.00 \\
.00 \\
.00 \\
.00 \\
.00\end{array}$ & $\begin{array}{l}.01 \\
.94 \\
.02 \\
.00 \\
.05\end{array}$ & $\begin{array}{l}.00 \\
.00 \\
.00 \\
.07 \\
.00\end{array}$ & $\begin{array}{l}.00 \\
.00 \\
.02 \\
.16 \\
.01\end{array}$ & $\begin{array}{l}.00 \\
.00 \\
.00 \\
.01 \\
.30\end{array}$ & $\begin{array}{l}.10 \\
.15 \\
.00 \\
.00 \\
.09\end{array}$ & $\begin{array}{l}.43 \\
.22 \\
.00 \\
.00 \\
.00\end{array}$ & $\begin{array}{l}.07 \\
.00 \\
.00 \\
.00 \\
.00\end{array}$ & $\begin{array}{l}- \\
\cdots \\
\cdots \\
\cdots\end{array}$ & & & \\
\hline $\begin{array}{l}21 \\
22 \\
23 \\
24 \\
25\end{array}$ & $\begin{array}{l}.00 \\
.00 \\
.00 \\
.00 \\
.00\end{array}$ & $\begin{array}{r}.00 \\
.00 \\
.03 \\
.29 \\
2.26\end{array}$ & $\begin{array}{l}.00 \\
.62 \\
.11 \\
.00 \\
.00\end{array}$ & $\begin{array}{l}.02 \\
.00 \\
.00 \\
.19 \\
.07\end{array}$ & $\begin{array}{l}.00 \\
.01 \\
.02 \\
.00 \\
.00\end{array}$ & $\begin{array}{l}.02 \\
.32 \\
.07 \\
.00 \\
.00\end{array}$ & $\begin{array}{l}.00 \\
.00 \\
.00 \\
.00 \\
.34\end{array}$ & $\begin{array}{l}.00 \\
.00 \\
.47 \\
.02 \\
.00\end{array}$ & $\begin{array}{l}\ldots \\
\cdots \\
\cdots \\
\cdots\end{array}$ & & & \\
\hline $\begin{array}{l}26 \\
27 \\
28 \\
29 \\
30 \\
31\end{array}$ & $\begin{array}{l}.00 \\
.00 \\
.00 \\
.15 \\
.00 \\
.00\end{array}$ & $\begin{array}{l}.00 \\
.00 \\
.00 \\
.60 \\
.63 \\
.-\end{array}$ & $\begin{array}{l}.00 \\
.00 \\
.85 \\
.05 \\
.00 \\
.00\end{array}$ & $\begin{array}{l}.03 \\
.05 \\
.09 \\
.02 \\
.04 \\
.53\end{array}$ & $\begin{array}{l}.31 \\
.01 \\
.65 \\
.42 \\
.- \\
.-\end{array}$ & $\begin{array}{l}.00 \\
.00 \\
.00 \\
.00 \\
.00 \\
.00\end{array}$ & $\begin{array}{l}.00 \\
.00 \\
.00 \\
.00 \\
.00 \\
.-\end{array}$ & $\begin{array}{l}.10 \\
.00 \\
.00 \\
.89 \\
.87 \\
.94\end{array}$ & $\begin{array}{l}\cdots \\
\cdots \\
\cdots \\
\cdots \\
\cdots\end{array}$ & & & \\
\hline Total & 2.27 & 7.82 & 6.19 & 1.78 & 3.95 & 2.52 & 2.10 & 5.91 & - & & & \\
\hline
\end{tabular}


Table 7.-- Daily precipitation totals at continuous-recording gages--Continued

4643500684414 Rain Gage 2 at Bald Mountain, ME

\begin{tabular}{|c|c|c|c|c|c|c|c|c|c|c|c|c|}
\hline \multirow[b]{2}{*}{ Day } & & \multicolumn{10}{|c|}{ Precipitation totals, in inches } & \\
\hline & OCT & NOV & DEC & JAN & FEB & MAR & APR & MAY & JUN & JUL & AUG & SEP \\
\hline $\begin{array}{l}1 \\
2 \\
3 \\
4 \\
5\end{array}$ & & $\begin{array}{l}-- \\
-- \\
-- \\
--\end{array}$ & $\begin{array}{r}0.06 \\
.24 \\
1.22 \\
.09 \\
.00\end{array}$ & $\begin{array}{r}0.00 \\
.29 \\
.04 \\
.01 \\
.00\end{array}$ & $\begin{array}{r}0.14 \\
.91 \\
.00 \\
.00 \\
.00\end{array}$ & $\begin{array}{r}0.15 \\
.00 \\
.01 \\
.00 \\
.06\end{array}$ & $\begin{array}{r}0.03 \\
.29 \\
.00 \\
.03 \\
.25\end{array}$ & $\begin{array}{r}0.00 \\
.14 \\
.00 \\
.00 \\
.00\end{array}$ & $\begin{array}{r}0.02 \\
.00 \\
.00 \\
.08 \\
.01\end{array}$ & $\begin{array}{r}0.00 \\
.00 \\
.00 \\
.00 \\
.00\end{array}$ & $\begin{array}{r}0.00 \\
.00 \\
.00 \\
.00 \\
1.15\end{array}$ & $\begin{array}{r}0.00 \\
.00 \\
.00 \\
.00 \\
.00\end{array}$ \\
\hline $\begin{array}{r}6 \\
7 \\
8 \\
9 \\
10\end{array}$ & & $\begin{array}{l}-- \\
-- \\
-- \\
--\end{array}$ & $\begin{array}{l}.00 \\
.01 \\
.40 \\
.04 \\
.05\end{array}$ & $\begin{array}{l}.02 \\
.37 \\
.03 \\
.00 \\
.17\end{array}$ & $\begin{array}{l}.00 \\
.00 \\
.23 \\
.17 \\
.00\end{array}$ & $\begin{array}{l}.08 \\
.07 \\
.00 \\
.00 \\
.09\end{array}$ & $\begin{array}{l}.03 \\
.00 \\
.00 \\
.23 \\
.00\end{array}$ & $\begin{array}{l}.01 \\
.00 \\
.00 \\
.00 \\
.00\end{array}$ & $\begin{array}{l}.18 \\
.04 \\
.00 \\
.16 \\
.00\end{array}$ & $\begin{array}{l}.63 \\
.03 \\
.14 \\
.00 \\
.03\end{array}$ & $\begin{array}{r}3.59 \\
.51 \\
.07 \\
.04 \\
.00\end{array}$ & $\begin{array}{l}.00 \\
.00 \\
.00 \\
.50 \\
.07\end{array}$ \\
\hline $\begin{array}{l}11 \\
12 \\
13 \\
14 \\
15\end{array}$ & & $\begin{array}{l}-- \\
-- \\
-- \\
-- \\
--\end{array}$ & $\begin{array}{l}.00 \\
.03 \\
.07 \\
.07 \\
.00\end{array}$ & $\begin{array}{l}.17 \\
.00 \\
.20 \\
.00 \\
.00\end{array}$ & $\begin{array}{l}.33 \\
.12 \\
.00 \\
.00 \\
.03\end{array}$ & $\begin{array}{l}.00 \\
.00 \\
.02 \\
.05 \\
.02\end{array}$ & $\begin{array}{l}.00 \\
.05 \\
.00 \\
.37 \\
.00\end{array}$ & $\begin{array}{l}.05 \\
.22 \\
.83 \\
.02 \\
.03\end{array}$ & $\begin{array}{l}.00 \\
.00 \\
.00 \\
.00 \\
.09\end{array}$ & $\begin{array}{l}.03 \\
.00 \\
.16 \\
.22 \\
.03\end{array}$ & $\begin{array}{l}.42 \\
.11 \\
.00 \\
.00 \\
.21\end{array}$ & $\begin{array}{l}.00 \\
.05 \\
.00 \\
.22 \\
.01\end{array}$ \\
\hline $\begin{array}{l}16 \\
17 \\
18 \\
19 \\
20\end{array}$ & & $\begin{array}{c}\cdots \\
\cdots \\
0 \\
0.00 \\
.00\end{array}$ & $\begin{array}{l}.48 \\
.03 \\
.02 \\
.02 \\
.00\end{array}$ & $\begin{array}{l}.00 \\
.00 \\
.00 \\
.00 \\
.00\end{array}$ & $\begin{array}{l}.00 \\
.00 \\
.00 \\
.04 \\
.04\end{array}$ & $\begin{array}{r}.00 \\
.10 \\
22 \\
.19 \\
.11\end{array}$ & $\begin{array}{l}.00 \\
.01 \\
.20 \\
.01 \\
.01\end{array}$ & $\begin{array}{l}.55 \\
.42 \\
.00 \\
.00 \\
.00\end{array}$ & $\begin{array}{l}.23 \\
.07 \\
.00 \\
.00 \\
.16\end{array}$ & $\begin{array}{l}.00 \\
.00 \\
.02 \\
.00 \\
.00\end{array}$ & $\begin{array}{r}1.28 \\
2.35 \\
.10 \\
.00 \\
.00\end{array}$ & $\begin{array}{l}.01 \\
.00 \\
.03 \\
.61 \\
.15\end{array}$ \\
\hline $\begin{array}{l}21 \\
22 \\
23 \\
24 \\
25\end{array}$ & & $\begin{array}{l}.00 \\
.00 \\
.00 \\
.36 \\
.58\end{array}$ & $\begin{array}{l}.00 \\
.02 \\
.06 \\
.25 \\
.05\end{array}$ & $\begin{array}{l}.00 \\
.03 \\
.13 \\
.03 \\
.02\end{array}$ & $\begin{array}{l}.09 \\
.01 \\
.03 \\
.01 \\
.01\end{array}$ & $\begin{array}{l}.01 \\
.00 \\
.00 \\
.00 \\
.00\end{array}$ & $\begin{array}{l}.00 \\
.00 \\
.00 \\
.45 \\
.02\end{array}$ & $\begin{array}{l}.00 \\
.00 \\
.03 \\
.00 \\
.03\end{array}$ & $\begin{array}{l}.44 \\
.27 \\
.30 \\
.00 \\
.14\end{array}$ & $\begin{array}{l}.04 \\
.01 \\
.00 \\
.00 \\
.00\end{array}$ & $\begin{array}{l}.00 \\
.00 \\
.35 \\
.00 \\
.00\end{array}$ & $\begin{array}{r}.00 \\
.05 \\
1.19 \\
.85 \\
.03\end{array}$ \\
\hline $\begin{array}{l}26 \\
27 \\
28 \\
29 \\
30 \\
31\end{array}$ & & $\begin{array}{l}.15 \\
.01 \\
.74 \\
.33 \\
.00 \\
.-\end{array}$ & $\begin{array}{l}.00 \\
.00 \\
.01 \\
.16 \\
.04 \\
.01\end{array}$ & $\begin{array}{l}.01 \\
.01 \\
.01 \\
.00 \\
.00 \\
.00\end{array}$ & $\begin{array}{l}.40 \\
.21 \\
.10 \\
-- \\
-- \\
-.\end{array}$ & $\begin{array}{r}.00 \\
.02 \\
.01 \\
0.30 \\
.65 \\
.57\end{array}$ & $\begin{array}{l}.00 \\
.00 \\
.00 \\
.73 \\
.00 \\
.-\end{array}$ & $\begin{array}{l}.12 \\
.16 \\
.41 \\
.08 \\
.00 \\
.42\end{array}$ & $\begin{array}{l}.86 \\
.01 \\
.00 \\
.00 \\
.00 \\
.-\end{array}$ & $\begin{array}{l}.00 \\
.05 \\
.00 \\
.25 \\
.55 \\
.05\end{array}$ & $\begin{array}{l}.00 \\
.00 \\
.00 \\
.00 \\
.00 \\
.00\end{array}$ & $\begin{array}{l}.00 \\
.17 \\
.03 \\
.05 \\
.00 \\
.-\end{array}$ \\
\hline Total & & - & 3.43 & 1.54 & 2.87 & 3.73 & 2.71 & 3.52 & 3.06 & 2.64 & 10.18 & 4.02 \\
\hline
\end{tabular}


Table 7.-- Daily precipitation totals at continuous-recording gages--Continued

4643500684414 Rain Gage 2 at Bald Mountain, ME.--Continued

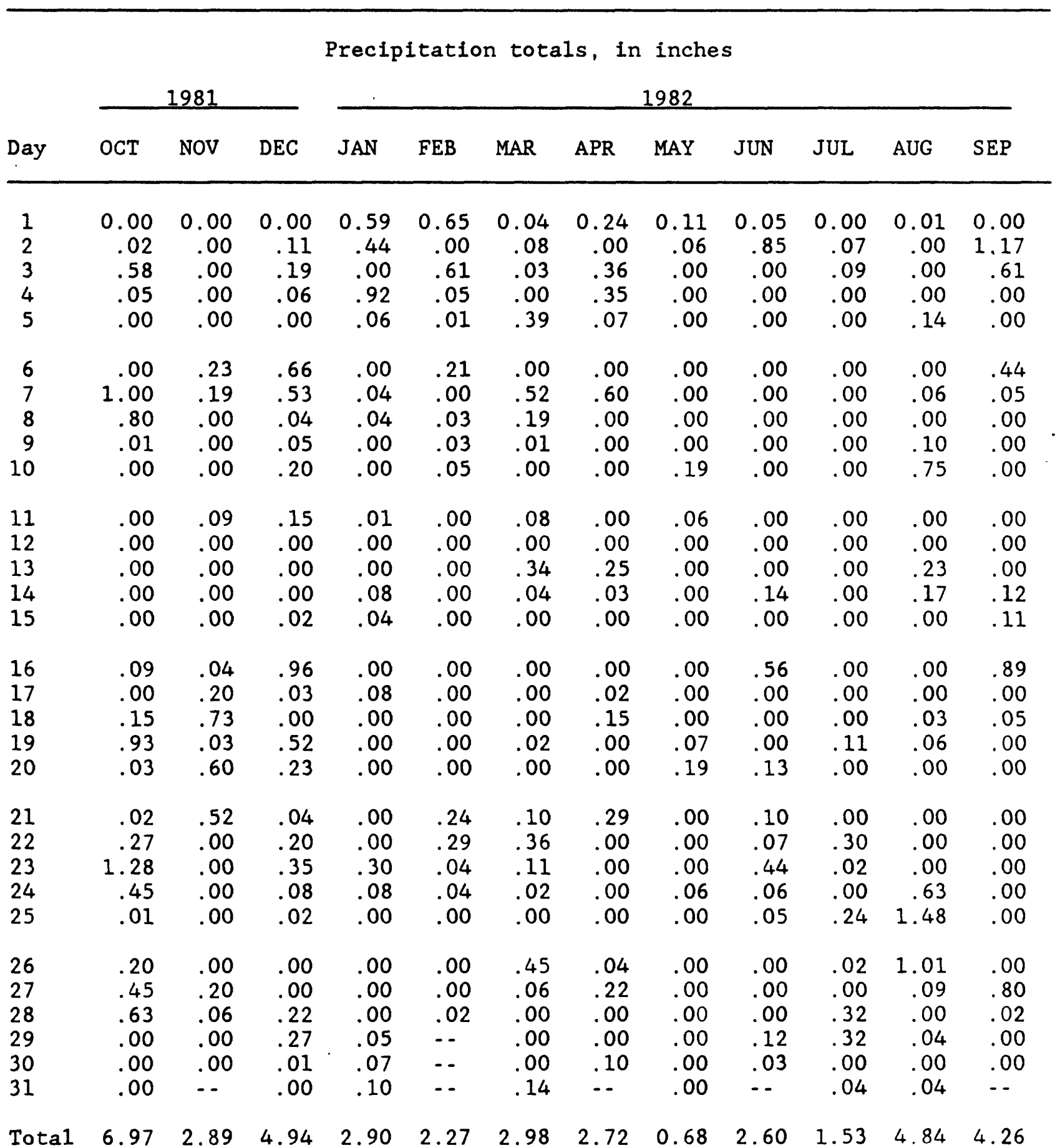


Table 7.-- Daily precipitation totals at continuous-recording gages--Continued

4643500684414 Rain Gage 2 at Bald Mountain, ME.--Continued

\begin{tabular}{|c|c|c|c|c|c|c|c|c|c|c|c|c|}
\hline \multirow[b]{2}{*}{ Day } & \multicolumn{12}{|c|}{$\begin{array}{c}\text { Precipitation totals, in inches } \\
1983 \\
\end{array}$} \\
\hline & OCT & NOV & DEC & JAN & FEB & MAR & APR & MAY & JUN & JUL & AUG & SEP \\
\hline $\begin{array}{l}1 \\
2 \\
3 \\
4 \\
5\end{array}$ & $\begin{array}{r}0.66 \\
.00 \\
.00 \\
.00 \\
.00\end{array}$ & $\begin{array}{r}0.00 \\
.39 \\
.03 \\
.00 \\
1.63\end{array}$ & $\begin{array}{r}0.13 \\
.00 \\
.24 \\
.06 \\
.06\end{array}$ & $\begin{array}{r}0.00 \\
.00 \\
.00 \\
.00 \\
.00\end{array}$ & $\begin{array}{r}0.04 \\
.00 \\
.18 \\
.09 \\
.07\end{array}$ & $\begin{array}{r}0.00 \\
.60 \\
.47 \\
.00 \\
.00\end{array}$ & $\begin{array}{r}0.00 \\
.00 \\
.00 \\
.44 \\
.00\end{array}$ & $\begin{array}{r}0.02 \\
.50 \\
.03 \\
.20 \\
.00\end{array}$ & $\begin{array}{r}0.12 \\
.00 \\
.07 \\
.00 \\
.00\end{array}$ & $\begin{array}{r}0.00 \\
.44 \\
.00 \\
.00 \\
.77\end{array}$ & $\begin{array}{r}0.00 \\
.00 \\
.06 \\
.00 \\
.00\end{array}$ & $\begin{array}{r}0.00 \\
.12 \\
.03 \\
.00 \\
.00\end{array}$ \\
\hline $\begin{array}{r}6 \\
7 \\
8 \\
9 \\
10\end{array}$ & $\begin{array}{l}.00 \\
.00 \\
.44 \\
.06 \\
.00\end{array}$ & $\begin{array}{l}.00 \\
.00 \\
.10 \\
.00 \\
.00\end{array}$ & $\begin{array}{l}.02 \\
.00 \\
.00 \\
.00 \\
.00\end{array}$ & $\begin{array}{l}.00 \\
.19 \\
.02 \\
.00 \\
.02\end{array}$ & $\begin{array}{l}.00 \\
.38 \\
.85 \\
.00 \\
.00\end{array}$ & $\begin{array}{l}.00 \\
.00 \\
.00 \\
.03 \\
.00\end{array}$ & $\begin{array}{l}.00 \\
.04 \\
.06 \\
.00 \\
.00\end{array}$ & $\begin{array}{l}.07 \\
.00 \\
.00 \\
.30 \\
.11\end{array}$ & $\begin{array}{l}.51 \\
.07 \\
.00 \\
.00 \\
.00\end{array}$ & $\begin{array}{l}.03 \\
.01 \\
.11 \\
.04 \\
.00\end{array}$ & $\begin{array}{l}.00 \\
.00 \\
.21 \\
.00 \\
.07\end{array}$ & $\begin{array}{l}.00 \\
.39 \\
.00 \\
.10 \\
.00\end{array}$ \\
\hline $\begin{array}{l}11 \\
12 \\
13 \\
14 \\
15\end{array}$ & $\begin{array}{l}.00 \\
.00 \\
.07 \\
.34 \\
.09\end{array}$ & $\begin{array}{l}.00 \\
.00 \\
.98 \\
.00 \\
.00\end{array}$ & $\begin{array}{l}.02 \\
.00 \\
.00 \\
.00 \\
.00\end{array}$ & $\begin{array}{r}1.07 \\
.00 \\
.00 \\
.00 \\
.04\end{array}$ & $\begin{array}{l}.00 \\
.00 \\
.00 \\
.00 \\
.00\end{array}$ & $\begin{array}{r}.04 \\
1.00 \\
.10 \\
.00 \\
.00\end{array}$ & $\begin{array}{l}.83 \\
.24 \\
.00 \\
.16 \\
.23\end{array}$ & $\begin{array}{l}.05 \\
.09 \\
.30 \\
.01 \\
.08\end{array}$ & $\begin{array}{l}.00 \\
.00 \\
.00 \\
.32 \\
.00\end{array}$ & $\begin{array}{l}.04 \\
.00 \\
.31 \\
.00 \\
.34\end{array}$ & $\begin{array}{l}.00 \\
.00 \\
.00 \\
.00 \\
.00\end{array}$ & $\begin{array}{l}.05 \\
.00 \\
.00 \\
.00 \\
.00\end{array}$ \\
\hline $\begin{array}{l}16 \\
17 \\
18 \\
19 \\
20\end{array}$ & $\begin{array}{l}.05 \\
.00 \\
.00 \\
.00 \\
.00\end{array}$ & $\begin{array}{l}.00 \\
.00 \\
.00 \\
.00 \\
.00\end{array}$ & $\begin{array}{r}1.08 \\
.00 \\
.00 \\
.00 \\
.25\end{array}$ & $\begin{array}{l}.95 \\
.00 \\
.00 \\
.00 \\
.00\end{array}$ & $\begin{array}{l}.00 \\
.06 \\
.00 \\
.00 \\
.00\end{array}$ & $\begin{array}{r}.05 \\
1.89 \\
.00 \\
.30 \\
.27\end{array}$ & $\begin{array}{l}.19 \\
.00 \\
.35 \\
.10 \\
.24\end{array}$ & $\begin{array}{l}.02 \\
.00 \\
.00 \\
.00 \\
.31\end{array}$ & $\begin{array}{l}.00 \\
.00 \\
.00 \\
.13 \\
.00\end{array}$ & $\begin{array}{l}.00 \\
.00 \\
.00 \\
.00 \\
.00\end{array}$ & $\begin{array}{l}.00 \\
.00 \\
.00 \\
.00 \\
.00\end{array}$ & $\begin{array}{l}.00 \\
.40 \\
.01 \\
.04 \\
.00\end{array}$ \\
\hline $\begin{array}{l}21 \\
22 \\
23 \\
24 \\
25\end{array}$ & $\begin{array}{l}.05 \\
.00 \\
.00 \\
.00 \\
.00\end{array}$ & $\begin{array}{l}.60 \\
.05 \\
.33 \\
.18 \\
.00\end{array}$ & $\begin{array}{l}.04 \\
.00 \\
.00 \\
.12 \\
.01\end{array}$ & $\begin{array}{l}.00 \\
.00 \\
.00 \\
.91 \\
.00\end{array}$ & $\begin{array}{l}.09 \\
.00 \\
.39 \\
.03 \\
.00\end{array}$ & $\begin{array}{l}.02 \\
.65 \\
.01 \\
.00 \\
.00\end{array}$ & $\begin{array}{l}.15 \\
.05 \\
.00 \\
.63 \\
.85\end{array}$ & $\begin{array}{l}.01 \\
.10 \\
.44 \\
.70 \\
.04\end{array}$ & $\begin{array}{l}.00 \\
.00 \\
.00 \\
.00 \\
.00\end{array}$ & $\begin{array}{r}1.85 \\
1.36 \\
.00 \\
.25 \\
.12\end{array}$ & $\begin{array}{l}.00 \\
.00 \\
.00 \\
.00 \\
.00\end{array}$ & $\begin{array}{r}.00 \\
1.21 \\
.00 \\
.00 \\
.00\end{array}$ \\
\hline $\begin{array}{l}26 \\
27 \\
28 \\
29 \\
30 \\
31\end{array}$ & $\begin{array}{l}.00 \\
.00 \\
.00 \\
.00 \\
.00 \\
.00\end{array}$ & $\begin{array}{l}.30 \\
.00 \\
.00 \\
.39 \\
.00 \\
.-\end{array}$ & $\begin{array}{l}.18 \\
.00 \\
.10 \\
.00 \\
.00 \\
.00\end{array}$ & $\begin{array}{l}.00 \\
.00 \\
.00 \\
.00 \\
.00 \\
.06\end{array}$ & $\begin{array}{l}.00 \\
.00 \\
.05 \\
-- \\
-- \\
--\end{array}$ & $\begin{array}{l}.00 \\
.00 \\
.70 \\
.14 \\
.00 \\
.00\end{array}$ & $\begin{array}{l}.11 \\
.00 \\
.00 \\
.05 \\
.05 \\
--\end{array}$ & $\begin{array}{r}.00 \\
.44 \\
.59 \\
.02 \\
1.05 \\
.27\end{array}$ & $\begin{array}{l}.00 \\
.17 \\
.00 \\
.00 \\
.00 \\
--\end{array}$ & $\begin{array}{l}.00 \\
.20 \\
.00 \\
.00 \\
.00 \\
.00\end{array}$ & $\begin{array}{l}.00 \\
.88 \\
.00 \\
.00 \\
.04 \\
.99\end{array}$ & $\begin{array}{l}.00 \\
.00 \\
.00 \\
.00 \\
.00 \\
.-\end{array}$ \\
\hline Total & 1.76 & 4.98 & 2.81 & 3.26 & 2.23 & 4.38 & 6.66 & 5.75 & 1.39 & 6.07 & 2.25 & 2.35 \\
\hline
\end{tabular}


Table 7.-. Daily precipitation totals at continuous-recording gages--Continued

4643500684414 Rain Gage 2 at Bald Mountain, ME.--Continued

Precipitation totals, in inches

1983

\begin{tabular}{lllllllll} 
Day OCT NOV & DEC JAN FEB MAR A \\
\hline & 0.00 & 0.00 & 0.00 & 0.00 & 0.00 & 0.01 & 0.0
\end{tabular}

1

2

3

4

5

7

8

9
10

10

11

12

13

14

15

16

17

18

19

20

2

2

23

24

25

26

27

28

29

30

31

Total $2.38 \quad 7.86$

$\begin{array}{rrr}0.00 & 0.00 & 0 . \\ .00 & .00\end{array}$

$.00 \quad .69$

$.08 \quad .15$

$1.20 \quad .36$

0.00

.00

$.00 \quad .00$

.00

0.00

0.01

.01

1.27

.00

.00

$.36 \quad .41$

$.02 \quad .06$

$.94 \quad .00$

$1.47 \quad .00$

.06

.00

.05

.00

.00

.00

.00

.06

.00

.00

$.00 \quad .00$

.00

.28

.01

.03

.00

.00

.00

$.00 \quad .94$

.00

.06

.01

.0

$.00 \quad .21$

$.45 . .02$

.07

.00

.24

.61

.00

1.00

.00

.05

.00

.01

.00

.12

.00

.35

1.25

.00

$00 \quad 01$

$.00 \quad .89$

$.00 \quad .00$

.05

$.05 \quad .00$

$.00 \quad .10$

.10

$.04 \quad .05$

.12

.05

.00

.00

.02

$.00 \quad .05$

.00

01

.00

03

.07

05

$.00 \quad .00$

$.00 \quad .03$

$.00 \quad .29$

.00

.00

.00

.00

.03

.16

.03

.28

.00

2.20

00

.09

00

31

.31
.02

.05

.00

00

$.00 \quad .00$

$.00 \quad .01 \quad .00$

$.00 \quad .00$

$.00 \quad .01$

$.13 \quad .74$

.00

.69

.00

00

$$
.35 \quad .00
$$

1.00

.00

.72

.02
.72

.72

.00

.00

.02

.39

$-$

.00

.00

.00

.00

2.41
$.00 \quad .00$

0.00

0.00
.00

.00

$.80 \quad .00$

.00

.00

.42

.00

.25

.29

.25

.00

.16

$.00 \quad .00$

$.00 \quad .04$

.27 .11

$\begin{array}{ll}.16 & .00 \\ .00 & .00\end{array}$

.00

.01

.00

$.00 \quad .02$

$.00 \quad .00$

.54

$.00 \quad .00$

$.00 \quad .00$

$.00 \quad .00$

.00

$.00 \quad .85$

-.

$\begin{array}{lll}6.59 & 1.65 \quad 3.64\end{array}$

2.13

$\begin{array}{ll}.00 & .00 \\ .37 & .00 \\ .00 & -- \\ .63 & -- \\ .00 & -\end{array}$

$54 \quad .07 \quad \ldots$

$\begin{array}{lll}.21 & .00 & .01 \\ .01 & .00\end{array}$

$01.00 \quad-$$$
.00 \quad .00
$$

$.00 \quad .00$

$00.43 \quad$.

$25 \quad .00$

$$
.00 \quad 10
$$

$00 \quad .10 \quad-$.

$.00 \quad .00$ 
Table 7.-- Daily precipitation totals at continuous-recording gages--Continued

4644350684416 Rain Gage 3 at Bishop Mountain, ME

Precipitation totals, in inches

1981

1982

Day OCT NOV DEC JAN FEB MAR APR MAY JUN JUL AUG SEP

\begin{tabular}{|c|c|c|c|c|c|c|c|c|c|c|c|}
\hline $\begin{array}{l}1 \\
2 \\
3 \\
4 \\
5\end{array}$ & $\begin{array}{c}\cdots \\
\cdots \\
0 \\
0.00 \\
.00\end{array}$ & $\begin{array}{r}0.00 \\
.07 \\
.14 \\
.04 \\
.01\end{array}$ & $\begin{array}{r}0.58 \\
.43 \\
.00 \\
.91 \\
.05\end{array}$ & $\begin{array}{r}0.71 \\
.00 \\
.60 \\
.05 \\
.05\end{array}$ & $\begin{array}{r}0.07 \\
.06 \\
.01 \\
.10 \\
.29\end{array}$ & $\begin{array}{r}0.31 \\
.00 \\
.36 \\
.35 \\
.07\end{array}$ & $\begin{array}{r}0.08 \\
.05 \\
.00 \\
.00 \\
.00\end{array}$ & $\begin{array}{r}0.00 \\
.86 \\
.00 \\
.00 \\
.00\end{array}$ & $\begin{array}{r}0.00 \\
.14 \\
.12 \\
.01 \\
.00\end{array}$ & $\begin{array}{r}0.16 \\
.00 \\
.00 \\
.00 \\
.14\end{array}$ & $\begin{array}{r}0.01 \\
1.22 \\
.62 \\
.00 \\
.00\end{array}$ \\
\hline $\begin{array}{r}6 \\
7 \\
8 \\
9 \\
10\end{array}$ & $\begin{array}{l}.20 \\
.09 \\
.13 \\
.00 \\
.00\end{array}$ & $\begin{array}{l}.63 \\
.50 \\
.00 \\
.07 \\
.24\end{array}$ & $\begin{array}{l}.00 \\
.06 \\
.02 \\
.01 \\
.01\end{array}$ & $\begin{array}{l}.18 \\
.00 \\
.01 \\
.04 \\
.11\end{array}$ & $\begin{array}{l}.00 \\
.55 \\
.18 \\
.02 \\
.01\end{array}$ & $\begin{array}{l}.00 \\
.34 \\
.01 \\
.00 \\
.00\end{array}$ & $\begin{array}{l}.00 \\
.00 \\
.00 \\
.00 \\
.19\end{array}$ & $\begin{array}{l}.00 \\
.00 \\
.00 \\
.00 \\
.00\end{array}$ & $\begin{array}{l}.00 \\
.00 \\
.00 \\
.00 \\
.00\end{array}$ & $\begin{array}{l}.00 \\
.07 \\
.02 \\
.10 \\
.71\end{array}$ & $\begin{array}{l}.45 \\
.03 \\
.00 \\
.00 \\
.00\end{array}$ \\
\hline $\begin{array}{l}11 \\
12 \\
13 \\
14 \\
15\end{array}$ & $\begin{array}{l}.03 \\
.01 \\
.00 \\
.00 \\
.00\end{array}$ & $\begin{array}{l}.07 \\
.02 \\
.00 \\
.00 \\
.06\end{array}$ & $\begin{array}{l}.00 \\
.00 \\
.00 \\
.09 \\
.01\end{array}$ & $\begin{array}{r}.00 \\
.0 \\
.00 \\
.01 \\
.00\end{array}$ & $\begin{array}{l}.05 \\
.00 \\
.35 \\
.05 \\
.00\end{array}$ & $\begin{array}{l}.00 \\
.00 \\
.20 \\
.00 \\
.00\end{array}$ & $\begin{array}{l}.04 \\
.00 \\
.00 \\
.00 \\
.00\end{array}$ & $\begin{array}{l}.00 \\
.00 \\
.00 \\
.12 \\
.00\end{array}$ & $\begin{array}{l}.00 \\
.00 \\
.00 \\
.00 \\
.00\end{array}$ & $\begin{array}{l}.00 \\
.00 \\
.26 \\
.19 \\
.00\end{array}$ & $\begin{array}{l}.00 \\
.00 \\
.00 \\
.10 \\
.07\end{array}$ \\
\hline $\begin{array}{l}16 \\
17 \\
18 \\
19 \\
20\end{array}$ & $\begin{array}{l}.04 \\
.23 \\
.27 \\
.00 \\
.16\end{array}$ & $\begin{array}{l}.90 \\
.02 \\
.02 \\
.62 \\
.05\end{array}$ & $\begin{array}{l}.03 \\
.04 \\
.00 \\
.00 \\
.00\end{array}$ & $\begin{array}{l}.00 \\
.00 \\
.00 \\
.02 \\
.00\end{array}$ & $\begin{array}{l}.00 \\
.00 \\
.00 \\
.00 \\
.00\end{array}$ & $\begin{array}{l}.00 \\
.00 \\
.13 \\
.00 \\
.00\end{array}$ & $\begin{array}{l}.00 \\
.00 \\
.00 \\
.05 \\
.18\end{array}$ & $\begin{array}{l}.06 \\
.00 \\
.00 \\
.01 \\
.10\end{array}$ & $\begin{array}{l}.00 \\
.00 \\
.00 \\
.11 \\
.00\end{array}$ & $\begin{array}{l}.00 \\
.00 \\
.00 \\
.00 \\
.07\end{array}$ & $\begin{array}{l}.80 \\
.02 \\
.02 \\
.01 \\
.00\end{array}$ \\
\hline $\begin{array}{l}21 \\
22 \\
23 \\
24 \\
25\end{array}$ & $\begin{array}{l}.58 \\
.01 \\
.00 \\
.00 \\
.00\end{array}$ & $\begin{array}{l}.01 \\
.00 \\
.12 \\
.00 \\
.01\end{array}$ & $\begin{array}{l}.00 \\
.00 \\
.21 \\
.13 \\
.01\end{array}$ & $\begin{array}{l}.16 \\
.22 \\
.06 \\
.02 \\
.00\end{array}$ & $\begin{array}{l}.08 \\
.35 \\
.08 \\
.02 \\
.00\end{array}$ & $\begin{array}{l}.25 \\
.00 \\
.00 \\
.00 \\
.00\end{array}$ & $\begin{array}{l}.00 \\
.00 \\
.00 \\
.00 \\
.00\end{array}$ & $\begin{array}{l}.13 \\
.06 \\
.48 \\
.08 \\
.03\end{array}$ & $\begin{array}{l}.00 \\
.34 \\
.00 \\
.00 \\
.25\end{array}$ & $\begin{array}{r}.00 \\
.00 \\
.02 \\
.67 \\
1.38\end{array}$ & $\begin{array}{l}.00 \\
.05 \\
.00 \\
.00 \\
.00\end{array}$ \\
\hline $\begin{array}{l}26 \\
27 \\
28 \\
29 \\
30 \\
31\end{array}$ & $\begin{array}{l}.00 \\
.00 \\
.00 \\
.00 \\
.00 \\
.-\end{array}$ & $\begin{array}{l}.01 \\
.02 \\
.08 \\
.18 \\
.00 \\
.00\end{array}$ & $\begin{array}{l}.00 \\
.00 \\
.02 \\
.00 \\
.08 \\
.16\end{array}$ & $\begin{array}{l}.00 \\
.01 \\
.00 \\
-- \\
-. \\
-.\end{array}$ & $\begin{array}{l}.41 \\
.07 \\
.00 \\
.00 \\
.00 \\
.14\end{array}$ & $\begin{array}{l}.08 \\
.22 \\
.00 \\
.00 \\
.08 \\
.-\end{array}$ & $\begin{array}{l}.00 \\
.00 \\
.00 \\
.00 \\
.00 \\
.00\end{array}$ & $\begin{array}{l}.00 \\
.00 \\
.07 \\
.16 \\
.00 \\
.-\end{array}$ & $\begin{array}{l}.01 \\
.00 \\
.27 \\
.45 \\
.00 \\
.03\end{array}$ & $\begin{array}{l}.87 \\
.13 \\
.03 \\
.01 \\
.00 \\
.05\end{array}$ & $\begin{array}{l}.00 \\
.76 \\
.02 \\
.02 \\
.00 \\
.-\end{array}$ \\
\hline Total & $\cdots$ & 3.89 & 2.85 & 2.25 & 2.89 & 2.40 & 0.59 & 2.70 & 1.73 & 4.88 & 4.20 \\
\hline
\end{tabular}


Table 7.-- Daily precipitation totals at continuous-recording gages--Continued

4644350684416 Rain Gage 3 at Bishop Mountain, ME.--Continued

\begin{tabular}{|c|c|c|c|c|c|c|c|c|c|c|c|c|}
\hline \multirow[b]{2}{*}{ Day } & \multicolumn{12}{|c|}{$\begin{array}{c}\text { Precipitation totals, in inches } \\
1983 \\
\end{array}$} \\
\hline & OCT & NOV & DEC & JAN & FEB & MAR & APR & MAY & JUN & JUL & AUG & SEP \\
\hline $\begin{array}{l}1 \\
2 \\
3 \\
4 \\
5\end{array}$ & $\begin{array}{r}0.60 \\
.00 \\
.00 \\
.00 \\
.00\end{array}$ & $\begin{array}{r}0.00 \\
.38 \\
.01 \\
.00 \\
1.67\end{array}$ & $\begin{array}{r}0.22 \\
.01 \\
.23 \\
.10 \\
.04\end{array}$ & $\begin{array}{r}0.00 \\
.00 \\
.01 \\
.00 \\
.00\end{array}$ & $\begin{array}{r}0.03 \\
.03 \\
.22 \\
.13 \\
.01\end{array}$ & $\begin{array}{r}0.04 \\
.58 \\
.52 \\
.01 \\
.00\end{array}$ & $\begin{array}{r}0.00 \\
.00 \\
.00 \\
.45 \\
.00\end{array}$ & $\begin{array}{r}0.04 \\
.48 \\
.00 \\
.21 \\
.01\end{array}$ & $\begin{array}{r}0.11 \\
.00 \\
.06 \\
.00 \\
.00\end{array}$ & $\begin{array}{r}0.00 \\
.49 \\
.00 \\
.00 \\
.80\end{array}$ & $\begin{array}{r}0.00 \\
.00 \\
.00 \\
.00 \\
.00\end{array}$ & $\begin{array}{r}0.00 \\
.13 \\
.03 \\
.00 \\
.00\end{array}$ \\
\hline $\begin{array}{r}6 \\
7 \\
8 \\
9 \\
10\end{array}$ & $\begin{array}{l}.00 \\
.03 \\
.38 \\
.08 \\
.00\end{array}$ & $\begin{array}{l}.00 \\
.00 \\
.13 \\
.00 \\
.00\end{array}$ & $\begin{array}{l}.04 \\
.02 \\
.00 \\
.07 \\
.00\end{array}$ & $\begin{array}{l}.00 \\
.11 \\
.13 \\
.00 \\
.01\end{array}$ & $\begin{array}{l}.00 \\
.41 \\
.93 \\
.03 \\
.00\end{array}$ & $\begin{array}{l}.00 \\
.00 \\
.00 \\
.02 \\
.00\end{array}$ & $\begin{array}{l}.00 \\
.07 \\
.02 \\
.01 \\
.01\end{array}$ & $\begin{array}{l}.09 \\
.00 \\
.00 \\
.31 \\
.11\end{array}$ & $\begin{array}{l}.49 \\
.16 \\
.00 \\
.00 \\
.00\end{array}$ & $\begin{array}{l}.03 \\
.00 \\
.11 \\
.04 \\
.00\end{array}$ & $\begin{array}{l}.00 \\
.00 \\
.00 \\
.00 \\
.00\end{array}$ & $\begin{array}{l}.00 \\
.34 \\
.00 \\
.12 \\
.00\end{array}$ \\
\hline $\begin{array}{l}11 \\
12 \\
13 \\
14 \\
15\end{array}$ & $\begin{array}{l}.00 \\
.00 \\
.06 \\
.32 \\
.08\end{array}$ & $\begin{array}{r}.00 \\
.00 \\
1.00 \\
.00 \\
.00\end{array}$ & $\begin{array}{l}.00 \\
.00 \\
.00 \\
.00 \\
.02\end{array}$ & $\begin{array}{r}1.08 \\
.00 \\
.01 \\
.00 \\
.00\end{array}$ & $\begin{array}{l}.00 \\
.00 \\
.00 \\
.00 \\
.00\end{array}$ & $\begin{array}{l}.04 \\
.97 \\
.09 \\
.00 \\
.02\end{array}$ & $\begin{array}{l}.92 \\
.18 \\
.02 \\
.00 \\
.00\end{array}$ & $\begin{array}{l}.05 \\
.09 \\
.31 \\
.00 \\
.08\end{array}$ & $\begin{array}{l}.00 \\
.00 \\
.00 \\
.65 \\
.00\end{array}$ & $\begin{array}{l}.04 \\
.00 \\
.43 \\
.00 \\
.39\end{array}$ & $\begin{array}{l}.00 \\
.00 \\
.00 \\
.00 \\
.00\end{array}$ & $\begin{array}{l}.00 \\
.00 \\
.00 \\
.00 \\
.00\end{array}$ \\
\hline $\begin{array}{l}16 \\
17 \\
18 \\
19 \\
20\end{array}$ & $\begin{array}{l}.00 \\
.00 \\
.00 \\
.00 \\
.02\end{array}$ & $\begin{array}{l}.03 \\
.00 \\
.00 \\
.00 \\
.00\end{array}$ & $\begin{array}{r}1.04 \\
.02 \\
.00 \\
.00 \\
.24\end{array}$ & $\begin{array}{l}.85 \\
.02 \\
.03 \\
.03 \\
.00\end{array}$ & $\begin{array}{l}.00 \\
.08 \\
.00 \\
.00 \\
.00\end{array}$ & $\begin{array}{l}.03 \\
.01 \\
.01 \\
.43 \\
.12\end{array}$ & $\begin{array}{r}.11 \\
1.90 \\
.35 \\
.12 \\
.26\end{array}$ & $\begin{array}{l}.04 \\
.00 \\
.00 \\
.00 \\
.19\end{array}$ & $\begin{array}{l}.00 \\
.00 \\
.00 \\
.13 \\
.00\end{array}$ & $\begin{array}{l}.00 \\
.00 \\
.00 \\
.00 \\
.12\end{array}$ & $\begin{array}{l}.00 \\
.00 \\
.00 \\
.00 \\
.00\end{array}$ & $\begin{array}{l}.00 \\
.43 \\
.01 \\
.08 \\
.00\end{array}$ \\
\hline $\begin{array}{l}21 \\
22 \\
23 \\
24 \\
25\end{array}$ & $\begin{array}{l}.04 \\
.00 \\
.00 \\
.00 \\
.00\end{array}$ & $\begin{array}{l}.70 \\
.02 \\
.41 \\
.19 \\
.01\end{array}$ & $\begin{array}{l}.06 \\
.01 \\
.00 \\
.22 \\
.43\end{array}$ & $\begin{array}{l}.00 \\
.00 \\
.00 \\
.99 \\
.04\end{array}$ & $\begin{array}{l}.08 \\
.00 \\
.36 \\
.04 \\
.01\end{array}$ & $\begin{array}{l}.47 \\
.20 \\
.02 \\
.00 \\
.01\end{array}$ & $\begin{array}{l}.23 \\
.05 \\
.00 \\
.75 \\
.94\end{array}$ & $\begin{array}{l}.03 \\
.11 \\
.41 \\
.73 \\
.03\end{array}$ & $\begin{array}{l}.00 \\
.00 \\
.00 \\
.00 \\
.00\end{array}$ & $\begin{array}{r}2.35 \\
.98 \\
.00 \\
.33 \\
.01\end{array}$ & $\begin{array}{l}.00 \\
.00 \\
.00 \\
.00 \\
.00\end{array}$ & $\begin{array}{r}.00 \\
1.26 \\
.00 \\
.00 \\
.00\end{array}$ \\
\hline $\begin{array}{l}26 \\
27 \\
28 \\
29 \\
30 \\
31\end{array}$ & $\begin{array}{l}.00 \\
.00 \\
.00 \\
.00 \\
.00 \\
.00\end{array}$ & $\begin{array}{l}.29 \\
.01 \\
.00 \\
.33 \\
.00 \\
.-\end{array}$ & $\begin{array}{l}.22 \\
.00 \\
.03 \\
.08 \\
.00 \\
.00\end{array}$ & $\begin{array}{l}.01 \\
.01 \\
.00 \\
.00 \\
.00 \\
.12\end{array}$ & $\begin{array}{l}.01 \\
.00 \\
.00 \\
-- \\
-- \\
--\end{array}$ & $\begin{array}{l}.00 \\
.00 \\
.77 \\
.09 \\
.01 \\
.00\end{array}$ & $\begin{array}{l}.12 \\
.06 \\
.01 \\
.00 \\
.04 \\
.-\end{array}$ & $\begin{array}{r}.00 \\
.44 \\
.61 \\
.00 \\
1.13 \\
.32\end{array}$ & $\begin{array}{l}.00 \\
.10 \\
.00 \\
.00 \\
.00 \\
.-\end{array}$ & $\begin{array}{l}.00 \\
.30 \\
.00 \\
.00 \\
.00 \\
.00\end{array}$ & $\begin{array}{r}.00 \\
1.06 \\
.00 \\
.00 \\
.05 \\
1.01\end{array}$ & $\begin{array}{l}.00 \\
.00 \\
.00 \\
.00 \\
.00 \\
-.\end{array}$ \\
\hline Total & 1.61 & 5018 & 3.10 & 3.45 & 2.37 & 4.46 & 6.62 & 5.82 & 1.70 & 6.42 & 2.12 & 2.40 \\
\hline
\end{tabular}


Table 7.-- Daily precipitation totals at continuous-recording gages--Continued

4644350684416 Rain Gage 3 at Bishop Mountain, ME.--Continued

Precipitation totals, in inches

1983

1984

Day OCT NOV DEC JAN FEB MAR APR MAY JUN JUL AUG SEP

\begin{tabular}{|c|c|c|c|c|c|c|c|c|c|}
\hline $\begin{array}{l}1 \\
2 \\
3 \\
4 \\
5\end{array}$ & $\begin{array}{r}0.00 \\
.00 \\
.00 \\
.10 \\
1.12\end{array}$ & $\begin{array}{r}0.00 \\
.00 \\
.70 \\
.10 \\
.21\end{array}$ & $\begin{array}{r}0.00 \\
.00 \\
.00 \\
.00 \\
.14\end{array}$ & $\begin{array}{r}0.00 \\
.00 \\
.00 \\
.00 \\
.00\end{array}$ & $\begin{array}{r}0.00 \\
.00 \\
.11 \\
.05 \\
.41\end{array}$ & $\begin{array}{r}0.00 \\
.02 \\
.00 \\
.00 \\
.08\end{array}$ & $\begin{array}{r}0.00 \\
.00 \\
.00 \\
.00 \\
.17\end{array}$ & $\begin{array}{r}0.00 \\
.00 \\
.00 \\
.82 \\
.34\end{array}$ & $\begin{array}{r}0.08 \\
.00 \\
.00 \\
.00 \\
.00\end{array}$ \\
\hline $\begin{array}{r}6 \\
7 \\
8 \\
9 \\
10\end{array}$ & $\begin{array}{l}.35 \\
.00 \\
.10 \\
.00 \\
.00\end{array}$ & $\begin{array}{l}.46 \\
.13 \\
.00 \\
.00 \\
.00\end{array}$ & $\begin{array}{r}.99 \\
1.46 \\
.00 \\
.00 \\
.00\end{array}$ & $\begin{array}{l}.03 \\
.07 \\
.03 \\
.02 \\
.23\end{array}$ & $\begin{array}{r}1.10 \\
.02 \\
.01 \\
.01 \\
.01\end{array}$ & $\begin{array}{l}.04 \\
.00 \\
.00 \\
.00 \\
.00\end{array}$ & $\begin{array}{l}.22 \\
.34 \\
.11 \\
.00 \\
.10\end{array}$ & $\begin{array}{l}.00 \\
.00 \\
.28 \\
.15 \\
.00\end{array}$ & $\begin{array}{l}.00 \\
.05 \\
.10 \\
.00 \\
.00\end{array}$ \\
\hline $\begin{array}{l}11 \\
12 \\
13 \\
14 \\
15\end{array}$ & $\begin{array}{l}.00 \\
.00 \\
.40 \\
.05 \\
.00\end{array}$ & $\begin{array}{l}.88 \\
.21 \\
.01 \\
.00 \\
.00\end{array}$ & $\begin{array}{l}.00 \\
.22 \\
.57 \\
.83 \\
.66\end{array}$ & $\begin{array}{l}.07 \\
.00 \\
.00 \\
.05 \\
.00\end{array}$ & $\begin{array}{l}.00 \\
.00 \\
.00 \\
.00 \\
.33\end{array}$ & $\begin{array}{r}.01 \\
.00 \\
.00 \\
1.25 \\
.09\end{array}$ & $\begin{array}{l}.00 \\
.03 \\
.01 \\
.00 \\
.00\end{array}$ & $\begin{array}{l}.00 \\
.40 \\
.00 \\
.64 \\
.01\end{array}$ & $\begin{array}{l}.00 \\
.00 \\
\cdots \\
\cdots \\
--\end{array}$ \\
\hline $\begin{array}{l}16 \\
17 \\
18 \\
19 \\
20\end{array}$ & $\begin{array}{l}.00 \\
.00 \\
.00 \\
.00 \\
.00\end{array}$ & $\begin{array}{l}.00 \\
.89 \\
.02 \\
.00 \\
.05\end{array}$ & $\begin{array}{l}.00 \\
.00 \\
.00 \\
.09 \\
.00\end{array}$ & $\begin{array}{l}.00 \\
.00 \\
.07 \\
.11 \\
.01\end{array}$ & $\begin{array}{l}.00 \\
.00 \\
.00 \\
.04 \\
.24\end{array}$ & $\begin{array}{l}.16 \\
.09 \\
.00 \\
.04 \\
.08\end{array}$ & $\begin{array}{l}.51 \\
.18 \\
.01 \\
.01 \\
.00\end{array}$ & $\begin{array}{l}.07 \\
.00 \\
.00 \\
.00 \\
.00\end{array}$ & $\begin{array}{l}\cdots \\
\cdots \\
\cdots \\
\cdots\end{array}$ \\
\hline $\begin{array}{l}21 \\
22 \\
23 \\
24 \\
25\end{array}$ & $\begin{array}{l}.00 \\
.00 \\
.00 \\
.00 \\
.00\end{array}$ & $\begin{array}{r}.00 \\
.00 \\
.03 \\
.29 \\
2.19\end{array}$ & $\begin{array}{l}.00 \\
.65 \\
.10 \\
.00 \\
.00\end{array}$ & $\begin{array}{l}.01 \\
.00 \\
.00 \\
.25 \\
.09\end{array}$ & $\begin{array}{l}.01 \\
.03 \\
.00 \\
.01 \\
.07\end{array}$ & $\begin{array}{l}.02 \\
.30 \\
.04 \\
.03 \\
.00\end{array}$ & $\begin{array}{l}.00 \\
.00 \\
.00 \\
.00 \\
.26\end{array}$ & $\begin{array}{l}.00 \\
.00 \\
.43 \\
.01 \\
.00\end{array}$ & $\begin{array}{l}\cdots \\
\cdots \\
\cdots \\
\cdots\end{array}$ \\
\hline $\begin{array}{l}26 \\
27 \\
28 \\
29 \\
30 \\
31\end{array}$ & $\begin{array}{l}.00 \\
.00 \\
.00 \\
.16 \\
.00 \\
.00\end{array}$ & $\begin{array}{l}.03 \\
.00 \\
.00 \\
.98 \\
.02 \\
.-\end{array}$ & $\begin{array}{l}.00 \\
.00 \\
.94 \\
.06 \\
.00 \\
.00\end{array}$ & $\begin{array}{l}.00 \\
.19 \\
.00 \\
.00 \\
.02 \\
.38\end{array}$ & $\begin{array}{l}.24 \\
.00 \\
.83 \\
.30 \\
-. \\
\ldots\end{array}$ & $\begin{array}{l}.00 \\
.00 \\
.00 \\
.00 \\
.01 \\
.00\end{array}$ & $\begin{array}{l}.00 \\
.00 \\
.00 \\
.00 \\
.00 \\
\cdots\end{array}$ & $\begin{array}{l}.09 \\
.00 \\
.00 \\
.86 \\
.85 \\
.94\end{array}$ & $\begin{array}{l}- \\
- \\
- \\
\cdots \\
\cdots\end{array}$ \\
\hline Total & 2.28 & 7.20 & 6.71 & 1.63 & 3.82 & 2.26 & 1.95 & 5.89 & - \\
\hline
\end{tabular}


Table 9.--Daily air-temperature data in the Bald Mountain watersheds

Air temperature, in degrees centigrade

Day

January 1982

February 1982

March 1982

April 1982

Max Min Mean Max Min Mean Max Min Mean

Max Min Mean

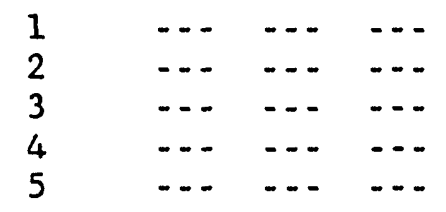

$\begin{array}{llll}6 & \cdots & \cdots & \cdots \\ 7 & \cdots & \cdots & \cdots\end{array}$

$8 \quad \cdots+\cdots+\cdots$

$9 \quad-\cdots+\cdots,-\cdots$

$10 \quad \ldots \quad \ldots \ldots$

11

12

13

14

15

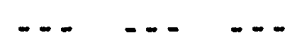

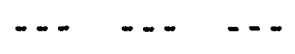

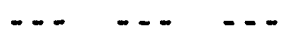

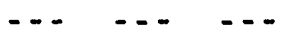

$\begin{array}{lll}-8 & -12 & -10\end{array}$

$\begin{array}{llll}16 & -6 & -16 & -11\end{array}$

$17 . \quad-7 \quad-33 \quad-20$

$\begin{array}{llll}18 & -21 & -34 & -28\end{array}$

$19 .-16 \quad-24 \quad-20$

$\begin{array}{llll}20 & -8 & -23 & -16\end{array}$

$\begin{array}{llll}21 & -15 & -25 & -20\end{array}$

$\begin{array}{llll}22 & -15 & -27 & -21\end{array}$

$\begin{array}{llll}23 & -11 & -32 & -22\end{array}$

$\begin{array}{llll}24 & -6 & -11 & -8\end{array}$

25

$\begin{array}{lll}-9 & -25 & -17\end{array}$

$\begin{array}{llll}26 & -16 & -32 & -24\end{array}$

$\begin{array}{llll}27 & -11 & -31 & -21\end{array}$

$\begin{array}{llll}28 & -4 & -28 & -16\end{array}$

$\begin{array}{llll}29 & -2 & -17 & -10\end{array}$

$\begin{array}{llll}30 & -5 & -25 & -15\end{array}$

$\begin{array}{llll}31 & -1 & -15 & -8\end{array}$

Mean -..

Max $\quad \cdots \quad \ldots \quad \ldots$

Min $\quad \ldots \quad \ldots, \ldots$

$\begin{array}{lll}-15 & -33 & -24\end{array}$ $\begin{array}{lll}-8 & -15 & -12\end{array}$

$\begin{array}{lll}-2 & -24 & -13\end{array}$

$\begin{array}{lll}1 & -6 & -2\end{array}$

$\begin{array}{lll}4 & -10 & -3\end{array}$

$\begin{array}{lll}-9 & -27 & -18\end{array}$

$\begin{array}{lll}-4 & -13 & -8\end{array}$

$\begin{array}{lll}-9 & -34 & -22\end{array}$

$\begin{array}{lll}-1 & -11 & -6\end{array}$

$\begin{array}{lll}-7 & -16 & -12\end{array}$

$\begin{array}{lll}-3 & -20 & -12 \\ 4 & -6 & -1\end{array}$

$\begin{array}{lll}3 & -17 & -7\end{array}$

$\begin{array}{lll}2 & -2 & 0\end{array}$

$\begin{array}{lll}1 & -13 & -6\end{array}$

$\begin{array}{lll}-7 & -27 & -17 \\ 1 & -26 & -12\end{array}$

$\begin{array}{lll}1 & -26 & -12\end{array}$

$\begin{array}{lll}6 & -2 & 2 \\ 5 & 3 & 4\end{array}$

$\begin{array}{lll}5 & 3 & 4 \\ 5 & 2 & 4\end{array}$

$\begin{array}{rrr}5 & 2 & 4 \\ 5 & -6 & 0\end{array}$

$\begin{array}{lll}-1 & -7 & -4\end{array}$

$\begin{array}{lll}4 & -10 & -3\end{array}$

$\begin{array}{lll}6 & -13 & -4\end{array}$

$\begin{array}{lll}8 & -14 & -3\end{array}$

$\begin{array}{lll}8 & -13 & -2\end{array}$

$\begin{array}{lll}5 & -12 & -4\end{array}$

$\begin{array}{lll}5 & -17 & -6\end{array}$

$\begin{array}{lll}-1 & -12 & -6\end{array}$

$\begin{array}{lll}-3 & -9 & -6\end{array}$

$\begin{array}{lll}-1 & -9 & -5\end{array}$

$\begin{array}{lll}-4 & -16 & -10\end{array}$

$\begin{array}{lll}-15 & -19 & -17\end{array}$

$\begin{array}{lll}-13 & -19 & -16\end{array}$

$\begin{array}{lll}-8 & -28 & -18\end{array}$

$\begin{array}{lll}-14 & -33 & -24\end{array}$

-. - -. - -.

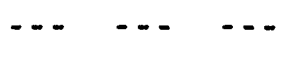

$\cdots+\cdots$

$\begin{array}{lll}-5 & -19 & -12\end{array}$ $\begin{array}{lll}1 & -3 & -1\end{array}$

$\begin{array}{lll}4 & -13 & -4\end{array}$

$\begin{array}{lll}7 & -18 & -6\end{array}$

$\begin{array}{lll}12 & -2 & 5\end{array}$

$\begin{array}{rrr}8 & -4 & 2 \\ -4 & -15 & -10\end{array}$

$\begin{array}{lll}-9 & -18 & -14\end{array}$

$\begin{array}{lll}5 & -10 & -2\end{array}$

$\begin{array}{rrr}12 & -3 & 4\end{array}$

$\begin{array}{rrr}3 & -11 & -4 \\ 12 & 3 & 5\end{array}$

$\begin{array}{lll}-9 & -34 & -22\end{array}$ $\begin{array}{lll}7 & 0 & 4\end{array}$

$\begin{array}{lll}1 & -7 & -3\end{array}$

$\begin{array}{lll}4 & -13 & -4\end{array}$

$\begin{array}{rrr}3 & -2 & 0 \\ 0 & -9 & -4\end{array}$

$\begin{array}{lll}-3 & -15 & -9\end{array}$

$\begin{array}{lll}-7 & -11 & -9\end{array}$

$\begin{array}{lll}-5 & -8 & -6\end{array}$

$\begin{array}{lll}-1 & -17 & -9\end{array}$

$\begin{array}{lll}3 & -19 & -8\end{array}$

$\begin{array}{lll}8 & -11 & -2\end{array}$

$\begin{array}{lll}6 & -10 & -2\end{array}$

$\begin{array}{lll}7 & -11 & -2\end{array}$

$\begin{array}{lll}3 & 0 & 2\end{array}$

$\begin{array}{lll}10 & -8 & 1\end{array}$

$\begin{array}{lll}16 & 1 & 8\end{array}$

$\begin{array}{lll}18 & 3 & 10\end{array}$

$\begin{array}{lll}13 & -1 & 6\end{array}$

$\begin{array}{rrr}7 & -2 & 2 \\ 7 & 1 & 4\end{array}$

$\begin{array}{lll}6 & -1 & 2\end{array}$

$\begin{array}{lll}4 & -2 & 1\end{array}$

$\begin{array}{lll}8 & -3 & 2\end{array}$

$\begin{array}{lll}10 & -6 & 2\end{array}$

$20 \quad 0 \quad 10$

$\begin{array}{lll}21 & 2 & 12\end{array}$

$\begin{array}{lll}14 & 2 & 8\end{array}$

$\begin{array}{lll}7 & -3 & 2\end{array}$

$\begin{array}{lll}8 & -5 & 2\end{array}$

$\begin{array}{lll}3 & -5 & -1\end{array}$

$\begin{array}{rrr}7 & -5 & 1 \\ 21 & 3 & 12\end{array}$ 
Table 9.--Daily air-temperature data in the Bald Mountain watersheds--Continued

Air temperature, in degrees centigrade

Day
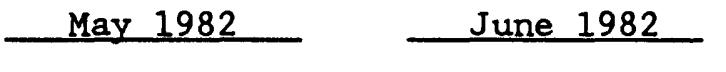

July 1982

August 1982

Max Min Mean Max Min Mean Max Min Mean Max Min Mean

$\begin{array}{llll}1 & 5 & -3 & 1\end{array}$

$2 \quad 15 \quad-4 \quad 6$

$3 \quad 14 \quad 0 \quad 7$

$\begin{array}{llll}4 & 12 & -4 & 4 \\ 5 & 17 & -5 & 6\end{array}$

5

$\begin{array}{lllr}6 & 22 & -4 & 9\end{array}$

$\begin{array}{llll}7 & 24 & 4 & 14\end{array}$

$\begin{array}{llll}8 & 26 & 3 & 14\end{array}$

$\begin{array}{rrrr}9 & 15 & 7 & 11 \\ 10 & 7 & 3 & 5\end{array}$

10

$11-6 \quad 2$

12

13

14

15

$\begin{array}{llll}16 & 20 & 3 & 12\end{array}$

$\begin{array}{llll}17 & 13 & -3 & 5\end{array}$

$\begin{array}{llll}18 & 17 & -6 & 6\end{array}$

$\begin{array}{llll}19 & 22 & -5 & 8\end{array}$

$\begin{array}{llll}20 & 21 & 2 & 12\end{array}$

$\begin{array}{llll}21 & 12 & -5 & 4\end{array}$

$\begin{array}{llll}22 & 15 & -7 & 4 \\ 23 & 19 & -7 & 6\end{array}$

$\begin{array}{llll}24 & 16 & -2 & 7\end{array}$

$\begin{array}{llll}25 & 25 & 8 & 16\end{array}$

$\begin{array}{llll}26 & 27 & 5 & 16\end{array}$

$\begin{array}{llll}27 & 23 & 2 & 12\end{array}$

$\begin{array}{llll}28 & 24 & 4 & 14\end{array}$

$\begin{array}{llll}29 & 28 & 12 & 20\end{array}$

$\begin{array}{llll}30 & 26 & 8 & 17\end{array}$

$\begin{array}{llll}31 & 25 & 9 & 17\end{array}$

$\begin{array}{llll}\text { Mean } & 18 & 1 & 9\end{array}$

$\begin{array}{llll}\operatorname{Max} & 28 & 12 & 20\end{array}$ $\begin{array}{lll}25 & 12 & 18\end{array}$

$\begin{array}{lll}17 & 6 & 12\end{array}$

$\begin{array}{lll}15 & 0 & 8\end{array}$

$\begin{array}{rrr}21 & -3 & 9\end{array}$

$\begin{array}{lll}24 & 10 & 17\end{array}$

$\begin{array}{lll}24 & 7 & 16\end{array}$

$22 \quad 4 \quad 13$

$\begin{array}{lll}24 & 3 & 14\end{array}$

$27 \quad 4 \quad 16$

$27 \quad 6 \quad 16$

$\begin{array}{lll}23 & 8 & 16\end{array}$

$21 \quad 11 \quad 16$

$\begin{array}{lll}14 & 6 & 10\end{array}$

$\begin{array}{lll}11 & 5 & 8\end{array}$

$\begin{array}{lll}17 & 8 & 12\end{array}$

$\begin{array}{lll}19 & 9 & 14\end{array}$

$21 \quad 5 \quad 13$

$23 \quad 2 \quad 12$

$\begin{array}{lll}22 & 3 & 12\end{array}$

$\begin{array}{lll}19 & 10 & 14\end{array}$

$\begin{array}{lll}21 & 8 & 14\end{array}$

$\begin{array}{lll}22 & 11 & 16\end{array}$

$\begin{array}{lll}21 & 10 & 16\end{array}$

$\begin{array}{lll}17 & 8 & 12\end{array}$

$\begin{array}{lll}20 & 5 & 12\end{array}$

$23 \quad 4 \quad 14$

$23 \quad 0 \quad 12$

$\begin{array}{lll}23 & 8 & 16\end{array}$

$\begin{array}{lll}18 & 14 & 16\end{array}$

$2199 \quad 15$

$\begin{array}{lll}21 & 6 & 14\end{array}$

$\begin{array}{lll}27 & 14 & 18\end{array}$

$\begin{array}{lll}11 & -3 & 8\end{array}$ $\begin{array}{lll}16 & 7 & 12\end{array}$

$\begin{array}{lll}21 & 10 & 16\end{array}$

$\begin{array}{lll}17 & 2 & 10\end{array}$

190010

$\begin{array}{lll}22 & 0 & 11\end{array}$

$\begin{array}{lll}27 & 7 & 17\end{array}$

$32 \quad 16 \quad 24$

$\begin{array}{lll}29 & 16 & 22\end{array}$

$\begin{array}{lll}25 & 11 & 18\end{array}$

$\begin{array}{lll}24 & 6 & 15\end{array}$

$\begin{array}{lll}29 & 2 & 16\end{array}$

$\begin{array}{lll}28 & 13 & 20\end{array}$

$\begin{array}{lll}22 & 12 & 17\end{array}$

$\begin{array}{lll}28 & 8 & 18\end{array}$

$\begin{array}{lll}29 & 7 & 18\end{array}$

$\begin{array}{lll}28 & 11 & 20\end{array}$

$30 \quad 10 \quad 20$

$\begin{array}{lll}31 & 17 & 24\end{array}$

$30 \quad 15 \quad 22$

$\begin{array}{lll}20 & 10 & 15\end{array}$

$\begin{array}{lll}24 & 7 & 16\end{array}$

$\begin{array}{lll}16 & 12 & 14\end{array}$

$2399 \quad 16$

$\begin{array}{lll}25 & 4 & 14\end{array}$

$\begin{array}{lll}23 & 13 & 18\end{array}$

$\begin{array}{lll}21 & 11 & 16\end{array}$

$\begin{array}{lll}26 & 6 & 16\end{array}$

$\begin{array}{lll}24 & 6 & 15\end{array}$

$\begin{array}{lll}15 & 10 & 12\end{array}$

$\begin{array}{lll}26 & 8 & 17 \\ 27 & 7 & 17\end{array}$

$\begin{array}{lll}27 & 7 & 17\end{array}$

$\begin{array}{lll}24 & 9 & 17\end{array}$

32. $17 \quad 24$

1500 $\begin{array}{lll}26 & 13 & 20\end{array}$

$\begin{array}{lll}19 & 4 & 12\end{array}$

$20 \quad 0 \quad 10$

$\begin{array}{lll}23 & 5 & 14 \\ 15 & 6 & 10\end{array}$

$\begin{array}{lll}22 & 8 & 15\end{array}$

$\begin{array}{lll}19 & 7 & 13\end{array}$

$\begin{array}{lll}23 & 9 & 16\end{array}$

$\begin{array}{lll}13 & 11 & 12\end{array}$

$25 \quad 14 \quad 20$

$\begin{array}{lll}19 & 10 & 14\end{array}$

$20 \quad 4 \quad 12$

$\begin{array}{lll}16 & 5 & 10\end{array}$

$\begin{array}{lll}19 & 13 & 16\end{array}$

$\begin{array}{lll}26 & 11 & 18\end{array}$

$\begin{array}{lll}29 & 8 & 18\end{array}$

$2310 \quad 16$

$22 \quad 4 \quad 13$

$\begin{array}{lll}23 & 8 & 16\end{array}$

$\begin{array}{lll}23 & 11 & 17\end{array}$

15510

$\begin{array}{rrr}16 & 0 & 8\end{array}$

$\begin{array}{lll}17 & 2 & 10\end{array}$

$\begin{array}{lll}21 & 9 & 15\end{array}$

$20 \quad 5 \quad 12$

$\begin{array}{lll}21 & 9 & 15\end{array}$

$\begin{array}{lll}26 & 7 & 16\end{array}$

$29 \quad 4 \quad 16$

$\begin{array}{lll}27 & 2 & 14\end{array}$

$\begin{array}{rrr}17 & 0 & 8 \\ 20 & 4 & 12\end{array}$

2042

$\begin{array}{lll}21 & 7 & 14\end{array}$

$\begin{array}{lll}29 & 14 & 20\end{array}$ 
Table 9.--Daily air-temperature data in the Bald Mountain watersheds--Continued

Air temperature, in degrees centigrade

Day September $1982 \quad$ October 1982 November 1982 December 1982

Max Min Mean Max Min Mean Max Min Mean Max Min Mean

\begin{tabular}{|c|c|c|c|c|c|c|c|c|c|c|c|c|}
\hline $\begin{array}{l}1 \\
2 \\
3 \\
4 \\
5\end{array}$ & $\begin{array}{l}18 \\
12 \\
20 \\
17 \\
11\end{array}$ & $\begin{array}{l}0 \\
9 \\
8 \\
7 \\
0\end{array}$ & $\begin{array}{r}9 \\
10 \\
14 \\
12 \\
6\end{array}$ & $\begin{array}{l}16 \\
11 \\
16 \\
16 \\
17\end{array}$ & $\begin{array}{r}7 \\
-1 \\
-1 \\
-2 \\
-3\end{array}$ & $\begin{array}{r}12 \\
5 \\
8 \\
7 \\
7\end{array}$ & $\begin{array}{r}14 \\
6 \\
12 \\
18 \\
18\end{array}$ & $\begin{array}{r}-2 \\
-2 \\
4 \\
9 \\
1\end{array}$ & $\begin{array}{r}6 \\
2 \\
8 \\
14 \\
10\end{array}$ & $\begin{array}{r}1 \\
3 \\
7 \\
12 \\
4\end{array}$ & $\begin{array}{r}-4 \\
0 \\
2 \\
1 \\
-3\end{array}$ & $\begin{array}{r}-2 \\
2 \\
4 \\
6 \\
0\end{array}$ \\
\hline $\begin{array}{r}6 \\
7 \\
8 \\
9 \\
10\end{array}$ & $\begin{array}{l}13 \\
15 \\
18 \\
18 \\
23\end{array}$ & $\begin{array}{r}8 \\
0 \\
-1 \\
6 \\
9\end{array}$ & $\begin{array}{r}10 \\
8 \\
8 \\
12 \\
16\end{array}$ & $\begin{array}{r}19 \\
11 \\
6 \\
7 \\
11\end{array}$ & $\begin{array}{r}5 \\
1 \\
3 \\
-3 \\
-6\end{array}$ & $\begin{array}{r}12 \\
6 \\
4 \\
2 \\
2\end{array}$ & $\begin{array}{l}4 \\
4 \\
8 \\
1 \\
3\end{array}$ & $\begin{array}{r}0 \\
-1 \\
0 \\
-2 \\
-5\end{array}$ & $\begin{array}{r}2 \\
2 \\
4 \\
0 \\
-1\end{array}$ & $\begin{array}{r}11 \\
9 \\
-4 \\
-5 \\
-11\end{array}$ & $\begin{array}{r}3 \\
-5 \\
-9 \\
-22 \\
-23\end{array}$ & $\begin{array}{r}7 \\
2 \\
-6 \\
-14 \\
-17\end{array}$ \\
\hline $\begin{array}{l}11 \\
12 \\
13 \\
14 \\
15\end{array}$ & $\begin{array}{l}22 \\
24 \\
28 \\
28 \\
11\end{array}$ & $\begin{array}{r}10 \\
5 \\
11 \\
14 \\
9\end{array}$ & $\begin{array}{l}16 \\
14 \\
20 \\
21 \\
10\end{array}$ & $\begin{array}{r}15 \\
14 \\
9 \\
12 \\
10\end{array}$ & $\begin{array}{r}-7 \\
-6 \\
-1 \\
7 \\
4\end{array}$ & $\begin{array}{r}4 \\
8 \\
4 \\
10 \\
7\end{array}$ & $\begin{array}{r}6 \\
14 \\
15 \\
-2 \\
-1\end{array}$ & $\begin{array}{r}-8 \\
6 \\
-4 \\
-7 \\
-5\end{array}$ & $\begin{array}{r}-1 \\
10 \\
6 \\
-4 \\
-3\end{array}$ & $\begin{array}{r}0 \\
-16 \\
-17 \\
-5 \\
-2\end{array}$ & $\begin{array}{r}-17 \\
-24 \\
-26 \\
-20 \\
-7\end{array}$ & $\begin{array}{r}-8 \\
-20 \\
-22 \\
-12 \\
-4\end{array}$ \\
\hline $\begin{array}{l}16 \\
17 \\
18 \\
19 \\
20\end{array}$ & $\begin{array}{l}10 \\
13 \\
13 \\
15 \\
17\end{array}$ & $\begin{array}{l}7 \\
9 \\
8 \\
3 \\
0\end{array}$ & $\begin{array}{r}8 \\
11 \\
10 \\
9 \\
8\end{array}$ & $\begin{array}{r}8 \\
9 \\
9 \\
16 \\
19\end{array}$ & $\begin{array}{r}3 \\
0 \\
-2 \\
0 \\
-1\end{array}$ & $\begin{array}{l}6 \\
4 \\
4 \\
8 \\
9\end{array}$ & $\begin{array}{l}0 \\
2 \\
3 \\
1 \\
3\end{array}$ & $\begin{array}{l}-12 \\
-11 \\
-13 \\
-15 \\
-14\end{array}$ & $\begin{array}{l}-6 \\
-4 \\
-5 \\
-7 \\
-6\end{array}$ & $\begin{array}{r}6 \\
-1 \\
-10 \\
-7 \\
-4\end{array}$ & $\begin{array}{r}-2 \\
-17 \\
-19 \\
-19 \\
-7\end{array}$ & $\begin{array}{r}2 \\
-9 \\
-14 \\
-13 \\
-6\end{array}$ \\
\hline $\begin{array}{l}21 \\
22 \\
23 \\
24 \\
25\end{array}$ & $\begin{array}{l}18 \\
15 \\
13 \\
21 \\
17\end{array}$ & $\begin{array}{l}4 \\
4 \\
7 \\
6 \\
6\end{array}$ & $\begin{array}{l}11 \\
10 \\
10 \\
14 \\
12\end{array}$ & $\begin{array}{r}11 \\
4 \\
7 \\
7 \\
13\end{array}$ & $\begin{array}{r}2 \\
-6 \\
-8 \\
-6 \\
-3\end{array}$ & $\begin{array}{r}6 \\
-1 \\
0 \\
0 \\
5\end{array}$ & $\begin{array}{r}8 \\
10 \\
6 \\
9 \\
-2\end{array}$ & $\begin{array}{r}-1 \\
2 \\
2 \\
-7 \\
-10\end{array}$ & $\begin{array}{r}4 \\
6 \\
4 \\
1 \\
-6\end{array}$ & $\begin{array}{r}-4 \\
-7 \\
-6 \\
-5 \\
3\end{array}$ & $\begin{array}{r}-8 \\
-17 \\
-22 \\
-22 \\
-5\end{array}$ & $\begin{array}{r}-6 \\
-12 \\
-14 \\
-14 \\
-1\end{array}$ \\
\hline $\begin{array}{l}26 \\
27 \\
28 \\
29 \\
30 \\
31\end{array}$ & $\begin{array}{r}21 \\
15 \\
15 \\
15 \\
21 \\
-\end{array}$ & $\begin{array}{r}9 \\
8 \\
-1 \\
-3 \\
4 \\
-\end{array}$ & $\begin{array}{r}15 \\
12 \\
7 \\
6 \\
12 \\
\ldots\end{array}$ & $\begin{array}{l}11 \\
13 \\
17 \\
14 \\
18 \\
17\end{array}$ & $\begin{array}{r}-6 \\
-7 \\
5 \\
2 \\
1 \\
2\end{array}$ & $\begin{array}{r}2 \\
3 \\
11 \\
8 \\
10 \\
10\end{array}$ & $\begin{array}{r}0 \\
-8 \\
-6 \\
-3 \\
-1 \\
---\end{array}$ & $\begin{array}{r}-8 \\
-16 \\
-19 \\
-8 \\
-4 \\
-.--\end{array}$ & $\begin{array}{r}-4 \\
-12 \\
-12 \\
-6 \\
-2 \\
--.\end{array}$ & $\begin{array}{r}9 \\
0 \\
9 \\
10 \\
-1 \\
-1\end{array}$ & $\begin{array}{r}-5 \\
-11 \\
-12 \\
-2 \\
-13 \\
-13\end{array}$ & $\begin{array}{r}2 \\
-6 \\
-2 \\
4 \\
-7 \\
-7\end{array}$ \\
\hline $\begin{array}{l}\text { Mean } \\
\text { Max } \\
\text { Min }\end{array}$ & $\begin{array}{l}17 \\
28 \\
10\end{array}$ & $\begin{array}{r}6 \\
14 \\
-3\end{array}$ & $\begin{array}{r}11 \\
21 \\
6\end{array}$ & $\begin{array}{r}12 \\
19 \\
4\end{array}$ & $\begin{array}{r}-1 \\
7 \\
-8\end{array}$ & $\begin{array}{r}6 \\
12 \\
-1\end{array}$ & $\begin{array}{r}5 \\
18 \\
-8\end{array}$ & $\begin{array}{r}-5 \\
9 \\
-19\end{array}$ & $\begin{array}{r}0 \\
14 \\
-12\end{array}$ & $\begin{array}{r}-1 \\
12 \\
-17\end{array}$ & $\begin{array}{r}-11 \\
3 \\
-26\end{array}$ & $\begin{array}{r}-6 \\
7 \\
-22\end{array}$ \\
\hline
\end{tabular}


Table 9.--Daily air-temperature data in the Bald Mountain watersheds--Continued

Air temperature, in degrees centigrade

\begin{tabular}{|c|c|c|c|c|c|c|c|c|c|c|c|c|}
\hline \multirow[t]{2}{*}{ Day } & \multicolumn{3}{|c|}{ January 1983} & \multicolumn{3}{|c|}{ February 1983} & \multicolumn{3}{|c|}{ March 1983} & \multicolumn{3}{|c|}{ April 1983} \\
\hline & $\operatorname{Max}$ & Min & Mean & $\operatorname{Max}$ & Min & Mean & $\operatorname{Max}$ & Min & Mean & $\operatorname{Max}$ & Min & Mean \\
\hline $\begin{array}{l}1 \\
2 \\
3 \\
4 \\
5\end{array}$ & $\begin{array}{r}1 \\
0 \\
-13 \\
-15 \\
-3\end{array}$ & $\begin{array}{r}-6 \\
-16 \\
-23 \\
-25 \\
-19\end{array}$ & $\begin{array}{r}-2 \\
-8 \\
-18 \\
-20 \\
-11\end{array}$ & $\begin{array}{r}0 \\
1 \\
9 \\
7 \\
-7\end{array}$ & $\begin{array}{r}-8 \\
-17 \\
1 \\
-7 \\
-18\end{array}$ & $\begin{array}{r}-4 \\
-8 \\
5 \\
0 \\
-12\end{array}$ & $\begin{array}{r}7 \\
1 \\
0 \\
-3 \\
-1\end{array}$ & $\begin{array}{r}-2 \\
-5 \\
-5 \\
-8 \\
-19\end{array}$ & $\begin{array}{r}2 \\
-2 \\
-2 \\
-6 \\
-10\end{array}$ & $\begin{array}{l}2 \\
3 \\
3 \\
2 \\
4\end{array}$ & $\begin{array}{r}-12 \\
-3 \\
-4 \\
-1 \\
0\end{array}$ & $\begin{array}{r}-5 \\
0 \\
0 \\
0 \\
2\end{array}$ \\
\hline $\begin{array}{r}6 \\
7 \\
8 \\
9 \\
10\end{array}$ & $\begin{array}{r}1 \\
-2 \\
-3 \\
-10 \\
2\end{array}$ & $\begin{array}{r}-4 \\
-10 \\
-17 \\
-25 \\
-27\end{array}$ & $\begin{array}{r}-2 \\
-6 \\
-10 \\
-18 \\
-12\end{array}$ & $\begin{array}{r}-11 \\
-10 \\
-5 \\
-7 \\
-13\end{array}$ & $\begin{array}{l}-21 \\
-23 \\
-11 \\
-17 \\
-20\end{array}$ & $\begin{array}{r}-16 \\
-16 \\
-8 \\
-12 \\
-16\end{array}$ & $\begin{array}{r}4 \\
-1 \\
-2 \\
4 \\
5\end{array}$ & $\begin{array}{r}-21 \\
-17 \\
-20 \\
-10 \\
-3\end{array}$ & $\begin{array}{r}-8 \\
-9 \\
-11 \\
-3 \\
1\end{array}$ & $\begin{array}{r}7 \\
10 \\
8 \\
9 \\
9\end{array}$ & $\begin{array}{r}-3 \\
-5 \\
1 \\
-4 \\
-6\end{array}$ & $\begin{array}{l}2 \\
2 \\
4 \\
2 \\
2\end{array}$ \\
\hline $\begin{array}{l}11 \\
12 \\
13 \\
14 \\
15\end{array}$ & $\begin{array}{r}10 \\
3 \\
-5 \\
-4 \\
-6\end{array}$ & $\begin{array}{r}2 \\
-5 \\
-13 \\
-17 \\
-15\end{array}$ & $\begin{array}{r}6 \\
-1 \\
-9 \\
-10 \\
-10\end{array}$ & $\begin{array}{r}-10 \\
-9 \\
-3 \\
3 \\
1\end{array}$ & $\begin{array}{r}-28 \\
-30 \\
-30 \\
-9 \\
-18\end{array}$ & $\begin{array}{r}-19 \\
-20 \\
-16 \\
-3 \\
-8\end{array}$ & $\begin{array}{l}0 \\
0 \\
2 \\
0 \\
4\end{array}$ & $\begin{array}{l}-3 \\
-1 \\
-2 \\
-4 \\
-1\end{array}$ & $\begin{array}{r}-2 \\
0 \\
0 \\
-2 \\
2\end{array}$ & $\begin{array}{l}2 \\
3 \\
8 \\
7 \\
6\end{array}$ & $\begin{array}{r}-1 \\
0 \\
-3 \\
-4 \\
0\end{array}$ & $\begin{array}{l}0 \\
2 \\
2 \\
2 \\
3\end{array}$ \\
\hline $\begin{array}{l}16 \\
17 \\
18 \\
19 \\
20\end{array}$ & $\begin{array}{r}-6 \\
-7 \\
-15 \\
-14 \\
-13\end{array}$ & $\begin{array}{l}-10 \\
-15 \\
-21 \\
-21 \\
-23\end{array}$ & $\begin{array}{r}-8 \\
-11 \\
-18 \\
-18 \\
-18\end{array}$ & $\begin{array}{r}1 \\
-2 \\
0 \\
-3 \\
-2\end{array}$ & $\begin{array}{l}-26 \\
-26 \\
-20 \\
-25 \\
-20\end{array}$ & $\begin{array}{l}-12 \\
-14 \\
-10 \\
-14 \\
-11\end{array}$ & $\begin{array}{l}1 \\
5 \\
5 \\
4 \\
7\end{array}$ & $\begin{array}{r}-1 \\
-1 \\
-3 \\
1 \\
-2\end{array}$ & $\begin{array}{l}0 \\
2 \\
1 \\
2 \\
2\end{array}$ & $\begin{array}{r}12 \\
11 \\
10 \\
12 \\
9\end{array}$ & $\begin{array}{r}-1 \\
3 \\
2 \\
2 \\
1\end{array}$ & $\begin{array}{l}6 \\
7 \\
6 \\
7 \\
5\end{array}$ \\
\hline $\begin{array}{l}21 \\
22 \\
23 \\
24 \\
25\end{array}$ & $\begin{array}{r}-11 \\
-3 \\
0 \\
2 \\
2\end{array}$ & $\begin{array}{r}-29 \\
-23 \\
-11 \\
-1 \\
-1\end{array}$ & $\begin{array}{r}-20 \\
-13 \\
-6 \\
0 \\
0\end{array}$ & $\begin{array}{r}0 \\
-4 \\
-9 \\
-4 \\
-2\end{array}$ & $\begin{array}{l}-18 \\
-20 \\
-21 \\
-15 \\
-22\end{array}$ & $\begin{array}{r}-9 \\
-12 \\
-15 \\
-10 \\
-12\end{array}$ & $\begin{array}{r}2 \\
3 \\
-3 \\
-10 \\
-6\end{array}$ & $\begin{array}{r}-7 \\
-3 \\
-13 \\
-16 \\
-21\end{array}$ & $\begin{array}{r}-2 \\
0 \\
-8 \\
-13 \\
-14\end{array}$ & $\begin{array}{r}5 \\
6 \\
12 \\
12 \\
12\end{array}$ & $\begin{array}{r}1 \\
-3 \\
-3 \\
-2 \\
4\end{array}$ & $\begin{array}{l}3 \\
2 \\
4 \\
5 \\
8\end{array}$ \\
\hline $\begin{array}{l}26 \\
27 \\
28 \\
29 \\
30 \\
31\end{array}$ & $\begin{array}{r}-1 \\
-10 \\
-4 \\
-1 \\
-3 \\
-1\end{array}$ & $\begin{array}{r}-24 \\
-29 \\
-28 \\
-28 \\
-13 \\
-5\end{array}$ & $\begin{array}{r}-12 \\
-20 \\
-16 \\
-14 \\
-8 \\
-3\end{array}$ & $\begin{array}{r}-5 \\
0 \\
2 \\
- \\
- \\
- \\
-\end{array}$ & $\begin{array}{r}-24 \\
-24 \\
-2 \\
-- \\
-.- \\
-.-\end{array}$ & $\begin{array}{r}-14 \\
-12 \\
0 \\
-- \\
-- \\
-\end{array}$ & $\begin{array}{r}-5 \\
7 \\
2 \\
0 \\
-2 \\
3\end{array}$ & $\begin{array}{r}-16 \\
-17 \\
-3 \\
-7 \\
-9 \\
-13\end{array}$ & $\begin{array}{r}-10 \\
-5 \\
0 \\
-4 \\
-6 \\
-5\end{array}$ & $\begin{array}{r}11 \\
8 \\
17 \\
20 \\
12 \\
-\ldots\end{array}$ & $\begin{array}{r}5 \\
3 \\
1 \\
3 \\
1 \\
-\end{array}$ & $\begin{array}{r}8 \\
6 \\
9 \\
12 \\
6 \\
-\end{array}$ \\
\hline $\begin{array}{l}\text { Mean } \\
\text { Max } \\
\text { Min }\end{array}$ & $\begin{array}{r}-4 \\
10 \\
-15\end{array}$ & $\begin{array}{r}-16 \\
2 \\
-29\end{array}$ & $\begin{array}{r}-10 \\
6 \\
-20\end{array}$ & $\begin{array}{r}-3 \\
9 \\
-13\end{array}$ & $\begin{array}{r}-19 \\
1 \\
-30\end{array}$ & $\begin{array}{r}-11 \\
5 \\
-20\end{array}$ & $\begin{array}{r}1 \\
7 \\
-10\end{array}$ & $\begin{array}{r}-8 \\
1 \\
-21\end{array}$ & $\begin{array}{r}-4 \\
2 \\
-14\end{array}$ & $\begin{array}{r}8 \\
20 \\
2\end{array}$ & $\begin{array}{r}-1 \\
5 \\
-12\end{array}$ & $\begin{array}{r}4 \\
12 \\
-5\end{array}$ \\
\hline
\end{tabular}


Table 9.--Daily alr-temperature data in the Bald Mountain watersheds--Continued

Air temperature, in degrees centigrade

Day
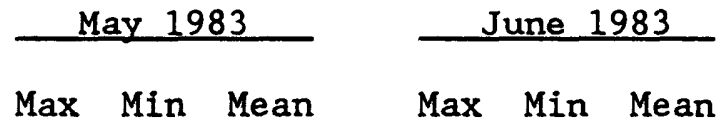

July 1983

August 1983

Max Min Mean Max Min Mean Max Min Mean Max Min Mean

\begin{tabular}{|c|c|c|c|c|c|c|c|c|c|c|c|c|}
\hline $\begin{array}{l}1 \\
2 \\
3 \\
4 \\
5\end{array}$ & $\begin{array}{r}18 \\
5 \\
19 \\
14 \\
15\end{array}$ & $\begin{array}{r}1 \\
-2 \\
3 \\
6 \\
-1\end{array}$ & $\begin{array}{r}10 \\
2 \\
11 \\
10 \\
7\end{array}$ & $\begin{array}{l}17 \\
16 \\
20 \\
17 \\
18\end{array}$ & $\begin{array}{r}5 \\
3 \\
-1 \\
1 \\
4\end{array}$ & $\begin{array}{r}11 \\
10 \\
10 \\
9 \\
11\end{array}$ & $\begin{array}{l}27 \\
21 \\
28 \\
33 \\
29\end{array}$ & $\begin{array}{l}10 \\
14 \\
12 \\
14 \\
15\end{array}$ & $\begin{array}{l}18 \\
18 \\
20 \\
24 \\
22\end{array}$ & $\begin{array}{l}22 \\
23 \\
29 \\
26 \\
28\end{array}$ & $\begin{array}{r}14 \\
10 \\
8 \\
12 \\
11\end{array}$ & $\begin{array}{l}18 \\
16 \\
18 \\
19 \\
20\end{array}$ \\
\hline $\begin{array}{r}6 \\
7 \\
8 \\
9 \\
10\end{array}$ & $\begin{array}{r}11 \\
14 \\
21 \\
12 \\
7\end{array}$ & $\begin{array}{r}-2 \\
-2 \\
7 \\
7 \\
1\end{array}$ & $\begin{array}{r}4 \\
6 \\
14 \\
10 \\
4\end{array}$ & $\begin{array}{l}20 \\
18 \\
17 \\
22 \\
22\end{array}$ & $\begin{array}{r}7 \\
5 \\
1 \\
-2 \\
-2\end{array}$ & $\begin{array}{r}14 \\
12 \\
9 \\
10 \\
10\end{array}$ & $\begin{array}{l}15 \\
21 \\
22 \\
20 \\
14\end{array}$ & $\begin{array}{l}4 \\
2 \\
8 \\
6 \\
5\end{array}$ & $\begin{array}{l}10 \\
12 \\
15 \\
13 \\
10\end{array}$ & $\begin{array}{l}29 \\
26 \\
27 \\
20 \\
17\end{array}$ & $\begin{array}{r}16 \\
11 \\
12 \\
8 \\
4\end{array}$ & $\begin{array}{l}22 \\
18 \\
20 \\
14 \\
10\end{array}$ \\
\hline $\begin{array}{l}11 \\
12 \\
13 \\
14 \\
15\end{array}$ & $\begin{array}{r}8 \\
8 \\
12 \\
18 \\
16\end{array}$ & $\begin{array}{l}1 \\
3 \\
6 \\
2 \\
1\end{array}$ & $\begin{array}{r}4 \\
6 \\
9 \\
10 \\
8\end{array}$ & $\begin{array}{l}22 \\
26 \\
27 \\
29 \\
27\end{array}$ & $\begin{array}{r}6 \\
7 \\
11 \\
14 \\
13\end{array}$ & $\begin{array}{l}14 \\
16 \\
19 \\
22 \\
20\end{array}$ & $\begin{array}{l}23 \\
27 \\
21 \\
26 \\
24\end{array}$ & $\begin{array}{r}4 \\
6 \\
13 \\
11 \\
15\end{array}$ & $\begin{array}{l}14 \\
16 \\
17 \\
18 \\
20\end{array}$ & $\begin{array}{l}19 \\
21 \\
24 \\
27 \\
27\end{array}$ & $\begin{array}{l}2 \\
2 \\
0 \\
3 \\
9\end{array}$ & $\begin{array}{l}10 \\
12 \\
12 \\
15 \\
18\end{array}$ \\
\hline $\begin{array}{l}16 \\
17 \\
18 \\
19 \\
20\end{array}$ & $\begin{array}{r}9 \\
10 \\
16 \\
22 \\
13\end{array}$ & $\begin{array}{r}1 \\
-3 \\
-5 \\
-2 \\
7\end{array}$ & $\begin{array}{r}5 \\
4 \\
6 \\
10 \\
10\end{array}$ & $\begin{array}{l}28 \\
29 \\
26 \\
24 \\
26\end{array}$ & $\begin{array}{r}16 \\
15 \\
15 \\
9 \\
5\end{array}$ & $\begin{array}{l}22 \\
22 \\
20 \\
16 \\
16\end{array}$ & $\begin{array}{l}25 \\
23 \\
25 \\
24 \\
21\end{array}$ & $\begin{array}{r}13 \\
10 \\
5 \\
5 \\
9\end{array}$ & $\begin{array}{l}19 \\
16 \\
15 \\
14 \\
15\end{array}$ & $\begin{array}{l}30 \\
24 \\
26 \\
25 \\
25\end{array}$ & $\begin{array}{l}13 \\
11 \\
16 \\
15 \\
11\end{array}$ & $\begin{array}{l}22 \\
18 \\
21 \\
20 \\
18\end{array}$ \\
\hline $\begin{array}{l}21 \\
22 \\
23 \\
24 \\
25\end{array}$ & $\begin{array}{r}16 \\
21 \\
12 \\
8 \\
11\end{array}$ & $\begin{array}{l}9 \\
8 \\
2 \\
5 \\
7\end{array}$ & $\begin{array}{r}12 \\
14 \\
7 \\
6 \\
9\end{array}$ & $\begin{array}{l}31 \\
31 \\
33 \\
26 \\
18\end{array}$ & $\begin{array}{r}7 \\
9 \\
13 \\
4 \\
1\end{array}$ & $\begin{array}{l}19 \\
20 \\
23 \\
15 \\
10\end{array}$ & $\begin{array}{l}18 \\
19 \\
26 \\
22 \\
22\end{array}$ & $\begin{array}{r}9 \\
10 \\
6 \\
9 \\
10\end{array}$ & $\begin{array}{l}14 \\
14 \\
16 \\
16 \\
16\end{array}$ & $\begin{array}{l}19 \\
15 \\
19 \\
21 \\
27\end{array}$ & $\begin{array}{r}7 \\
6 \\
2 \\
-1 \\
6\end{array}$ & $\begin{array}{l}13 \\
10 \\
10 \\
10 \\
16\end{array}$ \\
\hline $\begin{array}{l}26 \\
27 \\
28 \\
29 \\
30 \\
31\end{array}$ & $\begin{array}{r}13 \\
10 \\
9 \\
17 \\
10 \\
10\end{array}$ & $\begin{array}{l}6 \\
8 \\
4 \\
1 \\
7 \\
6\end{array}$ & $\begin{array}{r}10 \\
9 \\
6 \\
9 \\
8 \\
8\end{array}$ & $\begin{array}{r}24 \\
20 \\
20 \\
27 \\
27 \\
-\ldots\end{array}$ & $\begin{array}{r}0 \\
6 \\
0 \\
0 \\
3\end{array}$ & $\begin{array}{r}12 \\
13 \\
10 \\
14 \\
15 \\
-\end{array}$ & $\begin{array}{l}23 \\
24 \\
29 \\
26 \\
27 \\
26\end{array}$ & $\begin{array}{r}6 \\
3 \\
15 \\
19 \\
9 \\
4\end{array}$ & $\begin{array}{l}14 \\
14 \\
22 \\
22 \\
18 \\
15\end{array}$ & $\begin{array}{l}22 \\
24 \\
26 \\
24 \\
19 \\
15\end{array}$ & $\begin{array}{r}8 \\
9 \\
7 \\
10 \\
13 \\
13\end{array}$ & $\begin{array}{l}15 \\
16 \\
16 \\
17 \\
16 \\
14\end{array}$ \\
\hline $\begin{array}{l}\text { Mean } \\
\text { Max } \\
\text { Min }\end{array}$ & $\begin{array}{r}13 \\
22 \\
5\end{array}$ & $\begin{array}{r}3 \\
9 \\
-5\end{array}$ & $\begin{array}{r}8 \\
14 \\
2\end{array}$ & $\begin{array}{l}24 \\
33 \\
16\end{array}$ & $\begin{array}{r}6 \\
16 \\
-2\end{array}$ & $\begin{array}{r}15 \\
23 \\
9\end{array}$ & $\begin{array}{l}24 \\
33 \\
14\end{array}$ & $\begin{array}{r}9 \\
19 \\
2\end{array}$ & $\begin{array}{l}16 \\
24 \\
10\end{array}$ & $\begin{array}{l}23 \\
30 \\
15\end{array}$ & $\begin{array}{r}9 \\
16 \\
-1\end{array}$ & $\begin{array}{l}16 \\
22 \\
10\end{array}$ \\
\hline
\end{tabular}


Table 9.--Daily air-temperature data in the Bald Mountain watersheds--Continued

Air temperature, in degrees centigrade

Day September $1983 \quad$ October 1983 November 1983 December 1983

Max Min Mean Max Min Mean Max Min Mean Max Min Mean

\begin{tabular}{|c|c|c|c|c|c|c|c|c|c|c|c|c|}
\hline $\begin{array}{l}1 \\
2 \\
3 \\
4 \\
5\end{array}$ & $\begin{array}{l}19 \\
26 \\
24 \\
30 \\
29\end{array}$ & $\begin{array}{r}7 \\
7 \\
10 \\
10 \\
14\end{array}$ & $\begin{array}{l}13 \\
16 \\
17 \\
20 \\
22\end{array}$ & $\begin{array}{r}26 \\
24 \\
24 \\
18 \\
7\end{array}$ & $\begin{array}{l}6 \\
8 \\
7 \\
4 \\
3\end{array}$ & $\begin{array}{r}16 \\
16 \\
16 \\
11 \\
5\end{array}$ & $\begin{array}{r}13 \\
14 \\
6 \\
1 \\
0\end{array}$ & $\begin{array}{r}-6 \\
-7 \\
1 \\
-3 \\
-4\end{array}$ & $\begin{array}{r}4 \\
4 \\
4 \\
-1 \\
-2\end{array}$ & $\begin{array}{l}-1 \\
-3 \\
-2 \\
-7 \\
-3\end{array}$ & $\begin{array}{l}-10 \\
-11 \\
-18 \\
-22 \\
-10\end{array}$ & $\begin{array}{r}-6 \\
-7 \\
-10 \\
-14 \\
-6\end{array}$ \\
\hline $\begin{array}{r}6 \\
7 \\
8 \\
9 \\
10\end{array}$ & $\begin{array}{l}30 \\
25 \\
18 \\
21 \\
23\end{array}$ & $\begin{array}{r}13 \\
12 \\
7 \\
5 \\
9\end{array}$ & $\begin{array}{l}22 \\
18 \\
12 \\
13 \\
16\end{array}$ & $\begin{array}{r}11 \\
11 \\
9 \\
9 \\
11\end{array}$ & $\begin{array}{r}1 \\
0 \\
0 \\
-4 \\
-6\end{array}$ & $\begin{array}{l}6 \\
6 \\
4 \\
2 \\
2\end{array}$ & $\begin{array}{r}2 \\
11 \\
13 \\
15 \\
2\end{array}$ & $\begin{array}{r}0 \\
1 \\
-1 \\
-5 \\
-5\end{array}$ & $\begin{array}{r}1 \\
6 \\
6 \\
5 \\
-2\end{array}$ & $\begin{array}{r}0 \\
6 \\
-7 \\
-3 \\
2\end{array}$ & $\begin{array}{r}-7 \\
-10 \\
-16 \\
-18 \\
-18\end{array}$ & $\begin{array}{r}-4 \\
-2 \\
-12 \\
-10 \\
-8\end{array}$ \\
\hline $\begin{array}{l}11 \\
12 \\
13 \\
14 \\
15\end{array}$ & $\begin{array}{l}25 \\
21 \\
16 \\
16 \\
16\end{array}$ & $\begin{array}{r}6 \\
3 \\
-1 \\
-4 \\
-3\end{array}$ & $\begin{array}{r}16 \\
12 \\
8 \\
6 \\
6\end{array}$ & $\begin{array}{r}14 \\
16 \\
17 \\
18 \\
8\end{array}$ & $\begin{array}{r}-6 \\
-3 \\
13 \\
6 \\
3\end{array}$ & $\begin{array}{r}4 \\
6 \\
15 \\
12 \\
6\end{array}$ & $\begin{array}{r}2 \\
2 \\
0 \\
-2 \\
2\end{array}$ & $\begin{array}{r}-1 \\
-3 \\
-10 \\
-9 \\
-12\end{array}$ & $\begin{array}{r}0 \\
0 \\
-5 \\
-6 \\
-5\end{array}$ & $\begin{array}{r}-13 \\
-3 \\
0 \\
0 \\
4\end{array}$ & $\begin{array}{r}-24 \\
-21 \\
-6 \\
-5 \\
-2\end{array}$ & $\begin{array}{r}-18 \\
-12 \\
-3 \\
-2 \\
1\end{array}$ \\
\hline $\begin{array}{l}16 \\
17 \\
18 \\
19 \\
20\end{array}$ & $\begin{array}{l}15 \\
12 \\
15 \\
21 \\
24\end{array}$ & $\begin{array}{r}-4 \\
3 \\
8 \\
7 \\
6\end{array}$ & $\begin{array}{r}6 \\
8 \\
12 \\
14 \\
15\end{array}$ & $\begin{array}{r}9 \\
10 \\
12 \\
6 \\
3\end{array}$ & $\begin{array}{l}-2 \\
-1 \\
-2 \\
-7 \\
-9\end{array}$ & $\begin{array}{r}4 \\
4 \\
5 \\
0 \\
-3\end{array}$ & $\begin{array}{r}1 \\
2 \\
-9 \\
-2 \\
0\end{array}$ & $\begin{array}{l}-9 \\
-1 \\
-1 \\
-9 \\
-7\end{array}$ & $\begin{array}{r}-4 \\
0 \\
-5 \\
-6 \\
-4\end{array}$ & $\begin{array}{r}2 \\
-1 \\
-10 \\
-13 \\
-18\end{array}$ & $\begin{array}{r}-6 \\
-13 \\
-18 \\
-25 \\
-28\end{array}$ & $\begin{array}{r}-2 \\
-7 \\
-14 \\
-19 \\
-23\end{array}$ \\
\hline $\begin{array}{l}21 \\
22 \\
23 \\
24 \\
25\end{array}$ & $\begin{array}{l}29 \\
19 \\
15 \\
15 \\
18\end{array}$ & $\begin{array}{r}11 \\
2 \\
0 \\
-2 \\
-2\end{array}$ & $\begin{array}{r}20 \\
10 \\
8 \\
6 \\
8\end{array}$ & $\begin{array}{r}5 \\
9 \\
11 \\
10 \\
6\end{array}$ & $\begin{array}{r}-11 \\
-10 \\
-9 \\
-3 \\
-10\end{array}$ & $\begin{array}{r}-3 \\
0 \\
1 \\
4 \\
-2\end{array}$ & $\begin{array}{l}0 \\
1 \\
2 \\
1 \\
1\end{array}$ & $\begin{array}{r}-3 \\
-1 \\
-1 \\
-1 \\
0\end{array}$ & $\begin{array}{r}-2 \\
0 \\
0 \\
0 \\
0\end{array}$ & $\begin{array}{r}-15 \\
-3 \\
-3 \\
-9 \\
-17\end{array}$ & $\begin{array}{l}-26 \\
-24 \\
-16 \\
-20 \\
-19\end{array}$ & $\begin{array}{l}-20 \\
-14 \\
-10 \\
-14 \\
-23\end{array}$ \\
\hline $\begin{array}{l}26 \\
27 \\
28 \\
29 \\
30 \\
31\end{array}$ & $\begin{array}{r}15 \\
17 \\
15 \\
21 \\
21 \\
-\end{array}$ & $\begin{array}{r}-1 \\
3 \\
-3 \\
-4 \\
5 \\
---\end{array}$ & $\begin{array}{r}7 \\
10 \\
6 \\
8 \\
13 \\
-.-\end{array}$ & $\begin{array}{l}2 \\
7 \\
7 \\
7 \\
0 \\
9\end{array}$ & $\begin{array}{r}-12 \\
-7 \\
-4 \\
-3 \\
-4 \\
-5\end{array}$ & $\begin{array}{r}-5 \\
0 \\
2 \\
2 \\
-2 \\
2\end{array}$ & $\begin{array}{r}0 \\
-2 \\
-2 \\
-1 \\
1 \\
-\end{array}$ & $\begin{array}{r}-4 \\
-4 \\
-10 \\
-7 \\
-3 \\
-.-\end{array}$ & $\begin{array}{l}-2 \\
-3 \\
-6 \\
-4 \\
-1 \\
-.-\end{array}$ & $\begin{array}{r}-9 \\
-5 \\
0 \\
-1 \\
-14 \\
-9\end{array}$ & $\begin{array}{l}-27 \\
-16 \\
-20 \\
-16 \\
-24 \\
-17\end{array}$ & $\begin{array}{r}-18 \\
-10 \\
-10 \\
-8 \\
-19 \\
-13\end{array}$ \\
\hline $\begin{array}{l}\text { Mean } \\
\text { Max } \\
\text { Min }\end{array}$ & $\begin{array}{l}20 \\
30 \\
12\end{array}$ & $\begin{array}{r}4 \\
14 \\
-4\end{array}$ & $\begin{array}{r}12 \\
22 \\
6\end{array}$ & $\begin{array}{r}11 \\
26 \\
0\end{array}$ & $\begin{array}{r}-2 \\
13 \\
-12\end{array}$ & $\begin{array}{r}4 \\
16 \\
-5\end{array}$ & $\begin{array}{r}2 \\
15 \\
-9\end{array}$ & $\begin{array}{r}-4 \\
1 \\
-12\end{array}$ & $\begin{array}{r}-1 \\
6 \\
-6\end{array}$ & $\begin{array}{r}-5 \\
6 \\
-18\end{array}$ & $\begin{array}{r}-17 \\
-2 \\
-28\end{array}$ & $\begin{array}{r}-11 \\
1 \\
-23\end{array}$ \\
\hline
\end{tabular}


Table 9.--Daily air-temperature data in the Bald Mountain watersheds--Continued

Air temperature, in degrees centigrade

\begin{tabular}{|c|c|c|c|c|c|c|c|c|c|c|c|c|}
\hline \multirow[t]{2}{*}{ Day } & \multicolumn{3}{|c|}{ January 1984} & \multicolumn{3}{|c|}{ February 1984} & \multicolumn{3}{|c|}{ March 1984} & \multicolumn{3}{|c|}{ April 1984} \\
\hline & $\operatorname{Max}$ & Min & Mean & Max & Min & Mean & $\operatorname{Max}$ & Min & Mean & $\operatorname{Max}$ & Min & Mean \\
\hline $\begin{array}{l}1 \\
2 \\
3 \\
4 \\
5\end{array}$ & $\begin{array}{r}-12 \\
-11 \\
-8 \\
-3 \\
-2\end{array}$ & $\begin{array}{r}-27 \\
-28 \\
-24 \\
-13 \\
-6\end{array}$ & $\begin{array}{r}-20 \\
-20 \\
-16 \\
-8 \\
-4\end{array}$ & $\begin{array}{r}-12 \\
-8 \\
2 \\
2 \\
-1\end{array}$ & $\begin{array}{r}-28 \\
-28 \\
-30 \\
-6 \\
-13\end{array}$ & $\begin{array}{r}-20 \\
-18 \\
-14 \\
-2 \\
-7\end{array}$ & $\begin{array}{r}-12 \\
-14 \\
-14 \\
-11 \\
-5\end{array}$ & $\begin{array}{l}-19 \\
-25 \\
-27 \\
-29 \\
-23\end{array}$ & $\begin{array}{l}-16 \\
-20 \\
-20 \\
-20 \\
-14\end{array}$ & $\begin{array}{l}6 \\
4 \\
4 \\
6 \\
1\end{array}$ & $\begin{array}{r}-18 \\
-15 \\
-16 \\
-9 \\
-9\end{array}$ & $\begin{array}{l}-6 \\
-6 \\
-6 \\
-2 \\
-4\end{array}$ \\
\hline $\begin{array}{r}6 \\
7 \\
8 \\
9 \\
10\end{array}$ & $\begin{array}{r}-1 \\
-1 \\
-17 \\
-14 \\
-14\end{array}$ & $\begin{array}{r}-6 \\
-18 \\
-21 \\
-25 \\
-19\end{array}$ & $\begin{array}{r}-4 \\
-10 \\
-19 \\
-20 \\
-16\end{array}$ & $\begin{array}{r}-6 \\
-12 \\
-17 \\
-19 \\
-16\end{array}$ & $\begin{array}{l}-12 \\
-21 \\
-26 \\
-28 \\
-28\end{array}$ & $\begin{array}{r}-9 \\
-16 \\
-22 \\
-24 \\
-22\end{array}$ & $\begin{array}{r}4 \\
-3 \\
-12 \\
-11 \\
-9\end{array}$ & $\begin{array}{r}-9 \\
-20 \\
-24 \\
-30 \\
-25\end{array}$ & $\begin{array}{r}-2 \\
-12 \\
-18 \\
-20 \\
-17\end{array}$ & $\begin{array}{r}6 \\
4 \\
-5 \\
-2 \\
-1\end{array}$ & $\begin{array}{r}-1 \\
-5 \\
-10 \\
-10 \\
-9\end{array}$ & $\begin{array}{r}2 \\
0 \\
-8 \\
-6 \\
-5\end{array}$ \\
\hline $\begin{array}{l}11 \\
12 \\
13 \\
14 \\
15\end{array}$ & $\begin{array}{l}-13 \\
-20 \\
-13 \\
-15 \\
-14\end{array}$ & $\begin{array}{l}-28 \\
-31 \\
-28 \\
-28 \\
-28\end{array}$ & $\begin{array}{l}-20 \\
-26 \\
-20 \\
-22 \\
-21\end{array}$ & $\begin{array}{l}2 \\
4 \\
5 \\
1 \\
5\end{array}$ & $\begin{array}{r}-22 \\
-3 \\
-9 \\
-9 \\
-4\end{array}$ & $\begin{array}{r}-10 \\
0 \\
-2 \\
-4 \\
0\end{array}$ & $\begin{array}{r}-4 \\
-13 \\
-12 \\
-12 \\
-2\end{array}$ & $\begin{array}{l}-25 \\
-24 \\
-29 \\
-17 \\
-12\end{array}$ & $\begin{array}{r}-14 \\
-18 \\
-20 \\
-14 \\
-7\end{array}$ & $\begin{array}{l}6 \\
4 \\
7 \\
9 \\
9\end{array}$ & $\begin{array}{r}-1 \\
-3 \\
-4 \\
-7 \\
-10\end{array}$ & $\begin{array}{l}2 \\
0 \\
2 \\
1 \\
0\end{array}$ \\
\hline $\begin{array}{l}16 \\
17 \\
18 \\
19 \\
20\end{array}$ & $\begin{array}{r}-10 \\
-13 \\
-9 \\
-9 \\
-15\end{array}$ & $\begin{array}{l}-29 \\
-28 \\
-25 \\
-18 \\
-28\end{array}$ & $\begin{array}{l}-20 \\
-20 \\
-17 \\
-14 \\
-22\end{array}$ & $\begin{array}{l}2 \\
4 \\
3 \\
3 \\
0\end{array}$ & $\begin{array}{r}-16 \\
-20 \\
-15 \\
-9 \\
-3\end{array}$ & $\begin{array}{l}-7 \\
-8 \\
-6 \\
-3 \\
-2\end{array}$ & $\begin{array}{r}4 \\
-1 \\
-2 \\
0 \\
1\end{array}$ & $\begin{array}{r}-7 \\
-11 \\
-15 \\
-8 \\
-4\end{array}$ & $\begin{array}{r}-2 \\
-6 \\
-8 \\
-4 \\
-2\end{array}$ & $\begin{array}{r}3 \\
3 \\
3 \\
10 \\
12\end{array}$ & $\begin{array}{l}-2 \\
-1 \\
-1 \\
-2 \\
-4\end{array}$ & $\begin{array}{l}0 \\
1 \\
1 \\
4 \\
4\end{array}$ \\
\hline $\begin{array}{l}21 \\
22 \\
23 \\
24 \\
25\end{array}$ & $\begin{array}{r}-19 \\
-16 \\
-10 \\
-1 \\
0\end{array}$ & $\begin{array}{r}-29 \\
-29 \\
-31 \\
-30 \\
-7\end{array}$ & $\begin{array}{r}-24 \\
-22 \\
-20 \\
-16 \\
-4\end{array}$ & $\begin{array}{r}-3 \\
-6 \\
6 \\
1 \\
6\end{array}$ & $\begin{array}{r}-9 \\
-11 \\
-10 \\
-13 \\
-6\end{array}$ & $\begin{array}{r}-6 \\
-8 \\
-2 \\
-6 \\
0\end{array}$ & $\begin{array}{r}2 \\
6 \\
2 \\
-2 \\
-4\end{array}$ & $\begin{array}{r}-2 \\
-1 \\
-5 \\
-15 \\
-21\end{array}$ & $\begin{array}{r}0 \\
2 \\
-2 \\
-8 \\
-12\end{array}$ & $\begin{array}{r}5 \\
7 \\
20 \\
19 \\
6\end{array}$ & $\begin{array}{l}-4 \\
-7 \\
-2 \\
-1 \\
-1\end{array}$ & $\begin{array}{l}0 \\
0 \\
9 \\
9 \\
2 .\end{array}$ \\
\hline $\begin{array}{l}26 \\
27 \\
28 \\
29 \\
30 \\
31\end{array}$ & $\begin{array}{r}-7 \\
-8 \\
-14 \\
-13 \\
-7 \\
-7\end{array}$ & $\begin{array}{l}-27 \\
-30 \\
-35 \\
-28 \\
-30 \\
-17\end{array}$ & $\begin{array}{l}-17 \\
-19 \\
-24 \\
-20 \\
-18 \\
-12\end{array}$ & $\begin{array}{r}1 \\
-6 \\
-7 \\
0 \\
-. . \\
. .\end{array}$ & $\begin{array}{l}-10 \\
-21 \\
-25 \\
-12 \\
\ldots . \\
\ldots .\end{array}$ & $\begin{array}{r}-4 \\
-14 \\
-16 \\
-6 \\
-.- \\
-.-\end{array}$ & $\begin{array}{r}-1 \\
-3 \\
1 \\
0 \\
0 \\
2\end{array}$ & $\begin{array}{r}-18 \\
-16 \\
-20 \\
-8 \\
-5 \\
-14\end{array}$ & $\begin{array}{r}-10 \\
-10 \\
-10 \\
-4 \\
-2 \\
-6\end{array}$ & $\begin{array}{r}13 \\
17 \\
20 \\
20 \\
22 \\
--\end{array}$ & $\begin{array}{r}-1 \\
-2 \\
-1 \\
-3 \\
10 \\
--\end{array}$ & $\begin{array}{r}6 \\
8 \\
10 \\
8 \\
16 \\
-\end{array}$ \\
\hline $\begin{array}{l}\text { Mean } \\
\text { Max } \\
\text { Min }\end{array}$ & $\begin{array}{r}-10 \\
0 \\
-19\end{array}$ & $\begin{array}{r}-24 \\
-6 \\
-35\end{array}$ & $\begin{array}{r}-17 \\
-4 \\
-26\end{array}$ & $\begin{array}{r}-2 \\
6 \\
-19\end{array}$ & $\begin{array}{r}-15 \\
-3 \\
-30\end{array}$ & $\begin{array}{r}-9 \\
0 \\
-24\end{array}$ & $\begin{array}{r}-4 \\
6 \\
-14\end{array}$ & $\begin{array}{r}-16 \\
-1 \\
-30\end{array}$ & $\begin{array}{r}-10 \\
2 \\
-20\end{array}$ & $\begin{array}{r}8 \\
22 \\
-5\end{array}$ & $\begin{array}{r}5 \\
10 \\
-18\end{array}$ & $\begin{array}{r}1 \\
16 \\
-8\end{array}$ \\
\hline
\end{tabular}


Table 9.--Dally air-temperature data in the Bald Mountain watersheds--Continued

Afr temperature, In degrees centigrade

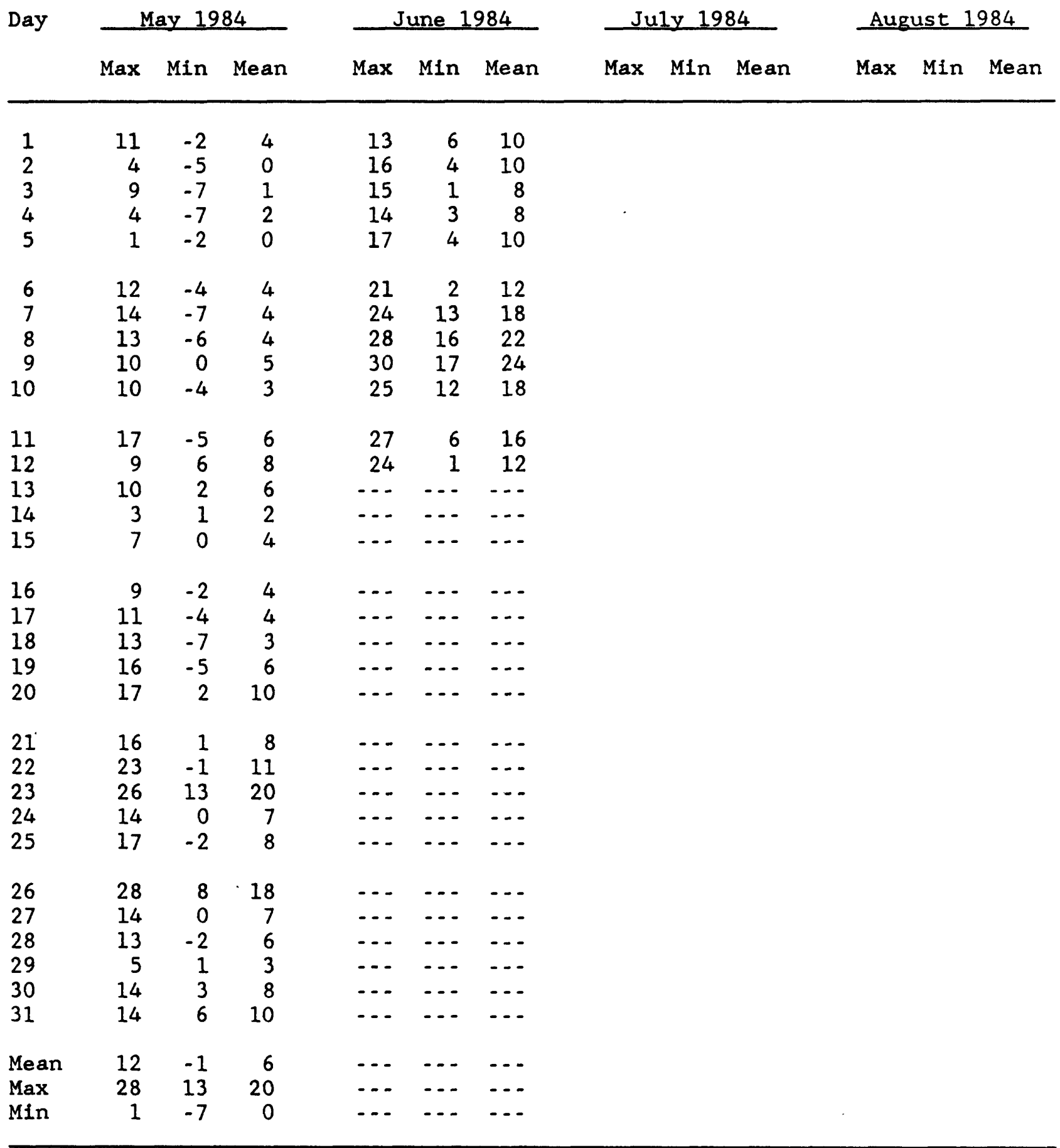


Table 10.-- Daily global-radiation data in the Bald Mountain watersheds

\begin{tabular}{|c|c|c|c|c|c|c|c|c|c|c|c|c|}
\hline \multirow[b]{2}{*}{ Day } & \multicolumn{11}{|c|}{$\begin{array}{l}\text { Global radiation, in langleys } \\
\text { (gram-calories per square centimeter) }\end{array}$} & \multirow[b]{2}{*}{ DEC } \\
\hline & JAN & FEB & MAR & APR & MAY & JUN & JUL & AUG & SEP & OCT & NOV & \\
\hline $\begin{array}{l}1 \\
2 \\
3 \\
4 \\
5\end{array}$ & $\begin{array}{l}\ldots \\
\ldots \\
\cdots \\
\cdots \\
\cdots\end{array}$ & $\begin{array}{r}26 \\
60 \\
17 \\
60 \\
179\end{array}$ & $\begin{array}{r}341 \\
193 \\
369 \\
344 \\
68\end{array}$ & $\begin{array}{l}162 \\
537 \\
315 \\
235 \\
273\end{array}$ & $\begin{array}{l}324 \\
400 \\
443 \\
434 \\
562\end{array}$ & $\begin{array}{r}451 \\
94 \\
690 \\
673 \\
656\end{array}$ & $\begin{array}{l}443 \\
417 \\
511 \\
664 \\
588\end{array}$ & $\begin{array}{l}485 \\
571 \\
613 \\
502 \\
136\end{array}$ & $\begin{array}{r}349 \\
77 \\
400 \\
349 \\
494\end{array}$ & $\begin{array}{r}68 \\
170 \\
375 \\
383 \\
383\end{array}$ & $\begin{array}{r}170 \\
43 \\
51 \\
77 \\
26\end{array}$ & $\begin{array}{r}26 \\
51 \\
17 \\
145 \\
136\end{array}$ \\
\hline $\begin{array}{r}6 \\
7 \\
8 \\
9 \\
10\end{array}$ & $\begin{array}{l}\cdots \\
\cdots \\
\cdots \\
\cdots \\
\cdots\end{array}$ & $\begin{array}{l}60 \\
51 \\
85 \\
77 \\
43\end{array}$ & $\begin{array}{r}358 \\
68 \\
34 \\
43 \\
51\end{array}$ & $\begin{array}{l}494 \\
204 \\
315 \\
349 \\
400\end{array}$ & $\begin{array}{l}477 \\
485 \\
605 \\
179 \\
128\end{array}$ & $\begin{array}{l}698 \\
681 \\
579 \\
366 \\
605\end{array}$ & $\begin{array}{l}375 \\
605 \\
732 \\
417 \\
554\end{array}$ & $\begin{array}{l}477 \\
204 \\
460 \\
102 \\
502\end{array}$ & $\begin{array}{r}60 \\
494 \\
409 \\
221 \\
375\end{array}$ & $\begin{array}{r}281 \\
290 \\
51 \\
170 \\
383\end{array}$ & $\begin{array}{r}128 \\
111 \\
26 \\
77 \\
153\end{array}$ & $\begin{array}{r}77 \\
85 \\
94 \\
111 \\
60\end{array}$ \\
\hline $\begin{array}{l}11 \\
12 \\
13 \\
14 \\
15\end{array}$ & $\begin{array}{c}\cdots \\
\cdots \\
\cdots \\
43 \\
85\end{array}$ & $\begin{array}{r}60 \\
77 \\
102 \\
94 \\
85\end{array}$ & $\begin{array}{r}60 \\
128 \\
119 \\
273 \\
468\end{array}$ & $\begin{array}{l}477 \\
519 \\
281 \\
273 \\
528\end{array}$ & $\begin{array}{l}145 \\
383 \\
375 \\
468 \\
468\end{array}$ & $\begin{array}{l}647 \\
460 \\
596 \\
145 \\
477\end{array}$ & $\begin{array}{l}375 \\
383 \\
298 \\
579 \\
519\end{array}$ & $\begin{array}{l}247 \\
324 \\
145 \\
273 \\
554\end{array}$ & $\begin{array}{r}247 \\
426 \\
307 \\
383 \\
68\end{array}$ & $\begin{array}{r}383 \\
315 \\
60 \\
60 \\
128\end{array}$ & $\begin{array}{r}128 \\
51 \\
17 \\
196 \\
26\end{array}$ & $\begin{array}{r}68 \\
68 \\
111 \\
85 \\
26\end{array}$ \\
\hline $\begin{array}{l}16 \\
17 \\
18 \\
19 \\
20\end{array}$ & $\begin{array}{l}196 \\
128 \\
230 \\
230 \\
162\end{array}$ & $\begin{array}{l}290 \\
332 \\
341 \\
119 \\
281\end{array}$ & $\begin{array}{l}494 \\
460 \\
443 \\
477 \\
349\end{array}$ & $\begin{array}{l}290 \\
400 \\
187 \\
622 \\
221\end{array}$ & $\begin{array}{l}579 \\
613 \\
690 \\
443 \\
281\end{array}$ & $\begin{array}{l}230 \\
537 \\
511 \\
605 \\
358\end{array}$ & $\begin{array}{l}579 \\
519 \\
605 \\
605 \\
315\end{array}$ & $\begin{array}{l}571 \\
315 \\
545 \\
392 \\
443\end{array}$ & $\begin{array}{r}43 \\
111 \\
136 \\
375 \\
392\end{array}$ & $\begin{array}{l}145 \\
196 \\
213 \\
298 \\
264\end{array}$ & $\begin{array}{l}179 . \\
153 \\
145 \\
136 \\
153\end{array}$ & $\begin{array}{r}17 \\
128 \\
128 \\
119 \\
34\end{array}$ \\
\hline $\begin{array}{l}21 \\
22 \\
23 \\
24 \\
25\end{array}$ & $\begin{array}{r}187 \\
238 \\
68 \\
105 \\
192\end{array}$ & $\begin{array}{r}102 \\
94 \\
162 \\
290 \\
290\end{array}$ & $\begin{array}{l}494 \\
279 \\
312 \\
502 \\
434\end{array}$ & $\begin{array}{l}170 \\
307 \\
358 \\
647 \\
605\end{array}$ & $\begin{array}{l}698 \\
690 \\
690 \\
579 \\
732\end{array}$ & $\begin{array}{l}409 \\
579 \\
119 \\
426 \\
562\end{array}$ & $\begin{array}{l}571 \\
119 \\
417 \\
639 \\
145\end{array}$ & $\begin{array}{l}511 \\
502 \\
145 \\
273 \\
264\end{array}$ & $\begin{array}{r}298 \\
153 \\
85 \\
434 \\
204\end{array}$ & $\begin{array}{l}102 \\
247 \\
196 \\
315 \\
273\end{array}$ & $\begin{array}{r}111 \\
77 \\
26 \\
34 \\
77\end{array}$ & $\begin{array}{r}68 \\
94 \\
128 \\
43 \\
17\end{array}$ \\
\hline $\begin{array}{l}26 \\
27 \\
28 \\
29 \\
30 \\
31\end{array}$ & $\begin{array}{r}198 \\
208 \\
119 \\
136 \\
60 \\
111\end{array}$ & $\begin{array}{l}426 \\
409 \\
332 \\
\ldots \ldots \\
\ldots \\
\ldots\end{array}$ & $\begin{array}{l}225 \\
281 \\
392 \\
230 \\
502 \\
187\end{array}$ & $\begin{array}{l}349 \\
187 \\
477 \\
656 \\
196 \\
-\ldots\end{array}$ & $\begin{array}{l}690 \\
622 \\
656 \\
698 \\
673 \\
622\end{array}$ & $\begin{array}{l}511 \\
630 \\
375 \\
128 \\
537 \\
\ldots-\end{array}$ & $\begin{array}{l}417 \\
579 \\
238 \\
187 \\
656 \\
434\end{array}$ & $\begin{array}{l}349 \\
443 \\
528 \\
451 \\
426 \\
417\end{array}$ & $\begin{array}{l}332 \\
102 \\
153 \\
417 \\
298 \\
\ldots-\end{array}$ & $\begin{array}{l}298 \\
298 \\
264 \\
128 \\
204 \\
213\end{array}$ & $\begin{array}{r}34 \\
51 \\
9 \\
34 \\
77 \\
\ldots-\end{array}$ & $\begin{array}{r}34 \\
170 \\
34 \\
51 \\
187 \\
119\end{array}$ \\
\hline $\begin{array}{l}\text { Mean } \\
\text { Max } \\
\text { Min }\end{array}$ & $\begin{array}{ll}-\cdots \\
\cdots \\
\cdots\end{array}$ & $\begin{array}{r}162 \\
426 \\
17\end{array}$ & $\begin{array}{r}290 \\
502 \\
34\end{array}$ & $\begin{array}{l}368 \\
656 \\
162\end{array}$ & $\begin{array}{l}511 \\
732 \\
128\end{array}$ & $\begin{array}{r}478 \\
698 \\
94\end{array}$ & $\begin{array}{l}467 \\
732 \\
119\end{array}$ & $\begin{array}{l}393 \\
613 \\
102\end{array}$ & $\begin{array}{r}273 \\
494 \\
43\end{array}$ & $\begin{array}{r}231 \\
383 \\
51\end{array}$ & $\begin{array}{r}86 \\
196 \\
9\end{array}$ & $\begin{array}{r}82 \\
187 \\
17\end{array}$ \\
\hline
\end{tabular}


Table 10.-- Daily global-radiation data in the Bald Mountain watersheds-Continued

\begin{tabular}{|c|c|c|c|c|c|c|c|c|c|c|c|c|}
\hline \multirow[b]{2}{*}{ Day } & \multicolumn{10}{|c|}{$\begin{array}{l}\text { Global radiation, in langleys } \\
\text { (gram-calories per square centimeter) }\end{array}$} & \multirow[b]{2}{*}{ NOV } & \multirow[b]{2}{*}{$\mathrm{DEC}$} \\
\hline & JAN & FEB & MAR & APR & MAY & JUN & JUL & AUG & SEP & OCT & & \\
\hline $\begin{array}{l}1 \\
2 \\
3 \\
4 \\
5\end{array}$ & $\begin{array}{r}60 \\
145 \\
196 \\
170 \\
111\end{array}$ & $\begin{array}{r}34 \\
156 \\
34 \\
51 \\
281\end{array}$ & $\begin{array}{r}179 \\
43 \\
136 \\
409 \\
409\end{array}$ & $\begin{array}{l}333 \\
268 \\
205 \\
111 \\
187\end{array}$ & $\begin{array}{l}307 \\
102 \\
349 \\
119 \\
349\end{array}$ & $\begin{array}{l}400 \\
400 \\
630 \\
307 \\
579\end{array}$ & $\begin{array}{l}366 \\
204 \\
528 \\
656 \\
400\end{array}$ & $\begin{array}{l}375 \\
511 \\
230 \\
529 \\
383\end{array}$ & $\begin{array}{l}392 \\
383 \\
392 \\
460 \\
392\end{array}$ & $\begin{array}{r}358 \\
324 \\
324 \\
85 \\
43\end{array}$ & $\begin{array}{r}204 \\
153 \\
34 \\
34 \\
43\end{array}$ & $\begin{array}{l}70 \\
43 \\
60 \\
86 \\
43\end{array}$ \\
\hline $\begin{array}{r}6 \\
7 \\
8 \\
9 \\
10\end{array}$ & $\begin{array}{r}94 \\
95 \\
148 \\
94 \\
26\end{array}$ & $\begin{array}{r}281 \\
77 \\
111 \\
230 \\
111\end{array}$ & $\begin{array}{l}247 \\
349 \\
162 \\
213 \\
187\end{array}$ & $\begin{array}{l}453 \\
428 \\
136 \\
562 \\
434\end{array}$ & $\begin{array}{r}443 \\
255 \\
443 \\
60 \\
170\end{array}$ & $\begin{array}{l}264 \\
409 \\
562 \\
681 \\
639\end{array}$ & $\begin{array}{l}290 \\
502 \\
324 \\
443 \\
375\end{array}$ & $\begin{array}{l}588 \\
613 \\
230 \\
392 \\
639\end{array}$ & $\begin{array}{l}358 \\
264 \\
460 \\
204 \\
400\end{array}$ & $\begin{array}{r}153 \\
281 \\
85 \\
162 \\
332\end{array}$ & $\begin{array}{r}60 \\
128 \\
196 \\
68 \\
111\end{array}$ & $\begin{array}{r}43 \\
103 \\
85 \\
95 \\
46\end{array}$ \\
\hline $\begin{array}{l}11 \\
12 \\
13 \\
14 \\
15\end{array}$ & $\begin{array}{r}102 \\
94 \\
153 \\
187 \\
51\end{array}$ & $\begin{array}{l}179 \\
298 \\
204 \\
255 \\
349\end{array}$ & $\begin{array}{r}102 \\
26 \\
213 \\
85 \\
153\end{array}$ & $\begin{array}{r}60 \\
145 \\
579 \\
460 \\
417\end{array}$ & $\begin{array}{l}213 \\
196 \\
153 \\
434 \\
218\end{array}$ & $\begin{array}{l}630 \\
571 \\
417 \\
460 \\
494\end{array}$ & $\begin{array}{l}537 \\
664 \\
196 \\
622 \\
307\end{array}$ & $\begin{array}{l}519 \\
511 \\
528 \\
596 \\
477\end{array}$ & $\begin{array}{l}238 \\
383 \\
502 \\
494 \\
494\end{array}$ & $\begin{array}{r}324 \\
238 \\
111 \\
68 \\
196\end{array}$ & $\begin{array}{r}34 \\
17 \\
128 \\
128 \\
187\end{array}$ & $\begin{array}{r}140 \\
60 \\
34 \\
25 \\
26\end{array}$ \\
\hline $\begin{array}{l}16 \\
17 \\
18 \\
19 \\
20\end{array}$ & $\begin{array}{r}26 \\
137 \\
115 \\
121 \\
135\end{array}$ & $\begin{array}{r}269 \\
92 \\
217 \\
290 \\
417\end{array}$ & $\begin{array}{r}255 \\
315 \\
341 \\
94 \\
136\end{array}$ & $\begin{array}{l}426 \\
111 \\
162 \\
426 \\
255\end{array}$ & $\begin{array}{l}443 \\
614 \\
664 \\
622 \\
111\end{array}$ & $\begin{array}{l}562 \\
477 \\
502 \\
596 \\
656\end{array}$ & $\begin{array}{l}554 \\
554 \\
647 \\
511 \\
213\end{array}$ & $\begin{array}{l}545 \\
255 \\
290 \\
571 \\
383\end{array}$ & $\begin{array}{l}358 \\
111 \\
179 \\
196 \\
264\end{array}$ & $\begin{array}{l}307 \\
111 \\
119 \\
273 \\
264\end{array}$ & $\begin{array}{l}17 \\
43 \\
26 \\
60 \\
34\end{array}$ & $\begin{array}{r}26 \\
131 \\
130 \\
93 \\
132\end{array}$ \\
\hline $\begin{array}{l}21 \\
22 \\
23 \\
24 \\
25\end{array}$ & $\begin{array}{r}171 \\
111 \\
128 \\
102 \\
68\end{array}$ & $\begin{array}{r}102 \\
213 \\
43 \\
204 \\
290\end{array}$ & $\begin{array}{r}366 \\
85 \\
255 \\
102 \\
451\end{array}$ & $\begin{array}{l}230 \\
238 \\
298 \\
238 \\
111\end{array}$ & $\begin{array}{r}290 \\
639 \\
136 \\
51 \\
179\end{array}$ & $\begin{array}{l}605 \\
477 \\
579 \\
664 \\
571\end{array}$ & $\begin{array}{l}187 \\
230 \\
400 \\
375 \\
571\end{array}$ & $\begin{array}{l}426 \\
153 \\
485 \\
545 \\
528\end{array}$ & $\begin{array}{l}298 \\
281 \\
324 \\
307 \\
434\end{array}$ & $\begin{array}{l}281 \\
255 \\
255 \\
238 \\
281\end{array}$ & $\begin{array}{r}17 \\
17 \\
26 \\
102 \\
51\end{array}$ & $\begin{array}{r}94 \\
85 \\
120 \\
114 \\
102\end{array}$ \\
\hline $\begin{array}{l}26 \\
27 \\
28 \\
29 \\
30 \\
31\end{array}$ & $\begin{array}{r}196 \\
60 \\
187 \\
128 \\
153 \\
43\end{array}$ & $\begin{array}{l}375 \\
136 \\
102 \\
-\ldots \\
-\ldots \\
-\ldots\end{array}$ & $\begin{array}{l}444 \\
438 \\
102 \\
143 \\
273 \\
462\end{array}$ & $\begin{array}{l}213 \\
170 \\
383 \\
460 \\
204 \\
\cdots\end{array}$ & $\begin{array}{l}230 \\
136 \\
204 \\
511 \\
102 \\
153\end{array}$ & $\begin{array}{l}630 \\
102 \\
690 \\
681 \\
485 \\
-\end{array}$ & $\begin{array}{r}494 \\
485 \\
400 \\
94 \\
460 \\
341\end{array}$ & $\begin{array}{r}341 \\
392 \\
366 \\
145 \\
128 \\
85\end{array}$ & $\begin{array}{r}196 \\
247 \\
426 \\
460 \\
68 \\
-\end{array}$ & $\begin{array}{r}136 \\
204 \\
68 \\
94 \\
111 \\
221\end{array}$ & $\begin{array}{r}102 \\
87 \\
150 \\
40 \\
106 \\
\ldots\end{array}$ & $\begin{array}{r}82 \\
88 \\
77 \\
128 \\
138 \\
138\end{array}$ \\
\hline $\begin{array}{l}\text { Mean } \\
\text { Max } \\
\text { Min }\end{array}$ & $\begin{array}{r}116 \\
196 \\
26\end{array}$ & $\begin{array}{r}193 \\
417 \\
34\end{array}$ & $\begin{array}{r}231 \\
462 \\
26\end{array}$ & $\begin{array}{r}290 \\
579 \\
60\end{array}$ & $\begin{array}{r}287 \\
664 \\
51\end{array}$ & $\begin{array}{l}524 \\
690 \\
102\end{array}$ & $\begin{array}{r}417 \\
664 \\
94\end{array}$ & $\begin{array}{r}412 \\
639 \\
85\end{array}$ & $\begin{array}{r}332 \\
502 \\
68\end{array}$ & $\begin{array}{r}203 \\
358 \\
43\end{array}$ & $\begin{array}{r}80 \\
204 \\
17\end{array}$ & $\begin{array}{r}85 \\
140 \\
25\end{array}$ \\
\hline
\end{tabular}


Table 10. - Daily global-radiation data in the Bald Mountain watersheds-Continued

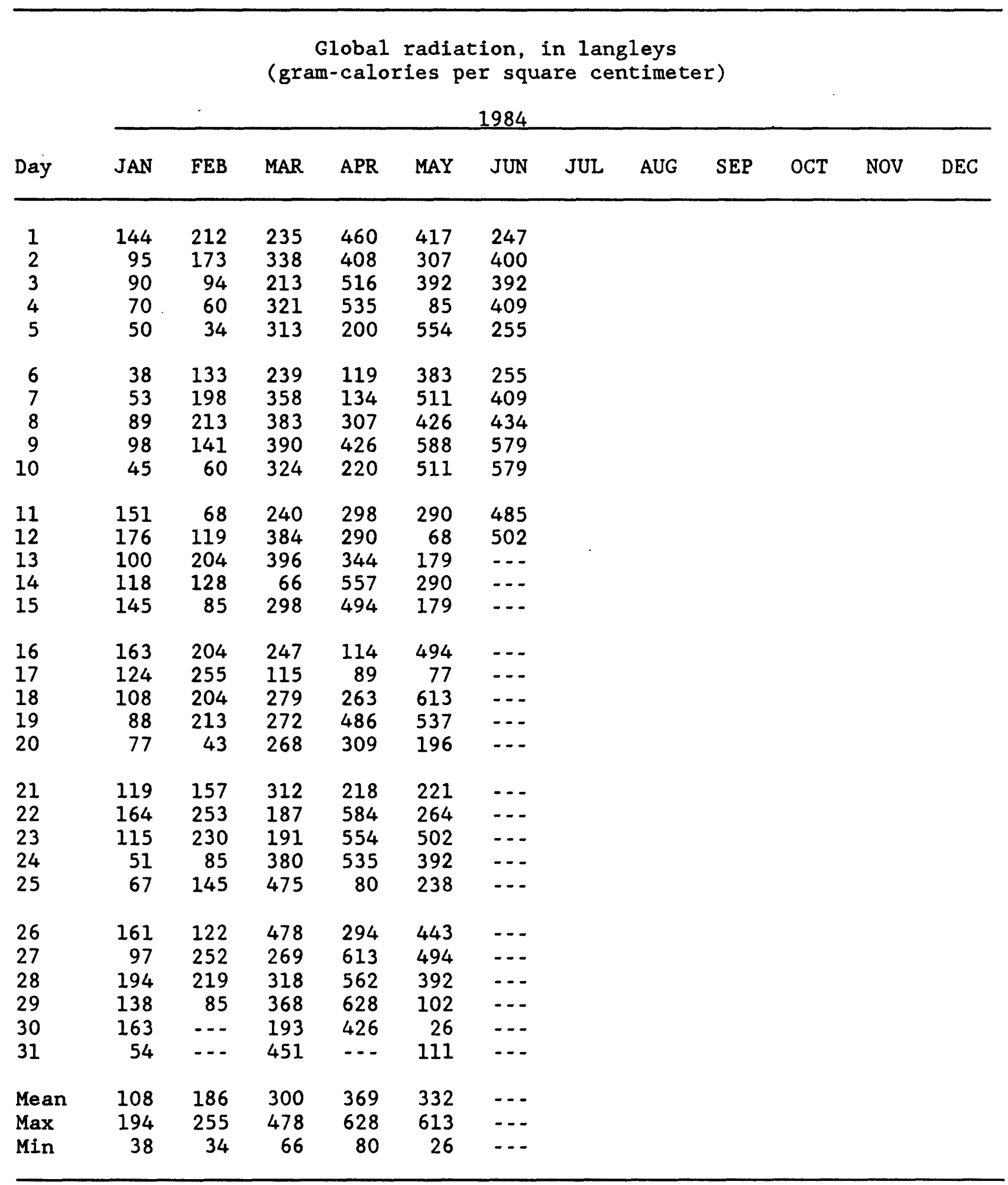


Table 11.--Snow-survey data in the Bald Mountain watersheds

\begin{tabular}{|c|c|c|c|c|c|c|}
\hline \multirow[b]{2}{*}{ Date } & \multicolumn{2}{|c|}{ Site 1} & \multicolumn{2}{|c|}{ site 2} & \multicolumn{2}{|c|}{ Site 3} \\
\hline & $\begin{array}{l}\text { snow } \\
\text { depth } \\
\text { (inches) }\end{array}$ & $\begin{array}{l}\text { water } \\
\text { equivalent } \\
\text { (inches) }\end{array}$ & $\begin{array}{l}\text { snow } \\
\text { depth } \\
\text { (inches) }\end{array}$ & $\begin{array}{l}\text { water } \\
\text { equivalent } \\
\text { (inches) }\end{array}$ & $\begin{array}{l}\text { snow } \\
\text { depth } \\
\text { (inches) }\end{array}$ & $\begin{array}{l}\text { water } \\
\text { equivalent } \\
\text { (inches) }\end{array}$ \\
\hline $\begin{array}{l}12-12-80 \\
12-22-80 \\
01-06-81 \\
02-06-81 \\
02-16-81 \\
03-25-81 \\
12-29-81 \\
02-04-82 \\
03-04-82 \\
03-17-82 \\
04-22-82 \\
01-29-83 \\
03-05-83 \\
12-27-83 \\
12-28-83 \\
02-03-84 \\
02-28-84 \\
03-29-84 \\
04-12-84\end{array}$ & $\begin{array}{l}\ldots . . \\
13.0 \\
19.6 \\
16.5 \\
11.4 \\
12.3 \\
17.2 \\
37.2 \\
\ldots . . \\
33.0 \\
18.4 \\
-\ldots \\
17.2 \\
26.2 \\
\ldots \ldots \\
\ldots \ldots \\
\ldots \ldots \\
\ldots \ldots \\
\ldots . .\end{array}$ & $\begin{array}{l}2 . . \\
2.1 \\
3.6 \\
4.2 \\
3.4 \\
4.5 \\
3.0 \\
6.9 \\
\ldots .- \\
7.9 \\
5.2 \\
-\ldots \\
3.4 \\
5.2 \\
\ldots . \\
\ldots . \\
\ldots . \\
\ldots . \\
\ldots\end{array}$ & $\begin{array}{r}16.7 \\
20.5 \\
19.4 \\
21.6 \\
17.8 \\
6.4 \\
-. .- \\
30.8 \\
40.1 \\
41.6 \\
14.6 \\
8.9 \\
20.2 \\
28.5 \\
-2 .- \\
37.7 \\
32.0 \\
41.4 \\
34.4\end{array}$ & $\begin{array}{r}3.4 \\
3.4 \\
3.5 \\
4.8 \\
5.1 \\
2.0 \\
-. . \\
9.6 \\
8.4 \\
12.7 \\
5.2 \\
1.0 \\
3.9 \\
4.6 \\
-.- \\
6.0 \\
9.6 \\
13.6 \\
9.8\end{array}$ & $\begin{array}{r}3.8 \\
9.3 \\
9.8 \\
10.8 \\
-. .- \\
6.4 \\
22.2 \\
35.6 \\
33.6 \\
35.8 \\
11.6 \\
6.7 \\
9.8 \\
-2 .- \\
16.0 \\
28.8 \\
24.2 \\
-2 .- \\
25.3\end{array}$ & $\begin{array}{r}0.4 \\
1.9 \\
2.4 \\
3.0 \\
-. . \\
2.0 \\
3.0 \\
10.6 \\
9.1 \\
9.9 \\
4.0 \\
1.7 \\
2.2 \\
-. . \\
4.4 \\
7.6 \\
7.2 \\
-. . \\
9.7\end{array}$ \\
\hline & \multicolumn{2}{|c|}{ Site 4} & \multicolumn{2}{|c|}{ Site 5} & \multicolumn{2}{|c|}{ Site 6} \\
\hline $\begin{array}{l}12-12-80 \\
12-22-80 \\
01-06-81 \\
02-06-81 \\
02-16-81 \\
03-25-81 \\
12-29-81 \\
02-04-82 \\
03-04-82 \\
03-17-82 \\
03-18-82 \\
04-22-82 \\
01-29-83 \\
03-05-83 \\
12-27-83 \\
12-28-83 \\
02-02-84 \\
02-03-84 \\
02-28-84 \\
03-29-84 \\
04-12-84\end{array}$ & $\begin{array}{r}9.5 \\
14.1 \\
19.6 \\
18.0 \\
9.8 \\
9.9 \\
25.0 \\
38.0 \\
40.2 \\
38.1 \\
-. .- \\
5.6 \\
8.8 \\
19.2 \\
22.0 \\
-\ldots . . \\
-\ldots \\
36.8 \\
30.6 \\
37.1 \\
29.7\end{array}$ & $\begin{array}{r}1.7 \\
2.7 \\
3.9 \\
4.8 \\
3.1 \\
3.6 \\
6.6 \\
10.6 \\
5.2 \\
11.1 \\
-. . \\
1.6 \\
0.9 \\
3.3 \\
5.2 \\
-. . \\
-. . \\
7.1 \\
8.7 \\
10.4 \\
8.0\end{array}$ & $\begin{array}{r}9.6 \\
12.8 \\
14.0 \\
10.4 \\
-. . \\
6.6 \\
18.4 \\
32.8 \\
31.1 \\
-. . \\
33.0 \\
-. . \\
6.8 \\
18.0 \\
-\ldots \\
17.0 \\
31.1 \\
-\ldots \\
23.9 \\
31.8 \\
26.7\end{array}$ & $\begin{array}{r}2.1 \\
2.4 \\
3.4 \\
3.7 \\
-. . \\
2.0 \\
3.6 \\
9.2 \\
8.0 \\
-. . \\
12.0 \\
--. \\
1.1 \\
3.9 \\
-. . \\
3.2 \\
8.6 \\
-. . \\
6.2 \\
11.0 \\
7.4\end{array}$ & 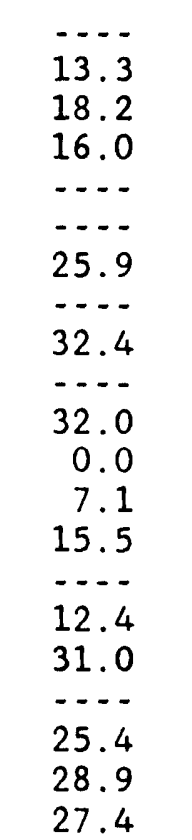 & $\begin{array}{l}-. . \\
2.4 \\
4.8 \\
4.5 \\
-. . \\
-. . \\
5.3 \\
-.- \\
8.2 \\
-. . \\
8.7 \\
0.0 \\
0.9 \\
4.1 \\
-.- \\
2.4 \\
6.2 \\
-. . \\
8.7 \\
9.6 \\
9.4\end{array}$ \\
\hline
\end{tabular}


Table 11.--Snow-survey data in the Bald Mountain watersheds--Continued

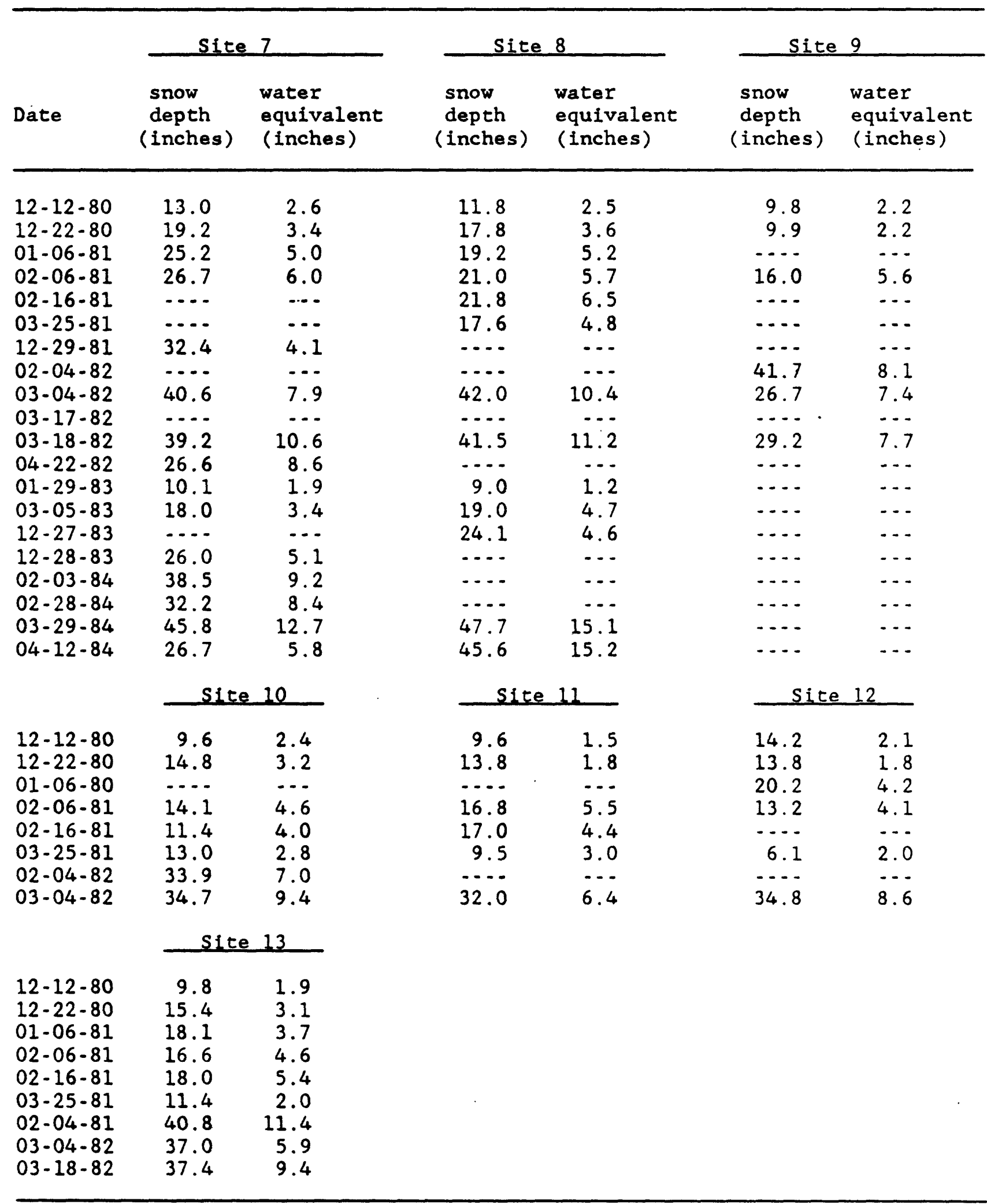

